\title{
Optimisation and Scale-up of the Synthesis of Gold Nanoparticle-Wool Fibre Composites
}

\author{
by \\ Thomas Wade Nilsson
}
Victoria
UNIVERSITY OF WELLINGTON
Te Whare Wānanga
o te Üpoko o te Ika a Māui

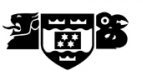

\author{
A thesis \\ submitted to Victoria University of Wellington \\ in fulfilment of the requirements for the degree of \\ Doctor of Philosophy \\ in Chemistry
}

Victoria University of Wellington

2015 


\section{Abstract}

Gold nanoparticles are known for their remarkable optical properties; they exhibit localised surface plasmon resonance bands in the visible region of the electromagnetic spectrum. This has led to their use as luxury dyes for the colouring of wool fibres. Gold is associated with wealth and desire, and as such, gold nanoparticlewool fibre composites may be fabricated into high-quality garments, apparel, textiles and carpets for international markets.

Novel proprietary approaches for the laboratory-scale synthesis of gold nanoparticlewool fibre composites have previously been developed by Professor James Johnston and Dr Kerstin Lucas. The innovative nanotechnology utilises the affinity of gold for sulfur-containing cystine residues in wool fibres, to attract and bind the gold nanoparticles. One approach involves the absorption of gold ions by wool fibres and the nucleation of gold nanoparticles in-situ. In an alternative method, gold nanoparticle colloids are synthesised ex-situ, and are then used to colour wool fibres.

The reaction conditions of the in-situ and ex-situ approaches were optimised with respect to cost-effectiveness and scalability. The gold content of the in-situ composites was minimised, and the range of possible colours widened, via the use of heat and external reducing agents. In the ex-situ process, the formation and stability of the gold nanoparticle colloids was studied, and the reaction conditions of the synthesis were optimised. The rate of uptake of gold nanoparticles to wool was controlled by manipulating the $\mathrm{pH}$, concentration, volume, and wool to liquor ratio of the gold colloids, and by introducing auxiliary agents into the dyeing reactions. A range of chemical treatments and alternative stabilising agents were investigated to improve the washfastness properties of ex-situ gold nanoparticle-wool fibre composites.

There are numerous size-controllable syntheses of gold nanoparticle colloids at the laboratory-scale. However, when the process is scaled-up, gold nanoparticle synthesis is no longer trivial. A barrel reactor with a high velocity mixer was utilised to achieve uniform mixing and heating in the synthesis of gold nanoparticle colloids of 
up to $90 \mathrm{~L}$ in volume. The ratios of gold to stabilising agents in the colloidal gold syntheses were optimised to result in more stable and reproducible gold colloids for subsequent dyeing reactions.

The uniform colouring of small quantities of wool is easily achieved in the laboratory, but preventing colour variation across a kilogram of wool is a significant challenge. Initial kilogram-scale dyeing reactions in static tank reactors resulted in unevenly coloured gold nanoparticle-wool fibre composites. To overcome this, conventional hank dyeing equipment was used to colour felted merino yarn, in collaboration with the wool dyeing industry. Modified hank dyeing procedures were recreated in the laboratory, and composites with remarkable colour uniformity were produced. Industrial package dyeing reactors were then used to colour fine merino yarn with gold nanoparticle colloids. The uptake of gold nanoparticles was controlled by manipulating the flowrates, flow direction and amounts of auxiliary agents that were employed in the dyeing reactions.

Based upon the success of the industrial dyeing reactions, novel dyeing reactors were developed for the colouring of hanks of wool fibres and yarns in the laboratory. These reactors utilised rapid dye circulation and pressure to produce gold nanoparticlewool fibre composites with remarkable colour uniformity. The composites were used to fabricate luxury apparel and carpets for international trade expositions.

The pathway from synthesis in the laboratory to pilot-scale production of gold nanoparticle-wool fibre composites is presented. The PhD research was an integral step in the successful commercialisation of this innovative nanotechnology, and will assist in scaling-up the synthesis of metal nanoparticle colloids and nanocomposites in the future. 


\section{Acknowledgements}

First and foremost, the supervision of Professor James Johnston is gratefully acknowledged. Jim not only provided excellent direction on the chemistry side of the research, but also shared knowledge and expertise of the commercial aspects of the project. His industrial linkages allowed extensive collaborations. This is all greatly appreciated. Dr Gerald Smith is also acknowledged for his co-supervision.

The guidance of Dr Kerstin Lucas and Dr Mathew Cairns has been invaluable throughout my $\mathrm{PhD}$ program. Many thanks are due to the other members of The JJ research group, particularly Dr Maria Parry, Dr Matthias Herzog, Eldon Tate, Michelle Cook and Ahmet Kitap, who became good friends and worthy travel companions. The expertise of David Flynn in electron microscopy is appreciated. Thanks to Jackie King and Teresa Gen for lending laboratory equipment and huge volumes of distilled water.

My family; Wade, Viv, Alex and Olivia are owed a huge debt for financial and emotional support for the entirety of my university studies. Tyrel, Caroline and Max helped to keep scurvy at bay. Huge thanks also go to Sam, Elliot, Kieran, Oreo and Jaimee, non-scientists who dutifully sat through confusing practice seminars. Thanks to the members of the CMFC scotch and squash clubs who provided welcome distractions. Finally, heartfelt thanks to the lovely Kate for helping me reach the finish line (and teaching me decent English in the process!).

Funding was provided by the MacDiarmid Institute of Advanced Materials and Nanotechnology PhD Scholarship and Travel Funds, the Victoria University Science Faculty Strategic Research Grant and the Curtis Gordon Research Scholarship. These agencies are all gratefully acknowledged. 


\section{Table of Contents}

Abstract $\quad$ ii

Acknowledgements

Table of Contents $\quad$ v

List of Figures viii

List of Tables $\quad$ xxiii

Glossary xxviii

1 Introduction $\quad 1$

1.1 Wool Fibres . . . . . . . . . . . . . . . . . . . 2

1.1.1 The Chemical and Morphological Structure of Wool Fibres . . 3

1.1.2 Wool Fibre Chemistry . . . . . . . . . . . . . . 8

1.1.3 Wool Processing. . . . . . . . . . . . . . . 14

1.1.4 Wool Dyeing . . . . . . . . . . . . . . . 17

1.2 Gold Nanoparticles . . . . . . . . . . . . . . . . . . . . . . 23

1.2 .1 Synthesis Methods . . . . . . . . . . . . . . . 23

1.2.2 Optical Properties of Gold Nanoparticles . . . . . . . . . . . . 27

1.3 Gold Nanoparticle-Wool Fibre Composites . . . . . . . . . . . . . . . 32

1.3.1 In-situ Procedure . . . . . . . . . . . . . . . . . . . . . 32

1.3.2 Ex-situ Procedure . . . . . . . . . . . . . . . . . . 37

1.3.3 Seed-mediated Approach . . . . . . . . . . . . . . . . 40

1.4 Research Overview . . . . . . . . . . . . . . . . . . . . . . . 42

2 Experimental $\quad 44$

2.1 Materials . . . . . . . . . . . . . . . . . . . . . 44

2.1.1 Wool Fibres . . . . . . . . . . . . . . . . . . 44

2.1 .2 Gold . . . . . . . . . . . . . . . . . . . . 46

2.1.3 Other Reagents . . . . . . . . . . . . . . . 46

2.2 Preparatory Methods . . . . . . . . . . . . . . . . . . . . . . 47

2.2.1 In-situ procedure . . . . . . . . . . . . . . . . . . . . . 47 
$2.2 .2 \quad$ Ex-situ Procedure . . . . . . . . . . . . . . . . . 48

2.2 .3 Wool Fibre Pre-Treatment . . . . . . . . . . . . . 51

2.2 .4 Scale-up Procedures . . . . . . . . . . . . . . . 53

2.2 .5 Washfastness Testing . . . . . . . . . . . . 56

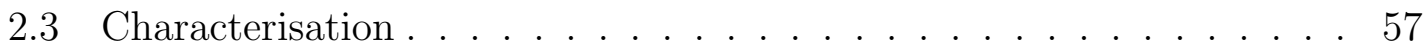

2.3.1 Atomic Absorption Spectroscopy _. . . . . . . . . . . 57

2.3.2 Ultraviolet-visible Absorption Spectroscopy . . . . . . . . . . 58

2.3 .3 CIE Colour Coordinates . . . . . . . . . . . . . . 59

2.3.4 Scanning Electron Microscopy . . . . . . . . . . . . . . 59

2.3.5 Energy Dispersive X-ray Spectroscopy _... . . . . . . . 60

2.3.6 Transmission Electron Microscopy . . . . . . . . . . . . . 60

3 In-situ Synthesis of Gold Nanoparticle-wool Fibre Composites $\quad 62$

3.1 In-situ Composites of Cariaggi Wool . . . . . . . . . . . . . . 63

3.1.1 Uptake Process . . . . . . . . . . . . . . . . . . . . . . 64

3.1 .2 Gold Nanoparticle Formation . . . . . . . . . . . . . 65

3.1.3 Discussion of the Importance of These Results . . . . . . . . . 68

3.2 Characterisation of the Reaction Parameters of the In-situ Procedure 70

3.2 .1 Wool Type . . . . . . . . . . . . . . . 71

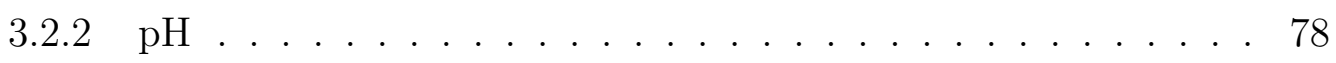

3.2 .3 Temperature . . . . . . . . . . . . . . . . 84

3.2 .4 Auxiliary Agents . . . . . . . . . . . . . . . . . 88

3.2 .5 Volume of Gold Solution . . . . . . . . . . . . . . 97

3.3 Manipulating the Rate of Gold Nanoparticle Formation . . . . . . . 102

3.3.1 External Energy Sources . . . . . . . . . . . . . . . . . 102

3.3.2 External Reducing and Stabilising Agents _.. . . . . . . 108

3.4 Conclusions . . . . . . . . . . . . . . . . . . . 117

4 Ex-situ Synthesis of Gold Nanoparticle-wool Fibre Composites $\mathbf{1 1 8}$

4.1 Ex-situ Composites of Cariaggi Wool . . . . . . . . . . . . . . . . . 119

4.1.1 Gold Nanoparticle Synthesis . . . . . . . . . . . . . . . . . . . 119

4.1.2 Dyeing Cariaggi Wool with Gold Colloids . . . . . . . . . . . . 123

4.2 Gold Nanoparticle Synthesis . . . . . . . . . . . . . . . . . . . . 127

4.2 .1 Formation Studies . . . . . . . . . . . . . . . . . . . 128

4.2.2 pH Stability of Gold Colloids . . . . . . . . . . . . . . . 133

4.2.3 Ratio of Gold to Stabilising Agents . . . . . . . . . . . . . . . 141

4.3 Dyeing Wool with Gold Nanoparticles . . . . . . . . . . . . . . . . . . 144

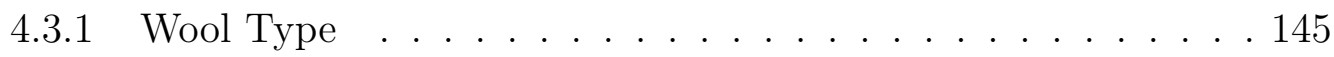

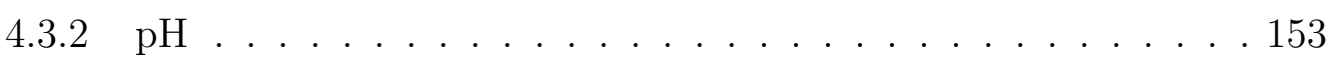

4.3.3 Auxiliary Agents . . . . . . . . . . . . . . . . 156

4.3.4 Concentration and Volume of Dye Solution . . . . . . . . . . 171 
4.3.5 Drying of Composites . . . . . . . . . . . . 176

4.4 Conclusions . . . . . . . . . . . . . . . . . . . 178

5 Scale-up of Ex-situ Gold Nanoparticle-wool Fibre Composites $\quad \mathbf{1 8 0}$

5.1 Scale-up of Gold Nanoparticle Synthesis . . . . . . . . . . . . . . . . 181

5.1 .1 Materials for Gold Nanoparticle Syntheses . . . . . . . . . . . 181

5.1.2 Scale-up Gold Nanoparticle Syntheses . . . . . . . . . . . . . . 184

5.2 Scale-up Hank Dyeing Procedures . . . . . . . . . . . . . . . . . . . . 190

5.2.1 Hank Dyeing of Top Form Wool . . . . . . . . . . . . . . . . . 191

5.2.2 Hank Dyeing of Felted Merino Yarn . . . . . . . . . . . . . . . 196

5.3 Package Dyeing of Merino Yarn . . . . . . . . . . . . . . . . . 208

5.3.1 Package Dyed TA-mauve TU Yarn Composites . . . . . . . . . 210

5.3.2 TSC-grey TU Yarn Composites . . . . . . . . . . . . . . . . 218

5.4 Novel Hybrid Flow Reactors for Wool Dyeing in the Laboratory . . . 220

5.4.1 Benchtop Flow Reactor . . . . . . . . . . . . . . . . . 221

5.4 .2 First Flow Reactor . . . . . . . . . . . . . . . . . . . . 232

5.4 .3 Pressurised Flow Reactors . . . . . . . . . . . . . . . . 240

5.5 Conclusions . . . . . . . . . . . . . . . . . . . . . 258

6 Improving the Washfastness Properties of Ex-situ Composites $\mathbf{2 6 1}$

6.1 Washfastness of Ex-situ Composites . . . . . . . . . . . . . . . . 262

6.2 Chemical Treatments . . . . . . . . . . . . . . . . . 268

6.2.1 Chemical Pre-treatments . . . . . . . . . . . . . 268

6.2.2 Chemical Post-treatments . . . . . . . . . . . . . . 274

6.3 Alternative Reducing and Stabilising Agents . . . . . . . . . . . . . 276

6.3.1 Sulfur-containing Stabilisers . . . . . . . . . . . . . . 277

6.3.2 Polyethylenimine . . . . . . . . . . . . . . . 280

6.3 .3 Reactive Dyes . . . . . . . . . . . . . . . . . . 289

6.4 Conclusions . . . . . . . . . . . . . . . . . . 300

$\begin{array}{lll}7 & \text { Conclusions } & 301\end{array}$

$\begin{array}{ll}\text { References } & 305\end{array}$ 


\section{List of Figures}

1.1 New Zealand wool and sheepmeat export values from 1953-2011. . . . 1

1.2 The helical structure of $\alpha$-keratins. . . . . . . . . . . . . . . . . 3

1.3 Crosslinking between peptide chains in wool . . . . . . . . . . 5

1.4 The physical structure of wool . . . . . . . . . . . . . 6

1.5 The organisation of the ortho- and para-cortex of merino fibres . . . . 6

1.6 SEM micrograph of overlapping cuticle cells on a crossbred wool fibre 7

1.7 18-methyleicosanioc acid (18-MEA) . . . . . . . . . 8

1.8 The structure of cystine. . . . . . . . . . . . . . . . . . . . . 8

1.9 The structure of tryptophan and tyrosine . . . . . . . . . . . . . . . 12

1.10 The structure of lanthionine and lysinoalanine . . . . . . . . . . . . . 13

1.11 Loose wool, top form wool and woollen yarn. . . . . . . . . . . . . . 15

1.12 The proposed removal of 18-MEA from wool fibres . . . . . . . . . 16

1.13 Antishrink polyamide Hercosett 125 . . . . . . . . . . . . . 17

1.14 The absorption pathway of dyes into wool fibres . . . . . . . . . . 18

1.15 Acid, reactive and $1: 1$ metal complex dyes . . . . . . . . . . . . 20

1.16 The uptake of dyes and counter ions by wool fibres . . . . . . . . . 20

1.17 A hank of felted woollen yarn (a) and a cone of fine merino yarn (b). 21

1.18 Industrial-scale hank (a) and package (b) dyeing reactors. . . . . . . . 22

1.19 Gold nanoparticles stabilised by thioalkanes . . . . . . . . . . . . . 24

1.20 Redox coupling in the TSC reduction of $\mathrm{Au}^{3+}$ to $\mathrm{Au}^{0} \ldots$. . . . . . . 24

1.21 The different colours of gold nanoparticles that are stabilised by Tannic acid $(\mathrm{TA})$ and TSC. . . . . . . . . . . . . . . . 25

1.22 TEM micrograph of gold nanorods that were synthesised in the presence of CTAB . . . . . . . . . . . . . . . . 2 26

1.23 Proposed growth mechanism for the growth of gold nanorods . . . . . 26

1.24 The Lycurgus cup . . . . . . . . . . . . . . . . . . . . . 27

1.25 Michael Faraday's glass slide with a ruby-red gold colloid . . . . . . . 28

1.26 Representation of the surface plasmons of bulk metals and metal nanoparticles. . . . . . . . . . . . . . . . . . . . . . . 29

1.27 The change in SPR with increasing gold nanoparticle size . . . . . . . 30

1.28 A representation of the dipole oscillation of a spherical metal nanoparticle and the transverse and longitudinal oscillations of a metal nanorod. 31 
1.29 In-situ produced gold nanoparticle-wool fibre composites . . . . . . . 33

1.30 The Pourbaix diagram of gold. . . . . . . . . . . . . . . . . . 34

1.31 The uptake of $\mathrm{AuCl}_{4}^{-}$to merino wool fibres at different $\mathrm{pH}$ levels . . . 35

1.32 An SEM micrograph in backscatter mode (a) and an Au EDS map (b) of the surface of a merino in-situ composite. . . . . . . . . . . . 35

1.33 EDS elemental maps of the cross-section of an in-situ composite of merino wool $(1.6 \mathrm{wt} \% \mathrm{Au})$ for sulfur and gold. . . . . . . . . . . 36

1.34 The binding of gold nanoparticles to sulfur groups in wool fibres . . . 37

1.35 The structure of Tannic acid (TA) . . . . . . . . . . . . . . . 38

1.36 A range of colloids that were synthesised with different concentration ratios of Au to either TSC or TA, and the ex-situ composites that were produced with these gold colloids. . . . . . . . . . . . . . . 39

1.37 An SEM micrograph in backscatter mode of the cross-section of an ex-situ composite, and an EDS elemental Au map of the same area . 39

1.38 Composites that were synthesised with the seed-mediated approach. . 41

2.1 The stock solution of $\mathrm{AuCl}_{4}^{-}$(4 wt \%) that was prepared from metallic gold for the synthesis of gold nanoparticle-wool fibre composites. . . . 46

2.2 The Benchtop flow reactor that was used for studying the uptake of TA-Au NPs to TU yarn. . . . . . . . . . . . . . . . . . 55

2.3 The "dunking machine" that was used to dunk composite samples in and out of a solution of Kindness wool wash. . . . . . . . . . . . . . . 57

2.4 CIE colour coordinates . . . . . . . . . . . . . . . . . . . . 59

3.1 The change in the world gold spot price in USD per troy ounce from $1990-2014 \ldots \ldots \ldots \ldots \ldots$

3.2 Uptake of $\mathrm{Au}^{3+}$ to Cariaggi wool synthesised with the standard purple in-situ method conditions. . . . . . . . . . . . . . . . . . . . . . . . 65

3.3 Plot of $\ln \left(\right.$ residual $\mathrm{Au}^{3+}$ conc) over time during the uptake of $\mathrm{AuCl}_{4}^{-}$ to Cariaggi wool synthesised with the standard purple in-situ method, to illustrate approximate 1 st order kinetics. . . . . . . . . . . . . . . 66

3.4 Image of a Cariaggi in-situ composite synthesised via the standard purple method (a), SEM micrographs of the surface of a composite fibre in secondary electron imaging (SEI) mode (b), in backscatter (BS) mode at different magnifications (c)-(e) and an EDS elemental Au map (of the area in (e)) (f) . . . . . . . . . . . . . 667

3.5 SEM and EDS analysis of the cross section of a Cariaggi in-situ composite synthesised with the standard purple in-situ method; SEI image (a), BS images of increasing magnification (b)-(d), S elemental map (e), Au elemental map (f) and a line profile of Au intensity across the cut fibre edge. . . . . . . . . . . . . . . . . . . . . . 69 
3.6 Uptake of $\mathrm{Au}^{3+}$ to Cariaggi, Ashford and Crossbred wool at RT. . . . 72

3.7 In-situ composites of Crossbred (a), Ashford (b) and Cariaggi (c) wool after 180 min uptake at RT (photograph taken 2 years after synthesis, allowing time for the gradual formation of gold nanoparticles. . . . . 72

3.8 SEM micrographs in BS mode of Crossbred (a)-(b), Ashford (c)-(d) and Cariaggi (e)-(f) in-situ composites synthesised using standard purple in-situ method. . . . . . . . . . . . . . . . . . . . . . . . . 74

3.9 Image of the composites of TU (a), TS (b), S19 (c) and S14 (d) yarn synthesised with the standard purple in-situ method. . . . . . . . . . 76

3.10 SEM micrographs in BS mode of the surfaces of in-situ composites of TU (a)-(b), TS (c)-(d) and S19 (e)-(f) yarns, that were synthesised with the standard purple in-situ method. . . . . . . . . . . . . . . 77

3.11 Ashford2 in-situ composites synthesised with the standard purple insitu method and a reaction $\mathrm{pH}$ of 2 (a), 3 (b) and 4 (c) . . . . . . . 79

$3.12 \mathrm{UV}$-visible reflectance spectra of in-situ Ashford2 composites synthesised at $\mathrm{pH} \mathrm{2,3}$ and 4, and unreacted Ashford2 wool. . . . . . . . . 80

3.13 SEM micrographs in BS mode of the surface of Ashford2 composites synthesised with the standard purple in-situ method at pH 2 (a), 3 (b) and 4(c) . . . . . . . . . . . . . . . . . . . 81

3.14 SEM and EDS analysis of the cross-section of an Ashford2 in-situ composite fibre synthesised at $\mathrm{pH} 2$; an SEM micrograph in BS mode (a), EDS elemental Au (b) and $\mathrm{Cl}$ (c) maps of the cross-section and an overlay of $\mathrm{Au}$ and $\mathrm{Cl}$ elemental maps (d). . . . . . . . . . . . . 82

3.15 SEM micrographs in BS mode of the cross-sections of in-situ composites synthesised at pH 3 (a) and 4 (c), and Au elemental EDS maps

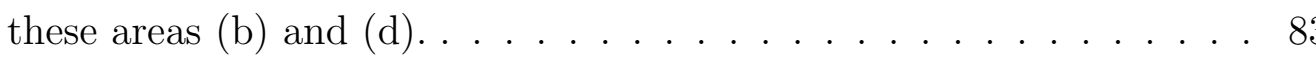

3.16 Uptake of $\mathrm{Au}^{3+}$ to Ashford2 wool with the standard purple in-situ method at different temperatures. . . . . . . . . . . . . . 85

3.17 Ashford2 in-situ composites removed after 120 min or overnight uptake of $160 \mathrm{mg} \mathrm{L}^{-1} \mathrm{Au}^{3+}$ at different temperatures (the photograph was taken soon after the uptake). . . . . . . . . . . . . 85

3.18 EDS Au elemental maps and line profiles of the cross-sections of Ashford2 in-situ composites removed after 10 min uptake at RT (a), 60 min uptake at RT (b), 10 min uptake at $50^{\circ} \mathrm{C}$ (b) and 10 min uptake at $75{ }^{\circ} \mathrm{C}(\mathrm{d}) \ldots \ldots \ldots \ldots \ldots$. . . . . . . . . . . . . 86

3.19 In-situ composites of TU, TS, S19 and S14 yarns synthesised without Albaflow FFA (a)-(d) respectively and with $1 \mathrm{~g} \mathrm{~L}^{-1}$ Albaflow FFA in

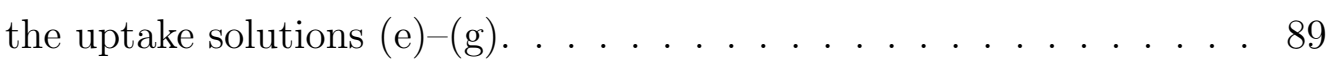

3.20 SEM and EDS analysis of an in-situ composite of TU yarn with $1 \mathrm{~g} \mathrm{~L}^{-1}$ Albaflow FFA. 
3.21 SEM micrographs in BS mode of in-situ composites of TU (a)-(b), TS (c)-(d), S19 (e)-(f) and S14 (g)-(h) yarn synthesised with $1 \mathrm{~g} \mathrm{~L}^{-1}$ Albaflow FFA. The rectangles indicate areas with high amounts of Albaflow FFA. . . . . . . . . . . . . . . . . . . . . . . . . . . . . . . 92

$3.22 \mathrm{AAS}$ uptake of $\mathrm{AuCl}_{4}^{-}$to Cariaggi wool with and without the presence of levelling agents.

3.23 Cariaggi in-situ composites synthesised after pre-soaking in $10 \mathrm{~g} \mathrm{~L}^{-1}$ $\mathrm{Na}_{2} \mathrm{SO}_{4}$ (a), with $1 \mathrm{~g} \mathrm{~L}^{-1} \mathrm{Na}_{2} \mathrm{SO}_{4}$ (b) or $\mathrm{NaCl}$ (c) present in the uptake solution or with no levelling agents $(\mathrm{d}) \ldots \ldots$. . . . . . . . . . 94

3.24 SEM and EDS analysis of a Cariaggi in-situ composite synthesised with $\mathrm{Na}_{2} \mathrm{SO}_{4}$ in the uptake solution; SEM images of the fibre surface in backscatter mode (a) and (b), SEM image of a fibre cross-section (c) and EDS maps of elemental S (d), Na (e) and Au (f) of the crosssection. . . . . . . . . . . . . . . . . . 9

3.25 SEM and EDS analysis of the cross section of a Cariaggi in-situ composite fibre synthesised with $\mathrm{NaCl}$ in the uptake solution; SEM image in backscatter mode (a), EDS elemental maps of $\mathrm{Na}$ (b), $\mathrm{Cl}$ (c) and $\mathrm{Au}(\mathrm{d}) \ldots \ldots \ldots \ldots \ldots$. . . . . . . . . . . . . . . . . . . . .

3.26 In-situ composites of finished wool yarns synthesised using 100, 80, 60, 40, 20 or $10 \mathrm{~mL}$ of $160 \mathrm{mg} \mathrm{L}^{-1} \mathrm{Au}^{3+}$ and $1 \mathrm{~g} \mathrm{~L}^{-1}$ Albaflow FFA (all composite samples are $1 \mathrm{~g}$ and illustrate a wide variation in colour). . 98

$3.27 \mathrm{~L}^{*}$ colour coordinates of in-situ finished wool yarn composites synthesised with different volumes of $160 \mathrm{mg} \mathrm{L}^{-1} \mathrm{Au}^{3+}$ solutions. . . . . . 99

$3.28 \mathrm{a}^{*}$ colour coordinates of in-situ finished wool yarn composites synthesised with different volumes of $160 \mathrm{mg} \mathrm{L}^{-1} \mathrm{Au}^{3+}$ solutions. . . . . . . 101

$3.29 \mathrm{~b}^{*}$ colour coordinates of in-situ finished wool yarn composites synthesised with different volumes of $160 \mathrm{mg} \mathrm{L}^{-1} \mathrm{Au}^{3+}$ solutions. . . . . . 101

3.30 In-situ composites of finished yarns synthesised at $80^{\circ} \mathrm{C}$, with starting solutions of 100, 80, 60, 40 and $20 \mathrm{~mL} 160 \mathrm{mg} \mathrm{L}^{-1} \mathrm{Au}^{3+} \ldots$. . . . . . 103

3.31 Converted UV-visible reflectance spectra of in-situ composites of finished wool yarns synthesised at $80^{\circ} \mathrm{C}$. . . . . . . . . . . . 104

3.32 SEM micrographs in BS mode and EDS elemental Au maps of the surface of in-situ composites of TU (a)-(b), TS (c)-(d) and S19 (e)(f) wool yarns synthesised at $80^{\circ} \mathrm{C}$ with $100 \mathrm{~mL}$ of $160 \mathrm{mg} \mathrm{L}^{-1} \mathrm{Au}^{3+} \ldots 105$

3.33 Ashford2 in-situ composites synthesised after uptake at RT and exposure to microwave radiation without and with $1 \mathrm{~g} \mathrm{~L}^{-1}$ Albaflow FFA in the uptake solution (a) and (b) respectively and after exposure to dry heat in the oven without and with $1 \mathrm{gL}^{-1}$ Albaflow FFA (c) and (d) . . . . . . . . . . . . . . . . . . 107 
3.34 Image of Ashford2 composites synthesised after soaking in $\mathrm{NaBH}_{4}$ solutions after $2 \mathrm{~min}(\mathrm{a}), 5 \mathrm{~min}(\mathrm{~b}), 10 \mathrm{~min}$ (c) and $60 \mathrm{~min}$ (d) of RT uptake of $160 \mathrm{mg} \mathrm{L}^{-1} \mathrm{Au}^{3+}$, and $\mathrm{NaBH}_{4}$ only (e). . . . . . . . . . . 109

3.35 SEM and EDS analysis of an Ashford2 composite synthesised with $\mathrm{NaBH}_{4}$ after $10 \mathrm{~min}$ of uptake of $160 \mathrm{mg} \mathrm{L}^{-1} \mathrm{Au}^{3+}$ at RT: BS micrographs of the fibre surface (a)-(c) and a cross-section (d), and EDS elemental $\mathrm{Au}(\mathrm{e})$ and $\mathrm{Na}$ (f) maps of the cross-section. . . . . . . . . 110

3.36 SEM and EDS analysis of an Ashford2 composite synthesised with $\mathrm{NaBH}_{4}$ after $60 \mathrm{~min}$ of uptake of $160 \mathrm{mg} \mathrm{L}^{-1} \mathrm{Au}^{3+}$ at RT: BS micrographs of the fibre surface (a)-(d) and cross-section (e), and an EDS elemental Au map of the cross-section (f) . . . . . . . . . . . . . . . . 112

3.37 Image of composites synthesised with immersion in a boiling solution of aqueous TSC after no uptake (a), and $5 \mathrm{~min}$ (b), $10 \mathrm{~min}$ (c) or $60 \mathrm{~min}(\mathrm{~d})$ uptake of $160 \mathrm{mg} \mathrm{L}^{-1} \mathrm{Au}^{3+}$ at RT. . . . . . . . . . . . . 113

3.38 SEM micrographs in BS mode of the surface of an Ashford2 composite synthesised with TSC as an external reducing agent after 10 min of $160 \mathrm{mg} \mathrm{L}^{-1} \mathrm{Au}^{3+}$ uptake at RT with increasing magnification from (a) $-(d)$.

3.39 Image of composites synthesised with immersion in an aqueous solution of TA (1 wt \%) after $5 \mathrm{~min}(\mathrm{a}), 10 \mathrm{~min}$ (b) and $60 \mathrm{~min}$ of uptake of $160 \mathrm{mg} \mathrm{L}^{-1} \mathrm{Au}^{3+}$ at RT. . . . . . . . . . . . . . . . . . . . . . . 115

3.40 SEM and EDS analysis of an Ashford2 composite synthesised with TA after 10 min of uptake of $160 \mathrm{mg} \mathrm{L}^{-1} \mathrm{Au}^{3+}$ at RT: SEM micrographs in BS mode at increasing magnification (a)-(c), and an EDS elemental Au map $(\mathrm{d}) \ldots \ldots \ldots \ldots 116$

4.1 TA-mauve and TSC-grey colloids. . . . . . . . . . . . . . . 120

4.2 Normalised UV-visible absorbance spectra of typical colloids of TA$\mathrm{Au}$ NPs and TSC-Au NPs. . . . . . . . . . . . . . . . . . . . . . 120

4.3 TEM micrographs of TA-Au NPs at different magnifications. . . . . . 121

4.4 TEM micrographs of TSC-Au NPs at different magnifications. . . . . 122

4.5 TA-mauve, TSC-pink and TSC-grey ex-situ Cariaggi composites. . . . 123

4.6 SEM micrographs of the surface of a TA-mauve Cariaggi composite in SEI mode (a), in backscatter mode at different magnifications (b) and (c), and a cross-sectional image in backscatter mode (d) . . . . . 125

4.7 SEM micrographs in backscatter mode of two different TSC-pink Cariaggi composite fibres; a relatively common "light fibre" (a) and (b), and an atypically seen "dark fibre" (c) and (d). . . . . . . . . . 126

4.8 SEM micrographs in backscatter mode of the surface of a TSC-grey Cariaggi composite fibre of increasing magnification from (a)-(c), and an Au elemental EDS map of area (b) shown in (d) . . . . . . . . . . 127 
4.9 UV-vis spectra measured during the formation of a TA-mauve colloid (10 $\mathrm{mg} \mathrm{L}^{-1} \mathrm{Au}^{3+}, 300 \mathrm{~mL}$ ), where the spectra are measured after every minute from $0-15 \mathrm{~min}$ and after $60 \mathrm{~min}$ of the formation. . . . . . . . 129

4.10 UV-vis spectra of the formation of the TA-mauve colloid inside the $\mathrm{UV}$-vis cuvette $\left(10 \mathrm{mg} \mathrm{L}^{-1} \mathrm{Au}^{3+}, 3 \mathrm{~mL}\right)$, where each spectrum is measured every minute for the first $30 \mathrm{~min}$ of the formation. . . . . . . . . 129

4.11 UV-vis spectra of the formation of a solution of four times the concentration of the TA-mauve colloid $\left(40 \mathrm{mg} \mathrm{L}^{-1} \mathrm{Au}^{3+}, 300 \mathrm{~mL}\right) \quad$. . . 130

4.12 UV-vis spectra of the formation of the TSC-grey concentration of TSC-Au NPs ( $40 \mathrm{mg} \mathrm{L}{ }^{-1} \mathrm{Au}^{3+}, 3 \mathrm{~mL}$ ) in-situ, where the time of each spectrum is measured after the addition of TSC to a solution of $\mathrm{AuCl}_{4}^{-}$ at $95{ }^{\circ} \mathrm{C}$ in a quartz cuvette. . . . . . . . . . . . . . . . . . . 131

4.13 UV-vis spectra of the formation of the TSC-pink concentration of TSC-Au NPs (10 mg L $\left.{ }^{-1} \mathrm{Au}^{3+}, 3 \mathrm{~mL}\right)$ in-situ, where the time of each spectrum is measured after the addition of TSC to a solution of $\mathrm{AuCl}_{4}^{-}$ at $95{ }^{\circ} \mathrm{C}$ in a quartz cuvette. . . . . . . . . . . . . . . . . . 133

4.14 UV-vis spectra of TA-mauve colloid illustrating the stability upon $\mathrm{pH}$ lowering with $\mathrm{HCl}$ addition, measured 30 min after the $\mathrm{pH}$ adjustment.134

4.15 UV-vis spectra of a TSC-pink colloid when the $\mathrm{pH}$ is lowered from $\mathrm{pH}$ 3.6-2.0 with $\mathrm{HCl}$ addition. The UV-vis spectra are measured $30 \mathrm{~min}$ after the $\mathrm{pH}$ adjustment. . . . . . . . . . . . . . . . . . 135

4.16 The chemical structure of PMA . . . . . . . . . . . . . . . 136

4.17 UV-vis spectra of a TSC-pink colloid synthesised with the addition of PMA in the colloid and the $\mathrm{pH}$ is lowered from 4.6-2.6. The UV-vis spectra are measured $30 \mathrm{~min}$ after the $\mathrm{pH}$ adjustment. . . . . . . . . . 137

4.18 TSC-pink Cariaggi composites synthesised with and without PMA in the colloid. . . . . . . . . . . . . . . . . . . . 137

4.19 SEM micrographs of TSC-pink Cariaggi composites synthesised without PMA that are relatively light (a) and dark (b), and with PMA (c)-(f), where (e) and (f) are higher magnification images of the white square areas in $(\mathrm{d}) . \ldots . . \ldots 139$

4.20 UV-vis spectra of a TSC-grey colloid upon lowering the $\mathrm{pH}$ from 3.01.4 with $\mathrm{HCl}$ addition. The UV-vis spectra were measured 2 min after the $\mathrm{pH}$ adjustment. . . . . . . . . . . . . . . . . 140

4.21 UV-vis spectra of TA-stabilised colloids with different ratios of Au:TA.142

$4.22 \mathrm{UV}$-vis spectra of TSC-grey colloids synthesised with higher amounts of TSC in the reaction solution. . . . . . . . . . . . . . . . . . . . . 144

4.23 Uptake of the TA-mauve colloid to different wool types. . . . . . . . . 146

4.24 TA-mauve composites of different wool types. . . . . . . . . . . . . 147 
4.25 TA-mauve and TSC-grey ex-situ composites synthesised with 15, 27 and $36 \mu \mathrm{m}$ loose wool. . . . . . . . . . . . . . . . . . . . . 149

4.26 SEM micrographs in backscatter mode of the surface of TA-mauve ex-situ composites of 15, 27 and $36 \mu \mathrm{m}$ loose wool, including both a relatively dark and light coloured fibre of the $27 \mu \mathrm{m}$ wool. . . . . . . . 151

4.27 SEM micrographs in backscatter mode of the surface of TSC-grey ex-situ composites of 15, 27 and $36 \mu \mathrm{m}$ loose wool. . . . . . . . . . . 152

4.28 UV-vis spectra of the uptake of TA-Au NPs from in the TA-mauve reaction to Cariaggi wool fibres at a reaction $\mathrm{pH}$ of 2.8 over time. The inset represents the uptake curve from these spectra. . . . . . . . 153

4.29 UV-vis spectra of the uptake of TA-Au NPs to Cariaggi wool from $\mathrm{pH} 2.8-3.6$, where the absorbance of the $\lambda_{\max }=530 \mathrm{~nm}$ decreases as the TA-Au NPs are absorbed by the wool. . . . . . . . . . . . . . 154

$4.30 \mathrm{UV}$-vis spectra of the uptake of TA-Au NPs to $16 \mu \mathrm{m}$ open top wool from $\mathrm{pH} 2.8-3.6$, where the absorbance of the $\lambda_{\max }=530 \mathrm{~nm}$ decreases as the TA-Au NPs are absorbed by the wool. . . . . . . . . . . . . 155

$4.31 \mathrm{UV}$-vis spectra of the uptake of TA-Au NPs to $18 \mu \mathrm{m}$ open top wool from $\mathrm{pH} 2.8-3.6$, where the absorbance of the $\lambda_{\max }=530 \mathrm{~nm}$ decreases as the TA-Au NPs are absorbed by the wool. . . . . . . . . . . . . 156

4.32 Uptake of TA-mauve to different wool types with $1 \mathrm{~g} \mathrm{~L}^{-1}$ of Albaflow FFA in the pH 2.2 buffer and pH 2.2 colloid (normalised so that the original SPR peak $\left(\lambda_{\max }\right.$ of $\left.\left.530 \mathrm{~nm}\right)=0.22 \mathrm{abs}\right)$. . . . . . . . . . 157

4.33 TA-mauve composites of different wool types with and without $1 \mathrm{~g} \mathrm{~L}^{-1}$ Albaflow in the glycine buffer and colloid. . . . . . . . . . . . . 158

4.34 Uptake of the TA-mauve colloid to TU yarn different amounts of Albaflow FFA in the $\mathrm{pH} 2.2$ buffer and $\mathrm{pH} 2.2$ colloid. . . . . . . . . . 159

4.35 TA-mauve composites of TU yarn synthesised with different amounts of Albaflow FFA in the pH 2.2 buffer and colloid. . . . . . . . . . . . 160

4.36 UV-vis spectra of TSC-grey colloids after 2 min of uptake to TU wool with different amounts of Albaflow FFA in the uptake solutions. . . . 161

4.37 The reaction solutions after $10 \mathrm{~min}$ of uptake of the TSC-grey colloid to TU yarn with different amounts of Albaflow FFA in the colloids. . 162

$4.38 \mathrm{UV}$-vis spectra of TSC-grey colloids after 4 days of uptake to TU wool with different amounts of Albaflow FFA in the uptake solutions. . . . 162

4.39 A TSC-grey TU yarn composites synthesised with different amounts of Albaflow FFA in the uptake solution. . . . . . . . . . . . . 163

4.40 SEM micrographs in backscatter mode of TSC-grey composites synthesised with different amounts of Albaflow FFA in the TSC-grey colloid. . . . . . . . . . . . . . . . . . . . . 164 
4.41 UV-vis spectra of the uptake of TSC-grey to TU yarn of TSC-grey colloid after pre-soaking in a pH 2.0 glycine buffer (without Albaflow FFA).

4.42 Uptake of the TSC-grey colloid to TU yarn after pre-soaking in a $\mathrm{pH}$ 2.0 glycine buffer with different amounts of Albaflow FFA in both the buffer and colloid. . . . . . . . . . . . . . . . . 166

4.43 TSC-grey composites of TU yarn synthesised with different amounts of Albaflow FFA in the pH 2.0 buffer and colloid. . . . . . . . . . . . 167

4.44 TSC-grey composites of TU yarn synthesised at an uptake temperature of $80^{\circ} \mathrm{C}$ with different amounts of Albaflow FFA in the $\mathrm{pH} 2.0$ buffer and colloid. . . . . . . . . . . . . . . . . . 168

4.45 The uptake of the TA-mauve colloid to TU yarn, with one quarter of the recommended amount of the levelling agents present in the buffer only. The uptake with no levelling agent is provided for reference. . . 170

4.46 TA-mauve TU yarn composites synthesised with the addition of levelling agents to the uptake solutions, where the uptake of the Albegal B and Lyogen MF solutions was elevated to $80^{\circ} \mathrm{C}$. . . . . . . . . . . 170

4.47 TA-mauve TU yarn composites synthesised with different concentrations of TA-Au NPs and $1 \mathrm{~g} \mathrm{~L}^{-1}$ Albaflow FFA. . . . . . . . . . . . . 172

4.48 SEM and EDS analysis of TA-mauve TU yarn composites synthesised with 1 and $6 \mathrm{x}$ the concentration of TA-Au NPs in the standard TA-mauve method, and a composite with $6 \mathrm{x}$ the concentration synthesised without Albaflow FFA. . . . . . . . . . . . . . . . . 173

4.49 TA-mauve composites of different finished merino yarns synthesised with different volumes of the TA-mauve colloid. . . . . . . . . . . . . 175

4.50 Cryo-SEM micrographs in backscatter mode of TSC-pink Cariaggi wool fibres frozen at the completion of the uptake (before drying) in (a) and (b).

4.51 Cryo-SEM images of a TSC-grey Ascend felted yarn composite fibre frozen at the completion of the uptake at different magnifications in (a) and (b).

5.1 The interior of stainless steel containers that stored an aqueous solution of $10 \mathrm{mg} \mathrm{L}^{-1} \mathrm{AuCl}_{4}^{-}$(a) and the standard TA-mauve colloid for 5 days $($ b) . . . . . . . . . . . . . . . . . . . . . . . . 183

5.2 UV-vis spectra of a TA-mauve stored in glass or stainless steel vessels after $60 \mathrm{~min}$ and 5 days. . . . . . . . . . . . . . . . . . . . . . . . . . 184

5.3 Images of the interior of the barrel reactor with the overhead stirrer beside it (a) and fitted (b) . . . . . . . . . . . . . . . . 185 
5.4 Images of the synthesis of a $60 \mathrm{~L}$ TA-mauve colloid in the barrel reactor during the preparation (a), and the TA-mauve colloid in a white bucket where the purple colour of the colloid is seen more easily (b). . 186

5.5 UV-vis spectra of a $600 \mathrm{~mL}$ TA-mauve colloid synthesised in a glass beaker and a $60 \mathrm{~L}$ TA-mauve colloid synthesised in the barrel reactor. 187

5.6 TSC-grey colloids synthesised in glass beakers: a $2 \mathrm{~L}$ stable colloid (a) and a 5 L destabilised colloid (b). . . . . . . . . . . . . . 188

5.7 The synthesis of the TSC-grey colloid in the barrel reactor during the synthesis (a) and the resulting stable colloid (b) . . . . . . . . . . . 189

5.8 A 100 g TA-mauve Cariaggi composite before (a) and after (b) drying after the dyeing reaction. . . . . . . . . . . . . . . . . . . . . . . 192

5.9 A $500 \mathrm{~g}$ 'TSC-pink' Cariaggi composite synthesised in a hank dyeing reaction. . . . . . . . . . . . . . . . . . . 193

5.10 Images of the synthesis of a $1.5 \mathrm{~kg}$ TSC-grey Cariaggi composite: the wool soaking in the barrel reactor early in the reaction (a), after the agglomeration of TSC-Au NPs (b) and the resulting composite (c). . 194

5.11 A $1 \mathrm{~kg}$ TSC-grey Cariaggi composite synthesised with vigorous agitation in the fish tank reactor (a) and exposing the interior of some of the more matted areas (b). . . . . . . . . . . . . . . . . . . . . . 194

5.12 Aulana demonstration products fabricated from Cariaggi ex-situ composites, a TA-mauve merino jersey (a) and a bespoke suit with TAmauve pin-stripes.

5.13 The hank dyeing reactor at Wool Yarns Ltd (a), where hanks of wool are held in place (b) before being lowered in to a gently circulating dye solution. . . . . . . . . . . . . . . . . . . . . . . . . 198

$5.141 \mathrm{~kg}$ TA-mauve felted yarn composites synthesised in the hank dyeing reactor: illustrating the colour differences between the inside and outside of the relatively dark hank (a) and between the two different hanks (b).

$5.15 \mathrm{UV}$-vis spectra of the uptake of the TA-mauve colloid to felted yarn in the fish tank reactor. . . . . . . . . . . . . . . . 200

5.16 The synthesis of a $1 \mathrm{~kg}$ TA-mauve felted yarn composite: the dyeing reaction near the beginning of the uptake (a), after approximately 60 min of the uptake (b) and the resulting composite (c). . . . . . . . 202

5.17 The uptake of the TSC-middle grey colloid to Ascend felted yarn $(1 \mathrm{~kg})$ in the fish tank reactor. . . . . . . . . . . . . . . 203

5.18 The TSC-dark grey composite synthesised in fish tank: the uptake solutions (a), the dyeing reaction soon after the addition of the felted yarn hank (b) and the dyeing reaction near the completion of the uptake (c) . . . . . . . . . . . . . . . . . . . . . . . . . . . 204 
5.19 TA-mauve and different shades of TSC-grey Ascend felted yarn composites $(1 \mathrm{~kg})$ synthesised in the fish tank reactor with modified hank dyeing procedures.

5.20 Aulana rugs fabricated from ex-situ felted yarn composites that were synthesised in modified hank dyeing procedures. . . . . . . . . . . . 207

5.21 The package dyeing reactor from Tararua Yarns Ltd, where a cone of fine yarn is compressed in the tank and a dye solution is circulating through the cone and out into the overflow tank (a), a $250 \mathrm{~g}$ cone of Tararua Untreated (TU) yarn (b), the possible flow directions in the dyeing tank (c) and the clamped top of the dyeing tank (d). . . . . . 209

5.22 Images of the overflow tank of the package dyeing reactor during the hot water washing step (a) and near the beginning of the uptake of the TA-mauve colloid (b). . . . . . . . . . . . . . . . . . . 210

5.23 Images of a $750 \mathrm{~g}$ TA-mauve TU yarn composite that was synthesised in the package dyeing reactor and a levelness test showing areas of yarn from the inside, middle and outside of the dyepack. . . . . . . . 211

5.24 The uptake of the TA-mauve colloid to TU yarn in package dyeing reactions with different amounts of Albaflow FFA in the pH 2.2 glycine buffer and colloid. . . . . . . . . . . . . . . . . . . . 213

5.25 Levelness tests of TA-mauve TU yarn composites synthesised in the package dyeing reactor with $1 \mathrm{gL}^{-1}$ (a), $2 \mathrm{gL}^{-1}$ (b) and $4 \mathrm{gL}^{-1}$ (c) Albaflow FFA in the buffer and colloid. . . . . . . . . . . . . . . . . . 214

5.26 Levelness tests of TA-mauve TU yarn composites resulting from Experiments $1,2,3$ and 4 in the package dyeing reactor. . . . . . . . 217

5.27 A TU yarn TSC-grey composite synthesised in the package dyeing reactor (a) and SEM micrographs in fibres from the inside (b), middle (c) and outside (d) of the dyepack. . . . . . . . . . . . . . . 218

5.28 A TU yarn TSC-grey composite synthesised in the package dyeing reactor (a) and a levelness sock of the dyepack (b) . . . . . . . . . . . 219

5.29 The Benchtop flow reactor that was used to study the uptake of gold nanoparticles to TU yarn, where the colloid flows through wool that is compressed between needles in PVC tubing, into the overflow beaker and back up through the pump. The inset is a diagram of the wool in the tubing. . . . . . . . . . . . . . . . . . . . . . 222

5.30 Uptake of TA-mauve colloid to TU in the Benchtop flow reactor with different amounts of Albaflow FFA (WA) in the buffer and colloid. . . 223

5.31 TA-mauve TU yarn composites synthesised in the Benchtop flow reactor without Albaflow FFA (a), and with $0.5,1,2$, and $4 \mathrm{gL}^{-1}$ (b)(e) Albaflow FFA in the buffer and colloid, and the composites are aligned as they were in the Benchtop flow reactor. . . . . . . . . . . . 224 
5.32 Uptake of TA-mauve colloid to TU with different amounts of Croscolor DH2 in the Benchtop flow reactor. . . . . . . . . . . . . . . 226

5.33 TA-mauve TU yarn composites synthesised in the Benchtop flow reactor with different amounts of Croscolor DH2 in the synthesis: $0.33 \mathrm{~g} \mathrm{~L}^{-1}$ in the buffer (a), $0.33 \mathrm{~g} \mathrm{~L}^{-1}$ in the colloid (b) and $0.083 \mathrm{~g} \mathrm{~L}^{-1}$ in the colloid (c). . . . . . . . . . . . . . . . . . . . . 227

5.34 Uptake of TA-mauve colloid to TU with different amounts of Tecarapid CBF in the colloid. . . . . . . . . . . . . . . . . . . 228

5.35 TA-mauve TU yarn composites synthesised in the Benchtop flow reactor with $0.5 \mathrm{gL}^{-1}$ (a), $1 \mathrm{gL}^{-1}$ (b) and $2 \mathrm{gL}^{-1}$ (c) Tecarapid CBF and $1 \mathrm{~g} \mathrm{~L}^{-1}$ Tecarapid CBF and Albaflow FFA in the buffer and colloid (d).230

5.36 TSC-grey TU yarn composites synthesised in the Benchtop flow reactor with no auxiliary agents (a), $1 \mathrm{~g} \mathrm{~L}^{-1}$ Tecarapid CBF (b), $0.5 \mathrm{~g} \mathrm{~L}^{-1}$ Tecarapid CBF and Albaflow FFA (c) and $1 \mathrm{gL}^{-1}$ Tecarapid CBF and Albaflow FFA $(\mathrm{d}) . \ldots 231$

5.37 First flow reactor set-up . . . . . . . . . . . . . . . 233

5.38 Cariaggi (a) and Ashford (b) TA-mauve ex-situ composites of $40 \mathrm{~g}$ size that were synthesised in the first flow reactor. . . . . . . . . . 234

$5.39 \mathrm{UV}$-vis spectra of the uptake of a diluted TA-mauve colloid to TU yarn in the first flow reactor. The inset represents the uptake curve from these spectra . . . . . . . . . . . . . . . . . 235

5.40 The top (a) and bottom (b) of a $50 \mathrm{~g}$ TA-mauve composite synthesised in the first flow reactor with the equivalent reaction conditions of the standard TA-mauve method. . . . . . . . . . . . . . . . . 235

5.41 SEM micrographs in backscatter mode of the surface of TA-mauve composite fibres synthesised in the first flow reactor from relatively dark (a)-(b) and light (c)-(d) areas of the dyepack. . . . . . . . . 236

$5.42 \mathrm{UV}$-vis spectra of the uptake of a diluted TA-mauve colloid to TU yarn in the first hybrid reactor with the gradual lowering of the $\mathrm{pH}$ of the uptake solution with $\mathrm{HCl}$ addition $\left(1 \mathrm{~g} \mathrm{~L}^{-1}\right.$ Albaflow FFA). . . 237

5.43 The top (a) and bottom (b) of a $50 \mathrm{~g}$ TA-mauve composite synthesised in the first flow reactor with the gradual $\mathrm{pH}$ lowering of the dye solution.238

5.44 The top (a) and bottom (b) of a $50 \mathrm{~g}$ TA-mauve composite synthesised in the first flow reactor with a $\mathrm{pH} 2.2$ buffer and colloid $\left(1 \mathrm{gL}^{-1}\right.$ Albaflow FFA).

5.45 The Baby Guinness (BG) reactor set-up, where dye liquor is pumped through the inflow, up the cylindrical reactor and exits through the outflow. The outlet tap allows sampling of the dye solution during the dyeing reactions and the rate of dye circulation is dictated by the control valve. . . . . . . . . . . . . . . . . . . . . . . . . 241 
5.46 Images of the interior of the Baby Guinness reactor: the coiled configuration of the top form wool before dyeing (a), the stamp used to compress the wool (b) and the open reactor after a TA-mauve dyeing

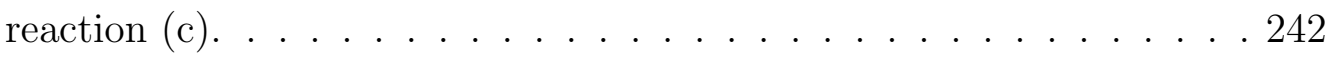

5.47 UV-vis spectra and images of the uptake of the concentrated TAmauve colloid to loose wool in the BG flow reactor $\left(\mathrm{pH} 2.2,0.5 \mathrm{~g} \mathrm{~L}^{-1}\right.$ Albaflow FFA, $0.1 \mathrm{~mol} \mathrm{~L}^{-1}$ glycine buffer $120 \mathrm{~min}$, hot water wash). . 244

5.48 TA-mauve loose wool composites synthesised in the BG flow reactor with the conditions of the standard TA-mauve method ( $\mathrm{pH} \mathrm{2.2,}$ $0.5 \mathrm{gL}^{-1}$ Albaflow FFA, glycine buffer circulation for $120 \mathrm{~min}$, hot water wash) before (a) and after (b) drying. . . . . . . . . . . . . . . 244

5.49 Uptake curves of the dyeing reactions of loose wool with the TAmauve colloid in the BG flow reactor with a hot water wash and 120 min buffer circulation or no hot water wash and 15 min buffer circulation. . . . . . . . . . . . . . . . . . 245

5.50 TA-mauve loose wool composites synthesised in the BG flow reactor immediately after dyeing (a) and when dried (b). . . . . . . . . . . 246

5.51 SEM micrographs in backscatter mode of a TA-mauve loose wool composite synthesised in the BG flow reactor (with no hot water wash and 15 min buffer circulation). . . . . . . . . . . . . . . . . . . 247

5.52 Ascend felted yarn TA-mauve composites synthesised in the BG flow reactor, where Experiment 1 is dark mauve, Experiments 2 and 3 are light mauve and Experiment 4 is purple-mauve in colour. . . . . . . . 249

5.53 A TSC-light grey loose wool composite synthesised in the BG reactor with a pH 2.0 glycine buffer and colloid (pH 2.0, $1 \mathrm{~g} \mathrm{~L}^{-1}$ Albaflow FFA).250

5.54 Images of a TSC-light grey loose wool composite synthesised in the BG reactor with a pH 2.8 glycine buffer and colloid directly removed from the reactor after the dyeing reaction (a) and the resulting composite (b), where the darker grey areas are circled. . . . . . . . . . . . 251

5.55 Uptake of the TSC grey colloid to loose wool with different reaction $\mathrm{pH}$ levels of the glycine buffer and colloid. . . . . . . . . . . . . 252

5.56 Images of the pH 2.4 TSC-light grey loose wool composite synthesised in the BG reactor: the top face of the dyepack immediately after the dyeing reaction (a) and the resulting composite (b). . . . . . . . . 253

5.57 Images of a new stamp (a), the wet TSC-grey composites in the BG reactor post-dyeing (b) and the resulting composite (c) . . . . . . . . 254

5.58 Image of the circulation of a TSC-grey colloid during the dyeing reaction in the BG reactor (approximately $1 \mathrm{~min}$ after the beginning of the reaction). . . . . . . . . . . . . . . . 256 
5.59 Images of the sacrificial filter used in the synthesis of the TSC-grey loose wool composite in the BG reactor after the dyeing reaction (a) and the resulting composite (b) . . . . . . . . . . . . . . . 257

5.60 A 660 g TSC-grey loose wool composites synthesised with the sacrificial filter in the Full Pint (FP) reactor, where the wool relates to the bottom (a), middle (b) and top of the dyepack (c). . . . . . . . 258

6.1 Cariaggi and TU yarn ex-situ composites of $1 \mathrm{~g}$ size before and after washing on the Chiltern shaker for $24 \mathrm{~h}$. . . . . . . . . . . . 263

6.2 SEM micrographs in backscatter (BS) mode of TA-mauve (a)-(b), TSC-pink (c)-(d) and TSC-grey (e)-(f) TU yarn composites after washing on the Chiltern shaker for $24 \mathrm{~h}$. . . . . . . . . . . 265

6.3 TA-mauve and TSC-pink composites of Cariaggi wool, before and after washing (three times with a 1:20 wool to liquor ratio of Kindness wool wash). . . . . . . . . . . . . . . . . . . . . 267

6.4 UV-vis spectra of solutions resulting from the washing of TA-mauve and TSC-pink composites of Cariaggi wool 3 times. . . . . . . . . . 267

6.5 Polydiallyldimethylammonium chloride (PDADMAC). . . . . . . . 269

6.6 TA-mauve composites of Cariaggi wool that were synthesised after different pre-treatments and with a colloid $\mathrm{pH}$ of 2.7 or 11 . . . . . . 271

6.7 Cariaggi TA-mauve composites synthesised after pre-soaking the wool in $1 \mathrm{wt} \%$ solutions of different reagents prior to the dyeing process (not washed). . . . . . . . . . . . . . . . . . . . . 272

6.8 UV-vis spectra of the wash solutions of Cariaggi TA-mauve composites synthesised after pre-soaking the wool in 1,3-propane dithiol at pH 8 or 10 and an untreated composite. . . . . . . . . . . . 273

6.9 TA-mauve composites of Cariaggi wool that were synthesised after PEI pre-treatments with different reaction conditions, before and af-

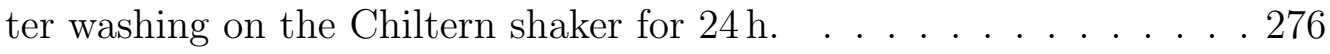

6.10 A schematic illustrating the possible interaction between MSA and gold nanoparticles in their synthesis. . . . . . . . . . . . . . 278

6.11 A schematic illustrating the possible interaction between 1,3-propane dithiol and gold nanoparticles in their synthesis. . . . . . . . . . . . . 279

6.12 An illustration of the chemical structure of a possible fraction of branched PEI. . . . . . . . . . . . . . . . . . . . . . . . . . 280

6.13 UV-vis spectra of PEI-stabilised gold colloids that were synthesised at different reaction $\mathrm{pH}$ levels. . . . . . . . . . . . . . . . 281

6.14 PEI-grey Cariaggi and TU yarn composites synthesised at RT (a) and (d), $50{ }^{\circ} \mathrm{C}(\mathrm{b})$ and $(\mathrm{e})$ and $100^{\circ} \mathrm{C}(\mathrm{c})$ and $(\mathrm{f}) \ldots . . . . . . . .282$ 
6.15 UV-vis spectra of the wash solutions of PEI-grey Cariaggi and TU yarn composites synthesised at different temperatures (1:20 ratio of wool to Kindness wool wash). . . . . . . . . . . . . . . . . . . . 283

6.16 PEI-grey Cariaggi (a) and TU (b) composites and the composites after washing and TU yarn after washing on the Chiltern shaker for

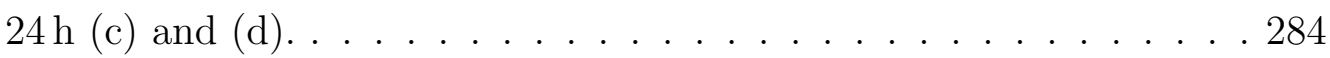

6.17 SEM micrographs in backscatter mode of an unwashed Cariaggi PEIgrey composite (a)-(c), an EDS elemental Au map (d), and SEM images of two composite fibres after washing on the Chiltern shaker

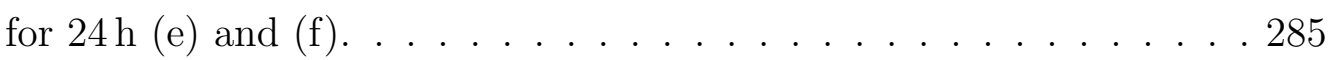

6.18 The structure of DMTMM . . . . . . . . . . . . . . . 286

6.19 PEI-grey composites of TU yarn before (top) and after (bottom) washing, synthesised without DMTMM (a) and (d), with DMTMM in the uptake solution (b) and (e) and with a DMTMM post-treatment

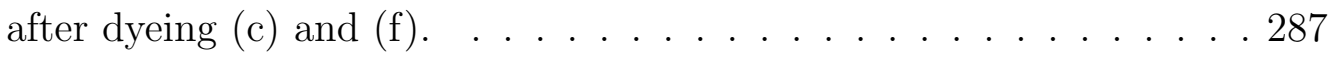

6.20 TU yarn composites synthesised PEI-grey (a) TSC-grey (b) and TAmauve (c) colloids with an uptake temperature of $100^{\circ} \mathrm{C}$ (without DMTMM), and the composites after washing on the dunking machine

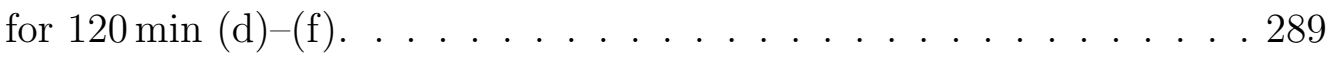

6.21 The UV-vis spectrum and chemical structure of Remazol Blue (RB), and the UV-vis spectra of gold colloids that were synthesised with different ratios of $\mathrm{AuCl}_{4}^{-}$to RB. . . . . . . . . . . . . . . . . . . . 291

6.22 The UV-vis spectrum and chemical structure of Lanasol Red (LR), and the UV-vis spectra of gold colloids that were synthesised with different ratios of $\mathrm{AuCl}_{4}^{-}$to LR. . . . . . . . . . . . . . . . . . . 292

6.23 The UV-vis spectrum and chemical structure of Lanasol Yellow (LY), and the UV-vis spectra of gold colloids that were synthesised with different ratios of $\mathrm{AuCl}_{4}^{-}$to $\mathrm{LY}$.

6.24 The UV-vis spectrum and chemical structure of Acomet Yellow (AY), and the UV-vis spectra of gold colloids that were synthesised with different ratios of $\mathrm{AuCl}_{4}^{-}$to AY . . . . . . . . . . . . . . . 293

6.25 RB composites of Cariaggi wool (a) and TU yarn (b), and the composites after washing on the Chiltern shaker for $24 \mathrm{~h}$ (c) and (d). . . . 294

6.26 SEM micrographs in backscatter mode of the surfaces of RB-Au NPs composites of Cariaggi wool before washing (a)-(b) and TU yarn (c)(d) before washing, and TU yarn composite fibres after washing in excess Kindness wool wash on the Chiltern shaker for $24 \mathrm{~h}$ (e)-(f) . . . 296

6.27 TU yarn composites dyed with LR-stabilised gold colloids that were synthesised with weight ratios of $\mathrm{AuCl}_{4}^{-}$to LR of 1:2.5 (a), 1:3.33 (b)

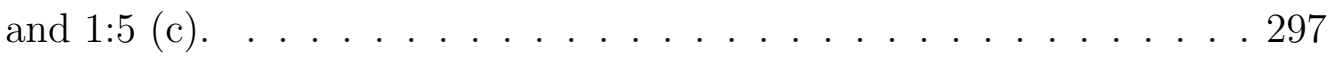


6.28 SEM micrographs in backscatter mode of the surfaces of LR TU yarn composites synthesised with an $\mathrm{AuCl}_{4}^{-}$:LR ratio of 1:2.5 (a)-(b) and

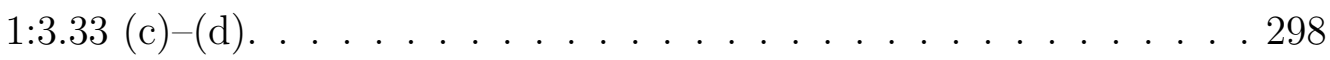

6.29 LR TU yarn composites dyed with colloids that incorporated different amounts of PMA in their syntheses (and a ratio of $\mathrm{AuCl}_{4}^{-}$to $\mathrm{LR}$ of 1:3.33): no PMA (a), $10 \mathrm{mg} \mathrm{L}^{-1}$ (b), $25 \mathrm{mg} \mathrm{L}^{-1}$ (c) and $50 \mathrm{mg} \mathrm{L}^{-1}$ (d). 299 


\section{List of Tables}

1.1 Amino acid composition of merino wool . . . . . . . . . . . . . . 4

1.2 Common steps in the processing of wool fibres and the name of the type of wool that results from the processing steps, which occur sequentially from scouring to knitting. . . . . . . . . . . . 15

1.3 The different approaches for the synthesis of gold nanoparticle-wool fibre composites developed by Lucas and Johnston . . . . . . . . . . . 32

2.1 The different types of wool fibres used in this research project. . . . . 45

2.2 Standard reaction conditions of the in-situ procedure to synthesise purple coloured gold nanoparticle-wool fibre composites. . . . . . . . 48

2.3 Standard reaction conditions of the ex-situ procedures. . . . . . . . . 49

2.4 Standard reaction conditions of the ex-situ procedures. . . . . . . . . 50

2.5 Levelling agents that were employed in the ex-situ procedure. . . . . . 53

2.6 A summary of the different scale-up dyeing reactions. . . . . . . . . . 54

2.7 A summary of the reaction parameters of the pressurised flow reactors. 56

3.1 Standard reaction conditions of the in-situ procedure to synthesise purple coloured gold nanoparticle-wool fibre composites. . . . . . . . 64

3.2 CIE colour coordinates of loose wool in-situ composites synthesised with different types of wool in top form (that were pictured in Figure 3.7. 73

3.3 Fine merino yarns used in the in-situ synthesis of gold nanoparticlewool fibre composites. . . . . . . . . . . . . . . . 75

3.4 CIE colour coordinates of in-situ composites of merino wool yarns. . . 76

3.5 CIE colour coordinates of Ashford2 in-situ composites synthesised at a different reaction $\mathrm{pH}$. . . . . . . . . . . . . . . . . . 79

3.6 The amount of gold in Ashford2 in-situ composites that were removed from uptake solutions at different temperatures and times. . . . . . . 87

3.7 CIE colour coordinates of in-situ composites of finished wool yarns synthesised with and without $1 \mathrm{~g} \mathrm{~L}^{-1}$ Albaflow FFA wetting agent. . . 90

3.8 CIE colour coordinates of Cariaggi in-situ composites synthesised with or without levelling agents in the uptake solution. . . . . . . . 95 
3.9 CIE colour coordinates of Ashford2 in-situ composites synthesised with exposure to microwave radiation or dry heat in the oven with different amounts of Albaflow FFA in the uptake solution. . . . . . . 107

3.10 CIE colour coordinates of Ashford2 composites synthesised with the application of $\mathrm{NaBH}_{4}$ after different times of RT uptake of $160 \mathrm{mg} \mathrm{L}^{-1}$ $\mathrm{Au}^{3+}$. . . . . . . . . . . . . . . . . . 109

3.11 CIE colour coordinates of Ashford2 composites synthesised with the application of TSC . . . . . . . . . . . . . . . . . . 113

3.12 CIE colour coordinates of Ashford2 composites synthesised with TA after the uptake of $160 \mathrm{mg} \mathrm{L}^{-1} \mathrm{Au}^{3+}$ at RT. . . . . . . . . . . . . 115

3.13 The colour shade and uniformity of in-situ composites that were synthesised with heat and external reducing agents. . . . . . . . . 117

4.1 Standard reaction conditions of the ex-situ procedures. . . . . . . . . 119

4.2 CIE colour coordinates of Cariaggi wool and ex-situ composites. . . . 124

4.3 CIE colour coordinates of TA-mauve composites of different wool types. 138

4.4 The different concentration ratios of $\mathrm{AuCl}_{4}^{-}$to $\mathrm{TA}$ that were used in the synthesis of TA-Au NPs. . . . . . . . . . . . . . . . . . 141

4.5 The different concentration ratios of $\mathrm{AuCl}_{4}^{-}$to TSC that were used in the synthesis of TSC-Au NPs. . . . . . . . . . . . . . . . 143

4.6 The different wool types used in ex-situ optimisation experiments. . . 145

4.7 CIE colour coordinates of TA-mauve composites of different wool types. 147

4.8 CIE colour coordinates of TA-mauve and TSC-grey composites of different sources of loose wool. . . . . . . . . . . . . . . . . . . . . 149

4.9 CIE colour coordinates of TA-mauve composites of different wool types different amounts of Albaflow FFA (WA) in the buffer and colloid. . . . . . . . . . . . . . . . . . . . 158

4.10 CIE colour coordinates of TU yarn TA-mauve composites synthesised with different amounts of Albaflow FFA (WA) employed in the buffer and the colloid. . . . . . . . . . . . . . . . 160

4.11 CIE colour coordinates of TU yarn TSC-grey composites synthesised with different amounts of Albaflow FFA (WA) added prior to the $\mathrm{pH}$ adjustment of the colloid. . . . . . . . . . . . . . . 163

4.12 CIE colour coordinates of TU yarn TSC-grey composites synthesised with different levels of Albaflow FFA (WA) in the buffer and colloid. . 167

4.13 CIE colour coordinates of TU yarn TSC-grey composites synthesised with different amounts of Albaflow FFA (WA) added after pH adjustment. . . . . . . . . . . . . . . . . . . . 168

4.14 Levelling agents that were employed in the ex-situ procedure. . . . . . 169 
4.15 CIE colour coordinates of TU yarn TA-mauve composites synthesised with different levelling agents in the buffer prior to the uptake of TA$\mathrm{Au}$ NPs. . . . . . . . . . . . . . . . . . . . . . . 171

4.16 The concentrations TA-Au NPs that were used to colour TU yarn. . . 172

4.17 CIE colour coordinates of TA-mauve TU yarn composites synthesised with different concentrations of the colloid. . . . . . . . . . . . . . . 172

4.18 CIE colour coordinates of TU yarn TA-mauve composites synthesised with different colloid concentrations. . . . . . . . . . . . . . . 175

4.19 The conditions of the standard ex-situ methods. . . . . . . . . . . . . 179

5.1 Properties of different materials for the composition of gold nanoparticle synthesis reactors. . . . . . . . . . . . . . . . . . . . . . . . 182

5.2 The concentration of $\mathrm{Au}^{3+}$ remaining in solution after storage in glass or stainless steel reaction vessels measured with AAS. . . . . . . . . . 182

5.3 The reaction conditions of the dyeing of Ascend felted yarn hanks in the laboratory. . . . . . . . . . . . . . . . . . . . . . . . . . 199

5.4 CIE colour coordinates of the Ascend felted yarn composites. . . . . . 206

5.5 CIE colour coordinates of inside, middle and outside of the $750 \mathrm{~g}$ TAmauve TU yarn composite dyepack synthesised in the package dyeing reactor (the areas of the dyepack are separated by white lines) . . . . 212

5.6 CIE colour coordinates measured from different areas of the $250 \mathrm{~g}$ TA-mauve TU yarn composites synthesised with different amounts of Albaflow FFA. . . . . . . . . . . . . . . . . . . . . . . . 215

5.7 Summary of the reaction conditions that were manipulated to attempt to increase the colour uniformity of TA-mauve TU yarn composites in the package dyeing reactor. . . . . . . . . . . . . . 216

5.8 The reaction conditions and function of the suite of novel flow reactors for the ex-situ synthesis of gold nanoparticle-wool fibre composites.

5.9 CIE colour coordinates of different areas of the $5 \mathrm{~g}$ TA-mauve TU yarn composites synthesised in the Benchtop flow reactor. . . . . . . . 225

5.10 CIE colour coordinates of TA-mauve TU yarn composites that were synthesised in the Benchtop flow reactor with different amounts of Croscolor DH2. . . . . . . . . . . . . . . . . . . . 226

5.11 CIE colour coordinates of $5 \mathrm{~g}$ TA-mauve TU yarn composites that were synthesised in the Benchtop flow reactor with different amounts of Tecarapid CBF.

5.12 CIE colour coordinates of $5 \mathrm{~g}$ TSC-grey TU yarn composites that were synthesised in the Benchtop flow reactor with different amounts of Tecarapid CBF.

5.13 CIE colour coordinates a TA-mauve TU yarn composite synthesised in the first flow reactor. . . . . . . . . . . . . . . . . . . 236 
5.14 CIE colour coordinates a TA-mauve TU yarn composite synthesised in the first flow reactor with gradual $\mathrm{pH}$ adjustment. . . . . . . . . 238

5.15 CIE colour coordinates a TA-mauve TU yarn composite synthesised in the first flow reactor with a $\mathrm{pH} 2.2$ buffer and colloid $\left(1 \mathrm{gL}^{-1}\right.$ Albaflow FFA). . . . . . . . . . . . . . . . . . . . . . . 239

5.16 A summary of the reaction parameters of the pressurised flow reactors. 240

5.17 Details of reaction parameter employed in different experiments carried out in the BG reactor. . . . . . . . . . . . . . . . . . . . . . . . . 248

6.1 Types of washfastness experiments. . . . . . . . . . . . . . . 262

6.2 CIE colour coordinates of Cariaggi ex-situ composites before and after

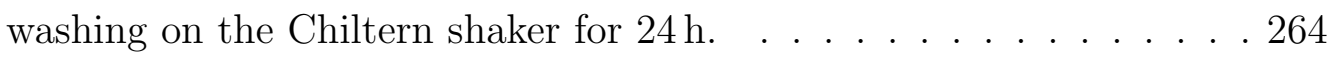

6.3 CIE colour coordinates of TU yarn ex-situ composites before and after washing on the Chiltern shaker for 24 h. . . . . . . . . . . . . . 264

6.4 Chemical reagents used for pre-treating wool fibres prior to dyeing with gold nanoparticles. . . . . . . . . . . . . . . . 268

6.5 The effects of pre-treatment chemical reagents on the uptake of the TA-mauve colloid to Cariaggi wool (where $\mathrm{X}=$ prevented uptake, $\mathrm{Y}$ $=$ complete uptake, $\mathrm{A}=$ agglomerated colloid and $-=$ not performed).270

6.6 CIE colour coordinates of TA-mauve Cariaggi composites with and without an MSA pre-treatment before and after washing in Kindness wool wash. . . . . . . . . . . . . . . . . . . . . . 272

6.7 Chemical reagents that were used in post-treatment experiments, and the reaction conditions of their use. . . . . . . . . . . . . . 274

6.8 The effects of pre-treatment chemical reagents on the TA-mauve Cariaggi composites (where $\mathrm{L}=$ leaching of Au NPs and A = agglomerated Au NPs). . . . . . . . . . . . . . . . . . . . . . 275

6.9 Chemical reagents used in the synthesis of gold nanoparticles for washfastness experiments. . . . . . . . . . . . . . . . . 277

6.10 CIE colour coordinates of Cariaggi and TU yarn PEI-grey composites before and after washing in Kindness wool wash on the Chiltern shaker for $24 \mathrm{~h}$.

6.11 CIE colour coordinates of PEI-grey TU yarn composites synthesised with and without the amide formation catalyst DMTMM before and after simulated washing. . . . . . . . . . . . . . . 287

6.12 CIE colour coordinates of TU yarn ex-situ composites synthesised with an uptake temperature of $100^{\circ} \mathrm{C}$ before and after washing. . . . 289

6.13 Reactive dyes that were used in the gold nanoparticle syntheses. . . . 290

6.14 CIE colour coordinates of Cariaggi and TU yarn RB composites before and after washing. 
6.15 CIE colour coordinates of TU yarn dyed with LR colloids that were synthesised with different weight ratios of $\mathrm{AuCl}_{4}^{-}: \mathrm{LR}$. . . . . . . 297

6.16 CIE colour coordinates of LR TU yarn composites dyed with colloids synthesised with different amounts of PMA in the colloid synthesis. . 299

7.1 The advantages and disadvantages of the $i n$-situ and ex-situ procedures.302 


\section{Glossary}

18-MEA

$a^{*}$ value

AAS

Ashford

Aqua regia

$\mathrm{Au} \mathrm{NPs}$

$\mathrm{b}^{*}$ value

BS

carding

Cariaggi

conc

DMTMM

Dyebath

Dyepack

ex-situ procedure

HDPE

in-situ procedure

$\mathrm{L}^{*}$ value

levelling agents

loose wool

MSA

NPs

open top

PVC

PEI 18-methyleicosanoic acid, a fatty acid that is covalently bound to the wool surface CIE colour coordinate indicating redness (more positive $\mathrm{a}^{*}$ ) versus greenness (more negative $\mathrm{a}^{*}$ )

atomic absorption spectroscopy

to form a cluster of multiple nanoparticles

top form wool sourced from Ashford Handicrafts

a 3:1 mixture of concentrated $\mathrm{HCl}$ and $\mathrm{HNO}_{3}$

gold nanoparticles

CIE colour coordinate indicating yellowness (more positive

$\left.\mathrm{b}^{*}\right)$ versus blueness (more negative $\mathrm{b}^{*}$ )

backscatter mode in SEM imaging

the process of removing matter from scoured wool

a type of wool in top form sourced from Cariaggi, Italy

concentration

4(4,6-dimethoxy-1,3,5-triazin-2-yl)-4-methylmorpholinium

chloride hydrate; a catalyst used in amide bond formation

dye solution that wool is immersed in wool dyeing

a sample of wool fibres that is being dyed

synthesising gold nanoparticle-wool fibre composites via

dyeing wool with gold nanoparticle colloids

high-density polyethylene

synthesising gold nanoparticle-wool fibre composites by

wool fibres absorbing $\mathrm{AuCl}_{4}^{-}$and forming gold nanoparticles

CIE colour coordinate indicating brightness versus darkness wool dyeing auxiliaries that are used to control

the uptake of dye molecules

wool after scouring and carding processes

mercaptosuccinic acid, a thiol-based reagent used to

modify wool fibres

nanoparticles

wool that results from the carding of loose wool

polyvinylchloride

polyethylenimine, a polymer with amine functionality that is used in wool fibre modification and Au NP synthesis 
RT

scouring

SEI

SEM

TA

TA-Au NPs

TGA

TSC

TS yarn

TSC-Au NPs

TU yarn

TEM

UV-vis

WA

washfastness

"wet out"

wetting agents

wool dyeing auxiliaries wool in top form

wt $\%$

XPS room temperature (typically $18-20^{\circ} \mathrm{C}$ )

the process of washing shorn wool to remove grease

secondary electron imaging in SEM imaging

scanning electron microscopy

Tannic acid, a relatively strong reducing and stabilising

agent

gold nanoparticles stabilised by TA

Thioglycollic acid, a thiol-based reagent used to

modify wool fibres

Trisodium citrate, a relatively weak reducing and stabilising agent

Wool yarn sourced from Tararua Yarns Ltd. with a surface treatment that prevents damage during washing cycles Gold nanoparticles stabilised by TSC

Fine merino yarn sourced from Tararua Yarns Ltd.

transmission electron microscopy

UV-visible absorption (spectroscopy or spectra)

wetting agent (typically Albaflow FFA)

the resistance of wool fibres to colour changes

during washing processes

the process of ensuring that all wool fibres are wet

wool dyeing auxiliaries that are used to help

to wet out a dyepack

chemical reagents that are used to improve dye uniformity

aligned wool fibres that result from the combing

of open top wool

weight percentage (mass/total mass)

X-ray photoelectron spectroscopy 


\section{Chapter 1}

\section{Introduction}

The New Zealand wool industry has generated valuable exports since the 1850s; from 1850s to the 1900s, wool was New Zealand's highest grossing export earner. However, by 2011 it had fallen to $1.6 \%$ of the total value of exports. ${ }^{1}$ The wool export price peaked in 1989, when it encompassed a total export value of $\$ 1.8$ billion, however this has fallen around $60 \%$ to $\$ 720$ million in $2011 .{ }^{1}$ The reduction in the value of New Zealands wool exports is related to the decrease in global demand due to the increased prominence of synthetic fibres, and the deregulation of the agricultural sector from 1984 onwards (which included removing farming subsidies). ${ }^{2}$ The lower profitability of wool resulted in a conversion of agricultural capital to the growing dairy industry, and an increase in sheepmeat exports compared to wool (Figure 1.1).

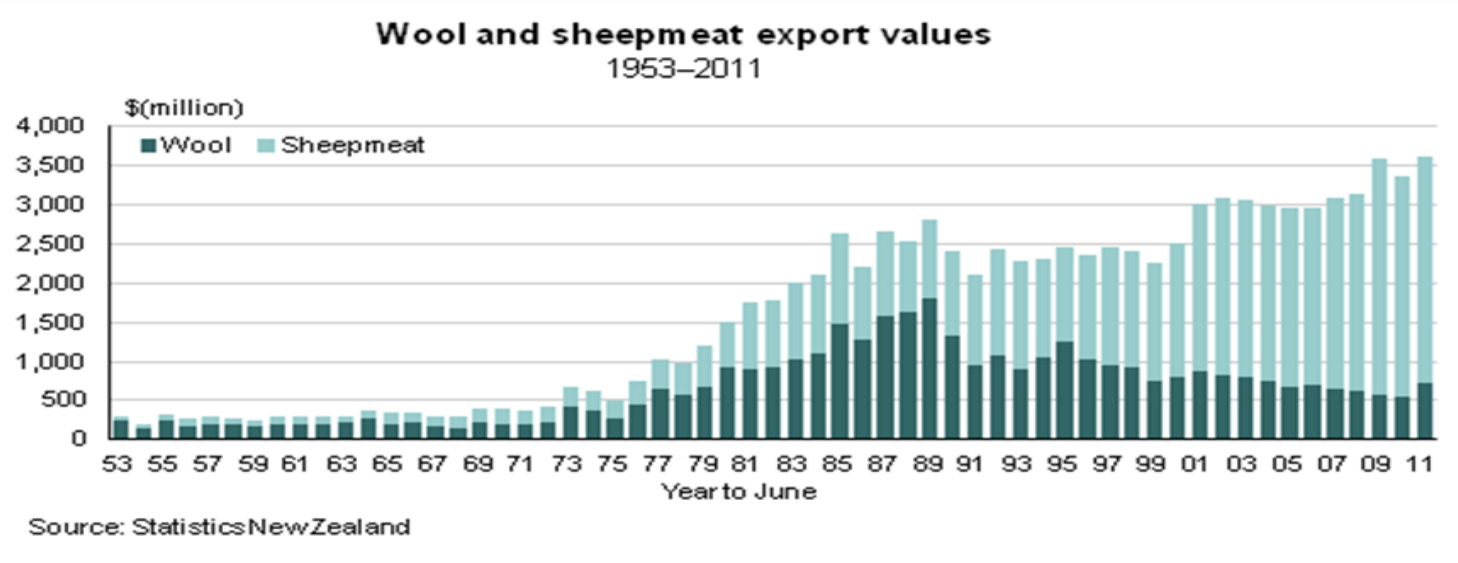

Figure 1.1 New Zealand wool and sheepmeat export values from 1953-2011. ${ }^{3}$

Given the rich economic history of wool production in New Zealand, and the country's ability to produce large quantities of wool, the opportunity to innovate and 
add value to New Zealand's wool exports is of significant interest to New Zealanders. One such innovation involves the synthesis of novel gold nanoparticle-wool fibre composites. This novel nanotechnology utilises gold nanoparticles as luxury dyes to colour premium New Zealand wool. The nanocomposites will be used in the fabrication of high quality apparel and luxury textiles for international markets.

Gold nanoparticle-wool fibre composites were first developed by Professor James Johnston and Dr Kerstin Lucas (neé Burridge) from Victoria University of Wellington. ${ }^{4,5}$ The innovative nanotechnology is currently being commercialised by Noble Bond Ltd in partnership with Wools of New Zealand, ${ }^{6}$ and operates under the internationally registered trademark Aulana ${ }^{\circledR}$.

A detailed market study on the commercial viability of gold nanoparticle-wool fibre composites was carried out by Noble Bond Ltd and Wools of New Zealand over recent years (while the technology was being developed). This study showed that there was major interest from international companies that were concerned with the sale of premium woollen apparel and rugs in luxury markets. A point of distinction was identified as a crucial driver for commercial success, which the Aulana technology had as it is a world first. The details of the market study are proprietary to Noble Bond Ltd and Wools of New Zealand. However, the essence of this market information was conveyed to the author, and provided confidence for the need to scale-up the synthesis of gold nanoparticle-wool fibre composites.

This $\mathrm{PhD}$ project presents research that relates to the optimisation and scale-up of the synthesis of gold nanoparticle-wool fibre composites. In order for this to be achieved, a comprehensive study of wool fibres, gold nanoparticles and the previous research into the synthesis of gold nanoparticle-wool fibre composites was carried out.

\subsection{Wool Fibres}

Research into the optimisation and scale-up of gold nanoparticle-wool fibre composites required the development of a comprehensive understanding of the structure and chemistry of wool fibres, and an in-depth knowledge of the industrial processing and dyeing practices that wool fibres are subjected to. 


\subsubsection{The Chemical and Morphological Structure of Wool Fibres}

Wool fibres are strong, flame resistant, hydrophobic and thermally insulating fibres, which makes them desirable for the use in clothing and textiles. The various properties of wool fibres exist due to the structural characteristics of wool fibres.

Wool is a proteinaceous material, consisting of keratins (protein molecules in a regular conformation), in a complex mixture with proteins of irregular structure. ${ }^{7}$ Keratins are characterised as hard keratins, for example wool, hair, nails and hooves, and soft keratins, such as those in skin. ${ }^{8}$ Hard keratins are stronger than soft keratins, due to their characteristically high sulfur content. ${ }^{8}$ Keratins are also classified as either $\alpha$ - or $\beta$-keratins, which exhibit a helical winding of proteins (as is represented in Figure 1.2), or a pleated sheet conformation respectively. Wool, skin and fingernails are $\alpha$-keratins, while examples of $\beta$-keratins include silk and feathers. ${ }^{8}$
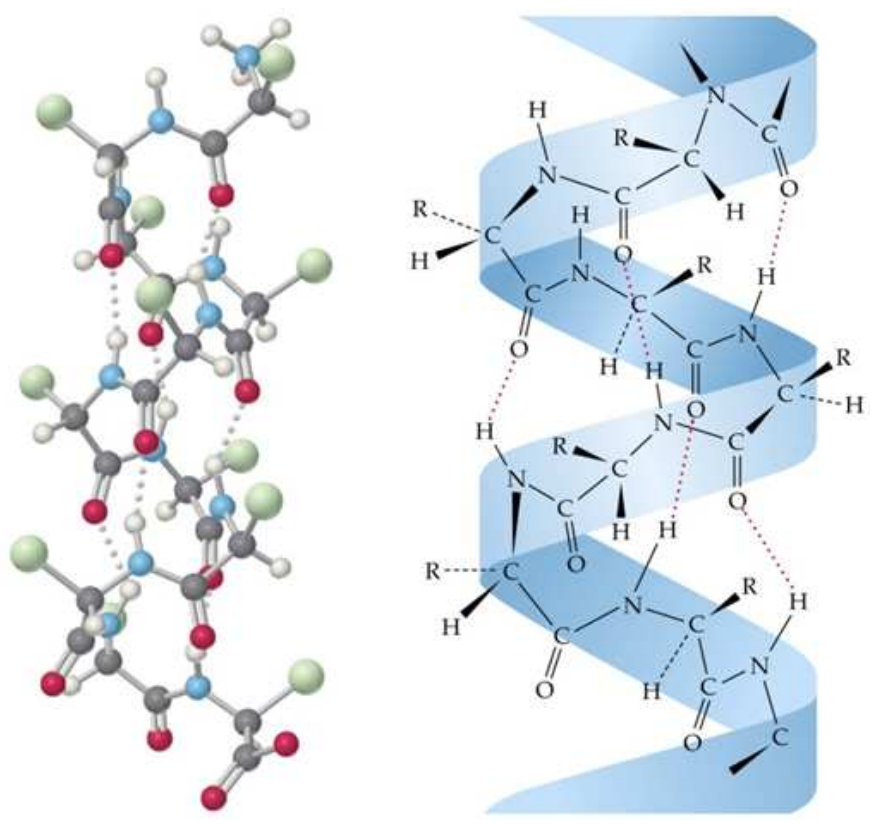

Figure 1.2 The helical structure of $\alpha$-keratins.

The elemental composition of the proteins in wool includes sequentially decreasing amounts of carbon, oxygen, nitrogen, hydrogen and sulfur, ${ }^{7}$ where the sulfur content is typically $4-5 \mathrm{wt} \% .{ }^{9}$ Wool fibres also contain lipids, mineral salts, nucleic acid residues and carbohydrates (which comprises approximately $3 \mathrm{wt} \%$ of the fibres). ${ }^{7}$ Table 1.1 lists the 24 amino acids that comprise the structure of merino wool. The relative composition of amino acids varies in wool fibres that are sourced from different sheepbreeds and is also influenced by the husbandry conditions of the sheep. ${ }^{7}$ The characteristically high sulfur content of wool fibres is principally due to the 
prevalence of cystine residues.

Table 1.1 Amino acid composition of merino wool. ${ }^{7}$

\begin{tabular}{|c|c|c|c|}
\hline Group & Name & Side chain & Conc $\left(\mu \mathrm{molg}^{-1}\right)$ \\
\hline $\begin{array}{l}\text { 'Acidic' amino acids and } \\
\text { their } \omega \text {-amides }\end{array}$ & $\begin{array}{l}\text { Aspartic acid } \\
\text { Glutamic acid } \\
\text { Asparagine } \\
\text { Glutamine }\end{array}$ & $\begin{array}{l}-\mathrm{CH}_{2}-\mathrm{COOH} \\
-\left(\mathrm{CH}_{2}\right)_{2}-\mathrm{COOH} \\
-\mathrm{CH}_{2}-\mathrm{CONH}_{2} \\
-\left(\mathrm{CH}_{2}\right)_{2}-\mathrm{CONH}_{2}\end{array}$ & $\begin{array}{l}200 \\
600 \\
360 \\
450\end{array}$ \\
\hline \multirow[t]{2}{*}{$\begin{array}{l}\text { 'Basic' amino acids } \\
\text { and tryptophan }\end{array}$} & $\begin{array}{l}\text { Arginine } \\
\text { Lysine } \\
\text { Histidine }\end{array}$ & $\begin{array}{l}-\left(\mathrm{CH}_{2}\right)_{3}-\mathrm{C}\left(\mathrm{NH}_{2}\right)=\mathrm{NH} \\
-\left(\mathrm{CH}_{2}\right)_{4}-\mathrm{NH}_{2} \\
\mathrm{H}_{2} \mathrm{C}-\end{array}$ & $\begin{array}{c}600 \\
250 \\
80\end{array}$ \\
\hline & Tryptophan & - & 40 \\
\hline $\begin{array}{l}\text { Amino acids with hydroxyl } \\
\text { groups in the side chain }\end{array}$ & $\begin{array}{l}\text { Serine } \\
\text { Threonine } \\
\text { Tyrosine }\end{array}$ & $\begin{array}{l}-\mathrm{CH}_{2}-\mathrm{OH} \\
-\mathrm{CH}\left(\mathrm{CH}_{2}\right)-\mathrm{OH} \\
-\mathrm{CH}_{2}-\mathrm{C}_{6} \mathrm{H}_{4}-\mathrm{OH}\end{array}$ & $\begin{array}{l}900 \\
570 \\
350\end{array}$ \\
\hline Sulfur containing amino acids & $\begin{array}{l}\text { Cysteine } \\
\text { Thiocysteine } \\
\text { Cysteic acid } \\
\text { Cystine } \\
\text { Lanthionine } \\
\text { Methionine }\end{array}$ & $\begin{array}{l}-\mathrm{CH}_{2}-\mathrm{SH} \\
-\mathrm{CH}_{2}-\mathrm{S}-\mathrm{SH} \\
-\mathrm{CH}_{2}-\mathrm{SO}_{3} \mathrm{H} \\
-\mathrm{CH}_{2}-\mathrm{S}-\mathrm{S}-\mathrm{CH}_{2}- \\
-\mathrm{CH}_{2}-\mathrm{S}-\mathrm{CH}_{2}- \\
-\left(\mathrm{CH}_{2}\right)_{2}-\mathrm{S}-\mathrm{CH}_{2}\end{array}$ & $\begin{array}{c}10 \\
5 \\
10 \\
460 \\
5 \\
50\end{array}$ \\
\hline \multirow[t]{2}{*}{$\begin{array}{l}\text { Amino acids without reactive } \\
\text { groups in the side chain }\end{array}$} & $\begin{array}{l}\text { Glycine } \\
\text { Alanine } \\
\text { Valine } \\
\text { Proline }\end{array}$ & $\begin{array}{l}-\mathrm{H} \\
-\mathrm{CH}_{3} \\
-\mathrm{CH}\left(\mathrm{CH}_{3}\right)_{2} \\
-\mathrm{CH}_{2}\end{array}$ & $\begin{array}{l}760 \\
470 \\
490 \\
520\end{array}$ \\
\hline & $\begin{array}{l}\text { Leucine } \\
\text { Isoleucine } \\
\text { Phenylalanine }\end{array}$ & $\begin{array}{l}-\mathrm{CH}_{2}-\mathrm{CH}\left(\mathrm{CH}_{2}\right)_{2} \\
-\mathrm{CH}\left(\mathrm{CH}_{2}\right)_{2}-\mathrm{CH}_{2}-\mathrm{CH}_{3} \\
-\mathrm{CH}_{2}-\mathrm{C}_{6} \mathrm{H}_{5}\end{array}$ & $\begin{array}{l}680 \\
270 \\
260\end{array}$ \\
\hline
\end{tabular}

The amino acids of wool fibres make up polypeptide chains of irregular structure, and the side chains of the amino acids determine the chemical reactivities of the fibres. Crosslinks occur between the side chains of the amino acid residues of different polypeptide chains, examples of which are presented in Figure 1.3. ${ }^{9}$ The disulfide bridges of cystine can occur both in the same polypeptide chain, and between different polypeptide chains. The disulfide crosslinks impart the greatest amount strength to the wool fibres. ${ }^{8}$ Isopetide bonds are also strong, but are rarer than the disulfide bridges. Hydrogen, hydrophobic and ionic bonds exist between peptide chains and provide additional stabilisation. 


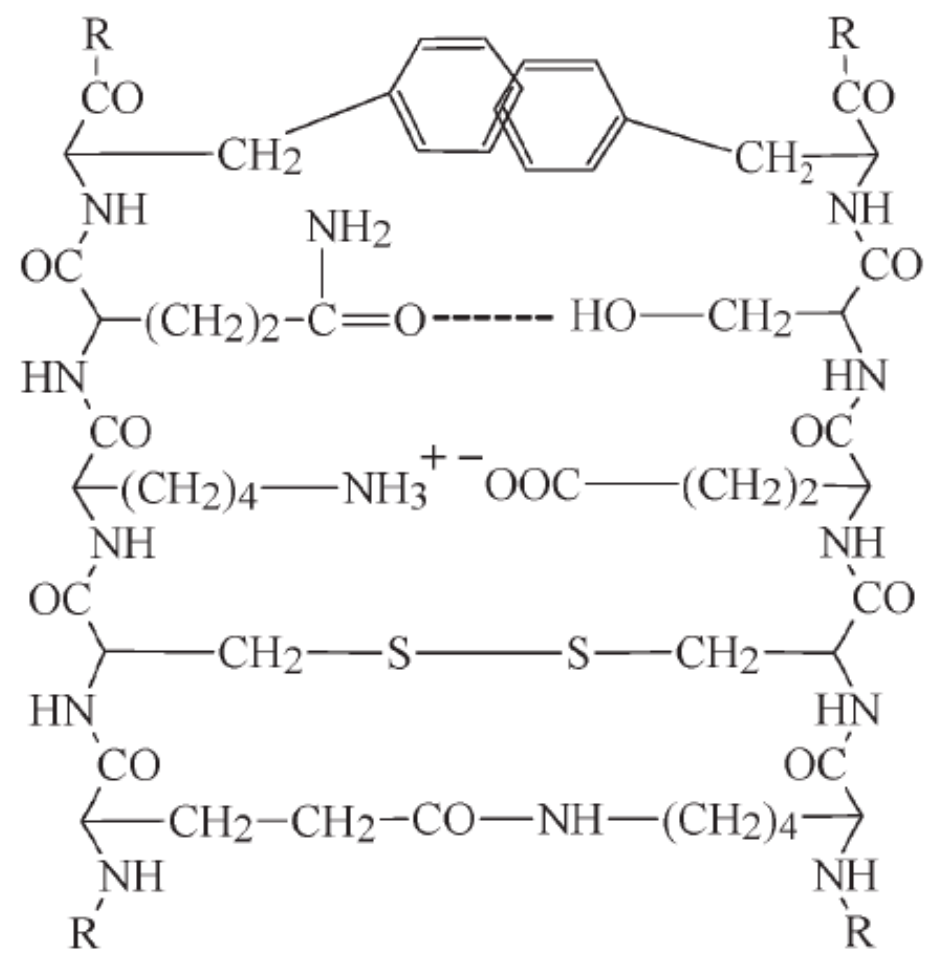

Figure 1.3 Possible crosslinks between different peptide chains in wool; $\pi-\pi$ interactions, hydrogen bonding, ionic, disulfide and isopeptide crosslinks (from top to bottom). ${ }^{9}$

The physical structure of wool fibres consists of a fibril reinforced matrix, and is illustrated in Figure 1.4, ${ }^{7}$ where the $\alpha$-helix of the keratin can be seen. The flattened, cuticle cells on the exterior of the wool protect the long, polyhedral cortical cells in the fibre interior. The cuticle and cortical cells are separated by (and embedded in) a cell membrane complex, which is composed of proteins and internal lipids. ${ }^{7}$ The intercellular proteins establish strong cohesion between the cells, and account for the fibril reinforced matrix structure of the fibres.

The cortex comprises approximately $90 \%$ of the composition of wool fibres, and is made up of two different types of cells; the ortho- and paracortical cells. ${ }^{7}$ The paracortex contains a comparitively high sulfur content, and therefore is more heavily crosslinked than the orthocortex. In fine wool types such as merino wool, there is a bilateral arrangement of the orthocortex and paracortex cells, which winds helically along the axis of the fibres. This arrangement is illustrated in Figure 1.5, where the more tightly bound paracortex is situated in the inner part of the curvature. ${ }^{7}$ This arrangement causes a stable crimp in merino wool, allowing the entrapment of air between the fibres, and aiding the insulating properties of wool fibres.

The cuticle cells are present on the exterior of wool fibres in the shape of rectangular sheets, with dimensions of approximately 20 by 30 microns and a cell thickness of 


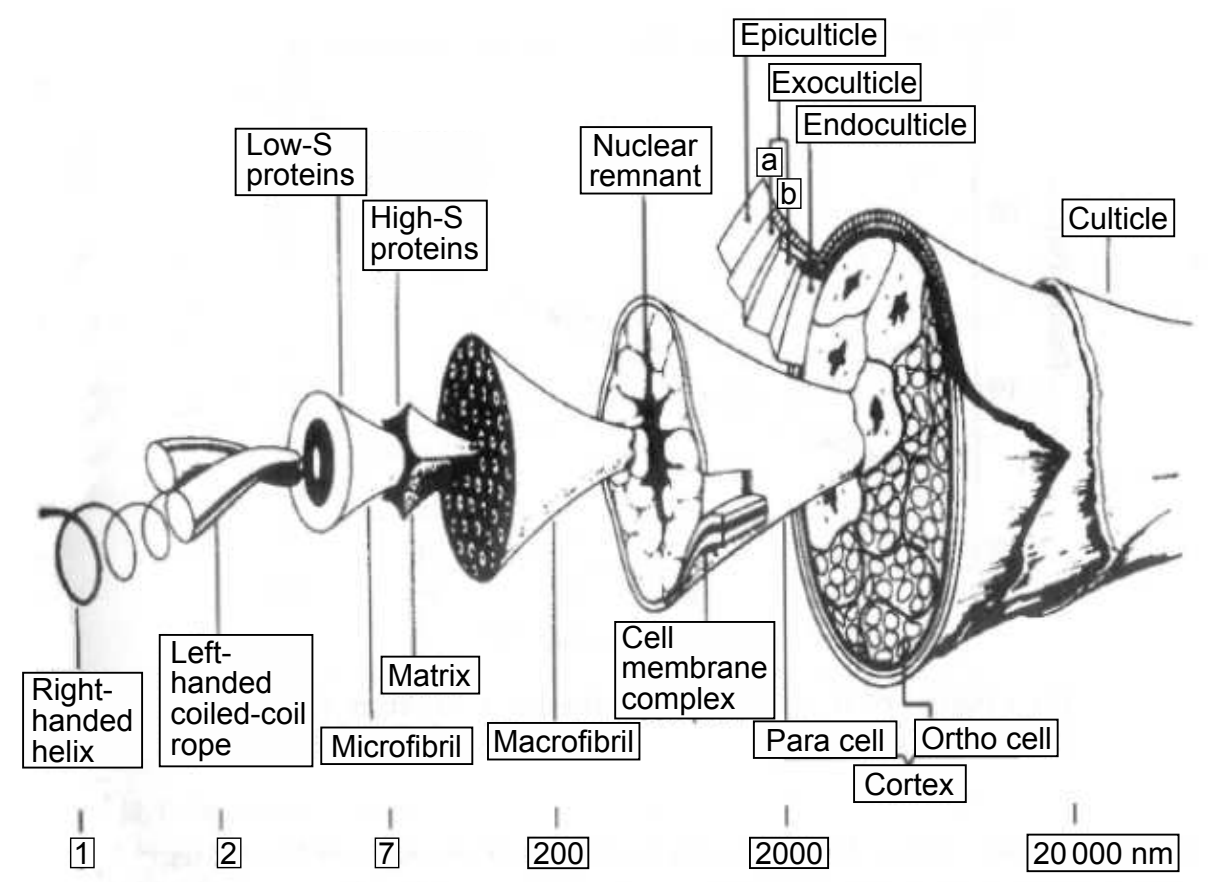

Figure 1.4 Illustration of the physical structure of wool. ${ }^{7}$

0.5-0.8 $\mu \mathrm{m} .{ }^{7}$ The cuticle cells make up between $6-16 \%$ of the total composition of wool fibres. ${ }^{7}$ Ultrafine wool fibres that have very small fibre diameters (typically $<15 \mu \mathrm{m}$ ) are surrounded by only one layer of cuticle cells, while thicker fibres can be surrounded by multiple cuticle layers.

The cuticle cells overlap one another in the longitudinal direction on the exterior of the wool fibres. An SEM micrograph of a Crossbred wool fibre is presented in Figure 1.6, where the overlapping cuticle cells are oriented from the root to the tip of the fibre, in a way which is analogous to roof tiling. This orientation imparts a self-cleaning mechanism to the wool fibres, as dirt is expelled towards

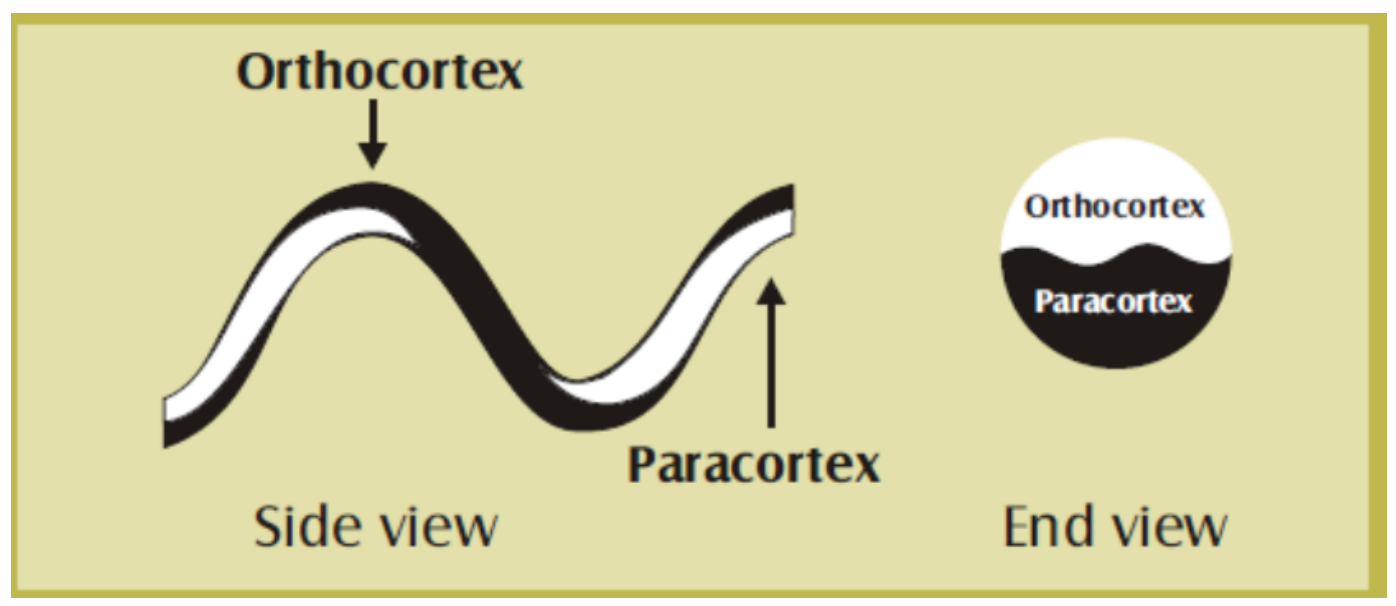

Figure 1.5 Representation of the organisation of the ortho- and para-cortex of merino wool fibres. ${ }^{7}$ 
the tip when fibres rub together. This structural characteristic also allows for the felting of wool fibres; when wool fibres rub together, they move preferentially in one direction, and the edges of the cuticle scales can interlock. ${ }^{8}$ Extensive agitation of wool fibres (particularly when wet), leads to felting and fibre shrinkage, and as such, is an important consideration during dyeing or laundering processes. The edges of cuticle scales may also provide more attractive surfaces for the adsorption of gold nanoparticles, due to these areas allowing access to the wool surface from two planes.

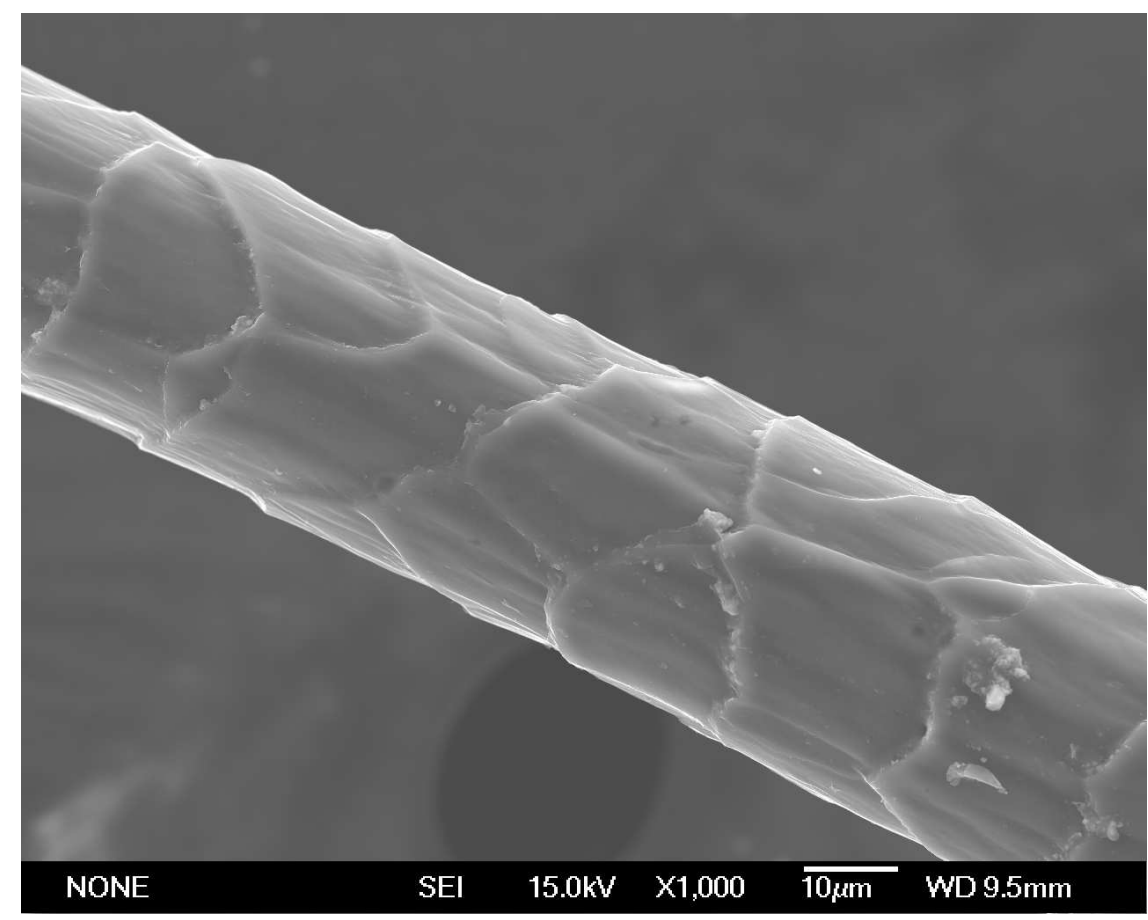

Figure 1.6 SEM micrograph in SEI mode of a crossbred wool fibre, illustrating the overlapping nature of exterior cuticle cells.

Cuticle cells consist of four different layers; the epicuticle, the A-, and B-layers of the exocuticle and the endocuticle (Figure 1.4). The outer epicuticle layer surrounds each cuticle cell distinctly, and is only $3-6 \mathrm{~nm}$ thick, making up only approximately $0.1 \%$ of the wool fibre's total composition. ${ }^{8}$ The epicuticle consists of $80 \%$ proteins, with the remainder comprising a relatively high lipid and membrane component, and is highly resistant to alkali, acidic, oxidising and proteolytic enzymatic attack. ${ }^{10} \mathrm{~A}$ lipid, namely 18-methyleicosanoic acid (18-MEA), is considered to be attached to the wool fibres via a thioester bond with cysteine residues of the epicuticle. ${ }^{11}$ The presence of 18-MEA, the structure of which is shown in Figure 1.7, adds to the hydrophobicity of the wool fibres.

The exocuticle is sulfur-rich; the A- and B-layers comprising 35 and $20 \%$ sulfur respectively. ${ }^{7}$ In contrast, the endocuticle has a very low sulfur content, and a corresponding low-degree of disulfide crosslinking. ${ }^{7}$ As such, the endocuticle is the most readily permeable of the cuticle layers, and the diffusion of water and chemical 


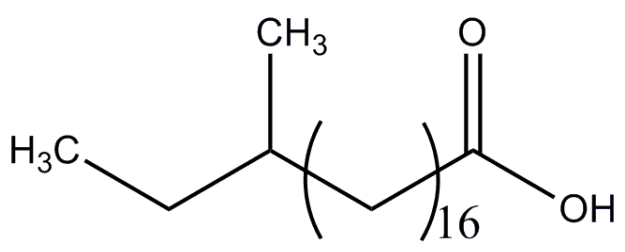

Figure 1.7 18-methyleicosanoic acid (18-MEA), the fatty acid which is covalently bound to the exterior of wool fibres.

reagents inside the wool fibres preferentially occurs through the endocuticle. ${ }^{7}$

The structural characteristics of wool fibres determine their chemical and physical properties, are influenced by the type of wool (through sheepbreed and husbandry conditions), and are important to consider when synthesising gold nanoparticle-wool fibre composites.

\subsubsection{Wool Fibre Chemistry}

Wool fibres undergo chemical reactions when exposed to acids, alkalis, oxidising and reducing agents, electromagnetic radiation and heat. Wool fibres are often exposed to these conditions during the husbandry or industrial processing and dyeing of wool fibres. These reactions can result in damage to the wool fibres, and as such, the effects of these reactions are important to consider in relation to this research project. This is especially so where the chemistry of cystine is concerned (the structure of which is presented in Figure 1.8), as it provides the greatest amount of strength to the wool fibres and has a high affinity for gold.

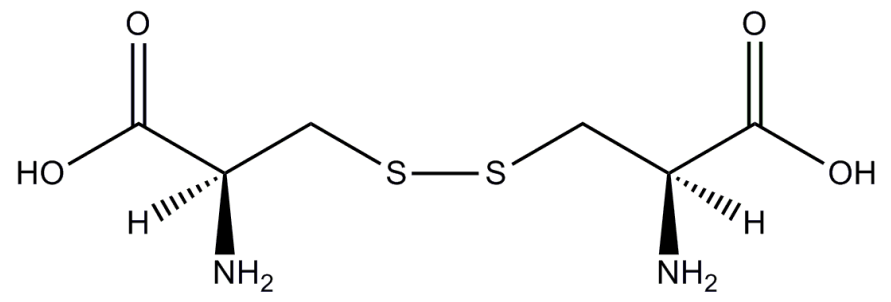

Figure 1.8 The structure of cystine.

\section{Selective Reduction of Wool Fibres}

The selective reduction of cystine in wool fibres is commonly employed in the industrial processing of wool, for example in the permanent creasing in woollen fabrics. This involves the reductive cleavage of the disulfide crosslinks of cystine, reshaping 
of the wool fibres, and the reformation of the disulfide crosslinks in the new shape (the same principles apply to the permanent waving of human hair). ${ }^{7}$ The selective reduction of cystine is also used in shrink resistive treatments. However, these treatments also reduce the strength of the wool fibres, due to an associated loss of stabilising disulfide linkages in the wool protein.

The selective reduction of cystine residues is typically achieved with the use of thiolbased reducing agents, for example thioglycollic acid (TGA). Other reducing agents, for example sodium borohydride $\left(\mathrm{NaBH}_{4}\right)$, are non-specific to cystine and cause the unwanted hydrolysis of peptide bonds. ${ }^{12}$ The reductive cleavage of the disulfide bond in cystine occurs via two reversible nucleophilic displacement reactions, as is seen in Equation 1.1 (where W refers to a polypeptide chain in wool). ${ }^{12}$

$$
\begin{aligned}
& \mathrm{WSSW}+\mathrm{RS}^{-} \rightleftharpoons \mathrm{WSSR}+\mathrm{WS}^{-} \\
& \mathrm{WSSR}+\mathrm{RS}^{-} \rightleftharpoons \mathrm{RSSR}+\mathrm{WS}^{-}
\end{aligned}
$$

The reactivities of different types of thiol-reagents are influenced by the $\mathrm{pK}_{a} \mathrm{~s}$ and redox potential values. ${ }^{12}$ Thiols typically have $\mathrm{pK}_{a}$ values of $9-10$, and the reactivity of thiols can be substantially increased if the reduction takes place at a $\mathrm{pH}$ at which the thiol is deprotonated. ${ }^{12}$ In contrast, the extensive reduction of disulfides in wool under acidic conditions requires a large excess of thiols. ${ }^{12}$ Additionally, the thiol groups that are formed upon the reduction of cystine are rapidly re-oxidised to disulfides in air, so subsequent reactions must be undertaken quickly. ${ }^{12}$

\section{Sulfitolysis of Wool Fibres}

Sulfitolysis reactions are commonly employed in the industrial processing of wool fibres, typically to aid in the setting of wool fibres or to provide additional reactive sites for the attachment of dye molecules. ${ }^{12}$ Sulfitolysis is the reversible cleavage of a disulfide group by sulfite to give a thiol and S-sulfonate anion $\left(\mathrm{SSO}_{3}^{-}\right){ }^{12}$ When cystine residues in wool fibres undergo sulfitolysis, it results in the deprotonated forms of cysteine and cysteic acid residues (Equation 1.2). ${ }^{12}$

$$
\mathrm{WSSW}+\mathrm{SO}_{3}{ }^{2-} \rightleftharpoons \mathrm{WS}^{-}+\mathrm{WSSO}_{3}^{-}
$$


Sulfitolysis is carried out with sulfite $\left(\mathrm{SO}_{3}{ }^{2-}\right)$, bisulfite $\left(\mathrm{HSO}_{3}^{-}\right)$or disulfite $\left(\mathrm{S}_{2} \mathrm{O}_{3}{ }^{2-}\right)$, which exist together in equilibrium in aqueous media. ${ }^{7}$ Bisulfite is the predominant species at an acidic $\mathrm{pH}$, and sulfite is most common in alkaline conditions $(\mathrm{pH}>7)$. Sulfitolysis occurs more readily when the $\mathrm{pH}$ of the reaction is increased up to $\mathrm{pH} 9$, due to a greater reactivity of sulfite over bisulfite. However, at a reaction $\mathrm{pH}$ of $>9$ a reversible bimolecular displacement reaction occurs in place of sulfitolysis, and is shown in Equation 1.3. ${ }^{7}$

$$
\mathrm{WSSW}+2 \mathrm{SO}_{3}^{2-} \rightleftharpoons 2 \mathrm{WSSO}_{3}^{-}
$$

Similar to the selective reduction of cystine, the sulfitolysis products are also rapidly re-oxidised to disulfides upon exposure to air. Thus, it is imperative that subsequent dyeing reactions take place immediately following this treatment.

\section{Oxidation of Wool Fibres}

Wool fibres are oxidised industrially, either to bleach the wool or to impart shrink resistance to the fibres. Oxidative bleaching degrades yellow-coloured chromophores that are present in the wool protein, and when amino acids that are located in the cuticle scales are oxidised, they soften and hence have a lesser propensity to felt. ${ }^{12}$ Certain amino acids in wool, namely cystine, cysteine, methionine and tryptophan, are most likely to undergo oxidation. ${ }^{12}$ The extensive oxidation of cystine results in a corresponding loss of fibre strength, therefore industrial oxidative processes only partially oxidise the cystine residues (for example to $\mathrm{W}-\mathrm{CH}_{2}-\mathrm{SO}_{2} \mathrm{H}$ ), or limit the amount of cystine residues that are oxidised.

Oxidative bleaching is typically achieved with hydrogen peroxide under alkaline conditions. ${ }^{12}$ Hydrogen peroxide liberates reactive oxygen species (ROS) in alkaline conditions, as is represented in Equation 1.4. The ROS degrade yellow coloured chromophores, in addition to oxidising cystine to cysteic acid. As such, there is a corresponding trade-off between whiteness and fibre strength.

$$
\mathrm{H}_{2} \mathrm{O}_{2}+\mathrm{OH}^{-} \rightleftharpoons \mathrm{OOH}^{-}+\mathrm{H}_{2} \mathrm{O} \longrightarrow \mathrm{OH}+\mathrm{O}_{2}^{-}+\mathrm{H}_{2} \mathrm{O}
$$

The complete oxidation of a cystine residue results in a cysteic acid residue, as can be seen in Equation 1.5. ${ }^{12}$ The oxidation is thought to occur through a number of 
steps, and as such partial oxidation products result if the oxidation is incomplete.

$$
\text { WSSW } \stackrel{R O S}{\longrightarrow} 2 \mathrm{WSO}_{3} \mathrm{H}
$$

Oxidative shrink resistive treatments are carried out either to swell the cuticle scales, or as a pre-treatment to the attachment of polymers to wool fibres (this is explained in more detail in Section 1.1.3). Again, there is a trade-off between the extent of oxidation and the corresponding loss in fibre strength that is caused by the treatment.

The chemistry of the reduction, sulfitolysis and oxidation of wool fibres has two important implications. Firstly, the wool fibres that were used in this research project may exhibit different properties, in terms of surface chemistry and reactive sites, due to being potentially subjected to such chemical treatments. Secondly, these reactions may be employed in laboratory experiments, to attempt to increase the reactive sites that are available for the attachment of gold nanoparticle colourants.

\section{The Interaction of Wool with Acids and Bases}

The industrial processing or dyeing of wool fibres often involves acidic or alkaline media. As such, the interaction of wool with acids and bases is important to consider in this research project, and is illustrated in Equation 1.6 below. ${ }^{7}$ The isoelectric region of wool, the $\mathrm{pH}$ at which the surface of the fibres do not exhibit excess charge (or have a zeta potential of zero), typically occurs in the range of $\mathrm{pH} 2-5 .{ }^{7}$ In the isoelectric region, the wool fibres are zwitterionic, where the positively charged amine groups balance the negative charge of the carboxylate groups of the wool protein.

$$
\mathrm{H}_{3} \mathrm{~N}^{+}-\mathrm{W}-\mathrm{COOH} \underset{\mathrm{H}^{+}}{\stackrel{\mathrm{OH}^{-}}{\rightleftharpoons}} \mathrm{H}_{3} \mathrm{~N}^{+}-\mathrm{W}-\mathrm{COO}^{-} \underset{\mathrm{H}^{+}}{\stackrel{\mathrm{OH}^{-}}{\rightleftharpoons}} \mathrm{H}_{2} \mathrm{~N}-\mathrm{W}-\mathrm{COO}^{-}
$$

When wool fibres are immersed in strongly acidic or alkaline solutions, the amine and carboxylate groups of the amino acids exhibit a buffering effect to stabilise the proteinaceous structure. ${ }^{7}$ An electrostatic barrier forms at the fibre surface after the initial absorption of $\mathrm{H}^{+}$or $\mathrm{OH}^{-}$ions, and as such equilibration is slow (up to $1 \mathrm{~h}$ for wool in solutions in the $\mathrm{pH}$ range of $3-10) .{ }^{7}$ Increasing the ionic strength of a solution (via the addition of a neutral salt such as $\mathrm{NaCl}$ ), increases the rate of ion 
exchange, due to the masking of the electrostatic barrier at the fibre surfaces. ${ }^{7}$ As such, electrolytes can be added to dyeing reactions to increase the rate of absorption of dye molecules.

Wool fibres can degrade when soaked in strongly acidic or alkaline media. Immersion of wool fibres in an excess of strong acid can hydrolyse the amide groups in the wool protein, which liberates ammonium from the wool and forms additional carboxylate groups. ${ }^{7}$ The degradation of wool fibres in alkaline solutions is comparatively more damaging, and involves the nucleophilic attack of the disulfide bond in cystine residues by hydroxide ions. ${ }^{12}$ This is illustrated in Equation 1.7, and results in the conversion of cystine residues to lanthionine residues, and a corresponding reduction in the sulfur content and loss of strength of the fibres. ${ }^{12}$

$$
\mathrm{WSSW}+\mathrm{OH}^{-} \rightarrow \mathrm{W}-\mathrm{S}-\mathrm{S}^{-}+\mathrm{W}-\mathrm{OH} \rightarrow \mathrm{W}-\mathrm{S}-\mathrm{W}+\mathrm{S}+\mathrm{OH}^{-}
$$

This implies that wool fibres should preferably be soaked in strongly acidic solutions for short periods of time, and only in mildly alkaline solutions, so that this degradation can be avoided during this research project.

\section{The Photodegradation of Wool Fibres}

Wool fibres undergo chemical reactions, including photodegradation, as a result of exposure to ultraviolet (UV) and visible light during sheep husbandry and the outdoor use of woollen products. Amino acid residues in wool, specifically tryptophan and tyrosine, are considered to absorb significant amounts of UV radiation. ${ }^{13}$ The aromatic structures of tryptophan and tyrosine are presented in Figure 1.9. The absorption of sunlight by these amino acid residues can lead to the formation of photo-decomposition products from degradation of the wool protein, which result in lower strength and can also change the colour of the wool.
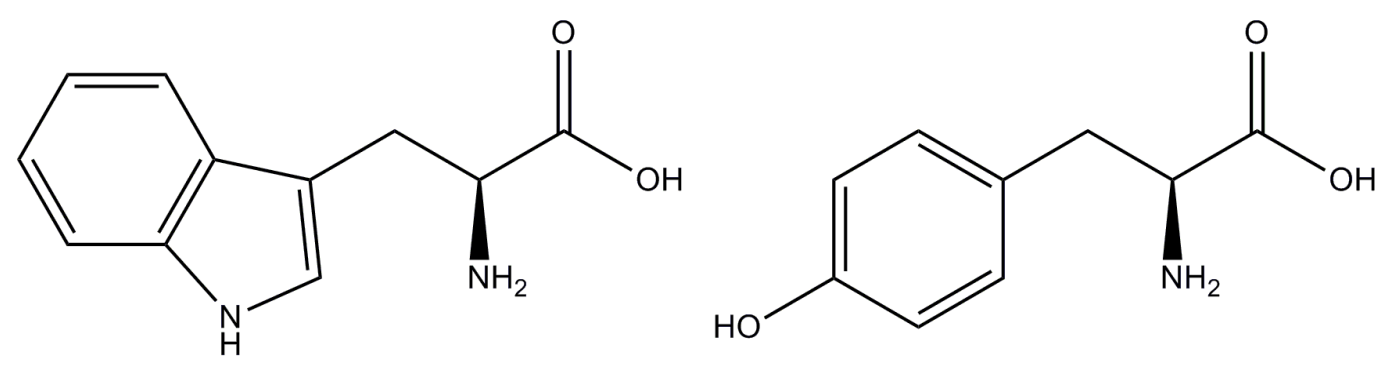

Figure 1.9 The structure of tryptophan (left) and tyrosine (right), the aromatic amino acid residues in wool fibres that absorb significant amounts of sunlight. 
When tryptophan or tyrosine absorb UV radiation, electrons are excited from the ground state to an singlet or triplet excited state. The excited triplet state of tryptophan is able to transfer its energy to ambient molecular oxygen, which forms singlet oxygen (an excited state of molecular oxygen). ${ }^{14}$ Singlet oxygen is a potent oxidising agent, and degrades nearby amino acid residues (including tryptophan, tyrosine and cystine). ${ }^{13}$ Tyrosine is considered to be able to transfer energy (gained from the absorption of UV light) to tryptophan, which is able to then produce additional singlet oxygen molecules. ${ }^{13}$

The photo-decomposition products that result from the exposure of wool to UV light are sometimes yellow chromophores. However, these yellow degradation products are further degraded by visible light. As such, photo-bleaching wool fibres with visible light has been considered as an industrial alternative to chemical oxidative bleaching methods for wool fibres. ${ }^{7}$

The photodegradation of wool during weathering primarily affects the tip of the fibres due to greater sunlight exposure over time. The cuticles become damaged, which allows dye molecules to diffuse through the cuticle scales (as well as diffusing through the junctions between cuticle cells). ${ }^{8}$ Photostabilisers may be added to wool to attempt to reduce the photodegradation of finished woollen products, for example $\mathrm{ZnO}_{2}$, which absorbs UV light in place of the wool fibres.

\section{The Heating of Wool Fibres}

Wool fibres are heated during industrial processing, particularly in scouring and dyeing stages. ${ }^{12}$ This heat can damage the wool fibres. When wet wool fibres are heated up to $55^{\circ} \mathrm{C}$, hydrogen sulfide is slowly liberated from wet wool fibres as cystine residues slowly degrade into lanthionine residues. At temperatures of $>100{ }^{\circ} \mathrm{C}$, ammonia is also liberated from wet wool fibres, as lysine and alanine residues are degraded to lysinoalanine. ${ }^{12}$ The structures of lanthionine and lysinoalanine are shown in Figure 1.10.
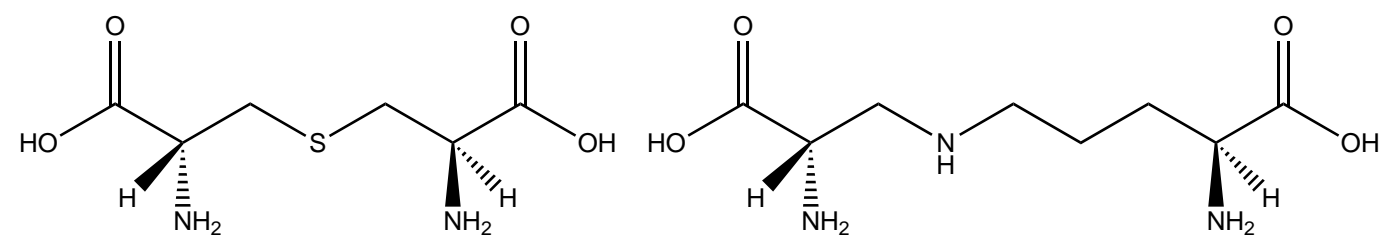

Figure 1.10 The structures of lanthionine (left) and lysinoalanine (right), which are produced when wet wool is heated for extended periods of time. 
The chemical changes that occur when wet wool is heated above $100{ }^{\circ} \mathrm{C}$ cause a corresponding loss in tensile strength, ${ }^{12}$ and can also form yellow coloured degradation products. ${ }^{7}$ This is called hydrothermal yellowing. Despite this, industrial dyeing procedures often involve temperatures of up to $120^{\circ} \mathrm{C}$ with acidic dyebaths, typically for 15-30 min. The degradation is substantially enhanced under alkaline conditions, and as such, these conditions are generally avoided. Wool fibres in this research project will only be heated to $100^{\circ} \mathrm{C}$ for $<1 \mathrm{~h}$, and under mildly acidic or neutral conditions, to avoid such damage. Wool fibres also undergo thermal degradation in the dry state, but require higher temperatures, or longer heating times, to result in similar chemical changes to that which occurs when wet fibres are heated. ${ }^{12}$

Wool fibres that are used in this research project may have been exposed to UV and visible radiation or heat during weathering on the sheep or in industrial processing. As such, wool types that are sourced from different processing routes may exhibit different dyeing chemistries. Additionally, radiative exposure of wool fibres during laboratory experiments (for example UV-light or microwave radiation) may alter the chemistry of the wool fibres, intentionally or as a side effect of the experiments.

\subsubsection{Wool Processing}

There is a diverse range of sheepbreeds in New Zealand, which produce different types of wool fibres. Of these sheepbreeds, Merino and Crossbred wool types are of particular interest to this research project. New Zealand Merino wool is soft and fine, with fibre thickness between $14-22 \mu \mathrm{m}$, and have a soft feel. ${ }^{7}$ In contrast, Crossbred wool is relatively strong and the fibres exhibit diameters of 30-39 $\mu \mathrm{m}$. Merino and Crossbred wool are used in garment and carpet manufacture, respectively. The comparatively fine Merino fibres have higher surface area to volume (or weight) ratios compared to Crossbred wool types, and are generally less porous. Additionally, wool that is sourced from different sheepbreeds exhibit unique amino acid composition and cuticle cell layer thickness (which was mentioned in Section 1.1.1). Further variation in the chemistry and properties of wool fibres are caused by the industrial

processing that occurs in the supply chain of wool. A summary of the different processes steps, which occur sequentially, is presented in Table 1.2.

The dyeing of wool may be carried out with wool that is sourced from different stages of the supply chain. There are comparative advantages and disadvantages of dyeing wool in different forms, which will be discussed further in Section 1.1.4. The forms of wool that were most extensively used in this research project are loose wool, 
Table 1.2 Common steps in the processing of wool fibres and the name of the type of wool that results from the processing steps, which occur sequentially from scouring to knitting.

\begin{tabular}{ccc}
\hline Processing step & Description & Wool type \\
\hline Scouring & Washing wool with hot water and detergents & Loose wool \\
$\downarrow$ & Removing vegetable matter & $\downarrow$ \\
Carding & Open top \\
$\downarrow$ & & $\downarrow$ \\
Combing & Spinning wool fibres into yarn & Top form \\
$\downarrow$ & & $\downarrow$ \\
Spinning & Yarn \\
$\downarrow$ & & $\downarrow$ \\
Knitting & Knitting finished products from yarn & Piece \\
\hline
\end{tabular}

top form wool and finished yarn, samples of which are presented in Figure 1.11.

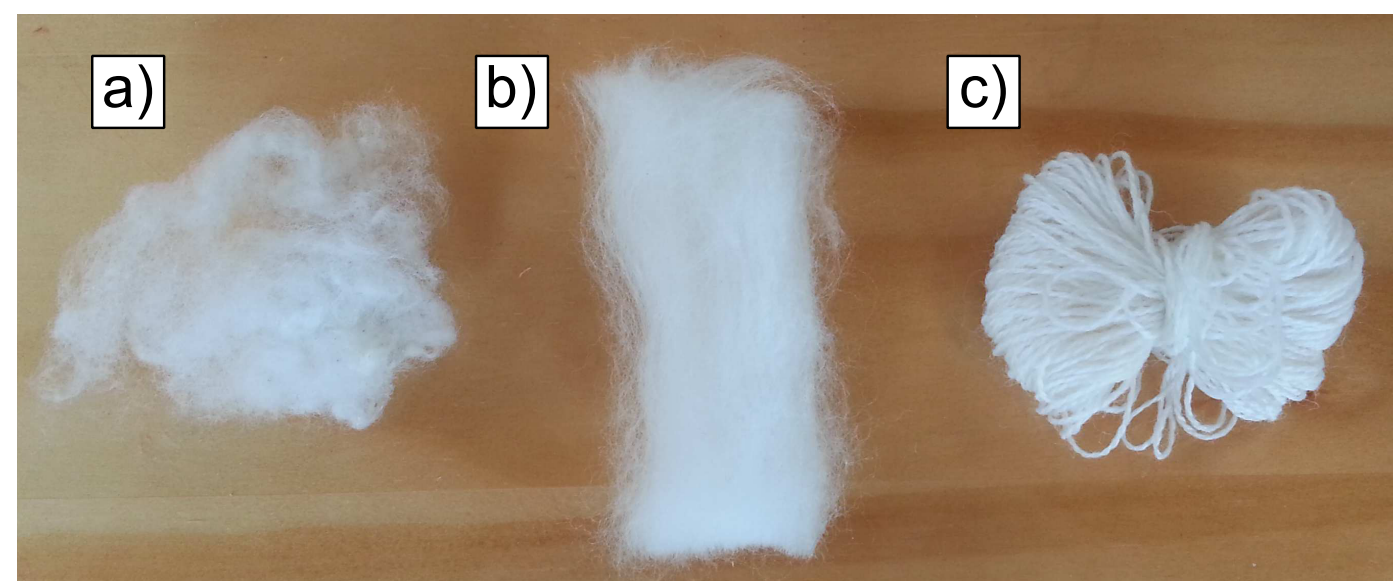

Figure 1.11 Different forms of wool fibres; loose wool (a), top form wool (b) and woollen yarn (c).

Lubricants are often applied to wool fibres in the different processing stages (particularly during carding and spinning). The presence of such lubricants can alter the surface chemistry of the wool fibres, and is an important consideration in this research project. Additionally, the processing of New Zealand wool is typically outsourced to China, and the specifications of the processing are unknown. Surface treatments, such as the removal of the 18-MEA layer or the attachment of shrink resistive polymers, may also be applied. 


\section{Surface Treatments}

One of the most commonly employed surface treatments involves the removal of the 18-MEA layer from wool fibres. As was mentioned in Section 1.1, 18-MEA is covalently bound to the epicuticle of wool fibres, and imparts hydrophobic properties. Thus, the 18-MEA layer is often removed to make the wool more hydrophilic and increase the ease of wetting the fibres. The 18-MEA layer is removed by soaking wool fibres in a solution of $\mathrm{KOH}\left(0.1 \mathrm{~mol} \mathrm{~L}^{-1}\right)$ in methanol, where the $\mathrm{CH}_{3} \mathrm{O}^{-}$nucleophile is considered to attack the carbonyl group of the thioester, ${ }^{15}$ an illustration of which is shown in Figure 1.12.
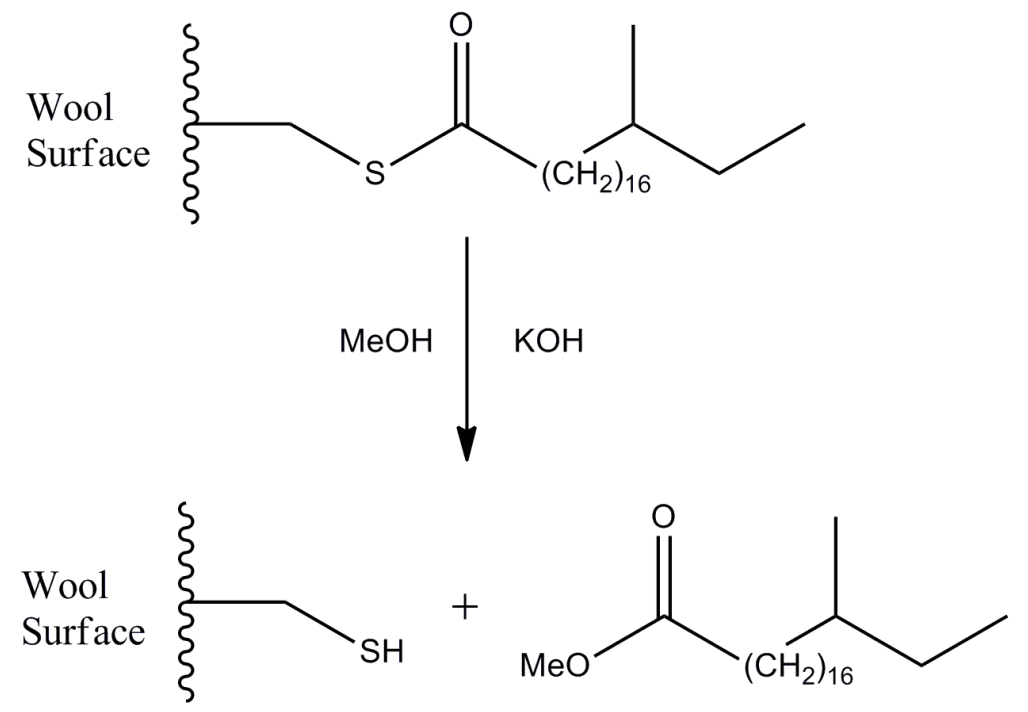

Figure 1.12 The proposed removal of 18-MEA from the surface of wool fibres. ${ }^{15}$ The application of a shrink resistive polymer to wool fibres is another common surface treatment, which is employed as an alternative to oxidative shrink resist treatment. The cationic polyamide Hercosett 125 is typically employed, the structure of which is shown in Figure 1.13. ${ }^{7}$ This treatment involves oxidation by chlorinating the wool fibres and then soaking in an alkaline solution (to provide additional negatively charged sites), before the cationic polymer is attached to the wool. Hercosett 125 has a propensity to swell to ten times its volume when immersed in water. ${ }^{7}$ The swelling imparts a smooth surface to the wool (that covers the cuticle scales), and as such, the felting and shrinkage of fibres during wet agitation is considerably diminished.

There are more than 100 different shrink resistive polymers, ${ }^{7}$ which demonstrates the extensive diversity in potential surface treatments that can be applied to wool fibres. This, together with the variation in the amino acid composition of wool that is sourced from different sheepbreeds, husbandry conditions and processing routes result in substantial differences in the surface chemistry of wool fibres that 


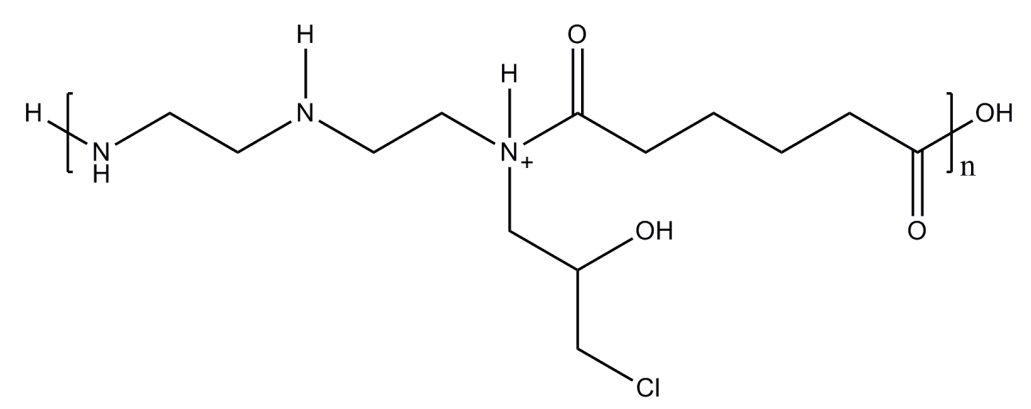

Figure 1.13 Shrink resist polyamide Hercosett $125 .^{7}$

are obtained from different sources. Care is thus needed when selecting a source and form of wool that is used in this research project. Loose wool will be free of surface lubricants, however will retain vegetable matter. Alternatively, the woollen yarn may exhibit a more complicated surface chemistry, but will also require fewer processing stages after the dyeing reactions to produce the finished woollen textile.

\subsubsection{Wool Dyeing}

The dyeing of wool fibres dates back to the days of ancient Egypt, where coloured woven woollen cloths survive to this day. ${ }^{8}$ The wool dyeing industry has evolved to be globally significant. The transfer of dye molecules from an aqueous dyebath to wool fibres is described by the following three stages in wool dyeing theory: ${ }^{12}$

1. Migration of dyes through the aqueous dyebath to the wool fibre surface.

2. Transfer of dye across the fibre surface.

3. Diffusion of dye from the surface into and throughout the whole fibre.

The uniform migration of dye molecules from the dyebath to the wool (in Stage 1) is afforded through the circulation of the dye liquor. ${ }^{12}$ If there is no dye circulation or agitation of the wool fibres, then dye molecules will be absorbed preferentially by the wool fibres that are located on the exterior of the dyepack. Wool dyeing auxiliaries are also employed to slow the rate of uptake of dye molecules, so that uniform absorption and colour of the fabrics can be achieved.

The diffusion of dye molecules from the dyebath into the wool fibres is illustrated in Figure 1.14. In Stage 2, dye molecules migrate across the epicuticle (the outermost layer of wool fibres) to the junctions between the cuticle scales. As mentioned in Section 1.1.1, the epicuticle is resistant to the absorption of dye molecules, and so, 
preferential absorption occurs at the junctions between the cuticle scales. Complications arise when the epicuticle is damaged, and dye molecules are able to diffuse through the cuticle scales (this is called transcellular absorption). The cuticle scales can be damaged when sheep are exposed to harsh weathering conditions, or when wool fibres are subjected to some chemical treatments. ${ }^{8}$

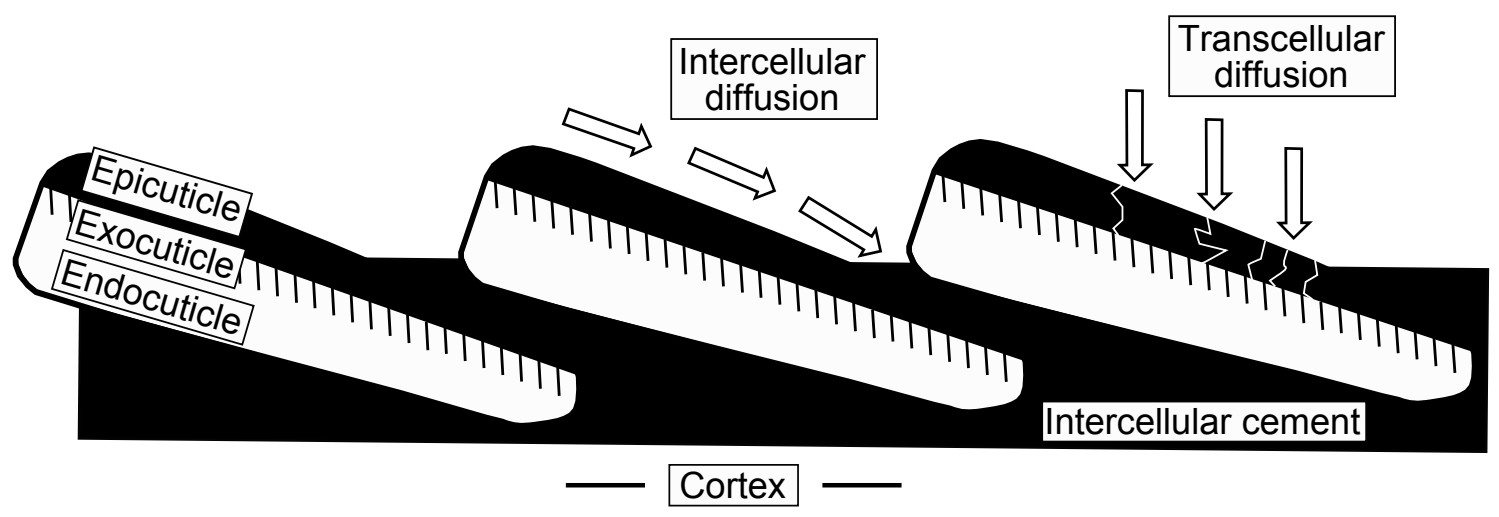

Figure 1.14 The diffusion pathways for dye molecules into wool fibres, where intercellular diffusion occurs at junctions between cuticle scales and transcellular diffusion can occur through damaged cuticle scales. ${ }^{8}$

Stage 3 involves the diffusion of dye molecules from the cuticle cell junctions or damaged epicuticles into the wool fibre interior. The movement occurs sequentially through the endocuticle, the regions of non-keratinous proteins and the regions of the cell membrane complex that are located between cortical cellular macrofibrils. ${ }^{7}$ Finally, the dye molecules progressively transfer into the sulfur-rich matrix proteins surrounding the microfibrils that are located in the cortical cells. ${ }^{7}$

The explanation of the uptake of dye molecules to wool fibres has been attempted by a number of absorption theories. The absorption does not follow Fick's laws of diffusion, which predict that a plot of dye uptake versus the square root of time will be linear for most of the uptake, ${ }^{8}$ and treats the wool fibres as cylinders which do not have barriers to absorption. ${ }^{8}$ Thus, the non-Ficksian diffusion is due to the presence of the epicuticle, which is a barrier to dyestuff absorption. The Langmuir, Freundlich and Nernst isotherms (that are used to describe the adsorption of gases on metal surfaces), have also been used to attempt to model the absorption of dye molecules by wool fibres with limited success. ${ }^{8}$ These models assume that the wool is homogeneous, rather than the heterogeneous, rough surfaced natural fibres. The uptake of gold nanoparticle colloids to wool is considered to be different to the uptake of dye molecules in solution, and hence will be studied in this research programme, 


\section{Types of Dyestuffs}

Industrial wool dyeing processes utilise four different classes of dye molecules; acid, reactive, chrome and metal complex dyes. ${ }^{8}$ Figure 1.15 shows examples of some of these dyes. Acid dyes are organic chromophoric molecules that are applied to wool fibres under acidic (or sometimes neutral) conditions. Acid dyes encompass functional groups that make them more soluble in aqueous solutions, typically sulfonic acid (or occasionally carboxylic acid groups), and are manufactured as stable sodium salts. ${ }^{8}$ Metal-complex, chrome and reactive dyes are types of acid dyes, that also contain metal ions or reactive side groups. The chromophore that gives dyes their colour is typically an azo group that is conjugated to aromatic functionality. ${ }^{8}$

Chrome dyes are acid dyes that chelate to chromium ions that covalently bond to carboxylate groups in the wool protein. ${ }^{8}$ Metal complex dyes incorporate transition metal ions that alter the colour of the molecule, but do not bond to the wool fibres (the bonding occurs through the dye ligands). The metal ion is typically $\mathrm{Cr}^{3+}$ or $\mathrm{Co}^{3+}$, and the dye exists in a either a 1:1 or 1:2 ratio of metal to dye molecule (which acts as a ligand). ${ }^{8}$ The colour of metal complex dyes is influenced by the crystal field splitting of the $3 \mathrm{~d}$ electron orbitals of the transition metal complexes.

Reactive dyes incorporate functional groups that are able to covalently bond to the wool protein. The reactive group of the dye in Figure 1.15 (b) exhibits an electron withdrawing chloride that activates a reactive carbon group for nucleophilic attack by a primary amine in the wool protein, providing excellent washfastness properties to the dyed material. ${ }^{8}$

The uptake of acid dyes to wool is typically carried out below the isoelectric region (usually at $\mathrm{pH} 2-3$ ), where there is an electrostatic attraction between the anionic dye molecules and the net positive charge of the wool surface. The uptake of acid dyes by wool in an $\mathrm{HCl}$ dyebath is illustrated in Figure 1.16. This involves the relatively small $\mathrm{H}^{+}$and $\mathrm{Cl}^{-}$ions being rapidly absorbed by the wool, after which the chloride ions are gradually replaced by the larger, anionic dye molecules. The uptake of $\mathrm{AuCl}_{4}^{-}$to wool is considered to follow a similar profile to that of acid dye absorption. 
a)<smiles>CC(=O)Nc1cc(S(=O)(=O)[O-])cc2ccc(N=Nc3ccccc3)c(O)c12</smiles>

b)<smiles>O=C(Cl)Nc1ccc2c(O)c(N=Nc3ccccc3)c([N+](=O)[O-])cc2c1</smiles>

C)

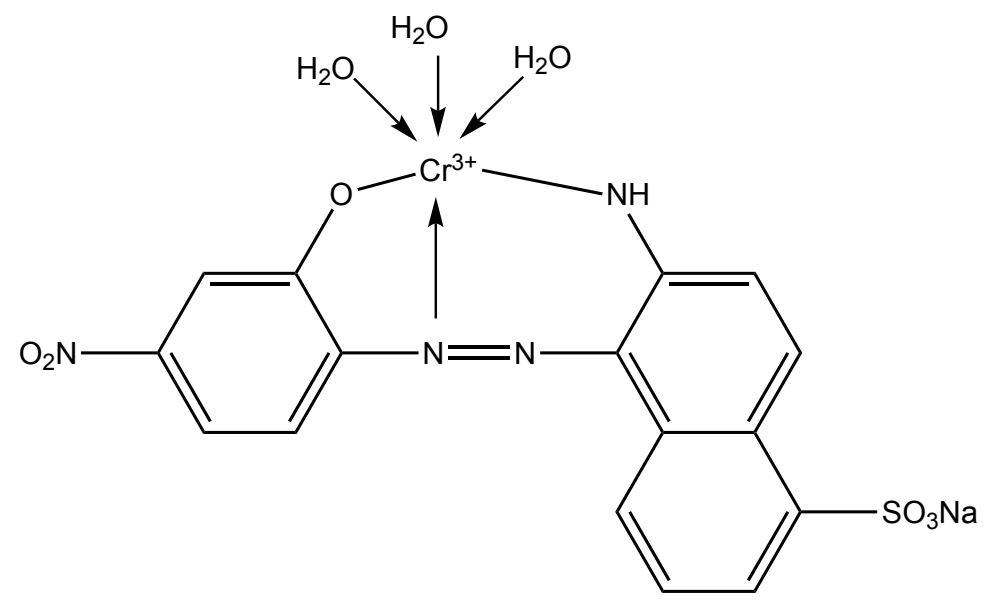

Figure 1.15 Different dyes of dye molecules; acid (a), reactive (b) and 1:1 metal complex dyes (c). ${ }^{8}$

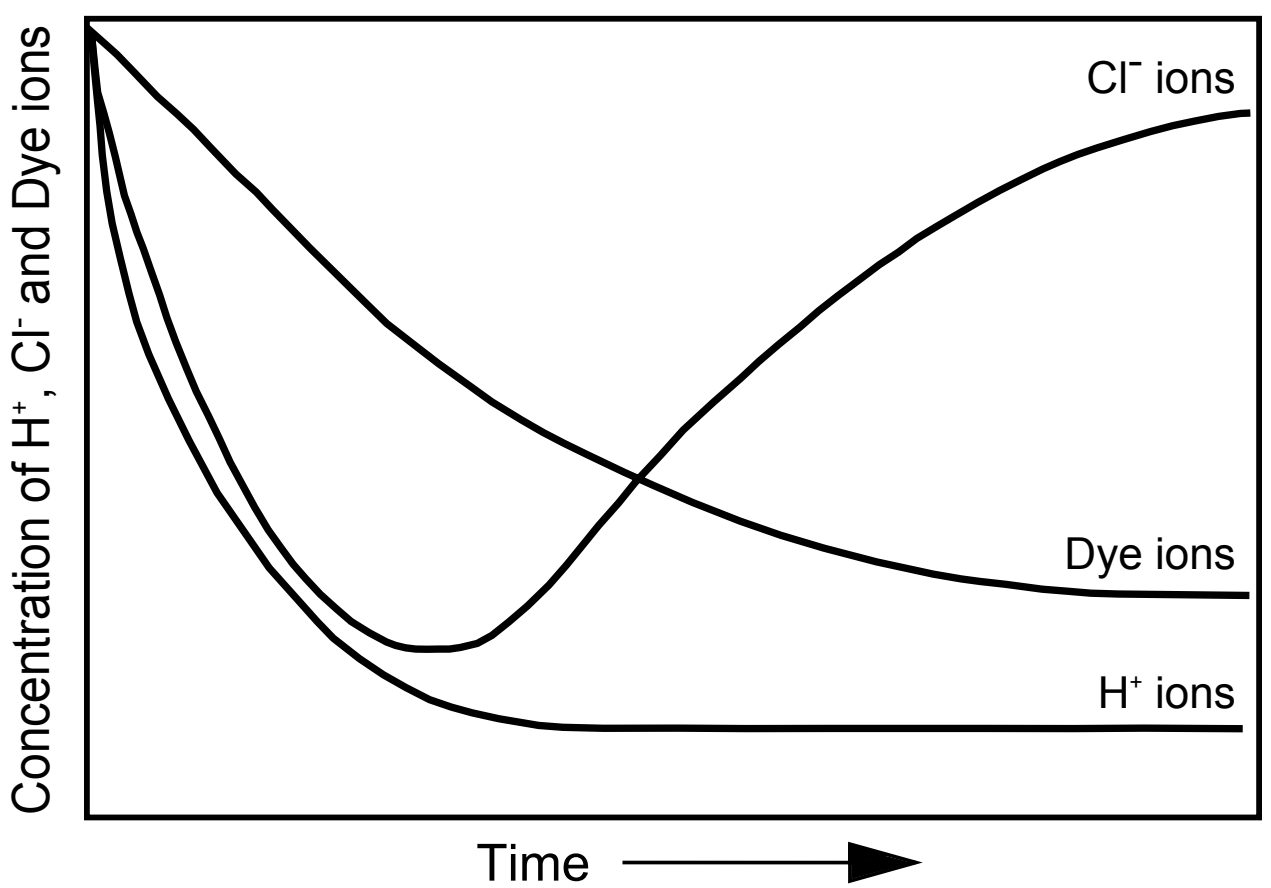

Figure 1.16 Representation of the uptake of $\mathrm{H}^{+}, \mathrm{Cl}^{-}$and dye ions to wool fibres in an acidic dyebath. ${ }^{8}$ 


\section{Types of Dyeing}

Wool fibres may be dyed in top form, as loose wool, as yarn or as a finished garment. ${ }^{8}$ Ideally, wool fibres will be dyed as yarn in this project (if the surface chemistry of the yarn allows it). Woollen yarns that have been dyed may be immediately fabricated into finished products without further industrial processing. Woollen yarns are typically coloured in the form of a loosely wound hank, or a tightly wound cone, which are shown in Figure 1.17.

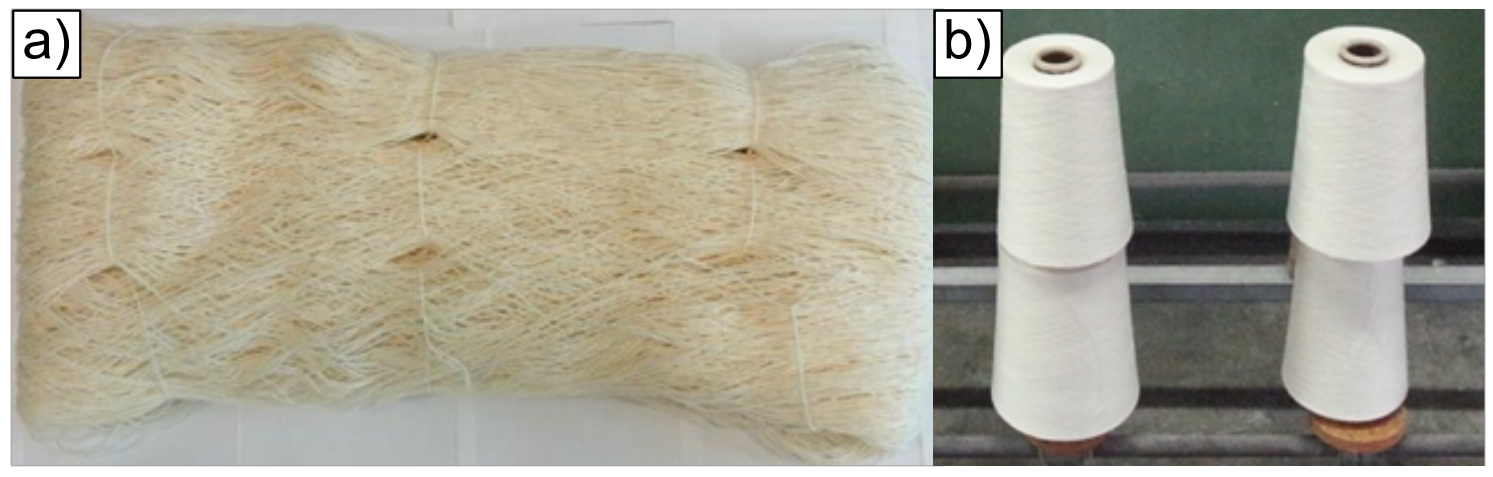

Figure 1.17 A hank of felted woollen yarn (a) and a cone of fine merino yarn (b).

\section{Hank dyeing}

Hank dyeing procedures involve hanging hanks of wool on rods, which are lowered (or sometimes wheeled) into large dye vats (an industrial-scale hank dyeing reactor is shown in Figure 1.18 (a)). The dye liquor is circulated around the system to promote uniform absorption of dye molecules. The flowrates of dye circulation are necessarily gentle, as the hanks are relatively loosely held in place, and vigorous agitation of the hanks will damage and felt the wool fibres. ${ }^{8}$ Hank dyeing reactors are composed of stainless steel, and often employ temperature and $\mathrm{pH}$ gradients during the dyeing reactions (through gradual heating and acid addition) to control the rate of dye uptake.

\section{Package dyeing}

Package dyeing involves the tight winding of woollen yarn onto perforated cones, that are then loaded onto spindles (this can be seen Figure $1.18(\mathrm{~b})$ ). Dye liquor is pumped up out of the holes in the spindle, through the perforated cones and around the dyeing reactor at flowrates that are significantly faster compared to those of hank dyeing procedures. The tight winding of the yarn prevents fibre felting, although some mechanical fibre damage is still possible with this dyeing method. ${ }^{8}$ Again, the 


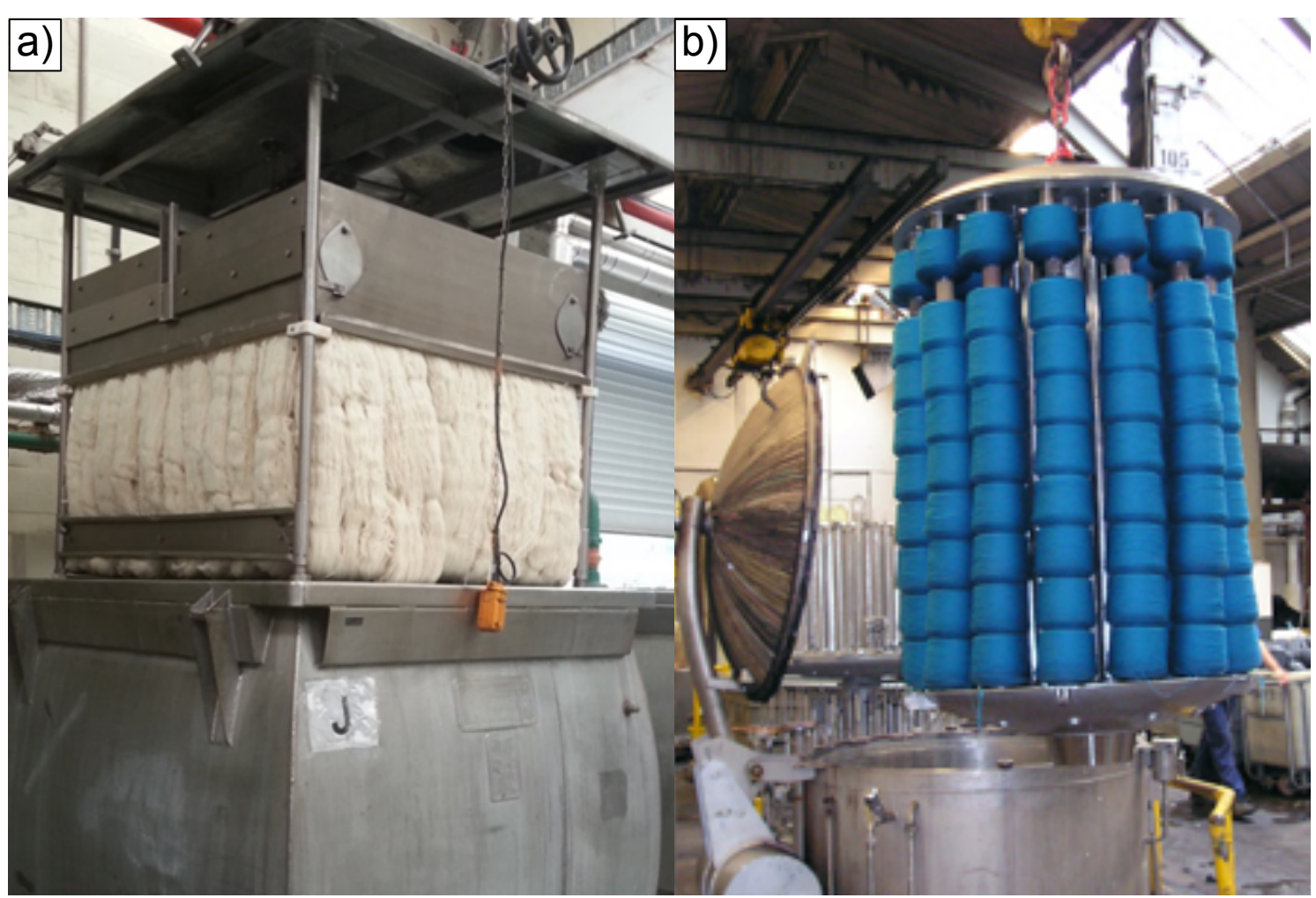

Figure 1.18 Industrial-scale hank (a) and package (b) dyeing reactors.

temperature and $\mathrm{pH}$ of the dyeing reactions, in addition to flow direction, can be manipulated during the package dyeing reactions.

\section{Auxiliary Agents}

Attaining uniformly coloured wool samples is a recurring challenge in the wool dyeing industry, and is a key issue that is faced in the synthesis of gold nanoparticle-wool fibre composites. Wool dyeing auxiliaries are often employed in industrial dyeing procedures to increase the uniformity of dye uptake. Auxiliary agents are typically either electrolytes or surfactant-like molecules, and the chemical composition of the commercial reagents is proprietary information, and can be anionic, cationic, nonionic or amphoteric in nature. ${ }^{8}$

There are two classes of auxiliary agents: wetting and levelling agents, which associate to the surface of wool fibres and aid their ability to absorb water, or to control the rate of uptake of dyes respectively. Wetting agents are chemically and structurally similar to modern household detergents, but incorporate comparatively short alkyl chains. ${ }^{8}$ Organic levelling agents associate to the surface of wool fibres, and slow the access of dye molecules to the wool fibres. Salt-based levelling agents, for example $\mathrm{Na}_{2} \mathrm{~S}_{2} \mathrm{O}_{4}$, are rapidly absorbed by wool fibres and provide ionic repulsion to the absorption of anionic dye molecules. 


\subsection{Gold Nanoparticles}

Gold nanoparticles, particles of gold on the length scale of 1-100 nm, exhibit unique properties compared to bulk gold. ${ }^{16}$ These properties are a consequence of high surface area to volume ratios and surface plasmon resonance effects (which are discussed in Section 1.2.2). These properties afford potential applications of gold nanoparticles in catalysis ${ }^{17}$ drug delivery ${ }^{18}$ and sensing. ${ }^{19}$ Gold nanoparticles are resistant to oxidation and photodegradation, and exhibit intense surface plasmon resonances in the visible spectrum of light, which are responsible for their colour. As such, the use of gold nanoparticles as stable and colourfast dyes for the colouring of premium New Zealand wool is an additional exciting and innovative application. This is discussed in Section 1.3.

\subsubsection{Synthesis Methods}

The development of cost-effective and efficient syntheses of metal nanoparticles has been an important focus of nanotechnological research in recent years. Scaling-up the syntheses of nanomaterials is required for the realisation of the extensive application potential of these materials, and efficient syntheses are particularly important for nanoparticles of expensive metals like gold. Gold nanoparticles can be synthesised by top-down approaches, such as laser ablation ${ }^{20}$ and thermal decomposition of metals. ${ }^{21}$ However, bottom-up syntheses in the solution-phase are scalable, and thus more relevant to this research project.

The solution-phase synthesis of gold nanoparticles requires three different components; metallic ions, organic reducing and stabilising agents and a solvent. ${ }^{22}$ The metal precursor is generally a metal ion or coordination complex, in this case $\mathrm{AuCl}_{4}^{-}$. The reducing agent, for example sodium borohydride $\left(\mathrm{NaBH}_{4}\right)$, couples its oxidation to the reduction of the metal precursor. The stabilising agent, such as a thioalkane, associates to the surface of the nanoparticle, and restricts particle growth through some or all of the crystal facets. An illustration of the solution-phase synthesis of gold nanoparticles with these reagents is presented in Figure 1.19. Stabilising agents provide electrostatic or steric repulsion to prevent the aggregation of nanoparticles. ${ }^{23}$

In the synthesis of gold nanoparticles, a reagent which acts as both a reducing agent and a stabilising agent is often employed, for example trisodium citrate (TSC). TSC is able to couple its oxidation with the reduction of $\mathrm{Au}^{3+}$ to $\mathrm{Au}^{0}$, and stabilises the 


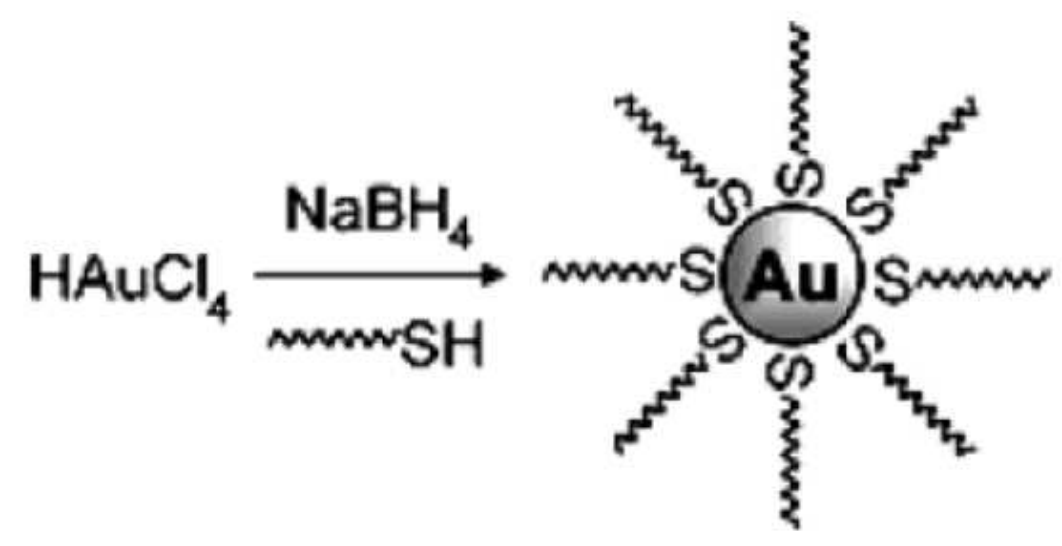

Figure 1.19 The solution-phase synthesis of gold nanoparticles that are stabilised by thioalkanes. ${ }^{24}$

resulting nanoparticles (Figure 1.20). ${ }^{5}$ The use of TSC as a dual reducing and stabilising agent in the solution-phase synthesis of gold nanoparticles was popularised by Turkevitch and co-workers in $1951 .{ }^{25}$ The Turkevitch method involves the reduction of an aqueous solution of $\mathrm{AuCl}_{4}^{-}$via the addition of TSC at $95^{\circ} \mathrm{C}$, developing the ruby-red colour common to spherical gold nanoparticles in less than $10 \mathrm{~min}$. The particle size may be tuned from $10-100 \mathrm{~nm}$ by varying the concentration ratio of $\mathrm{AuCl}_{4}^{-}$to TSC. A variation of the Turkevitch method is used extensively in this research project, where TSC-stabilised gold nanoparticles are used to colour wool fibres.

$$
\begin{aligned}
& \mathrm{HO}-\underset{\mathrm{C}}{\mathrm{C}}-\mathrm{COOH} \rightarrow \underset{\mathrm{C}}{\mathrm{C}}=\mathrm{O}+\mathrm{CO}_{2(\mathrm{~g})}+2 \mathrm{H}_{(\mathrm{aq})}^{+}+2 \mathrm{e}^{-} \\
& \mathrm{Au}^{3+}{ }_{(\mathrm{aq})}+3 \mathrm{e}^{-} \rightarrow \mathrm{Au}_{(\mathrm{s})}^{0} \\
& \text { Overall Reaction } \quad 3 \mathrm{C}_{6} \mathrm{O}_{7} \mathrm{H}_{8(\mathrm{aq})}+2 \mathrm{Au}^{3+}{ }_{(\mathrm{aq})} \rightarrow 3 \mathrm{C}_{5} \mathrm{O}_{5} \mathrm{H}_{6}+2 \mathrm{Au}^{0}{ }_{(\mathrm{s})}+ \\
& 3 \mathrm{CO}_{2(\mathrm{~g})}+6 \mathrm{H}^{+}(\mathrm{aq})
\end{aligned}
$$

Figure 1.20 Redox coupling in the TSC reduction of $\mathrm{Au}^{3+}$ to nanoparticulate $\mathrm{Au}^{0}{ }^{5}$

Another popular method for the solution-phase synthesis of gold nanoparticles was developed by Brust and co-workers. ${ }^{26}$ The Brust method employs a two-phase liquidliquid reduction system, where aqueous dissolved $\mathrm{AuCl}_{4}^{-}$is transferred to a toluene phase via the use of the phase transfer catalyst tetraoctylammonium bromide. The organic phase is then mixed through an aqueous solution of $\mathrm{NaBH}_{4}$, in the presence of thioalkane or aminoalkane stabilising agents. The particle size can be tuned between 1 and $10 \mathrm{~nm}$ by altering the concentration ratio of the $\mathrm{AuCl}_{4}^{-}$to the stabilising agents. The complexity, and the requirement of organic solvents and catalysts, of the Brust method makes it difficult to scale-up. As such, approaches that are similar 
to the Turkevitch method will be investigated in this research project.

\section{Size and Shape Control in Solution-phase Gold Nanoparticle Synthesis}

The optical properties of gold nanoparticles, which are detailed in Section 1.2.2, are dependent on both the size and shape of the particles. As such, controlling the size, shape and polydispersity of gold nanoparticles is an important consideration when colouring wool with gold nanoparticle colloids.

Figure 1.21 shows two gold nanoparticle colloids that were synthesised with the use of different dual reducing and stabilising agents; Tannic acid (TA) and TSC. The gold nanoparticles are predominantly spherical in shape, and thus the different colours are due to the different sizes of the resulting gold nanoparticles.
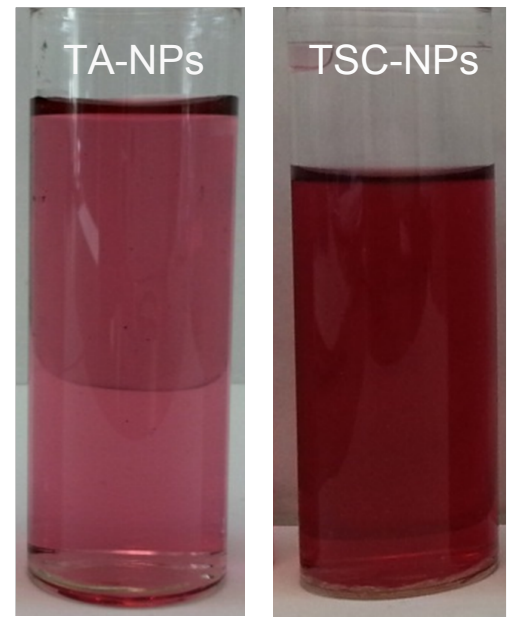

Figure 1.21 The different colours of gold nanoparticles that are stabilised by Tannic acid (TA) and TSC.

The formation of spherical gold nanoparticles is thermodynamically favourable in the absence of anisotropic shape-inducing reagents, due to this conformation exhibiting lowest possible surface area to mass ratio. Gold nanorods may be formed with the use of cetyltrimethylammmonium bromide (CTAB), which directs material addition to the $\{111\}$ plane. In the method developed by Murphy and coworkers, ${ }^{27}$ a solution of gold nanoparticle seeds is first synthesised, via the reduction of $\mathrm{AuCl}_{4}^{-}$with $\mathrm{NaBH}_{4}$ in the presence of TSC at room temperature (RT). A growth solution is prepared that contains $\mathrm{AuCl}_{4}^{-}, \mathrm{CTAB}$ and ascorbic acid (which reduces $\mathrm{Au}^{3+}$ to $\mathrm{Au}^{+}$in the presence of CTAB. The seed and growth solutions are combined, and CTAB-stabilised gold nanorods form. ${ }^{27}$

The shape directing activity of CTAB is illustrated in Figure $1.23,{ }^{27}$ where CTAB preferentially associates to the $\{100\}$ facets. This is due to the relatively large 


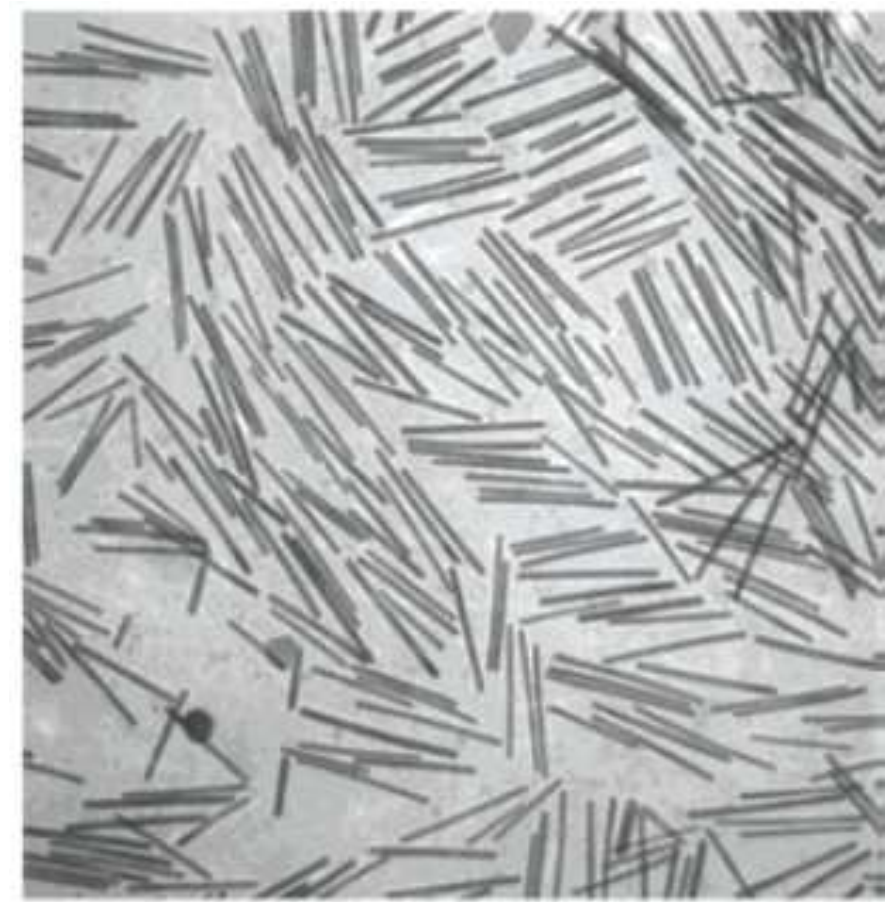

Figure 1.22 TEM micrograph of gold nanorods that were synthesised in the presence of shape-directing stabilising agent CTAB. ${ }^{27}$

spacing of the $\mathrm{CTA}^{+}$head groups, which is comparable with the space between gold atoms in the $\{100\}$ crystal facets. Thus, the $\{111\}$ pentatetrahedral twin ends of the nanorod (which exhibit closer packing of gold atoms) are left relatively bare, and growth occurs in this direction. The aspect ratio (the ratio of length to width) affects the colours of the nanorods, and may be tuned by manipulating the concentration ratio of the seed to growth solution that is employed in the synthesis.

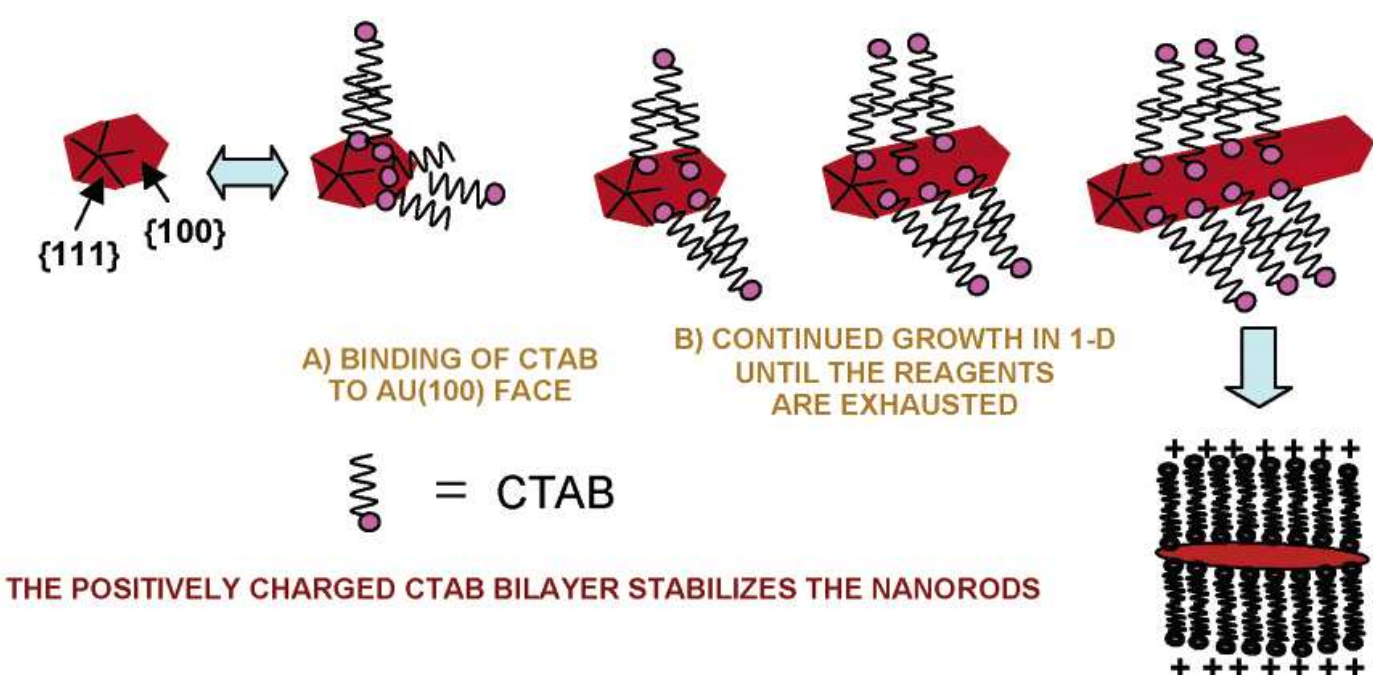

Figure 1.23 Proposed mechanism for the use of CTAB in the synthesis of gold nanorods. ${ }^{27}$

Other geometries of gold nanoparticles, for example nanotriangles ${ }^{28}$ or nanostars, ${ }^{29}$ 
are also possible with the use of alternative shape-directing reagents. In the solutionphase synthesis of gold nanoparticles, size and shape control is important when considering the dependence it has on the colour of the composites. Extending the boutique colour range of gold nanoparticle-wool fibre composites may be achieved by developing scalable and cost-effective syntheses of gold nanoparticle of different sizes and shapes.

\subsubsection{Optical Properties of Gold Nanoparticles}

The optical properties of metal nanoparticles have been unwittingly exploited as early as the 4th century AD, where gold, silver and copper nanoparticles were used as pigments in the Lycurgus Cup. ${ }^{30}$ As is seen in Figure 1.24, the colour of the Lycurgus Cup differs, depending on where the source of light is in relation to the cup.

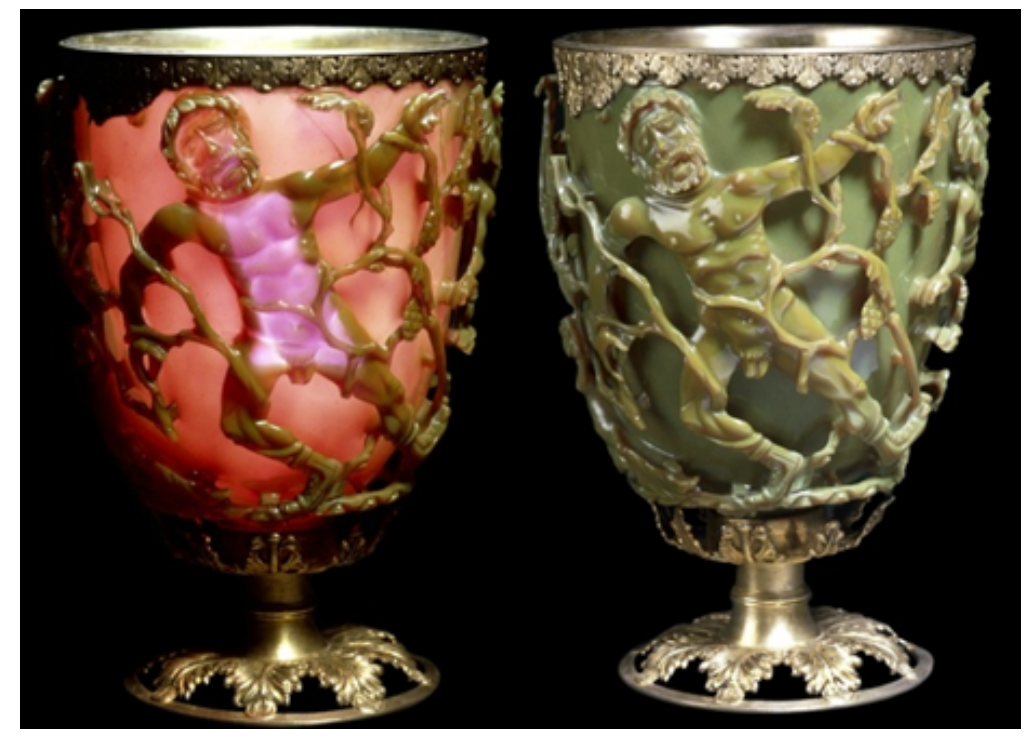

Figure 1.24 The Lycurgus cup, which appears red when the light source originates from inside or behind the cup, or green when light is reflected from the front of the cup. $^{31}$

Gold nanoparticles were also used as colourants in stained glass windows in churches throughout the Middle Ages, where the ruby-red colour that is common to spherical gold nanoparticles is often observed. At the time the dependence of particle size on colour was not understood.

The chemistry behind this phenomenon was investigated in 1857, when Michael Faraday conducted a study on the synthesis and colour of colloidal gold. ${ }^{32}$ The study involved running a strong electrical current through gold wire between two gold plates, and saw a range of colours (green, grey violet, green and ruby-red) 
deposited on the glass surfaces. ${ }^{32}$ Some larger gold particles were observable under a optical microscope, however, generally the particles were too small to be seen. ${ }^{32}$ The ruby-red colour common to spherical gold nanoparticles was seen at the part of the gold wire that was subjected to the most intense heat, and Faraday concluded that this colour was due to very fine particles of gold. ${ }^{32}$ An image of the glass slide containing gold nanoparticles that Faraday used to presented to the scientific community in 1858 is shown in Figure 1.25.

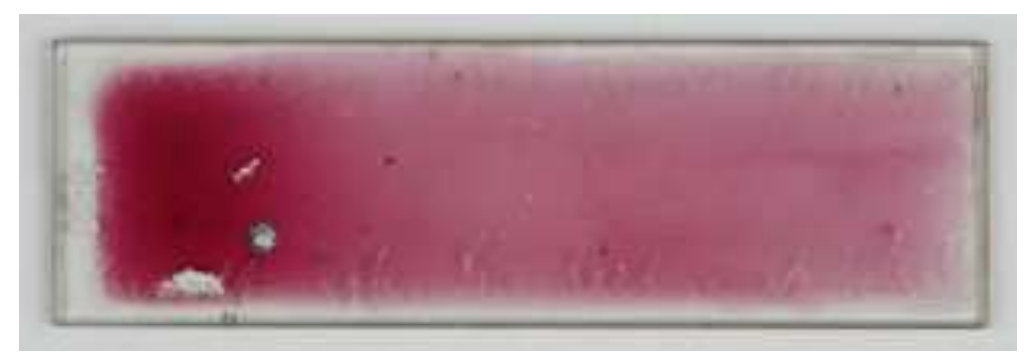

Figure 1.25 A glass slide containing a ruby-red gold colloid that Michael Faraday presented to explain the change in the colour of gold with particle size. ${ }^{33}$

An understanding of the dependence of particle size on colour was achieved with the development of Mie Theory in 1908. ${ }^{34}$ Gustav Mie solved Maxwell's equations for the interaction of light with spherical metal particles. ${ }^{35}$ The intense colours of gold nanoparticles are attributed to the surface plasmon resonance (SPR) of the particles. $\mathrm{SPR}$ is the resonance coupling of collective oscillations of conduction band electrons with certain frequencies of incident electromagnetic radiation. ${ }^{36}$

When light interacts with a metal surface, the conduction band electrons collectively oscillate in phase with the electric field of the incoming light. A representation of these oscillations on the surfaces of a bulk metal and a spherical metal nanoparticle is presented in Figure 1.26. ${ }^{37}$ The collective oscillation of free electrons of a spherical metal nanoparticle induces a polarisation of the conduction band electrons with respect to the much heavier ionic core of the particle. ${ }^{38}$ A net charge difference occurs at the metal surface particle surface, which acts as a restoring force on the non-equilibrium position of the electrons. ${ }^{39}$ When the frequency of the electromagnetic field is resonant with the coherent motion of the electrons, an intense SPR absorbance occurs. ${ }^{40}$

In a bulk metal, the frequency of the surface plasmon is dependent only on the free electron density of the metal. ${ }^{37}$ In the case of a spherical metal nanoparticle however, the SPR is influenced by the size, shape and dielectric constant of the metal particle, as well as the dielectric function or refractive index of the surrounding medium (that the nanoparticle is dispersed in or bound to). ${ }^{16}$ Additionally, the spacing 


\section{a}
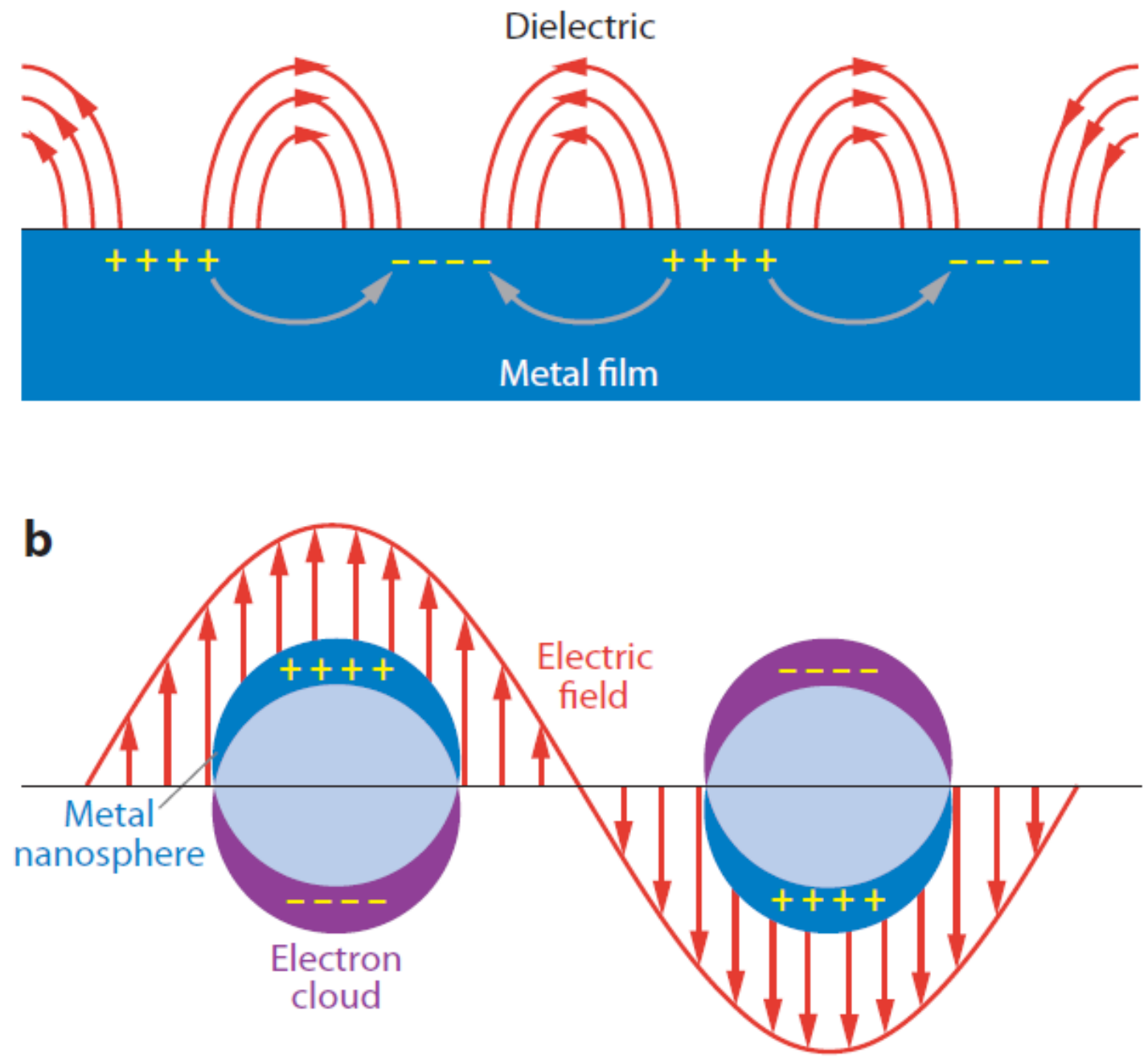

Figure 1.26 Depiction of propagating surface plasmons of a bulk metal surface (a), and localised surface plasmons of a spherical metal nanoparticle (b). ${ }^{37}$ 
between neighbouring metal nanoparticles affects the SPR absorbance; when two metal nanoparticles are in close proximity to one another, their SPRs can hybridise, and a red-shift of the SPR absorbance to higher wavelengths results. ${ }^{37}$

When the radius of a metal nanoparticle is less than 1-2 nm, SPR bands are no longer observed due to the quantisation of energy states inside the conduction band of the material. ${ }^{41}$ When the size of a metal nanoparticle increases, higher order modes become more dominant, which causes the SPR band to broaden and red-shift (or absorb at higher wavelengths). ${ }^{40}$ This is due to light being unable to homogeneously polarise the nanoparticles as retardation effects become more prominent. ${ }^{40}$

Figure 1.27 presents the UV-vis absorbance spectra of spherical gold nanoparticle colloids with different particle sizes. ${ }^{40}$ The SPR peak of a colloid of $22 \mathrm{~nm}$ gold nanoparticles occurs at approximately $525 \mathrm{~nm}$. An increase in the size of the gold nanoparticles to 48 and then $99 \mathrm{~nm}$ has caused a red-shift and broadening of SPR absorbance. The agglomeration of individual gold nanoparticles causes a similar change in the UV-visible absorption spectra, and is exploited in the synthesis of grey-coloured gold nanoparticle-wool fibre composites.

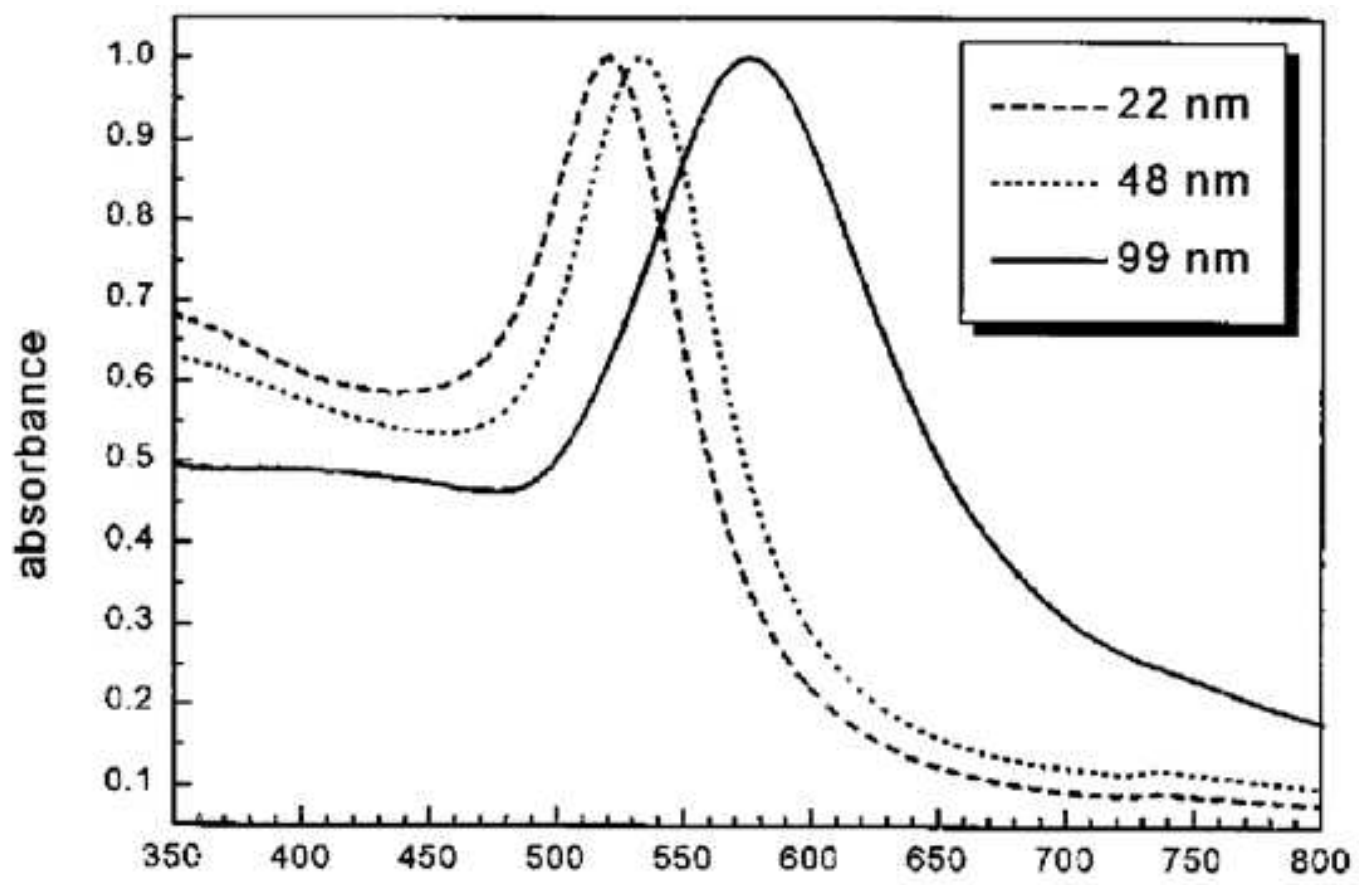

Figure 1.27 Normalised absorption spectra of colloids of 22, 48, and $99 \mathrm{~nm}$ spherical gold nanoparticles. ${ }^{40}$

All metal particles exhibit SPRs, however, the plasmon frequency that they resonate with typically lies outside of the visible spectrum. ${ }^{39}$ Nanoparticles of the alkali and group 10 and 11 metals exhibit SPRs in the visible spectrum, ${ }^{42}$ due to the presence of free valence electrons, which redshifts the plasmon frequency into the visible region 
of the electromagnetic spectrum. Copper, silver and gold display the most intense SPR bands in the visible region. ${ }^{42}$ Oxide layers can complicate SPRs, for example silver nanoparticles are extremely sensitive to oxidation, and the presence of a silver oxide outer surface redshifts its SPR absorption. ${ }^{43}$ However, gold is resistant to oxidation, and as such, oxide layers do not complicate its optical properties. ${ }^{39}$

A representation of the dipole oscillation that occurs in spherical metal nanoparticles is shown in Figure 1.28. ${ }^{39}$ Spherical particles exhibit a single SPR band, as the boundaries of the plasmons are equivalent regardless of the orientation of the particle to the electric field of light. Anisotropic shapes however, such as metal nanorods, exhibit multiple SPR bands. Gold nanorods exhibit dipoles in the transverse and longitudinal directions, which are also represented in Figure 1.28, and are responsible for two different SPR bands in the optical absorption spectra of gold nanorods ${ }^{39}$ The longitudinal oscillation is sensitive to the aspect ratio (the ratio of length to width) of the nanorod, rather than the length of the nanorod. ${ }^{16}$ Thus, manipulating the aspect ratio of gold nanorods allows for tuning of the colour (gold nanorods with an aspect ratio of 2 are green and green nanorods have an aspect ratio of $3^{27}$ ).

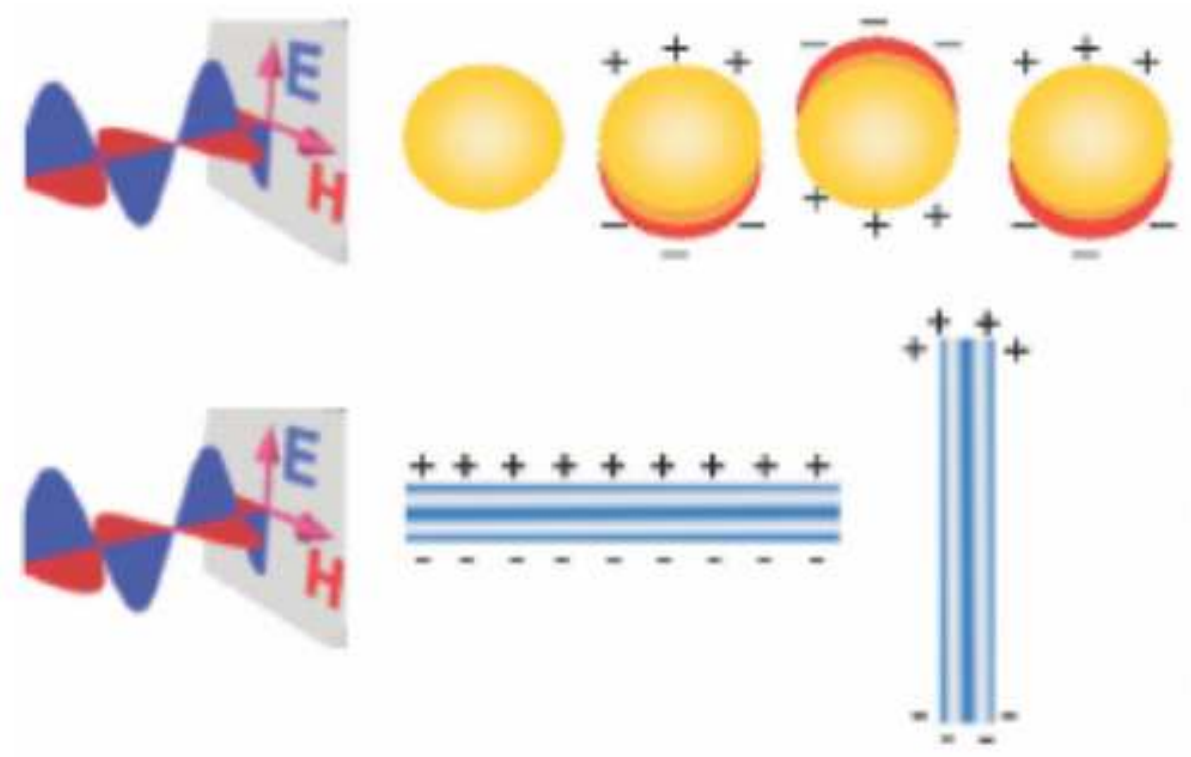

Figure 1.28 A representation of the dipole oscillation of a spherical metal nanoparticle and the transverse and longitudinal oscillations of a metal nanorod. ${ }^{39}$

An understanding of the optical properties of metal nanoparticles is integral to manipulate gold nanoparticles to fit a certain purpose. It shows that by controlling the size and shape of the gold nanoparticles that are used in the synthesis of gold nanoparticle-wool fibre composites, the colour of the composites can be tuned. 


\subsection{Gold Nanoparticle-Wool Fibre Composites}

The research group of Professor James Johnston has focused on the synthesis and characterisation of novel nanocomposites in recent years. Such composites include silver nanoparticle-wool fibre composites, ${ }^{44}$ conducting polymer-wool fibre composites, ${ }^{45}$ conducting polymer-clay composites, ${ }^{46}$ silver nanoparticle-clay composites, ${ }^{47}$ conducting polymer-cellulose fibre composites ${ }^{48}$ and gold and silver nanoparticlecellulose fibre composites. ${ }^{49}$

The synthesis of gold nanoparticle-wool fibre composites was developed by Dr Kerstin Lucas and Professor James Johnston, who carried out extensive research on laboratory-scale procedures. ${ }^{5}$ The composites have been documented in the literature since $2007,{ }^{4,50,51}$ and the intellectual property is protected with a provisional patent. ${ }^{52}$ The nanotechnology is commercialised through the start-up company Noble Bond Ltd, under the registered international trademark Aulana ${ }^{\circledR}$. Three distinct approaches were developed by Lucas and Johnston for the synthesis of gold nanoparticle-wool fibre composites, which are outlined in Table 1.3.

Table 1.3 The different approaches for the synthesis of gold nanoparticle-wool fibre composites developed by Lucas and Johnston. ${ }^{5}$

\begin{tabular}{cc}
\hline Approach & Description \\
In-situ & $\begin{array}{c}\text { uses wool fibres as redox active biotemplates that absorb } \\
\mathrm{AuCl}_{4}^{-} \text {ions and form gold nanoparticles in-situ }\end{array}$ \\
Ex-situ & wool fibres absorb gold nanoparticle colloids \\
Seed-mediated & $\begin{array}{r}\text { gold nanoparticle-wool fibre composites are used } \\
\text { as seed substrates for nanoparticle growth. }\end{array}$ \\
\hline \hline
\end{tabular}

The approaches in Table 1.3 result in composites that exhibit significantly different properties, and require varying quantities of expensive gold precursors reagents.

\subsubsection{In-situ Procedure}

The in-situ procedure was the first method that was developed to synthesise gold nanoparticle-wool fibre composites. It involves the absorption of $\mathrm{AuCl}_{4}^{-}$by wool, whereby the electron-rich functionality in the wool protein is able to facilitate the 
reduction of $\mathrm{Au}^{3+}$ to nanoparticulate $\mathrm{Au}^{0}$, and stabilise the nanoparticles that have formed. ${ }^{5}$ In-situ composites exhibit gold nanoparticles on the surface and throughout the interior of the wool fibres. A range of in-situ composites of merino wool that were synthesised under different reaction conditions are shown in Figure 1.29, where the possible colours are pinks, purples and greys. ${ }^{5}$

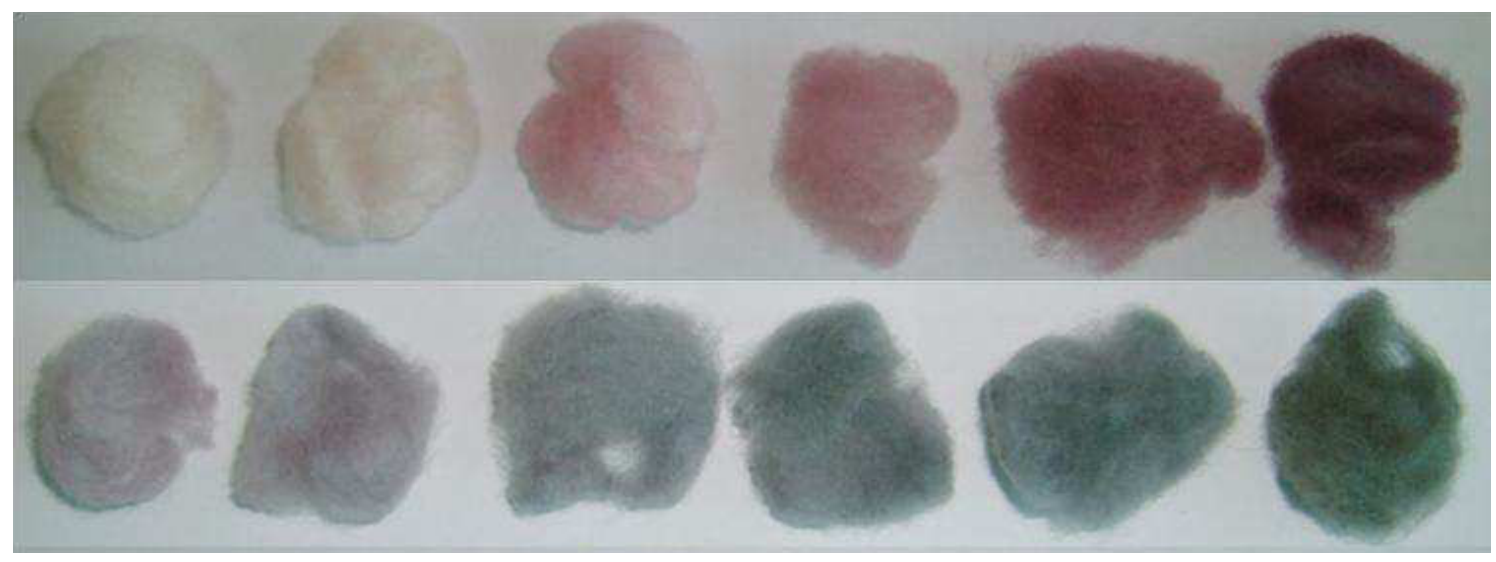

Figure 1.29 In-situ gold nanoparticle-wool fibre composites that were synthesised under different reaction conditions. ${ }^{5}$

The in-situ procedure first involves the uptake of $\mathrm{AuCl}_{4}^{-}$to wool fibres, which is followed by an ageing process where gold nanoparticles form in the wool matrix over time. In this one-pot synthesis, the uptake of gold ions to wool fibres occurs by simply soaking wool in an aqueous solution of $\mathrm{AuCl}_{4}^{-}$. In the ageing process, the uptake reaction vessels are transferred to a water bath, where heat aids the gradual formation of nanoparticles of gold over time.

\section{The Uptake Process}

Lucas and Johnston manipulated a number of reaction conditions to optimise the uptake of $\mathrm{Au}^{3+}$ to wool fibres. These included the reaction $\mathrm{pH}$, time, temperature, concentration of $\mathrm{AuCl}_{4}^{-}$and the wool to liquor ratio that was employed. The Pourbaix diagram of gold is shown in Figure 1.30, where $\mathrm{Au}^{3+}$ ions exist as $\mathrm{AuCl}_{4}^{-}$ complexes at a reaction $\mathrm{pH}$ of $<6$. Similar to the acid dyeing of wool (which was described in Section 1.1.4), the uptake of the negatively charged $\mathrm{AuCl}_{4}^{-}$complex to wool fibres occurs readily if the wool surface is positively charged. Thus, the uptake of $\mathrm{AuCl}_{4}^{-}$to wool is carried out at a reaction $\mathrm{pH}$ that is below the isoelectric point of wool, to provide an electrostatic driving force for the uptake.

The uptake of $\mathrm{AuCl}_{4}^{-}$to wool may be characterised with the use of atomic absorption spectroscopy (AAS), which is used to measure the residual concentration of $\mathrm{Au}^{3+}$ 


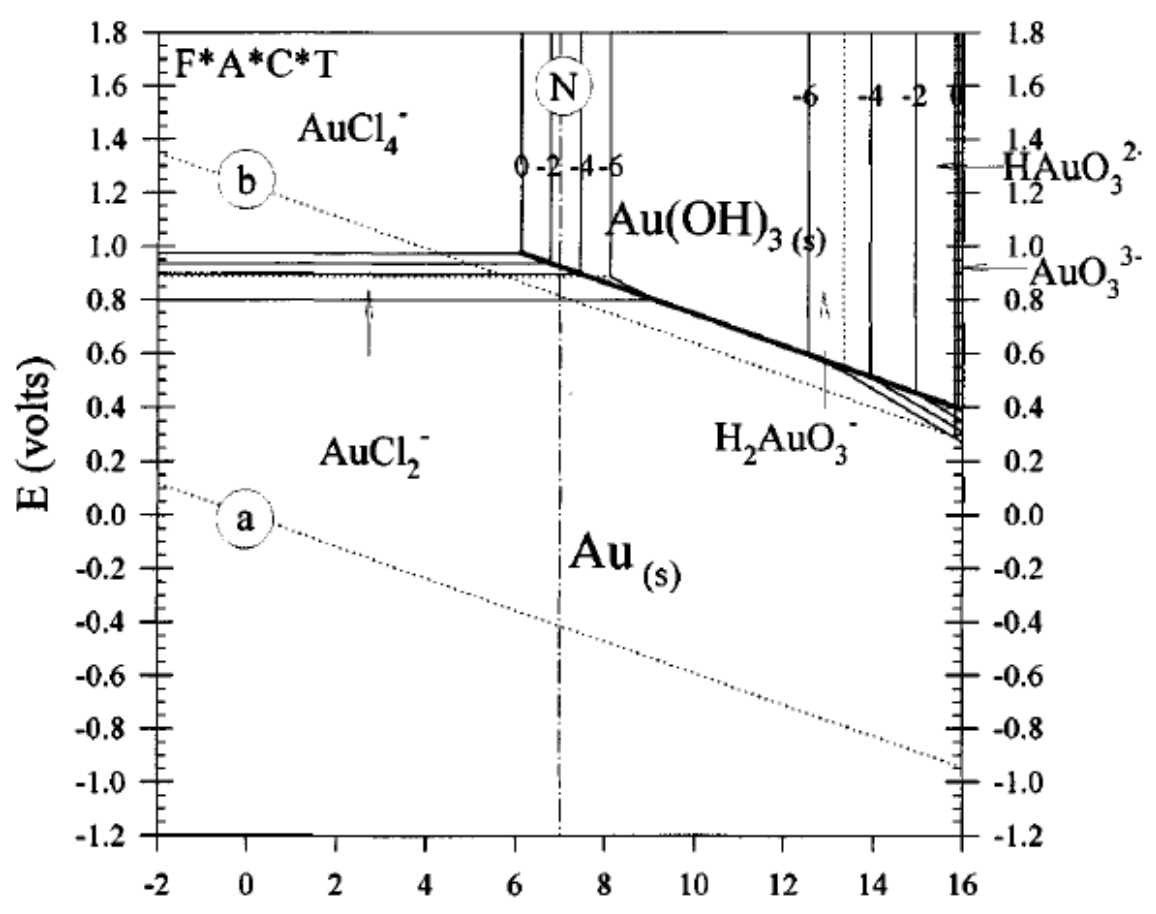

Figure 1.30 The Pourbaix diagram of gold.

in the uptake solutions over time. Figure 1.31 presents the uptake of $160 \mathrm{mg} \mathrm{L}^{-1}$ solutions of $\mathrm{AuCl}_{4}^{-}$to merino wool at $\mathrm{RT}$ and different reaction $\mathrm{pH}$ levels. ${ }^{5}$ The uptake at pH 2.7 is rapid; it is complete after approximately $60 \mathrm{~min}$. When the $\mathrm{pH}$ of the reaction is increased to 5 or 7 , the uptake of $\mathrm{AuCl}_{4}^{-}$is much slower, and is incomplete over the time period. The rate of uptake of $\mathrm{AuCl}_{4}^{-}$to wool fibres may also be increased by elevating the reaction temperature, increasing the concentration of $\mathrm{AuCl}_{4}^{-}$and decreasing the wool to liquor ratio that is employed. ${ }^{5}$

\section{The Ageing Process}

The ageing process involves transferring the uptake reaction vessels to a water bath, where heat is used to increase the rate of gold nanoparticle formation in the wool fibres. The purple colour that is common to gold nanoparticles in wool fibres gradually develops over one week at $50^{\circ} \mathrm{C}$, after which time there is no further colour development. ${ }^{5}$ Scanning electron microscopy (SEM) and energy dispersive X-ray spectroscopy (EDS) analysis of the surface of an in-situ composite fibres are shown in Figure 1.32. ${ }^{5}$ The SEM micrograph in backscatter mode shows the presence of gold nanoparticles on the surface of the wool fibre, which appear as bright dots on the comparatively dark wool surface (the reason for this is explained in Section 2.3.4). The gold content of the nanoparticles is confirmed in the EDS map of elemental $\mathrm{Au}$, where the areas of high concentration of gold correspond to the bright areas in the SEM micrograph. 


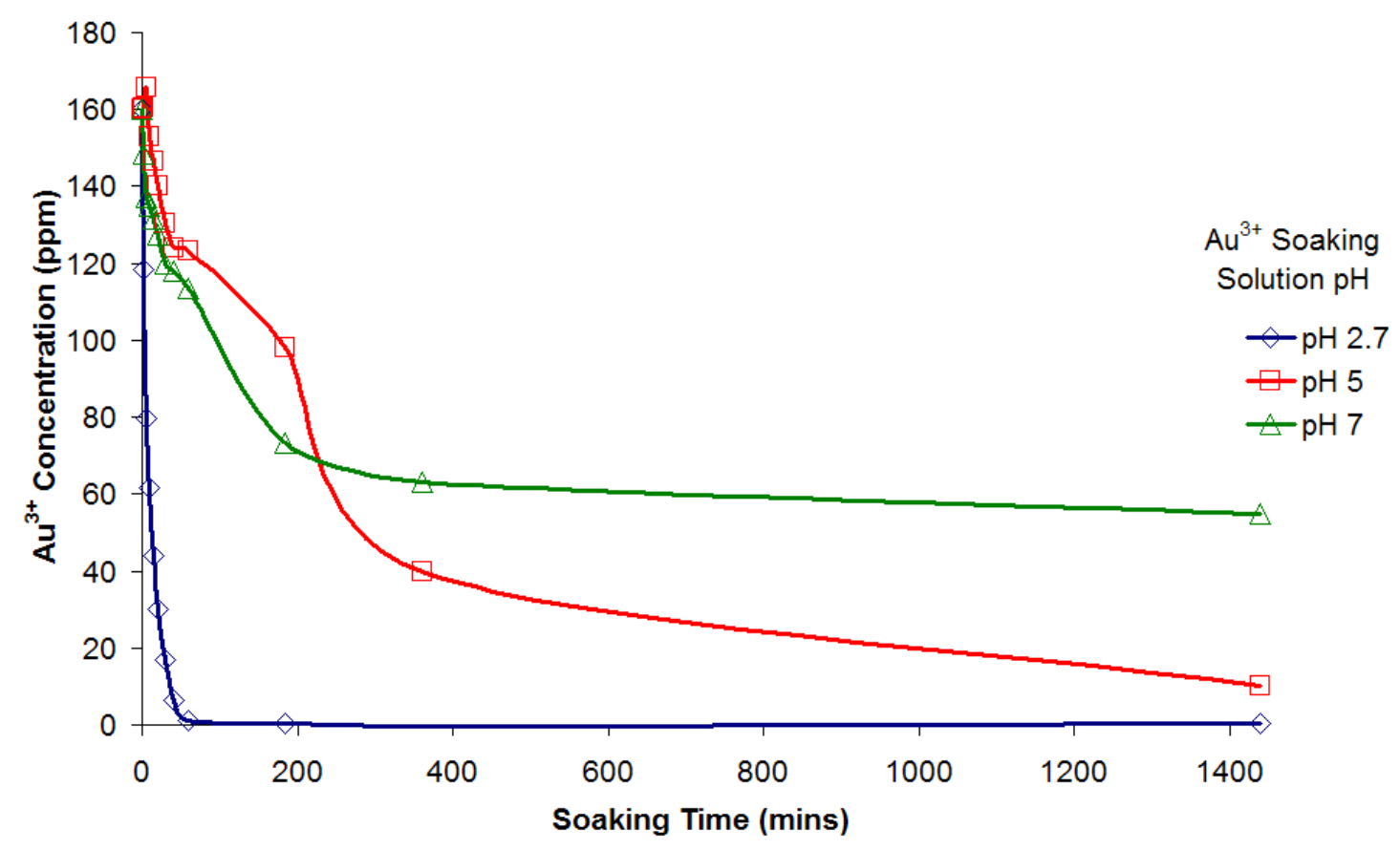

Figure 1.31 The uptake of $\mathrm{AuCl}_{4}^{-}$to merino wool fibres at different $\mathrm{pH}$ levels. ${ }^{5}$
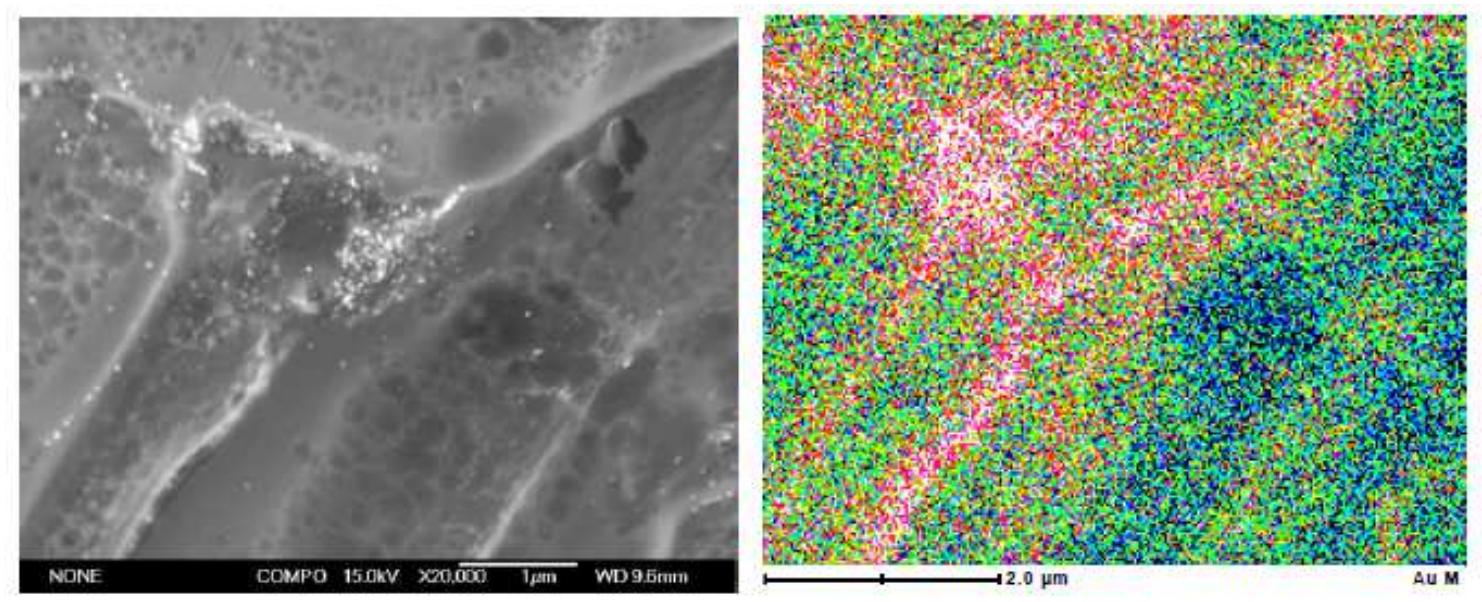

Figure 1.32 An SEM micrograph in backscatter mode of the surface of an in-situ composite of merino wool $(1.6 \mathrm{wt} \% \mathrm{Au})$ and an Au EDS map of the same area (where the white and red areas indicate relatively high gold concentrations). ${ }^{5}$

\section{Discussion of the Mechanism of Nanoparticle Formation}

The uptake process in the in-situ procedure is considered to involve the diffusion of $\mathrm{AuCl}_{4}^{-}$throughout the wool fibres via a similar absorption pathway to that of the conventional dye molecules. As was mentioned in Section 1.1.4, dye molecules diffuse through the junctions between cuticle scales (or alternatively through damaged cuticle scales), into the fibre interior where they eventually accumulate in the sulfur-rich proteins in wool. ${ }^{8}$ Evidence of this occurrence is seen in the EDS ele- 
mental analysis of the cross-section of an in-situ composite. This is presented in Figure $1.33,{ }^{5}$ where the relative concentrations of elemental $\mathrm{S}$ and $\mathrm{Au}$ maps are very similar, indicating a preference for $\mathrm{AuCl}_{4}^{-}$to migrate into the paracortex of the wool fibres, which contains a relatively high sulfur content.
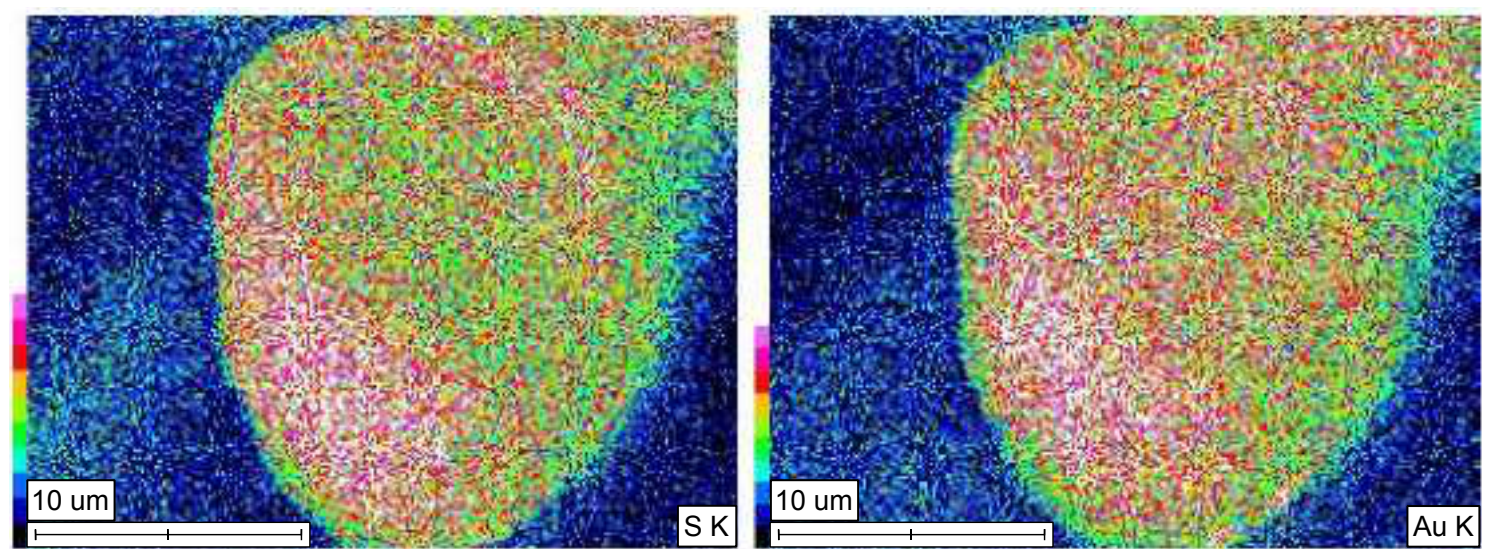

Figure 1.33 EDS elemental maps of the cross-section of an in-situ composite of merino wool (1.6 wt \% Au) for sulfur and gold. ${ }^{5}$

Once $\mathrm{AuCl}_{4}^{-}$has been absorbed by the wool fibres, $\mathrm{AuCl}_{4}^{-}$is believed to dissociate to $\mathrm{Au}^{3+}$, which is then reduced to nanoparticulate $\mathrm{Au}^{0}$. Dr Lucas used X-ray photoelectron spectroscopy (XPS) to investigate the chemical environment of the in-situ composite fibres. By comparing the XPS spectra of different elements in merino wool fibres that had been removed from uptake solutions at different time periods, Dr Lucas observed a decrease in the peak relating to binding energy of $\mathrm{Au}^{3+}$, and corresponding increases in the peaks relating to $\mathrm{Au}-\mathrm{S}$ and $\mathrm{Au}^{0}$ over time. ${ }^{5}$ This indicates that during the uptake there are $\mathrm{Au}-\mathrm{S}$ bonds are forming as the $\mathrm{Au}^{0}$ entities form in the wool fibre. During the ageing process, the $\mathrm{Au}^{0}$ peak increases in intensity, while the $\mathrm{Au}^{3+}$ peak decreases as gold nanoparticles are formed. ${ }^{5}$ Thus, the gold nanoparticles were considered to be bound to the sulfur-containing amino acid residues in wool. Such an interaction between a gold nanoparticle and cystine and cysteine residues is illustrated in Figure 1.34). ${ }^{5}$

There are many possible electron-rich functional groups in the wool protein that have the potential to couple their oxidation to the reduction of $\mathrm{Au}^{3+}$ to $\mathrm{Au}^{0}$. One such redox reaction involves the oxidation of cystine to cysteic acid, and is shown 


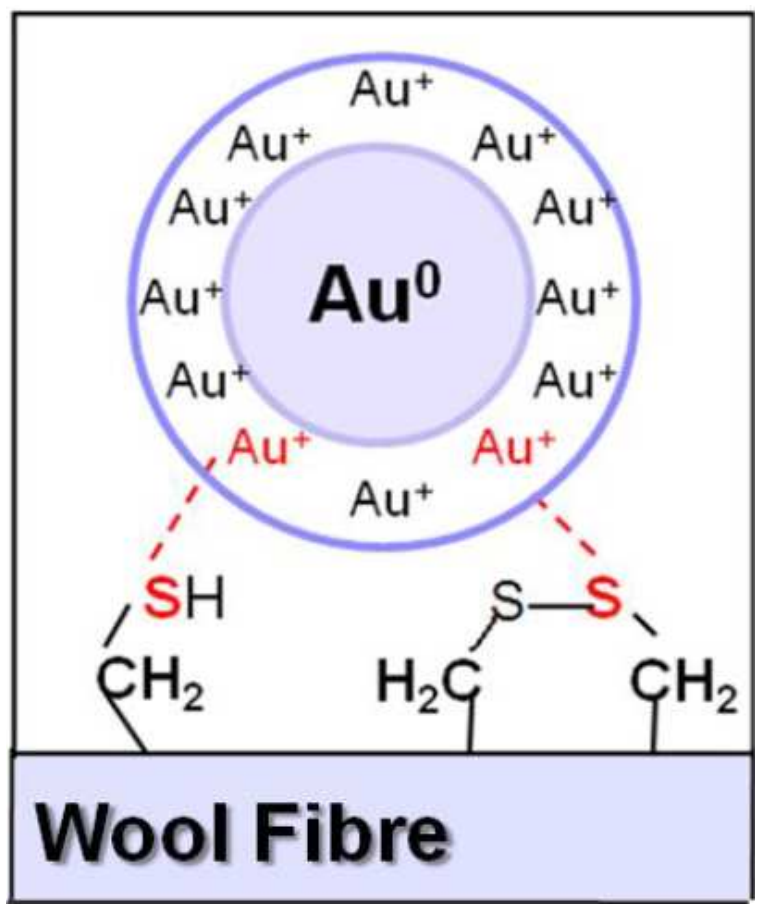

Figure 1.34 The proposed bonding between gold nanoparticles and sulfur atoms in cysteine and cystine residues in wool of an in-situ composite. ${ }^{5}$

in Equation 1.8, where $\mathrm{W}$ refers to a polypeptide chain in wool. ${ }^{5}$

$$
\begin{gathered}
\mathrm{Au}^{3+}+3 \mathrm{e}^{-} \rightarrow \mathrm{Au}^{0} \\
\mathrm{~W}-\mathrm{S}-\mathrm{S}-\mathrm{W}+6 \mathrm{H}_{2} \mathrm{O} \rightarrow 2 \mathrm{~W}-\mathrm{SO}_{3} \mathrm{H}+10 \mathrm{H}^{+}+10 \mathrm{e}^{-} \\
3 \mathrm{~W}-\mathrm{S}-\mathrm{S}-\mathrm{W}+18 \mathrm{H}_{2} \mathrm{O}+10 \mathrm{Au}^{3+} \rightarrow 6 \mathrm{~W}-\mathrm{SO}_{3} \mathrm{H}+30 \mathrm{H}^{+}+\mathrm{Au}^{0}
\end{gathered}
$$

The in-situ procedure is used to synthesise gold nanoparticle-wool fibre composites that exhibit deep, colourfast shades of pink, purple and grey. However, a major drawback of the in-situ procedure is the requirement of a high gold content, which is on the order of hundreds of dollars of gold to colour one kilogram of wool. Additionally, as will become clear in Chapter 3, the quality of composites that are produced is highly dependent on the type of wool that is employed in the in-situ procedure. As such, an alternative synthesis method that is relatively robust and produces composites with a significantly lower gold content, was developed.

\subsubsection{Ex-situ Procedure}

The ex-situ procedure involves the solution-phase synthesis of gold nanoparticles, that are then used to colour wool fibres. With this process, a greater range of 
composite colours are theoretically possible, by varying the size and shape of gold nanoparticles. Lucas and Johnston utilised TSC and TA (Figure 1.35) in the gold nanoparticle synthesis. ${ }^{5}$ A range of gold colloids with different colours were synthesised using different concentration ratios of $\mathrm{AuCl}_{4}^{-}$to TSC or TA. A selection of these gold colloids, and the composites that resulted from colouring wool with the colloids, are shown in Figure 1.36. ${ }^{5}$

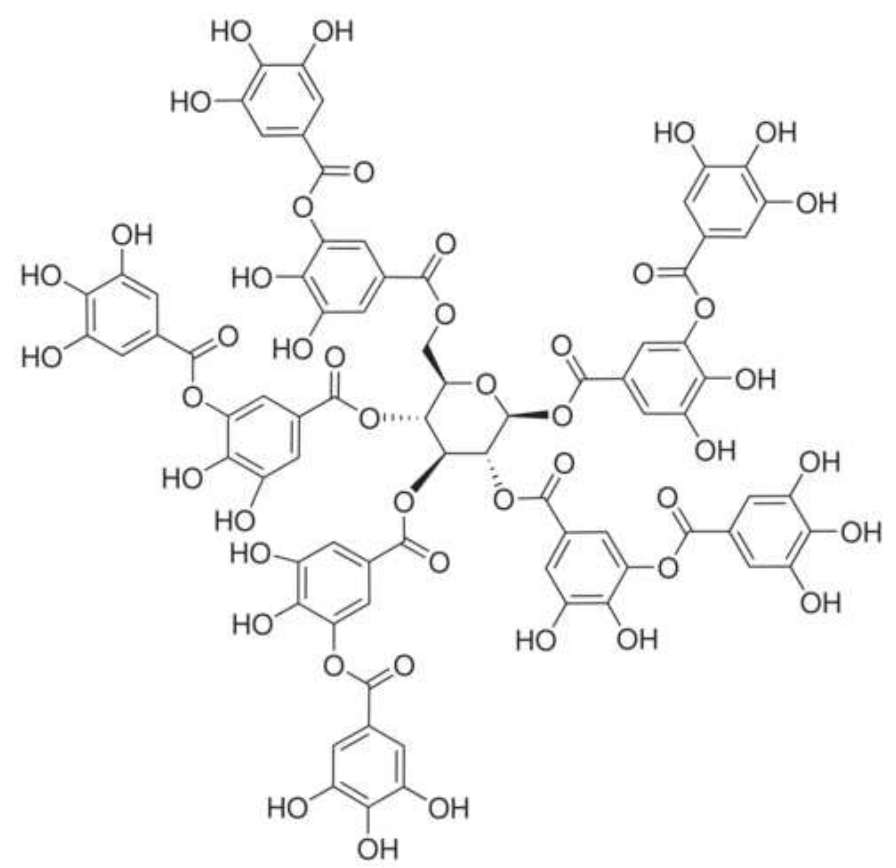

Figure 1.35 The structure of Tannic acid (TA).

In the ex-situ procedure results in the attachment of gold nanoparticles only on the surfaces of the wool fibres; the gold nanoparticles are too large to penetrate into the interior of the fibres. This is illustrated in the SEM image and elemental $\mathrm{Au}$ EDS map of the cross-section of an ex-situ composite Figure $1.37,{ }^{5}$ where gold nanoparticles only appear on the outside of the wool fibre. The Au EDS image confirms the absence of gold nanoparticles in the fibre interior. The absence of gold in the interior of the wool fibre indicates that the gold content of an ex-situ composite can be significantly lower than that of an in-situ composite (which contain a high amount of gold in the interior of the fibre). However, the deep and intense hues that are typical of in-situ composites are not possible with the ex-situ procedure.

Similar to the in-situ procedure, the ex-situ procedure exploits a pH-related driving force for the absorption of gold nanoparticles. TSC- and TA-stabilised gold nanoparticles are negatively charged, and are attracted to wool fibres when the $\mathrm{pH}$ of the gold colloid is below the isoelectric point of wool (where the wool fibre surface has a net positive charge). However, the attachment of gold nanoparticles to wool fibres 


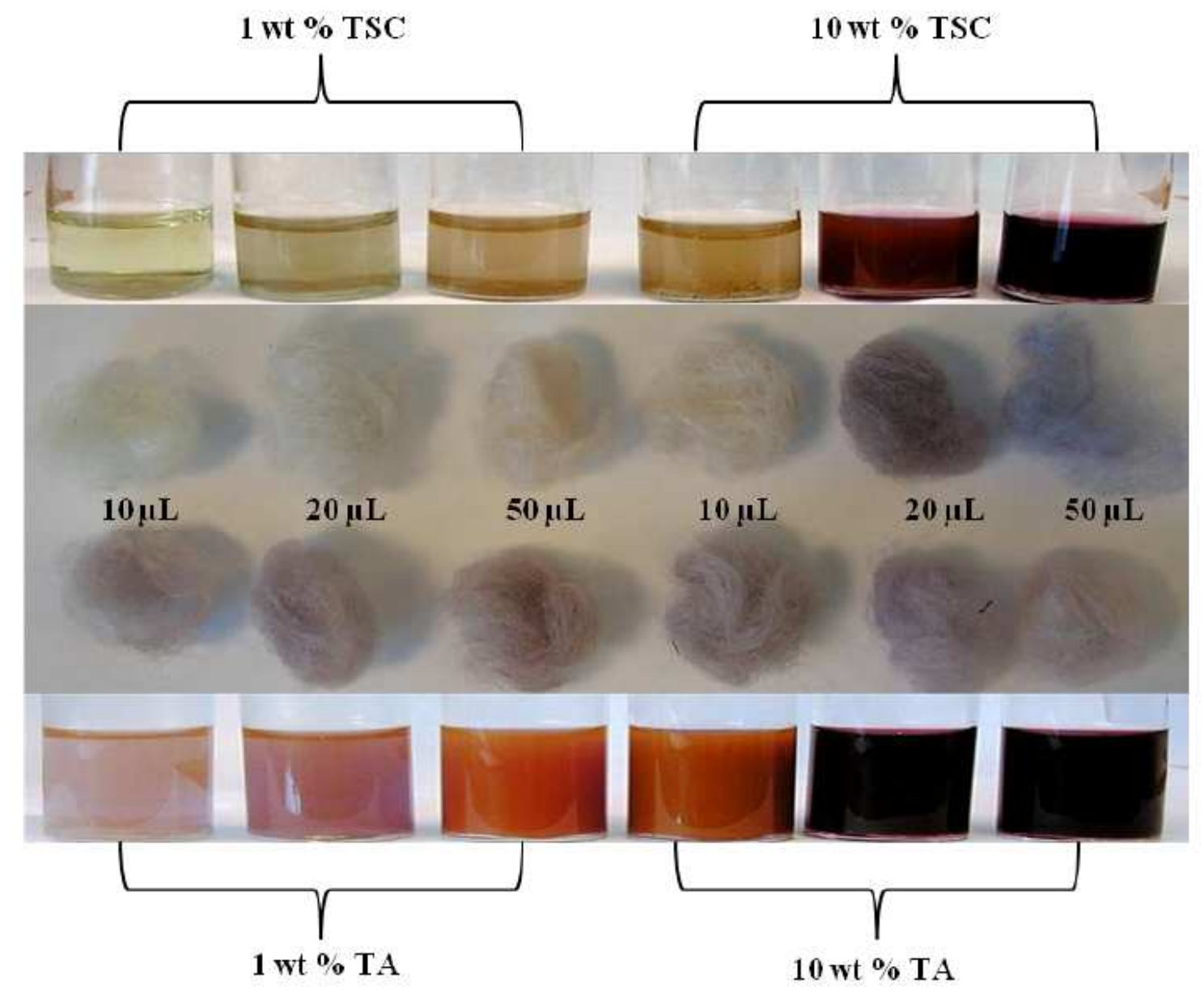

Figure 1.36 A range of colloids that were synthesised with different concentration ratios of $\mathrm{Au}$ to $\mathrm{TSC}$ or TA, and the resulting ex-situ composites. ${ }^{5}$
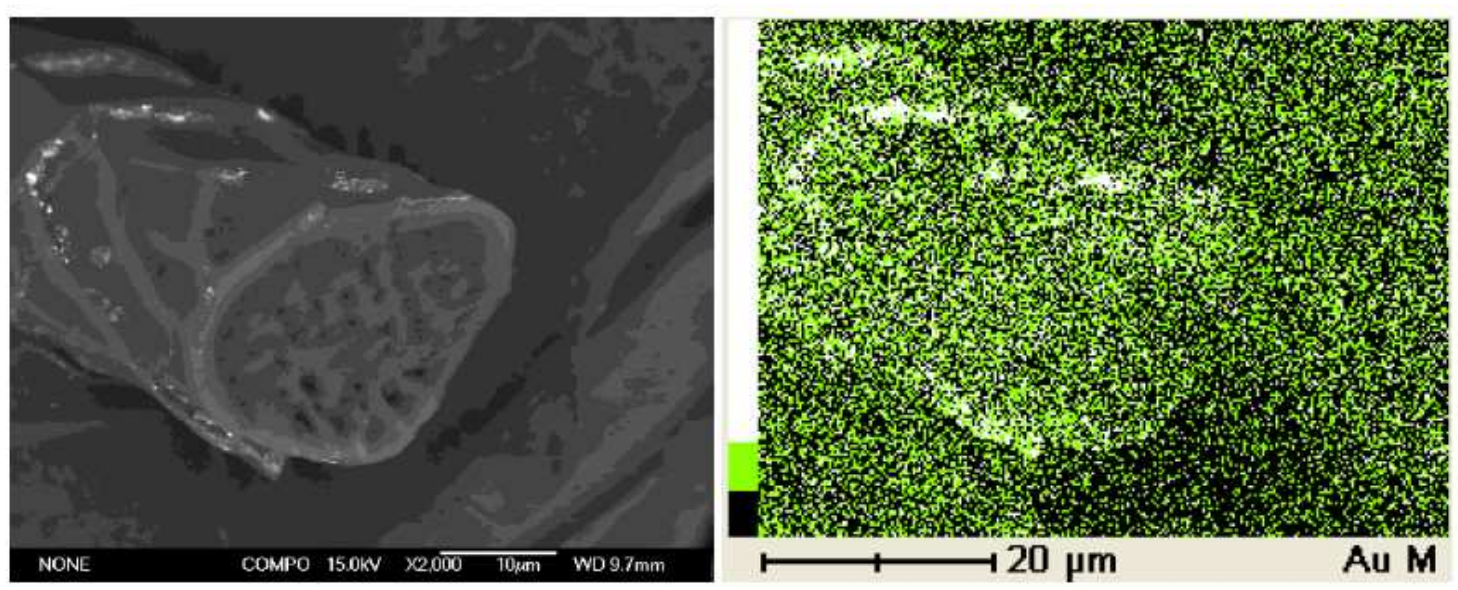

Figure 1.37 An SEM micrograph in backscatter mode of the cross-section of an ex-situ composite, and an EDS elemental Au map of the same area. ${ }^{5}$ 
in ex-situ composites is only electrostatic in nature, and as such, ex-situ composites have significantly poorer washfastness properties than in-situ composites.

\subsubsection{Seed-mediated Approach}

The seed-mediated approach was developed to attempt to form grey coloured insitu composites, where the presence of large particles of gold or agglomerates of gold nanoparticles were to impart a grey colouration to the wool fibres. The approach utilised in-situ composite fibres that contained relatively low gold contents as "seed" substrates for the growth of gold nanoparticles. ${ }^{5}$ For this, in-situ composites were soaked in growth solutions that contained $\mathrm{AuCl}_{4}^{-}$and hydroxylamine $\left(\mathrm{NH}_{2} \mathrm{OH}\right)$, which is a mild reducing agent that reduces $\mathrm{Au}^{3+}$ to $\mathrm{Au}^{0}$ very slowly in the absence of catalysts. ${ }^{5}$ Metallic gold surfaces catalyse the reduction of $\mathrm{AuCl}_{4}^{-}$by $\mathrm{NH}_{2} \mathrm{OH}$, and as such, $\mathrm{AuCl}_{4}^{-}$in the growth solution was considered to preferentially increase the size of gold nanoparticles that are present on the "seed" composites, rather than nucleating new gold nanoparticles. The growth of the nanoparticles into larger particles (or agglomerates) was anticipated to result in grey coloured composites.

Figure 1.38 presents a range of composites that were synthesised with the seedmediated approach, where seed composites with different gold contents on the left hand side of the image were soaked in growth solutions of different concentration ratios of $\mathrm{AuCl}_{4}^{-}$to $\mathrm{NH}_{2} \mathrm{OH}$. Grey or purple-grey coloured composites were able to be synthesised under some of the reaction conditions, however, most of the composites were purple in colour. The seed-mediated approach allowed for a range of different coloured composites, however the gold content of these composites was greater than those in the in-situ approach, and far greater than those in the ex-situ approach. ${ }^{5}$ As such, the optimisation research in this research project will focus on the in-situ and ex-situ procedures. 


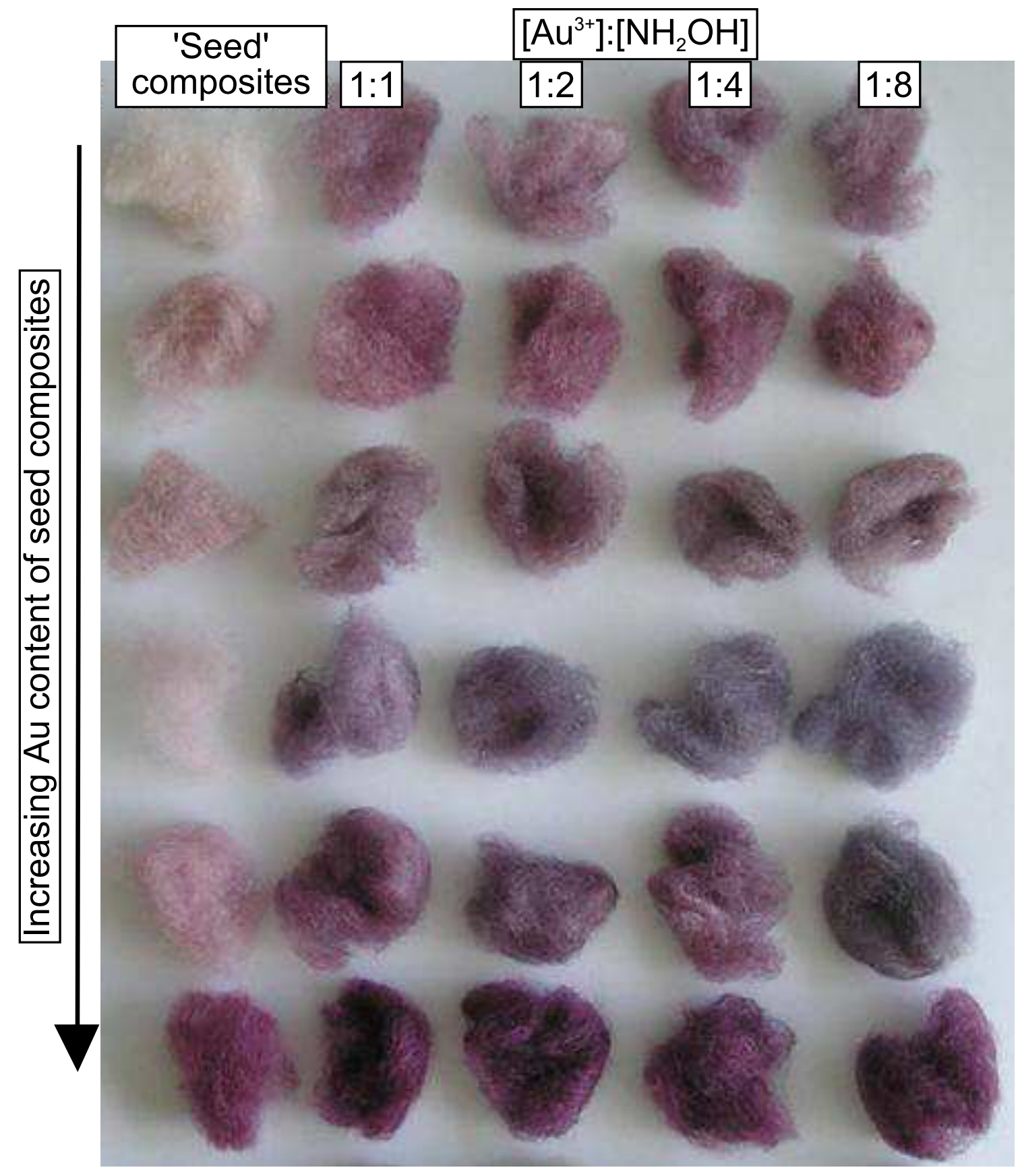

Figure 1.38 A range of composites that were synthesised with the seed-mediated approach, where seed composites containing different gold contents were soaked in growth solutions of different concentration ratios of $\mathrm{AuCl}_{4}^{-}$to $\mathrm{NH}_{2} \mathrm{OH} .{ }^{5}$ 


\subsection{Research Overview}

The research into the optimisation and scale-up of the synthesis of gold nanoparticlewool fibre composites is divided into four chapters. Chapter 3 describes experiments that were carried out to reduce the gold content of the in-situ procedure, by developing more cost-effective methodologies that result in in-situ composites with significantly lower gold contents. Chapter 4 involves optimising the laboratory-scale chemistry of the ex-situ procedure, before scale-up syntheses of ex-situ composites are carried out in Chapter 5. Finally, Chapter 6 details experiments that were employed to attempt to improve the washfastness properties of ex-situ composites. A brief description of the components of the research carried out and detailed in the results chapters is as follows:

\section{In-situ Synthesis of Gold Nanoparticle-wool Fibre Composites}

1. An introduction of the chemistry of the in-situ procedure by characterising the uptake and ageing processes in the in-situ synthesis of composites of Cariaggi wool (a source of merino wool in top form).

2. A characterisation of the effects of manipulating the reaction conditions of the synthesis of in-situ composites, in terms of changing the wool type, $\mathrm{pH}$, temperature, use of auxiliary agents, volume and wool to liquor ratio of the syntheses.

3. An attempt to reduce the gold content of in-situ composites by changing the mentioned reaction conditions, by facilitating the formation of gold nanoparticles at or near the surface of the wool fibres.

4. The use of heat and external reducing agents to increase the rate of gold nanoparticle formation in wool fibres, to facilitate gold nanoparticle formation before the $\mathrm{AuCl}_{4}^{-}$can diffuse into the centre of the wool fibres.

\section{Ex-situ Synthesis of Gold Nanoparticle-wool Fibre Composites}

1. An introduction of the chemistry of the ex-situ procedure through the characterisation of the uptake of gold nanoparticles to Cariaggi wool, and the colour of the resulting ex-situ composites.

2. An optimisation of the solution-phase synthesis of gold nanoparticle colloids, achieved by characterising the formation of gold nanoparticles, the $\mathrm{pH}$ stability 
of gold colloids and the ratios of $\mathrm{AuCl}_{4}^{-}$to stabilising agents used in the colloidal syntheses.

3. A characterisation of the uptake of gold nanoparticle colloids to wool fibres, and the colours of the resulting composites, while varying the wool type, reaction pH, use of auxiliary agents, concentration and volumes of the gold colloids.

4. An attempt to elucidate the changes in the colour of ex-situ composites upon drying after the dyeing process with Cryo-SEM.

\section{Scale-up of Ex-situ Gold Nanoparticle-wool Fibre Composites}

1. The development of novel reactors for scale-up syntheses of gold nanoparticles, and a characterisation of the large volumes of gold colloids that were produced with these reactors.

2. The hank dyeing of kilogram quantities of wool with gold colloids at an industrial wool dyeing plant, and the recreation of these dyeing procedures in the laboratory.

3. The use of industrial package dyeing equipment to colour fine merino yarns with gold nanoparticle colloids.

4. The design and construction of a suite of flow reactors that were based upon industrial dyeing reactors, and the colouring of wool in top form, felted merino yarns and fine merino yarns with these reactors.

\section{Improving the Washfastness Properties of Ex-situ Composites}

1. A characterisation of the washfastness properties of ex-situ composites of Cariaggi wool and fine merino yarn.

2. An attempt to improve the washfastness properties of ex-situ composites via the use of different chemical pre- and post-treatments.

3. An investigation of the use of a range of reducing and stabilising agents that contain reactive groups in the synthesis of gold nanoparticle colloids, and the dyeing of wool fibres with these gold colloids. 


\section{Chapter 2}

\section{Experimental}

\subsection{Materials}

\subsubsection{Wool Fibres}

The wool fibres used in this research project were from merino or crossbred sheep and sourced through Professor James Johnston's industry contacts. ${ }^{53}$ Ultrafine merino wool $(16 \mu \mathrm{m}$ diameter) in top form was sourced from New Zealand Merino Ltd. via Cariaggi, an Italian company that processes fine merino wool and yarns. Fine merino wool $(22 \mu \mathrm{m}$ diameter $)$ in top form was supplied by Ashford Handicrafts Ltd., Ashburton, New Zealand. Crossbred wool (38 $\mu \mathrm{m}$ diameter) in top form was sourced from Hawkes Bay Woolscourers Ltd (Cavalier Woolscourers), New Zealand. Ultrafine New Zealand merino yarn (14.5 $\mu \mathrm{m}$ diameter) was supplied via Spectrum Yarns Ltd, Huddersfield, England. Fine merino yarn (20.5 $\mu$ m diameter) was sourced from Tararua Yarns Ltd, Levin, New Zealand. Felted merino yarn was provided by Ascend Rugs Ltd., a rug manufacturer from Christchurch, New Zealand. A summary of the different types of wool that were used in this research project are presented in Table 2.1. 
Table 2.1 The different types of wool fibres used in this research project.

\begin{tabular}{lccc}
\hline Wool name & Wool type & $\begin{array}{c}\text { Approx fibre } \\
\text { diameter }(\mu \mathrm{m})\end{array}$ & Application \\
\hline Cariaggi & Top form & 16 & Apparel \\
Ashford & Top form & 22 & Apparel \\
Ashford2* & Top form & 22 & Apparel \\
Crossbred & Top form & 38 & Carpets \\
Open top & Loose wool & 16,25 and 36 & Various \\
$16 \mu \mathrm{m}$ & Loose wool & 16 & Apparel \\
$18 \mu \mathrm{m}$ & Loose wool & 18 & Apparel \\
S14 & Merino yarn & 14.5 & Apparel \\
S19 & Merino yarn & 19.5 & Apparel \\
TS & Merino yarn & 22 & Apparel \\
TU & Merino yarn & 22 & Apparel \\
Ascend yarn & Felted merino yarn & $500^{* *}$ & Rugs \\
\hline \hline
\end{tabular}

*Ashford and Ashford2 wool were sourced from the same sheepbreeds and husbandry conditions, however were processed through different production routes. Thus, these wool types exhibit unique surface chemistries and dyeing properties. ${ }^{* *}$ Yarn thickness. 


\subsubsection{Gold}

The $\mathrm{AuCl}_{4}^{-}$that was used in the in-situ procedure and the synthesis of gold nanoparticles in the ex-situ procedure, was taken from a stock solution of $4 \mathrm{wt} \% \mathrm{AuCl}_{4}^{-}$. Stock solutions of $250 \mathrm{~mL}$ were prepared by dissolving metallic gold $(10 \mathrm{~g})$ in concentrated aqua regia (a 3:1 mixture of concentrated $\mathrm{HCl}$ and $\mathrm{HNO}_{3}$ ) $(1 \mathrm{~L}$ ) in a $2 \mathrm{~L}$ glass beaker overnight. Excess $\mathrm{HNO}_{3}$ was then removed via the addition of urea $\left(\mathrm{CO}\left(\mathrm{NH}_{2}\right)_{2}\right)$ to the solution, which converts $\mathrm{HNO}_{3}$ into $\mathrm{N}_{2}(\mathrm{~g}), \mathrm{CO}_{2}(\mathrm{~g})$ and $\mathrm{H}_{2} \mathrm{O}$. The solution was heated to expel dissolved $\mathrm{CO}_{2}(\mathrm{~g})$, and distilled to remove excess $\mathrm{HCl}$ down to approximately $100 \mathrm{~mL}$. The concentrated solution was made up to $250 \mathrm{~mL}$ in a volumetric flask with distilled water to give a solution of $4 \mathrm{wt} \% \mathrm{AuCl}_{4}^{-}$. The $\mathrm{pH}$ of the stock solution was adjusted with the addition of $\mathrm{NaOH}$ (in a saturated aqueous solution), so that a diluted solution of $160 \mathrm{mg} \mathrm{L}^{-1}$ was $\mathrm{pH} \mathrm{2.6-2.7.}$ An image of the $\mathrm{AuCl}_{4}^{-}$stock solution is presented in Figure 2.1

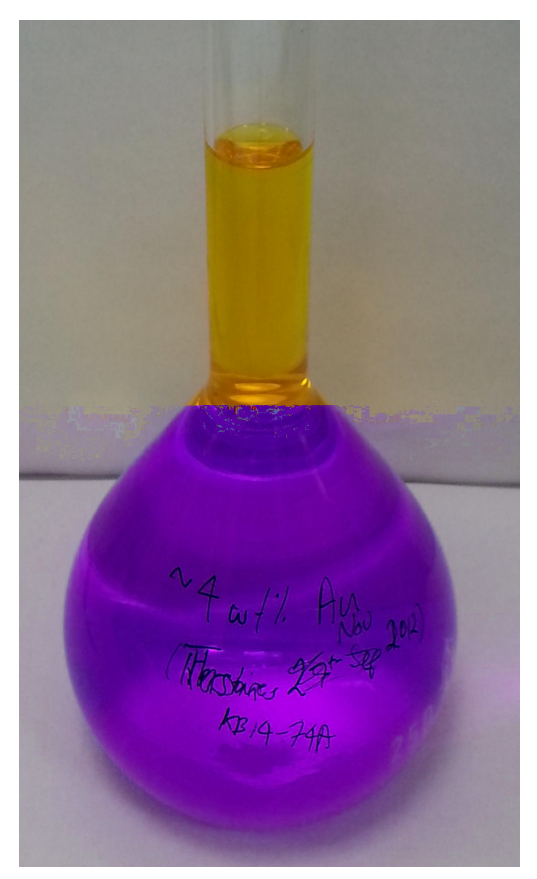

Figure 2.1 The stock solution of $\mathrm{AuCl}_{4}^{-}$(4 wt \%) that was prepared from metallic gold for the synthesis of gold nanoparticle-wool fibre composites.

\subsubsection{Other Reagents}

Wool dyeing auxiliaries, which were used to enhance the colour uniformity of gold nanoparticle-wool fibre composites, were sourced from Wool Yarns Ltd and Tararua Yarns Ltd, that are located in Lower Hutt and Levin, New Zealand, respectively. The recommended concentrations that of these reagents that were employed in dye- 
ing reactions were recommended by the local dyers. Reactive dyes were also supplied by Tararua Yarns Ltd. Kindness wool wash was purchased at a local supermarket.

All other chemicals were purchased from Sigma Aldrich.

\subsection{Preparatory Methods}

All of the glassware that was used in the synthesis of in-situ composites or gold nanoparticle colloids was cleaned in freshly prepared concentrated aqua regia and rinsed with distilled water prior to the synthesis. Contact between metallic surfaces and solutions of $\mathrm{AuCl}_{4}^{-}$were also avoided.

The preparatory methods in this section describe the general reaction parameters only. Specific details of the reaction conditions of experiments are provided in the proceeding chapters.

\subsubsection{In-situ procedure}

As mentioned previously, the in-situ procedure for the synthesis of gold nanoparticlewool fibre composites involves an uptake process where $\mathrm{AuCl}_{4}^{-}$is absorbed by wool fibres. This is followed by an ageing process where gold nanoparticles form in-situ. ${ }^{5}$

\section{The Uptake Process}

The uptake process involves soaking wool in $\mathrm{AuCl}_{4}^{-}$solutions. A typical laboratoryscale sample size was $0.1 \mathrm{~g}$ wool that was soaked in a $10 \mathrm{~mL}$ solution of $\mathrm{AuCl}_{4}^{-}$ $\left(160 \mathrm{mg} \mathrm{L}^{-1} \mathrm{Au}^{3+}\right)$, in a glass sample vial. This equates to a wool to liquor ratio of 1:100. The sample vials were agitated on an orbital shaker table at room temperature (RT) for $24 \mathrm{~h}$, by which time all of the $\mathrm{AuCl}_{4}^{-}$in the solution was typically absorbed by the wool fibres. This was determined via Atomic absorption spectroscopy. The standard conditions of the synthesis of an in-situ composite are listed in Table 2.2.

Uptake curves were determined to characterise the absorption of $\mathrm{AuCl}_{4}^{-}$by wool over time. This involved the synthesis of a number of duplicate samples, where the reaction was halted at different times. For example, 10 samples were made up, and 
Table 2.2 Standard reaction conditions of the in-situ procedure to synthesise purple coloured gold nanoparticle-wool fibre composites.

\begin{tabular}{lc}
\hline Reaction parameter & Standard amount \\
\hline Concentration of $\mathrm{Au}^{3+*}$ & $160 \mathrm{mg} \mathrm{L}^{-1}$ \\
Sample size & $0.1 \mathrm{~g}$ \\
Wool to liquor ratio & $1: 100$ \\
$\mathrm{pH}$ & 2.7 \\
Uptake conditions & $24 \mathrm{~h} \mathrm{@} \mathrm{RT}$ \\
Ageing conditions & $168 \mathrm{~h} \mathrm{@} \mathrm{50}{ }^{\circ} \mathrm{C}$ \\
Gold content of composite & $1.6 \mathrm{wt} \%$ \\
\hline \hline
\end{tabular}

*Present as $\mathrm{AuCl}_{4}^{-}$

wool was removed after $0,2,5,10,30,45,60,90,120$ and 180 min of uptake. The solutions were filtered and the residual concentration of $\mathrm{Au}^{3+}$ in the solutions were measured with atomic absorption spectroscopy (AAS).

\section{The Ageing Process}

The ageing process involved heating the uptake reaction vessels to promote the formation of gold nanoparticles in the wool fibres. This was achieved by transferring the uptake reaction vessels to a thermostatted water bath, where the fibres gradually develop the purple colour common to gold nanoparticles over 1 week. The rate of colour development in the composites was determined by removing samples at different times from the ageing and analysing the colour of the composites with UV-visible reflectance spectroscopy or CIE colour coordinate measurement.

\subsubsection{Ex-situ Procedure}

\section{Gold Nanoparticle Synthesis}

The ex-situ procedure involved the solution-phase synthesis of gold nanoparticles, which were then used to colour wool fibres. The standard conditions of the syntheses of TA-, TSC- and polyethylenimine (PEI)-stabilised gold nanoparticles are presented in Table 2.3. 
Table 2.3 Standard reaction conditions of the ex-situ procedures.

\begin{tabular}{lcccc}
\hline Method & TA-mauve & TSC-pink & TSC-grey & PEI-grey \\
\hline Amount of $\mathrm{Au}^{3+}\left(\mathrm{mg} \mathrm{L}^{-1}\right)$ & 10 & 10 & 40 & 40 \\
Conc of $\mathrm{Au}^{3+}\left(\mathrm{mol} \mathrm{L}^{-1}\right)$ & $5.08 \times 10^{-5}$ & $5.08 \times 10^{-5}$ & $2.03 \times 10^{-5}$ & $2.03 \times 10^{-5}$ \\
Temperature & $\mathrm{RT}$ & $95^{\circ} \mathrm{C}$ & $95^{\circ} \mathrm{C}$ & $95^{\circ} \mathrm{C}$ \\
Ratio of $\mathrm{AuCl}_{4}^{-}$to stabiliser & $1: 0.39$ & $1: 2.1$ & $1: 2.1$ & $1: 33$ \\
\hline \hline
\end{tabular}

\section{Synthesis of TA-Au NPs}

The synthesis of the volume of TA-stabilised gold nanoparticles that was used to colour $1 \mathrm{~g}$ of wool involved the addition of $\mathrm{Au}^{3+}(15 \mu \mathrm{L}, 4 \mathrm{wt} \%)$ to distilled water $(60 \mathrm{~mL})$ under rapid stirring at RT. TA was then added $(200 \mu \mathrm{L}, 1 \mathrm{wt} \%)$, and the purple-mauve colour common to TA-stabilised gold nanoparticles began to form in less than $2 \mathrm{~min}$. The colloid was left stirring for a further 30-60 min until the formation of TA-Au NPs was complete.

\section{Synthesis of TSC-Au NPs}

The synthesis of the TSC-stabilised gold colloid that was used to produce a $1 \mathrm{~g}$ pinkcoloured composite sample involved heating distilled water $(60 \mathrm{~mL})$ to $95^{\circ} \mathrm{C}$ under rapid stirring on a thermostatted hot plate with a temperature probe. Once the temperature has stabilised, $\mathrm{Au}^{3+}(15 \mu \mathrm{L}, 4 \mathrm{wt} \%)$ was added, followed by the addition of $\operatorname{TSC}(37.5 \mu \mathrm{L}, 5 \mathrm{wt} \%)$. The solution became dark after approximately $3 \mathrm{~min}$ of the reaction, and the ruby-red colour common to TSC-stabilised gold nanoparticles develops for the next $30-60 \mathrm{~min}$. After this time, the solution was removed from the hot plate before being left to cool to RT.

Varying the concentration of the TSC-stabilised gold colloids allows the preparation of different coloured composites (while retaining the same concentration ratio of $\mathrm{AuCl}_{4}^{-}$to TSC). For example, the addition of $\mathrm{Au}^{3+}(60 \mu \mathrm{L}, 4 \mathrm{wt} \%)$ and TSC $(150 \mu \mathrm{L}$, $5 \mathrm{wt} \%$ ) results in the grey coloured composites when used to colour a $1 \mathrm{~g}$ quantity of wool.

\section{Synthesis of PEI-Au NPs}

The synthesis of PEI-stabilised gold nanoparticles involved the same procedure as the TSC-grey colloid; distilled water $(60 \mathrm{~mL})$ was heated to $95^{\circ} \mathrm{C}$ under rapid stirring, before the addition of $\mathrm{Au}^{3+}(60 \mu \mathrm{L}, 4 \mathrm{wt} \%)$ and PEI $(1.728 \mathrm{~mL}, 1 \mathrm{wt} \%)$. Low molecular weight branched PEI was utilised in the synthesis. The formation of PEI-Au NPs occurs over a time period of $30 \mathrm{~min}$. 


\section{Gold Colloid Syntheses with Reactive Dyes}

The synthesis of gold colloids that were stabilised with reactive dyes was carried out at $95^{\circ} \mathrm{C}$, and involved the addition of different amounts of $1 \mathrm{wt} \%$ solutions of the dye molecules to a $40 \mathrm{mg} \mathrm{L}^{-1}$ solution of $\mathrm{Au}^{3+}$.

\section{Characterisation of Gold Nanoparticle Formation}

The rate of formation of TA-Au NPs and TSC-Au NPs was studied with UV-vis spectroscopy (Section 4.2.1). The formation of TA-Au NPs involved the synthesis of $300 \mathrm{~mL}$ gold colloid. $3 \mathrm{~mL}$ of the reaction solution was removed for UV-vis analysis and then rapidly returned to the reaction solution.

Due to the variation in temperatures caused upon removing a sample from a solution at $95^{\circ} \mathrm{C}$ for UV-vis analysis, an alternative method was required. As such, the formation of TSC-Au NPs was studied inside a quartz cuvette that was heated in a UV-vis thermostatted cuvette holder that was attached to a water bath. The total volume of the reaction was $3 \mathrm{~mL}$. An Agilent Diode Array Spectrometer was used for studying the formation of TA-Au NPs and TSC-Au NPs in all cases except the in-situ formation of TA-Au NPs, which employed an HP diode array spectrophotometer.

\section{Uptake of Gold Nanoparticles to Wool Fibres}

The uptake of gold nanoparticles to wool fibres involved adjusting the $\mathrm{pH}$ of the gold colloids, to provide an electrostatic driving force for the absorption, and then soaking the wool fibres in the solutions. The standard dyeing conditions that were employed in the ex-situ procedure are listed in Table 2.4 for TA-, TSC- and PEI stabilised gold colloids.

Table 2.4 Standard reaction conditions of the ex-situ procedures.

\begin{tabular}{lcccc}
\hline Method & TA-mauve & TSC-pink & TSC-grey & PEI-grey \\
\hline pH of dyeing & 2.2 & 2.7 & $2.0-2.7$ & 7 \\
Wool to liquor ratio & $1: 60$ & $1: 60$ & $1: 60$ & $1: 60$ \\
$\begin{array}{l}\text { Approx gold content of } \\
\text { composites (wt \%) }\end{array}$ & 0.06 & 0.06 & 0.24 & 0.24 \\
\hline \hline
\end{tabular}

The rate of uptake of the gold nanoparticles to the wool fibres was studied with UV-vis spectroscopy, as the intensity of colour of the nanoparticles is related to the number of nanoparticles in solution. For this, samples of the uptake solution were removed at different times for UV-vis analysis. Uptake curves were determined by 
plotting the decrease in intensity of the SPR peak of gold nanoparticles in the uptake solution over time. With the uptake of TSC-Au NPs, the gold nanoparticles often agglomerate during the uptake, which results in a shift in the absorption wavelength of the SPR peak. Thus, the uptake of TSC-Au NPs could sometimes not be studied with this method. In contrast, TA-Au NPs do not agglomerate during the dyeing process.

One of the aims of the uptake process was to attain as uniform a distribution of gold nanoparticles throughout the wool sample as possible, so that evenly coloured composites were produced. On the laboratory-scale (typically $0.1-10 \mathrm{~g}$ ), this was achieved by placing the reaction vessels on an orbital shaker table during the uptake. This was no longer possible when the process was scaled-up, and as such, alternative measures, including dye circulation, were employed.

\section{Gold Recovery}

The were times where gold colloids that were produced for the ex-situ procedure were destabilised. These colloids were not able to be used for dyeing procedures. In order to prevent wastage, the gold was recovered. The colloids were left in settling tanks to coagulate (typically for several weeks), the water was decanted off, the agglomerated colloids combined in a glass beaker, and evaporated to dryness on a hotplate. The beaker was then heated in a furnace at $550^{\circ} \mathrm{C}$ for $120 \mathrm{~min}$ to remove the organic stabilisers. The remaining material was gold or purple coloured, due to the presence of $\mathrm{Au}^{0}$ metal or nanoparticles. The gold was used to produce a precursor $\mathrm{AuCl}_{4}^{-}$solution using the method outlined in Section 2.1.2.

\subsubsection{Wool Fibre Pre-Treatment}

A number of wool fibre pre-treatments were employed to alter the chemistry of the wool fibres prior to the dyeing process. The pre-treatments were carried out to control the rate of uptake of gold nanoparticles, to remove surface lubricants and lipids that may interfere with dye chemistry, or to attempt to create additional reactive sites for the absorption and binding of $\mathrm{AuCl}_{4}^{-}$or $\mathrm{Au}$ NPs in the in-situ and ex-situ procedures, respectively. 


\section{Warm Water Washing}

Wool fibres were often soaked in warm tap water for at least $10 \mathrm{~min}$ to attempt to remove processing lubricants and wool grease that was present on the wool fibres. The warm water wash solutions typically became cloudy, which indicated the leaching of lubricants and lipids from the wool fibres. The warm water also made it easier to "wet out" the dyepack (the sample of wool that was being dyed) during subsequent dyeing reactions.

\section{Removal of 18-MEA}

The hydrophobic 18-MEA layer is covalently bound to the surface of wool fibres and was removed to make the wool fibres hydrophilic. The removal of the 18-MEA layer was discussed in Section 1.1.3, and involved wool $(1 \mathrm{~g})$ soaking in a solution of $\mathrm{KOH}$ in methanol $\left(0.1 \mathrm{~mol} \mathrm{~L}^{-1}, 20 \mathrm{~mL}\right)$ for $10 \mathrm{~min}$ at RT. If it was left for any longer then wool fibres would degrade in the alkaline solution.

\section{Selective Reduction of Cystine}

As was described in Section 1.1.2, the reduction of cystine results in additional thiol sites on the wool fibres. The selective reduction of cystine was achieved by soaking wool fibres in aqueous solutions of thiol-containing reagents, for example mercaptosuccinic acid (MSA) and thioglycolic acid (TGA). For this, wool (1 g) was soaked in a $1 \mathrm{wt} \% \mathrm{MSA}$ or TGA solution $(60 \mathrm{~mL})$ at $\mathrm{pH} 8$ and $50{ }^{\circ} \mathrm{C}$ for $120 \mathrm{~min}$. After this time, the wool was patted dry with paper towels and immediately immersed in dye solutions following this treatment. The wool was originally rinsed to remove excess thiol reagents prior to the dyeing process. However, after discussion with industrial collaborators, the rinsing step was removed, due to oxidation of the thiol groups that form. ${ }^{54}$

\section{Sulfitolysis of Cystine}

The sulfitolysis of cystine was carried out in an attempt to increase the number of reactive sites on the wool fibres. For this, wool $(1 \mathrm{~g})$ was soaked in a $\mathrm{Na}_{2} \mathrm{~S}_{2} \mathrm{O}_{5}$ solution $(60 \mathrm{~mL}, 1 \mathrm{wt} \%)$ at $\mathrm{pH} 8$ for either $120 \mathrm{~min}$ at $60^{\circ} \mathrm{C}$ or for $10 \mathrm{~min}$ at $90^{\circ} \mathrm{C}$. The wool was patted dry with paper towels and then immediately soaked in the dye 
solutions following this treatment. The chemistry of this reaction was explained in Section 1.1.2.

\section{Glycine Buffer}

The use of glycine buffers was introduced into the ex-situ procedure during this research project. This treatment involved soaking wool fibres in an aqueous glycine buffer $\left(0.1 \mathrm{~mol} \mathrm{~L}^{-1}\right)$ that was $\mathrm{pH}$ adjusted with $\mathrm{HCl}\left(6 \mathrm{~mol} \mathrm{~L}^{-1}\right)$ to the same $\mathrm{pH}$ as the gold colloid in the subsequent dyeing reaction (typically between $\mathrm{pH} 2-3$ ). This was undertaken to prevent the change in $\mathrm{pH}$ that would occur during dyeing reactions, where the $\mathrm{H}^{+}$ions in the dye solution would associate to the wool surface. This would increase the $\mathrm{pH}$ of the gold colloid and reduce the driving force for the absorption of gold nanoparticles by wool.

\section{Wool Dyeing Auxiliaries}

Wool dyeing auxiliaries were employed to increase the ease of wetting out a dyepack and to control the rate of uptake of $\mathrm{AuCl}_{4}^{-}$or gold nanoparticles to wool. As mentioned in Section 1.1.4, auxiliary agents are classed as wetting or levelling agents, and were introduced into dye solutions or wool fibre pre-treatments. Table 2.5 presents the wool dyeing auxiliary agents that were used in this research project, where the wetting agent was sourced via Wool Yarns Ltd, and Tararua Yarns Ltd provided the levelling agents.

Table 2.5 Levelling agents that were employed in the ex-situ procedure.

\begin{tabular}{lcc}
\hline Auxiliary agent & Type & Recommended level $\left(\mathrm{g} \mathrm{L}^{-1}\right)$ \\
\hline Albaflow FFA & Wetting agent & $0.5-1$ \\
Albegal B & Levelling agent & 2 \\
Lyogen MF & Levelling agent & 2 \\
Croscolor DH2 & Levelling agent & 0.33 \\
Tecarapid CBF & Levelling agent & 2 \\
\hline \hline
\end{tabular}

\subsubsection{Scale-up Procedures}

A range of scale-up syntheses of ex-situ gold nanoparticle-wool fibre composites were carried out and are detailed in Chapter 5. The syntheses of gold nanoparticle colloids 
that were used in these dyeing reactions are detailed in Section 5.1. A summary of the different scale-up syntheses is shown in Table 2.6

Table 2.6 A summary of the different scale-up dyeing reactions.

\begin{tabular}{lccc}
\hline Dyeing method & Wool type & Sample size $(\mathrm{g})$ & Wool to liquor ratio \\
\hline Static dyeing & Top form & $100-1500$ & $1: 60$ \\
Hank dyeing & Felted yarn & $500-1000$ & $1: 60$ \\
Package dyeing & Fine merino yarn & $250-750$ & $1: 12-1: 36$ \\
Pressurised & Top form and & $300-660$ & $1: 16.7-1: 20$ \\
flow reactors & felted yarn & & \\
\hline \hline
\end{tabular}

The static dyeing and pressurised flow reactors were carried out in the laboratory at Victoria University of Wellington (VUW). Hank dyeing reactions were first undertaken at Wool Yarns Ltd, with their $150 \mathrm{~L}$ stainless hank dyeing reactor. These reactions were then reproduced at VUW. Industrial package dyeing equipment was used on site at Tararua Yarns Ltd for the dyeing of fine merino yarns. The specific reaction parameters of the scale-up dyeing reactions are detailed in Chapter 5.

\section{Benchtop Flow Reactor}

The Benchtop flow reactor was designed for studies of the uptake of gold nanoparticles to wool fibres with dye circulation. This was carried out as uptake studies from static dyeing reactions were not representative of the rate of uptake that was observed in scale-up package dyeing reactions. The set-up of the Benchtop flow reactor is presented in Figure 2.2, where dye solutions were pumped from the overflow beaker through the PVC tubing, where wool yarn $(5 \mathrm{~g})$ was held in place between two needles. The inset in Figure 2.2 shows how the colloid flows through the wool sample more clearly. The dye solution was stirred constantly during the reaction and samples were removed from the overflow beaker during the uptake for subsequent $\mathrm{UV}$-vis analysis.

\section{Pressurised Flow Reactors}

The pressurised flow reactors were designed and used in collaboration with Ahmet Kitap, a process engineering intern student from Georg-Simon-Ohm University for Applied Sciences in Nuremberg, Germany. The reactors were built by Nick Grinter, a mechanical technician from the VUW workshop. Three reactors of different sizes were developed to colour a range of masses of wool. The reactors were sealed and 


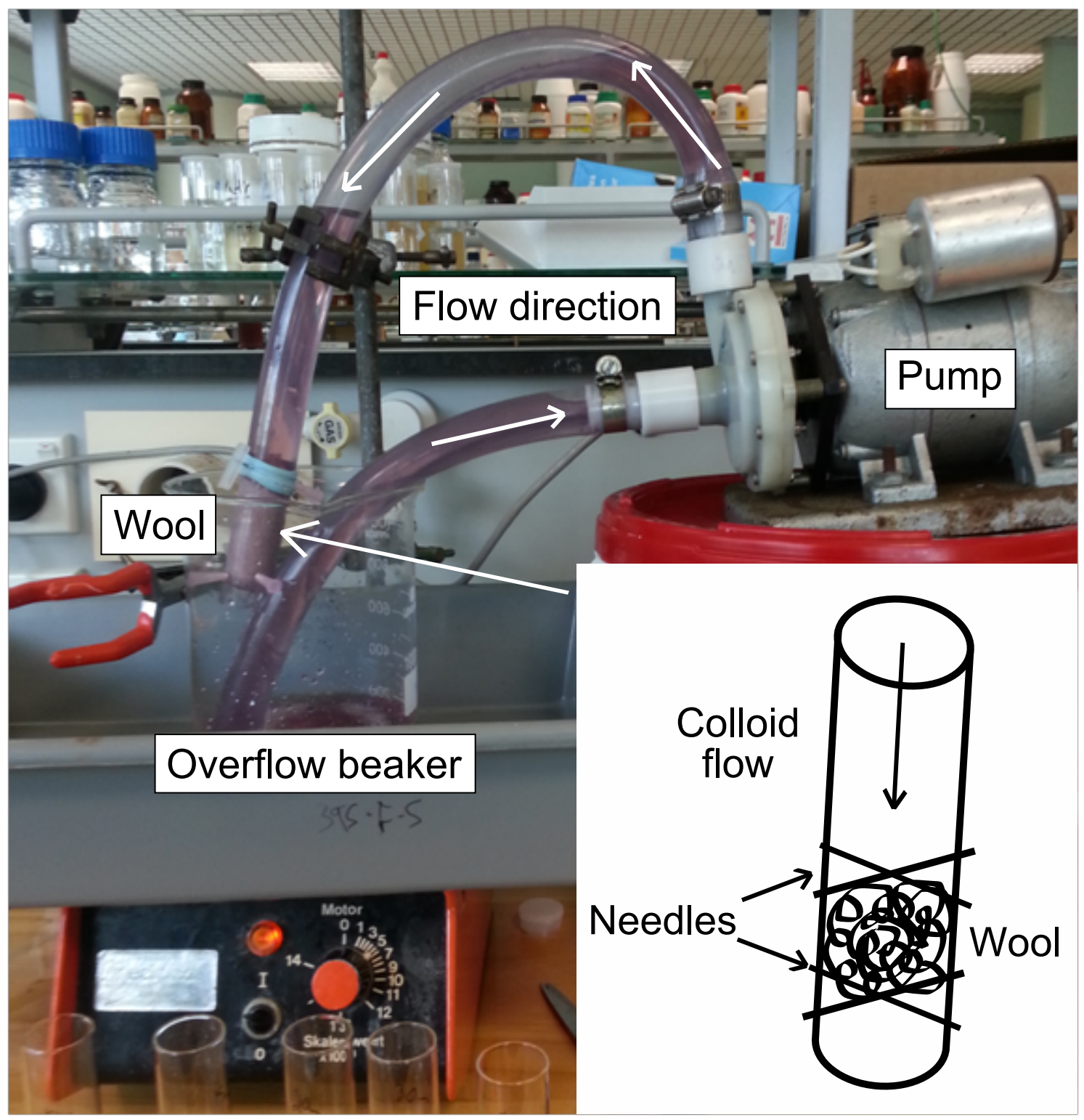

Figure 2.2 The Benchtop flow reactor that was used for studying the uptake of TA-Au NPs to TU yarn. 
do not employ overflow tanks. This set-up means that the volumes of dye liquor employed in the dyeing reactions were fixed. The relevant parameters of the reactors are summarised in Table 2.7 .

Table 2.7 A summary of the reaction parameters of the pressurised flow reactors.

\begin{tabular}{cccc}
\hline Reactor & Baby Guinness & Half Pint & Full Pint \\
\hline Abbreviation & BG & HP & FP \\
Volume of reactor $(\mathrm{L})$ & 6 & 8 & 10 \\
Weight of wool $(\mathrm{g})$ & $300-360$ & 500 & $600-660$ \\
Estimated flow with wool $\left(\mathrm{L} \mathrm{min}^{-1}\right)$ & 30 & 30 & 30 \\
\hline \hline
\end{tabular}

The set-up of the reactor is described in Section 5.4.3, where the flowrates that were employed are dictated by the pumping capabilities, the internal pressure and the sizes of the inlets and outlets that were used. An Onga model 413 pump was used, which operates at $2750 \mathrm{rpm}$. The flowrate was measured at $80 \mathrm{~L} \mathrm{~min}^{-1}$ for the circulation of water without wool inside the system. However, when wool was present inside the reactor, the internal pressure rises considerably, and can damage the structural integrity of the reactor. Thus, the conservative flowrates of $30 \mathrm{~L} \mathrm{~min}^{-1}$ were employed in the dyeing reactions, so as to avoid damage to the reactor through excessive pressure. The flowrate was limited by a valve that reduced the volume flow from the pump to the inlet tubing, as the pump speed was fixed.

\subsubsection{Washfastness Testing}

Washfastness testing was carried out to characterise the leaching of gold nanoparticles from composites synthesised with the ex-situ procedure. The testing involved the washing of wool in an aqueous dilution of Kindness wool wash, commercially available wool washing detergent that contains a mixture of cationic, anionic and non-ionic surfactants. The concentration of Kindness wool wash that was used was the commercially recommended level of $12.5 \mathrm{~mL} \mathrm{~L}^{-1}$, or approximately $0.3 \mathrm{wt} \%$, in cold tap water.

There were two different types of washfastness tests that were employed in Chapter 6 , the first of which involved soaking composites in a sample vial with Kindness wool wash. A wool to liquor ratio of $1: 20$ was employed (typically $0.5 \mathrm{~g}$ in a $10 \mathrm{~mL}$ wash solution), and the sample vials were agitated on an orbital shaker table $24 \mathrm{~h}$ at RT. The leaching of gold nanoparticles from the ex-situ composites into the wash solution was measured with UV-vis spectroscopy. 
The alternative washfastness test involved washing larger samples (1-2g) in excess Kindness wool wash for an extended period of time. Early washfastness tests utilised a Chiltern flask shaker, where wool was soaked in a wash solution (500 mL) and agitated for $24 \mathrm{~h}$. Experiments that were carried out later on in the $\mathrm{PhD}$ programme used a "dunking machine". This was developed to simulate hand washing and involved attaching composite samples to a dunking apparatus that was continuously lowered in and out of a solution of Kindness wool wash (20 L) for a period of $120 \mathrm{~min}$ (Figure 2.3). Samples were dunked in the wash solutions approximately 42 times a minute, which equated to around 5000 immersion cycles in $120 \mathrm{~min}$. The washfastness properties of the composites were characterised by measuring the differences in the CIE colour coordinates of the samples before and after the washing process.

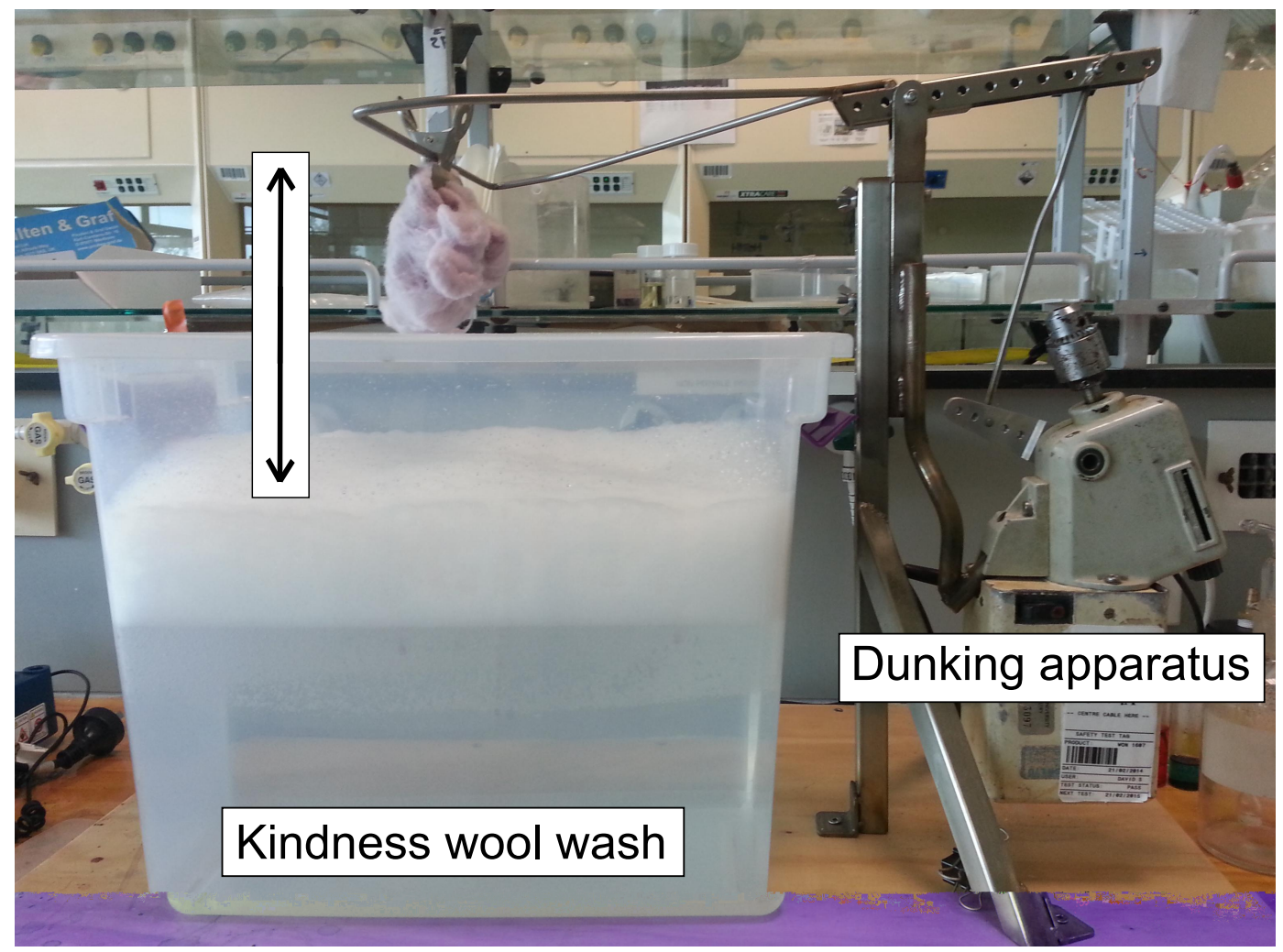

Figure 2.3 The "dunking machine" that was used to dunk composite samples in and out of a solution of Kindness wool wash.

\subsection{Characterisation}

\subsubsection{Atomic Absorption Spectroscopy}

Flame atomic absorption spectroscopy (AAS) was used to measure the concentration of residual $\mathrm{Au}^{3+}$ that was present in uptake reaction solutions. This allowed for uptake curves to be determined. A Flame Thermoscientific iCE 3000 series AA 
Spectrometer was used, which employed a $242.8 \mathrm{~nm}$ wavelength lamp that is specific to an electronic transition in $\mathrm{Au}^{3+}$. The absorption was referenced to calibration curves of standard solutions of $\mathrm{Au}^{3+}$ (present as $\mathrm{AuCl}_{4}^{-}$), with concentrations of 3.5, 7.5, 10, 12 and $14 \mathrm{mg} \mathrm{L}^{-1}$. The $\mathrm{AuCl}_{4}^{-}$standards were obtained by diluting a $997 \mathrm{mg} \mathrm{L}^{-1}$ calibration solution of $\mathrm{AuCl}_{4}^{-}$that was purchased from Sigma Aldrich. The uptake solutions were often diluted, as they were more concentrated than the highest standard solution of the calibration curve $\left(14 \mathrm{mg} \mathrm{L}^{-1} \mathrm{Au}^{3+}\right)$.

\subsubsection{Ultraviolet-visible Absorption Spectroscopy}

Ultraviolet-visible absorbance (UV-vis) spectroscopy was used to study the colour of both gold nanoparticle colloids and gold nanoparticle-wool fibre composites. The position, intensity and broadness of peaks in UV-vis spectra of gold colloids and composites gave information on the size, shape and the polydispersity of the size and shape of the gold nanoparticles. Uptake curves were also determined by plotting the absorbance maximum of the SPR peak of gold nanoparticles over time during the dyeing reactions. The reaction kinetics of the formation of gold nanoparticles were likewise measured. UV-vis spectroscopy was also used to characterise the leaching of gold nanoparticles into wash solutions.

The colours of gold nanoparticle-wool fibre composites were characterised using UVvisible reflectance spectroscopy, which is applicable to solid samples. The reflectance values were transformed to colour yield values according to Kubelka Munk theory. This is shown in Equation 2.1 below, where $\mathrm{R}$ is reflectance, and $\mathrm{K}$ and $\mathrm{S}$ relate to the absorption and scattering coefficients of the sample, respectively.

$$
K / S \approx(1-R)^{2} / 2 R
$$

An Agilent Diode Array Spectrometer was used to analyse liquid samples in absorbance mode and an A.I. Scientific Carey UV-visible Spectrophotometer was employed to analyse solid samples in \% Reflectance mode. The UV-vis spectra were generally normalised at $1000 \mathrm{~nm}=0$ intensity, as this absorbance was typically outside of the range of SPR absorbances of gold nanoparticles, and should remain constant. However, during the extensive agglomeration of TSC-Au NPs that occurs in the TSC-grey method, longer wavelength absorbances dominate. As such, the UV-vis spectra were not normalised. 


\subsubsection{CIE Colour Coordinates}

A Hunterlab ColorQuest spectrophotometer was used to measure the spatial reflectance of gold nanoparticle-wool fibre composites, where subtle differences in colouration were quantified by characterising changes in the CIE colour coordinates of the samples. $\mathrm{L}^{*}$ is a measure of brightness; a $\mathrm{L}^{*}$ value of 0 relates to complete blackness and 100 for complete whiteness. Colour ranges between red (positive) and green (negative) are denoted for $\mathrm{a}^{*}$, while $\mathrm{b}^{*}$ is between yellow (positive) and blue (negative) colouration. The $\mathrm{L}^{*}, \mathrm{a}^{*}$ and $\mathrm{b}^{*}$ values are illustrated in Figure 2.4 below.

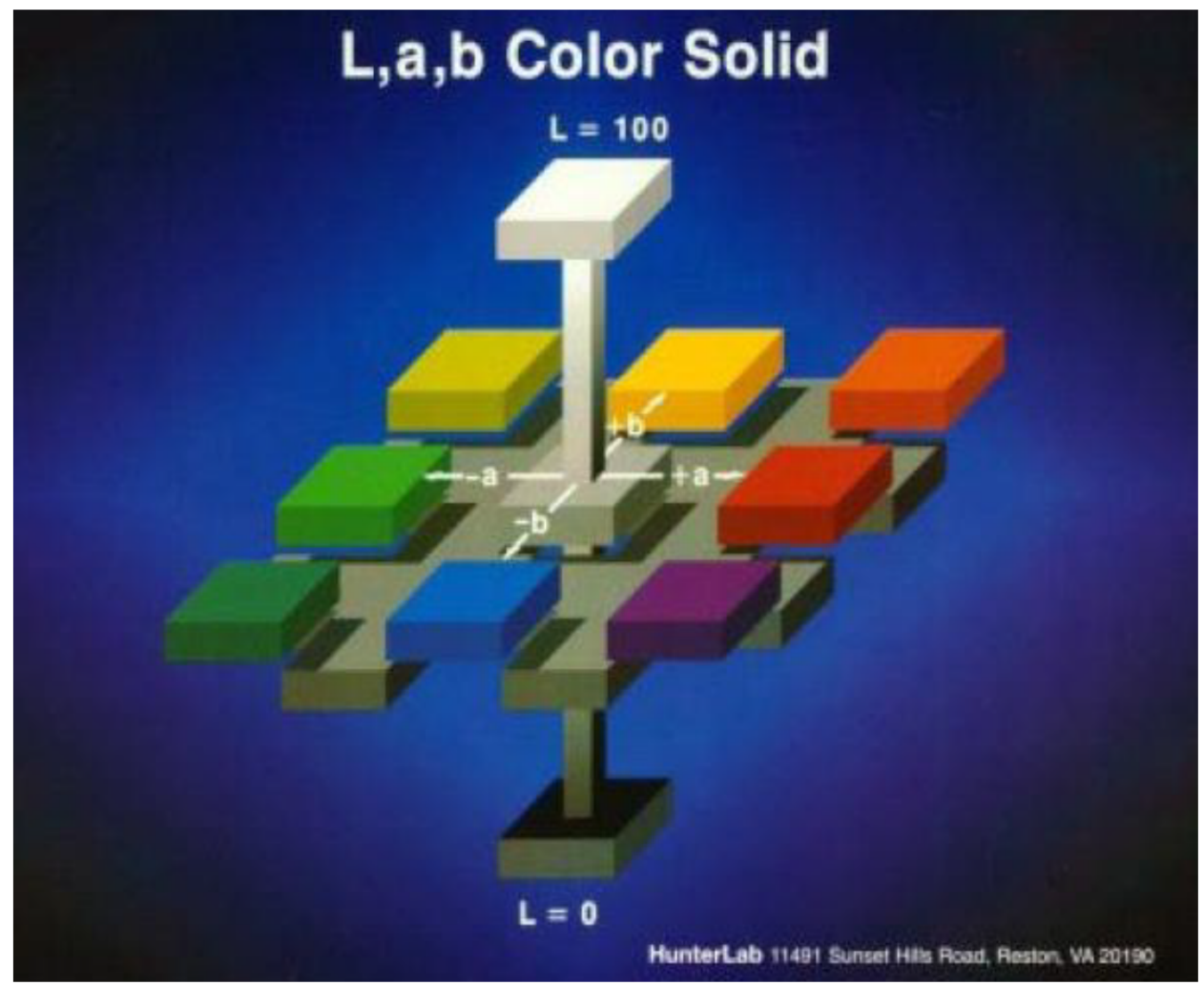

Figure 2.4 Representation of the $\mathrm{L}^{*}, \mathrm{a}^{*}$ and $\mathrm{b}^{*}$ CIE colour coordinate values that were measured with the ColorQuest spectrophotometer.

\subsubsection{Scanning Electron Microscopy}

Scanning electron microscopy (SEM) imaging was used to study the size, shape and distribution of gold nanoparticles in gold nanoparticle-wool fibre composites. SEM imaging can analyse the surfaces or cross-sections of individual composite fibres, where cross-sectional analysis determines the penetration of $\mathrm{AuCl}_{4}^{-}$or gold nanoparticles inside the wool fibres. Backscatter imaging was most commonly employed, where stark contrast differences were observed between gold nanoparticles and the wool fibres. This was due to the high atomic mass of gold appearing bright on the 
relatively dark organic wool fibres, which have much lower atomic masses.

A JEOL 6500 F field emission gun scanning electron microscope was used for SEM analysis. Sample preparation involved mounting wool fibres on aluminium stubs with double sided carbon tape. The samples were carbon coated with a JEOL JEC560 carbon coater, where 2-3 carbon coats provided optimal imaging conditions. Cross-sectional analysis was achieved by aligning fibres between strips of carbon tape, freezing the tape in liquid nitrogen, and cutting cleanly through the frozen fibres with a razor blade. The cut fibres were mounted on the vertical face of an aluminium stub for clear exposure of the fibre cross-sections.

\section{Cryo Scanning Electron Microscopy}

To determine differences in ex-situ composites before and after drying, Cryo SEM was utilised. For this, samples of composite fibres were frozen in liquid nitrogen while still in the dye solutions, and were carbon coated while frozen. The frozen samples were only imaged at relatively low resolution while under Cryo conditions, as when the energy of the electron beam was focused down to a smaller area (as occurs upon magnification), the samples melted. When compared with the SEM images of a dry composite sample, possible differences between the distribution of gold nanoparticles on the wool fibres were ascertained.

\subsubsection{Energy Dispersive X-ray Spectroscopy}

Energy dispersive X-ray spectroscopy (EDS) was employed to investigate the elemental composition of gold nanoparticle-wool fibre composites. EDS was able to illustrate the concentration of different elements on the surface and cross-sections of the composites when used with SEM. Areas of high concentration of elemental Au were used to show areas where gold nanoparticles were present on the composites. Cross-sectional analysis with EDS shows the extent of diffusion of $\mathrm{AuCl}_{4}^{-}$or gold nanoparticles into the wool fibre interior.

\subsubsection{Transmission Electron Microscopy}

Transmission electron microscopy (TEM) was used to study gold colloids. TEM is a technique which is complementary to SEM, and can image gold colloids at higher 
resolutions. In TEM images, the relatively high atomic weight gold nanoparticles appeared relatively darker than the organic elements that were present in stabilising agents of the gold colloids. A JEOL 2010 High Resolution TEM instrument with a $\mathrm{LaB}_{6}$ filament was employed. The sample preparation simply involved drop casting gold nanoparticle colloids onto copper grids, and evaporating the solvent, prior to TEM analysis. 


\section{Chapter 3}

\section{In-situ Synthesis of Gold Nanoparticle-wool Fibre Composites}

During the course of her PhD research, Dr Kerstin Lucas (neé Burridge), under the supervision of Professor James H Johnston, developed the in-situ procedure for the synthesis of gold nanoparticle-wool fibre composites. This approach utilises wool fibres as redox active biotemplates that facilitate the formation of gold nanoparticles in-situ (within the protein matrix of the fibres). ${ }^{5}$ The in-situ procedure was comprehensively reviewed in Section 1.3.1. The in-situ procedure is different from the ex-situ procedure which involves colouring wool fibres with gold nanoparticle colloids prepared ex-situ. Composites synthesised with the in-situ procedure possess excellent washfastness properties due to the chemical binding of gold nanoparticles to the wool fibres. However, in-situ composites also exhibit a significantly higher gold content relative to ex-situ composites (approximately $1.6 \mathrm{wt} \%$ for purple-coloured composites). Unfortunately, this meant that the in-situ approach developed by Lucas and Johnston was not economically viable for scale-up at this stage of the research.

The economic impact of the gold content of the in-situ composites has been enhanced by the rising world gold price over the past 12 years. Figure 3.1 highlights the dramatic increase in the world price of gold during the time of Dr Lucas's doctoral research from 2006-2010. ${ }^{55}$ The gold price remains strong at approximately 1500 NZD per troy ounce.

Chapter 3 details research relating to minimising the gold content of in-situ of a 


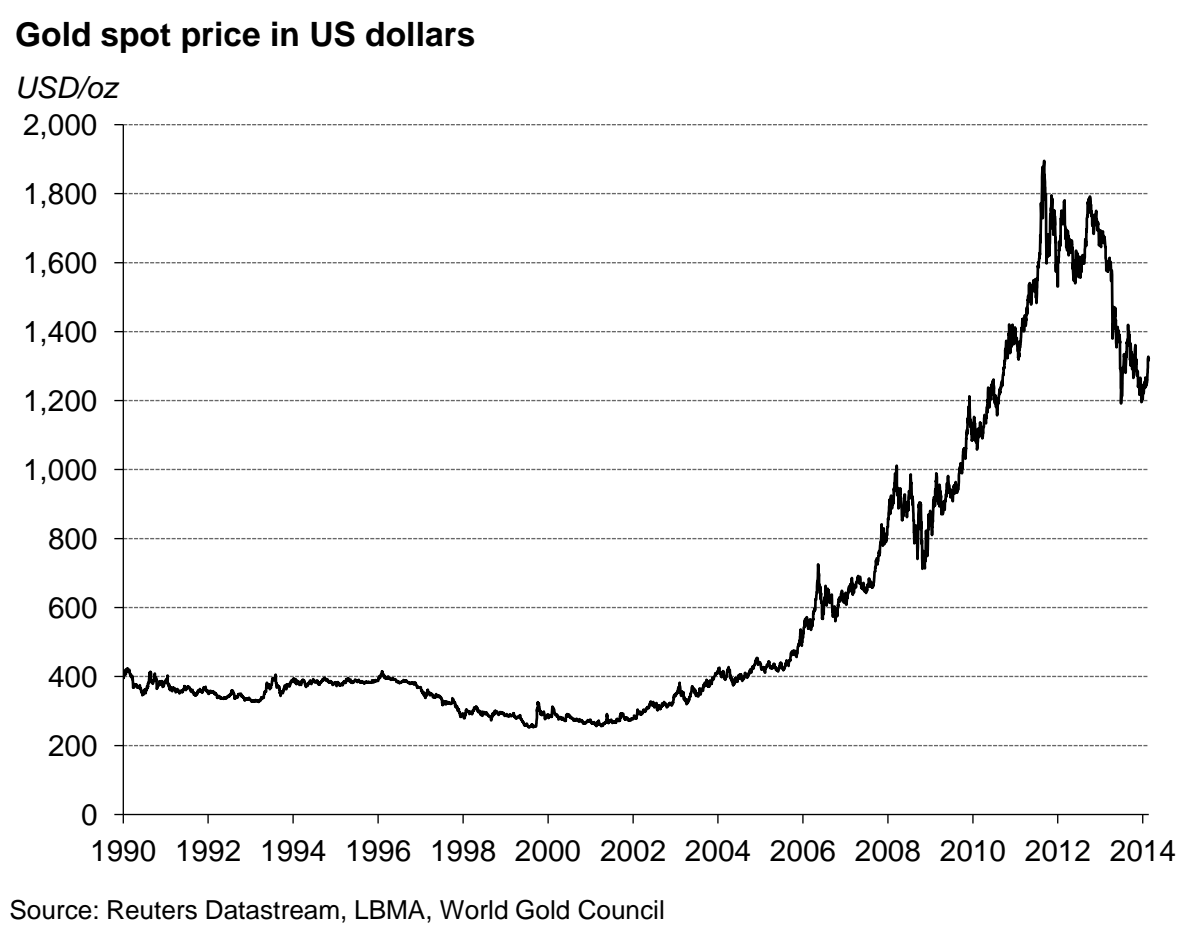

Figure 3.1 The change in the world gold spot price in USD per troy ounce from $1990-2014 .^{55}$

given colour, that were synthesised via the in-situ procedure. This involved initially expanding the understanding of the chemistry of the in-situ procedure developed by Lucas and Johnston.

Section 3.1 details the synthesis and characterisation of gold nanoparticle-wool fibre composites that were prepared with Cariaggi wool via the in-situ process. Section 3.2 characterises the effects of wool type, time, $\mathrm{pH}$, temperature and auxiliary agents on the chemistry of the in-situ procedure. Section 3.3 details how the rate of $\mathrm{AuCl}_{4}^{-}$ uptake to wool, the rate of gold nanoparticle formation and the distribution of gold nanoparticles in the wool were controlled. This was achieved by manipulating the reaction conditions and introducing external reducing agents and heat to the in-situ process.

\subsection{In-situ Composites of Cariaggi Wool}

The in-situ procedure will be introduced by detailing the synthesis and characterisation of a purple-coloured gold nanoparticle-wool fibre composite. The method 
used for the in-situ synthesis of a purple-coloured composites is outlined in Section 2.2.1. Dr Lucas optimised the reaction conditions that are detailed in Table 3.1 for the synthesis of purple-coloured composites. ${ }^{5}$

Table 3.1 Standard reaction conditions of the in-situ procedure to synthesise purple coloured gold nanoparticle-wool fibre composites.

\begin{tabular}{lc}
\hline Reaction parameter & Standard amount \\
\hline Concentration of $\mathrm{Au}^{3+}$ & $160 \mathrm{mg} \mathrm{L}^{-1}$ \\
Wool to liquor ratio & $1: 100$ \\
$\mathrm{pH}$ & 2.7 \\
Uptake conditions & $24 \mathrm{~h} @ \mathrm{RT}$ \\
Ageing conditions & $168 \mathrm{~h} @ 50^{\circ} \mathrm{C}$ \\
\hline \hline
\end{tabular}

The synthesis of composites with the above reaction conditions will be referred to as the standard purple in-situ method. As was described in Section 1.3.1, the in-situ procedure encompasses two processes; uptake and nanoparticle formation.

\subsubsection{Uptake Process}

The uptake process involves the absorption of $\mathrm{AuCl}_{4}^{-}$by wool. The uptake of $\mathrm{AuCl}_{4}^{-}$ by wool is similar to the chemistry of the conventional acid dyeing of wool with anionic dye molecules in a number of ways. As mentioned in Section 1.1.4, in wool dyeing, dye molecules gradually move from the dyebath to the surface of wool fibres. There, dye molecules migrate across the wool surface to the intercellular junctions between cuticle scales (or alternatively to damaged areas of the cuticle cells, which are relatively porous). Here, dye molecules are absorbed into the wool fibre, where they diffuse throughout the non-keratinous regions of the cortical cells, and eventually migrate to sulfur-rich proteins in the wool (see Figure 1.14). ${ }^{7}$

The uptake of $\mathrm{AuCl}_{4}^{-}$by wool is believed to follow a similar absorption route. ${ }^{5}$ The $\mathrm{pH}$ of the uptake solution is typically 2.7 , which is below the isoelectric region of wool, and hence there is a net positive charge on the wool fibre. This also occurs in the acid dyeing of wool with anionic dye molecules. As such, there is an electrostatic attraction between positively charged functional groups of the wool protein and the negatively charged $\mathrm{AuCl}_{4}^{-}$complex, which provides a driving force for the uptake.

The uptake of $\mathrm{AuCl}_{4}^{-}$to Cariaggi wool is measured with atomic absorption spectroscopy (AAS) and is presented in Figure 3.2 with a starting concentration of approximately $160 \mathrm{mg} \mathrm{L}^{-1}$. The y-axis represents the residual concentration of $\mathrm{Au}^{3+}$ 
remaining in the uptake solution as the reaction proceeds, as the AAS measures the concentration of $\mathrm{Au}^{3+}\left(\right.$ not $\left.\mathrm{AuCl}_{4}^{-}\right)$. The $\mathrm{Au}^{3+}$ that is removed from the reaction solution is considered to be completely absorbed by the wool. Although the AAS measures the concentration of $\mathrm{Au}^{3+}$ in solution, the gold in solution is typically referred to as $\mathrm{AuCl}_{4}^{-}$, as this is the form that the gold remains in during the uptake process.

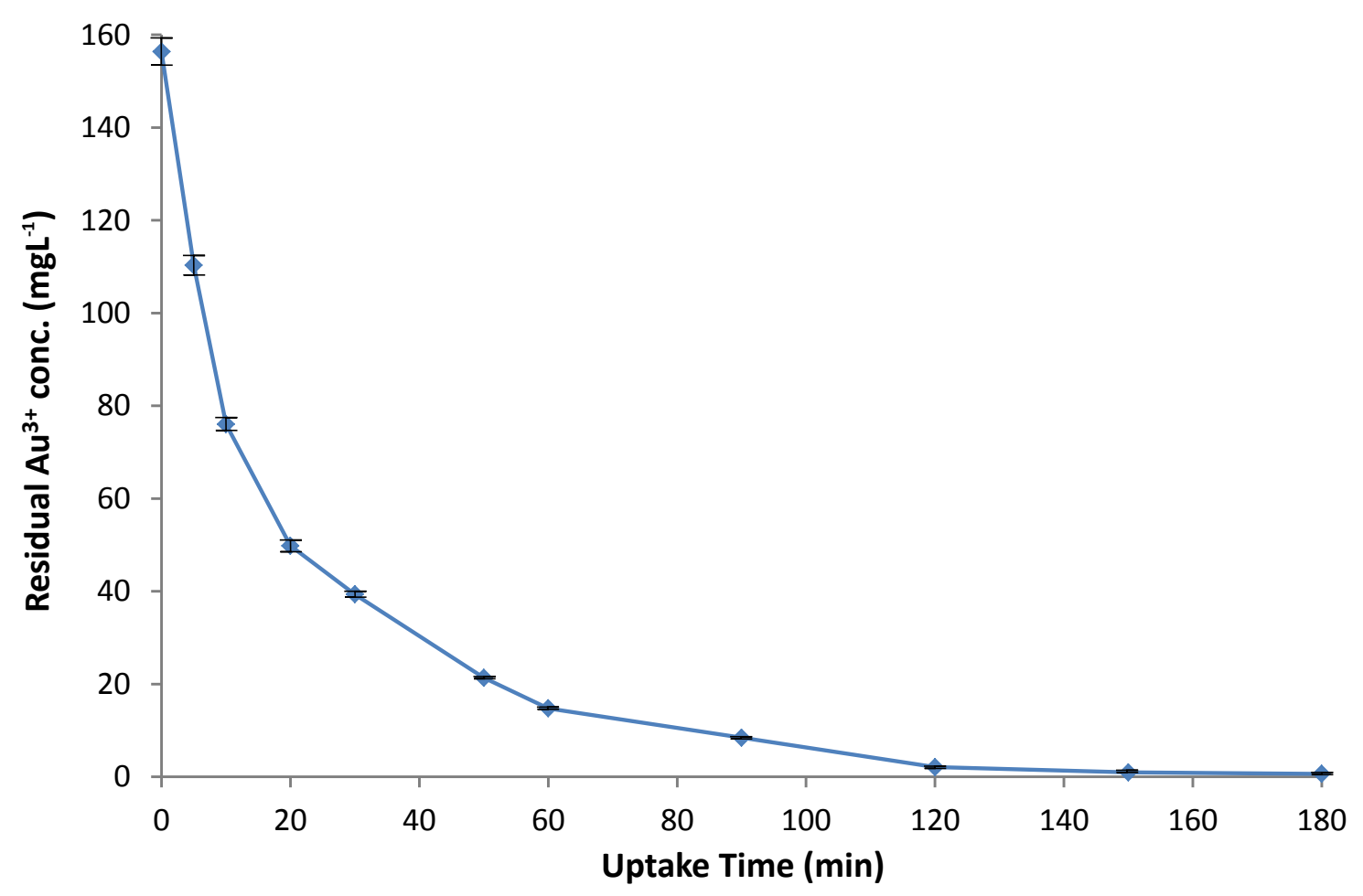

Figure 3.2 Uptake of $\mathrm{Au}^{3+}$ to Cariaggi wool synthesised with the standard purple in-situ method conditions.

The uptake of $\mathrm{AuCl}_{4}^{-}$into Cariaggi wool is rapid, with more than half of the $\mathrm{AuCl}_{4}^{-}$ in solution being absorbed by the wool after $10 \mathrm{~min}$, and the uptake is complete after approximately $150 \mathrm{~min}$. Figure 3.3 depicts a plot of $\ln \left(\right.$ residual $\mathrm{Au}^{3+}$ conc) versus time of the uptake curve shown in Figure 3.2 with a starting concentration of $160 \mathrm{mg} \mathrm{L}^{-1} \mathrm{Au}^{3+}$. The relationship between the two is approximately linear $\left(\mathrm{R}^{2}=0.9859\right)$, illustrating that the reaction is first order, and dependant on the concentration of $\mathrm{Au}^{3+}$ only.

\subsubsection{Gold Nanoparticle Formation}

The gold nanoparticle formation process involves electron-rich functional groups of the amino acids in wool facilitating the reduction of $\mathrm{Au}^{3+}$ and the formation of nanoparticulate $\mathrm{Au}^{0}$. Once $\mathrm{AuCl}_{4}^{-}$has been absorbed by wool, $\mathrm{AuCl}_{4}^{-}$is believed to 


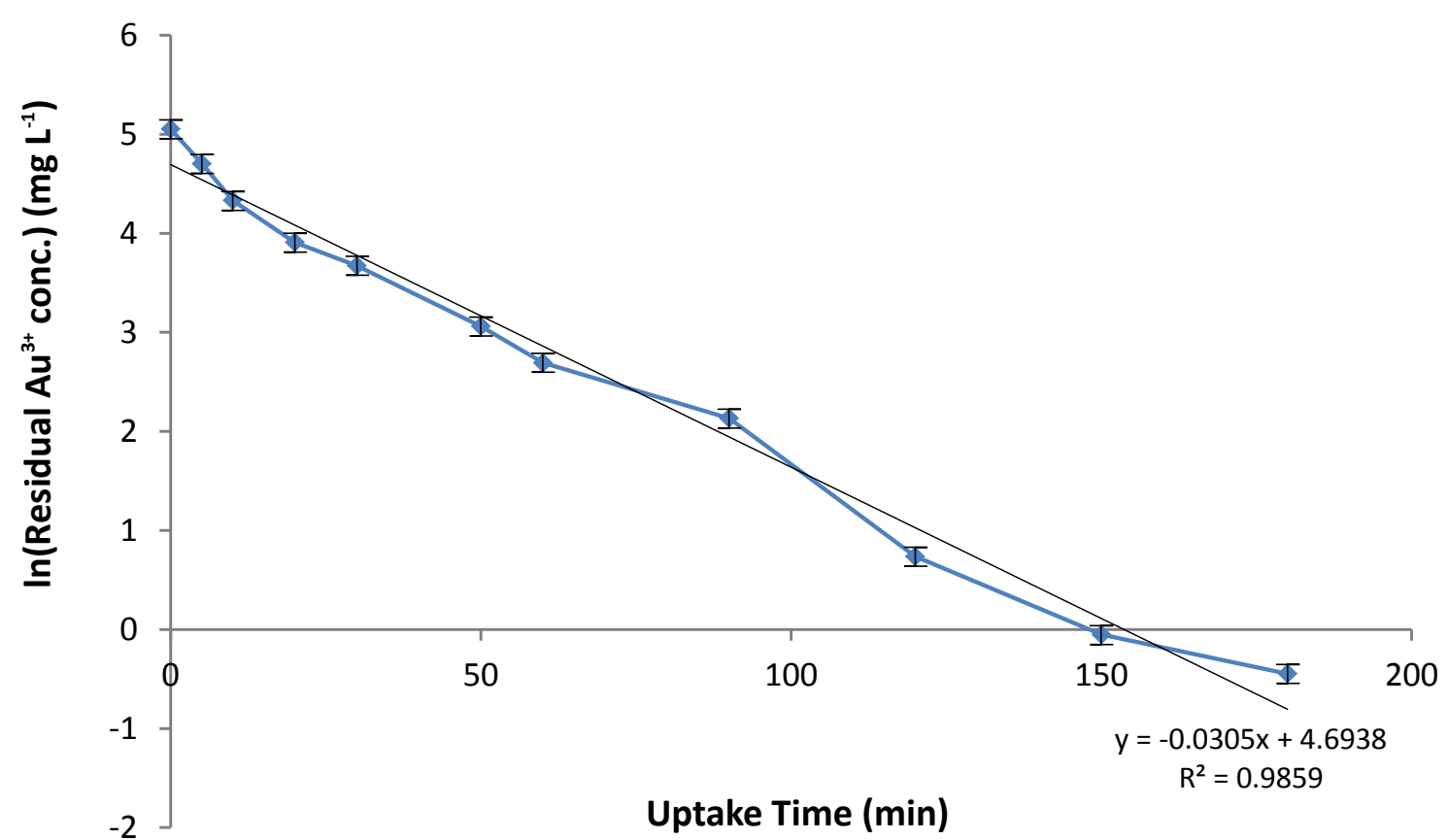

Figure 3.3 Plot of $\ln \left(\right.$ residual $\mathrm{Au}^{3+}$ conc) over time during the uptake of $\mathrm{AuCl}_{4}^{-}$ to Cariaggi wool synthesised with the standard purple in-situ method, to illustrate approximate 1st order kinetics.

dissociate to $\mathrm{Au}^{3+}$, which is then reduced to $\mathrm{Au}^{0}$ entities by electron-rich functional groups in the wool protein. ${ }^{5}$ The $\mathrm{Au}^{0}$ entities then coalesce to nucleate nanoparticles, the growth of which is restricted by the pore size and binding to the wool matrix. In this, the wool acts as a dual reducing and stabilising matrix, similar to the function of TA or TSC (by coupling its oxidation to the reduction of $\mathrm{Au}^{3+}$ to $\mathrm{Au}^{0}$, and stabilising the formed particles). This controls the size of the gold nanoparticles and hence the colour of the composites. Thus, the variability of the natural fibres leads to difficulties in controlling the colour of the composites, which is a recurring theme with the in-situ process.

The nucleation of gold nanoparticles in wool occurs very gradually at ambient temperatures; the purple colour that indicates gold nanoparticles in a wool matrix develops over a period of several months at RT. The nanoparticle formation process involves the heating of the uptake reaction vessels in a thermostatted water bath, to increase the rate of gold nanoparticle formation. At $50{ }^{\circ} \mathrm{C}$ the purple colour of the composites fully develops after 1 week, a time that is commercially viable for scale-up.

A Cariaggi gold nanoparticle-wool fibre composite synthesised with the in-situ approach is shown in Figure 3.4 (a), where the purple colour of gold nanoparticles in a wool matrix is clearly seen. Figure 3.4 also depicts SEM and EDS analysis of areas of the surface of the Cariaggi composite. The SEI micrograph (Figure 3.4 (b)) shows the composite fibre, but the gold is not visible. The image of the same 
area in backscatter (BS) mode (Figure $3.4(\mathrm{c})$ ) shows the presence of gold particles across the surface of the wool fibre. The gold appears as bright dots on the darker, organic wool fibres, as gold is relatively dense, and registers a higher BS intensity. The BS image at higher magnification (Figure $3.4(\mathrm{~d})$ ) shows that the nanoparticles are typically in the nano-dimensions.

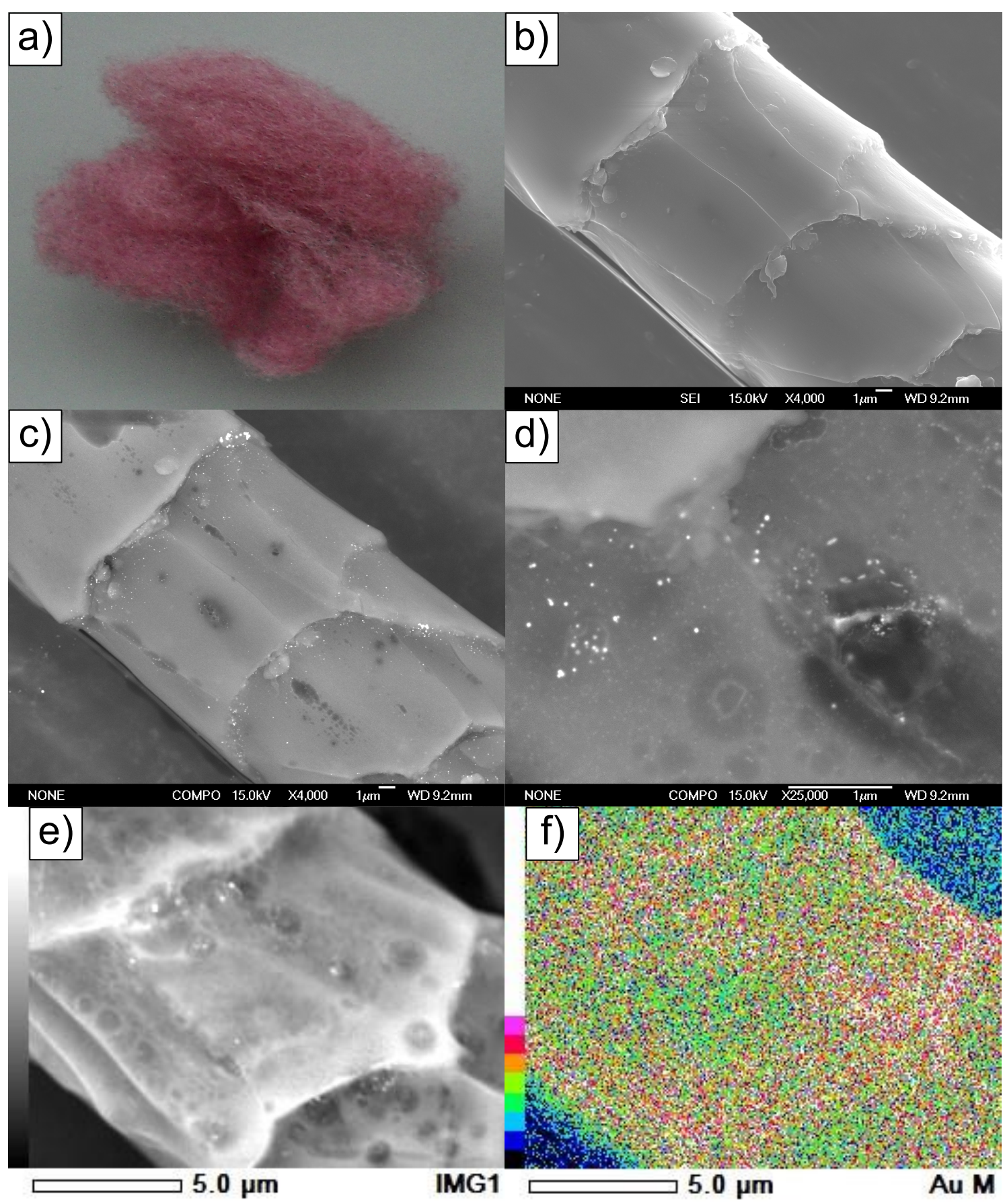

Figure 3.4 Image of a Cariaggi in-situ composite synthesised via the standard purple method (a), SEM micrographs of the surface of a composite fibre in secondary electron imaging (SEI) mode (b), in backscatter (BS) mode at different magnifications (c)-(e) and an EDS elemental Au map (of the area in (e)) (f).

The presence of gold nanoparticles on the wool fibre surface shows that some of the $\mathrm{AuCl}_{4}^{-}$interacts with functional groups present on the surface of the wool, before 
they are absorbed into the centre of the wool fibres, where they remain to form nanoparticles of gold. The SEM images also show that the surface gold nanoparticles are more commonly located near the intercellular junctions between overlapping cuticle scales, which is where dye molecules preferentially absorb into wool fibres. Additionally, at the edges of the cuticle scales there is a greater effective surface area of the wool fibre for the $\mathrm{AuCl}_{4}^{-}$ions to interact with, and for this reason it is also the kinetically favourable site for the adsorption of material by the wool. ${ }^{7}$ EDS analysis of the surface of an in-situ Cariaggi composite is shown in Figure 3.4 (f). The EDS elemental Au map shows relatively higher gold density at the edges of the cuticle scales. These areas of high Au density correspond to the distribution of gold nanoparticles observed across the fibre surface in Figure 3.4 (e).

SEM and EDS analysis of the cross-section of the same Cariaggi composite is presented in Figure 3.5. Cross-sectional analysis is useful to indicate the level of penetration of $\mathrm{AuCl}_{4}^{-}$into the fibres, and to determine whether the absorbed $\mathrm{AuCl}_{4}^{-}$forms gold nanoparticles. The SEM micrographs in BS mode (Figure 3.5 (b)-(d)) show that gold nanoparticles form in the wool fibre interior. Sequential magnification of the square areas in Figure 3.5 (b) and (c) shows a significant concentration of gold nanoparticles in the fibre interior.

The Au elemental map of the cross section (Figure 3.5 (f)) shows that gold is present throughout the fibre centre, with a higher density across the left hand side. This confirms the penetration of $\mathrm{AuCl}_{4}^{-}$throughout the wool fibre interior during the uptake process. The EDS elemental S map also shows high density of $\mathrm{S}$ on the left hand side of the fibre, which corresponds to the paracortex of the wool (Figure 3.5 (e)). The EDS elemental Au map shows a similar distribution of Au throughout the composite fibre. The overlap of the relative densities of $\mathrm{S}$ and $\mathrm{Au}$ shows the attraction of $\mathrm{AuCl}_{4}^{-}$to sulfur-containing amino acids and alludes to the role of these amino acids in the formation and stabilisation of gold nanoparticle. Finally, the line profile Figure $3.5(\mathrm{~g})$ depicts the change in the intensity of the elemental Au from the left to right across the outlined rectangular area. The line profile also shows a higher density of Au in the paracortex of the wool fibre.

\subsubsection{Discussion of the Importance of These Results}

The results presented in Section 3.1 showed the successful extension of the in-situ procedure to synthesise ultrafine Cariaggi gold nanoparticle-wool fibre composites, a new source of wool suitable for luxury apparel manufacture. However, the resulting Cariaggi composites have gold content of approximately $1.6 \mathrm{wt} \%$. The cost of the 

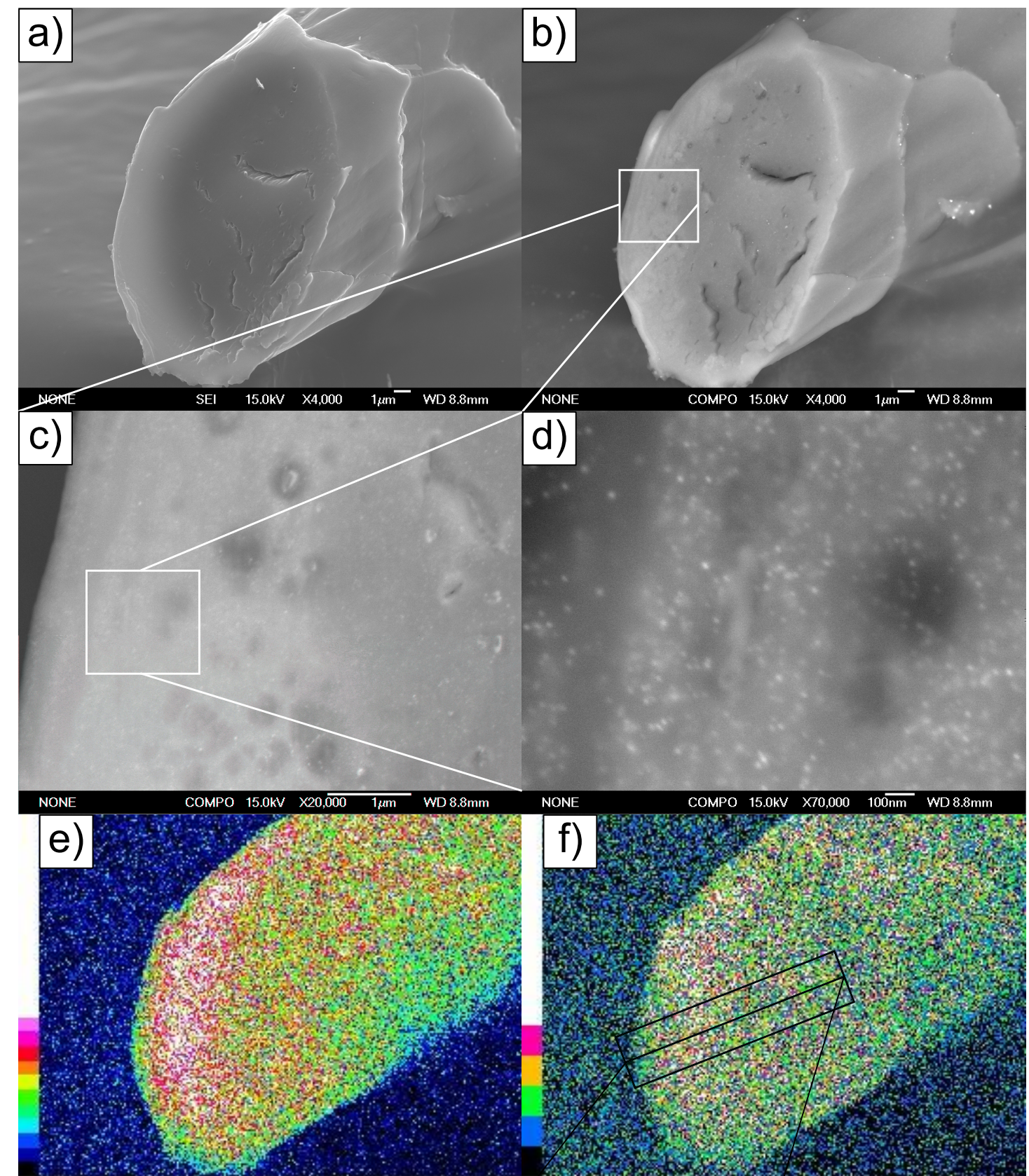

$10 \mu \mathrm{m}$

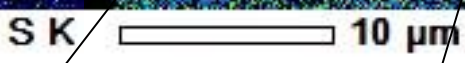

Au M

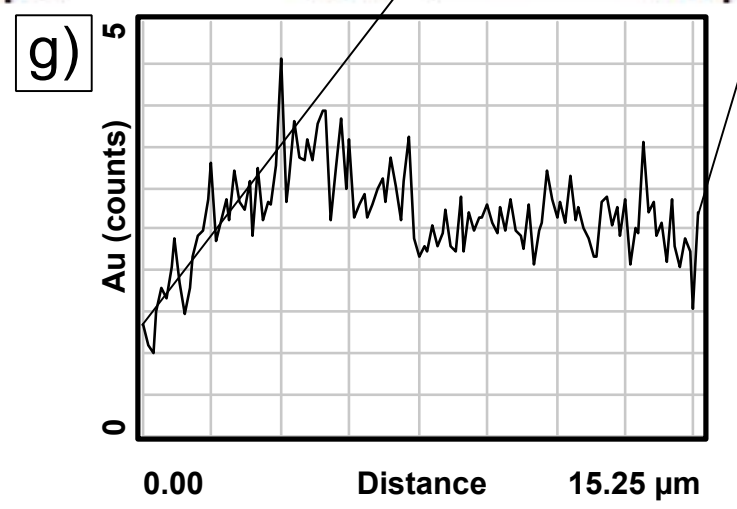

Figure 3.5 SEM and EDS analysis of the cross section of a Cariaggi in-situ composite synthesised with the standard purple in-situ method; SEI image (a), BS images of increasing magnification (b)-(d), S elemental map (e), Au elemental map (f) and a line profile of Au intensity across the cut fibre edge. 
gold required for the synthesis of the composites is around 700 USD per $\mathrm{kg}$ of composites produced, which is not economically viable for large-scale production.

SEM and EDS analysis of the in-situ Cariaggi composites showed that gold nanoparticles form both on the surface and interior of the wool fibres. It is considered that gold nanoparticles at or near the surface of the wool fibre add more to the overall colour of the composite, compared to those near the centre of the wool. This is due to gold nanoparticles in the wool interior being shielded from interaction with visible light due to absorption by the wool (and the refractive index of the wool). Thus, efforts were made to direct gold nanoparticle formation at or near the wool fibre surface.

This objective was attempted to be achieved by understanding the effects of the reaction conditions on the chemistry of the in-situ procedure, so that they could perhaps be manipulated to control the uptake and nanoparticle formation processes, while using less gold to achieve similar colours.

\subsection{Characterisation of the Reaction Parameters of the In-situ Procedure}

Dr Lucas found that the following reaction conditions affect the chemistry of the in-situ procedure: ${ }^{5}$

- wool type

- $\mathrm{pH}$

- temperature

- auxiliary agents

- volume of gold solution

The effects of these reaction conditions on the chemistry of the in-situ procedure are now characterised to allow the manipulation of the chemistry of the uptake and nanoparticle formation processes and the eventual lowering of the gold content of the resulting composites. 


\subsubsection{Wool Type}

The wool type used in the in-situ synthesis affects the chemistry of the in-situ procedure in a number of ways. Different wool types can exhibit unique fibre thickness, chemical composition, fibre damage, processing chemistry and have chemical pretreatments applied to them. Additionally, the form of the wool is important and there may or may not be further processing required post-dyeing. These parameters influence the chemistry of the in-situ procedure and the colour of the resulting composites. As such, a range of wool types were studied in this section, either in the form of top wool or finished wool yarns.

\section{Wool in Top Form}

The following three stocks of wool in top form; Cariaggi, Ashford and Crossbred wool, were used to investigate chemistry of the in-situ procedure. All were sourced through different supply chains, and their relevant properties were described in Section 2.1.1.

Cariaggi wool fibres are the thinnest, and hence have the highest effective surface area for a given mass of wool. Thus, Cariaggi fibres have the greatest number of available surface reactive sites for the absorption of $\mathrm{AuCl}_{4}^{-}$for a given mass of wool. Surface sites are the most accessible sites for interaction with $\mathrm{AuCl}_{4}^{-}$, as they do not require diffusion through the wool matrix to interact with. As such, the uptake of $\mathrm{AuCl}_{4}^{-}$should be the quickest with Cariaggi wool, assuming equivalent surface chemistries of the different wool types.

The uptake of $\mathrm{AuCl}_{4}^{-}$by Cariaggi, Ashford and Crossbred wool fibres is shown in Figure 3.6 with starting solutions of approximately $120 \mathrm{mg} \mathrm{L}^{-1} \mathrm{Au}^{3+}$. As expected, the uptake of $\mathrm{AuCl}_{4}^{-}$by Cariaggi wool is the most rapid, and the uptake to Ashford and Crossbred wool is sequentially slower. The uptake of $\mathrm{AuCl}_{4}^{-}$by Cariaggi wool is complete after $150 \mathrm{~min}$, however for Ashford and Crossbred wool a small amount of $\mathrm{AuCl}_{4}^{-}$remains in solution after $180 \mathrm{~min}$, and each wool type exhibits a distinct absorption profile.

The uptake appears to involve two stages of absorption; first rapid absorption occurs to the surface until it becomes saturated. Following this, absorption into the wool fibre interior takes place relatively gradually, as $\mathrm{AuCl}_{4}^{-}$must migrate through the inter-cuticle junctions, leaving surface absorption sites available for the $\mathrm{AuCl}_{4}^{-}$ remaining in the uptake solution. 


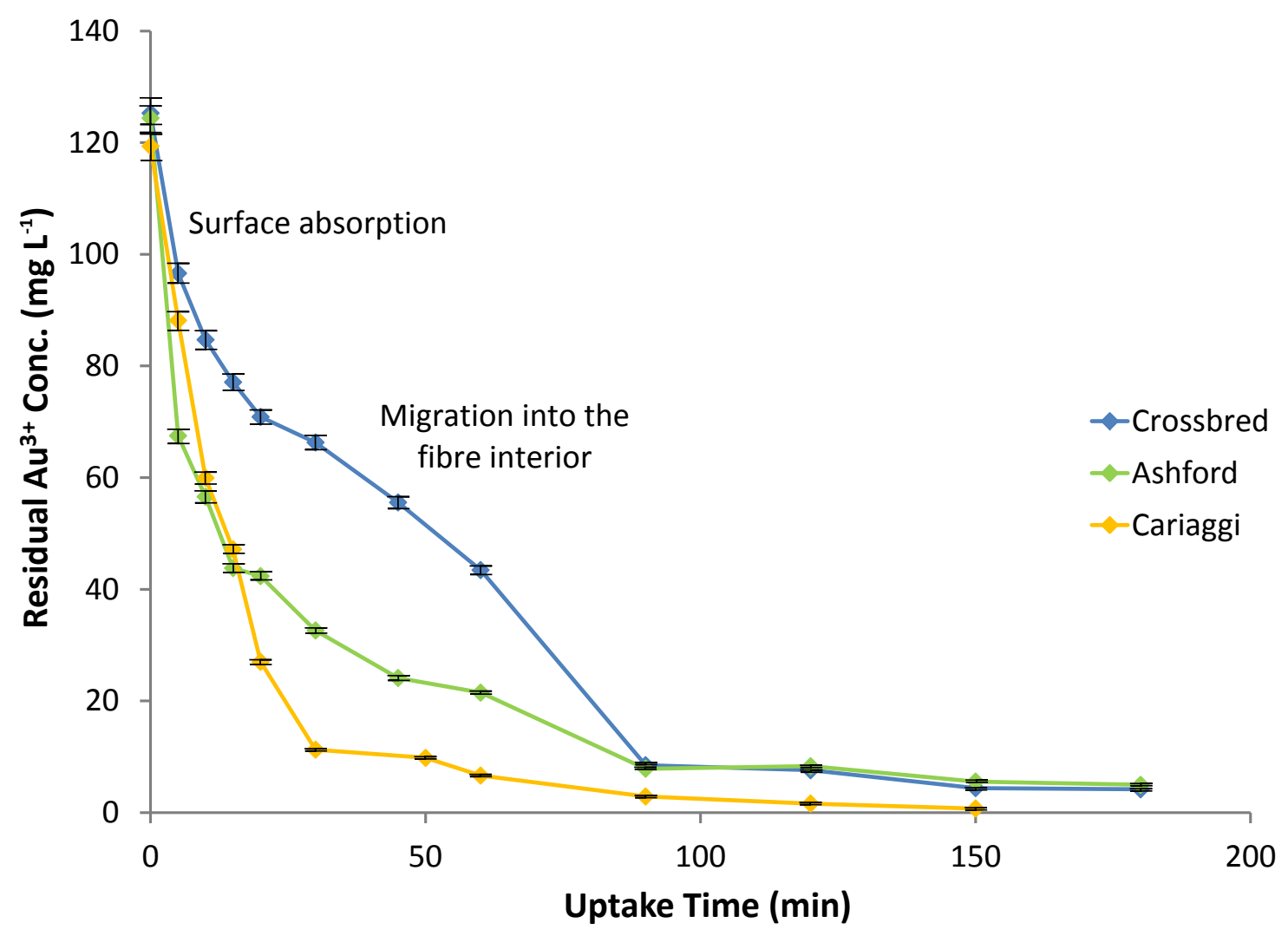

Figure 3.6 Uptake of $\mathrm{Au}^{3+}$ to Cariaggi, Ashford and Crossbred wool at RT.

Images of wool in top form removed from the uptake solution after $180 \mathrm{~min}$ are shown in Figure 3.7. The photographs were taken 2 years after the uptake experiment was carried out, over which time the purple colour of gold nanoparticles in a wool matrix gradually developed (this process is promoted by heat in the nanoparticle formation process, and the colour fully develops after $168 \mathrm{~h}$ at $50^{\circ} \mathrm{C}$ ). The Cariaggi composite is pink-purple, the Ashford composite is a darker purple and surprisingly, the Crossbred composite is light purple. There is significant colour variation across the Crossbred composite; many fibres remain completely white, while others are dark purple. This colour variation is unusual for small sample sizes such as this, and is possibly due to an unevenly applied surface treatment or a blend of two different wool types with different reactivities.

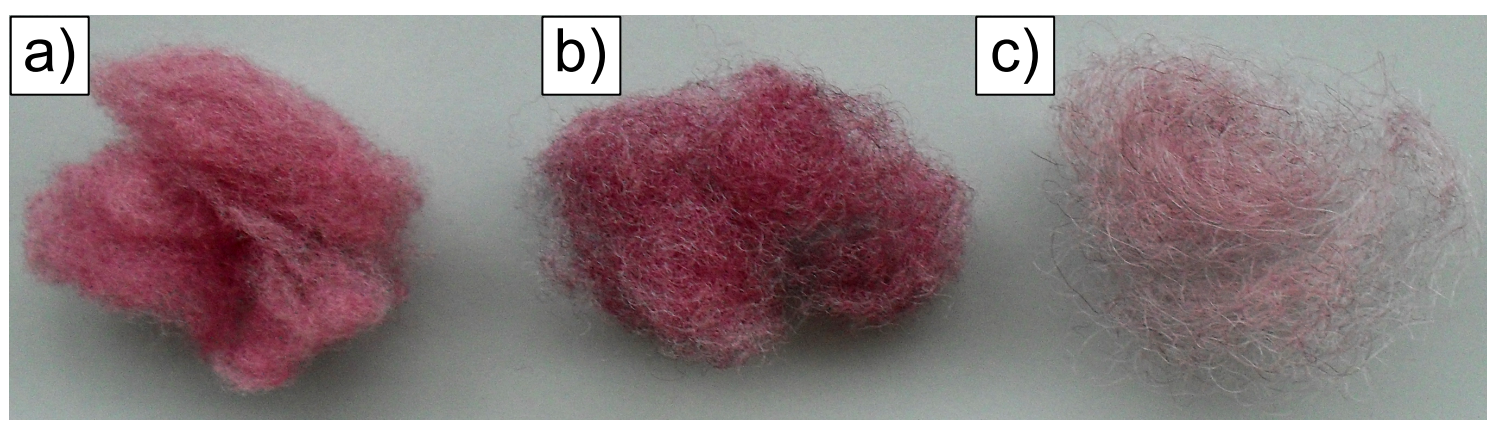

Figure 3.7 In-situ composites of Crossbred (a), Ashford (b) and Cariaggi (c) wool after 180 min uptake at RT (photograph taken 2 years after synthesis, allowing time for the gradual formation of gold nanoparticles. 
CIE colour coordinates of the in-situ loose wool composites that appear above are shown in Table 3.2. As explained in Section 2.3, the $\mathrm{L}^{*}, \mathrm{a}^{*}$, and $\mathrm{b}^{*}$ colour scale describes a colour as lighter/darker, redder/greener, or more yellow/blue respectively.

Table 3.2 CIE colour coordinates of loose wool in-situ composites synthesised with different types of wool in top form (that were pictured in Figure 3.7.

\begin{tabular}{cccc}
\hline Wool type & Crossbred & Ashford & Cariaggi \\
\hline $\mathrm{L}^{*}$ (brightness) & 57.49 & 45.51 & 55.64 \\
$\mathrm{a}^{*}$ (redness) & 11.78 & 16.81 & 16.24 \\
$\mathrm{~b}^{*}$ (yellowness) & 1.38 & -1.12 & 1.16 \\
\hline
\end{tabular}

The purple colouration of each composite registers a much lower $\mathrm{L}^{*}$ value, a significantly higher $a^{*}$ value and $a b^{*}$ value that is somewhat lower in comparison with the unreacted loose wool. This indicates the development of the purple colour of gold nanoparticles in a wool fibre matrix.

The $a^{*}$ value of the Crossbred composite is markedly lower than the Ashford and Cariaggi composites, indicating a lesser red contribution due to fewer purple coloured gold nanoparticles. This is expected considering that some Crossbred fibres remain uncoloured. The Ashford composite exhibits lower $\mathrm{L}^{*}$ and $\mathrm{b}^{*}$ values than the Cariaggi composite, which shows the darker purple colour of the Ashford composite, and the same concentration of gold being distributed across a smaller effective surface area in the Ashford composite (due to fewer fibres per given volume). The uneven colour of the Crossbred composites results in the disruption of the trend of darker purple coloured composites being seen with thicker fibres (with relatively lower effective surface areas).

SEM imaging of the loose wool in-situ composites is shown in Figure 3.8. The BS micrographs show the presence of gold particles, appearing as bright dots on the darker surfaces of each of the composites. The imaged Crossbred composite fibre exhibited a comparatively high concentration of gold across its surface, however, there were some fibres with little to no gold on the surface, which corresponded to the uncoloured fibres in the composite sample. In the imaged Crossbred wool, the gold nanoparticles are distributed across the fibre surface, with a higher density of gold particles compared to the Ashford and Cariaggi composite fibres. This is understandable given the higher effective surface area of the merino fibres compared to the relatively thick crossbred fibres, and that the gold nanoparticles are distributed across a higher number of fibres.

The gold nanoparticles congregate at and near the edges of the cuticle scales on each composite, as evidenced in the higher magnification images on the right hand side of Figure 3.8. This is the kinetically favourable site for the absorption of $\mathrm{AuCl}_{4}^{-}$, 
giving access to the interior of the fibres and providing a higher number of reactive sites for the formation of gold nanoparticles.

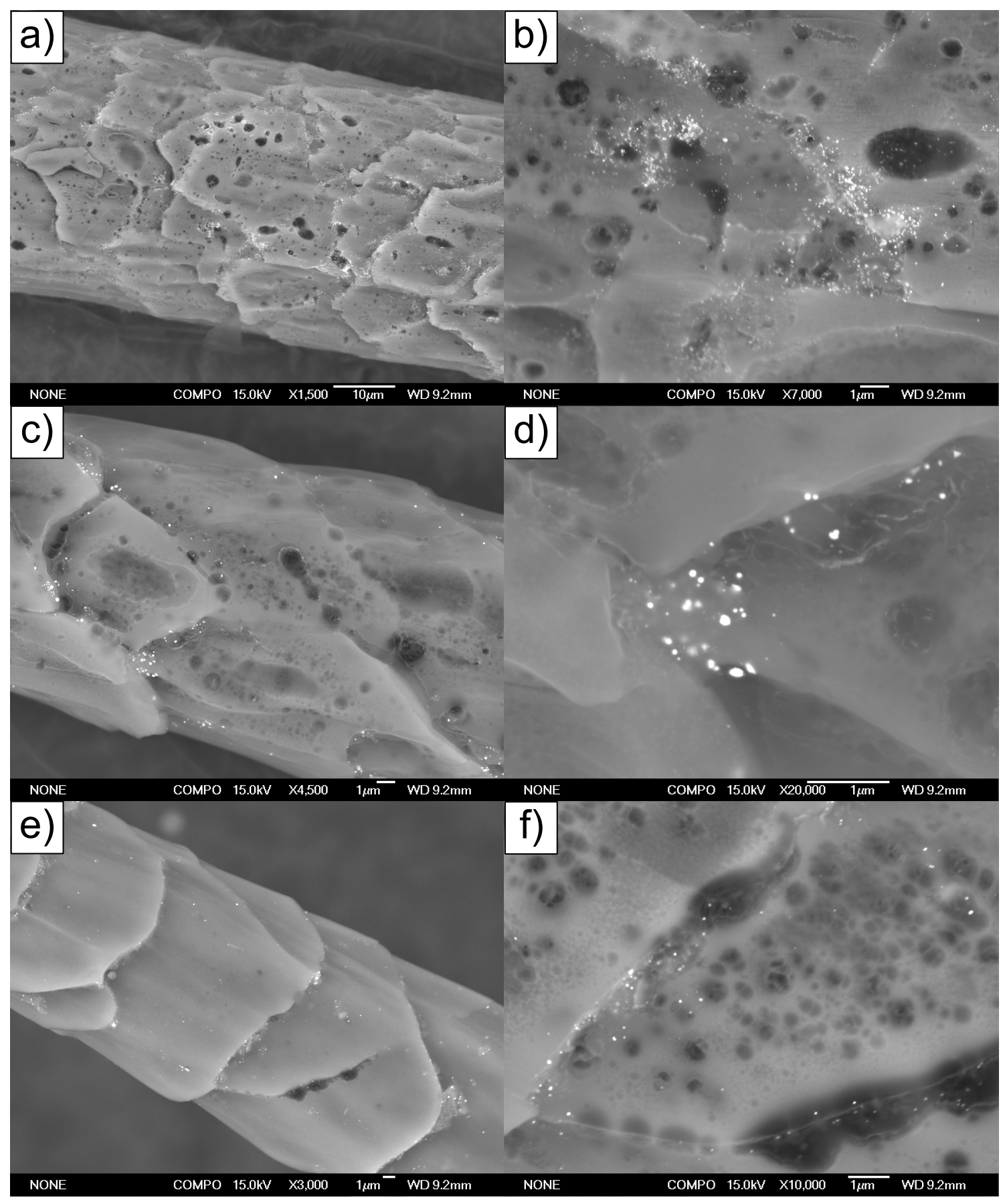

Figure 3.8 SEM micrographs in BS mode of Crossbred (a)-(b), Ashford (c)-(d) and Cariaggi (e)-(f) in-situ composites synthesised using standard purple in-situ method.

The synthesis of composites of different stocks of wool in top form shows that the in-situ approach can be expanded to incorporate fine merino wool for high-quality apparel manufacture. However, the approach was less effective for the colouring of the thicker yarn used in carpet manufacture, which illustrated the difficulty introduced by using wool types with different chemical and physical properties. If 
the in-situ procedure is able to be made more cost effective, future research into improving the colour uniformity of Crossbred composites will be carried out.

The synthesis of the in-situ composites is expensive, and the combing, carding and spinning stages required to process wool in top form into the finished yarn required for carpet and apparel fabrication adds further cost. These processing stages also result in the loss of usable wool, which further reduces the efficiency of colouring wool in top form. In the interest of improving the commercial potential of the insitu procedure, gold nanoparticle-wool fibre composites of finished wool yarns were synthesised.

\section{Merino Yarns}

Finished wool yarns represent the optimal form of wool to be coloured, as it requires no further processing before being knitted or woven into finished products. These processing stages (that are omitted with dyeing yarn) can include the mixing and aligning of wool fibres, which improves the colour uniformity of unevenly coloured composites. However, the processing stages can also interfere with the chemistry of the composite fibres, and result in an unwanted loss of wool. As such, the uniform colouring of finished wool yarns is paramount. The cost-saving potential of dyeing finished wool yarns (compared to wool in top form) lead to the following study.

Finished wool yarns were sourced from Tararua Yarns Ltd, Levin, New Zealand and Spectrum Yarns Ltd, Huddersfield, England. All yarns are twin twists of merino wool, with different fibre diameters that are listed in Table 3.3.

Table 3.3 Fine merino yarns used in the in-situ synthesis of gold nanoparticle-wool fibre composites.

\begin{tabular}{ccc}
\hline Yarn type & Abbreviation & Fibre diameter $(\mu \mathrm{m})$ \\
\hline Tararua Untreated & TU & 22 \\
Tararua Superwash & TS & 22 \\
Spectrum 19.5 & S19 & 19.5 \\
Spectrum 14.5 & S14 & 14.5 \\
\hline \hline
\end{tabular}

In-situ wool yarn composites were synthesised with the standard purple in-situ method, the results of which are shown in Figure 3.9. Although the reaction conditions are those optimised by Dr Lucas for the synthesis of purple-coloured composites, only the TS composite exhibits the typical purple colouration of this approach. The remaining yarns are all different shades of purplish-grey to grey, due to agglom- 
eration of gold nanoparticles on the fibre surfaces.

Additionally, the purple colouration of the TS composites is non-uniform; there are many areas that remain completely uncoloured. This is uncommon for samples of such a small size $(1 \mathrm{~g})$. It is likely that the superwash treatment, which is a shrink resistant coating, is unevenly applied to the yarn. It is also likely that the superwash treatment exhibits functional groups that are capable of forming gold nanoparticles, as there are more areas of TS yarn that are coloured than not.

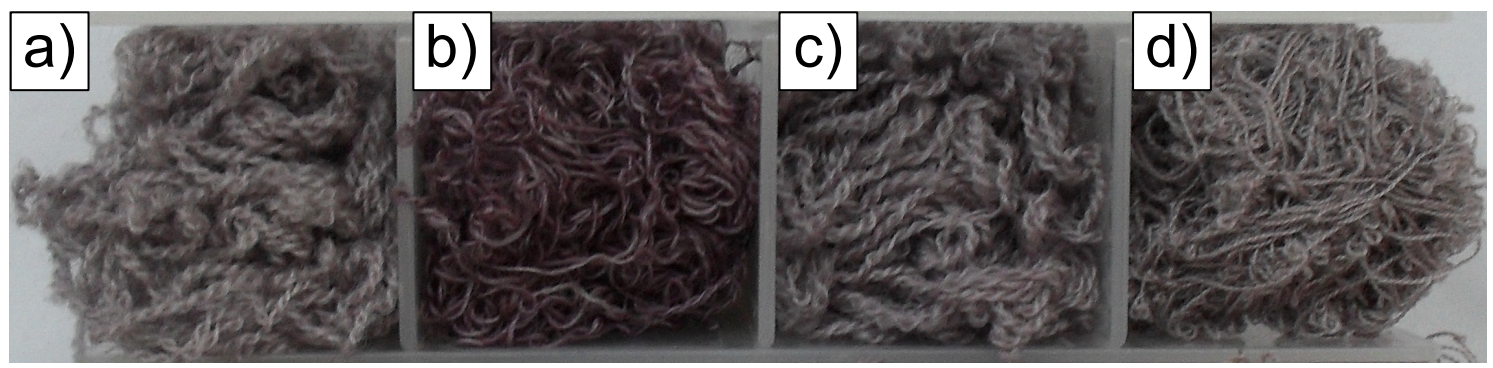

Figure 3.9 Image of the composites of TU (a), TS (b), S19 (c) and S14 (d) yarn synthesised with the standard purple in-situ method.

The CIE colour coordinates of the finished wool yarn in-situ composites are represented Table 3.4. All finished yarns exhibit much lower $\mathrm{L}^{*}$ (brightness) values than the original yarns. TS has the highest $a^{*}$ value and the lowest $b^{*}$ value, which is indicative of the contribution of purple-coloured gold nanoparticles in the wool matrix. The purplish-grey colouration of the remaining composites is represented by their lower a* values, with TU and S19, the greyest of the composites displaying the lowest $a^{*}$ values.

Table 3.4 CIE colour coordinates of in-situ composites of merino wool yarns.

\begin{tabular}{ccccc}
\hline Wool type & TU & TS & S19 & S14 \\
\hline $\mathrm{L}^{*}$ & 51.00 & 38.88 & 48.00 & 46.44 \\
$\mathrm{a}^{*}$ & 4.02 & 9.24 & 4.15 & 5.84 \\
$\mathrm{~b}^{*}$ & 0.20 & -2.42 & -1.02 & 1.76 \\
\hline \hline
\end{tabular}

SEM and EDS images of the finished yarn composites is presented in Figure 3.10. Interestingly, all of the finished yarns show a range of sizes and shapes of gold particles. In addition to particles of gold in the nano-dimensions, the TU and TS composites exhibit a significant quantity of much larger triangular platelet-shaped particles. These are up to $500 \mathrm{~nm}$ in width, and are observed in Figure 3.10 (b) and (d). The large, hexagon-like shapes that are also present appear to be shapes that would grow into triangular platelets in the presence of additional $\mathrm{AuCl}_{4}^{-}$. There are also agglomerates of gold nanoparticles that are located next to each other. As was 
mentioned in Section 1.2.2, the surface plasmon resonance of these nanoparticles will interact to act as those of larger particles, with the absorbance red-shifting and broadening. As such, the combination of agglomerates of gold nanoparticles and larger particles account for the grey colouration of the composites.

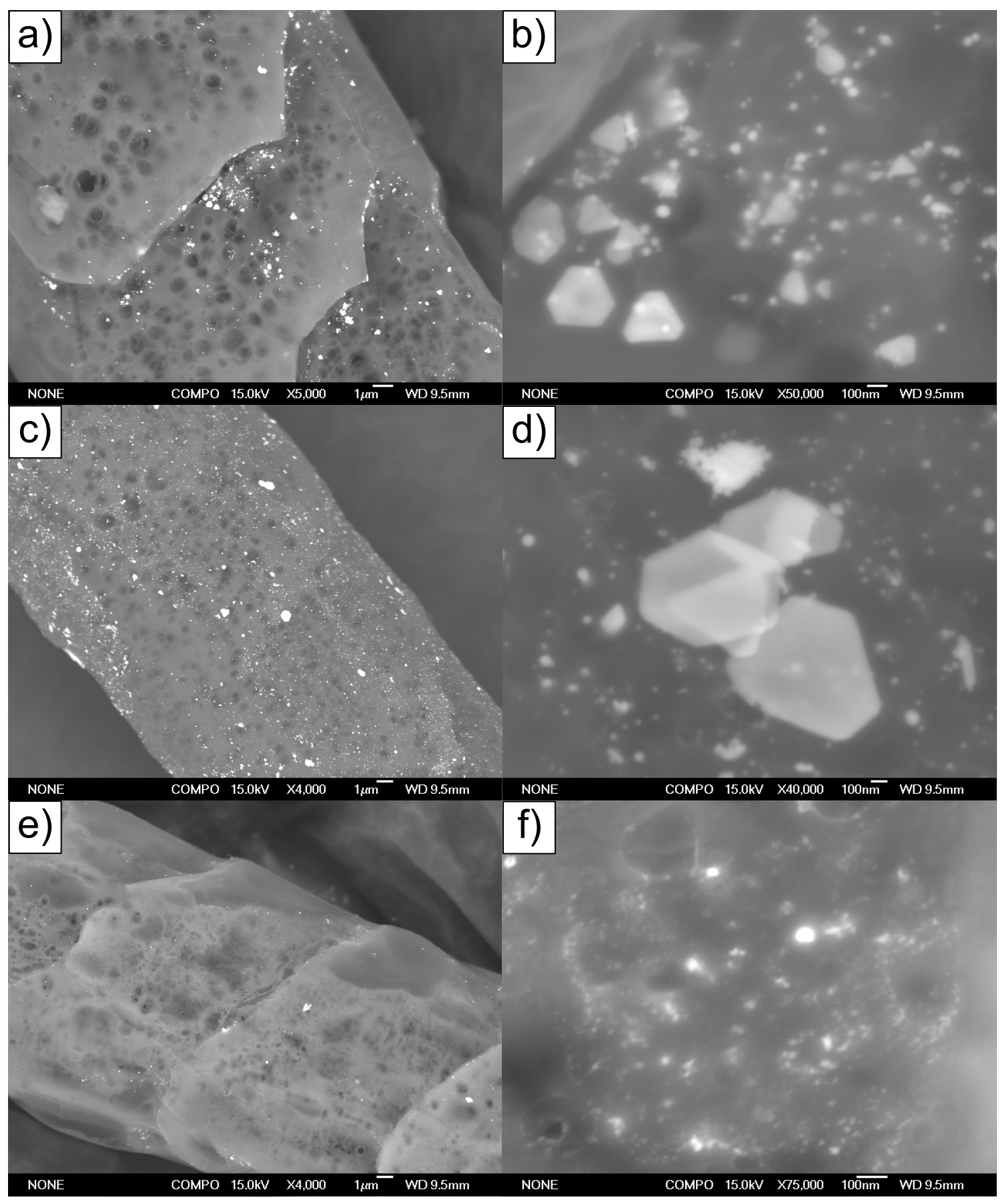

Figure 3.10 SEM micrographs in BS mode of the surfaces of in-situ composites of TU (a)-(b), TS (c)-(d) and S19 (e)-(f) yarns, that were synthesised with the standard purple in-situ method.

The S19 composite also exhibits the occasional triangular platelet-shaped gold particle. The bulk of the gold is present as individual nanoparticles, some of which are in close proximity to one and other and the occasional larger particle. The combi- 
nation of these particles succinctly explains the purple-grey colour of the S19 in-situ composite.

The standard purple in-situ method has shown a propensity to produce purplishgrey - grey coloured composites. This may be due to the relatively low surface area available for $\mathrm{AuCl}_{4}^{-}$absorption, due to the twisting nature of the merino fibres in the finished yarns that would block the access of $\mathrm{AuCl}_{4}^{-}$certain areas of the yarn. This essentially reduces the effective surface area of the wool, and relative concentration of $\mathrm{AuCl}_{4}^{-}$in the accessible areas of the yarn will be higher, causing the formation of larger particles of gold. The S14 yarn exhibits thinner wool fibres with a larger effective surface area, smaller particles of gold and hence a more purple colouration. The TS composite exhibited unique behaviour due to the effect of the superwash treatment. Other reaction parameters will be manipulated to optimise the in-situ synthesis of gold nanoparticle-wool fibre composites of finished wool yarns.

This section has shown that the wool type has a significant effect on the chemistry of the in-situ approach, or both the hue and uniformity of the resulting composites.

\subsection{2 $\mathrm{pH}$}

The effect of $\mathrm{pH}$ on the in-situ procedure is very important. The uptake process requires a pH-related driving force for the reaction to commence, involving an electrostatic attraction between the negatively charged $\mathrm{AuCl}_{4}^{-}$complex and the positively charged wool (as the reaction $\mathrm{pH}$ is lower than the isoelectric region of the wool). This attraction is also exploited in the acid dyeing of wool with anionic dye molecules. As such, developing an understanding of the effect of the $\mathrm{pH}$ on the rate of uptake of $\mathrm{AuCl}_{4}^{-}$, the rate of gold nanoparticle formation and the colour of the composites produced is integral to optimising the in-situ synthesis.

As mentioned in Section 1.3.1, Lucas found that when the $\mathrm{pH}$ of in-situ procedure is lowered, the rate of uptake of $\mathrm{AuCl}_{4}^{-}$to wool fibres increases. ${ }^{5}$ This is due to the presence of a greater number of positively charged functional groups on the wool to electrostatically attract $\mathrm{AuCl}_{4}^{-}$at a lower reaction $\mathrm{pH}$. The colour of the composites are also affected by the reaction $\mathrm{pH}$; in general Lucas synthesised gold nanoparticlewool fibre composites at a reaction $\mathrm{pH}$ of $2.7-3$ as these gave consistently the best colours. ${ }^{5}$

To investigate the effects of $\mathrm{pH}$ on the chemistry of the in-situ procedure, merino wool in top form was sourced from Ashford handicrafts. The merino wool will be 
referred to as Ashford2, and the wool exhibits the same approximate fibre diameter as Ashford wool $(22 \mu \mathrm{m})$. However, Ashford2 wool was processed through an alternative supply chain, and as such, Ashford2 fibres exhibited different surface chemistry and dyeing properties due to this different (and unknown) treatment.

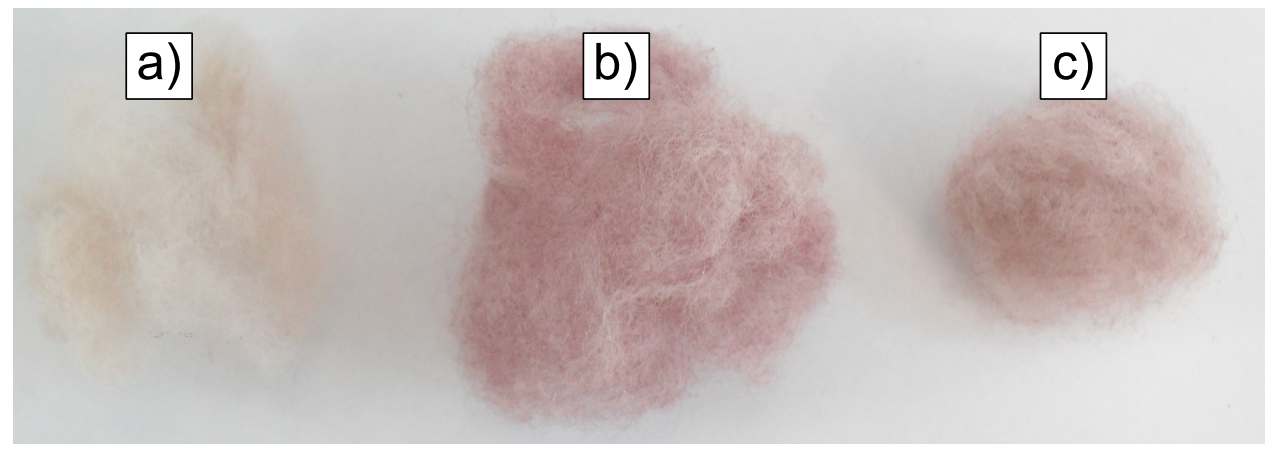

Figure 3.11 Ashford2 in-situ composites synthesised with the standard purple in-situ method and a reaction $\mathrm{pH}$ of 2 (a), 3 (b) and 4 (c).

Figure 3.11 shows in-situ Ashford2 composites synthesised at pH 2, 3 and 4 respectively. All other reaction conditions were those of the standard purple in-situ method. Interestingly, the $\mathrm{pH} 2$ composite lacks the purple colour of gold nanoparticles in a wool matrix. The variation in colour of the composites in Figure 3.11 was quantified with measurement of the composites' CIE colour coordinates in Table 3.5.

Table 3.5 CIE colour coordinates of Ashford2 in-situ composites synthesised at a different reaction $\mathrm{pH}$.

\begin{tabular}{cccc}
\hline Reaction $\mathrm{pH}$ & 2 & 3 & 4 \\
\hline $\mathrm{L}^{*}$ & 80.57 & 54.96 & 67.73 \\
$\mathrm{a}^{*}$ & 1.73 & 11.07 & 6.73 \\
$\mathrm{~b}^{*}$ & 10.65 & 4.17 & 3.66 \\
\hline \hline
\end{tabular}

The development of the purple colour of gold nanoparticles in a wool matrix is represented by the composites measuring lower $\mathrm{L}^{*}$, higher $\mathrm{a}^{*}$ and more negative $\mathrm{b}^{*}$ values. The $\mathrm{pH} 2$ composite is the lightest, the least red and the yellowest composite, indicating that gold nanoparticles are not contributing significantly to the hue of the composite prepared at $\mathrm{pH}$ 2. The composite synthesised at $\mathrm{pH} 3$ has the lowest $\mathrm{L}^{*}$ and highest $\mathrm{a}^{*}$ values. The $\mathrm{b}^{*}$ value is slightly higher than that of the $\mathrm{pH} 4$ composite, which is likely due to increased yellowing of the wool fibres occurring after 1 week spent at $50^{\circ} \mathrm{C}$ at a lower (and more damaging) $\mathrm{pH}$. The lower a* and higher $\mathrm{L}^{*}$ value of the of composite synthesised at $\mathrm{pH} 4$ quantifies the relatively lighter purple colouration and indicates the presence of fewer gold nanoparticles in the wool matrix.

It is also possible to use UV-visible reflectance spectroscopy to show the colour 
of the in-situ composites, as shown in Figure 3.12. However, as is often the case when the samples are not a flat surface, the spectra are very jagged and of low intensity. In spite of this, the surface plasmon resonance peak of gold nanoparticles between $500-600 \mathrm{~nm}$ is observable in all of the composites, although barely so in the composite prepared at $\mathrm{pH} 2$. In contrast, the unreacted Ashford2 wool exhibits no such absorbance. The most intense surface plasmon resonance peak is observed for the pH 3 composite. The pH 3 and pH 4 spectra are only slightly different due to the high signal to noise ratios of the spectra. The lower intensity of the colour yield of the $\mathrm{pH} 2$ composite reflects the light colouration of the composite and the poor development of gold nanoparticles in the fibre.

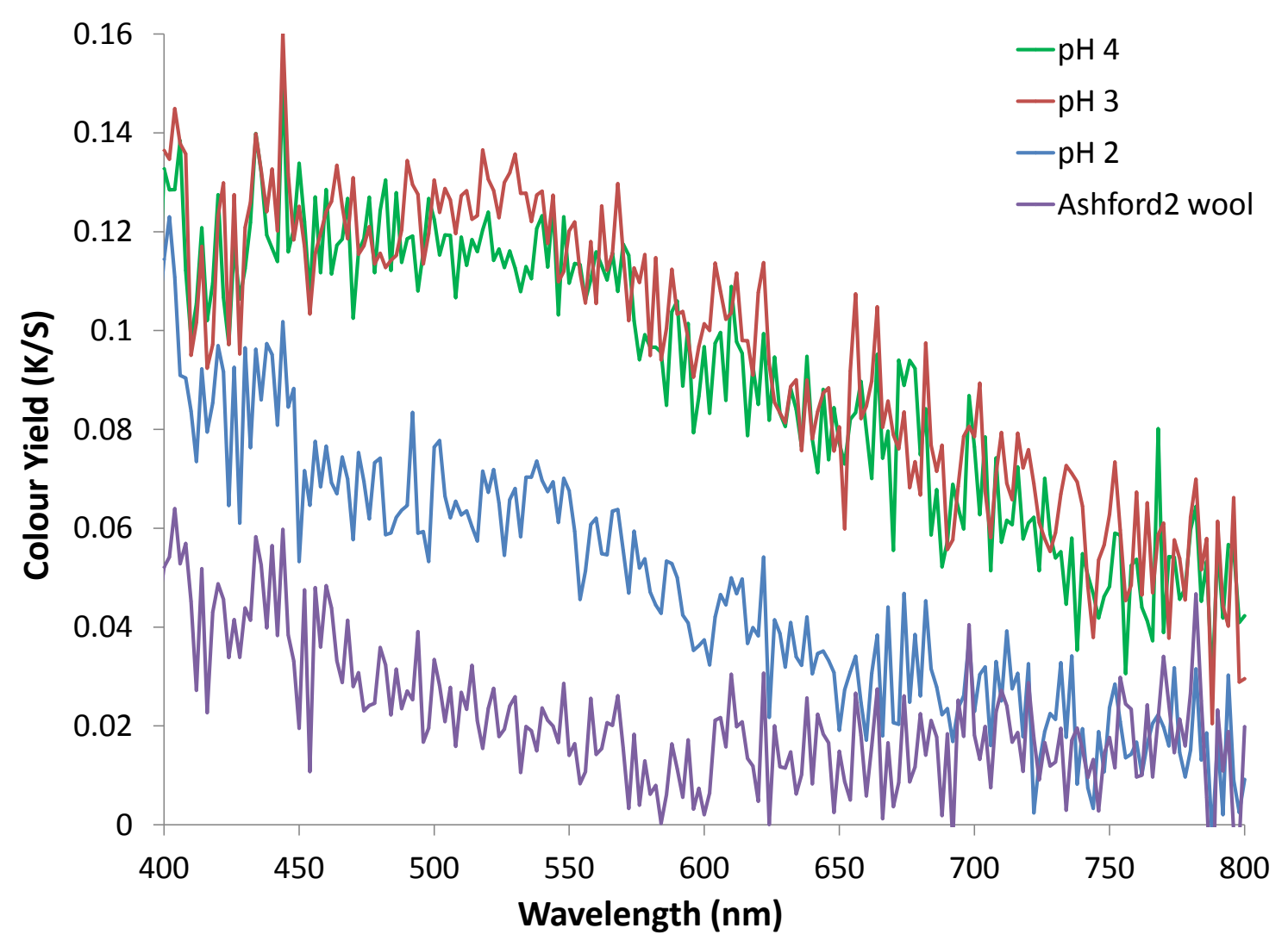

Figure 3.12 UV-visible reflectance spectra of in-situ Ashford2 composites synthesised at pH 2, 3 and 4, and unreacted Ashford2 wool.

SEM micrographs of the surfaces of in-situ composites synthesised at a reaction $\mathrm{pH}$ of 2, 3 and 4 is shown in Figure 3.13. All composites show the presence of gold nanoparticles (appearing as bright dots) on the surface of the wool. The pH 3 sample expectedly shows the greatest amount of gold. The gold is typically present at the cuticle junctions as individual nanoparticles, however a few larger particles and agglomerates are also present in the $\mathrm{pH} 3$ composite.

The uptake of $\mathrm{AuCl}_{4}^{-}$was the most rapid when the synthesis was carried out at $\mathrm{pH}$ 2 , however the pink-purple colour of gold nanoparticles in a wool matrix was barely 

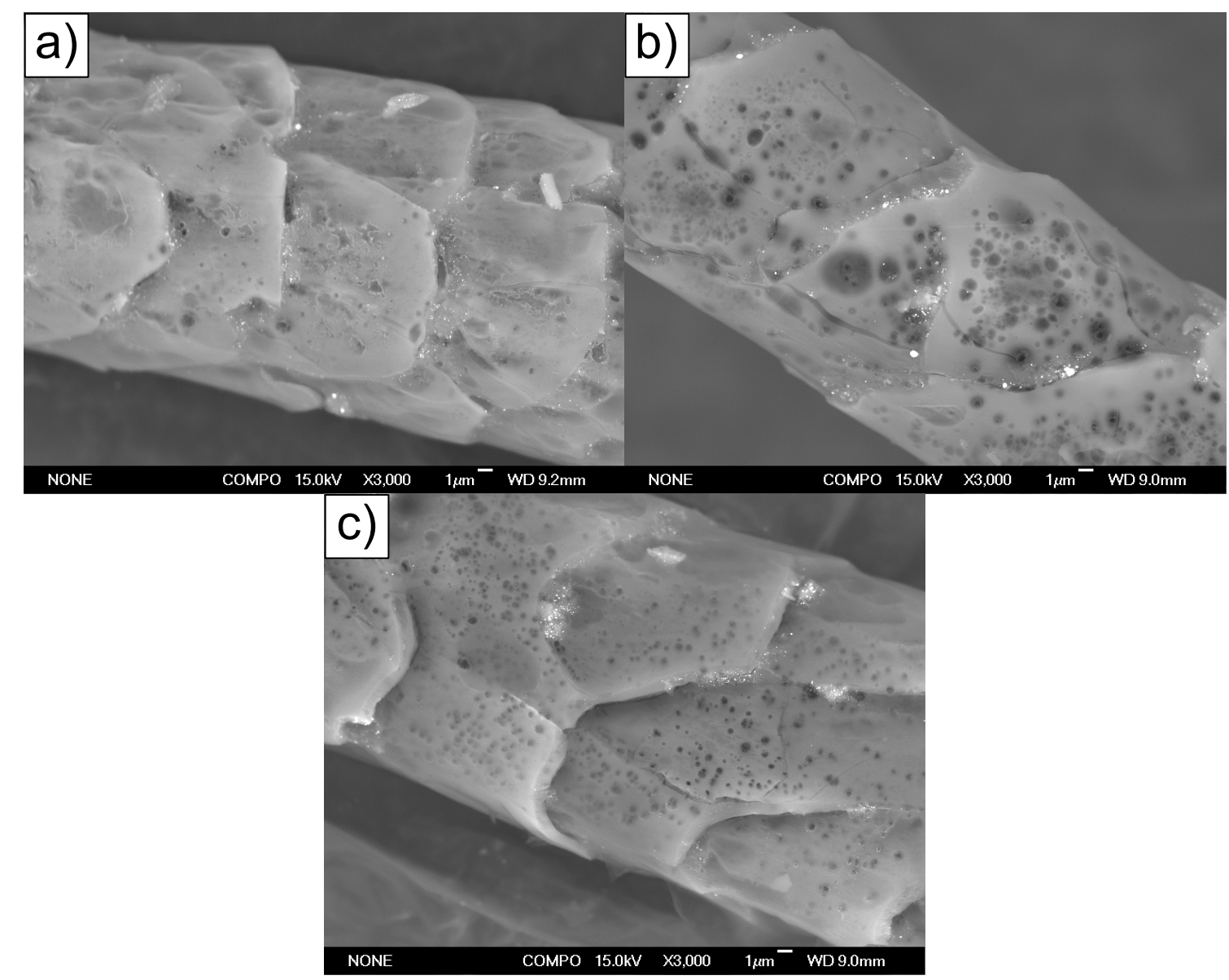

Figure 3.13 SEM micrographs in BS mode of the surface of Ashford2 composites synthesised with the standard purple in-situ method at $\mathrm{pH} 2$ (a), 3 (b) and 4(c).

observable in the resulting composite. This implies that when the $\mathrm{pH}$ is lowered below a certain threshold, there is a smaller driving force for the formation of gold nanoparticles. This is understandable considering that at a lower $\mathrm{pH}$, the amino acids in the wool protein are more likely to be protonated, and hence less capable of electron-donation to reduce $\mathrm{Au}^{3+}$ to $\mathrm{Au}^{0}$ and facilitate nanoparticle formation. As such, it is possible that the $\mathrm{AuCl}_{4}^{-}$absorbed by the wool remains as $\mathrm{AuCl}_{4}^{-}$. Additionally $\mathrm{HCl}$ was used to alter the $\mathrm{pH}$ of the reaction solution, and the concentration of $\mathrm{Cl}^{-}$ions at $\mathrm{pH} 2$ is the highest, resulting in further stabilisation of the $\mathrm{AuCl}_{4}^{-}$complex.

SEM and EDS analysis was used to investigate this; Figure 3.14 (b) and (c) show the EDS elemental maps of $\mathrm{Au}$ and $\mathrm{Cl}$ respectively of the cross-section of a composite fibre synthesised at $\mathrm{pH}$ 2. There is a significant density of both $\mathrm{Au}$ and $\mathrm{Cl}$ throughout the centre of the composite fibre. The overlay of these EDS maps in Figure 3.14 (d) shows that the areas of relatively high density of $\mathrm{Au}$ and $\mathrm{Cl}$ overlap each other (which is seen as the purple areas on the map). This indicates that some of the $\mathrm{AuCl}_{4}^{-}$absorbed into the centre of the fibre remains in the form of $\mathrm{AuCl}_{4}^{-}$. Further evidence for this is seen in the SEM micrograph (Figure 3.14 (a)), where bright spots 
that indicate the presence of gold nanoparticles are barely observable.

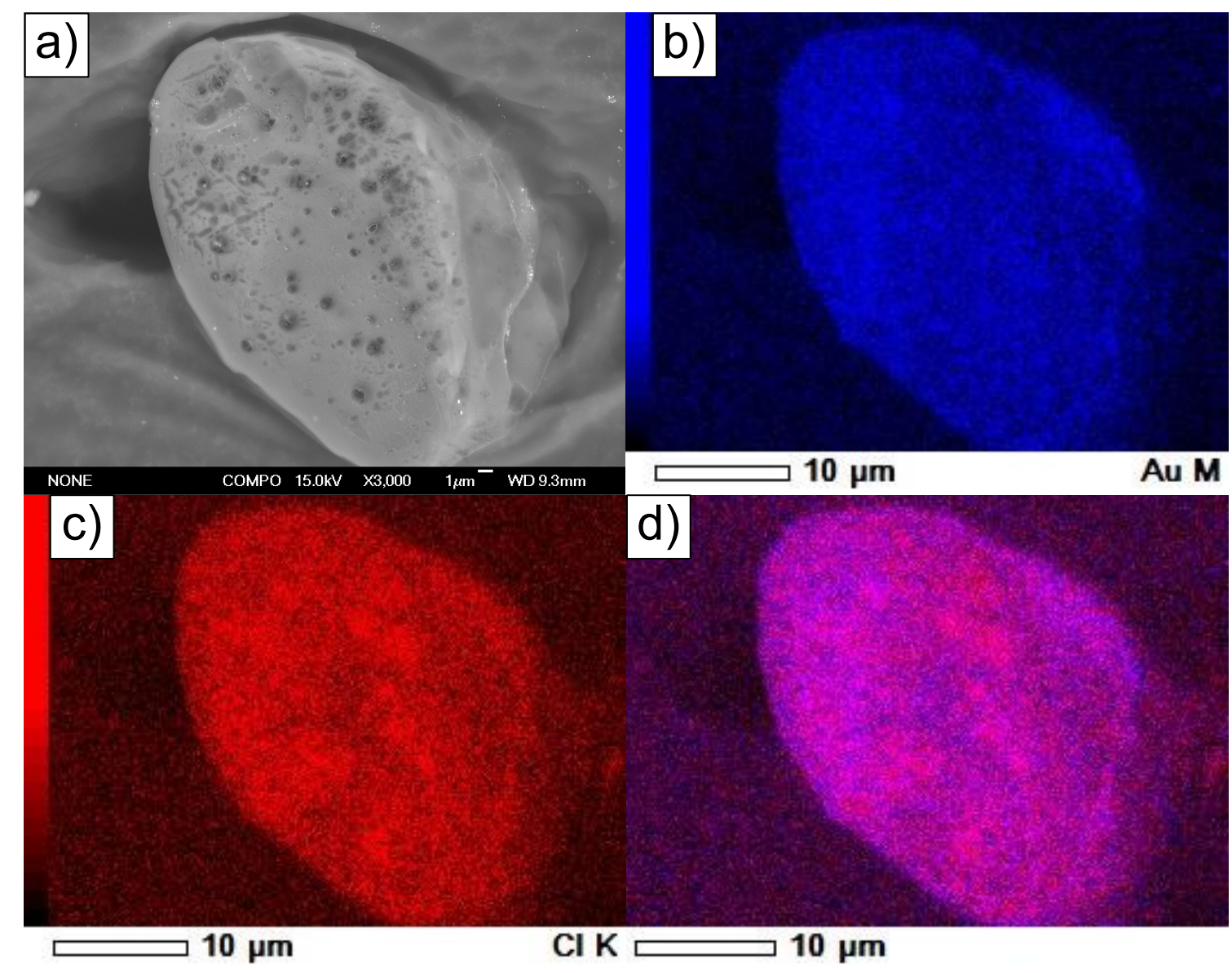

Figure 3.14 SEM and EDS analysis of the cross-section of an Ashford2 in-situ composite fibre synthesised at $\mathrm{pH} 2$; an SEM micrograph in BS mode (a), EDS elemental $\mathrm{Au}(\mathrm{b})$ and $\mathrm{Cl}$ (c) maps of the cross-section and an overlay of $\mathrm{Au}$ and $\mathrm{Cl}$ elemental maps $(\mathrm{d})$.

The relatively high density of $\mathrm{Au}$ throughout the interior of the composite fibres compared to those near the surface shows that the $\mathrm{AuCl}_{4}^{-}$complex is stable enough to be absorbed throughout the centre of the wool fibre before it is reduced to $\mathrm{Au}^{0}$ entities. As such, the faint purple colour of the composite synthesised at $\mathrm{pH} 2$ is considered to be due the formation of fewer surface gold nanoparticles. Additionally, the gold that is present throughout the interior of the fibres may be remain in the form of the $\mathrm{AuCl}_{4}^{-}$complex.

Cross-sectional SEM and EDS analysis of the composites synthesised at pH 3 and 4 are shown in Figure 3.15. The SEM micrographs again show few gold nanoparticles throughout the fibre interiors, however there are some near the surface (which are circled in the images). The EDS elemental Au maps show that the density of gold is higher near the surfaces as compared to the centre of the fibres (additionally, the there are no distinct areas of overlap of $\mathrm{Cl}$ and $\mathrm{Au}$ concentrations and as such, the $\mathrm{Cl}$ maps are not presented). This implies that $\mathrm{AuCl}_{4}^{-}$is not absorbed as far into 
the centre of the wool when the $\mathrm{pH}$ of the uptake solution is increased. Thus, the $\mathrm{pH}$ of the uptake solution significantly influences the extent of diffusion of $\mathrm{AuCl}_{4}^{-}$ into the centre of the wool fibres. This is considered to be due to the relatively slow uptake of $\mathrm{AuCl}_{4}^{-}$and the increased ease of reduction at a higher reaction $\mathrm{pH}$, causing increased formation of gold nanoparticles at or near the fibre surfaces.

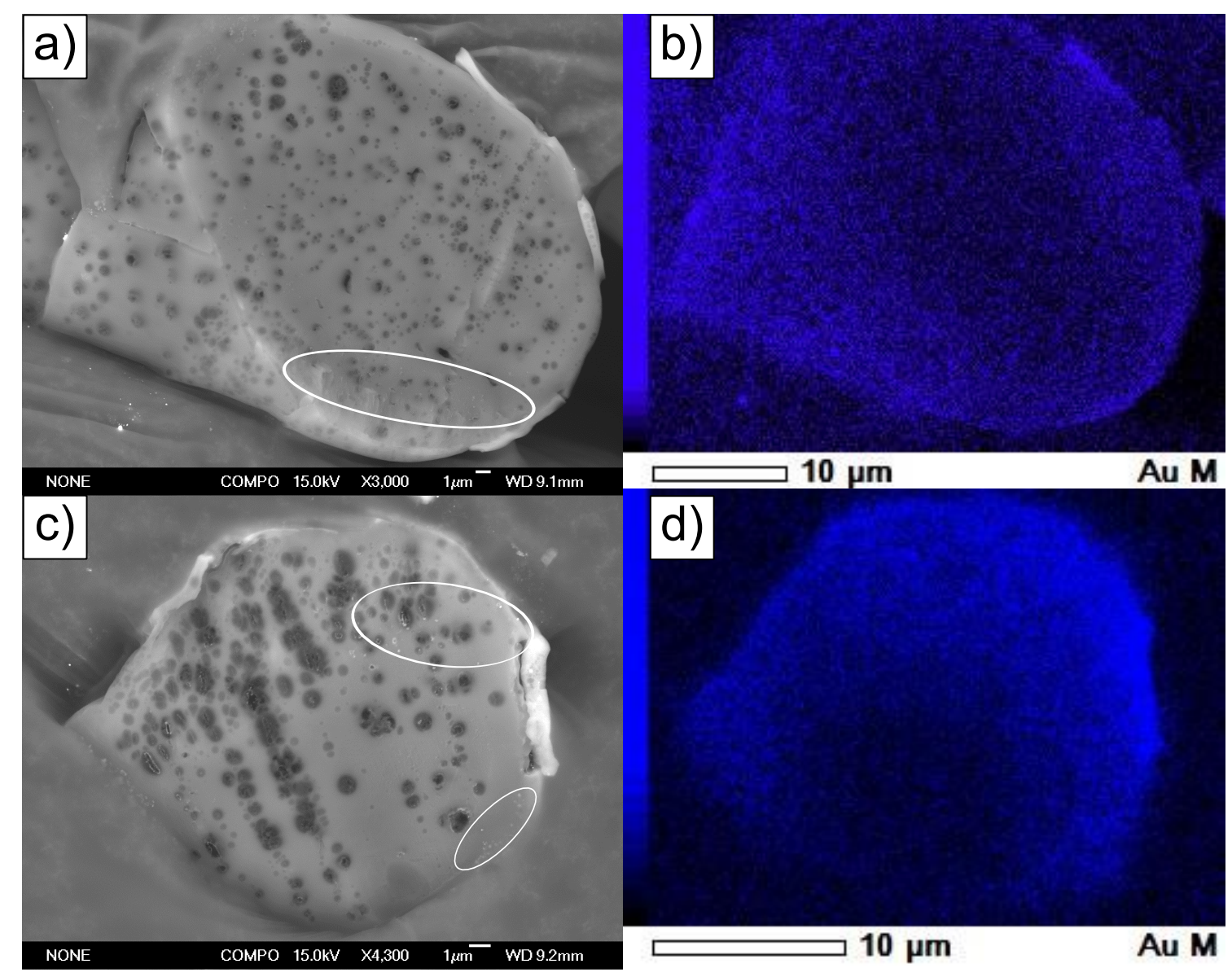

Figure 3.15 SEM micrographs in BS mode of the cross-sections of in-situ composites synthesised at pH 3 (a) and 4 (c), and Au elemental EDS maps these areas (b) and (d).

Continually increasing the $\mathrm{pH}$ of the in-situ synthesis is not a viable option for directing gold nanoparticle formation near the surface of the composites, as a $\mathrm{pH}$ driving force is required for the uptake of $\mathrm{AuCl}_{4}^{-}$to wool. Evidence of this was observed in the $\mathrm{pH} 4$ composite, where the uptake was incomplete after $24 \mathrm{~h}$, and the resulting composite is a lighter shade of purple than than the $\mathrm{pH} 3$ composite. Thus, the uptake $\mathrm{pH}$ employed in the in-situ process must be lower than the isoelectric region of wool fibres (where the wool exhibits a net positive surface charge) to attract the negatively charged $\mathrm{AuCl}_{4}^{-}$complex.

The study presented in this section has shown that altering the $\mathrm{pH}$ of the in-situ approach allows for manipulation of both the uptake of $\mathrm{AuCl}_{4}^{-}$and the colour of the 
composites produced. Further research into this area would include investigating the an optimal $\mathrm{pH}$ for each distinct wool type used in the in-situ procedure.

\subsubsection{Temperature}

Temperature is an important variable of the in-situ procedure. The uptake of $\mathrm{AuCl}_{4}^{-}$ to wool is typically carried out at RT, a temperature at which the nucleation of gold nanoparticles is very gradual. The in-situ procedure utilises heat to increase the rate of gold nanoparticle formation in the wool fibres, thus increasing the commercial viability of the approach. In-situ composites of Ashford2 wool were synthesised at a range of temperatures to study the influence of temperature on the rate of $\mathrm{AuCl}_{4}^{-}$ uptake and gold nanoparticle formation, and the distribution of gold throughout the composites.

Figure 3.16 shows the uptake of $\mathrm{AuCl}_{4}^{-}$to Ashford2 wool at a range of temperatures. In general, the rate of uptake increases when the uptake temperature is raised. The uptake of $\mathrm{AuCl}_{4}^{-}$to Ashford2 wool is remarkably slow compared to Ashford wool; at an uptake temperature of $5^{\circ} \mathrm{C}$ or RT, less than half of the $\mathrm{AuCl}_{4}^{-}$in solution has been absorbed by Ashford 2 wool after $180 \mathrm{~min}$. In contrast, the uptake of $\mathrm{AuCl}_{4}^{-}$by the original Ashford wool is approximately complete after 90 min at RT (this was shown in Figure 3.6). This highlights the differences in chemistry of wool types sourced through various processing routes. Increasing the absorption temperature to boiling causes the uptake to be complete after only $30 \mathrm{~min}$, whilst the temperature must be elevated to $50^{\circ} \mathrm{C}$ for the uptake to be approximately complete after $180 \mathrm{~min}$. After this time, the uptake of $\mathrm{AuCl}_{4}^{-}$to Ashford wool was complete at RT.

Figure 3.17 shows samples of Ashford2 wool that have been removed from the uptake solution and dried after $120 \mathrm{~min}$ and $24 \mathrm{~h}$ (the photograph was taken soon after the uptake was complete). With an uptake temperature of $50{ }^{\circ} \mathrm{C}$ or higher, the purple colour of gold nanoparticles in a wool matrix is seen after 120 min uptake. The higher the uptake temperature, the more rapid the development the purple colouration and hence the faster the rate of nanoparticle formation in the wool matrix. The purple colouration of the $50^{\circ} \mathrm{C}$ composite develops before the uptake is complete, indicating that at this temperature the uptake of $\mathrm{AuCl}_{4}^{-}$and the formation of gold nanoparticles occurs simultaneously.

In addition to the rates of $\mathrm{AuCl}_{4}^{-}$uptake and gold nanoparticle formation, the effect of the reaction temperature on the distribution of gold throughout the composite fibres was also investigated. EDS analyses of the cross-sections of Ashford2 compos- 


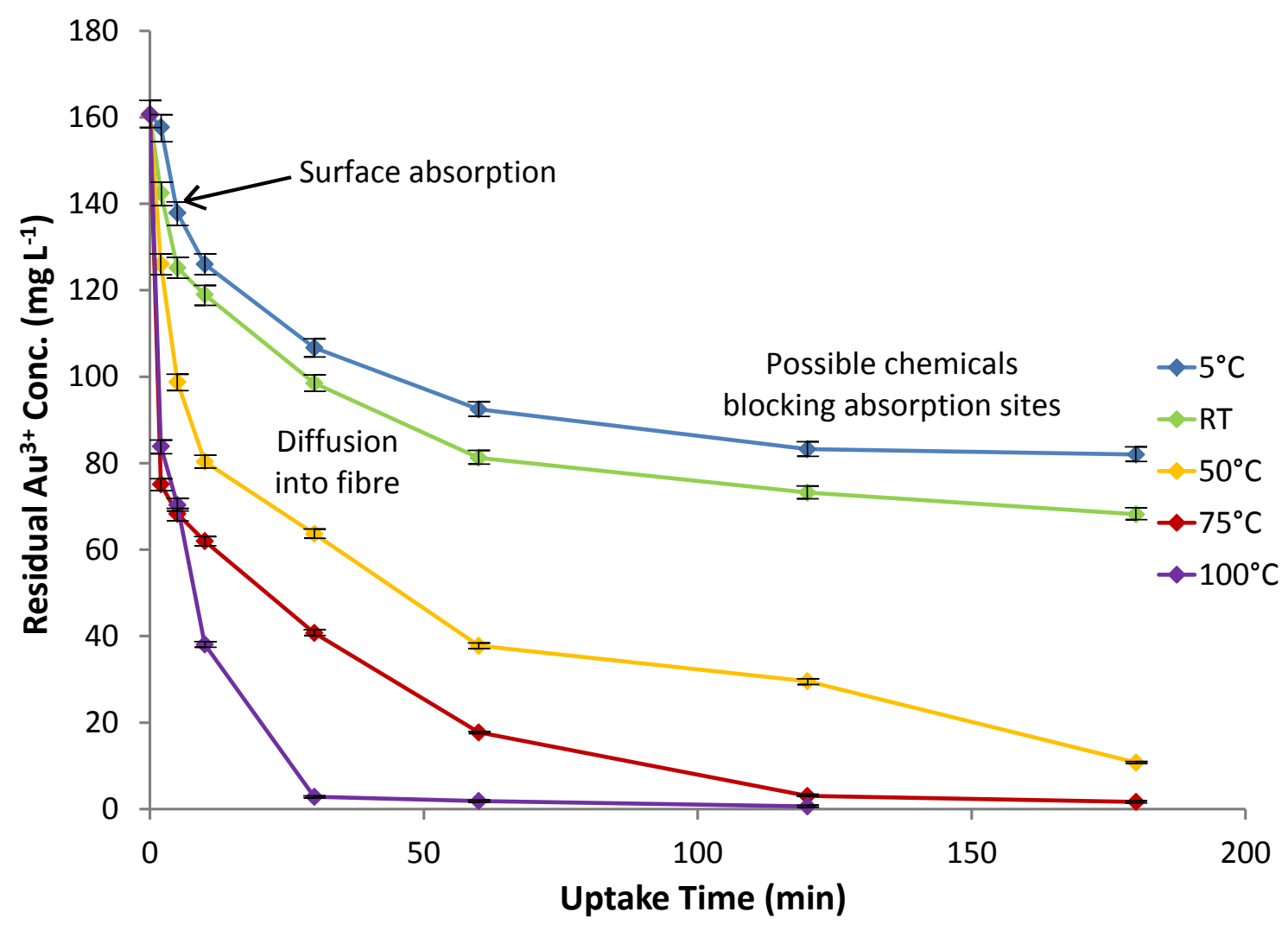

Figure 3.16 Uptake of $\mathrm{Au}^{3+}$ to Ashford2 wool with the standard purple in-situ method at different temperatures.

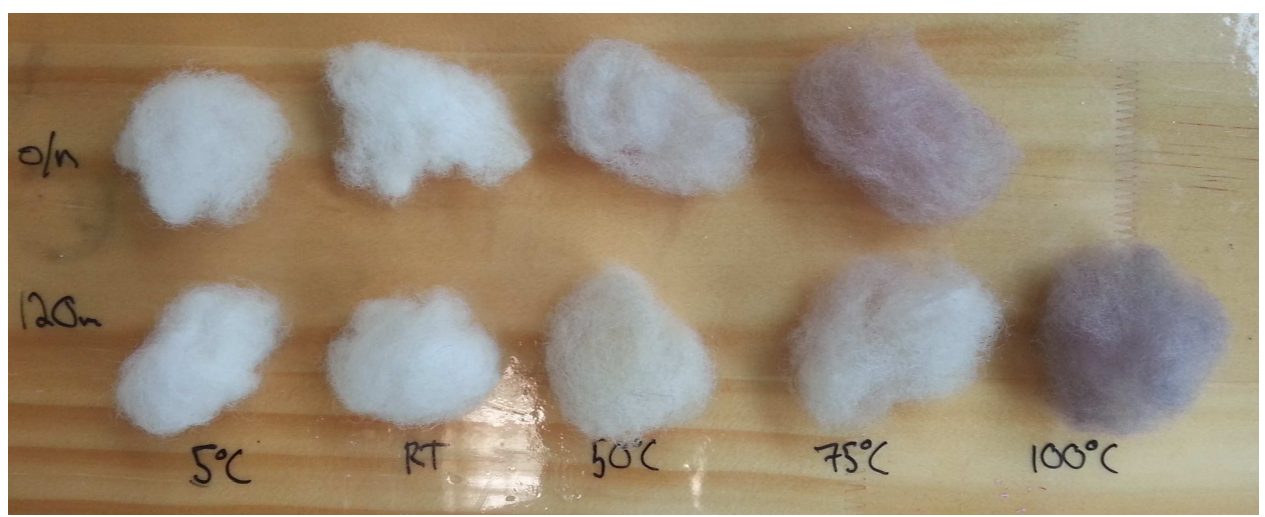

Figure 3.17 Ashford2 in-situ composites removed after 120 min or overnight uptake of $160 \mathrm{mg} \mathrm{L}^{-1} \mathrm{Au}^{3+}$ at different temperatures (the photograph was taken soon after the uptake).

ites removed from uptake solutions after $10 \mathrm{~min}$ and $60 \mathrm{~min}$ at RT, and after $10 \mathrm{~min}$ at $50^{\circ} \mathrm{C}$ and $75^{\circ} \mathrm{C}$ are shown in Figure 3.18. The approximate gold content of the samples imaged is shown in Table 3.6, where which is determined by AAS analysis of the residual $\mathrm{AuCl}_{4}^{-}$concentration in the uptake solutions. After $10 \mathrm{~min}$ of uptake at RT the Ashford2 wool has absorbed approximately $25 \mathrm{mg} \mathrm{L}^{-1}$ of $\mathrm{Au}^{3+}$, which relates to an approximate gold content of $0.25 \mathrm{wt} \%$ (given that the wool to liquor ratio is $1: 100)$. 

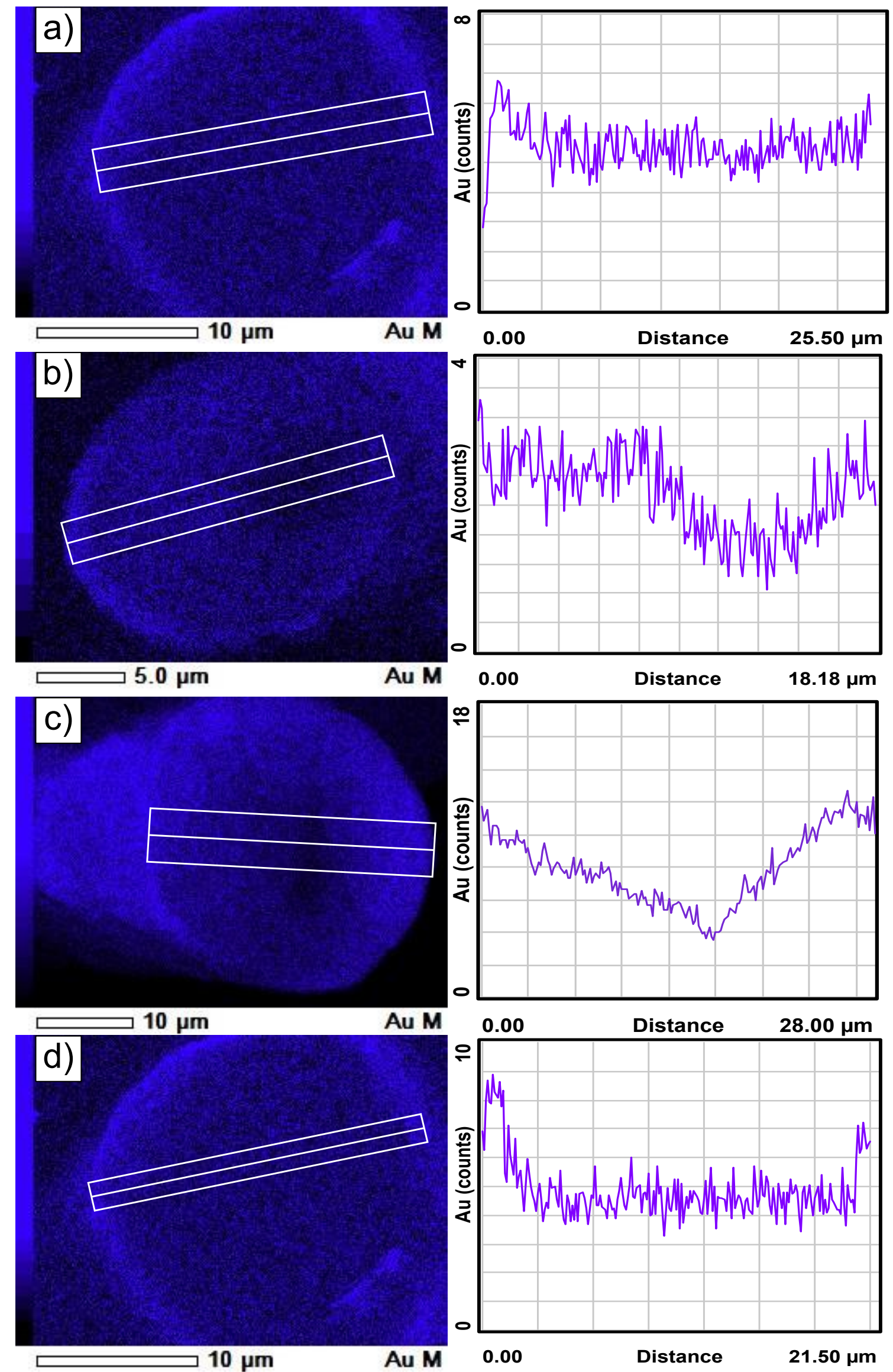

Figure 3.18 EDS Au elemental maps and line profiles of the cross-sections of Ashford2 in-situ composites removed after 10 min uptake at RT (a), 60 min uptake at RT (b), $10 \mathrm{~min}$ uptake at $50^{\circ} \mathrm{C}(\mathrm{b})$ and $10 \mathrm{~min}$ uptake at $75^{\circ} \mathrm{C}(\mathrm{d})$. 
Table 3.6 The amount of gold in Ashford2 in-situ composites that were removed from uptake solutions at different temperatures and times.

\begin{tabular}{lccc}
\hline Conditions & $\begin{array}{c}\text { Residual } \mathrm{Au}^{3+} \\
\text { conc }\left(\mathrm{mg} \mathrm{L}^{-1}\right)\end{array}$ & $\mathrm{Au}^{3+}$ absorbed & $\begin{array}{c}\text { Approximate } \\
\text { Au content (wt \%) }\end{array}$ \\
\hline RT 10 min & $135 \pm 2.7$ & $25 \pm 0.5$ & $0.25 \pm 0.005$ \\
RT 60 min & $90 \pm 1.8$ & $70 \pm 1.4$ & $0.7 \pm 0.014$ \\
$50^{\circ} \mathrm{C} 10 \mathrm{~min}$ & $100 \pm 2.0$ & $60 \pm 1.2$ & $0.6 \pm 0.012$ \\
$75^{\circ} \mathrm{C} 10 \mathrm{~min}$ & $70 \pm 1.4$ & $90 \pm 1.8$ & $0.9 \pm 0.018$ \\
\hline \hline
\end{tabular}

After 10 min of uptake of $\mathrm{AuCl}_{4}^{-}$by Ashford2 wool at RT, there is little absorption of $\mathrm{AuCl}_{4}^{-}$throughout the interior of the wool. The line profile of the composite cross-section shows relatively higher intensity of $\mathrm{Au}$ near the surface of the fibre. This indicates the preferential absorption of $\mathrm{AuCl}_{4}^{-}$at or near the surface of the composite fibre after $10 \mathrm{~min}$, as there is not time for the $\mathrm{AuCl}_{4}^{-}$to be absorbed into the centre of the wool.

After 60 min of uptake at RT, the $\mathrm{AuCl}_{4}^{-}$has absorbed further into the centre of the fibre. The concentration of $\mathrm{AuCl}_{4}^{-}$appears to be relatively high near the surface of the $60 \mathrm{~min}$ fibre (compared to the centre of the fibre), however there is a relatively high proportion of $\mathrm{Au}$ present in the fibre interior when compared with the $10 \mathrm{~min}$ composite. This shows that as the uptake proceeds at $\mathrm{RT}, \mathrm{AuCl}_{4}^{-}$gradually diffuses towards the centre of the wool, and that the ratio of $\mathrm{Au}$ present in the fibre interior compared to that near the surface increases. Additionally, the line profile displays a relatively high gold concentration on the left side of the fibre, which corresponds to the location of the paracortex of the fibres. This indicates an attraction and binding of $\mathrm{AuCl}_{4}^{-}$to sulfur (the paracortex has a relatively high sulfur content).

When the temperature of the uptake is increased, the rate of uptake is significantly enhanced. After $10 \mathrm{~min}$ of uptake at $50^{\circ} \mathrm{C}, \mathrm{AuCl}_{4}^{-}$has migrated into the fibre interior, but has not yet penetrated to the centre of the fibre. The line profile clearly shows a sequentially lower intensity of $\mathrm{Au}$ when moving from the outside to the the centre of the fibre. Again, the level of Au density at or near the surface of the wool is much greater than that near the fibre centre.

At an uptake temperature of $75^{\circ} \mathrm{C}$, the gold is concentrated at or near the surface of the wool fibre. The line profile shows that except for at the edges of the fibres, the distribution of $\mathrm{Au}$ is approximately equivalent throughout the fibre. It is considered that at such a high temperature, there is sufficient energy for the $\mathrm{AuCl}_{4}^{-}$complex to react with the functional groups of the wool protein, which it does so preferentially to penetrating into the fibre interior. After $10 \mathrm{~min}$ of uptake time at $75^{\circ} \mathrm{C}$, the Ashford2 wool has absorbed more $\mathrm{AuCl}_{4}^{-}$than after 60 min of RT uptake. This means that 
elevating the temperature is causing the difference in observed gold distribution, as although the rate of uptake increases, the extent of diffusion of $\mathrm{AuCl}_{4}^{-}$into the centre fibre decreases. The decrease in the extent of migration of $\mathrm{AuCl}_{4}^{-}$throughout the fibres at higher uptake temperatures is understandable given the requirement of heat to facilitate the reduction of $\mathrm{Au}^{3+}$ in the nanoparticle nucleation process.

This study has shown that the temperature of the in-situ procedure influences the rate of uptake and nanoparticle formation, and the distribution of gold throughout the composite fibres. As such, the temperature of the reaction can be manipulated to direct preferential formation of gold nanoparticles at or near the surface of the fibres. Further reduction of the uptake time at 75 or $100{ }^{\circ} \mathrm{C}$ has the potential to further direct gold nanoparticle formation near the surface of the composite fibres, and would be interesting to investigate in the future.

\subsubsection{Auxiliary Agents}

Auxiliary agents are commonly employed in the wool dyeing industry to promote the uniformity of colouring. They are typically split into two categories; wetting agents and levelling agents. Wetting agents aid in the wetting of wool fibres to allow for the complete penetration of dyestuffs throughout a dyepack. Levelling agents are used to control the absorption of dyes by the wool fibres.

In this section, a range of dyeing auxiliaries are experimented with to attempt to control the uptake of $\mathrm{AuCl}_{4}^{-}$to wool and inhibit the absorption of $\mathrm{AuCl}_{4}^{-}$into the centre of wool fibres.

\section{Wetting Agents}

Achieving colour uniformity in the dyeing of kilogram samples of wool is a significant challenge faced by the wool dyeing industry. On the laboratory-scale however, achieving uniform dyeing of wool is typically trivial. Despite this, the finished wool yarns dyed in Section 3.2.1 displayed significant colour variation in composites of $1 \mathrm{~g}$ size. As such, a wetting agent was used promote the uniform absorption of $\mathrm{AuCl}_{4}^{-}$by wool yarns. The wetting agent Albaflow FFA was used upon the recommendation of Professor Johnston's industry contacts at Wool Yarns Ltd, in Lower Hutt, New Zealand. Albaflow FFA is an anionic auxiliary agent is used to aid the wetting out of dyepacks in wool dyeing. 
In-situ composites of finished wool yarns synthesised with and without $1 \mathrm{~g} \mathrm{~L}^{-1} \mathrm{Al}$ baflow FFA are depicted in Figure 3.19. The conditions of the standard purple in-situ method were used $\left(160 \mathrm{mg} \mathrm{L}^{-1} \mathrm{AuCl}_{4}^{-}, \mathrm{pH} 2.7\right.$, RT uptake, ageing at $50^{\circ} \mathrm{C}$ for 1 week). The presence of the wetting agent was seen to improve the uniformity of colour of each composite. When the wetting agent was used, the rate of uptake of $\mathrm{AuCl}_{4}^{-}$was slower, allowing for a more uniform absorption of $\mathrm{AuCl}_{4}^{-}$. The wetting agent also resulted in slightly lighter-coloured composites and appears to somewhat prevent the agglomeration of the gold nanoparticles; the TS composite is more pink than purple and the S19 and S14 composites are more purple than grey.

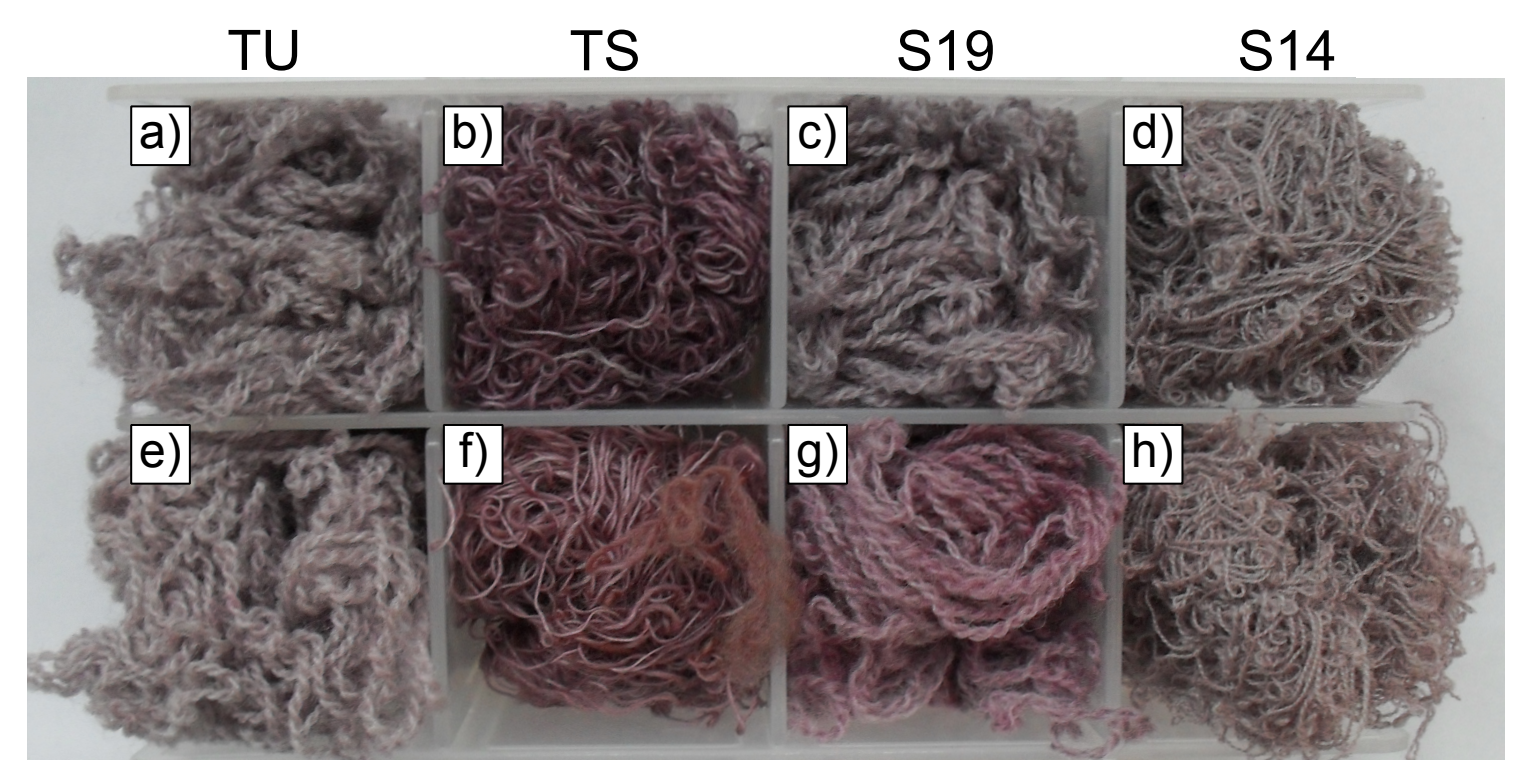

Figure 3.19 In-situ composites of TU, TS, S19 and S14 yarns synthesised without Albaflow FFA (a)-(d) respectively and with $1 \mathrm{gL}^{-1}$ Albaflow FFA in the uptake solutions (e)-(g).

Quantification of the colour differences are observed when comparing the CIE colour coordinates of the finished wool yarn composites, as shown in Table 3.7. The TU yarn composite is slightly brighter and redder when the wetting agent was employed. The TS composite is slightly more red and significantly less blue, alluding to the colour change from purple to pink when the wetting agent was used. S19 is slightly darker, much more red and blue, accounting for the grey-purple colour change. Finally, S14 exhibits a slightly higher $a^{*}$ and $b^{*}$, showing a greater contribution of purple-coloured gold nanoparticles present in the S14 composite.

SEM and EDS analysis of the surface of a TU yarn composite fibre synthesised with $1 \mathrm{gL}^{-1}$ Albaflow FFA in the uptake solution is shown in Figure 3.20. The SEM micrograph (Figure 3.20 (a)) shows that a significant amount of the wetting agent remains on the surface of the composite fibres. These are clumps and ricelike shapes that are typically $<1 \mu \mathrm{m}$ in size, which are generally associated with 
Table 3.7 CIE colour coordinates of in-situ composites of finished wool yarns synthesised with and without $1 \mathrm{~g} \mathrm{~L}^{-1}$ Albaflow FFA wetting agent.

\begin{tabular}{ccccccccc}
\hline Wool type & \multicolumn{2}{c}{ TU } & \multicolumn{2}{c}{ TS } & \multicolumn{2}{c}{ S19 } & \multicolumn{2}{c}{ S14 } \\
\hline WA $\left(\mathrm{g} \mathrm{L}^{-1}\right)$ & 0 & 1 & 0 & 1 & 0 & 1 & 0 & 1 \\
\hline $\mathrm{L}^{*}$ & 51.00 & 52.10 & 38.88 & 38.82 & 48.00 & 43.11 & 48.44 & 46.44 \\
$\mathrm{a}^{*}$ & 4.02 & 4.87 & 9.24 & 10.28 & 4.15 & 9.61 & 4.54 & 5.84 \\
$\mathrm{~b}^{*}$ & 0.20 & -0.11 & -2.42 & -0.27 & -1.02 & -2.86 & 0.72 & 1.76 \\
\hline
\end{tabular}

the junctions between overlapping cuticle scales. EDS elemental mapping of the fibres shows areas of high concentration of $\mathrm{Ca}$ and $\mathrm{Si}$, which may be due to calcium silicate from the laboratory. The Si may be due to the presence of silicone, which is commonly present in wetting agents (the organic components of Albaflow FFA are not observable, due to the carbon coating of samples prior to SEM imaging).
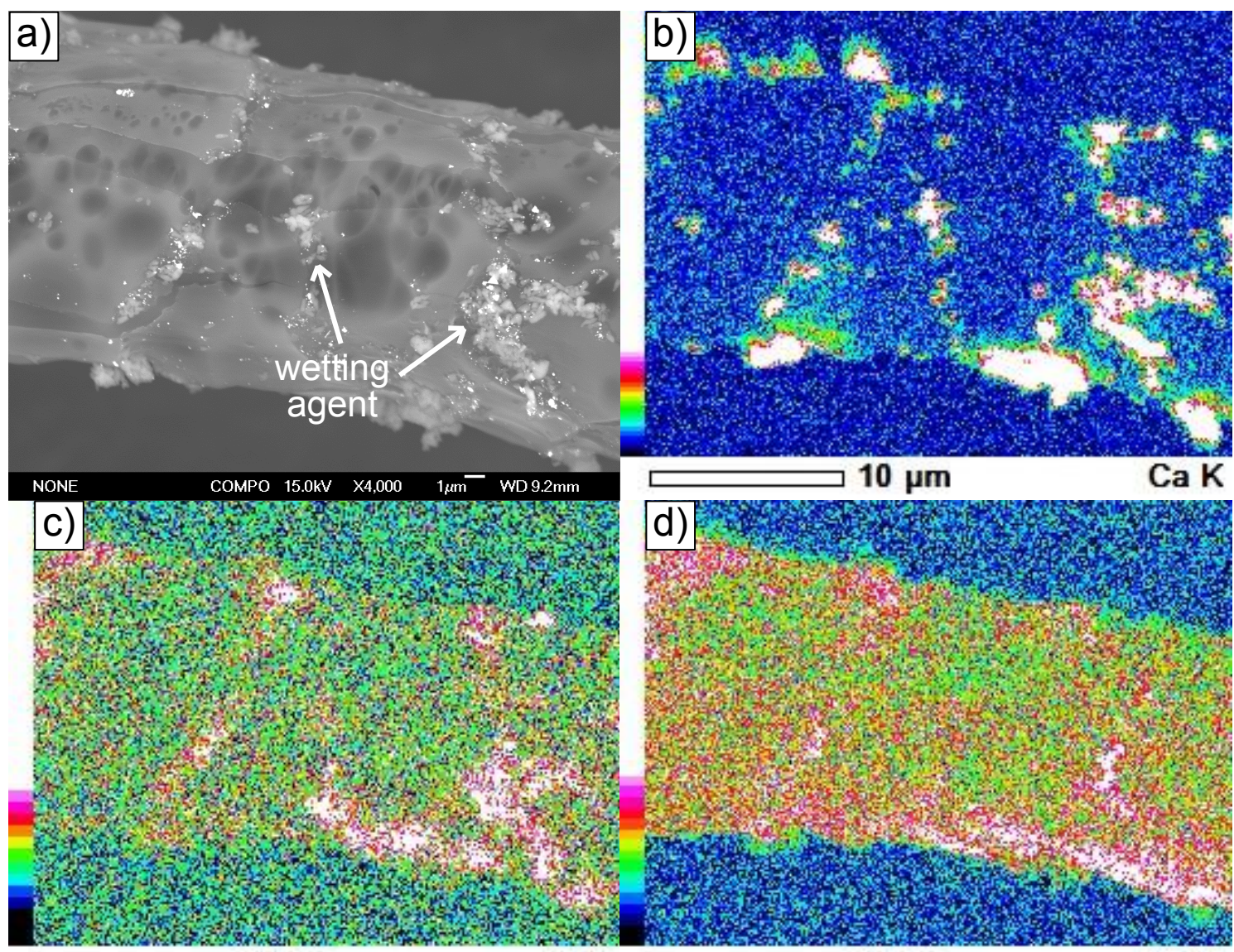

$10 \mu \mathrm{m}$

Si K

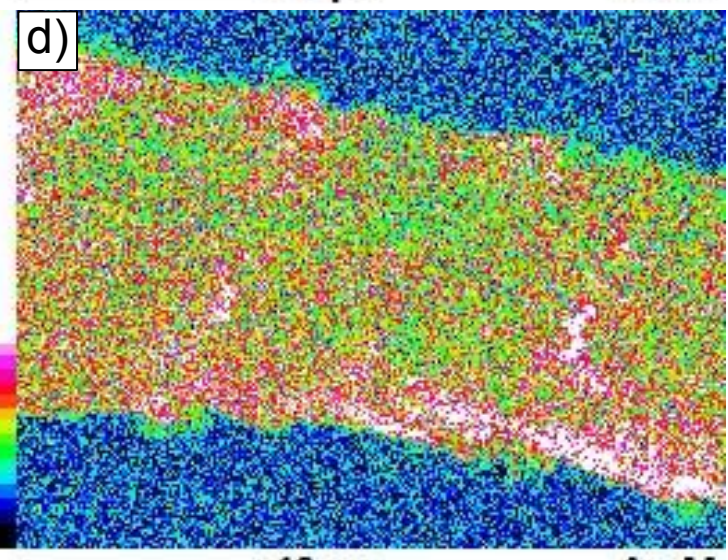

Figure 3.20 SEM and EDS analysis of an in-situ composite of TU yarn with $1 \mathrm{~g} \mathrm{~L}^{-1}$ Albaflow FFA.

SEM micrographs of in-situ composites of different types of yarn synthesised with the standard purple in-situ method and $1 \mathrm{~g} \mathrm{~L}^{-1}$ Albaflow FFA are presented in Figure 3.21. Similar to the distribution of $\mathrm{Au}$, the wetting agent is most commonly present at near the junctions between cuticle cells. The higher magnification micro- 
graph of the TU yarn composite (Figure $3.21(\mathrm{~b})$ ) shows the presence of triangular platelet shaped gold nanoparticle, in addition to predominantly spherical nanoparticles that are in close proximity to one and other. This is very similar to the size, shape and distribution of gold nanoparticles on the surface of the in-situ TU yarn composite synthesised without the wetting agent (that was presented in Figure 3.10), however it appears that there are relatively fewer gold nanoparticles present when the wetting agent is employed. This difference may be due to Albaflow FFA causing a more uniform distribution of gold nanoparticles across a greater number of wool fibres in the composite sample.

The TS composite exhibits both individual gold nanoparticles and larger particles of gold, many of which are triangular-platelets. Like the TU yarn composite, the colour difference is likely due to the presence of fewer gold nanoparticles of a similar size and shape profile to the composite with no wetting agent. Interestingly, the S19 and S14 composites exhibit large particles of gold on the fibre surfaces, which is unexpected due to the relatively purple colouration of the composites.

The SEM of the composites appear to display gold nanoparticles that are located relatively further apart from each other when the wetting agent is employed in the synthesis. As the colouration of the samples is more uniform, it is probable that the gold nanoparticles are more evenly distributed over a larger number of wool fibres. As such, implementation of Albaflow FFA has enhanced the uniformity of colouring of finished wool yarn in-situ composites, typically without influencing the size and shape of the gold particles that form. This is a significant accomplishment and has enhanced the potential to successfully scale-up the in-situ synthesis of finished wool yarn composites.

\section{Levelling Agents}

Levelling agents are commonly used wool dyeing auxiliaries that slow the absorption of dyestuffs, thus improving the colour uniformity of wool dyeing. Levelling agents are typically salts that are absorbed throughout the wool fibres, or otherwise surfactant-like molecules that adsorb to the surface of wool (similar to wetting agents), which block and slow the access of dye molecules to the wool fibre. Levelling agents are either added into the dyebath, or absorbed in a pre-dyeing process.

In this experiment, $\mathrm{Na}_{2} \mathrm{SO}_{4}$ and $\mathrm{NaCl}$ were incorporated into the standard purple in-situ method synthesis of Cariaggi gold nanoparticle-wool fibre composites. In this, $1 \mathrm{~g} \mathrm{~L}^{-1} \mathrm{Na}_{2} \mathrm{SO}_{4}$ or $\mathrm{NaCl}$ was added to uptake solutions, or the Cariaggi wool 

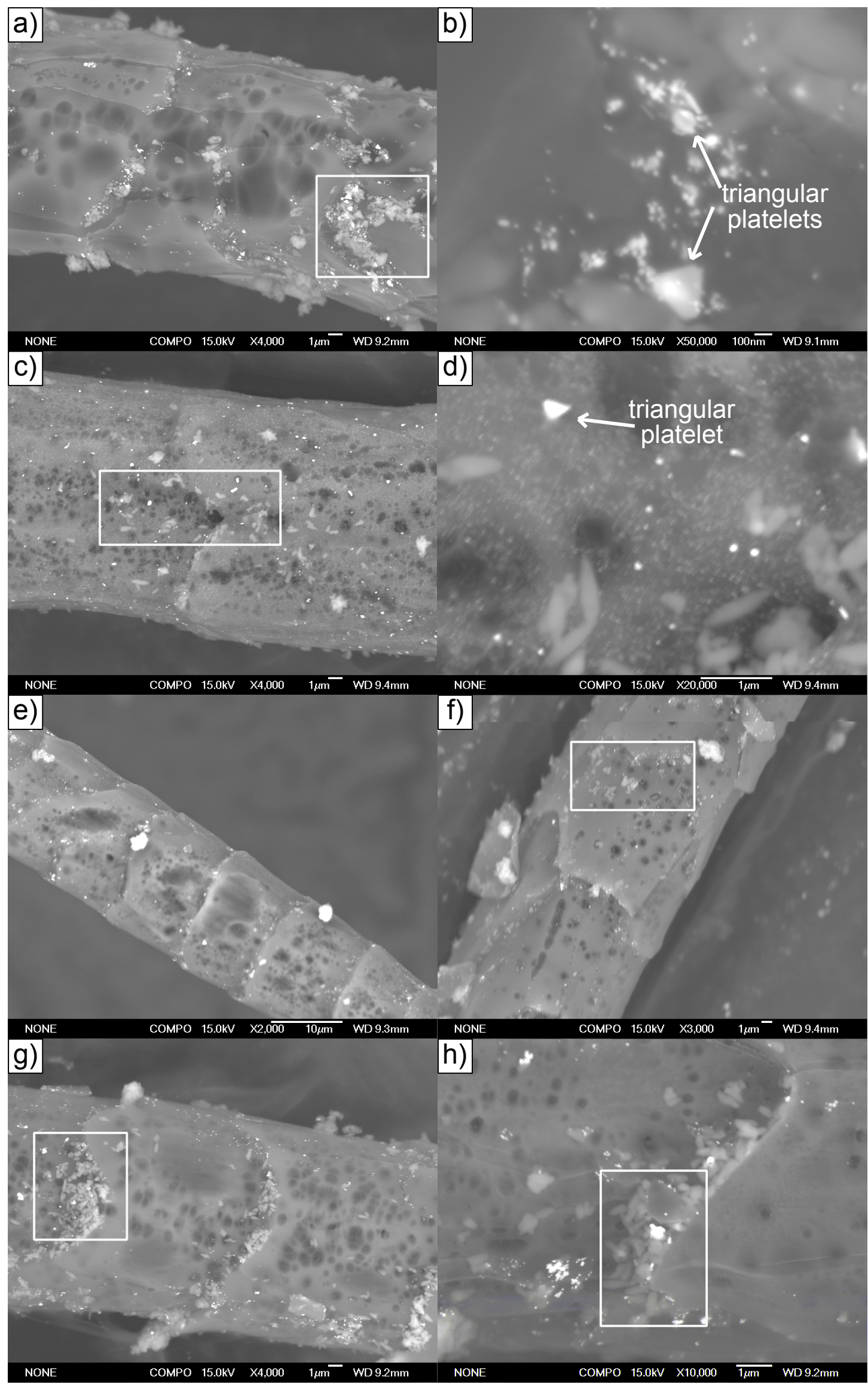

Figure 3.21 SEM micrographs in BS mode of in-situ composites of TU (a)-(b), TS (c)-(d), S19 (e)-(f) and S14 (g)-(h) yarn synthesised with $1 \mathrm{~g} \mathrm{~L}^{-1}$ Albaflow FFA. The rectangles indicate areas with high amounts of Albaflow FFA. 
was soaked in a solution of $10 \mathrm{~g} \mathrm{~L}^{-1} \mathrm{Na}_{2} \mathrm{SO}_{4}$ prior to the uptake of $\mathrm{AuCl}_{4}^{-}$. The effect of the levelling agents on the uptake process was first investigated; Figure 3.22 shows the uptake of approximately $160 \mathrm{mg} \mathrm{L}^{-1} \mathrm{AuCl}_{4}^{-}$to Cariaggi wool with and without the presence of levelling agents.

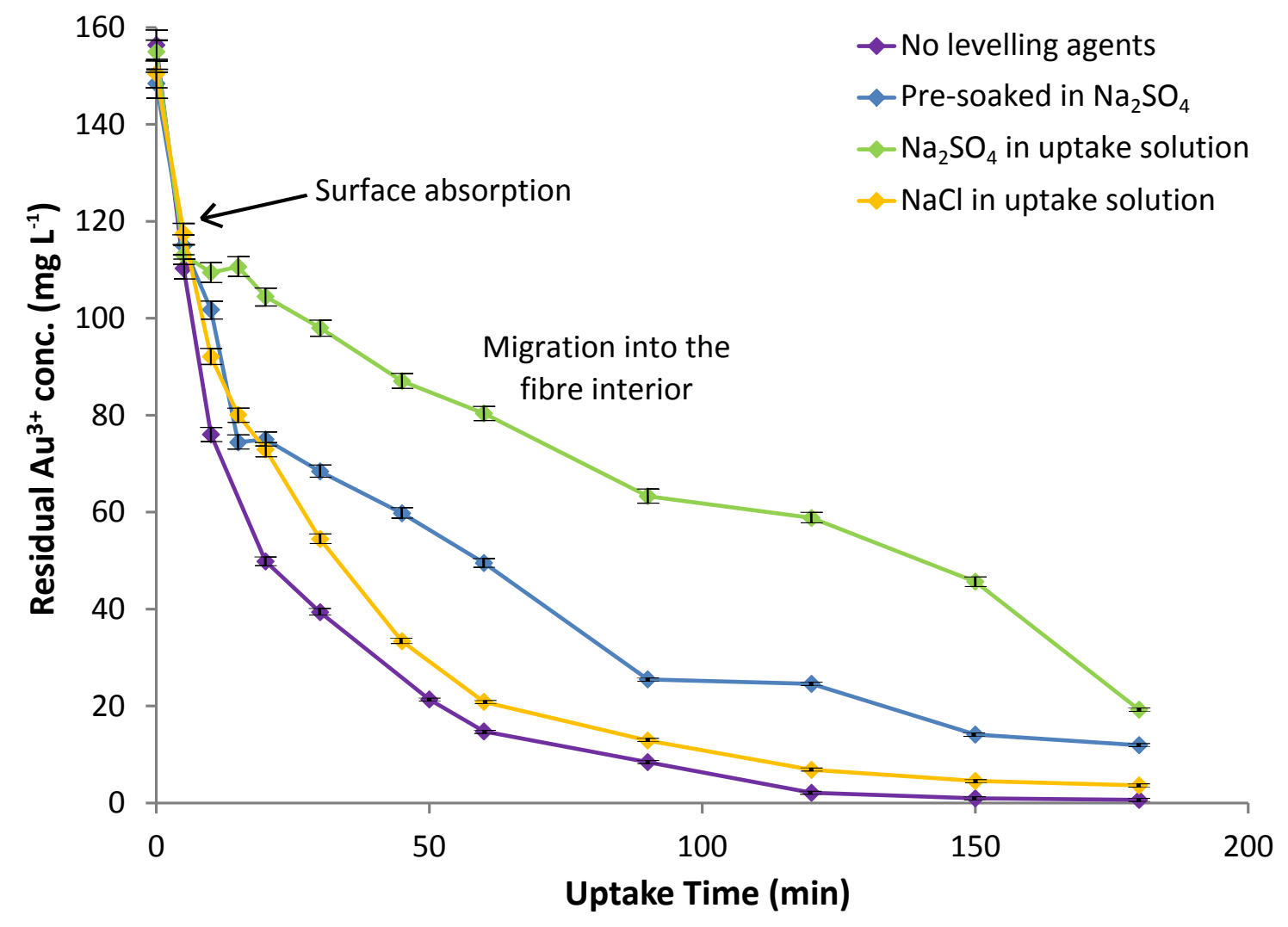

Figure 3.22 AAS uptake of $\mathrm{AuCl}_{4}^{-}$to Cariaggi wool with and without the presence of levelling agents.

The incorporation of salt-based levelling agents into the in-situ procedure has slowed the rate of $\mathrm{AuCl}_{4}^{-}$absorption by Cariaggi wool to some extent. The most significant change in uptake was observed when $\mathrm{Na}_{2} \mathrm{SO}_{4}$ is present in the uptake solution. In this, the absorption of $\mathrm{AuCl}_{4}^{-}, \mathrm{Na}^{+}$and $\mathrm{SO}_{4}{ }^{2-}$ ions by the wool are competing processes, and as such, interaction between the ions may lower the stability of the $\mathrm{AuCl}_{4}^{-}$complex.

However, when the wool is soaked in $\mathrm{Na}_{2} \mathrm{SO}_{4}$ prior to the uptake, there is a more subtle change in the uptake profile. There is likely a lower effective concentration of $\mathrm{Na}_{2} \mathrm{SO}_{4}$ in the uptake solution when the wool is pre-soaked, as it requires leaching from the fibres to interact with the $\mathrm{AuCl}_{4}^{-}$in the uptake solution. In both cases, the uptake curve is relatively jagged, at times there is an increase in $\mathrm{AuCl}_{4}^{-}$concentration in the uptake solution as the reaction proceeds. This suggests re-equilibration of $\mathrm{AuCl}_{4}^{-}$throughout the uptake; as $\mathrm{AuCl}_{4}^{-}$that has been absorbed by the wool leaches back into the uptake solution. When $\mathrm{NaCl}$ is present in the uptake solution, the uptake curve is only partially altered. The rate of uptake is slightly slowed due to 
the presence of $\mathrm{NaCl}$, but the shape of the curve is approximately equivalent. This may be due to the higher ionic strength of the $\mathrm{SO}_{4}{ }^{2-}$ solution compared to that of the $\mathrm{Cl}^{-}$solution.

In-situ composites of Cariaggi wool synthesised with the presence of levelling agents are shown in Figure 3.23, where the levelling agents have altered the colouration of the resulting composites. The presence $\mathrm{Na}_{2} \mathrm{SO}_{4}$ in the uptake solution resulted in grey coloured composites. The extent of agglomeration is more pronounced when the $\mathrm{Na}_{2} \mathrm{SO}_{4}$ is added to the uptake solution, presumably due to the relatively higher concentration of $\mathrm{Na}_{2} \mathrm{SO}_{4}$ being present. The $\mathrm{Na}_{2} \mathrm{SO}_{4}$ slows the diffusion of $\mathrm{AuCl}_{4}^{-}$ into the interior of the wool fibres, and hence the grey colour may be due to the formation of agglomerates of gold nanoparticles near the surfaces of the wool fibres. Alternatively, it may be due to the ionic strength of $\mathrm{Na}_{2} \mathrm{SO}_{4}$ destabilising the $\mathrm{AuCl}_{4}^{-}$ complex.

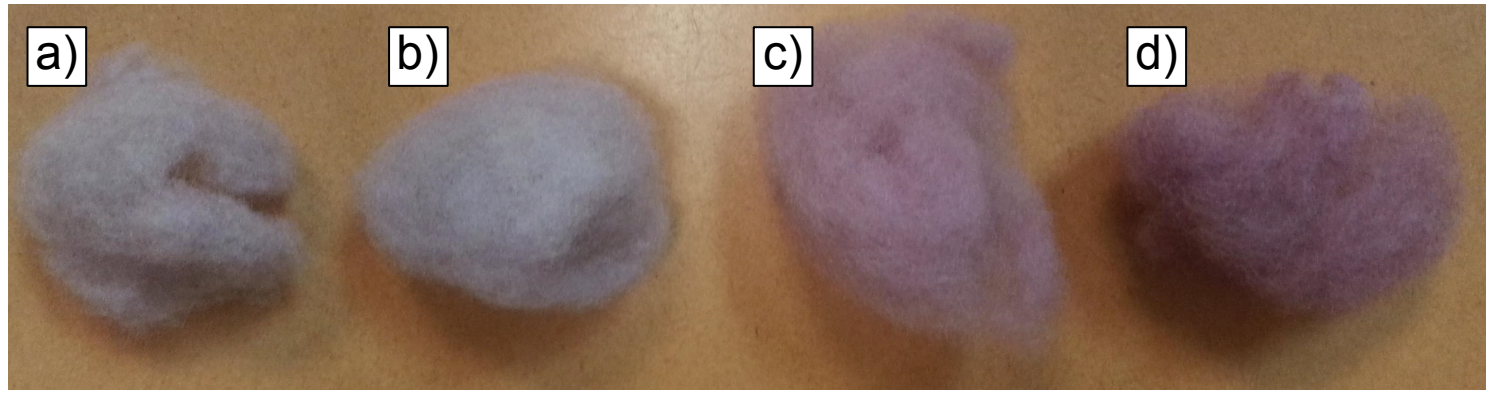

Figure 3.23 Cariaggi in-situ composites synthesised after pre-soaking in $10 \mathrm{~g} \mathrm{~L}^{-1}$ $\mathrm{Na}_{2} \mathrm{SO}_{4}$ (a), with $1 \mathrm{~g} \mathrm{~L}^{-1} \mathrm{Na}_{2} \mathrm{SO}_{4}$ (b) or $\mathrm{NaCl}$ (c) present in the uptake solution or with no levelling agents $(\mathrm{d})$.

When $\mathrm{NaCl}$ was added to the uptake solution, there is only a marginal effect on to rate of uptake and the colour of the resulting composite; only the $\mathrm{NaCl}$ composite exhibits the expected purple colouration.

CIE colour coordinates of the composites synthesised with and without salt-based levelling agents are shown in Table 3.8. The subtle change in colour with the addition of $\mathrm{NaCl}$ to the uptake solution is seen with a slightly brighter, less red composite and less blue colouration (compared to when no levelling agent is employed). The greying effect of $\mathrm{Na}_{2} \mathrm{SO}_{4}$ being present in the uptake solution is evidenced by the higher $\mathrm{L}^{*}$ brightness values and significantly lowered $\mathrm{a}^{*}$ and higher $\mathrm{b}^{*}$ values, due to less purple-coloured gold nanoparticles being formed.

SEM and EDS were used to analyse the composite synthesised when $\mathrm{Na}_{2} \mathrm{SO}_{4}$ was added to the uptake solution, as shown in Figure 3.24. The presence of $\mathrm{NaSO}_{4}$ 
Table 3.8 CIE colour coordinates of Cariaggi in-situ composites synthesised with or without levelling agents in the uptake solution.

\begin{tabular}{ccccc}
\hline & No levelling agent & $\mathrm{NaCl}$ (uptake) & $\mathrm{Na}_{2} \mathrm{SO}_{4}$ (uptake) & $\mathrm{Na}_{2} \mathrm{SO}_{4}$ (pre-soak) \\
\hline $\mathrm{L}^{*}$ & 59.32 & 64.58 & 70.56 & 70.55 \\
$\mathrm{a}^{*}$ & 15.63 & 14.18 & 4.20 & 5.74 \\
$\mathrm{~b}^{*}$ & 1.04 & 1.98 & 2.25 & 5.77 \\
\hline \hline
\end{tabular}

in the uptake solution has significantly influenced the size and distribution of gold nanoparticles on the surface of the composites. Figure 3.24 (a) and (b) show the presence of large particles of gold, in addition to a relatively lower coverage of gold across the fibre surface than is expected given the concentration of $\mathrm{AuCl}_{4}^{-}$in the uptake solution, which explains the greying of the composites colour.

The extent of penetration of $\mathrm{Na}^{+}$and $\mathrm{SO}_{4}{ }^{2-}$ into the wool is shown in the EDS elemental S and Na maps in Figure 3.24 (d) and (e)). It is difficult to ascertain whether the fibres retain a significant concentration of $\mathrm{SO}_{4}{ }^{2-}$ given the prevalence of $\mathrm{S}$ and $\mathrm{O}$ in chemical structure of wool, however level of $\mathrm{S}$ appears relatively high, and the density of $\mathrm{Na}$ throughout the fibre indicates the retention of some $\mathrm{Na}_{2} \mathrm{SO}_{4}$ in the fibre interior.

The elemental Au EDS map in Figure 3.24 (f) appears to show that $\mathrm{Au}$ is concentrated near the surface of the fibre to a greater extent than is typical for the standard purple in-situ method. Unfortunately, it is difficult to determine whether the presence of $\mathrm{Na}_{2} \mathrm{SO}_{4}$ in the fibre has blocked the access of $\mathrm{AuCl}_{4}^{-}$to the centre of the fibre, which was the aim of incorporating the levelling agents in the in-situ synthesis.

EDS analysis of the cross-section of a Cariaggi composite synthesised with $\mathrm{NaCl}$ in the uptake solution is shown in Figure 3.25. There is a significant level of $\mathrm{Na}$ and $\mathrm{Cl}$ throughout the fibre, indicating the retention of $\mathrm{NaCl}$ in the wool matrix. However, the $\mathrm{AuCl}_{4}^{-}$has penetrated throughout the composite fibre interior; the elemental $\mathrm{Au}$ map is similar to that of a composite synthesised without $\mathrm{NaCl}$. This is not surprising considering the minimal effect that $\mathrm{NaCl}$ had on the rate of uptake and colouration of the composite.

The use of salt-based levelling agents in the in-situ approach allowed the manipulation of the uptake of $\mathrm{AuCl}_{4}^{-}$to Cariaggi wool. The presence of $\mathrm{Na}_{2} \mathrm{SO}_{4}$ in the uptake had the most significant effect on the rate of uptake of $\mathrm{AuCl}_{4}^{-}$by wool and resulted in grey-coloured composites. This implied that $\mathrm{Na}_{2} \mathrm{SO}_{4}$ destabilised the 


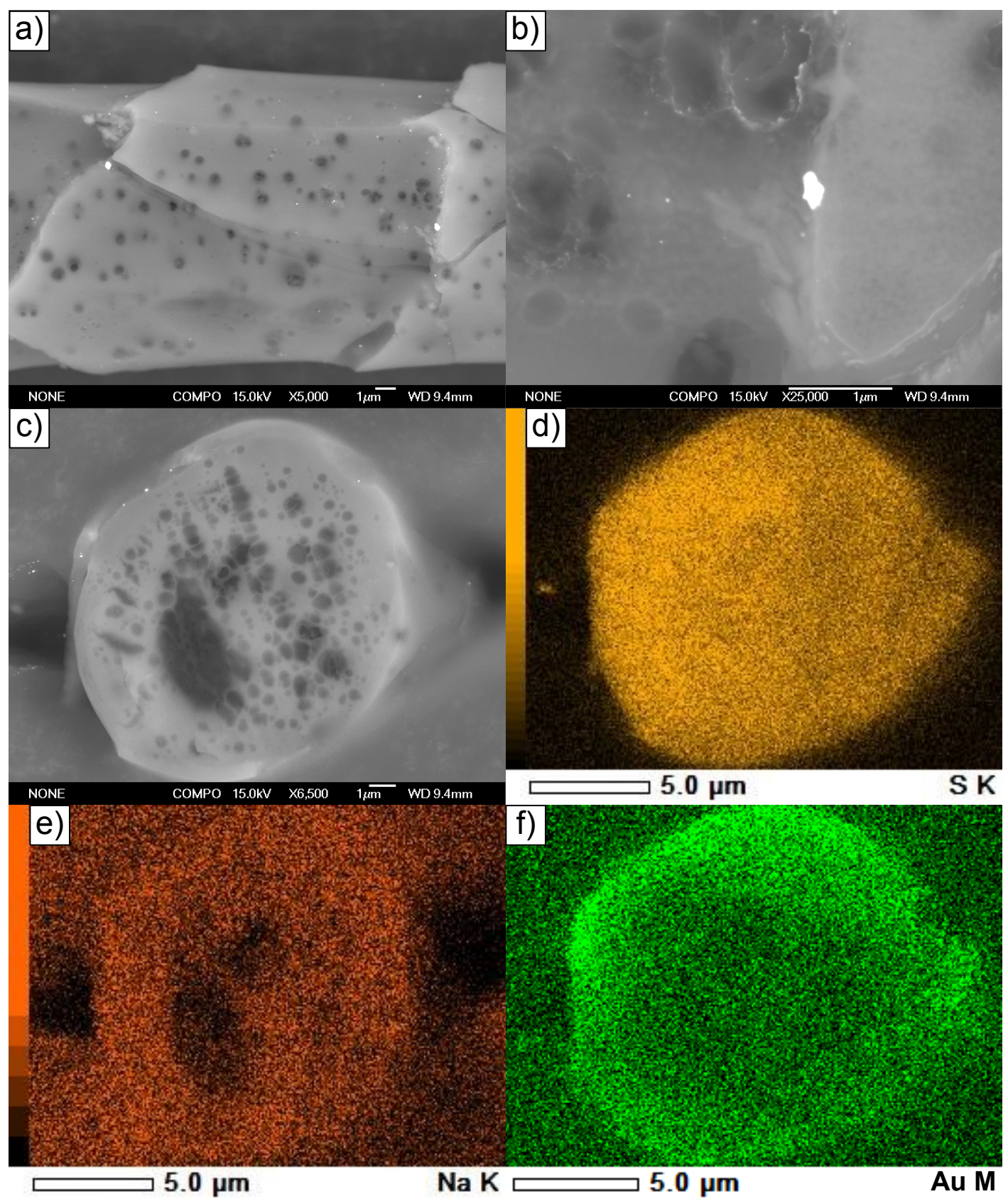

Figure 3.24 SEM and EDS analysis of a Cariaggi in-situ composite synthesised with $\mathrm{Na}_{2} \mathrm{SO}_{4}$ in the uptake solution; SEM images of the fibre surface in backscatter mode (a) and (b), SEM image of a fibre cross-section (c) and EDS maps of elemental $\mathrm{S}(\mathrm{d}), \mathrm{Na}(\mathrm{e})$ and $\mathrm{Au}(\mathrm{f})$ of the cross-section.

gold nanoparticles. However, purple hues may potentially be achieved by using lower concentrations of $\mathrm{AuCl}_{4}^{-}$in the process. In contrast, the presence of $\mathrm{NaCl}$ in the uptake solution had a minimal effect on the uptake profile and colour of the composite. As such, it is recommended that the use of $\mathrm{Na}_{2} \mathrm{SO}_{4}$ (and possibly other salt-based levelling agents) in the in-situ procedure be investigated in greater depth in future studies. 

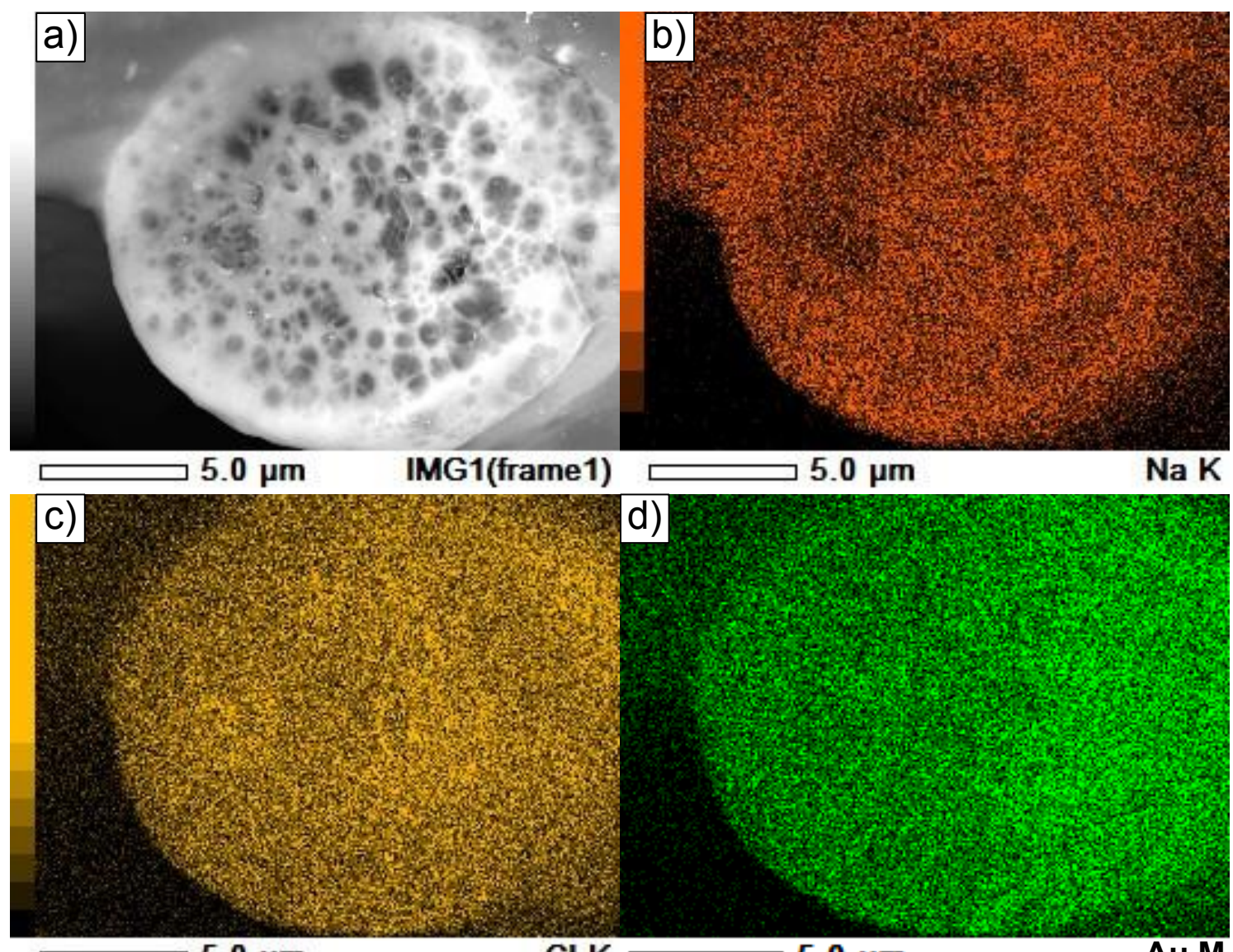

$5.0 \mu \mathrm{m}$

Cl K

$5.0 \mu \mathrm{m}$

Au M

Figure 3.25 SEM and EDS analysis of the cross section of a Cariaggi in-situ composite fibre synthesised with $\mathrm{NaCl}$ in the uptake solution; SEM image in backscatter mode (a), EDS elemental maps of $\mathrm{Na}(\mathrm{b}), \mathrm{Cl}$ (c) and $\mathrm{Au}(\mathrm{d})$.

\subsubsection{Volume of Gold Solution}

Dr Lucas and Professor Johnston found that reducing the volume of an uptake solution, while retaining the same amount of $\mathrm{AuCl}_{4}^{-}$(by using a higher effective concentration of $\mathrm{AuCl}_{4}^{-}$), can result in more intensely coloured composites. ${ }^{5}$ As such, the effect of the volume of $\mathrm{AuCl}_{4}^{-}$solution used in the in-situ synthesis was investigated further. The volume of an $\mathrm{AuCl}_{4}^{-}$solution used encompasses two reaction conditions; the concentration of $\mathrm{AuCl}_{4}^{-}$used and the wool:liquor ratio of the synthesis. In-situ composites of finished wool yarns were synthesised using different volumes of $\mathrm{AuCl}_{4}^{-}$. If a suitable composite colour was able to be produced with a low enough volume of $\mathrm{AuCl}_{4}^{-}$solution, the commercial potential of the in-situ procedure would be improved.

Figure 3.26 shows in-situ gold nanoparticle-wool fibre composites of finished wool yarns synthesised using starting solutions of different volumes of $160 \mathrm{mg} \mathrm{L}^{-1} \mathrm{Au}^{3+}$. The standard purple in-situ method conditions were employed with $1 \mathrm{~g} \mathrm{~L}^{-1}$ Albaflow FFA in the uptake solutions, to promote the uniform colouring of the wool yarns. 
As the volume of $\mathrm{AuCl}_{4}^{-}$in the starting solution was decreased, the purple or grey colouration of the composites decreased. Interestingly, the colours of the TU, S19 and S14 yarns are different shades of purple-grey colouration, with the $100 \mathrm{~mL}$ composites being the most purple. In contrast, the TS composites display a variety of pink-purple hues. At least $20 \mathrm{~mL}$ of starting solution was required for a slight colouration of the TU and TS yarns, and $40 \mathrm{~mL}$ of $\mathrm{AuCl}_{4}^{-}$solution was required for the spectrum yarns to exhibit a faint tinge of colour.

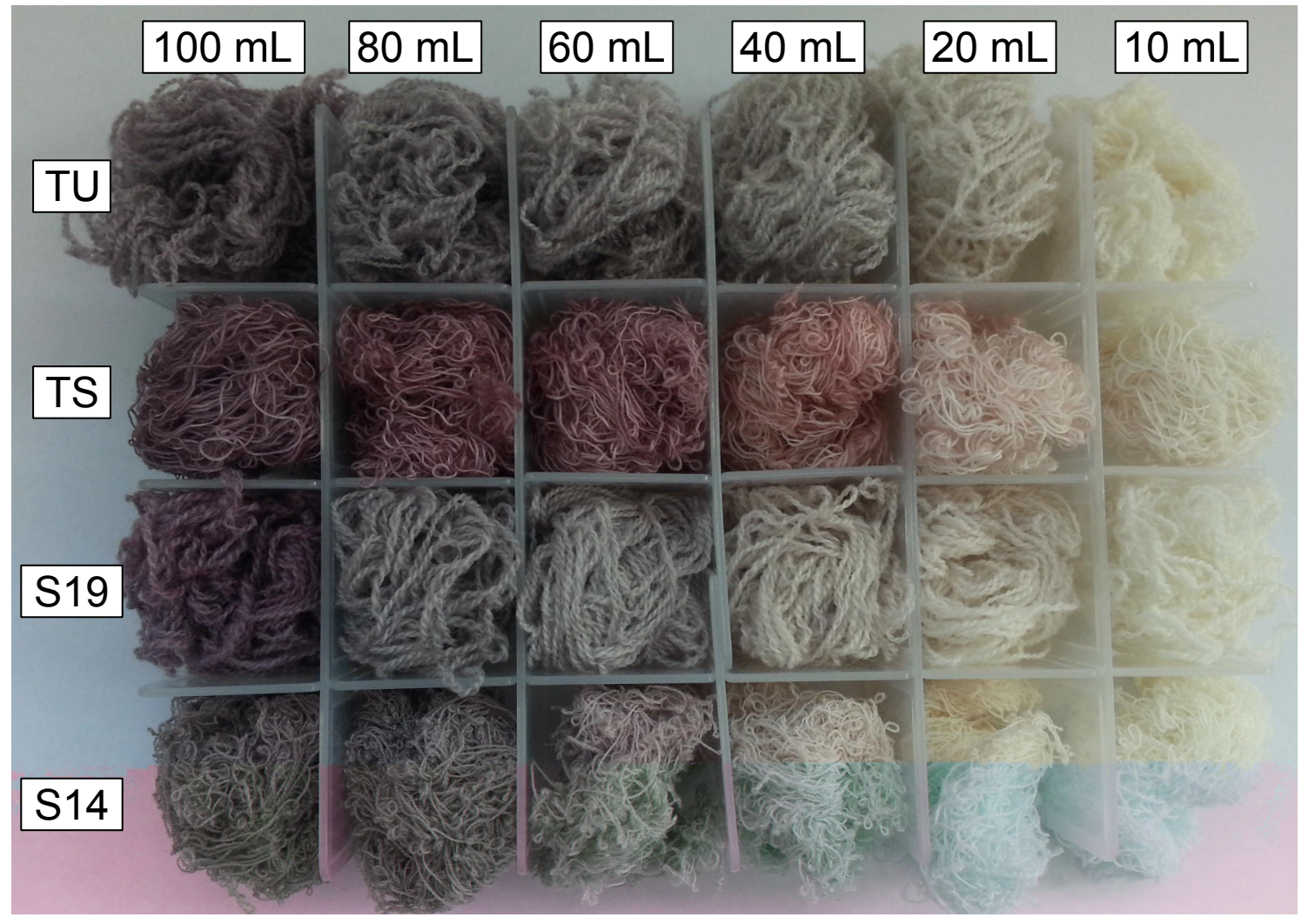

Figure 3.26 In-situ composites of finished wool yarns synthesised using 100, 80, 60, 40, 20 or $10 \mathrm{~mL}$ of $160 \mathrm{mg} \mathrm{L}^{-1} \mathrm{Au}^{3+}$ and $1 \mathrm{gL}^{-1}$ Albaflow FFA (all composite samples are $1 \mathrm{~g}$ and illustrate a wide variation in colour). 
Quantification of the colour changes of the composites are displayed in the graphical representations of the CIE colour coordinates that follow. Figure 3.27 shows the difference in $\mathrm{L}^{*}$ or brightness value of the composites produced with different volumes of $160 \mathrm{mg} \mathrm{L}^{-1} \mathrm{Au}^{3+}$. When the volume is increased, there is a general decrease in the $\mathrm{L}^{*}$ value across each of the finished yarns. The darkening represents the formation of gold nanoparticles as the volume of $\mathrm{AuCl}_{4}^{-}$in increased, and the darkening of the TS composites is the most pronounced compared to the other finished wool yarn composites.

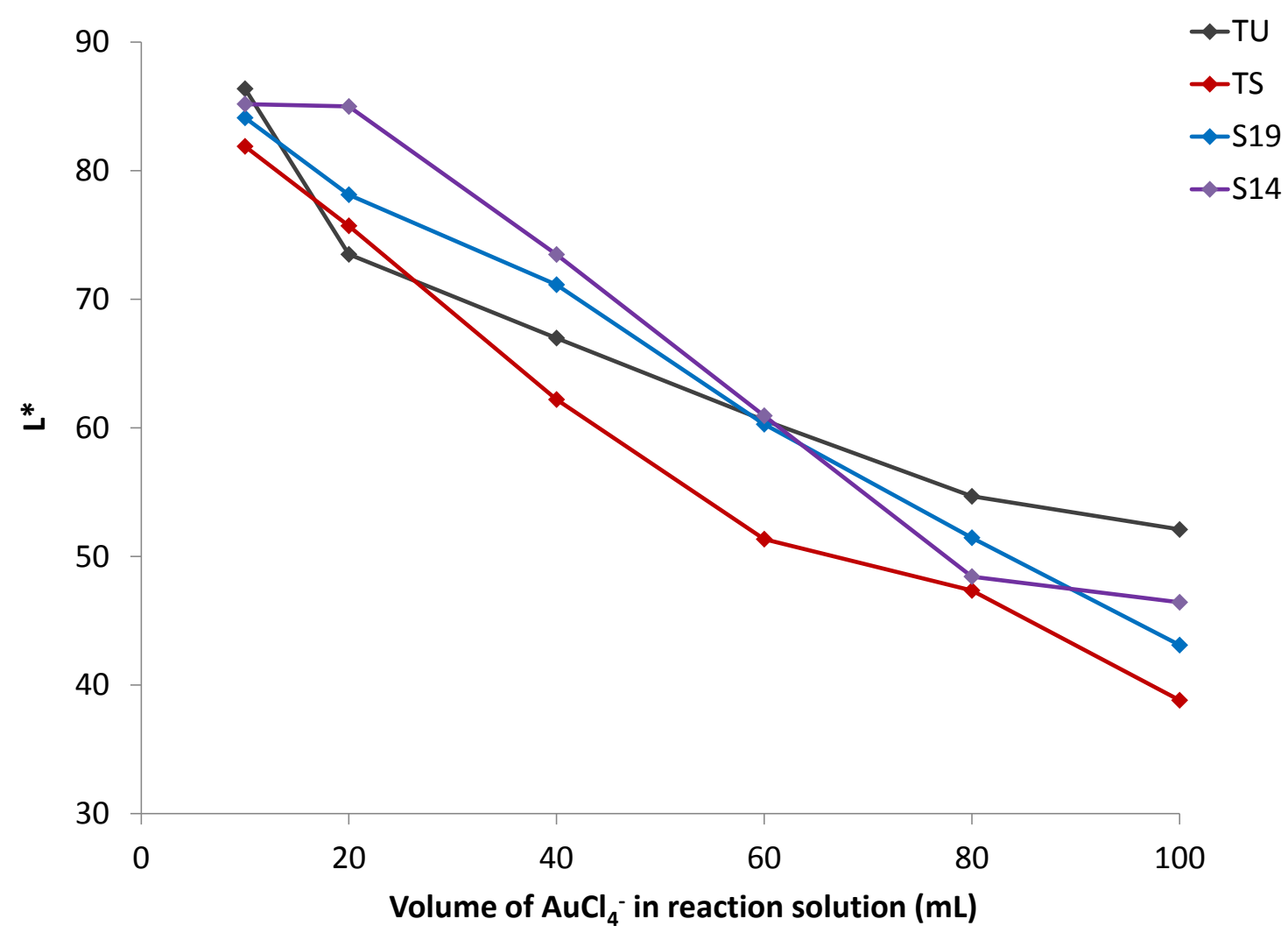

Figure 3.27 $\mathrm{L}^{*}$ colour coordinates of in-situ finished wool yarn composites synthesised with different volumes of $160 \mathrm{mg} \mathrm{L}^{-1} \mathrm{Au}^{3+}$ solutions.

The $\mathrm{a}^{*}$ or redness value typically increases as the volume of $\mathrm{AuCl}_{4}^{-}$increases, however there are significant differences between the types of finished wool yarns. The TU, S19 and S14 composites exhibit only a modest increase in redness as the $\mathrm{AuCl}_{4}^{-}$volume increases, representative of their purple-grey colouration, apart for the $100 \mathrm{~mL}$ S19 composite, which is completely purple. The TS composites exhibit a remarkable increase in redness from $10 \mathrm{~mL}$ to $40 \mathrm{~mL}$ as the pink colouration of the composites develops, followed by a moderate decrease in redness as the composites become darker purple.

There is a general decrease in $b^{*}$ for all of the finished wool yarn composites as the volume of $\mathrm{AuCl}_{4}^{-}$increases. This reflects the shielding of the yellowness of the 
composites as the purple or grey colouration of the composites develops; blue is a component of the purple colour of gold nanoparticles in a wool matrix, but is also a component of the grey colour of larger gold particles and agglomerates.

The CIE colour coordinates of composites of each of the different merino yarns show that the volume of $\mathrm{AuCl}_{4}^{-}$used in the in-situ procedure effects the intensity and hue of the composites. A modest reduction in volume of $\mathrm{AuCl}_{4}^{-}$solution from $100-80 \mathrm{~mL}$ caused a colour change in the resulting composites, and any further reduction in volume resulted in either very light or unevenly coloured composites. Thus, reducing the volume of the $\mathrm{AuCl}_{4}^{-}$solution may result in only a modest reduction of the gold content of in-situ composites of a given colour.

Section 3.2 outlined the effects of wool type, $\mathrm{pH}$, temperature, auxiliary agents and volume of $\mathrm{AuCl}_{4}^{-}$on the chemistry of the in-situ procedure. The presented studies have shown ways to preferentially direct the formation of gold nanoparticles at or near the surface of the wool fibres. This knowledge led to the chemistry carried out in the following section, where the reaction conditions were manipulated and combined with the application of external energy sources and reducing agents, to minimise the gold content of gold nanoparticle-wool fibre composites, thus increasing the economic viability of the in-situ procedure. 


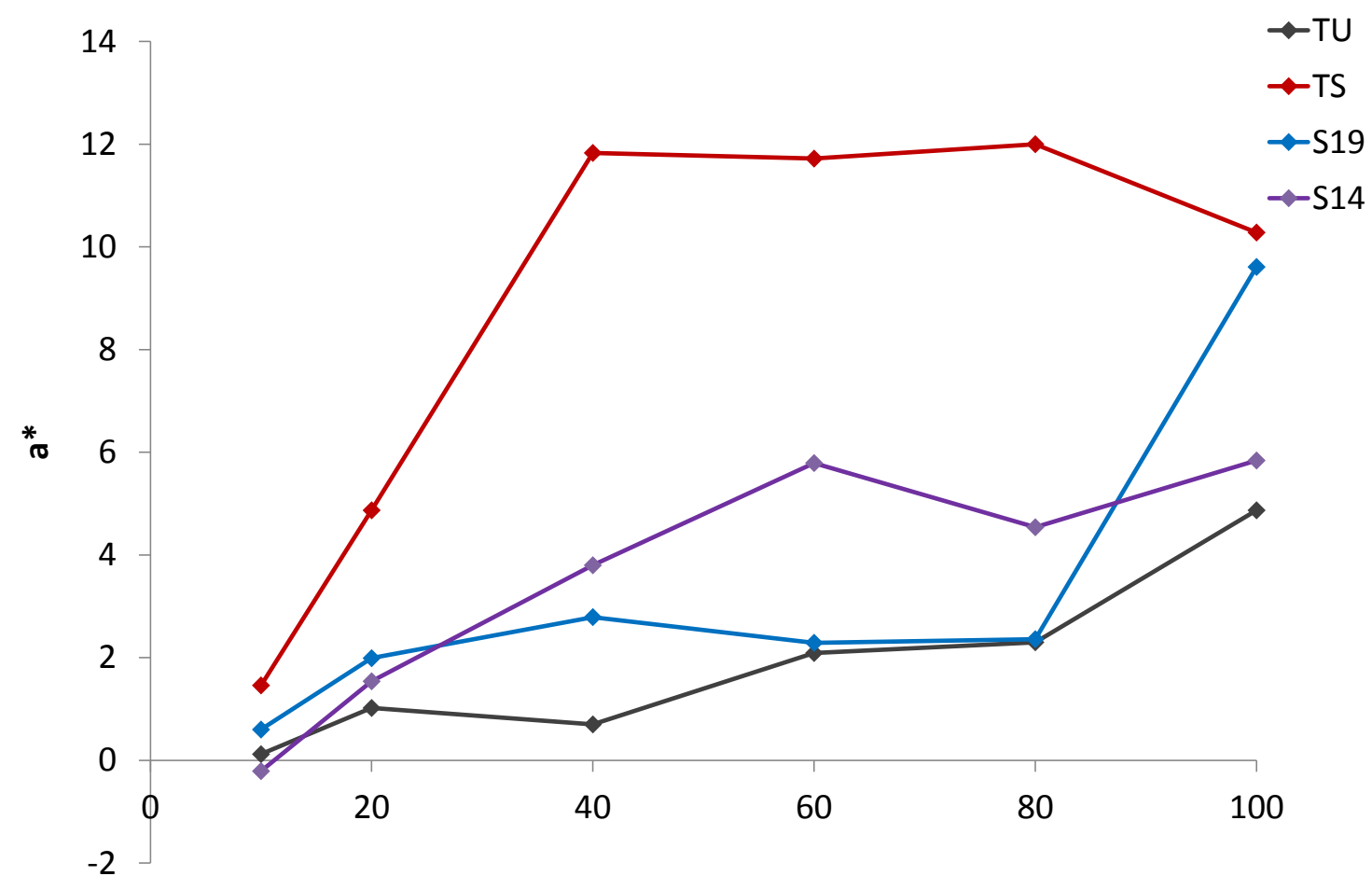

\section{Volume of $\mathrm{AuCl}_{4}^{-}$in reaction solution (mL)}

Figure 3.28 a* colour coordinates of in-situ finished wool yarn composites synthesised with different volumes of $160 \mathrm{mg} \mathrm{L}^{-1} \mathrm{Au}^{3+}$ solutions.

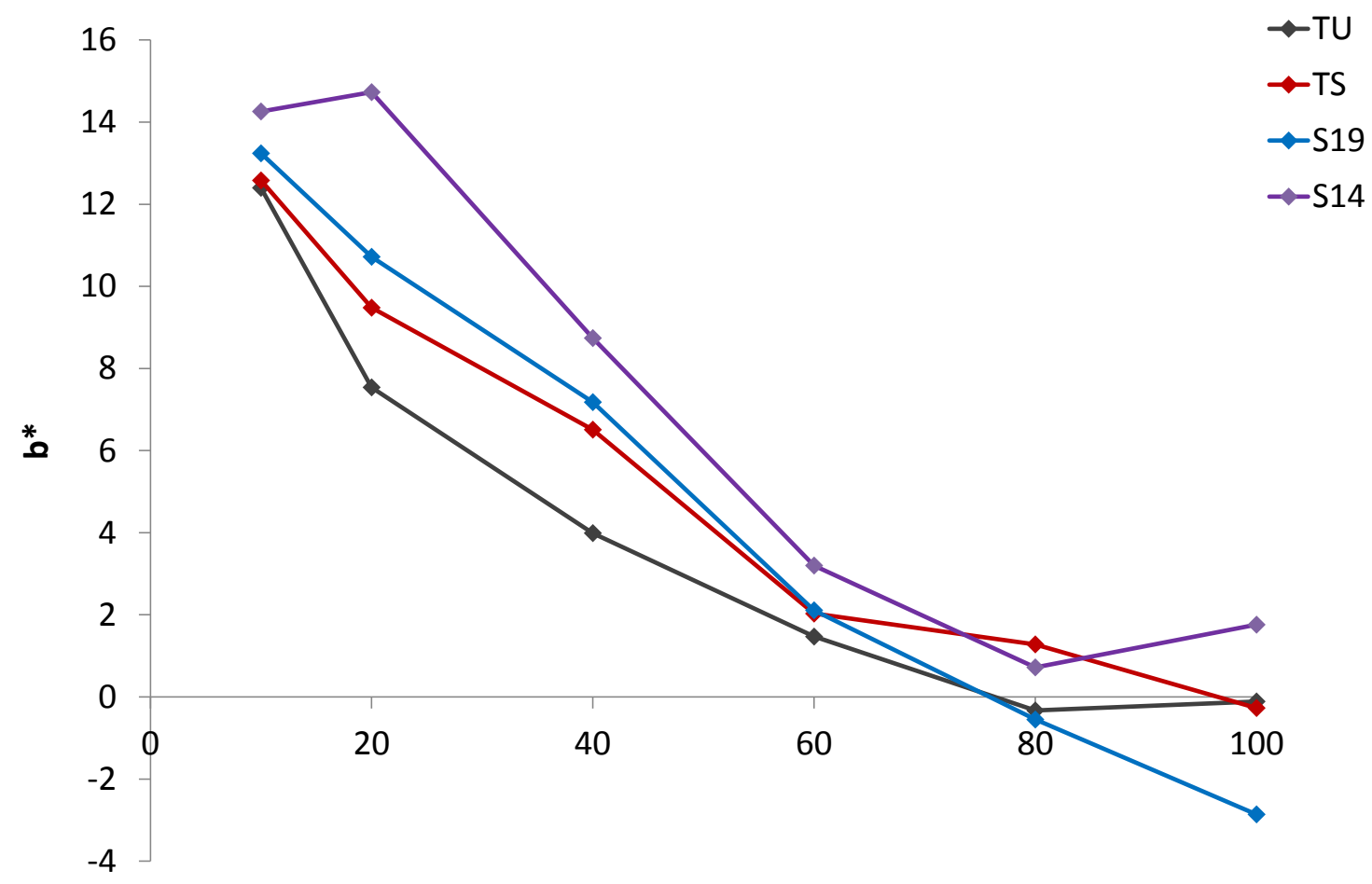

Volume of $\mathrm{AuCl}_{4}{ }^{-}$in reaction solution $(\mathrm{mL})$

Figure 3.29 $\mathrm{b}^{*}$ colour coordinates of in-situ finished wool yarn composites synthesised with different volumes of $160 \mathrm{mg} \mathrm{L}^{-1} \mathrm{Au}^{3+}$ solutions. 


\subsection{Manipulating the Rate of Gold Nanoparticle Formation}

This section investigates the use of external stimuli to manipulate the rate of formation of gold nanoparticles and their distribution throughout the wool fibre matrix. The knowledge gained from the Section 3.2 showed that such manipulation is possible through varying the temperature of the in-situ synthesis. As such, composites were exposed to heat and radiative energy sources, to increase the rate of gold nanoparticle formation in the wool. Incorporation of external reducing agents in the in-situ procedure was also explored, due to their use in the solution-phase synthesis of gold nanoparticles.

Additionally, Section 3.2.2 showed that the colour of gold nanoparticle-wool fibre composites is more dependent on the gold nanoparticles on the surface of the fibres, compared to those in the fibre interior. This was illustrated when in-situ composites synthesised at pH 2 exhibited gold nanoparticles throughout the wool fibre matrix, and yet only a slight purple tinge of colour. As such, gold nanoparticle formation was directed to the surface of the wool fibres.

The external stimuli were applied after removal of samples from different stages of the uptake process. This often involved halting the uptake and rapidly applying the stimuli, before the $\mathrm{AuCl}_{4}^{-}$could penetrate into the centre of the fibre.

\subsubsection{External Energy Sources}

The influence of external energy on the in-situ process may be investigated by increasing the temperature of the uptake, or alternatively by removing wool samples from an uptake solution (at a given temperature) and rapidly exposing them to an external energy source. The energy sources investigated were heat and microwave radiation.

\section{Application of Heat During the Uptake Process.}

The following experiment involved the uptake of $\mathrm{AuCl}_{4}^{-}$to finished wool yarns at $80^{\circ} \mathrm{C}$. At this temperature the uptake of $\mathrm{AuCl}_{4}^{-}$and the nucleation of gold nanoparticles occur simultaneously. Figure 3.30 shows composites of finished wool yarns 
synthesised at $80^{\circ} \mathrm{C}$ for $24 \mathrm{~h}$ with different volumes of $160 \mathrm{mg} \mathrm{L}^{-1} \mathrm{Au}^{3+}$. The conditions of the standard purple in-situ method were employed (including $1 \mathrm{~g} \mathrm{~L}^{-1} \mathrm{Al}$ baflow FFA).

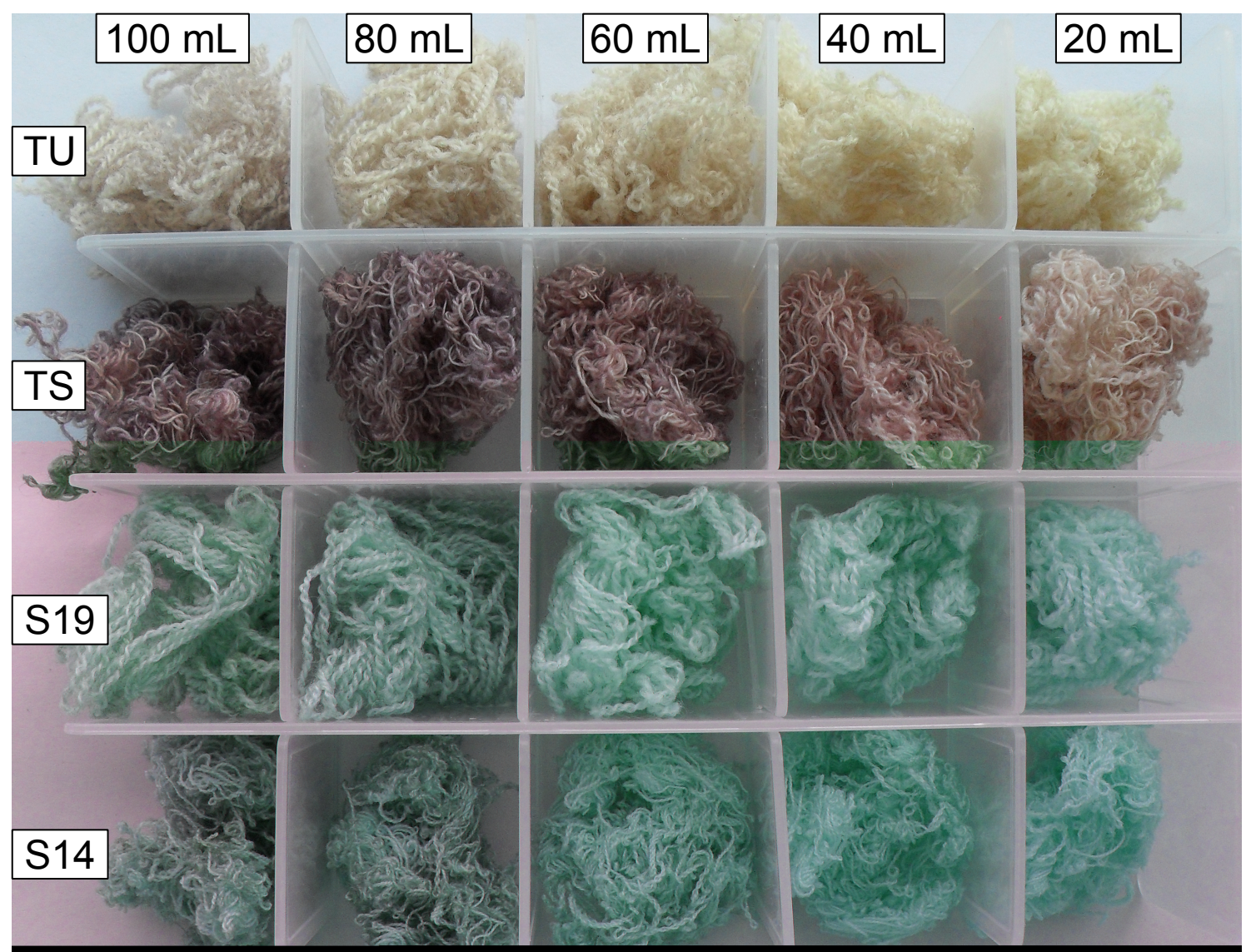

Figure 3.30 In-situ composites of finished yarns synthesised at $80^{\circ} \mathrm{C}$, with starting solutions of 100, 80, 60, 40 and $20 \mathrm{~mL} 160 \mathrm{mg} \mathrm{L}^{-1} \mathrm{Au}^{3+}$.

Unfortunately, only the TS composites exhibited the purple colour of gold nanoparticles in a wool matrix with any volume of $\mathrm{AuCl}_{4}^{-}$solution. The TU, S19 and S14 composites were unevenly grey coloured when 100 or $80 \mathrm{~mL}$ of $160 \mathrm{mg} \mathrm{L}^{-1} \mathrm{Au}^{3+}$ was used, and when a lower volume of $\mathrm{AuCl}_{4}^{-}$solution was used in the synthesis there was was little to no indication of gold nanoparticles in the composites. This implies that $\mathrm{AuCl}_{4}^{-}$is absorbed rapidly throughout the TU, S19 and $\mathrm{S} 14$ fibres at $80^{\circ} \mathrm{C}$, at a faster rate than the reduction of $\mathrm{AuCl}_{4}^{-}$to $\mathrm{Au}^{0}$ by the amino acids on the surface of the yarns.

The UV-visible reflectance spectra of the finished wool yarn composites synthesised are shown in Figure 3.31. Only the TS composite spectrum shows evidence of the peak between 500-600 nm relating to the surface plasmon resonance band of gold nanoparticles (which is very broad). The TU, S19 and S14 composites display a broad absorbance of relatively low intensity, as expected given the yellow-grey 
colouration of the composites.

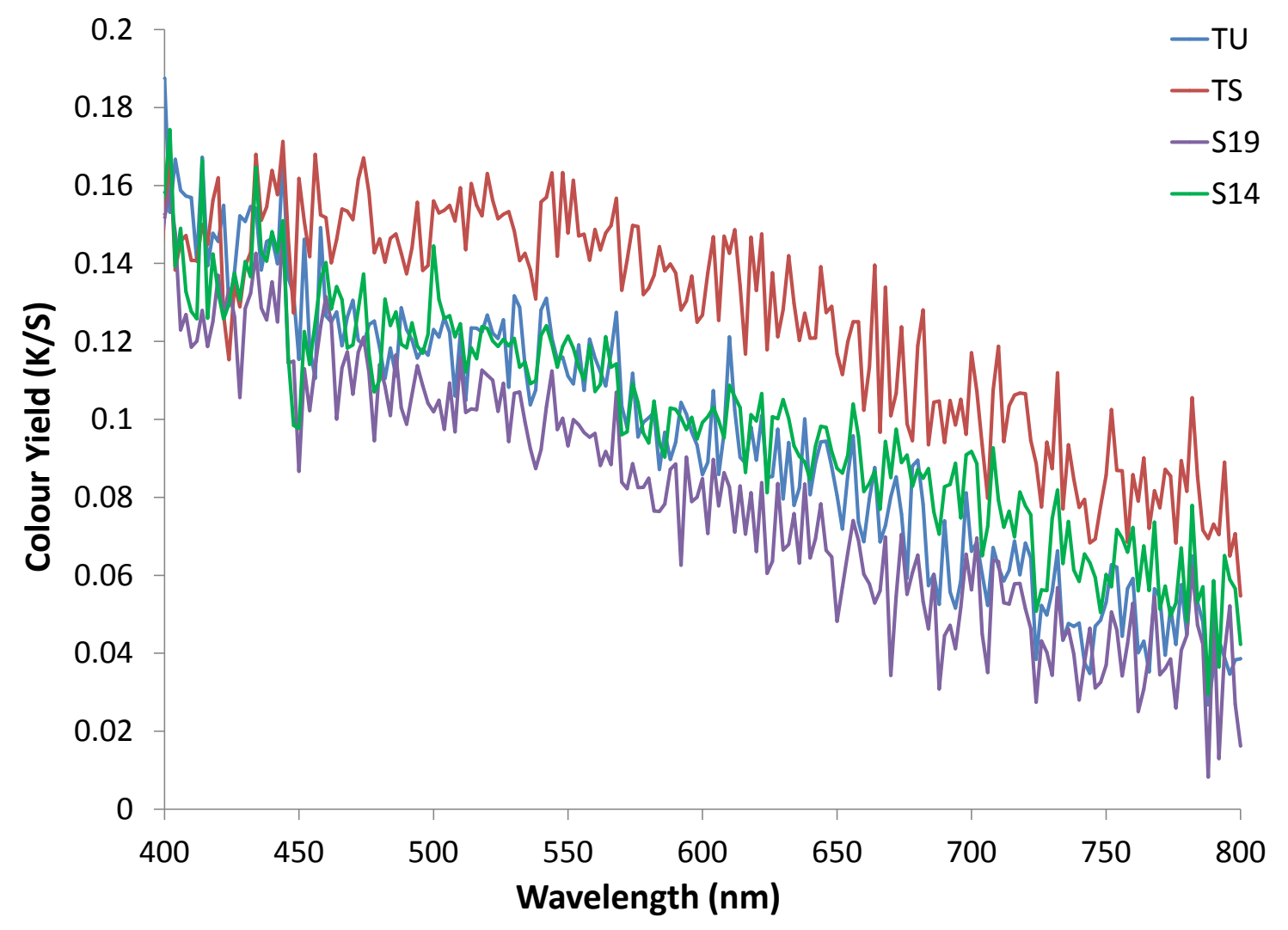

Figure 3.31 Converted UV-visible reflectance spectra of in-situ composites of finished wool yarns synthesised at $80^{\circ} \mathrm{C}$ with $100 \mathrm{~mL}$ of $160 \mathrm{mg} \mathrm{L}^{-1} \mathrm{Au}^{3+}$.

SEM and EDS analysis of the finished wool yarn composites synthesised at $80^{\circ} \mathrm{C}$ with $100 \mathrm{~mL}$ of $160 \mathrm{mgL}^{-1} \mathrm{Au}^{3+}$ are shown in Figure 3.32. The SEM micrograph of the TU yarn composite at relatively low magnification display gold density preferentially present near the edges of cuticle cells. The higher magnification image shows that the gold is present as agglomerates of gold nanoparticles that are in close enough proximity to one another for their surface plasmon resonance bands to couple, which accounts for the grey colouration of the $100 \mathrm{~mL}$ TU yarn composite.

The TS composite also has agglomerates of gold nanoparticles and larger particles of gold near the cuticle edges, in addition to a high density of gold nanoparticles covering the cuticle scales. This accounts for the dark purple colour of the composite. The colour of the TS composite is uneven, like that seen in 3.2.5, and the imaged fibre was one with a relatively high gold density. The gold coverage on the surface of the S19 composite is typically sparse, with the occasional area of density larger gold particles and agglomerates of nanoparticles, accounting for the composite's grey colouration. The EDS elemental Au maps of the TS and S19 composites confirm the gold content of the nanoparticles. 


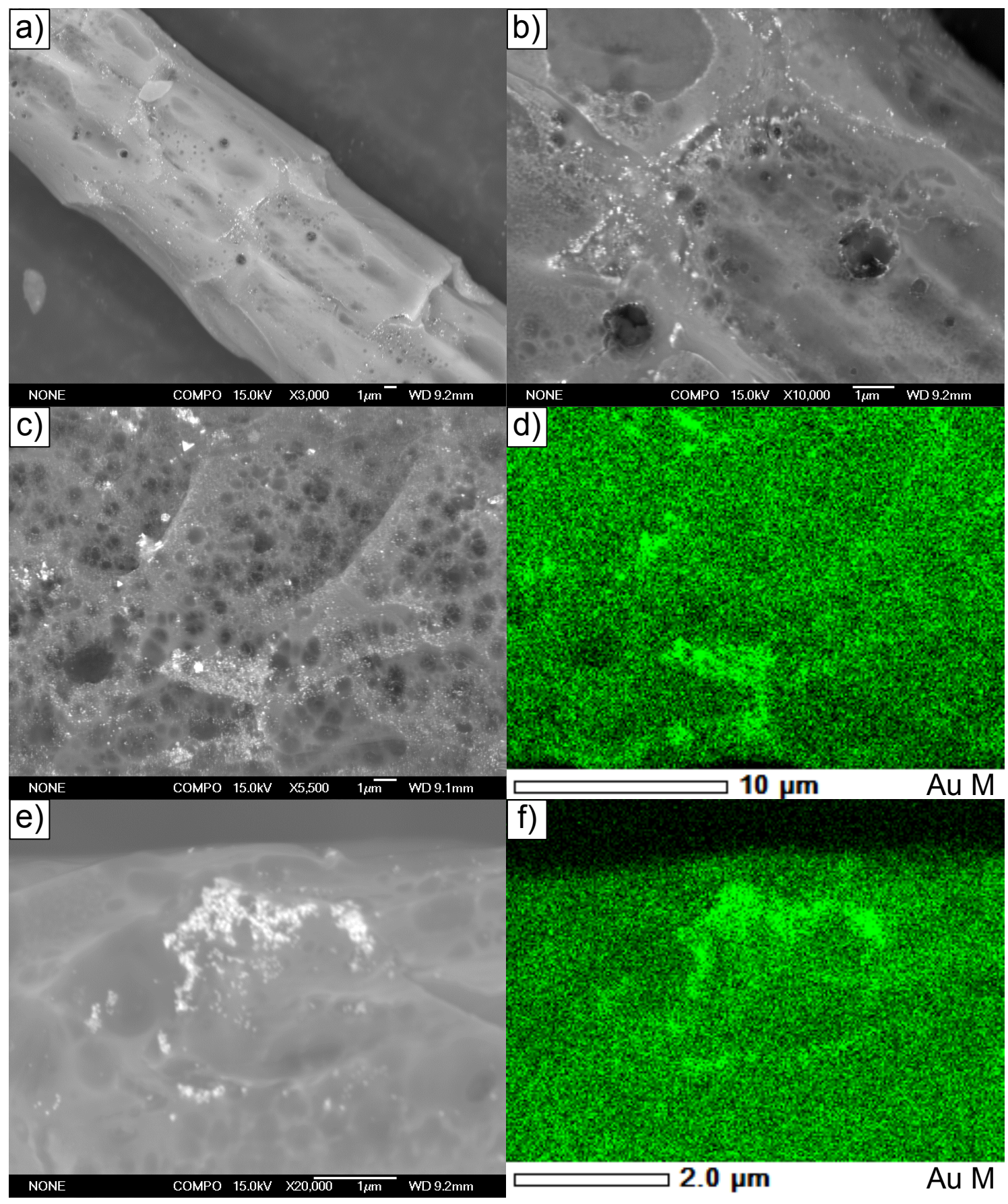

Figure 3.32 SEM micrographs in BS mode and EDS elemental Au maps of the surface of in-situ composites of TU (a)-(b), TS (c)-(d) and S19 (e)-(f) wool yarns synthesised at $80^{\circ} \mathrm{C}$ with $100 \mathrm{~mL}$ of $160 \mathrm{mg} \mathrm{L}^{-1} \mathrm{Au}^{3+}$.

The presented study has shown that increasing the temperature of the uptake of $\mathrm{AuCl}_{4}^{-}$to finished wool yarns causes a non-uniform colouring of the composites. This result is in contrast to that seen with loose wool uptake at a higher temperature in Section 3.2.3, where Ashford2 composites at $75^{\circ} \mathrm{C}$ and boiling temperatures were purple-coloured. There is also a lower colour yield attained per volume of $\mathrm{AuCl}_{4}^{-}$ used in the synthesis compared to the uptake at RT (as seen in 3.2.5); $80 \mathrm{~mL}$ of $160 \mathrm{mgL}^{-1} \mathrm{Au}^{3+}$ solution is required to observe the colour of gold nanoparticles present in the yarn composites. This implies that at a higher uptake temperature, 
a relatively higher concentration of $\mathrm{AuCl}_{4}^{-}$is present throughout the interior of the fibres, as compared to the surface particles.

It was considered that the grey colours of the TU, S19 and S14 yarns may be due to the lower effective surface area of the yarns relative to that of wool in top form resulting in the agglomeration of the gold nanoparticles. The rapid uptake at $80^{\circ} \mathrm{C}$ has lead to the accumulation of $\mathrm{AuCl}_{4}^{-}$at the junctions between cuticle scales, which is the pathway into the fibre interior, and some of the $\mathrm{AuCl}_{4}^{-}$to be reduced to $\mathrm{Au}^{0}$ entities in close proximity to one another. This would account for the relatively high density of agglomerates at the cuticle edges of the composite, as while $\mathrm{AuCl}_{4}^{-}$can diffuse into the fibres along the cuticles, gold nanoparticles are too large to do this.

Due to the lack of success of improving the commercial potential of the in-situ approach by applying heat during the uptake process, composites were next exposed to heat at the completion of the uptake.

\section{Application of External Energy After the Uptake}

The effects of the following energy sources on the rate of gold nanoparticle formation and the distribution of gold nanoparticles in wool fibres were investigated:

\begin{tabular}{cc}
\hline Heat source & Time $(\min )$ \\
\hline Microwave & 3 \\
Oven $\left(120^{\circ} \mathrm{C}\right)$ & 120 \\
\hline \hline
\end{tabular}

The following experiment involved the uptake of $\mathrm{AuCl}_{4}^{-}$to Ashford2 wool, followed by patting the samples dry, and then microwaving them for $3 \mathrm{~min}$ or heating in an oven at $120^{\circ} \mathrm{C}$ for $120 \mathrm{~min}$. The conditions of the standard purple in-situ method were used in the uptake. Figure 3.33 shows that when microwaved composites are pink and the composites heated in the oven are purple-coloured. The colouration of both composites is relatively uneven for samples of $1 \mathrm{~g}$ size. In an effort to increase the colour uniformity of the composites, the experiment was repeated with $1 \mathrm{~g} \mathrm{~L}^{-1}$ Albaflow FFA present in the uptake solution.

However, the colouration of the composites is even less uniform when the wetting agent was employed. A possible reason for the uneven colour of the composites is that the $\mathrm{AuCl}_{4}^{-}$absorbed into the wool migrates with the moisture that evaporates from the wool matrix when the composites are dried. If this were the case, the presence of the wetting agent would do little to aid in colour uniformity. The further 


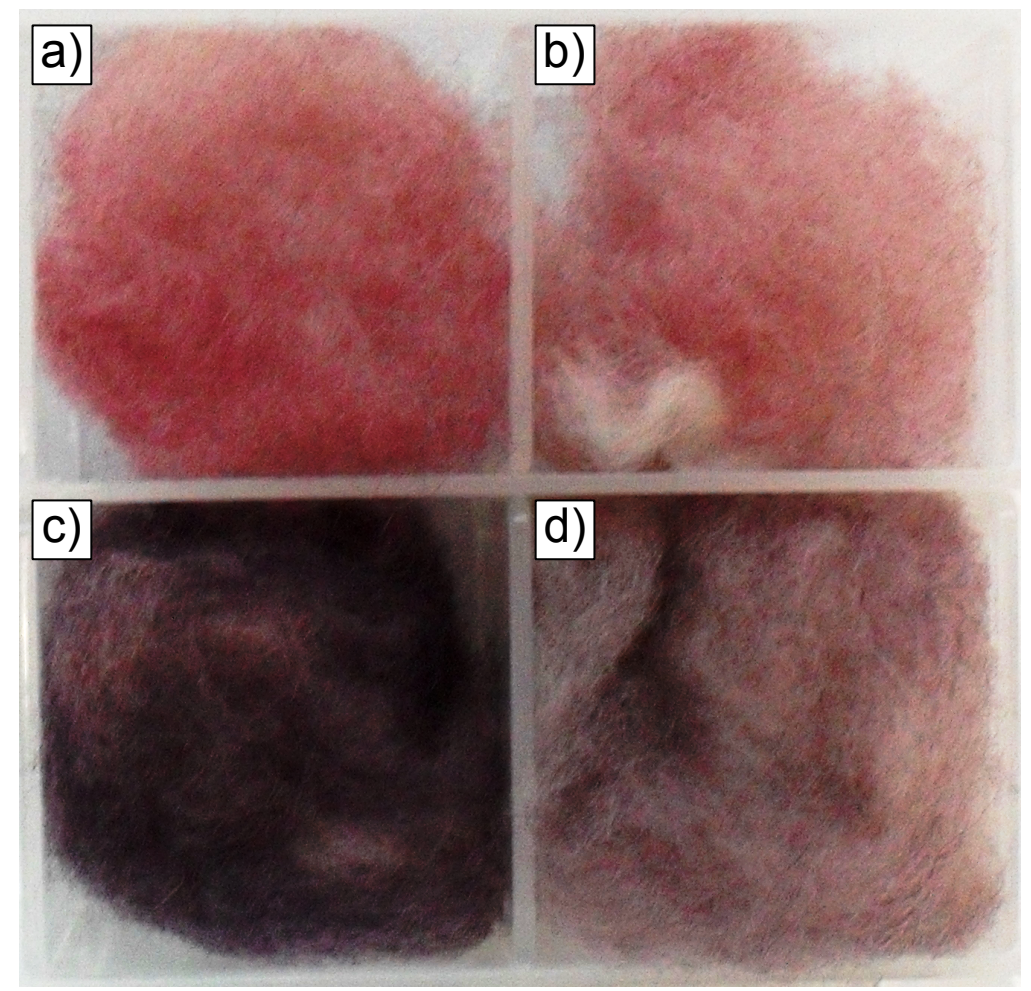

Figure 3.33 Ashford2 in-situ composites synthesised after uptake at RT and exposure to microwave radiation without and with $1 \mathrm{~g} \mathrm{~L}^{-1}$ Albaflow FFA in the uptake solution (a) and (b) respectively and after exposure to dry heat in the oven without and with $1 \mathrm{gL}^{-1}$ Albaflow FFA (c) and (d).

reduction in colour uniformity when Albaflow FFA is added to the uptake solution may be due to an unfavourable interaction between the composites and the wetting agent upon the exposure of such high energy.

Quantification of the colours of the composites is shown in Table 3.9. The pink colour of the microwave composites is evidenced by the relatively high $\mathrm{a}^{*}$ and $\mathrm{b}^{*}$ values compared to the oven composites. Introduction of the wetting agent into the uptake solution has caused less of the pink and purple colour of the microwave and oven composites respectively, evidenced by the lower $\mathrm{L}^{*}$ values of both, the lower $a^{*}$ of the microwave composite and the higher $b^{*}$ of the oven composite.

Table 3.9 CIE colour coordinates of Ashford2 in-situ composites synthesised with exposure to microwave radiation or dry heat in the oven with different amounts of Albaflow FFA in the uptake solution.

\begin{tabular}{ccccc}
\hline & \multicolumn{2}{c}{ Microwave } & \multicolumn{2}{c}{ Oven $\left(120^{\circ} \mathrm{C}\right)$} \\
\hline WA $\left(\mathrm{g} \mathrm{L}^{-1}\right)$ & 0 & 1 & 0 & 0 \\
\hline $\mathrm{L}^{*}$ & 59.94 & 65.44 & 43.65 & 60.00 \\
$\mathrm{a}^{*}$ & 15.53 & 10.58 & 8.89 & 8.34 \\
$\mathrm{~b}^{*}$ & 3.59 & 4.38 & 0.20 & 6.62 \\
\hline \hline
\end{tabular}

The exposure of the wool fibres to the external energy sources increased the rate of 
gold nanoparticle formation, however the resulting colours were undesirable. The heating of finished yarn samples during the uptake and the exposure of Ashford2 composites to microwave and dry heat resulted in the unevenly coloured composites. As such, the use of external reducing agents to increase the rate of gold nanoparticle formation in wool was then investigated.

\subsubsection{External Reducing and Stabilising Agents}

External reducing agents were employed to increase the rate of gold nanoparticle formation during the in-situ synthesis of gold nanoparticle-wool fibre composites. The reducing agents employed were those commonly used in the solution-phase synthesis of gold nanoparticles, and are shown below together with the temperatures of their syntheses:

\begin{tabular}{cc}
\hline Reducing agent & Temperature \\
\hline $\mathrm{NaBH}_{4}$ & $\mathrm{RT}$ \\
$\mathrm{TSC}$ & $100^{\circ} \mathrm{C}$ \\
$\mathrm{TA}$ & $\mathrm{RT}$ \\
\hline \hline
\end{tabular}

$\mathrm{NaBH}_{4}$ has the ability to reduce gold, but not stabilise the formed $\mathrm{Au}^{0}$ entities. In contrast, TSC and TA are dual-reducing and stabilising agents, and have the ability to influence the size and shape of the formed nanoparticles. Both will require chemical interaction between the wool protein and the gold to chemically bind the gold nanoparticles to the wool matrix, as typically occurs in the in-situ approach.

In the following experiments, the uptake of $\mathrm{AuCl}_{4}^{-}$to Ashford2 wool was carried out at RT. Samples of wool were removed from the uptake solutions after different times, and then immediately soaked in aqueous solutions of $\mathrm{NaBH}_{4}$, TSC or TA (to increase the rate of gold nanoparticle formation). The uptake was carried out for 2-60 min, and the quicker uptake times were used to attempt to prevent the penetration of $\mathrm{AuCl}_{4}^{-}$into the wool, thus promoting the nucleation of gold nanoparticles at or near the surface of the fibres.

\section{Sodium Borohydride}

Ashford2 wool was soaked in aqueous solutions of $\mathrm{NaBH}_{4}\left(5 \times 10^{-3} \mathrm{~mol} \mathrm{~L}^{-1}\right)$ after the uptake of $\mathrm{AuCl}_{4}^{-}$(at too high a concentration of $\mathrm{NaBH}_{4}\left(\geq 0.18 \mathrm{~mol} \mathrm{~L}^{-1}\right.$ ), the wool fibres begin to dissolve). The application of $\mathrm{NaBH}_{4}$ to samples of Ashford2 wool removed from an uptake solution of $160 \mathrm{mg} \mathrm{L}^{-1} \mathrm{Au}^{3+}$ at $\mathrm{RT}$ after different reaction times yielded the composites shown in Figure 3.34. Interestingly, the composites 
all exhibit a yellow-brown colouration, which is darker when the uptake time, and relative gold content of the fibres, increases.

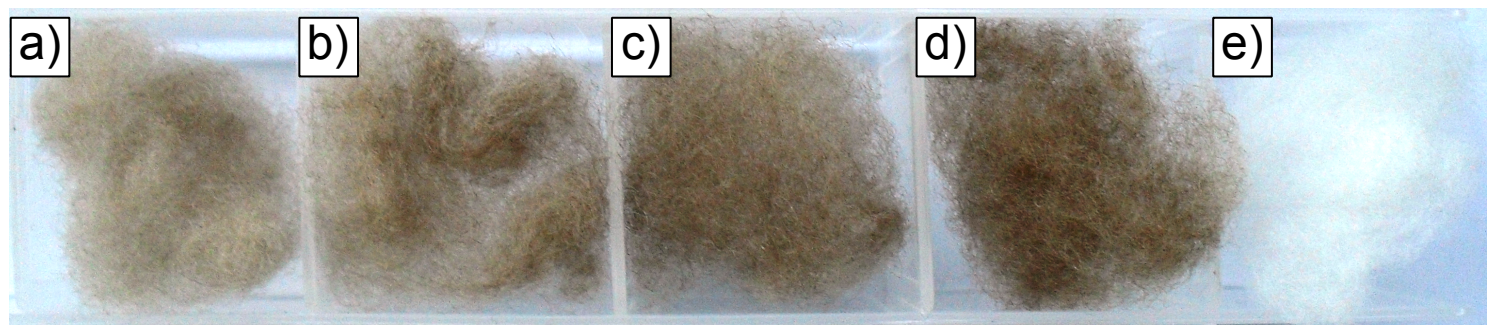

Figure 3.34 Image of Ashford2 composites synthesised after soaking in $\mathrm{NaBH}_{4}$ solutions after $2 \mathrm{~min}$ (a), $5 \mathrm{~min}$ (b), $10 \mathrm{~min}$ (c) and $60 \mathrm{~min}$ (d) of RT uptake of $160 \mathrm{mg} \mathrm{L}^{-1} \mathrm{Au}^{3+}$, and $\mathrm{NaBH}_{4}$ only (e).

Table 3.10 CIE colour coordinates of Ashford2 composites synthesised with the application of $\mathrm{NaBH}_{4}$ after different times of RT uptake of $160 \mathrm{mg} \mathrm{L}^{-1} \mathrm{Au}^{3+}$.

\begin{tabular}{cccccc}
\hline Uptake time (min) & 2 & 5 & 10 & 60 & $\mathrm{NaBH}_{4}$ only \\
\hline $\mathrm{L}^{*}$ & 69.33 & 66.52 & 57.96 & 53.09 & 91.76 \\
$\mathrm{a}^{*}$ & 4.22 & 4.85 & 6.43 & 7.14 & -0.80 \\
$\mathrm{~b}^{*}$ & 12.84 & 14.05 & 15.62 & 16.34 & 4.58 \\
\hline \hline
\end{tabular}

CIE colour coordinates of the $\mathrm{NaBH}_{4}$ composites are represented in Table 3.10. The composites display very high $b^{*}$ values, evidencing their yellow colouration. As the uptake time increases, the composites become relatively darker and more yellowed, however an increase in a* value is also observed, and this increase in redness indicates the formation of some nanoparticles of gold.

Initially, it was thought that the yellow-brown colouration could be due to amino acids in the wool protein being reduced by $\mathrm{NaBH}_{4}$. However, unreacted Ashford2 wool immersed in a solution of $\mathrm{NaBH}_{4}$, no such yellowing occurred (as was seen in Figure 3.34). Additionally, the extent of the yellow/brown colouration is enhanced with an increase in uptake time, further indicating that the colour is due to the presence of gold.

SEM and EDS analysis was carried out on a composite fibre synthesised with the application of $\mathrm{NaBH}_{4}$ after $10 \mathrm{~min}$ RT uptake of $160 \mathrm{mg} \mathrm{L}^{-1} \mathrm{Au}^{3+}$ is presented in Figure 3.35. It is difficult to detect the presence of gold on the relatively low magnification SEM image in Figure 3.35 (a). The higher magnification images in Figure 3.35 (b) and (c) however, show the presence of what appears to be very small nanoparticles of gold on the surface of the composite fibres.

Additionally, it is difficult to detect the presence of gold nanoparticles in the BS image of the cross-section (Figure 3.35 (d)). The EDS elemental Au map of this 

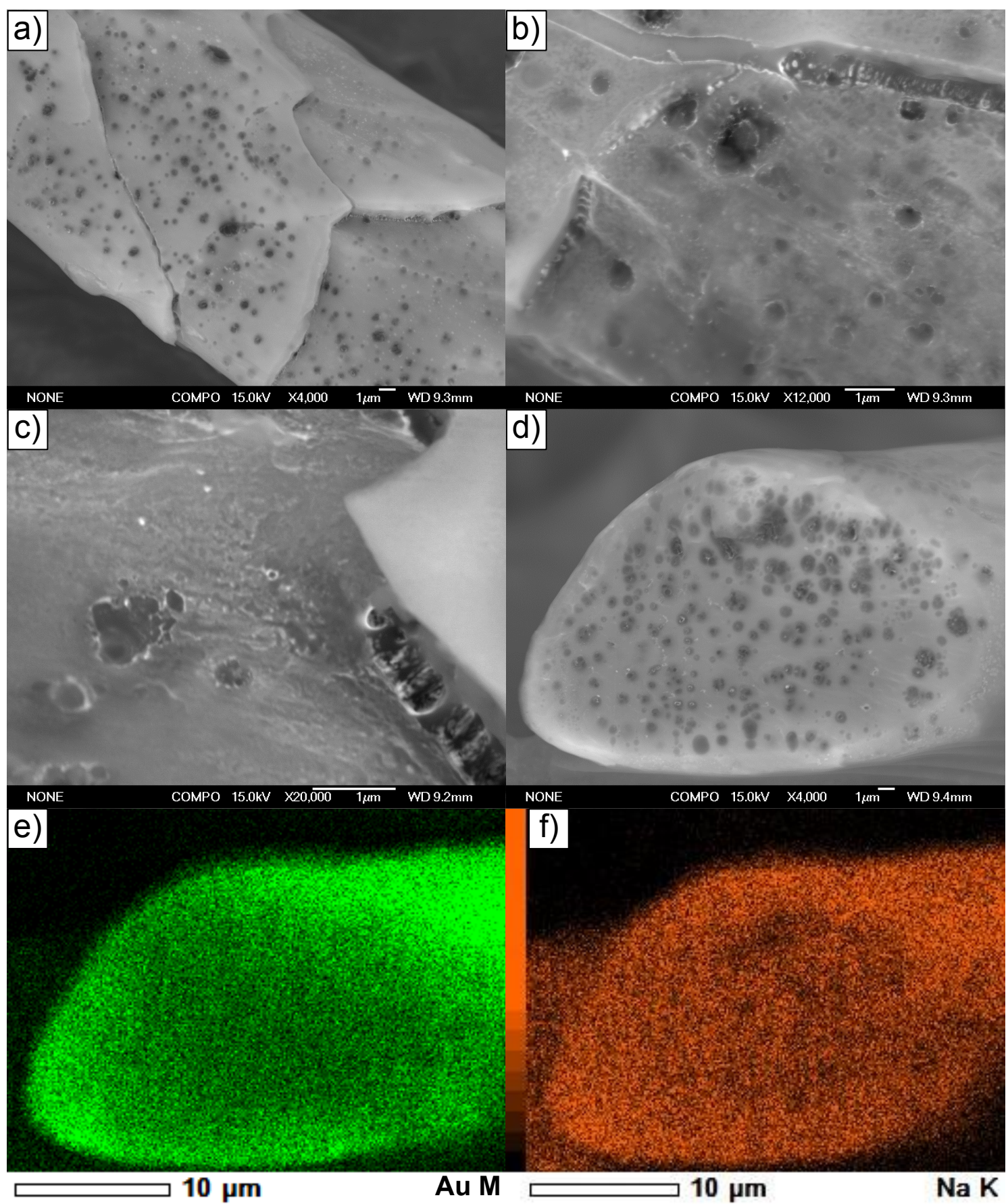

Figure 3.35 SEM and EDS analysis of an Ashford2 composite synthesised with $\mathrm{NaBH}_{4}$ after $10 \mathrm{~min}$ of uptake of $160 \mathrm{mg} \mathrm{L}^{-1} \mathrm{Au}^{3+}$ at RT: BS micrographs of the fibre surface (a)-(c) and a cross-section (d), and EDS elemental $\mathrm{Au}(\mathrm{e})$ and $\mathrm{Na}$ (f) maps of the cross-section.

area in Figure 3.35 (e) shows that the concentration of $\mathrm{Au}$ is much higher near the surface of the wool, compared to the fibre interior. This is expected after only 10 min of $\mathrm{AuCl}_{4}^{-}$uptake at RT. The EDS elemental map in Figure 3.35 (f) shows the presence of $\mathrm{Na}$ throughout the fibre interior, indicating that $\mathrm{NaBH}_{4}$ may have been absorbed into the fibre interior during the synthesis. 
To further investigate the size and distribution of gold on the in-situ composites synthesised with $\mathrm{NaBH}_{4}$, SEM and EDS analysis of the Ashford2 composite synthesised after $60 \mathrm{~min}$ of uptake at RT is presented in Figure 3.36. Again, it is difficult to observe gold on the relatively low magnification micrograph in Figure 3.36 (a). The higher magnification images exhibit an expectedly higher concentration of gold on the surface of the composites compared to the 10 min sample. Figure 3.36 (c) shows the presence of the occasional gold nanoparticle of the typical size that is seen in the standard purple in-situ method, however, the bulk of the gold is present as very small nanoparticles. These $\mathrm{Au}^{0}$ entities are sometimes located so closely together on the surface of the composites that the structure appears to be similar to a fractured layer of gold (this is seen in Figure $3.36(\mathrm{~b})$ ). The higher resolution image in Figure 3.36 (d) shows an area containing a range of sizes of gold nanoparticles.

The BS micrograph of the cross-section shown in Figure 3.36 (e) shows evidence of gold nanoparticles present near the surface of the fibre only. However, the Au elemental map of this area (in Figure 3.36 (f)) indicates that there is gold present throughout the interior of the fibre, with what appear to be a higher proportion of gold in the centre of the fibre compared to the surface as compared to the $10 \mathrm{~min}$ composite. The 60 min $\mathrm{NaBH}_{4}$ composite is a darker brown colour than the $10 \mathrm{~min}$ composite, and as such, the very small particles of gold are likely yellow-brown in colour. There is evidence of brown coloured gold nanoparticles that are between $1-3 \mathrm{~nm}$ size (and do not have a stabiliser). ${ }^{56}$

This experiment showed that $\mathrm{NaBH}_{4}$ can be used to aid the reduction of $\mathrm{Au}^{3+}$ to $\mathrm{Au}^{0}$ on the surface of wool fibres. The rapid reduction resulted in both small and very small nanoparticles of gold on the composites. The $\mathrm{NaBH}_{4}$ composites were different shades of yellow-brown, a novel colour which expands the boutique colour palate of the composites. This research has also shown that synthesis of purple coloured composites with $\mathrm{NaBH}_{4}$ at this stage. 


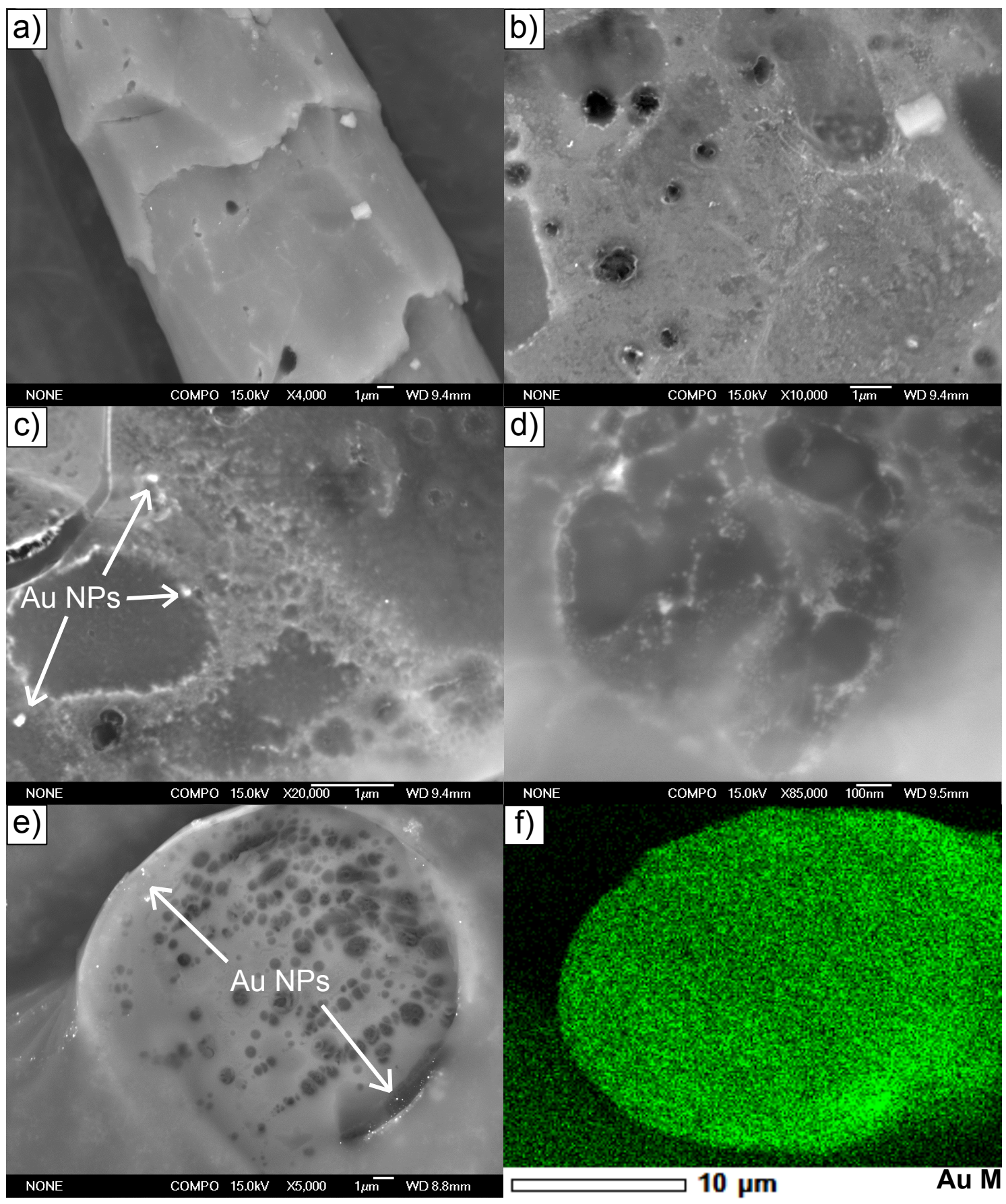

Figure 3.36 SEM and EDS analysis of an Ashford2 composite synthesised with $\mathrm{NaBH}_{4}$ after $60 \mathrm{~min}$ of uptake of $160 \mathrm{mg} \mathrm{L}^{-1} \mathrm{Au}^{3+}$ at RT: BS micrographs of the fibre surface (a)-(d) and cross-section (e), and an EDS elemental Au map of the cross-section (f).

\section{Trisodium Citrate}

TSC was the first dual reducing and stabilising agent to be utilised in the synthesis of gold colloids, ${ }^{25}$ and TSC colloids are used to produce the grey and pink composites with the ex-situ procedure. TSC is a much weaker reducing agent than $\mathrm{NaBH}_{4}$, and requires $95^{\circ} \mathrm{C}$ to nucleate gold nanoparticles in solution (as compared to RT reduction with $\mathrm{NaBH}_{4}$ ). As such, samples of Ashford2 wool were removed from 
uptake solutions and immersed in boiling solutions of aqueous TSC (1 wt \%).

The resulting composites from the application of TSC as an external reducing agent after different times of uptake of $160 \mathrm{mg} \mathrm{L}^{-1} \mathrm{Au}^{3+}$. Similar to the composites synthesised with $\mathrm{NaBH}_{4}$, TSC has caused a yellow-brown colouration of the composites.

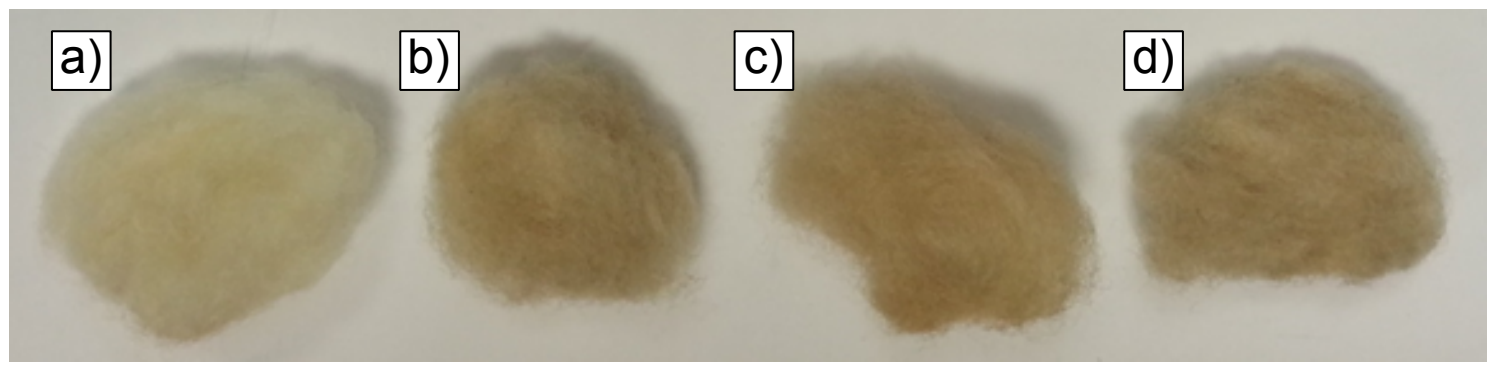

Figure 3.37 Image of composites synthesised with immersion in a boiling solution of aqueous TSC after no uptake (a), and $5 \mathrm{~min}$ (b), $10 \mathrm{~min}$ (c) or $60 \mathrm{~min}$ (d) uptake of $160 \mathrm{mg} \mathrm{L}^{-1} \mathrm{Au}^{3+}$ at RT.

CIE colour coordinates of the composites shown in Figure 3.37 are represented in Table 3.11. Interestingly, the yellowness of the composites seems to be unaffected by the uptake time, or the gold content, of the composites. Additionally, there is only a gradual increase in the $\mathrm{a}^{*}$ value of the composites with increasing gold content. When Ashford2 wool (with no $\mathrm{AuCl}_{4}^{-}$) is boiled in a solution of TSC, significant yellowing of the fibres also occurs. As such, the yellow colour of the TSC composites is likely due to the formation of yellow chromophores that are degradation products of the boiling of wool in TSC.

Table 3.11 CIE colour coordinates of Ashford2 composites synthesised with the application of TSC.

\begin{tabular}{ccccc}
\hline Uptake time $(\mathrm{min})$ & 5 & 10 & 60 & TSC only \\
\hline $\mathrm{L}^{*}$ & 74.18 & 71.93 & 67.75 & 86.96 \\
$\mathrm{a}^{*}$ & 0.54 & 1.59 & 2.43 & 0.02 \\
$\mathrm{~b}^{*}$ & 21.44 & 22.61 & 22.03 & 21.80 \\
\hline \hline
\end{tabular}

SEM micrographs of the surface of the TSC composite synthesised after $10 \mathrm{~min}$ of $\mathrm{AuCl}_{4}^{-}$uptake at RT is shown in Figure 3.38. Interestingly, the composite surface is covered with distinct gold nanoparticles, larger particles of gold and agglomerates of gold nanoparticles. The fibres see the typical higher concentration of $\mathrm{Au}$ at the edges of cuticle scales. The SEM micrographs do not look dissimilar to composites that have been synthesised with the standard in-situ procedure. As such, the fibre damage sustained during the boiling of the composites likely dominates the purplecoloured gold nanoparticles on the surface of the composites. 


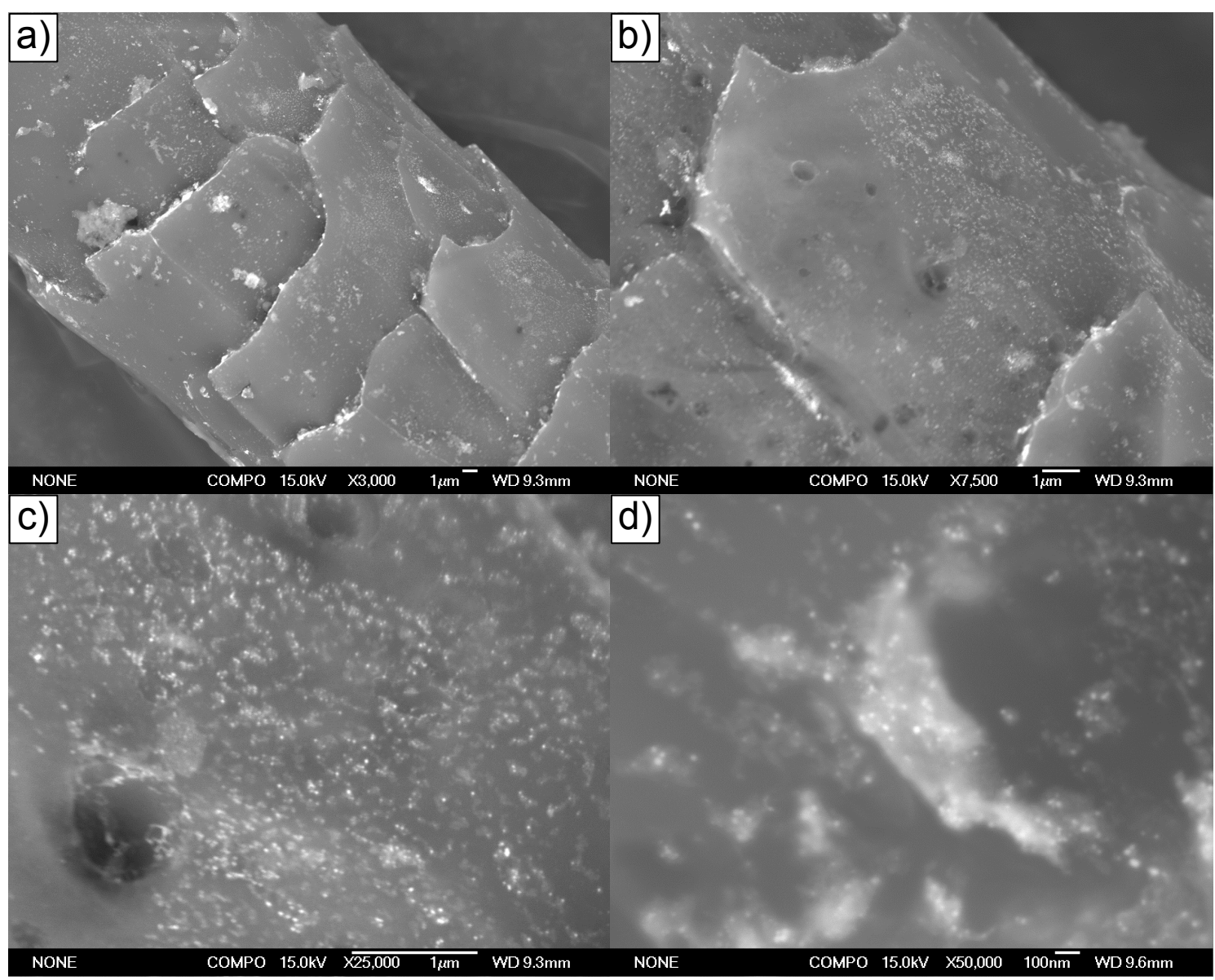

Figure 3.38 SEM micrographs in BS mode of the surface of an Ashford2 composite synthesised with TSC as an external reducing agent after $10 \mathrm{~min}$ of $160 \mathrm{mg} \mathrm{L}^{-1}$ $\mathrm{Au}^{3+}$ uptake at RT with increasing magnification from (a)-(d).

The use of TSC in the synthesis of in-situ composites has caused a range of yellowbrown composites. However, due to the fact that the yellow colouration is due to the damaging of the wool fibres, the use of TSC in the in-situ procedure was not continued at this concentration.

\section{Tannic Acid}

TA is a dual-reducing and stabilising reagent that is used extensively in the solutionphase synthesis of gold nanoparticles in the ex-situ procedure. TA is a stronger reducing agent than TSC, and is able to nucleate gold nanoparticles at RT.

The immersion of Ashford2 wool samples in an aqueous solution of TA (1 wt \%) at RT after removal from uptake solutions yielded the composites shown in Figure 3.39. The composites are all different shades of grey or black, far different from the yellowbrown coloured $\mathrm{NaBH}_{4}$ and TSC composites, and the purple composites produced with the standard purple in-situ method. 


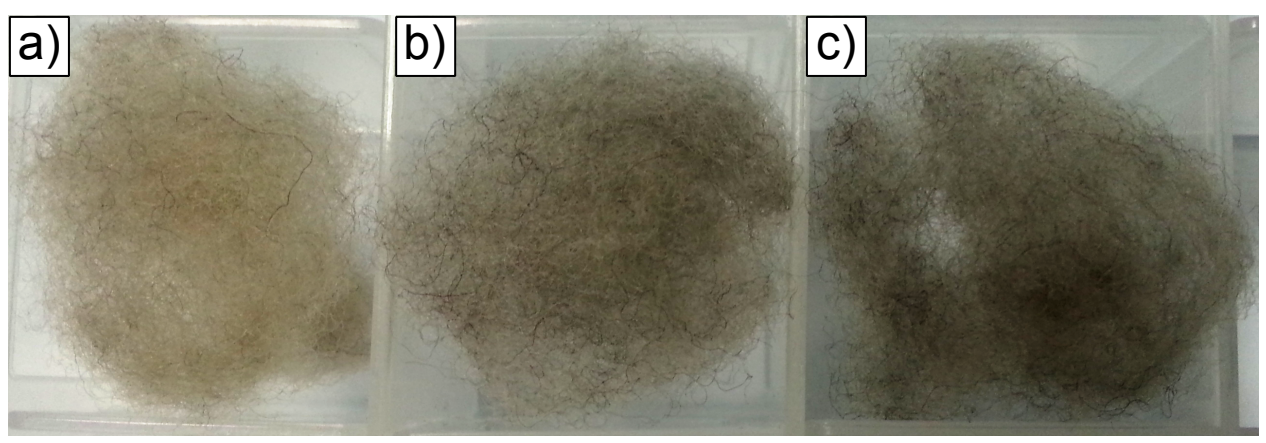

Figure 3.39 Image of composites synthesised with immersion in an aqueous solution of TA (1 wt \%) after $5 \mathrm{~min}(\mathrm{a}), 10 \mathrm{~min}$ (b) and $60 \mathrm{~min}$ of uptake of $160 \mathrm{mg} \mathrm{L}^{-1}$ $\mathrm{Au}^{3+}$ at RT.

Table 3.12 shows the CIE colour coordinates of the composites in Figure 3.39. As the uptake time is increased, composites are relatively darker, slightly less red and more blue. This is synonymous with the increase in grey/black colouration of the composites. Interestingly, this grey-black colouration is uncharacteristically nonuniform between different wool fibres. Additionally, increasing the uptake time of the composites shows an increase in intensity of the grey-black colour of some fibres, while retaining a similar proportion fibres that are relatively uncoloured. This could be due to the TA associating to some fibres preferentially.

Table 3.12 CIE colour coordinates of Ashford2 composites synthesised with TA after the uptake of $160 \mathrm{mg} \mathrm{L}^{-1} \mathrm{Au}^{3+}$ at $\mathrm{RT}$.

\begin{tabular}{cccc}
\hline Uptake time (min) & 5 & 10 & 60 \\
\hline $\mathrm{L}^{*}$ & 74.25 & 68.27 & 62.95 \\
$\mathrm{a}^{*}$ & 1.71 & 1.38 & 0.54 \\
$\mathrm{~b}^{*}$ & 10.86 & 8.37 & 6.61 \\
\hline \hline
\end{tabular}

SEM and EDS analysis of the surface of a TA in-situ composite synthesised after 10 min uptake of $\mathrm{AuCl}_{4}^{-}$is shown in Figure 3.40. The non-uniformity of colouration of the composites is reflected in this, with a substantial difference in gold coverage observed in the two imaged fibres in Figure 3.40 (a) and (b). The gold coverage on Figure 3.40 (b) is extraordinary; there is a diverse range of shapes and sizes of gold particles including a significant proportion of large gold particles. Magnification of the square area in Figure 3.40 (b) shows a significant number of gold particles that are larger than nano-sized (>100 $\mathrm{nm}$ in diameter) present amongst the nanoparticles. The gold content of the particles is confirmed in the elemental Au EDS map of this area in Figure 3.40 (d).

The large particles of gold, together with the range of sizes and shapes of gold nanoparticles present on the surface of the TA in-situ composites is considered to cause a major broadening of the surface plasmon resonance of the gold present on the 


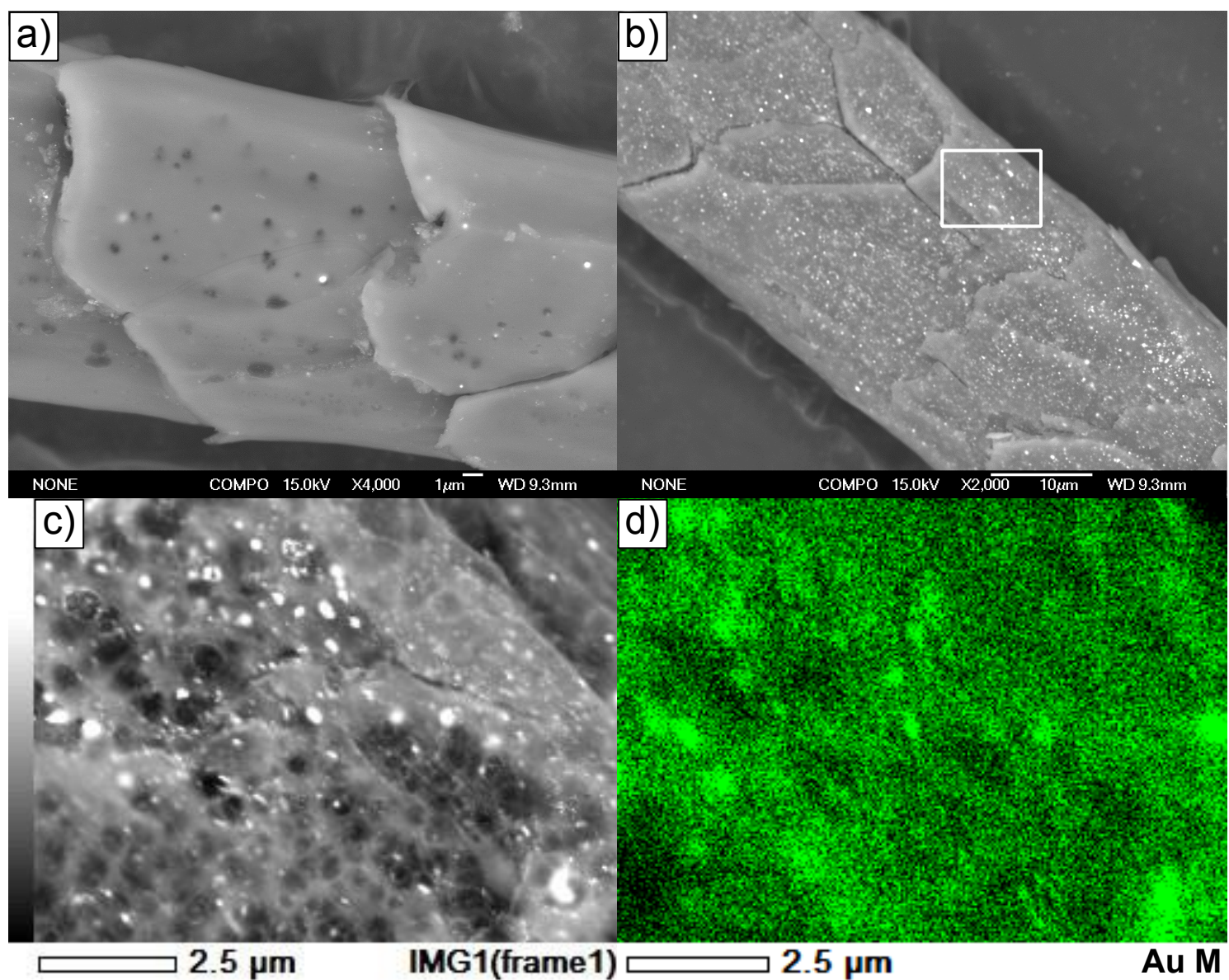

Figure 3.40 SEM and EDS analysis of an Ashford2 composite synthesised with TA after $10 \mathrm{~min}$ of uptake of $160 \mathrm{mg} \mathrm{L}^{-1} \mathrm{Au}^{3+}$ at RT: SEM micrographs in BS mode at increasing magnification (a)-(c), and an EDS elemental Au map (d).

surface of the composites, thereby accounting for their grey-black colouration. The development of a black coloured composite is very desirable, it allows the broadening of the boutique range of possible colours. However, before this can be scaled-up, more research is required to improve the uniformity of the colouration.

Section 3.3.2 has shown that the application of external energy sources and reducing agents can in many cases be used to manipulate the rate of gold nanoparticle nucleation, and also effect the size, shape and distribution of nanoparticles in the wool matrix. This has affected the colour of the composites, both in terms of the hue and uniformity. 


\subsection{Conclusions}

Section 3.1 introduced the chemistry of the in-situ procedure, by characterising the uptake and ageing processes in the synthesis of Cariaggi composites. The effects of a range of reaction conditions on the chemistry of the in-situ approach were then detailed in Section 3.2. The uptake of $\mathrm{AuCl}_{4}^{-}$to wool was influenced by the wool type, $\mathrm{pH}$, temperature, use of auxiliary agents and the volume and concentration of the $\mathrm{AuCl}_{4}^{-}$solutions. Increasing the temperature of the ageing process was found to substantially promote the nucleation of gold nanoparticles in the wool fibres.

Section 3.3 presented the use of external energy sources and reducing agents in the in-situ procedure. The heat and reducing agents manipulated of the rate of gold nanoparticle formation, the distribution of gold nanoparticles throughout the wool fibres, and the colours of the in-situ composites. A summary of the results of these experiments are listed in Table 3.13.

Table 3.13 The colour shade and uniformity of in-situ composites that were synthesised with heat and external reducing agents.

\begin{tabular}{cccc}
\hline Approach & Approx Au content (wt \%) & Colour & Gold coverage \\
\hline Microwave & 1.6 & purple & uneven \\
Oven & 1.6 & pink & uneven \\
Boiling & 0.7 & yellow & uniform \\
$\mathrm{NaBH}_{4}$ & 0.25 & yellow-brown & uniform \\
TSC & 0.25 & yellow-brown & uniform \\
TA & 0.7 & grey-black & uneven \\
\hline \hline
\end{tabular}

To date, a significant colour range of $i n$-situ composites is possible that encompasses pink, purple, yellow, brown, grey and black. However, in many cases the colours are uneven. Additionally, the colour uniformity of in-situ composites was found to be substantially influenced by the type of wool that was employed in the synthesis. This is a significant problem for scale-up procedures, where a range of wool types will be employed.

The gold content of in-situ composites could not be sufficiently reduced to an economically viable level. Thus, the in-situ approach is currently too expensive for larger-scale production, and scale-up syntheses of in-situ composites have not yet been carried out. The ex-situ procedure is a relatively cost-effective approach that can be used with a wider range of wool types. As such, laboratory-scale optimisation of the ex-situ procedure was carried out, and is presented in Chapter 4. 


\section{Chapter 4}

\section{Ex-situ Synthesis of Gold Nanoparticle-wool Fibre Composites}

The ex-situ approach for the synthesis of gold nanoparticle-wool fibre composites was originally developed by Lucas and Johnston, ${ }^{5}$ in response to the need for a more cost-effective alternative to the in-situ procedure. The ex-situ procedure results in gold nanoparticles attached to the surface of the wool fibres, with no gold present in the wool fibre interiors. As such, ex-situ composites exhibit a much lower gold content of the gold content of the in-situ composites (approximately one tenth). However, the downside of this relatively low gold content is that a covalent bond does not form between the gold nanoparticles and the wool fibre matrix. This results in composites with reduced washfastness properties, and is a significant issue relating to the commercialisation of these composites. Research involved in improving the washfastness of ex-situ composites is presented in Chapter 6.

In Chapter 4, the ex-situ procedure is first introduced by detailing the synthesis and characterisation of Cariaggi ex-situ composites in Section 4.1. Following this, the reaction conditions of the synthesis of TA- and TSC-stabilised gold colloid are optimised in Section 4.2. Finally, Section 4.3 details the optimisation of the reaction conditions involved in the colouring of wool with gold colloids. 


\subsection{Ex-situ Composites of Cariaggi Wool}

The ex-situ procedure involves the solution-phase synthesis of gold nanoparticles, that are then used to colour wool fibres. The standard colloidal syntheses are outlined in Section 2.2.2, and specific reaction conditions of each unique experiment are detailed in this chapter. There are three standard gold nanoparticle syntheses: the TA-mauve, TSC-pink and TSC-grey methods. A summary of the typical reaction conditions used in each method is Table 4.1 below:

Table 4.1 Standard reaction conditions of the ex-situ procedures.

\begin{tabular}{lccc}
\hline Synthesis method & TA-mauve & TSC-pink & TSC-grey \\
\hline Conc of $\mathrm{Au}^{3+}\left(\mathrm{mg} \mathrm{L}^{-1}\right)$ & 10 & 10 & 40 \\
Amount of $4 \mathrm{wt} \% \mathrm{Au}^{3+}$ in colloid $\left(\mathrm{mL} \mathrm{L}^{-1}\right)$ & 0.25 & 0.25 & 1 \\
Temperature of $\mathrm{NP}$ synthesis & $\mathrm{RT}$ & $95^{\circ} \mathrm{C}$ & $95^{\circ} \mathrm{C}$ \\
Ratio of $\mathrm{AuCl}_{4}^{-}$to stabiliser & $1: 0.39$ & $1: 2.1$ & $1: 2.1$ \\
Typical pH of dyeing & 2.2 & 2.7 & $2.0-2.7$ \\
$\begin{array}{l}\text { Wool to liquor ratio } \\
\begin{array}{l}\text { Approximate gold } \\
\text { content of composites (wt } \%)\end{array}\end{array}$ & $1: 60$ & $1: 60$ & $1: 60$ \\
& 0.06 & 0.06 & 0.24 \\
\hline
\end{tabular}

The synthesis and characterisation of TA- and TSC-stabilised gold colloids is first presented, followed by the chemistry of using these colloids to colour Cariaggi wool.

\subsubsection{Gold Nanoparticle Synthesis}

The synthesis of TSC-stabilised gold nanoparticles involves the reduction of a boiling solution of $\mathrm{AuCl}_{4}^{-}$upon the addition of TSC. TSC-Au NPs are used to produce both pink and grey coloured ex-situ composites, where the grey composites are produced with a four times more concentrated colloid. In contrast, TA-stabilised gold nanoparticles are synthesised at RT, and different concentrations of TA-Au NPs typically produce different shades of mauve-coloured composites. The typical gold colloids used to produce TA-mauve and TSC-grey composites are shown in Figure 4.1. 

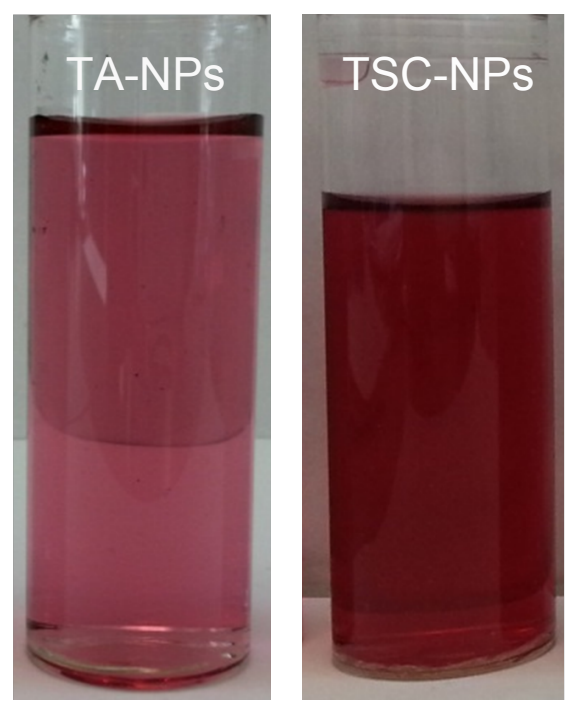

Figure 4.1 TA-mauve and TSC-grey colloids.

Figure 4.2 shows the UV-visible absorption spectra of the colloids of TSC-Au NPs and TA-Au NPs shown in Figure 4.1. The UV-vis spectra are typical of these colloids when synthesised on the laboratory-scale. The shape of the UV-visible absorption spectra of gold colloids gives an indication of the size, shape and polydispersity of the gold nanoparticles.

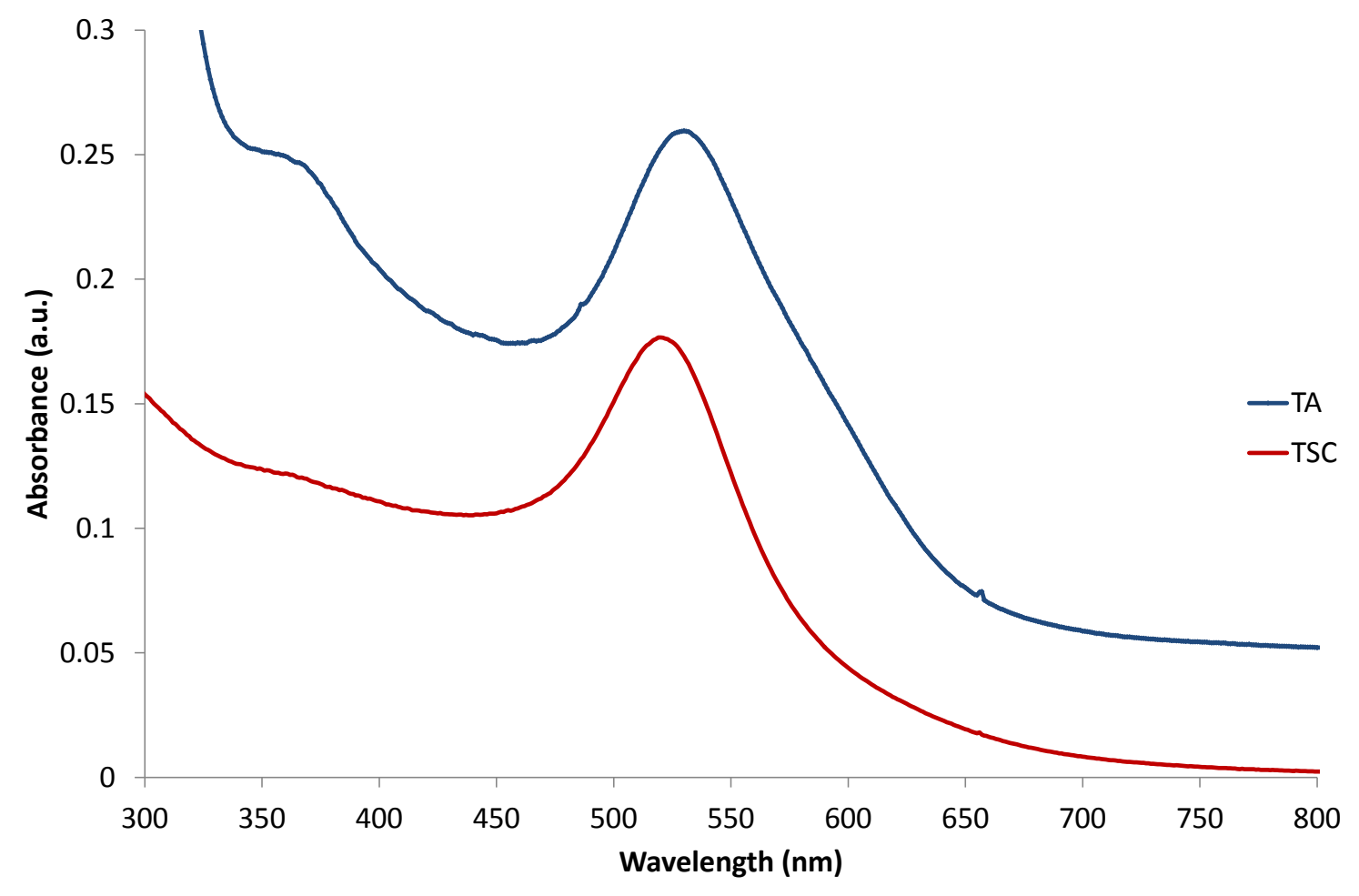

Figure 4.2 Normalised UV-visible absorbance spectra of typical colloids of TA-Au NPs and TSC-Au NPs.

The surface plasmon resonance (SPR) peaks $\left(\lambda_{\max }\right)$ of TA-Au NPs and TSC-Au NPs are at approximately 530 and $525 \mathrm{~nm}$ respectively. An increase in the size of 
gold nanoparticles (or agglomeration of nanoparticles) results in a red-shift (or a shift of the absorbance to higher wavelengths) and broadening of the SPR peak. The presence of anisotropic shapes can introduce additional SPR peaks. The TA$\mathrm{Au}$ NPs spectra is relatively broad, which indicates a relatively diverse range of sizes and shapes of gold nanoparticles in the colloid.

TEM is a complementary technique to UV-vis spectroscopy, which is used to investigate the size, shape and polydispersity of gold nanoparticles. In contrast to SEM, denser elements appear darker on TEM micrographs, as they shield more of the electron beam. TEM micrographs of TA-Au NPs are shown in Figure 4.3.

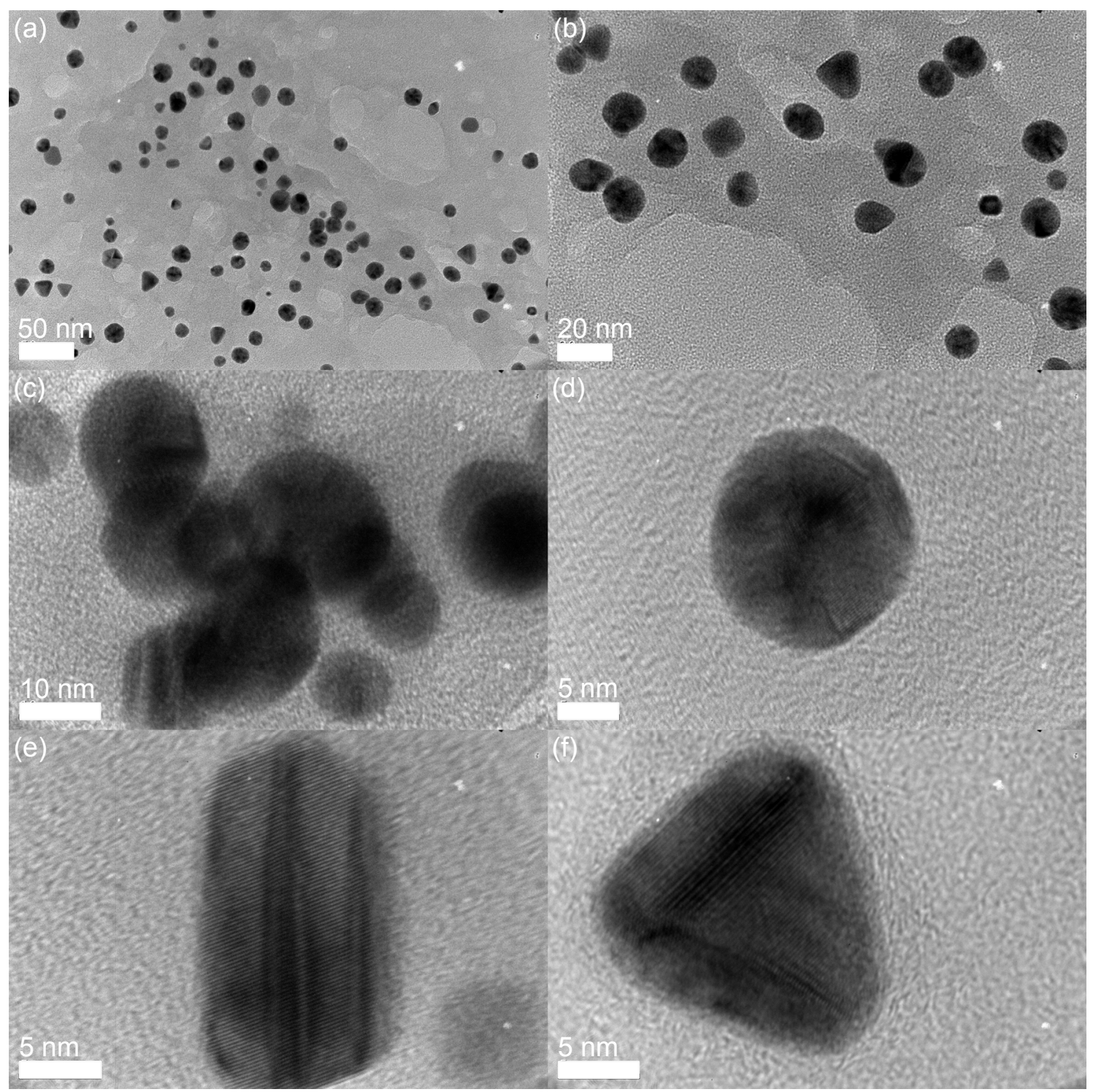

Figure 4.3 TEM micrographs of TA-Au NPs at different magnifications.

There is a range of different sizes and shapes of the TA-stabilised nanoparticles in Figure 4.3. Most of the TA-Au NPs are spherical in shape, with approximate diameters between $10-20 \mathrm{~nm}$. TA is a relatively strong stabilising agent, which allows anisotropic shapes (with higher surface area to volume ratios than spherical par- 
ticles), to form. The higher magnification TEM micrographs in Figure 4.3 (d)-(f) show some of the different shapes of TA-Au NPs, including triangular platelets and cuboid-type shapes. Additionally, there are some agglomerates of smaller nanoparticles shown in Figure 4.3 (c). The strong stabilising nature of TA is also evidenced in the large distances between the TA-Au NPs. The variability in the size and shape of TA-Au NPs explains the relative broadness of its SPR band compared to that of the TSC-stabilised colloid.

TEM micrographs of TSC-Au NPs are presented in Figure 4.4. The bulk of the gold nanoparticles are spherical and between 10-20 nm in diameter. The TSC-Au NPs are much more uniform in size and shape than the TA-Au NPs. There is evidence for the presence of small $\mathrm{Au}^{0}$ entities of approximately $2 \mathrm{~nm}$ diameter (circled in Figure 4.4 (b) and (c)). These may be nanocrystals that would coalesce together to form larger nanoparticles if the reaction was to proceed for longer.

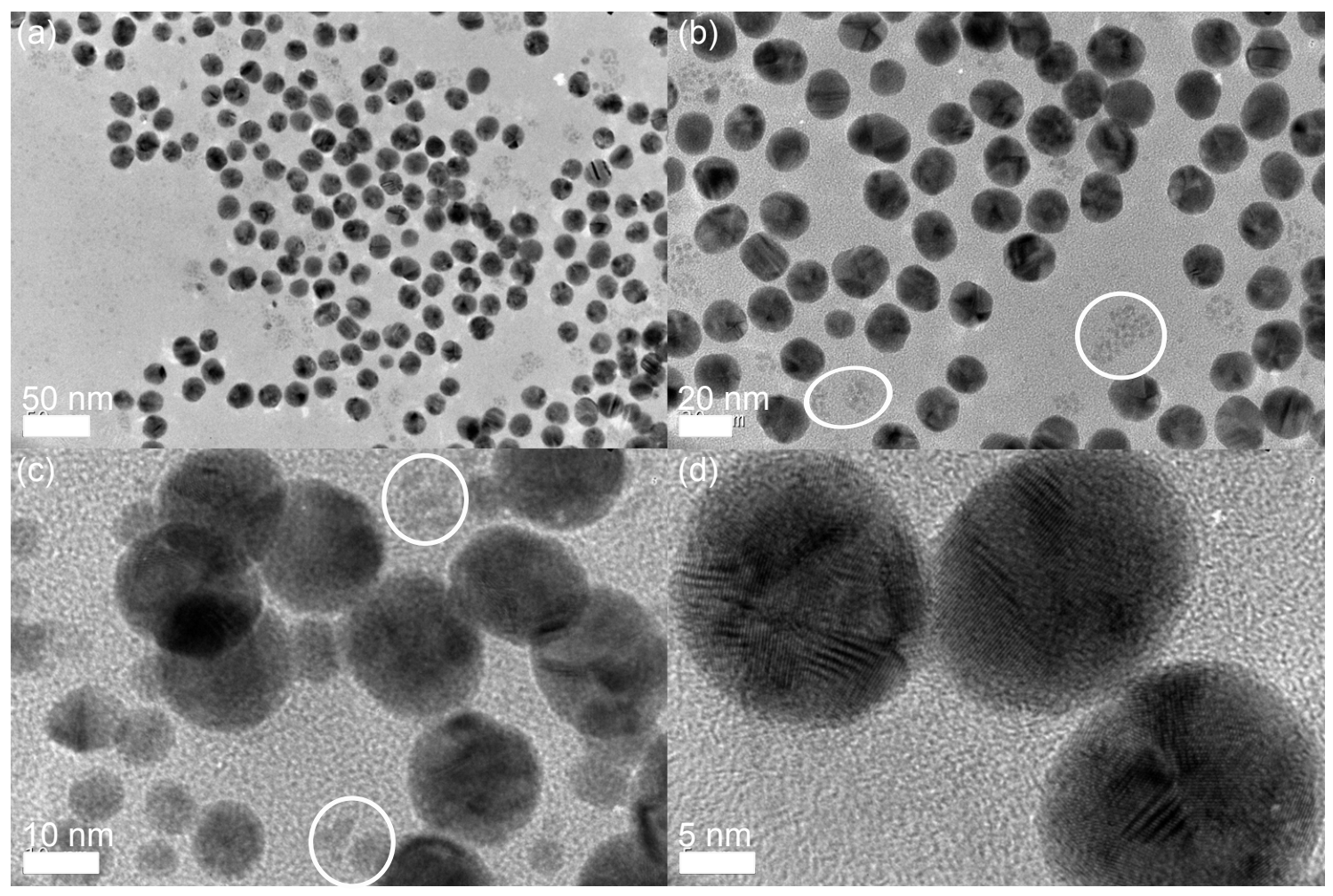

Figure 4.4 TEM micrographs of TSC-Au NPs at different magnifications.

The TSC-stabilised gold nanoparticles are in much closer proximity to one another than the TA-stabilised particles, due to the relatively weak stabilising nature of TSC. This highlights the potential of TSC-Au NPs to agglomerate together, and is exploited in the TSC-grey method. Additionally, the lack of higher energy shapes is likely due to the inability of TSC to stabilise the crystal facets that allow for the formation of asymmetric shapes (with higher surface area to volume ratios than spherical particles). The TA- and TSC-stabilised gold colloids synthesised in this 
research are then used to dye Cariaggi wool.

\subsubsection{Dyeing Cariaggi Wool with Gold Colloids}

The colouring of wool with gold colloids exploits a pH-dependent driving force, which is similar to the uptake of $\mathrm{AuCl}_{4}^{-}$to wool that occurs in the in-situ procedure. In this, the $\mathrm{pH}$ of the dye solution is maintained below the isoelectric point of wool, causing a net positive charge at the wool surface. This provides an electrostatic attraction for the absorption of negatively charged gold nanoparticles (or $\mathrm{AuCl}_{4}^{-}$).

There are two key differences that are unique to the ex-situ procedure compared to the in-situ procedure. The first is that the positively charged wool fibres are electrostatically attracted to the negatively charged stabilising agents surrounding the gold nanoparticles, not the gold itself. Secondly, the gold nanoparticles are too large to penetrate into the interior of the wool fibres, and hence can only associate to the surface of the wool.

Figure 4.5 shows TA-mauve, TSC-pink and TSC-grey Cariaggi wool composites of $1 \mathrm{~g}$ size, illustrating some of the different colours that may be achieved with the exsitu procedure. The TA-mauve and TSC-pink composites have the same gold content (approximately $0.06 \mathrm{wt} \%$ ), and the variation in colour is due to the differences in the size, shape and distribution of the gold nanoparticles across the composite fibres. In contrast, the TSC-grey composite encompasses a four times higher gold content (of approximately $0.24 \mathrm{wt} \%$ ) of the TSC-pink composite. This results in agglomerates of gold nanoparticles on the surface of the wool, which causes the grey colour of the composites.

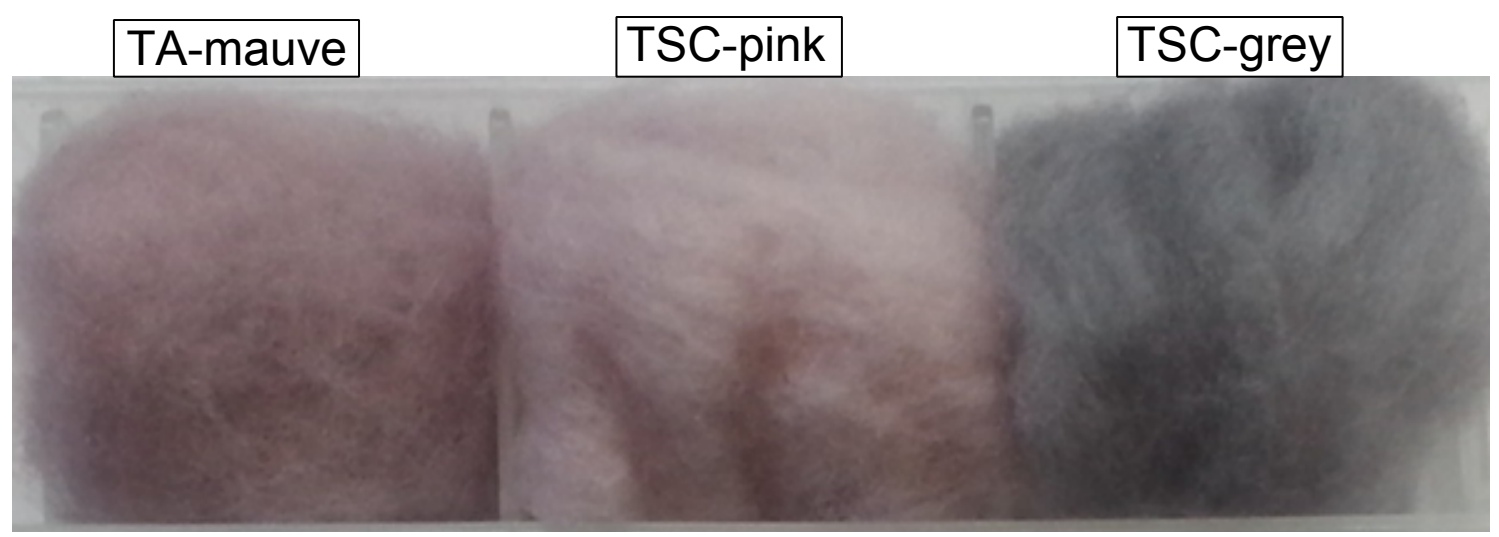

Figure 4.5 TA-mauve, TSC-pink and TSC-grey ex-situ Cariaggi composites. 
CIE colour coordinates are often provided to quantify colour differences between different composites. The colour differences are easily observable when physically holding the composites, but are difficult to observe in printed photographs. The $\mathrm{L}^{*}$ (brightness/darkness), a* (redness/greenness) and $b^{*}$ (yellowness/blueness) values of the ex-situ Cariaggi composites are tabulated in Table 4.2. The TA-mauve Cariaggi composite exhibits darker, less red and bluer colour coordinates compared to the TSC-pink Cariaggi composite. This represents the relatively purple colouration of the TA-mauve composite. The TSC-grey composite registers the lowest $\mathrm{L}^{*}$ and $\mathrm{a}^{*}$ values, which quantifies its dark grey colouration. As the blue colour is a component of both purple and grey coloured composites, the TA-mauve and TSC-grey composites exhibit similar $b^{*}$ values.

Table 4.2 CIE colour coordinates of Cariaggi wool and ex-situ composites.

\begin{tabular}{ccccc}
\hline Composite & Cariaggi wool & TA-mauve & TSC-pink & TSC-grey \\
\hline $\mathrm{L}^{*}$ (lightness) & 88.16 & 57.67 & 69.80 & 54.54 \\
$\mathrm{a}^{*}$ (redness) & -0.39 & 6.34 & 8.03 & 0.36 \\
$\mathrm{~b}^{*}$ (yellowness) & 4.98 & -3.38 & -1.13 & -3.21 \\
\hline \hline
\end{tabular}

SEM micrographs of the TA-mauve Cariaggi composite are shown in Figure 4.6. The SEI image of the composite surface shows the overlapping nature of the cuticle scales, but not the presence of gold nanoparticles. The backscatter image of this area (Figure 4.6 (b)) shows the gold nanoparticles appearing as bright spots on the wool fibre surface. As is typical for backscatter micrographs of wool, the organic fibres begin to degrade, such beam damage appears as the dark spots that are circled in Figure 4.6 (b). There is a relatively higher distribution of gold nanoparticles near the cuticle edges compared to the gold nanoparticles on the cuticle scales. This is clearly seen in the higher magnification micrograph in Figure 4.6 (c). The crosssectional micrograph of a TA-mauve composite fibre is shown in Figure 4.6 (d). It shows gold nanoparticles are located on the surface of the composite only (as seen in the circled areas), with none present in the interior of the fibre. This indicates that gold nanoparticles are too large to penetrate the cuticle layer of wool fibres. 


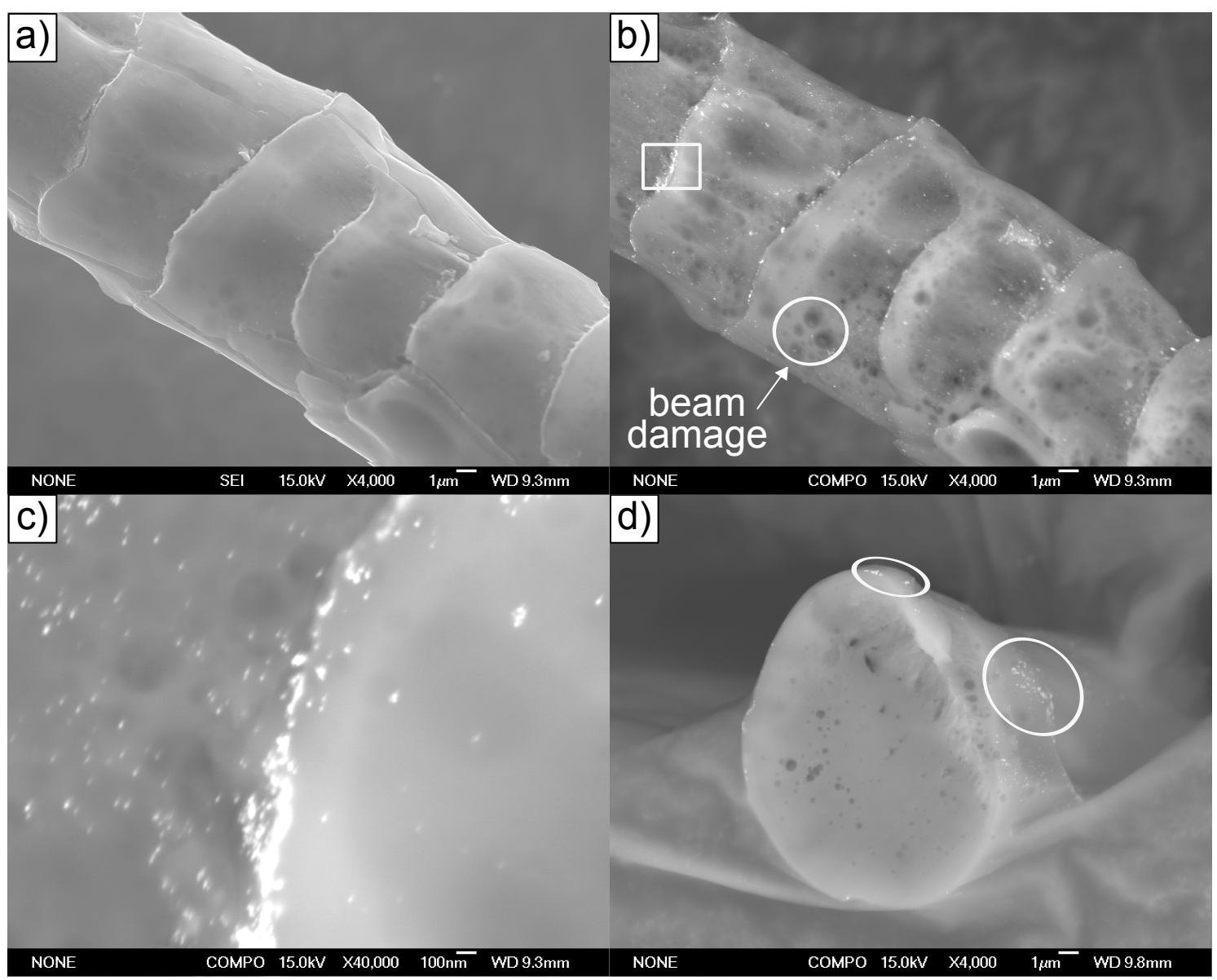

Figure 4.6 SEM micrographs of the surface of a TA-mauve Cariaggi composite in SEI mode (a), in backscatter mode at different magnifications (b) and (c), and a cross-sectional image in backscatter mode (d).

SEM micrographs of a TSC-pink Cariaggi composite are shown in Figure 4.7. The concentration of $\mathrm{AuCl}_{4}^{-}$used in the synthesis of the TA-mauve and TSC-pink colloids is equivalent, and as such a similar gold density on the composite fibres is expected. However, two distinct distributions of gold nanoparticles were observed on the surfaces of different composite fibres. The gold coverage observed on the surface of the relatively "light fibre" imaged in Figure 4.7 (a) and (b) is most commonly observed. The higher magnification image in Figure 4.7 (b) shows that the gold nanoparticles are present as individual particles or agglomerates of a few nanoparticles. Other composite fibres exhibit a relatively high coverage of gold nanoparticles, one of which is represented in Figure 4.7 (c) and (d). The "dark fibre" exhibits gold nanoparticles that have typically aggregated together at or near the edges of the cuticle scales. The combination of the relatively dark and light fibres explains the light pink-purple colouration of the TSC-pink composite, and indicates that uneven dye uptake can occur in samples as small as $1 \mathrm{~g}$.

The close proximity of TSC-Au NPs on the TSC-pink composite illustrates the relatively weak stabilising nature of TSC compared to TA. The potential for the 


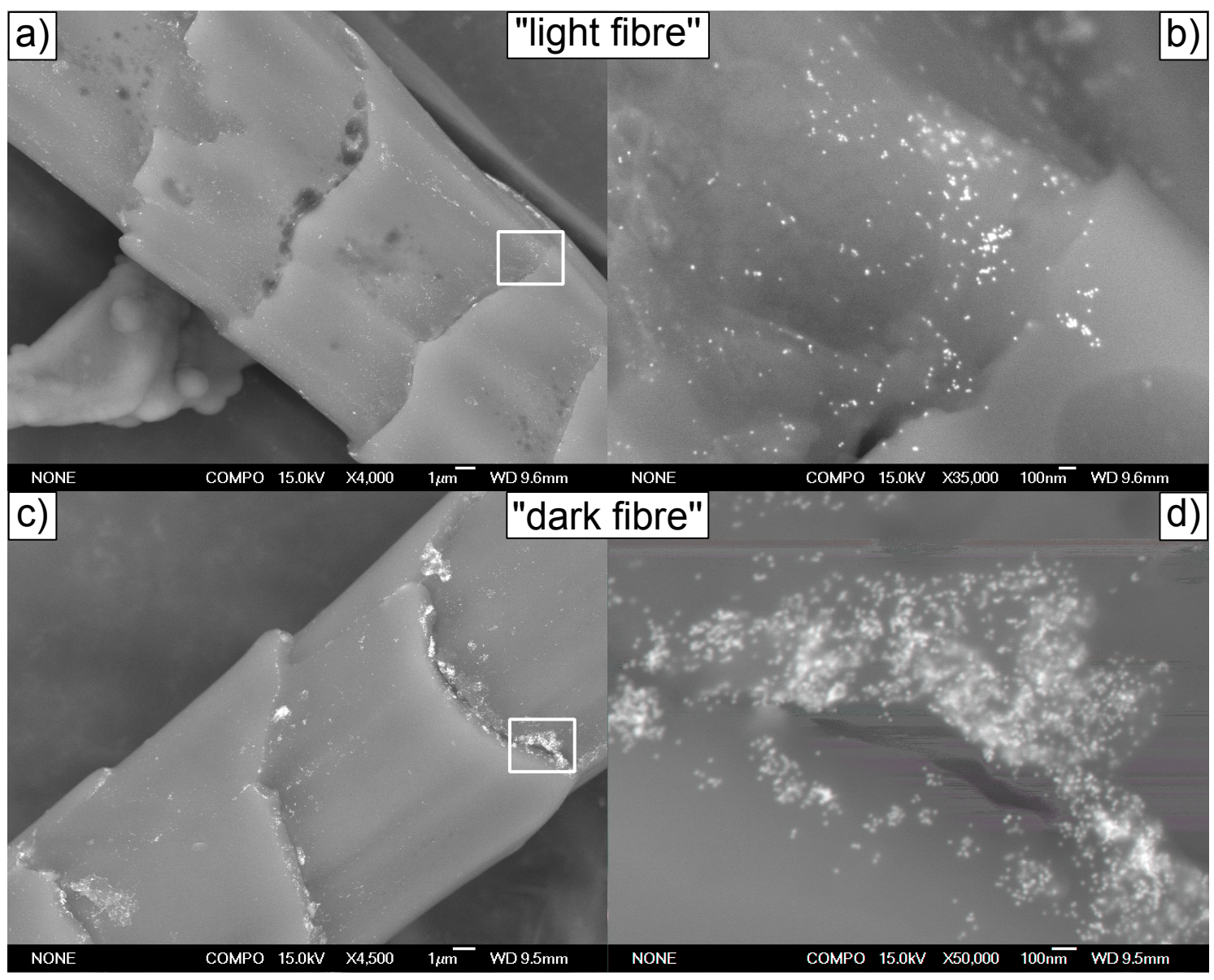

Figure 4.7 SEM micrographs in backscatter mode of two different TSC-pink Cariaggi composite fibres; a relatively common "light fibre" (a) and (b), and an atypically seen "dark fibre" (c) and (d).

aggregation of TSC-Au NPs represents the difficulty in controlling the colour of the TSC-pink procedure. This is discussed more in-depth in Section 4.2.2.

SEM micrographs of the surface of a TSC-grey composite fibre are shown in Figure 4.8. The TSC-grey method utilises a four times more concentrated solution of TSC-Au NPs compared to the TSC-pink method, and as such the exhibits a much higher coverage of gold nanoparticles on its surface. The gold coverage is also more prevalent near the junctions between cuticle scales.

The relatively low magnification image in Figure 4.8 (a) shows many bright areas that may consist of either large particles of gold or agglomerates of gold nanoparticles. Sequential magnification of the TSC-grey composite's surface shows that the areas of relatively dense gold coverage comprise agglomerates of gold nanoparticles. The SPR bands of the gold nanoparticles present in the agglomerates interact together, resulting in a red-shift and broadening of the SPR absorbance, and a grey colour of the composites. This agglomeration, that the TSC-grey method relies upon, is possibly due to the relatively weak stabilising nature of TSC. The gold 


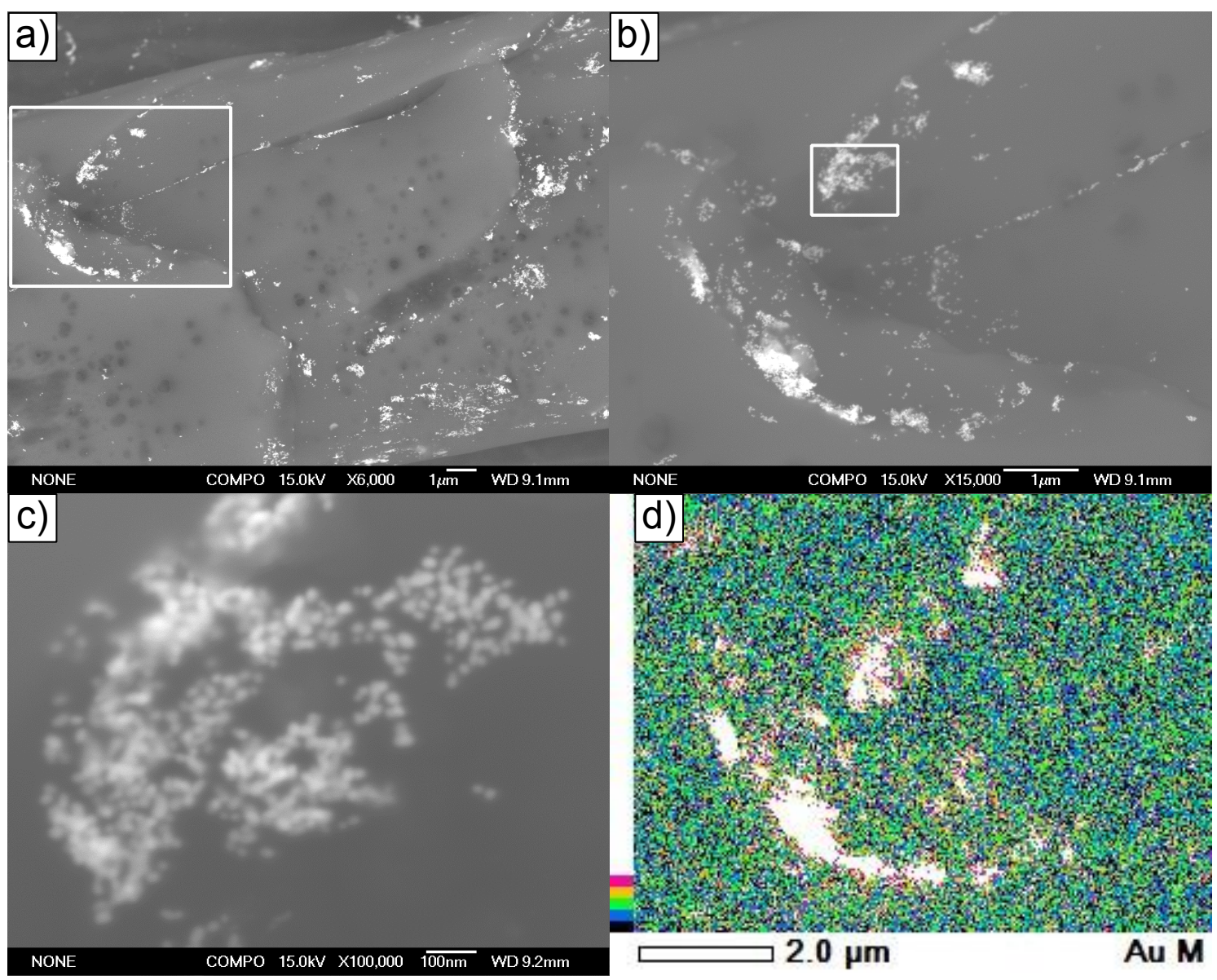

Figure 4.8 SEM micrographs in backscatter mode of the surface of a TSC-grey Cariaggi composite fibre of increasing magnification from (a)-(c), and an Au elemental EDS map of area (b) shown in (d).

content of the nanoparticles in Figure 4.8 (b) is confirmed by the EDS elemental Au map in Figure 4.8 (d), where the bright areas in the SEM image correspond to the areas of high concentration of $\mathrm{Au}$ in the EDS map.

The synthesis and characterisation of the TA-mauve, TSC-pink and TSC-grey Cariaggi composites succinctly introduced the chemistry of the ex-situ procedure. Scalingup the ex-situ synthesis of gold nanoparticle-wool fibre composites requires an indepth knowledge of the chemistry of the ex-situ procedure. This involves first optimising the synthesis of the gold colloids that are then used in to colour wool fibres.

\subsection{Gold Nanoparticle Synthesis}

This section presents the optimisation of the syntheses the TA- or TSC-stabilised gold colloids used in the ex-situ procedure. Chapter 6 investigates gold colloids that are synthesised with different reducing and stabilising agents. The research in this 
section involves characterising both the formation and stability of the TA-mauve, TSC-pink and TSC-grey colloids.

\subsubsection{Formation Studies}

The formation of TA-Au NPs and TSC-Au NPs were studied with UV-vis spectroscopy. The rate of formation of the nanoparticles, as well as the different sizes and shapes of particles that form during the reaction, were investigated. The reaction conditions of the TA-mauve, TSC-pink and TSC-grey colloid syntheses were optimised prior to scale-up dyeing reactions.

\section{Formation of TA-Au NPs}

A study of the formation of the TA-mauve colloid over time is shown in Figure 4.9. The UV-vis spectra were measured at different time intervals after the addition of TA to a stirred solution of $10 \mathrm{mgL}^{-1} \mathrm{Au}^{3+}(300 \mathrm{~mL})$ at room temperature (RT). A sample of the solution (approximately $2 \mathrm{~mL}$ ) was removed for immediate UV-vis analysis and then rapidly returned to the reaction solution.

Figure 4.9 shows that the surface plasmon resonance (SPR) peak (at $\lambda_{\max }=530 \mathrm{~nm}$ ) begins to form $2 \mathrm{~min}$ after the addition of $\mathrm{TA}$ to $\mathrm{AuCl}_{4}^{-}$. The SPR peak rapidly increases in intensity up to $15 \mathrm{~min}$ of the reaction, and then more gradually develops until the reaction is complete after approximately $60 \mathrm{~min}$. The low wavelength absorbance also increases in intensity during this time, and a shoulder between 350$375 \mathrm{~nm}$ eventually forms. The absorbance at $350-375 \mathrm{~nm}$ is explained in Section 4.2.3. There is a significant increase in the low wavelength absorbance between spectra measured after 10 and 11 min of the formation reaction.

An additional study of the formation of the TA-mauve colloid was carried out inside the UV-vis cuvette (of approximately $3 \mathrm{~mL}$ volume). This study was referred to as an in-situ formation study. The in-situ study was undertaken to provide a comparison for the formation of TSC-Au NPs, the formation of which was only able to be accurately studied in-situ (due to the elevated temperature of the synthesis of TSC-Au NPs). As the in-situ formation of TA-Au NPs was carried out directly in the cuvette, there is no stirring of the colloid, however the cuvette was vigorously shaken prior to the UV-vis measurements. The rate of formation of the SPR peak $\left(\lambda_{\max }=530 \mathrm{~nm}\right)$ was similar to that observed in Figure 4.9. Again, the shoulder between $350-375 \mathrm{~nm}$ gradually forms during the formation reaction. 


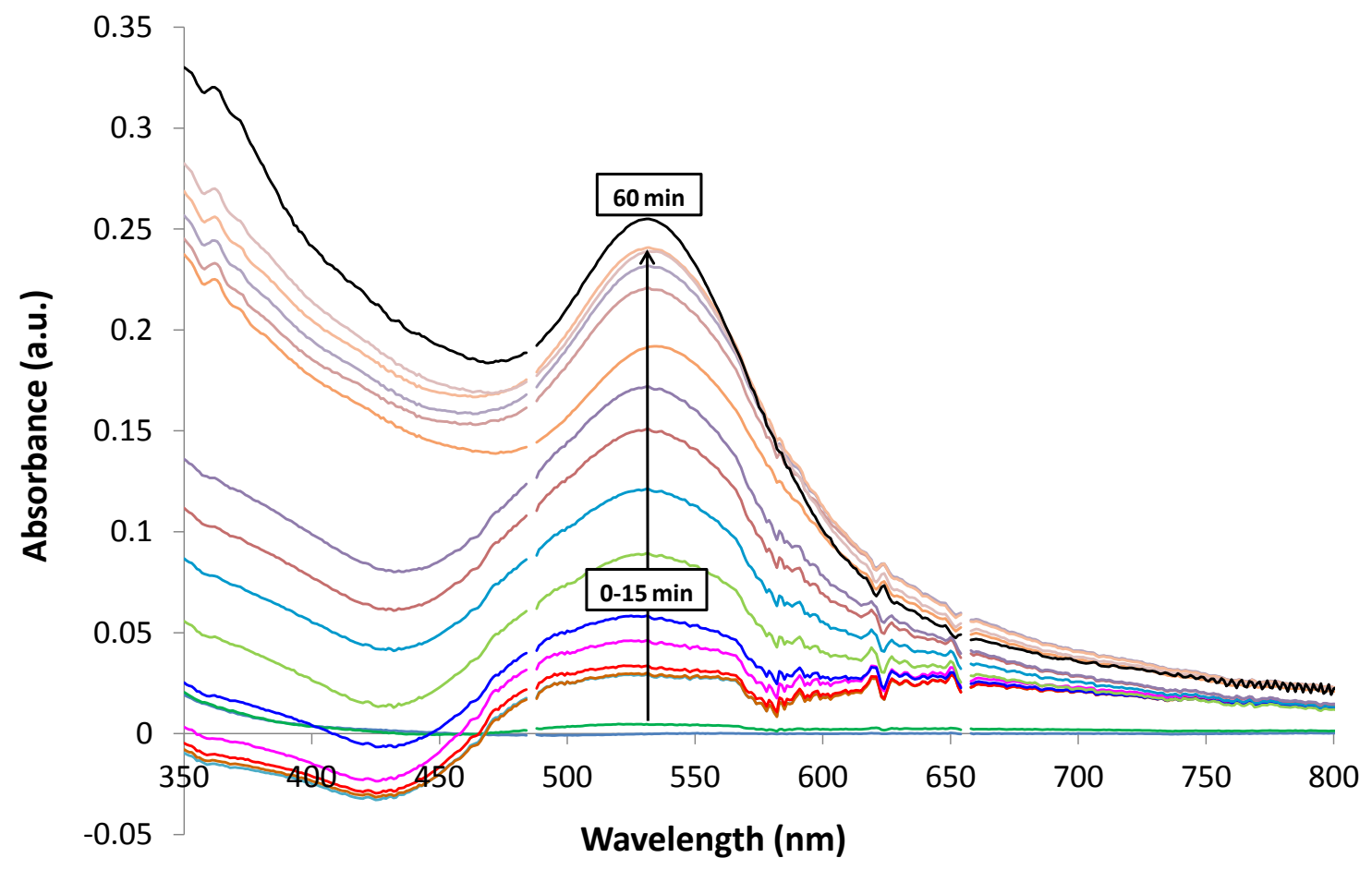

Figure 4.9 UV-vis spectra measured during the formation of a TA-mauve colloid $\left(10 \mathrm{mg} \mathrm{L}^{-1} \mathrm{Au}^{3+}, 300 \mathrm{~mL}\right)$, where the spectra are measured after every minute from $0-15 \mathrm{~min}$ and after $60 \mathrm{~min}$ of the formation.

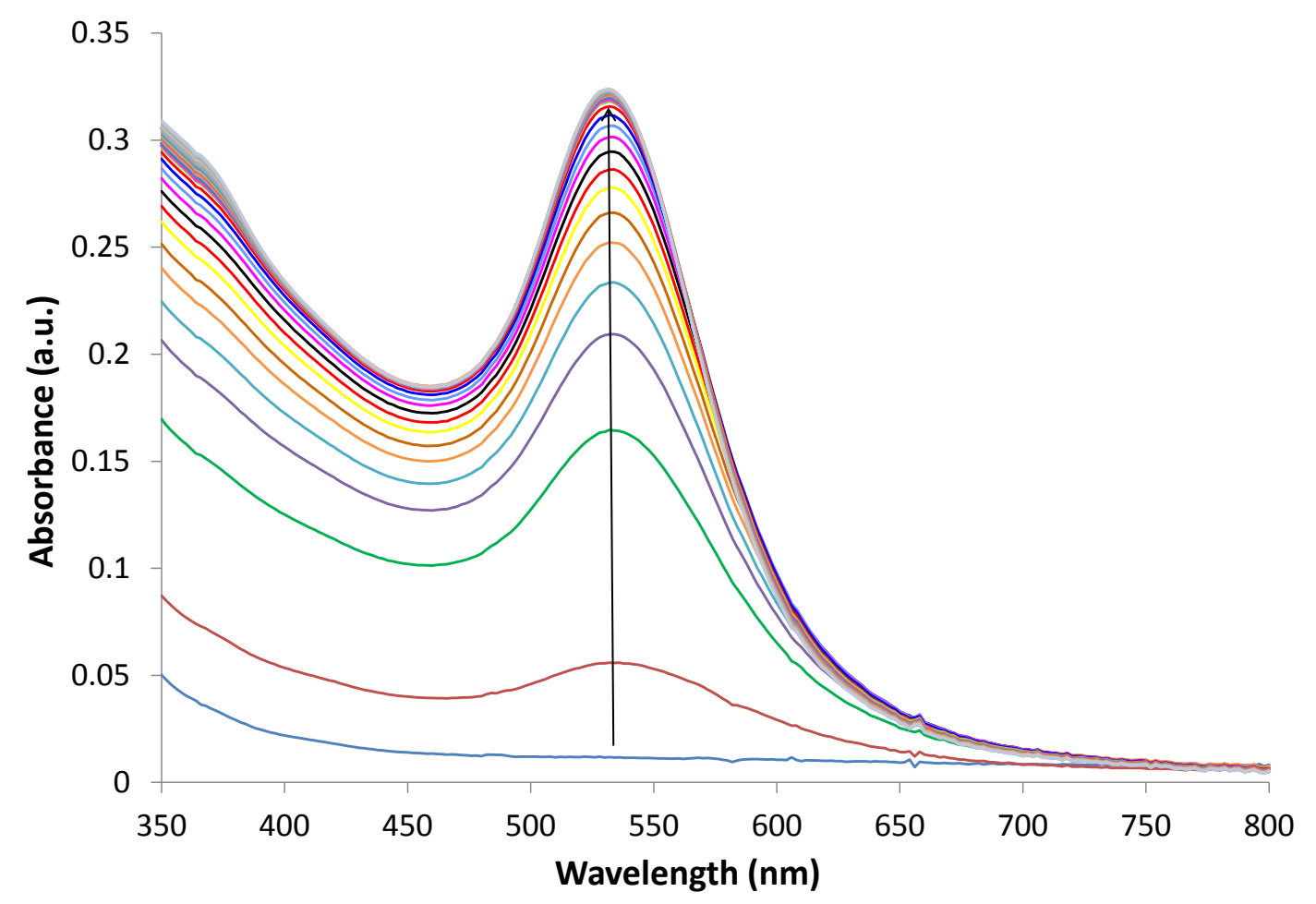

Figure 4.10 UV-vis spectra of the formation of the TA-mauve colloid inside the UV-vis cuvette $\left(10 \mathrm{mg} \mathrm{L}^{-1} \mathrm{Au}^{3+}, 3 \mathrm{~mL}\right)$, where each spectrum is measured every minute for the first $30 \mathrm{~min}$ of the formation. 
More concentrated colloids of TA-Au NPs are used to colour wool a deeper shade of mauve, and hence the formation of different concentrations of TA-stabilised colloids were also studied with UV-vis spectroscopy. Figure 4.11 shows the UV-vis spectra of a gold colloid with four times the concentration of both TA and $\mathrm{AuCl}_{4}^{-}\left(40 \mathrm{mg} \mathrm{L}^{-1}\right.$ $\mathrm{Au}^{3+}, 300 \mathrm{~mL}$ ) which equates to the equivalent concentration of $\mathrm{AuCl}_{4}^{-}$that is used in the TSC-grey method. At this concentration, the formation of TA-Au NPs is much more rapid, with the SPR peak $\left(\lambda_{\max }=530 \mathrm{~nm}\right)$ completely forming after $10 \mathrm{~min}$ of the reaction. Additionally, the SPR peak is approximately four times as intense as the that of the standard TA-mauve colloid. This implies that there is no loss of colour intensity of the gold nanoparticles due to the higher concentration of reactants employed in the synthesis.

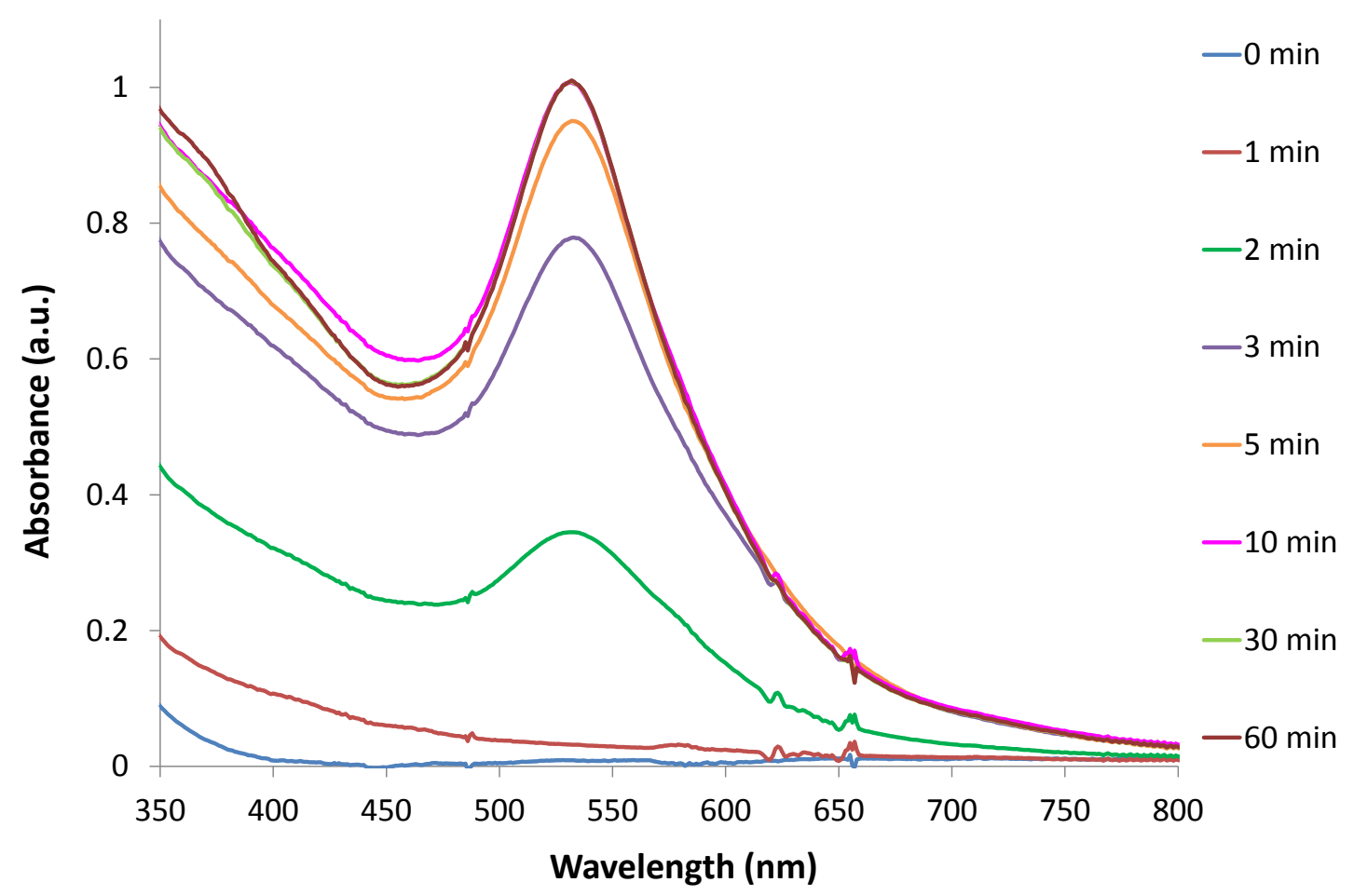

Figure 4.11 UV-vis spectra of the formation of a solution of four times the concentration of the TA-mauve colloid $\left(40 \mathrm{mg} \mathrm{L}^{-1} \mathrm{Au}^{3+}, 300 \mathrm{~mL}\right)$

During the synthesis of TA-Au NPs, $\mathrm{Au}^{3+}$ is reduced to $\mathrm{Au}^{0}$, and $\mathrm{Au}^{0}$ entities coalesce to form nanoparticulate gold that is stabilised by TA. Given that the rate of nanoparticle formation is much slower at this reaction concentration, the absorbances centred around 450 and $580 \mathrm{~nm}$ in the spectra from 0-9 min of the reaction may represent the formation of some anisotropic-shaped $\mathrm{Au}^{0}$ entities or nanocrystals, prior to the formation of nanoparticles. These anisotropic entities would then coalesce to form (predominantly spherical) nanoparticles as the reaction proceeds. 


\section{Formation of TSC-Au NPs}

The formation of TSC-Au NPs over time was also studied with UV-vis spectroscopy. As the synthesis of TSC-Au NPs requires elevated temperatures $\left(95^{\circ} \mathrm{C}\right)$, the formation reaction was undertaken in a quartz cuvette in a thermostatted sample holder which had boiling water pumping around it. Figure 4.12 shows the in-situ formation of a TSC-grey colloid of $3 \mathrm{~mL}$ volume over time.

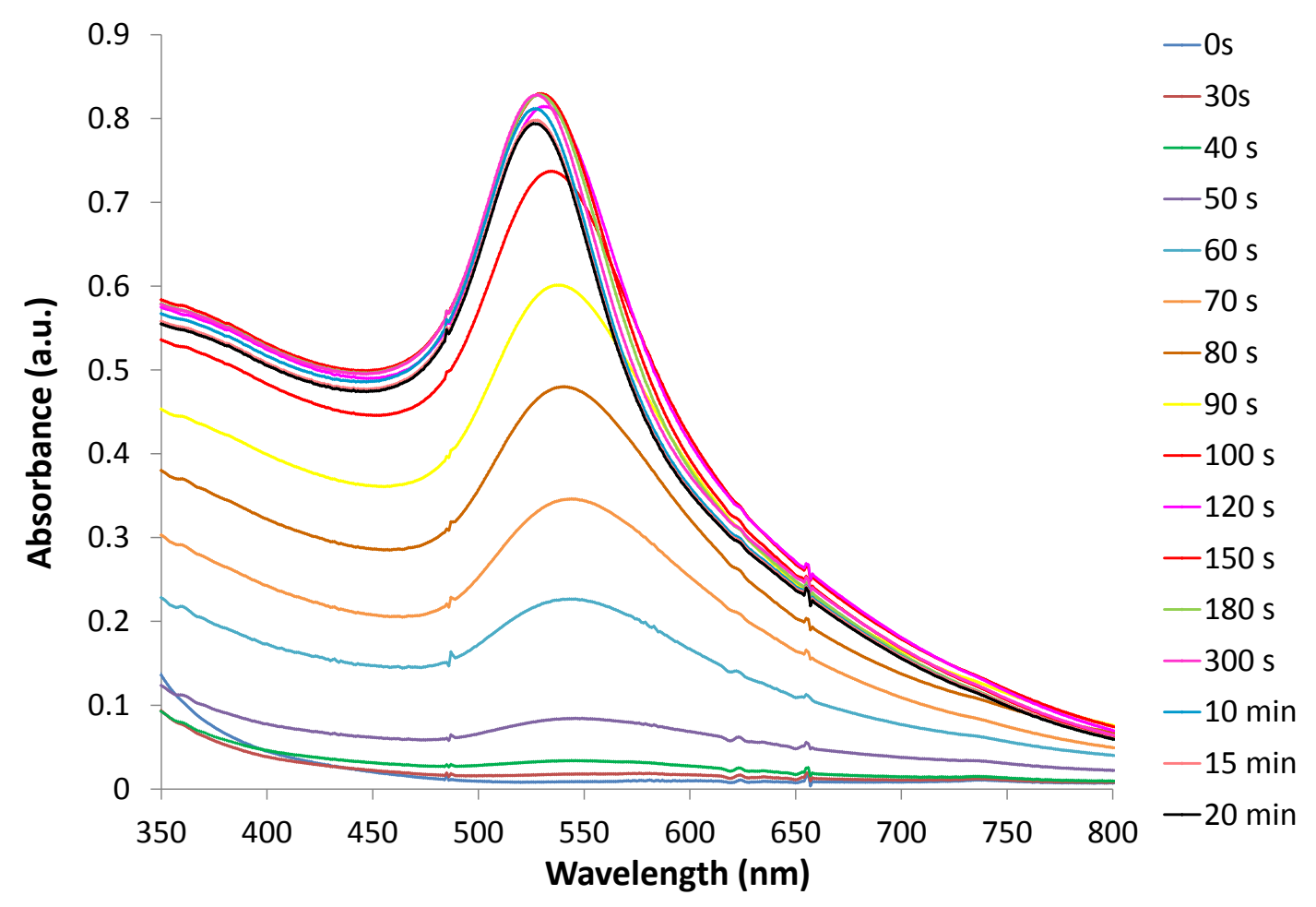

Figure 4.12 UV-vis spectra of the formation of the TSC-grey concentration of TSC-Au NPs $\left(40 \mathrm{mg} \mathrm{L}^{-1} \mathrm{Au}^{3+}, 3 \mathrm{~mL}\right)$ in-situ, where the time of each spectrum is measured after the addition of TSC to a solution of $\mathrm{AuCl}_{4}^{-}$at $95^{\circ} \mathrm{C}$ in a quartz cuvette.

The laboratory-scale synthesis of the TSC-grey colloid (which typically involves colloidal volumes of $60-600 \mathrm{~mL}$ ) involves the formation of a very dark, almost blackcoloured solution after approximately 2-3 min of the reaction. This colour then changes from black to purple, then red over approximately $30 \mathrm{~s}$. Interestingly, the formation of the TSC-grey colloid in Figure 4.12 is rapid, with the SPR peak relating to gold nanoparticles forming after $60 \mathrm{~s}$ of the reaction. The relatively broad absorbance occurring from 30-50 s indicates a possible brief appearance of the black colour that precedes the development of the deep red colloid.

The formation of the TSC-grey colloid is complete after 20 min; subsequent UV-vis spectra showed no further absorbance changes. The SPR peak reaches its maximum 
intensity after $150 \mathrm{~s}$, after which time there is a slight blue-shift (shift to lower absorption wavelength) and intensity loss of the SPR peak. This is an interesting feature, and could possibly be due to the formation of smaller particles of gold near the end of the reaction. The TEM micrographs of TSC-Au NPs in Figure 4.4 showed the presence of very small particles of gold (approximately $2 \mathrm{~nm}$ diameter). The formation of these small particles near the end of the reaction could explain the observable blue-shift of the UV-vis spectrum from $150 \mathrm{~s}-20 \mathrm{~min}$.

The lower intensity of the SPR peak in the 20 min spectrum implies a reduction in the number of nanoparticles in the colloid, and indeed some nanoparticles do coat the UV-vis cuvette as the reaction proceeds. The magnitude of this effect is much lower when larger volumes of TSC-Au NPs are synthesised, due to the lower ratio of liquid volume to glass surface area of larger reaction vessels. An alternative explanation for the reduction in intensity and blue-shift of the SPR peak is the preferential coating of larger nanoparticles on the quartz cuvette, leaving relatively smaller nanoparticles in the solution to be measured by the UV-vis spectrophotometer.

The formation of the TSC-pink colloid was also monitored with UV-vis spectroscopy in a similar study. Figure 4.13 shows the in-situ formation of the TSC-pink concentration of TSC-Au NPs (which is one quarter of the concentration of the TSC-grey colloid). The rate of the TSC-pink colloid formation is expectedly slower than that of the TSC-grey colloid (given the lower concentration). The maximum intensity of the SPR peak is reached after $240 \mathrm{~s}$, and the reaction is complete after $60 \mathrm{~min}$. A relatively broad absorbance develops from approximately $0-100 \mathrm{~s}$, which again may be due to the dark colour that is observed during the synthesis of TSCAu NPs. This broad absorbance then develops into the SPR peak of TSC-Au NPs $\left(\lambda_{\max }=525 \mathrm{~nm}\right)$. Again, the SPR absorbance becomes blue-shifted and less intense from $240 \mathrm{~s}$ to $60 \mathrm{~min}$ of the reaction. The intensity decrease is relatively more pronounced compared to that of the TSC-grey colloid, and again is likely due to the coating of the quartz cuvette with gold nanoparticles.

The formation of the TSC-pink colloid is likely mechanistically equivalent to that of the TSC-grey colloid, however the rate of formation is slower. Figure 4.13 shows the formation of a longer wavelength absorbance from 575-650 nm from 0-160 s. Again, this absorbance is potentially due to the initial formation of a diverse range of different sizes and shapes of gold particles that eventually coalesce to form larger nanoparticles of gold.

Studying the formation of the TA- and TSC-stabilised colloids used in the ex-situ procedure has provided a greater understanding of the chemistry involved in the 


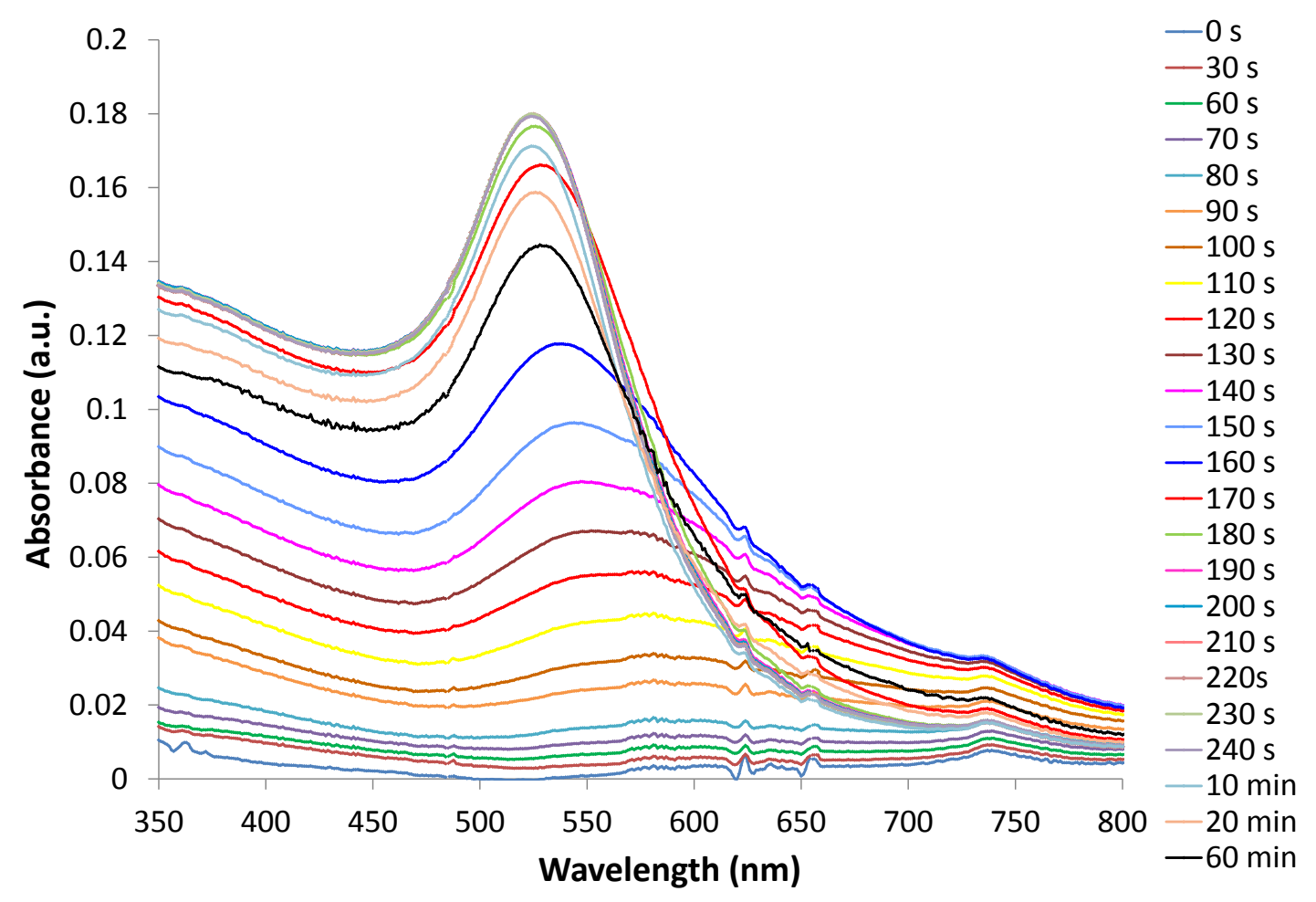

Figure 4.13 UV-vis spectra of the formation of the TSC-pink concentration of TSC-Au NPs $\left(10 \mathrm{mg} \mathrm{L}^{-1} \mathrm{Au}^{3+}, 3 \mathrm{~mL}\right)$ in-situ, where the time of each spectrum is measured after the addition of TSC to a solution of $\mathrm{AuCl}_{4}^{-}$at $95{ }^{\circ} \mathrm{C}$ in a quartz cuvette.

synthesis of these gold colloids. It has also shown that the concentration of the reactants affects the rate of nanoparticle formation. The use of different concentrations of TA-Au NPs and TSC-Au NPs allows for a boutique colour range of gold nanoparticle-wool fibre composites.

\subsection{2 pH Stability of Gold Colloids}

The ex-situ procedure involves the dyeing of wool with gold colloids at a typical $\mathrm{pH}$ range of 2.0-3.0. The reaction $\mathrm{pH}$ that is employed is dependent on the type of wool and colloid involved in the synthesis. As such, the stability of TA- and TSC-stabilised gold colloids were studied across this $\mathrm{pH}$ range.

\section{TA-mauve pH Stability}

The stability of the TA-mauve colloid to changes in $\mathrm{pH}$ was investigated with UV-vis spectroscopy. Figure 4.14 shows the UV-vis spectra of a $300 \mathrm{~mL}$ TA-mauve colloid as the $\mathrm{pH}$ is lowered from 3.0-1.4, where the UV-vis spectra were measured $30 \mathrm{~min}$ 
after the $\mathrm{pH}$ adjustment. There is only a slight reduction in the intensity of the SPR peak $\left(\lambda_{\max }=530 \mathrm{~nm}\right)$ of TA-Au NPs upon lowering the $\mathrm{pH}$ from 3.0-2.2. Adjusting the $\mathrm{pH}$ from $2.2-1.4$ causes a slight red-shift and a more pronounced reduction in intensity of the SPR peak. This is coupled with a slight increase in longer wavelength absorption at around $650 \mathrm{~nm}$. Lowering the $\mathrm{pH}$ of the TA-mauve colloid results in a slight destabilisation of the colloid, however the effect is minimal for the $\mathrm{pH}$ range typically employed in the ex-situ procedure ( $\mathrm{pH} 2.0-3.0)$.

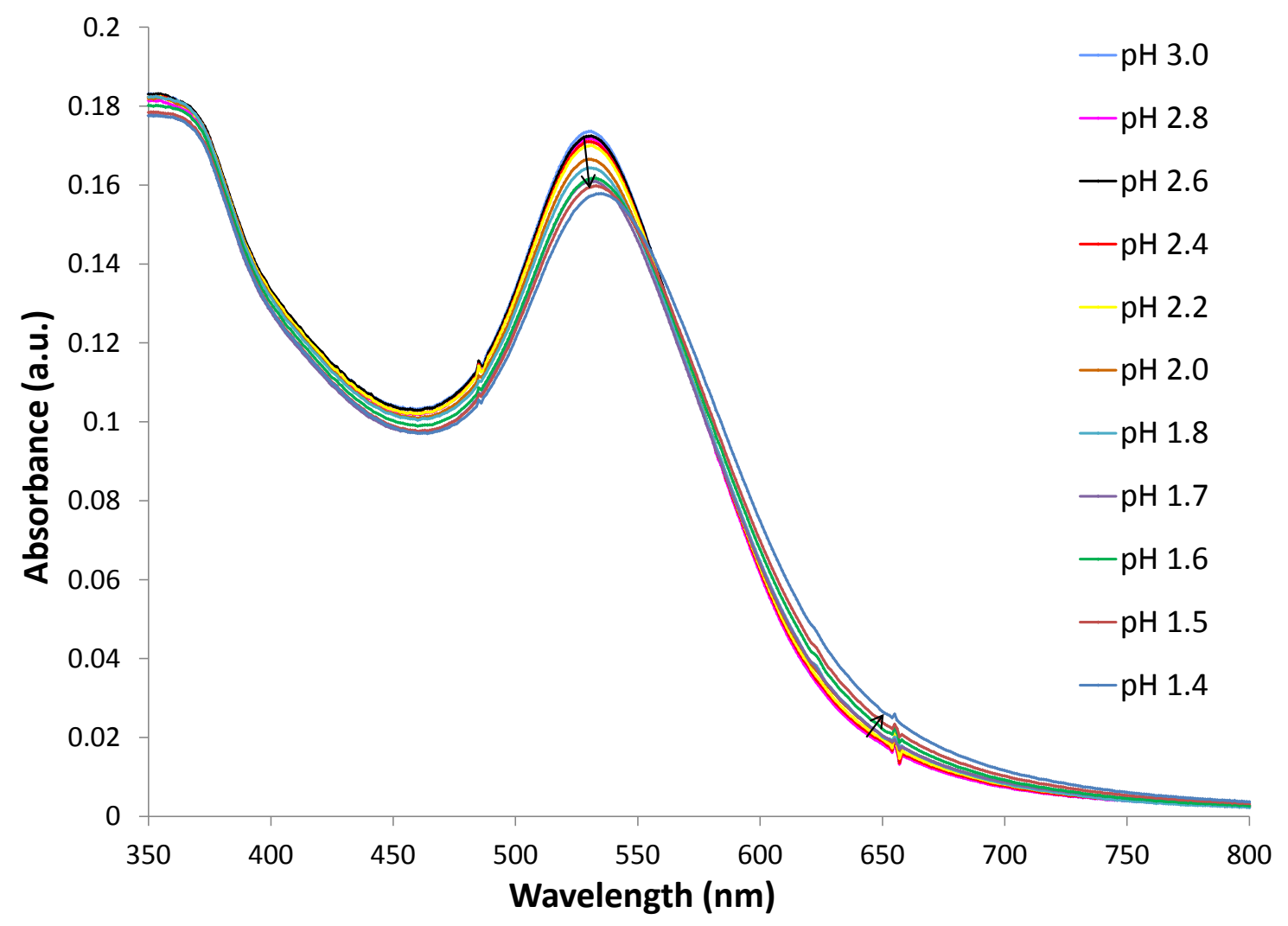

Figure 4.14 UV-vis spectra of TA-mauve colloid illustrating the stability upon $\mathrm{pH}$ lowering with $\mathrm{HCl}$ addition, measured 30 min after the $\mathrm{pH}$ adjustment.

Throughout the TA-mauve syntheses undertaken in this research, no colour changes of the colloid have been observed when the $\mathrm{pH}$ is adjusted prior to wool dyeing. This is consistent with the $\mathrm{pH}$ stability of TA-Au NPs that is observed in Figure 4.14, and highlights the strong stabilising nature of TA.

\section{TSC-pink pH Stability}

The TSC-pink method involves the uptake of TSC-Au NPs to wool fibres at a reaction $\mathrm{pH}$ of 2.6-2.8. The UV-vis spectra of a TSC-pink colloid from $\mathrm{pH}$ 3.6-2.0 are shown in Figure 4.15, where the UV-vis spectra are measured $30 \mathrm{~min}$ after the $\mathrm{pH}$ adjustment with $\mathrm{HCl}$ addition. The TSC-pink colloid forms at a reaction $\mathrm{pH}$ of 
3.6, and lowering the $\mathrm{pH}$ of the colloid to just $\mathrm{pH} 3.3$ causes an observable reduction in intensity of the SPR peak at approximately $525 \mathrm{~nm}$. Further lowering of the $\mathrm{pH}$ to 2.6 causes a more significant loss of the intensity of the SPR peak, and an accompanying increase in the longer wavelength absorbance from 600-800 nm. This indicates the destabilisation of the TSC-pink colloid, as the TSC surrounding the gold nanoparticles becomes less negatively charged and hence less electrostatically repulsive, and the nanoparticles begin to aggregate together.

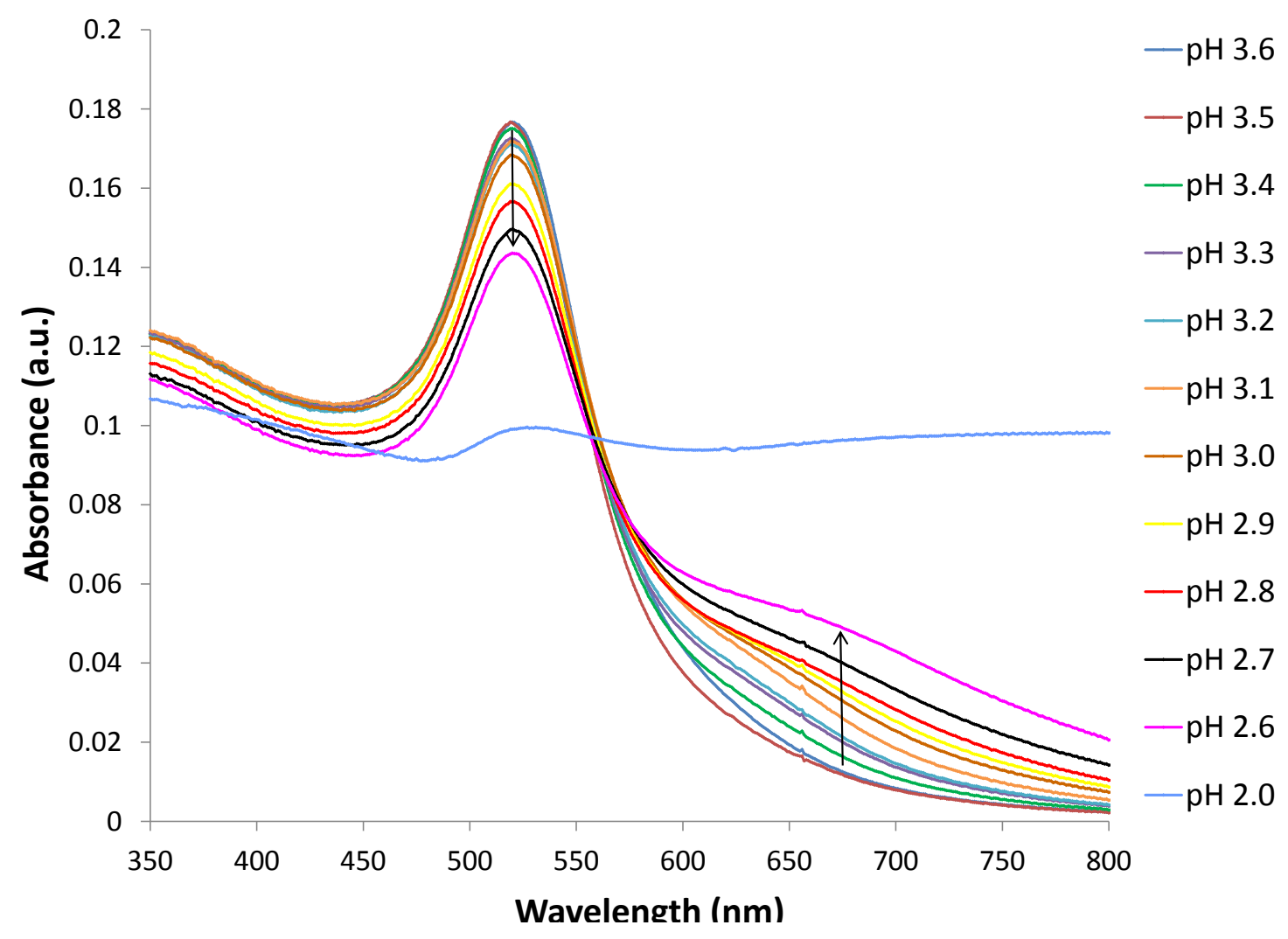

Figure 4.15 UV-vis spectra of a TSC-pink colloid when the $\mathrm{pH}$ is lowered from $\mathrm{pH} 3.6-2.0$ with $\mathrm{HCl}$ addition. The UV-vis spectra are measured $30 \mathrm{~min}$ after the $\mathrm{pH}$ adjustment.

The significant destabilisation of the TSC-pink colloid that occurs at the uptake $\mathrm{pH}$ of this method (2.6-2.8) may explain the agglomerates of TSC-Au NPs observed on the surface of the relatively "dark" Cariaggi TSC-pink composite fibres (as seen in the SEM micrographs in Figure 4.7). Additionally, when the $\mathrm{pH}$ of the TSC-pink colloid is lowered to 2.0 (the uptake $\mathrm{pH}$ commonly used in the TSC-grey method), a broad absorbance results, where the intensity of the original SPR peak at $525 \mathrm{~nm}$ and the intensity of the $600-800 \mathrm{~nm}$ absorbance are approximately equivalent.

In an attempt to prevent the destabilisation of the TSC-pink colloid that occurs at the uptake $\mathrm{pH}$ of 2.6-2.8, poly(methyl acrylate) (PMA) was employed in the synthesis of the TSC-pink colloid. The structure of PMA is shown in Figure 4.16. 
PMA (26 mg L $^{-1}$ ) was added to the TSC-pink colloid 3 min after the addition of TSC. This was carried out to provide additional steric repulsion to attempt to prevent the aggregation of the formed nanoparticles.

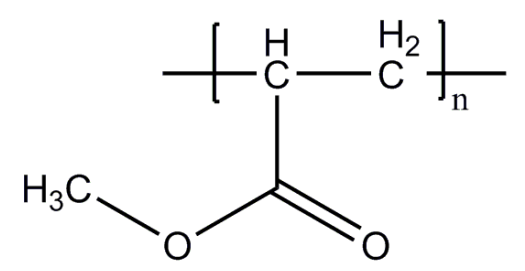

Figure 4.16 The chemical structure of PMA.

Figure 4.17 shows the UV-vis spectrum of the TSC-pink colloid synthesised with PMA and shows the changes that occur upon $\mathrm{pH}$ adjustment via $\mathrm{HCl}$ addition. The presence of PMA in the TSC-pink colloid resulted in a higher formation $\mathrm{pH}$ of 4.6, and lowering the $\mathrm{pH}$ of the colloid to 3.0 causes only a slight reduction in the intensity of the SPR peak. Further lowering of the pH to 2.6 however causes a red-shift of the SPR peak to approximately $600 \mathrm{~nm}$ and significant broadening of the SPR peak. This is in stark contrast to when no PMA is employed in the synthesis, which results in the reduction of the initial SPR peak and the growth of a longer wavelength absorption simultaneously, without shifting the maximum absorption wavelength of the original SPR peak. 


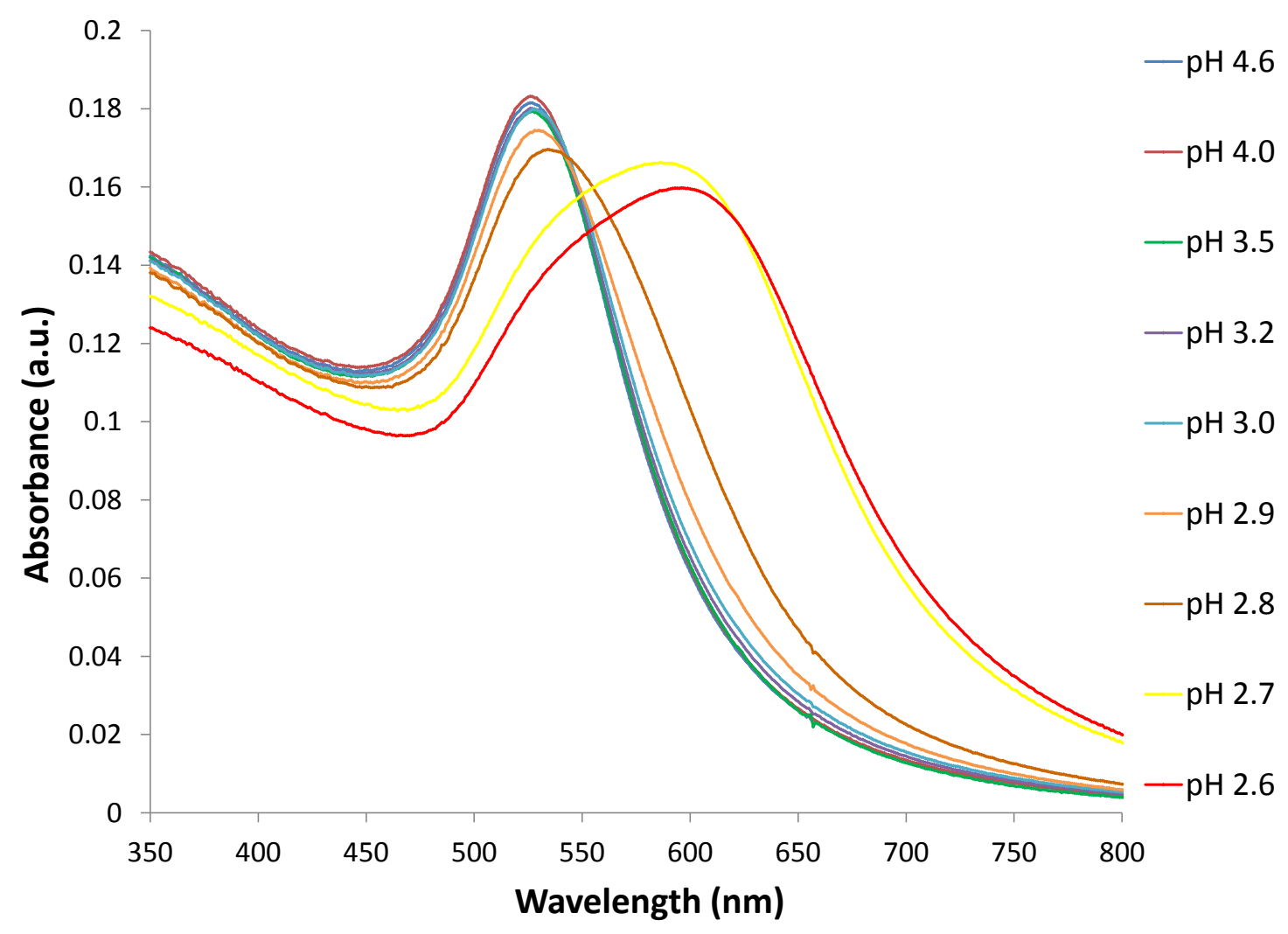

Figure 4.17 UV-vis spectra of a TSC-pink colloid synthesised with the addition of PMA in the colloid and the $\mathrm{pH}$ is lowered from 4.6-2.6. The UV-vis spectra are measured $30 \mathrm{~min}$ after the $\mathrm{pH}$ adjustment.

TSC-pink colloids synthesised with and without PMA in the solution were used to dye Cariaggi wool. The TSC-pink Cariaggi composites are shown in Figure 4.18. The composite synthesised without PMA is the typical pink-purple hue observed in the TSC-pink method and the PMA-TSC-pink composite is more purple coloured.

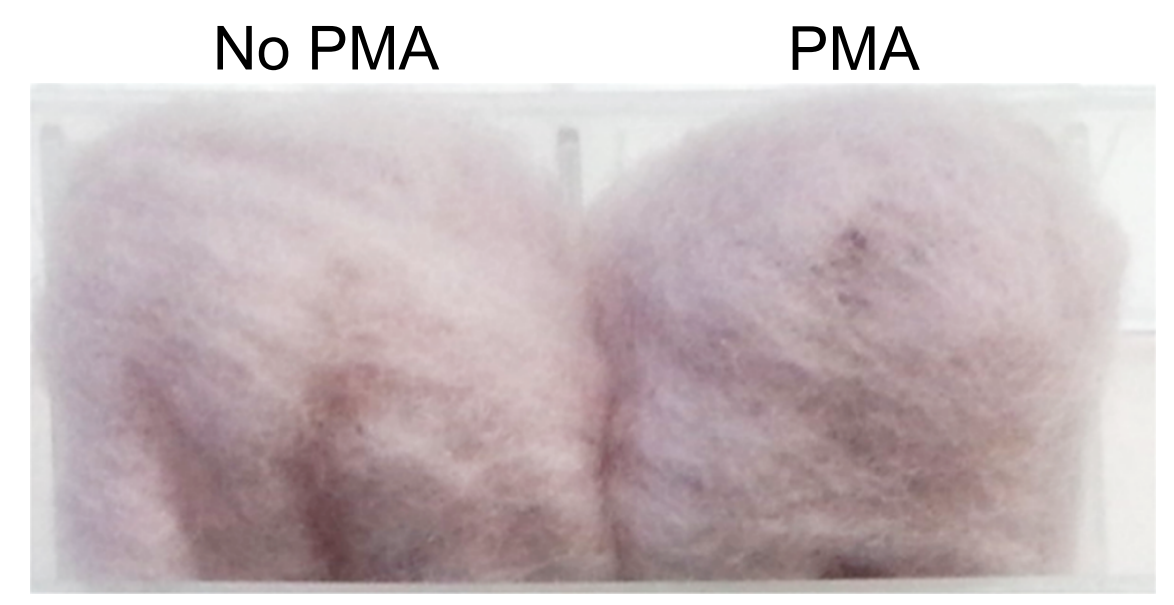

Figure 4.18 TSC-pink Cariaggi composites synthesised with and without PMA in the colloid. 
CIE colour coordinates of the TSC-pink composites with and without PMA in the colloid are shown in Table 4.3. The brightness values are very similar, however the PMA composite exhibits lower $\mathrm{a}^{*}$ and $\mathrm{b}^{*}$ values, which is indicative of a more purple colour. As such, the addition of PMA into the synthesis of the TSC-pink colloid has resulted in more purple-coloured composites.

Table 4.3 CIE colour coordinates of TA-mauve composites of different wool types.

\begin{tabular}{ccc}
\hline Method & No PMA & PMA \\
\hline $\mathrm{L}^{*}$ & 69.80 & 69.96 \\
$\mathrm{a}^{*}$ & 8.03 & 6.80 \\
$\mathrm{~b}^{*}$ & -0.53 & -1.13 \\
\hline \hline
\end{tabular}

SEM analysis of the TSC-pink Cariaggi composites was undertaken to investigate the effect of PMA on the distribution of gold nanoparticles across the composite fibres. Figure 4.19 shows SEM micrographs of TSC-pink Cariaggi composites synthesised with and without PMA in the colloid. The SEM micrographs of the TSC-pink composites synthesised without PMA (Figure 4.19 (a) and (b)) were also previously shown in Figure 4.7, where there were relatively light and dark composite fibres with different levels of gold nanoparticles on their surfaces.

The TSC-pink composite fibres synthesised with PMA (Figure 4.19 (c)-(f)) appear to exhibit a relatively higher proportion of agglomerates of gold nanoparticles compared to individual nanoparticles. The higher magnification images (Figure 4.19 (e) and (f)) predominantly show agglomerates of gold nanoparticles. The gold distribution of the PMA-containing composites appears to be similar to that of the "dark" fibres synthesised without PMA, which is shown in Figure 4.19 (b). As such, the relatively high proportion of agglomerates on the PMA-containing TSC-pink composite explains the relatively purple colouration of the composite.

The SEM micrographs have indicated a greater propensity for the gold nanoparticles to aggregate when PMA is present in the colloid. The agglomeration of TSC-Au NPs was also represented in the red-shift and broadening of the UV-vis spectrum of the PMA containing colloid. PMA has therefore caused the agglomeration of TSC-Au NPs, rather than preventing it, and as such, PMA not incorporated into future syntheses of the TSC-pink colloid. 


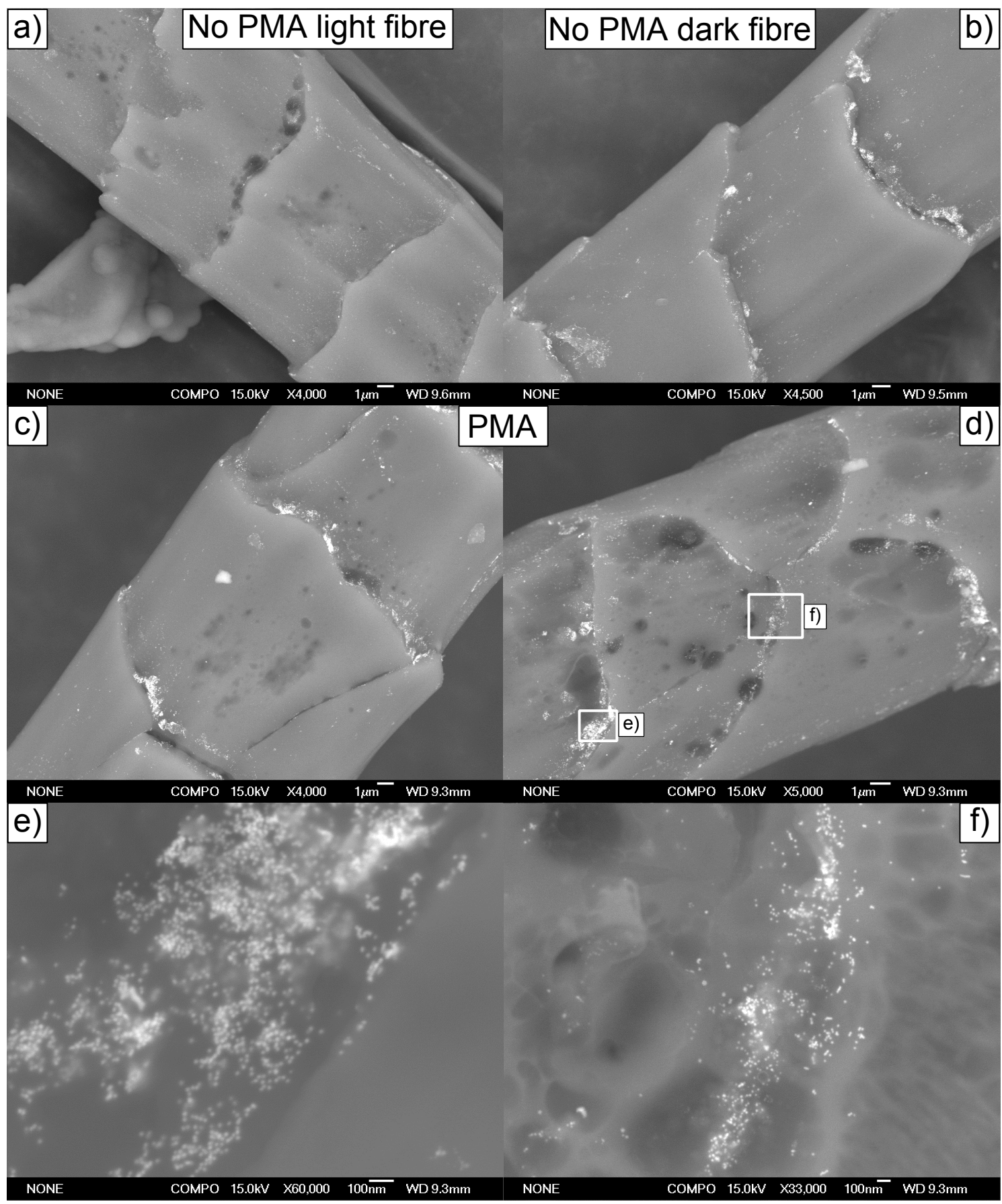

Figure 4.19 SEM micrographs of TSC-pink Cariaggi composites synthesised without PMA that are relatively light (a) and dark (b), and with PMA (c)-(f), where (e) and (f) are higher magnification images of the white square areas in (d).

\section{TSC-grey pH Stability}

The stability of the TSC-grey colloid to $\mathrm{pH}$ changes was also studied with UV-vis spectroscopy. Figure 4.20 shows the changes in the UV-vis spectra of a TSC-grey colloid as the $\mathrm{pH}$ is lowered from $\mathrm{pH}$ 3.0-1.4. The UV-vis spectra are measured 2 min after the $\mathrm{pH}$ adjustment, a time scale that is representative of the time it takes to immerse the wool in the dye solution. Interestingly, there is only a small 
reduction in the SPR peak when the UV-vis spectra are measured 2 min after $\mathrm{pH}$ adjustment from $\mathrm{pH}$ 3.0-2.0 (which encompasses the $\mathrm{pH}$ range that wool fibres are dyed at in the TSC-grey method). There is also an accompanying marginal increase in the longer wavelength absorption of the UV-vis spectra during the adjustment of $\mathrm{pH}$ 3.0-2.0. A more substantially reduction in the SPR peak (and simultaneous increase in the longer wavelength absorbance) occurs when the $\mathrm{pH}$ of the TSC-grey colloid is lowered from 2.0-1.4. This indicates the increasing agglomeration of TSC$\mathrm{Au}$ NPs as the colloid steadily destabilises due to acid addition. If left for longer at a particular $\mathrm{pH}$, the agglomeration continues.

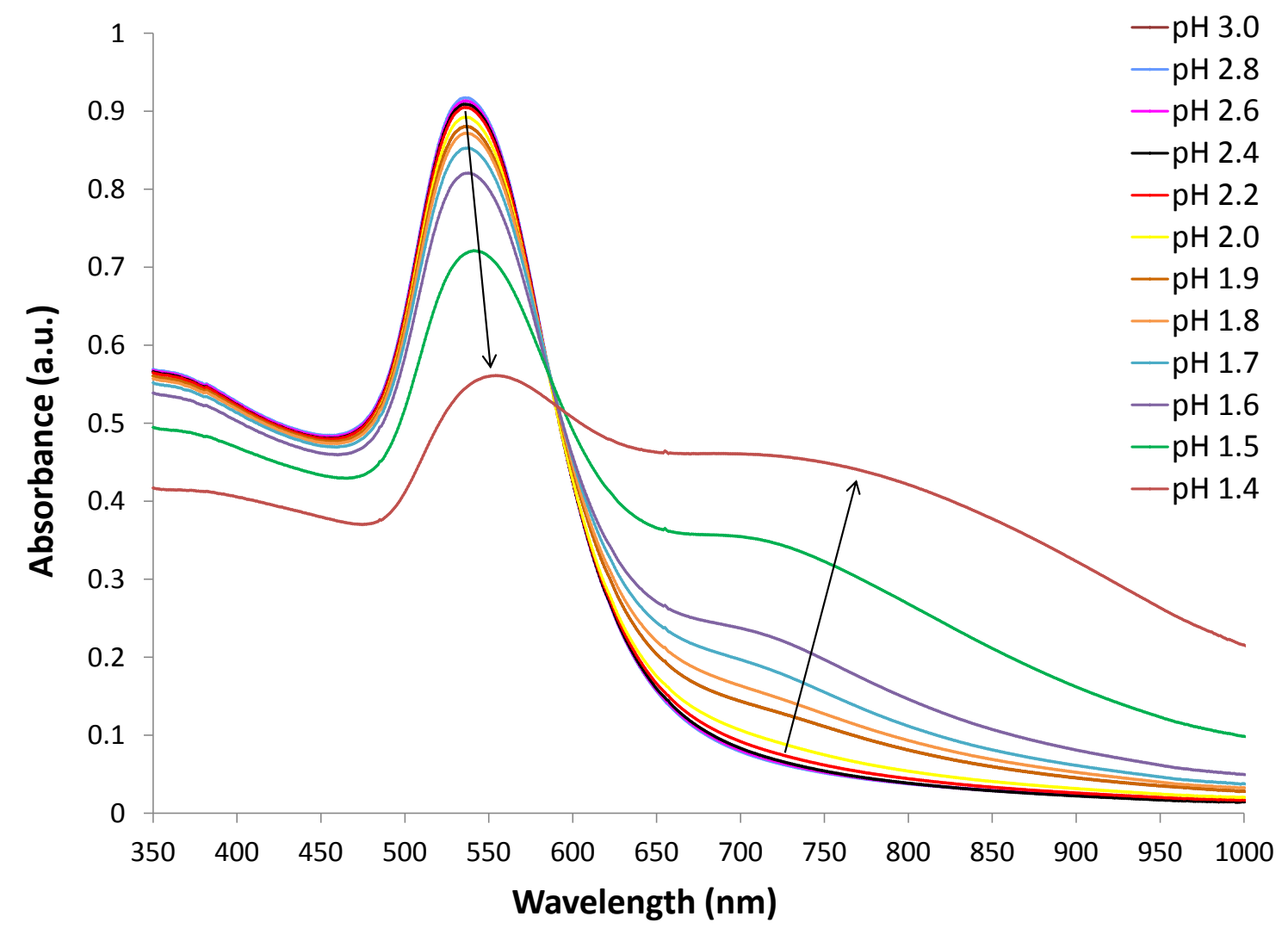

Figure 4.20 UV-vis spectra of a TSC-grey colloid upon lowering the $\mathrm{pH}$ from 3.0-1.4 with $\mathrm{HCl}$ addition. The $\mathrm{UV}$-vis spectra were measured $2 \mathrm{~min}$ after the $\mathrm{pH}$ adjustment.

Immersing wool in a TSC-stabilised gold colloid increases the rate of agglomeration of TSC-Au NPs at a given pH. In the TSC-pink method, the colloid typically becomes more purple over time, and in the TSC-grey method the colloid colour changes from purple to grey throughout the dyeing process. As the TSC-Au NPs are absorbed by the wool fibres, the local concentration of nanoparticles in solution decreases. This occurrence, with all other factors remaining constant, should increase the stability of the colloid (due to fewer collisions between gold nanoparticles and increasing the Debye-Huckel length between nanoparticles). However, the immersed wool may leach salts, lipids, processing lubricants or chemical reagents (that 
were applied in a pre-dying stage). This would likely destabilise the TSC-Au NPs (for example by increasing the counter ion concentration which reduces colloidal stability). Additionally, excess stabilising TSC in the colloid may be absorbed by the wool, which would most likely destabilise the nanoparticles. As such, controlling the agglomeration of TSC-Au NPs that the TSC-grey method relies upon is a recurring and often difficult issue faced in this research.

\subsubsection{Ratio of Gold to Stabilising Agents}

The effect of the ratio of $\mathrm{Au}$ to TA or TSC employed in the synthesis of gold nanoparticles was investigated with UV-vis spectroscopy, to optimise the amount of TA and TSC that would be employed in scale-up syntheses.

\section{TA-mauve Colloid}

In order to ascertain the reason for the peak at approximately $360 \mathrm{~nm}$ in the UV-vis spectra of TA-Au NPs, the relationship between the amount of TA and Au used in the synthesis was investigated. In this, the synthesis of TA-Au NPs was undertaken with a lower concentration of TA, while retaining the typical concentration of $\mathrm{AuCl}_{4}^{-}$ $\left(10 \mathrm{mg} \mathrm{L}^{-1}\right)$. The $\mathrm{AuCl}_{4}^{-}$:TA ratios that were employed are listed in Table 4.4.

Table 4.4 The different concentration ratios of $\mathrm{AuCl}_{4}^{-}$to $\mathrm{TA}$ that were used in the synthesis of TA-Au NPs.

\begin{tabular}{cc}
\hline TA amount & Molar ratio of $\mathrm{AuCl}_{4}^{-}$to TA \\
\hline Regular TA & $1: 0.386$ \\
1/2 TA & $1: 0.193$ \\
1/4 TA & $1: 0.0965$ \\
$1 / 6$ TA & $1: 0.0643$ \\
$1 / 8$ TA & $1: 0.0483$ \\
\hline \hline
\end{tabular}

Figure 4.21 shows the UV-vis spectra of TA-stabilised gold nanoparticles with the above molar ratios of $\mathrm{AuCl}_{4}^{-}$:TA. Reducing the amount of TA from the regular amount to $1 / 2$ and then $1 / 4$ causes the sequential reduction in the peak at approximately $360 \mathrm{~nm}$. This indicates that the $360 \mathrm{~nm}$ peak is due to the interaction between excess TA and the gold nanoparticles. One possible explanation is the formation of a charge-transfer state between the conduction band electrons of the gold nanoparticles and the stabilising TA (the UV-vis spectrum of TA alone does not exhibit this absorbance). 


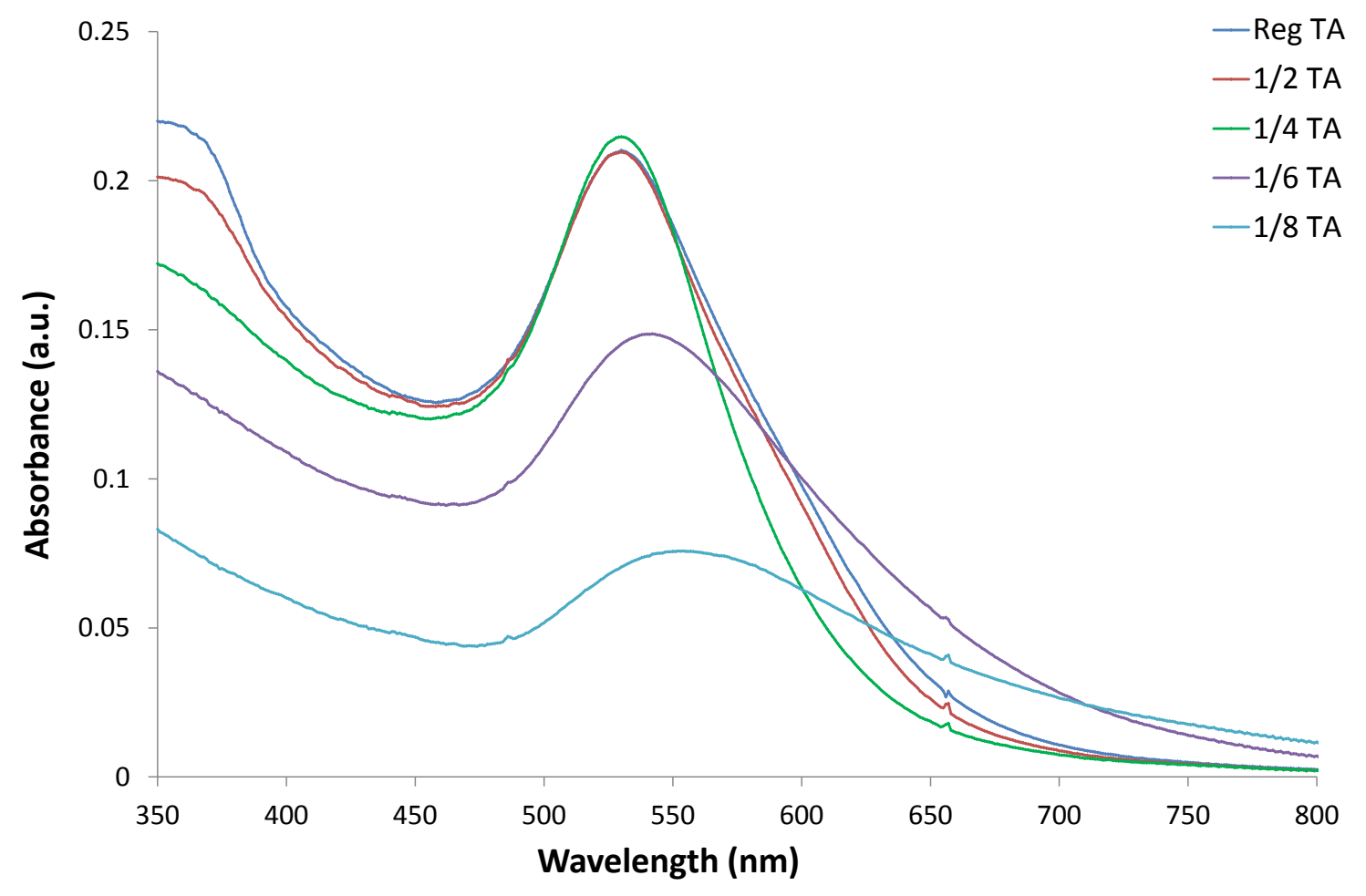

Figure 4.21 UV-vis spectra of TA-stabilised colloids with different ratios of $\mathrm{Au}: \mathrm{TA}$.

Interestingly, a reduction in the broadness of the SPR peak also occurs when the amount of TA is reduced from the regular amount to $1 / 4 \mathrm{TA}$, indicating the formation of a more monodisperse distribution of sizes and shapes of gold nanoparticles in the colloid. A further reduction in the amount of TA to $1 / 6$ or $1 / 8$ results in the formation of a less intense and broader SPR peak, as a much more polydisperse range of gold nanoparticles form, as there is insufficient TA in the solution to form a stable colloid. This experiment has shown that the optimal amount of TA in the synthesis of the TA-mauve colloid may be $1 / 4$ of that which is typically employed.

\section{TSC-grey Colloid}

The ratio of Au:TSC used in the syntheses of the TSC-pink and TSC-grey colloids was optimised by Lucas and Johnston, ${ }^{5}$ where the ratio of 1:2.09 of Au:TSC was utilised. Lucas found that lesser amounts of TSC produced unstable or agglomerated colloids. However, increasing the amount of TSC used in the synthesis of TSC-Au NPs had not yet been investigated.

Using larger amounts of TSC in the synthesis of the TSC-grey colloid was investigated due to difficulties encountered in scale-up procedures. When $5 \mathrm{~L}$ volumes of the TSC-grey colloid were synthesised, a destabilised colloid would sometimes form. 
This implied that the reaction conditions had a narrow range of success, and any change in mixing/heating efficiencies could prevent the formation of a stable colloid. As such, the different molar ratios of $\mathrm{AuCl}_{4}^{-}$to TSC that are shown in Table 4.5 were employed in the synthesis of the TSC-grey colloid, to attempt to increase the stability of the formed nanoparticles.

Table 4.5 The different concentration ratios of $\mathrm{AuCl}_{4}^{-}$to TSC that were used in the synthesis of TSC-Au NPs.

\begin{tabular}{cc}
\hline Amount of TSC & Molar ratio of $\mathrm{AuCl}_{4}^{-}$to TSC \\
\hline Regular TSC & $1: 2.09$ \\
2x TSC & $1: 4.18$ \\
4x TSC & $1: 8.36$ \\
6x TSC & $1: 12.54$ \\
8x TSC & $1: 16.72$ \\
\hline \hline
\end{tabular}

UV-vis spectra of TSC-grey colloids synthesised with different amounts of TSC in the reaction are presented in Figure 4.22. Interestingly, changing the amount of TSC in the synthesis has only marginally affected the shape of the UV-vis spectra of the colloids. Increasing the TSC amount to four or six times has resulted in a narrower SPR peak, with a slight reduction in the relatively long wavelength absorbance from $575-700 \mathrm{~nm}$. This indicates the formation of a more monodisperse distribution of the sizes and shapes of nanoparticles when four and six times the TSC is used. Using eight times the TSC amount causes a slight increase in the absorbance in the 575-700 nm wavelength range.

This experiment showed that higher amounts of TSC could be used in the synthesis of the TSC-grey colloid, and that manipulating the Au:TSC ratio can influence the polydispersity of the formed nanoparticles. Large-scale volumes of TSC-Au NPs were synthesised with higher amounts of TSC in scale-up procedures with enhanced reproducibly (Section 5.1.2) because of this research. 


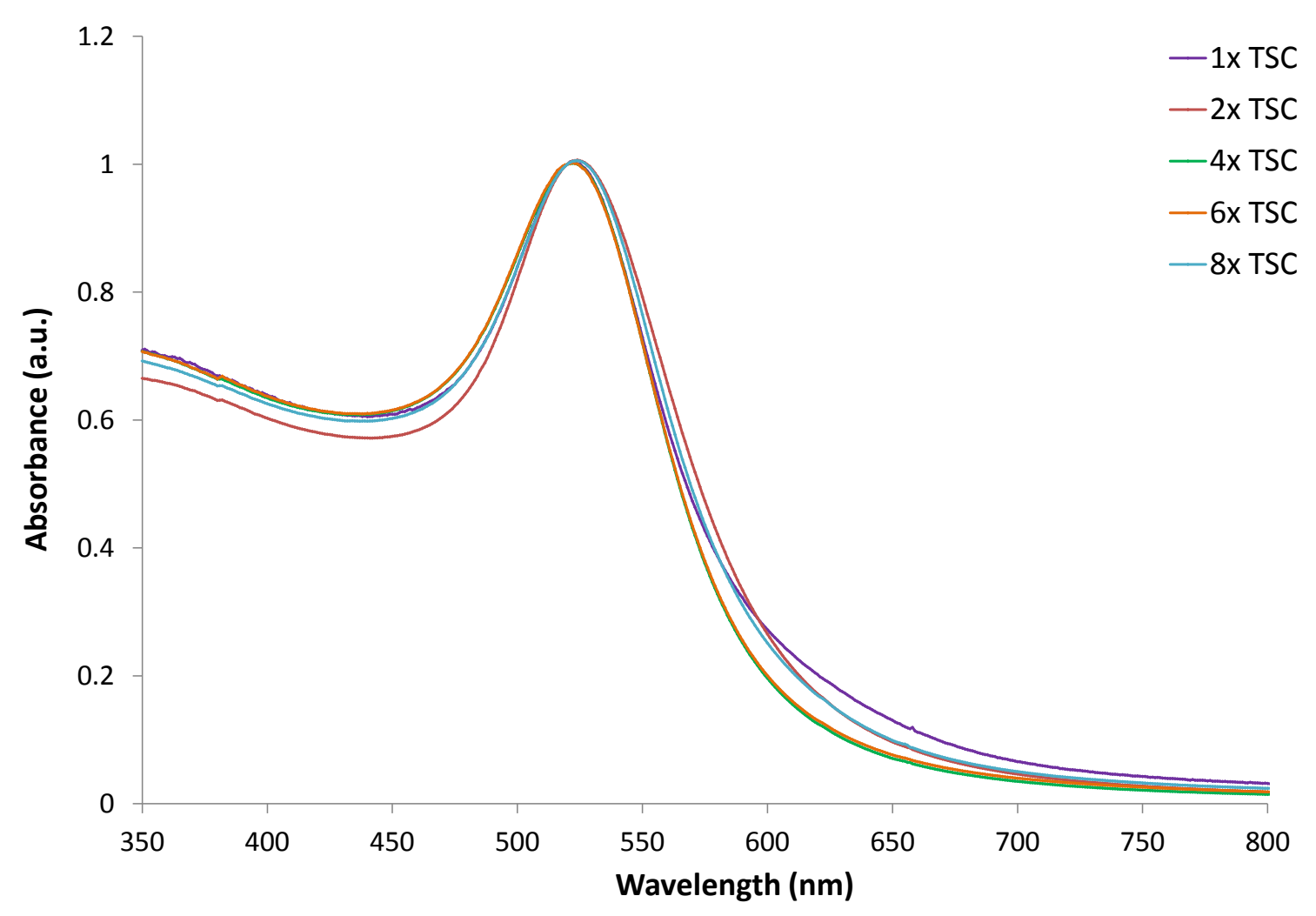

Figure 4.22 UV-vis spectra of TSC-grey colloids synthesised with higher amounts of TSC in the reaction solution.

\subsection{Dyeing Wool with Gold Nanoparticles}

This section encompasses the second-step of the ex-situ procedure; dyeing wool with gold nanoparticles. Manipulating the reaction conditions can affect both the rate of the uptake of gold nanoparticles to wool and the colouration of the resulting composites. One of the aims of this research is to slow the rate of uptake of gold nanoparticles to wool while not adversely affecting the colour of the composites. This is integral to scaling-up the ex-situ procedure, as the rate of uptake is directly related to the colour uniformity of large quantities of composites. The following reaction parameters are investigated in this section:

- Wool type

- $\mathrm{pH}$

- Auxiliary agents

- Concentration of gold nanoparticles

- Volume of dye solution 
The above reaction conditions are synonymous with those investigated in the in-situ Chapter 3 with the exception of temperature, as an increase in temperature is known to cause an increase in the rate of uptake. Additionally, the cooling of large-scale dyeing reactions is not feasible.

\subsubsection{Wool Type}

The type of wool used in the ex-situ procedure is important for the same reasons as for the in-situ procedure; it affects both the uptake of the gold dye (in this case gold nanoparticles) to the wool and the colour of the resulting composites. The wool fibre thickness (and hence effective surface area of a given mass of wool), influences the colour of the composites. Possible wool fibre pre-treatments also influence the hue and colour uniformity of the composites. One difference to the in-situ procedure is that as the gold nanoparticles do not penetrate the exterior of the wool fibres in the ex-situ procedure, the relative porosity of the fibres is less important.

To illustrate the effect of wool type on the ex-situ procedure, a range of loose wool, wool in top form and merino yarns were used in the ex-situ procedure. One of the goals of the following studies was to deduce the optimal form of wool to be used in scale-up syntheses of gold nanoparticle-wool fibre composites.

\section{Ex-situ Composites of Wool in Top Form and Woollen Yarn}

A study of the effect of wool type on the chemistry of the TA-mauve procedure was carried out with top form wool and an untreated yarn. The wool types that were employed are listed in Table 4.6, and were also used extensively in the synthesis of in-situ composites.

Table 4.6 The different wool types used in ex-situ optimisation experiments.

\begin{tabular}{cc}
\hline Wool type & Approximate fibre diameter $(\mu \mathrm{m})$ \\
\hline Cariaggi & 16 \\
Ashford & 22 \\
Crossbred & 38 \\
Tararua untreated yarn (TU) & 22 \\
\hline \hline
\end{tabular}

Figure 4.23 shows the uptake of the TA-mauve colloid to the different wool types over time. The typical conditions of the TA-mauve synthesis were used. These are pre-soaking the wool in a $\mathrm{pH} 2.2$ glycine buffer $\left(0.1 \mathrm{~mol} \mathrm{~L}^{-1}, 1: 60\right.$ wool to liquor 
ratio, $120 \mathrm{~min}$ ), followed by immersing the wool in the TA-mauve colloid (also at $\mathrm{pH}$ 2.2). The uptake of TA-Au NPs to each wool type is rapid and the uptake curves are all of a similar shape. The uptake approximately complete for all wool types after 120 min. Similar to the in-situ uptake, the rate of uptake decreases as the reaction proceeds. This indicates the initial rapid absorption of the colloid at the beginning of the uptake until the wool fibre surfaces are saturated, followed by the gradual electrostatic bonding of the gold nanoparticles to the wool fibres.

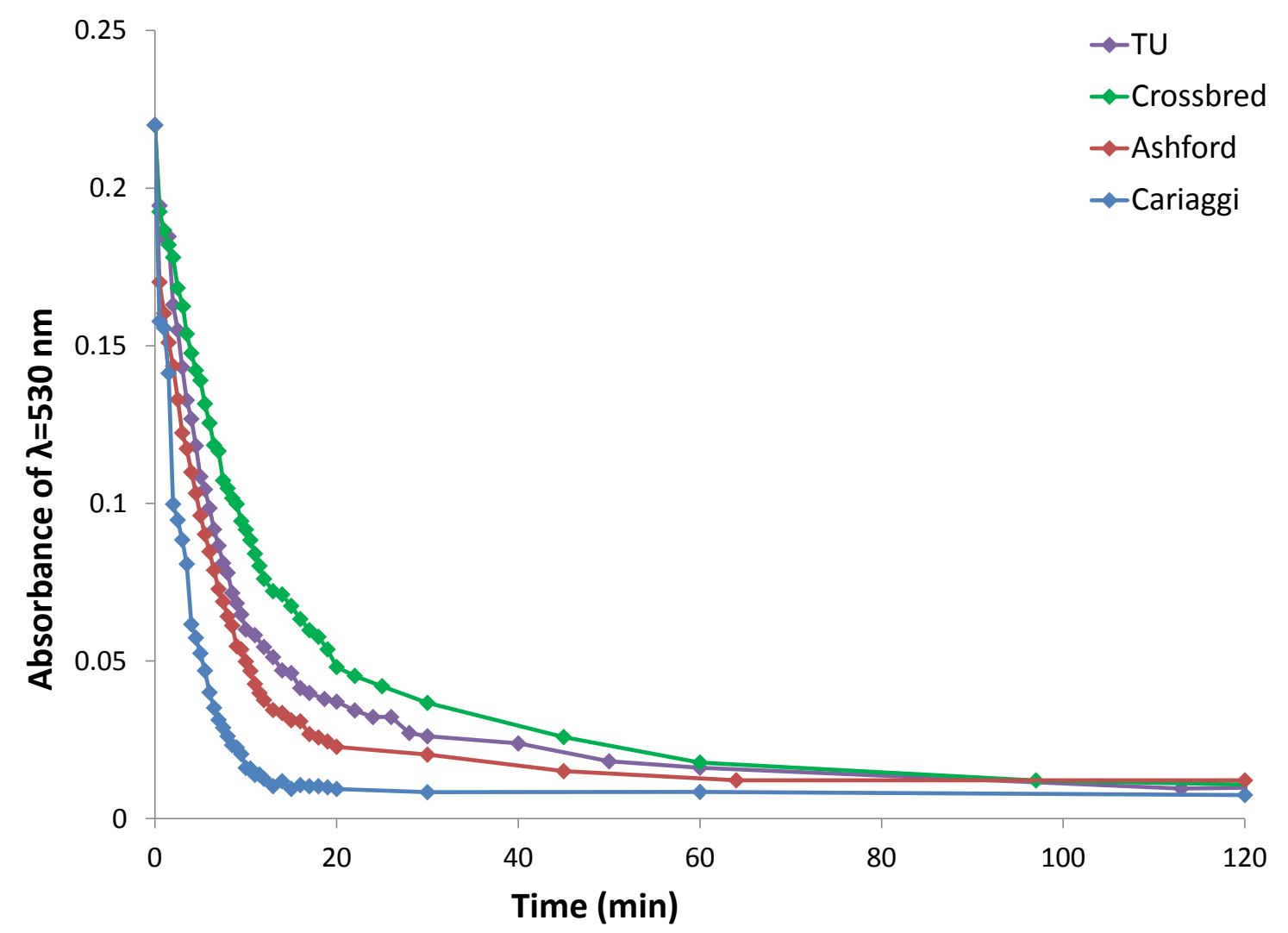

Figure 4.23 Uptake of the TA-mauve colloid to different wool types.

Cariaggi wool exhibits the fastest absorption, with the uptake approximately complete after $15 \mathrm{~min}$ of the reaction. The rate of uptake is sequentially slower for the Ashford and Crossbred wool fibres. This shows the expected general decrease in the rate of uptake of TA-Au NPs to top form wool types with larger fibre diameters (and hence lower effective surface areas available for TA-Au NPs absorption). The uptake of TA-Au NPs to TU yarn is slightly slower than the uptake to Ashford wool. This is likely due to the twisting structure of the yarn restricting access of gold nanoparticles to the surface of the wool, as Ashford and TU have approximately equivalent fibre diameters.

The composites that resulted at the completion of the uptake shown in Figure 4.23 are presented in Figure 4.24. The composites are different shades of purple-mauve 
in colour, where the thicker wool fibres are darker shades. The colouration of the TU composite is relatively uneven; there are completely white areas of yarn. This illustrates the difficulty of dying yarn samples without auxiliary agents, and will be elaborated on in Section 4.3.3.

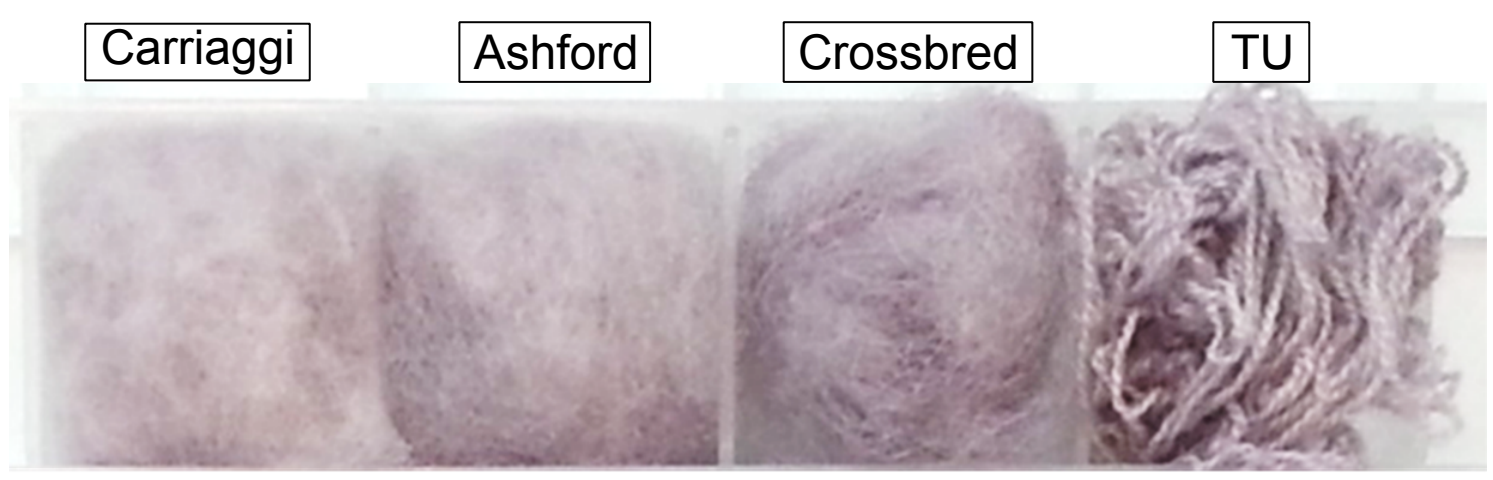

Figure 4.24 TA-mauve composites of different wool types.

Table 4.7 presents the CIE colour coordinates of the TA-mauve composites of different wool types. Each of the composites exhibit relatively similar $a^{*}$ and $b^{*}$ values compared to that which is typically observed for in-situ composites of different wool types. This represents the relative robustness of the colours achieved with the exsitu procedure compared to the in-situ procedure (in Section 3.2.1 the top form wool in-situ composites were purple and the TU yarn composites were grey coloured).

Table 4.7 CIE colour coordinates of TA-mauve composites of different wool types.

\begin{tabular}{ccccc}
\hline Wool type & Cariaggi & Ashford & Crossbred & TU \\
\hline L* (brightness) $^{*} 65.79$ & 62.12 & 57.14 & 56.14 \\
$\mathrm{a}^{*}$ (redness) & 6.39 & 6.24 & 5.88 & 5.28 \\
$\mathrm{~b}^{*}$ (yellowness) & -0.88 & -1.56 & -1.60 & -2.30 \\
\hline \hline
\end{tabular}

However, the wool type has significantly affected the intensity of the purple-mauve colouration of the composites. There is a decrease in the $\mathrm{L}^{*}, \mathrm{a}^{*}$ and $\mathrm{b}^{*}$ values of the composites of thicker top form wool fibres. Although Ashford and TU have similar fibre diameters, the TU composite is darker, less red and bluer than the Ashford composites, illustrating the relatively dark purple (and uneven) colour of the TU composite. This is likely due to the structure of the TU yarn facilitating preferential gold nanoparticle uptake to the more easily accessible areas of the yarn. 


\section{Loose Wool Ex-situ Composites}

Three sources of loose wool were provided by Professor James Johnston's industry contacts with fibre diameters of approximately 15, 27 and $36 \mu \mathrm{m}$. The TA-mauve and TSC-grey methods were used to synthesise composites of these loose wool types to access the feasibility of dyeing this type of wool. This further investigates potential colour shade and uniformity differences attained from using different wool types in the ex-situ procedure.

The TA-mauve composites were synthesised using the standard TA-mauve method; pre-soaking a glycine buffer $\left(0.1 \mathrm{~mol} \mathrm{~L}^{-1}, \mathrm{pH} 2.2,120 \mathrm{~min}\right)$ prior to immersion in the colloid (also at pH 2.2). The TSC-grey composites were synthesised without the use of a buffer and a colloid $\mathrm{pH}$ of 2.7. Figure 4.25 shows the TA-mauve and TSC-grey loose wool composites ( $5 \mathrm{~g}$ samples). In contrast to the ex-situ composites of top form wool and TU yarn investigated in the previous section, there are differences in the hue, intensity and uniformity of the colour of the loose wool composites.

The TA-mauve method resulted in 15 and $36 \mu \mathrm{m}$ composites that are different shades of mauve in colour and the $27 \mu \mathrm{m}$ composite is mauve-grey. The TSC-grey $15 \mu \mathrm{m}$ composite is purple-grey, and the 27 and $36 \mu \mathrm{m}$ composites are different shades of grey. These colour differences are unexpected for the ex-situ procedure, where the colour is typically dependent on the type and concentration of gold nanoparticles used in the synthesis (as opposed to the chemical interaction between $\mathrm{AuCl}_{4}^{-}$and the wool protein that occurs in the in-situ procedure). The colour uniformity of the composites is relatively uneven; each of the loose wool composites exhibit areas of completely undyed wool. These areas represent pockets of matted wool fibres which do not come into contact with the dye solution and require further processing to comb, mix and align the fibres into top form wool.

CIE colour coordinates of the TA-mauve and TSC-grey loose wool composites are shown in Table 4.8. The expected decrease in the $\mathrm{L}^{*}$ value of the TA-mauve and TSC-grey composites of thicker loose wool is observed, and the TSC-grey composites are darker than the TA-mauve composites. The mauve-grey colour of the $27 \mu \mathrm{m}$ TA-mauve composite is evident with its relatively low a* value compared to the 15 and $36 \mu \mathrm{m}$ TA-mauve composites. Additionally, the relatively high $\mathrm{a}^{*}$ values of the $15 \mu \mathrm{m}$ TSC-grey composite illustrates the purple tinge of the composite.

SEM micrographs of the surfaces of the TA-mauve loose wool composites are presented in Figure 4.26. The TA-mauve $27 \mu \mathrm{m}$ composite fibres exhibited two distinct 


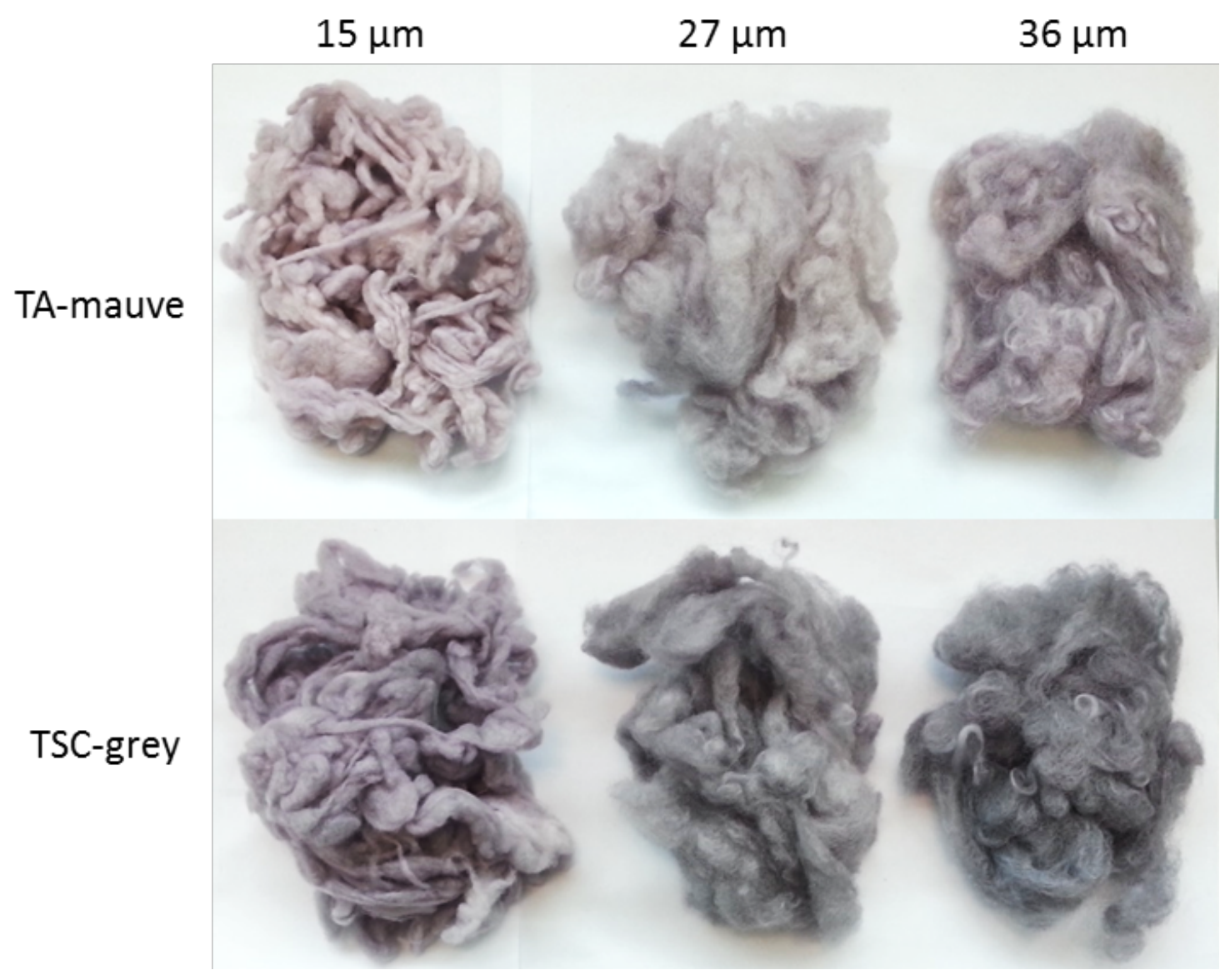

Figure 4.25 TA-mauve and TSC-grey ex-situ composites synthesised with 15, 27 and $36 \mu \mathrm{m}$ loose wool.

Table 4.8 CIE colour coordinates of TA-mauve and TSC-grey composites of different sources of loose wool.

\begin{tabular}{ccccccc}
\hline Method & \multicolumn{3}{c}{ TA-mauve } & \multicolumn{3}{c}{ TSC-grey } \\
\hline Wool type & $15 \mu \mathrm{m}$ & $27 \mu \mathrm{m}$ & $36 \mu \mathrm{m}$ & $15 \mu \mathrm{m}$ & $27 \mu \mathrm{m}$ & $36 \mu \mathrm{m}$ \\
\hline $\mathrm{L}^{*}$ & 67.41 & 62.33 & 56.45 & 55.39 & 54.00 & 50.42 \\
$\mathrm{a}^{*}$ & 5.31 & 3.08 & 4.70 & 4.46 & 2.36 & 2.30 \\
$\mathrm{~b}^{*}$ & 0.29 & -0.37 & -1.38 & -4.00 & -3.06 & -3.64 \\
\hline \hline
\end{tabular}

distributions of gold nanoparticles; most commonly appearing similar to the SEM images labelled "light fibre" in Figure 4.26. However, there were also some composite fibres with relatively high gold densities, one of which is labelled the $27 \mu \mathrm{m}$ "dark fibre". The $27 \mu \mathrm{m}$ dark fibres are likely responsible for the grey areas of the composites observed in Figure 4.25. The dark fibres in the $27 \mu \mathrm{m}$ blend were relatively thin (approximately $20 \mu \mathrm{m}$ diameter), compared to the light fibres that were approximately $30 \mu \mathrm{m}$ in diameter. This indicates that the $27 \mu \mathrm{m}$ loose wool contains a blend of wool fibres of different thicknesses and absorption properties.

Interestingly, there does not appear to be a significant difference between the coverage of gold nanoparticles across the surfaces of the TA-mauve composite fibres of 
different loose wool types (with the exception of the $27 \mu \mathrm{m}$ dark fibres). This is likely due to the relatively uneven colouring of the composites, which indicates varying gold distribution across different fibres of the same composite sample. The higher magnification images show that the gold nanoparticles are present as both agglomerates and discrete nanoparticles for both the 15 and $36 \mu \mathrm{m}$ composites, whereas the $27 \mu \mathrm{m}$ composite fibres (particularly the dark fibres) appear to encompass a higher proportion of agglomerates of gold nanoparticles. This explains the relative greyness of the $27 \mu \mathrm{m}$ composites.

SEM micrographs of the surfaces of the TSC-grey loose wool composites are presented in Figure 4.27. The TSC-grey composites of each wool type show the expected higher gold coverage on the fibres compared to the TA-mauve composites, and that the TSC-Au NPs are typically present as agglomerates of nanoparticles. The $15 \mu \mathrm{m}$ composite does display a significant number of discrete gold nanoparticles, which will contribute to the purple colour of the composite. The $27 \mu \mathrm{m}$ and $36 \mu \mathrm{m}$ composites appear to exhibit sequentially fewer discrete nanoparticles and a relatively higher proportion of agglomerates of nanoparticles. This is understandable given the lower effective surface areas of thicker wool fibres causing the relatively close proximity of the TSC-Au NPs.

This study has shown that the type of loose wool has affected the hue, intensity and uniformity of the colour of the ex-situ composites. It is understandable that this wool type could exhibit chemical functionality that could interact with the stability of the typically stable TA-mauve colloid, as there is remaining wool grease and vegetable matter throughout the wool in this form. Given that loose wool requires multiple processing stages before it may be fabricated into carpets or apparel, and that the colour variation across samples was greater than that of top form wool, loose wool was deemed to be unsuitable for the ex-situ synthesis of gold nanoparticle-wool fibre composites.

The presented results have shown the significant (and sometimes unpredictable) influence of the wool type on the chemistry of ex-situ procedure. As this is such an important and influential variable, later studies often investigated the effects of manipulating other reaction parameters across a range of wool types. The following section studies the effects of reaction $\mathrm{pH}$ on the ex-situ procedure while employing a range of wool types. 


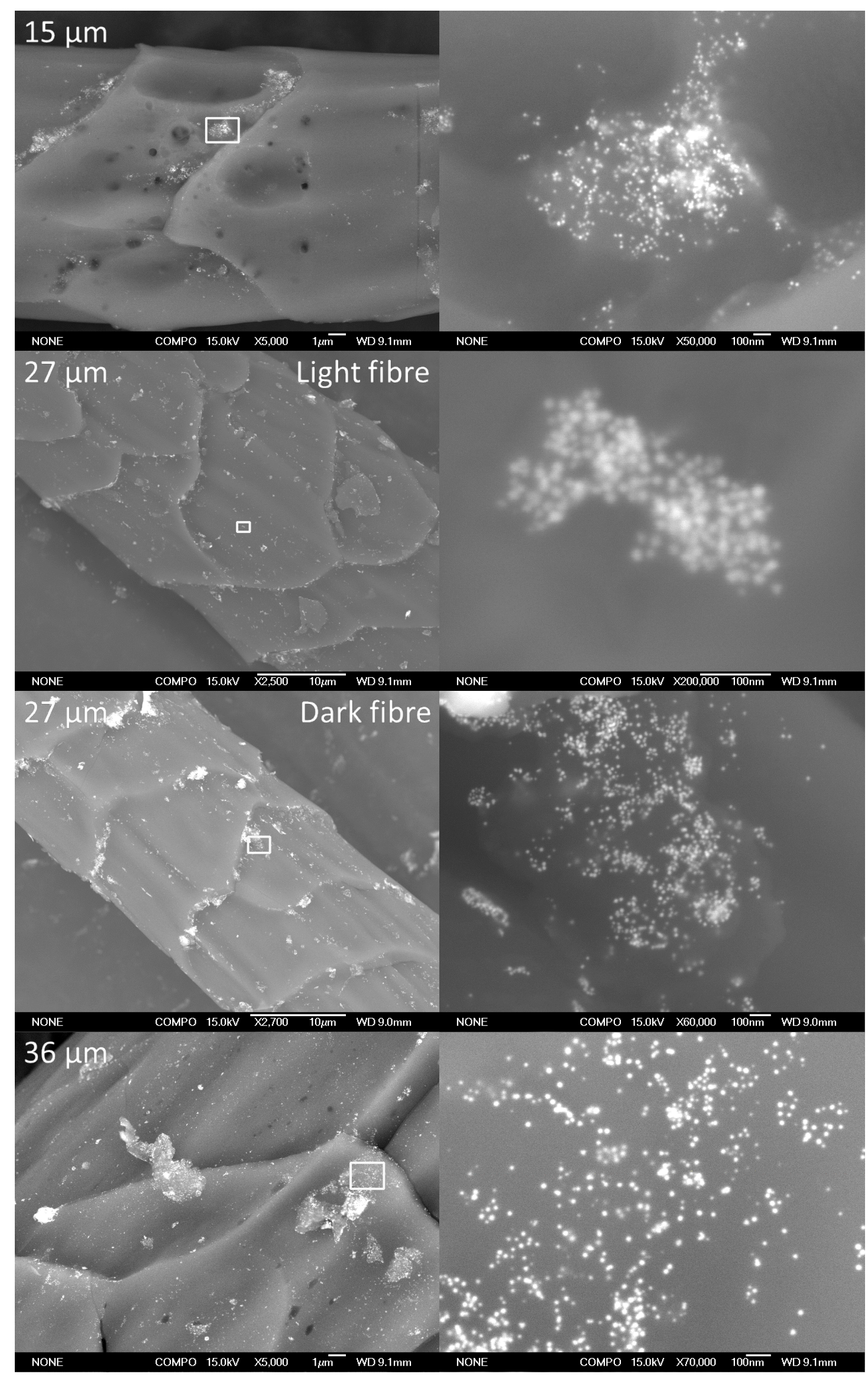

Figure 4.26 SEM micrographs in backscatter mode of the surface of TA-mauve ex-situ composites of 15, 27 and $36 \mu \mathrm{m}$ loose wool, including both a relatively dark and light coloured fibre of the $27 \mu \mathrm{m}$ wool. 


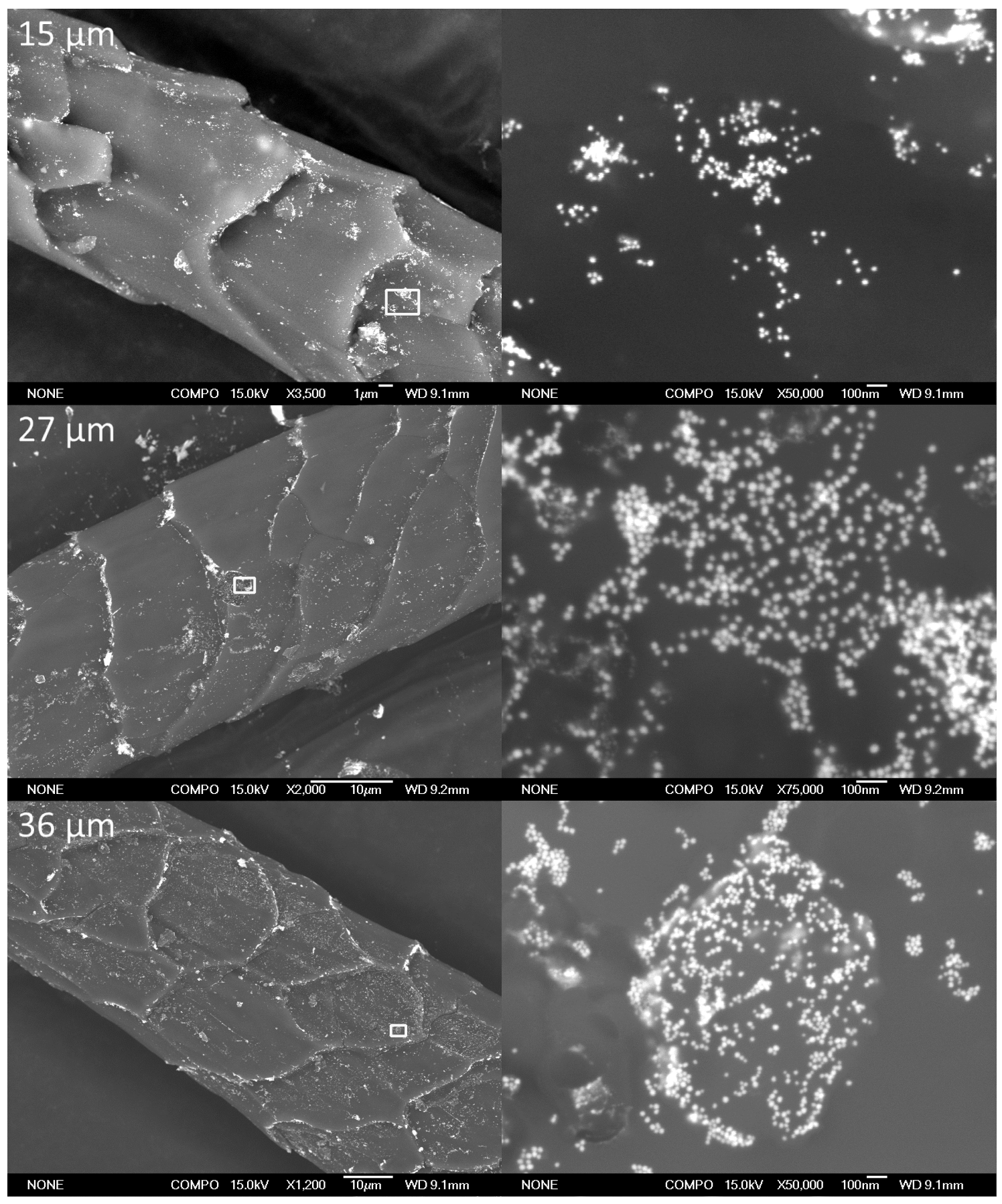

Figure 4.27 SEM micrographs in backscatter mode of the surface of TSC-grey ex-situ composites of 15, 27 and $36 \mu \mathrm{m}$ loose wool. 


\subsection{2 $\mathrm{pH}$}

The $\mathrm{pH}$ of the ex-situ procedure effects the rate of uptake of TA-Au NPs and TSC$\mathrm{Au}$ NPs to wool fibres, and in the case of the TSC-stabilised gold nanoparticles also influences the colour of the composites. Like the in-situ procedure, the reaction $\mathrm{pH}$ provides a driving force for the uptake of gold nanoparticles to wool, and lowering the $\mathrm{pH}$ provides more attractive sites for the uptake of negatively charged gold nanoparticles. When dyeing wool with TSC-stabilised nanoparticles, lowering the reaction $\mathrm{pH}$ destabilises the TSC-Au NPs, promoting their agglomeration and hence the grey colour of the composites. In contrast, TA-Au NPs are relatively stable to changes in $\mathrm{pH}$. As such, TA-Au NPs were used to study the effects of $\mathrm{pH}$ on the uptake of gold nanoparticles to wool.

UV-vis spectra of the uptake of TA-Au NPs to Cariaggi wool at pH 2.8 are shown in Figure 4.28. This experiment involved the pre-soaking of wool in an aqueous solution that was held at $\mathrm{pH} 2.8$ by continual $\mathrm{HCl}$ addition (a buffer was not used as buffer molecules may influence the uptake). This is done to prevent the usual increase in $\mathrm{pH}$ of the dye solution which occurs during the uptake of gold nanoparticles to the wool. When the reaction $\mathrm{pH}$ is maintained at 2.8, the uptake of TA-Au NPs to Cariaggi wool is approximately complete after $5 \mathrm{~min}$.

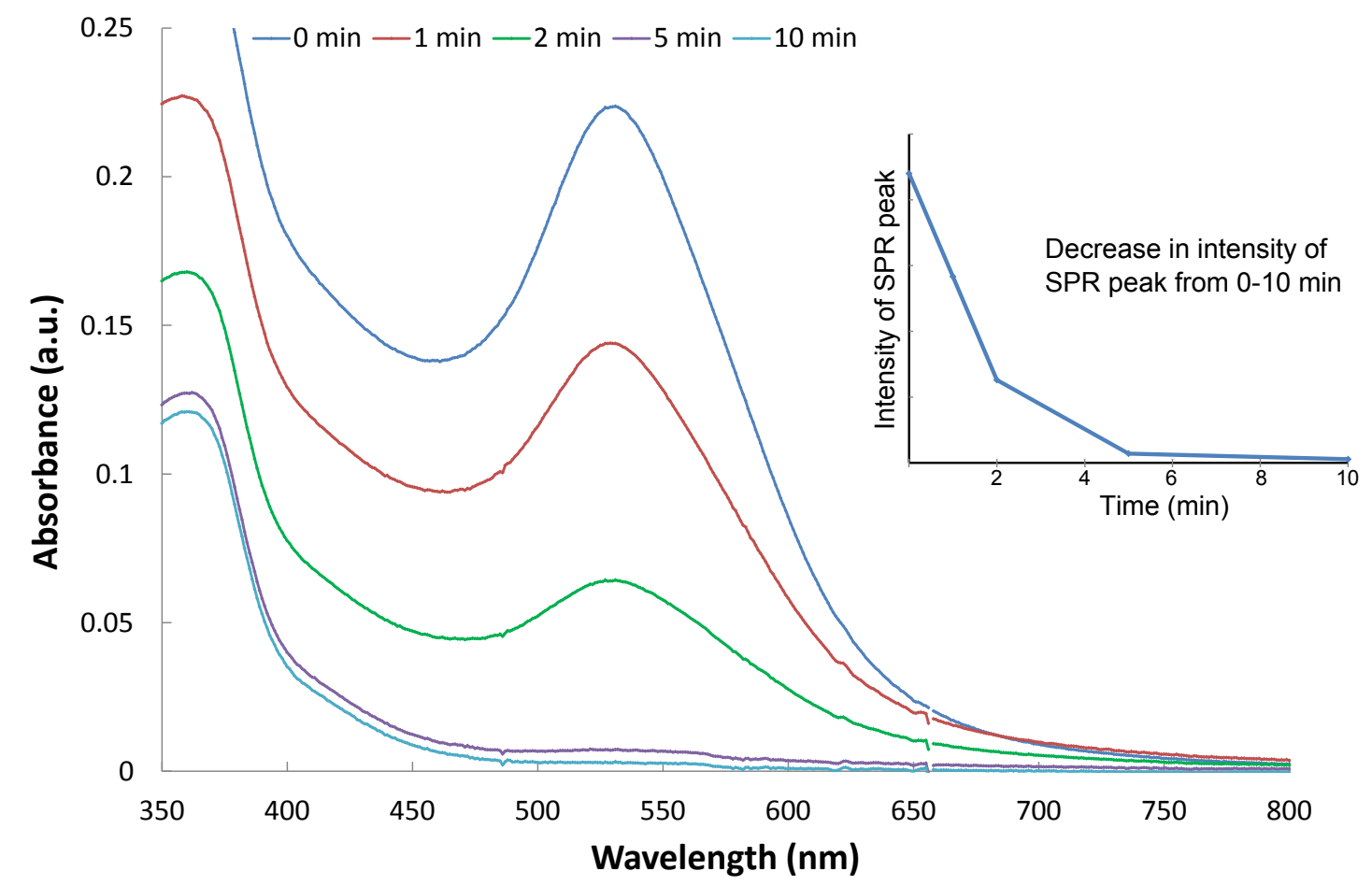

Figure 4.28 UV-vis spectra of the uptake of TA-Au NPs from in the TA-mauve reaction to Cariaggi wool fibres at a reaction $\mathrm{pH}$ of 2.8 over time. The inset represents the uptake curve from these spectra. 
Figure 4.29 illustrates the uptake of TA-Au NPs to Cariaggi wool at different reaction $\mathrm{pH}$ levels, where the uptake curves are determined from the UV-vis spectra (for example the $\mathrm{pH} 2.8$ uptake curve was plotted from the intensity of the SPR peak over time that is shown in Figure 4.28). Figure 4.29 shows that any uptake of TA-Au NPs requires a reaction $\mathrm{pH}$ of less than 3.6, implying that at this $\mathrm{pH}$ there is no net positive charge on the Cariaggi wool fibres to attract the nanoparticles. When the reaction $\mathrm{pH}$ is lowered to 3.4, approximately half of the TA-Au NPs in the colloid are eventually absorbed by the wool. At a reaction $\mathrm{pH}$ of $3.2-2.8$, the uptake is rapid; uptake of TA-Au NPs is complete after $30 \mathrm{~min}$ at $\mathrm{pH} 3.2$, and complete after only 5 min at $\mathrm{pH} 3.0-2.8$.

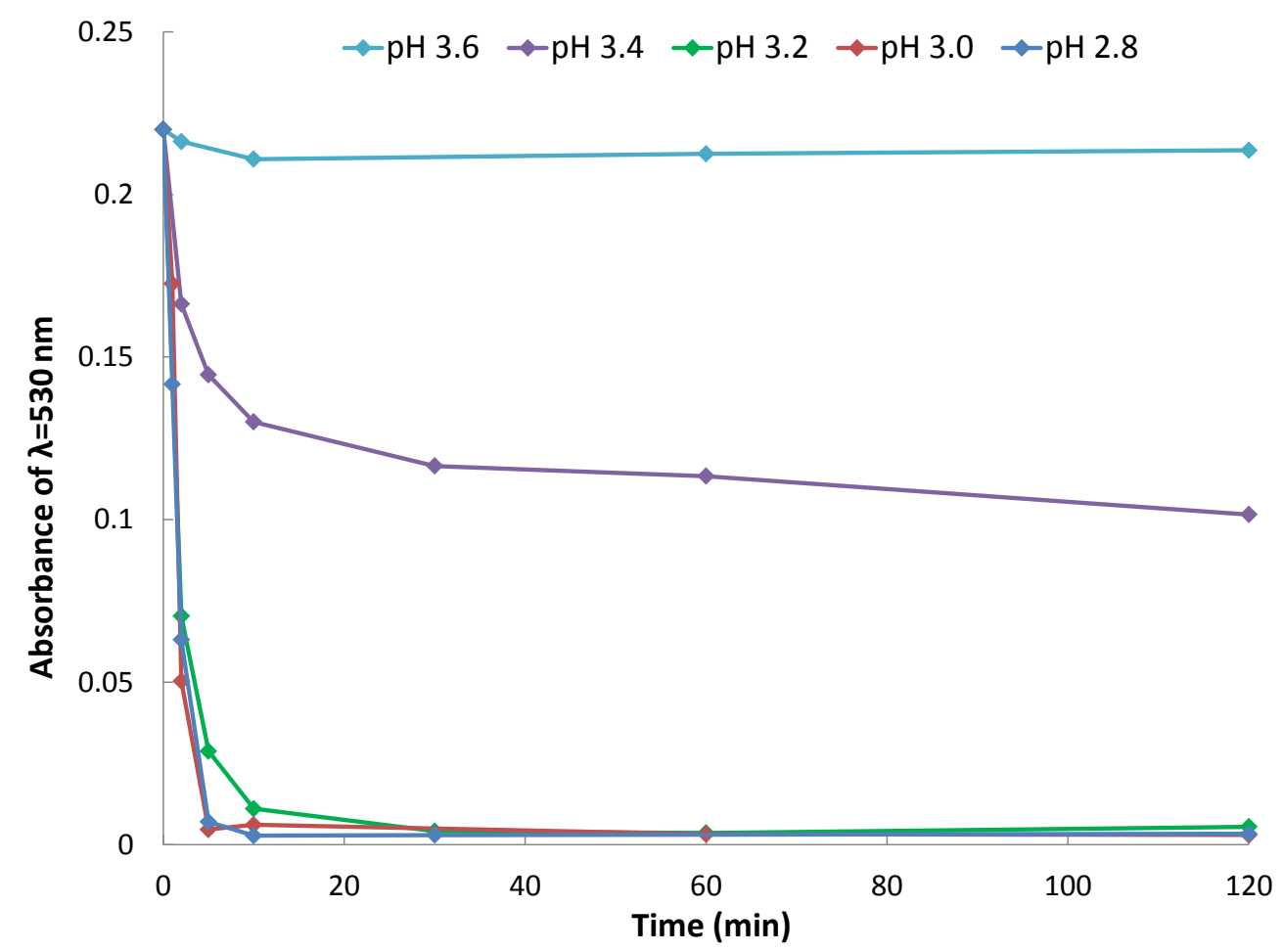

Figure 4.29 UV-vis spectra of the uptake of TA-Au NPs to Cariaggi wool from $\mathrm{pH} 2.8-3.6$, where the absorbance of the $\lambda_{\max }=530 \mathrm{~nm}$ decreases as the TA-Au NPs are absorbed by the wool.

To investigate the influence of wool type on the uptake of gold nanoparticles across a $\mathrm{pH}$ range of 3.6-2.8, the uptake of TA-mauve colloid was repeated with 16 and $18 \mu \mathrm{m}$ open top merino wool. Open top is loose wool that has been carded to remove vegetable matter. The wool was sourced by Professor James Johnston's contacts from AgResearch. Figure 4.30 shows the uptake of TA-Au NPs to $16 \mu \mathrm{m}$ open top wool. The uptake is only approximately complete after $120 \mathrm{~min}$ at $\mathrm{pH} 2.8$. This implies that the $16 \mu \mathrm{m}$ wool has a relatively low isoelectric region. At $\mathrm{pH} 3.4$ and 3.6, re-equilibration of the TA-Au NPs is observed, as the amount of TA-Au NPs in solution varies over time. 


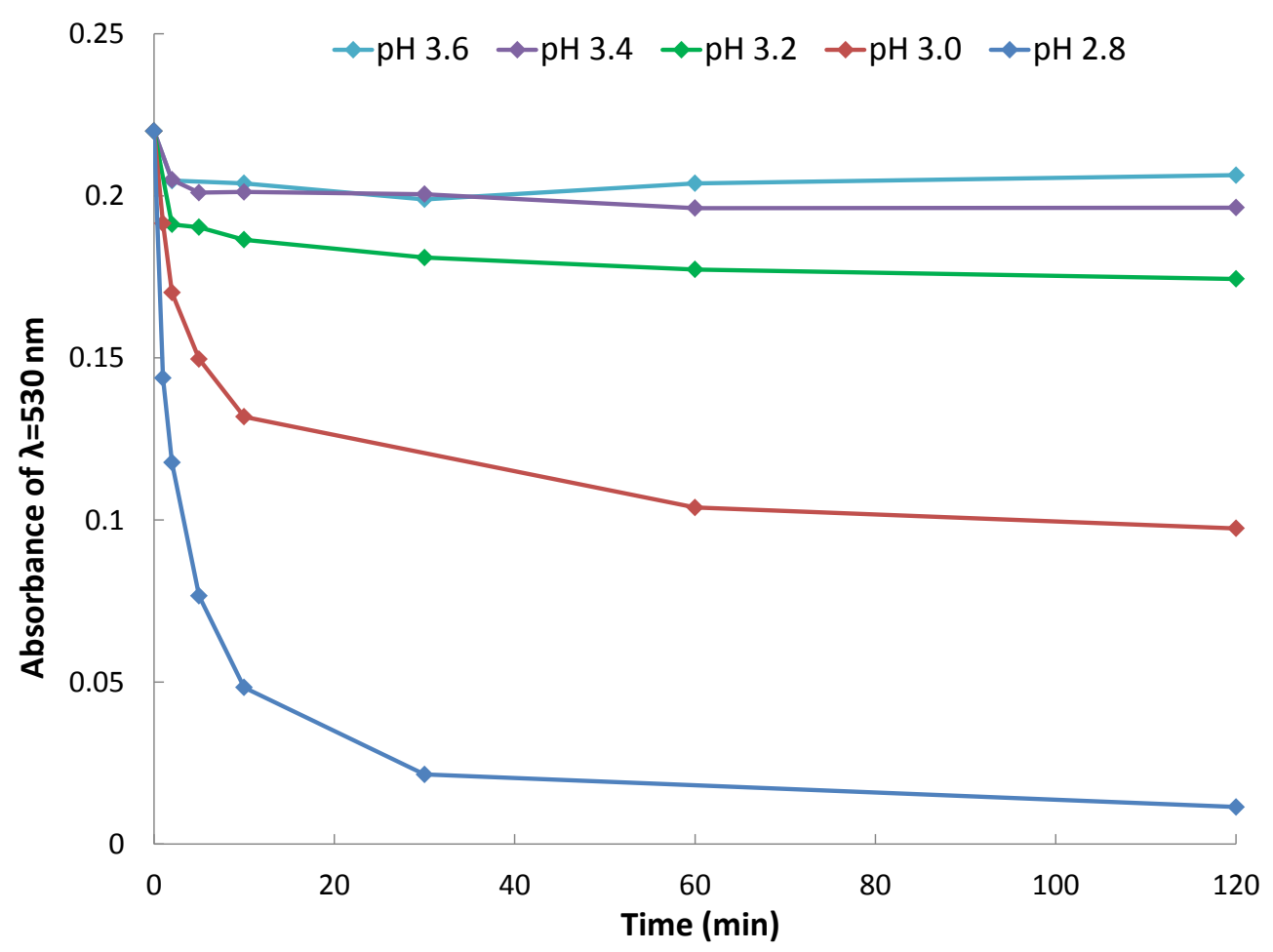

Figure 4.30 UV-vis spectra of the uptake of TA-Au NPs to $16 \mu \mathrm{m}$ open top wool from $\mathrm{pH} 2.8-3.6$, where the absorbance of the $\lambda_{\max }=530 \mathrm{~nm}$ decreases as the TA-Au NPs are absorbed by the wool.

The uptake of TA-Au NPs to $18 \mu \mathrm{m}$ open top wool from $\mathrm{pH} 2.8-3.6$ is presented in Figure 4.31. The uptake is much more rapid than that of Cariaggi or $16 \mu \mathrm{m}$ open top wool, with complete uptake of TA-Au NPs occurring at a reaction $\mathrm{pH}$ of just 3.4. Lowering the $\mathrm{pH}$ of the reaction further increases the rate of uptake to the point where the uptake is approximately complete after $10 \mathrm{~min}$ from $\mathrm{pH} 3.2-2.8$.

Each wool type has shown a unique, pH-dependent absorption profile, with unique isoelectric regions seen in each wool type. One common theme is that the amount of TA-Au NPs that will be absorbed by the wool at a specific reaction $\mathrm{pH}$ typically occurs after $30 \mathrm{~min}$ of the reaction. As such, lowering the $\mathrm{pH}$ of the reaction increases the amount of TA-Au NPs that are eventually absorbed, however, this will only influence the rate of absorption when lowered below the point where the complete of uptake of TA-Au NPs will eventually occur. The experiments in Section 4.3.2 have shown that each wool type used in large-scale ex-situ procedures will exhibit different absorption chemistries. Next, conventional wool dyeing auxiliary agents are used in the ex-situ procedure. 


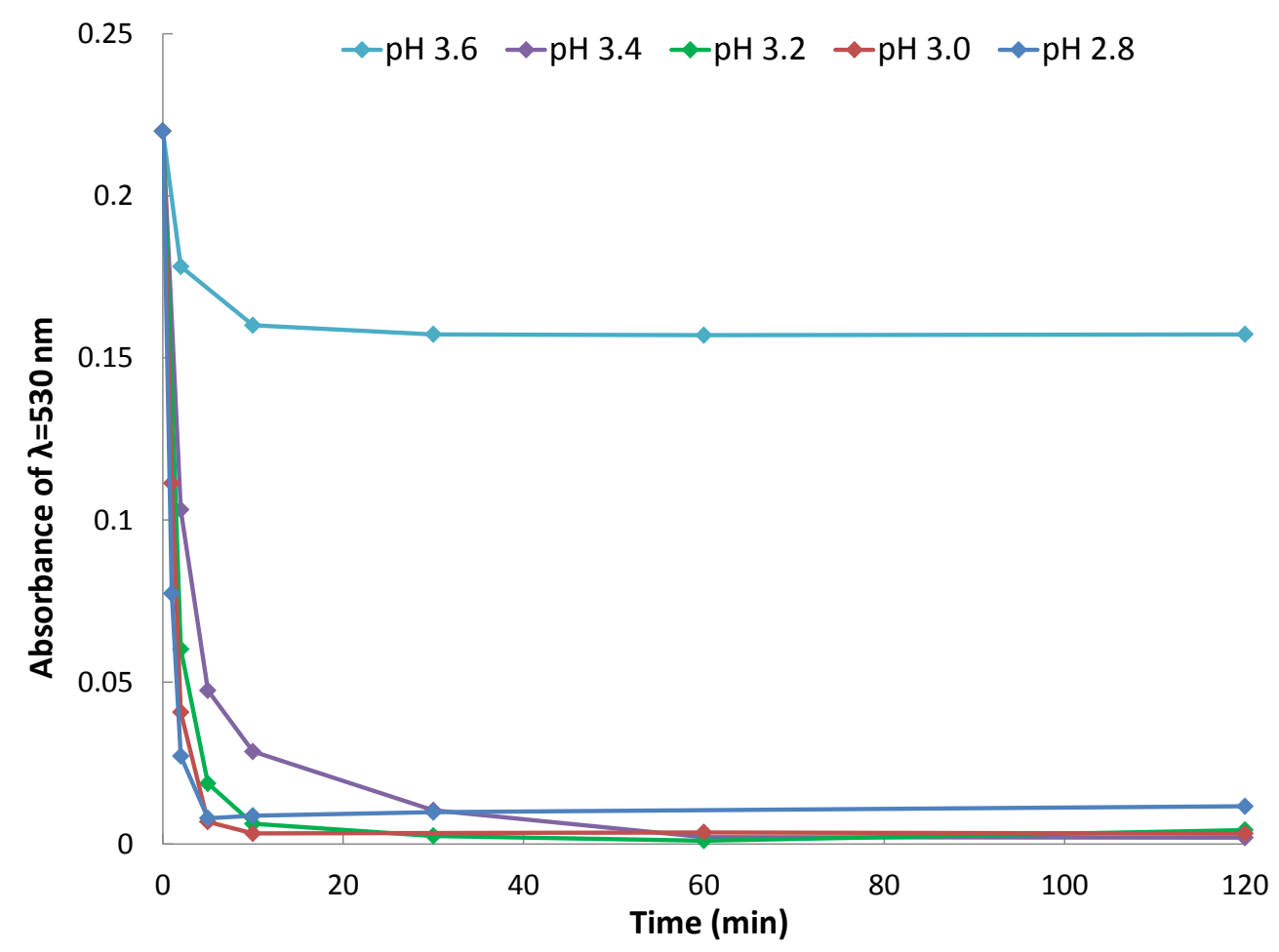

Figure 4.31 UV-vis spectra of the uptake of TA-Au NPs to $18 \mu \mathrm{m}$ open top wool from $\mathrm{pH} 2.8-3.6$, where the absorbance of the $\lambda_{\max }=530 \mathrm{~nm}$ decreases as the TA-Au NPs are absorbed by the wool.

\subsubsection{Auxiliary Agents}

The following experiments utilise conventional wool dyeing auxiliaries to attempt to enhance the colour uniformity of the ex-situ composites. The auxiliary agents are employed to improve the access of gold nanoparticles throughout the entire wool sample, and to control the rate of uptake of the gold nanoparticles. Similar to the in-situ procedure (in Section 3.2.4), both wetting agents and levelling agents were investigated.

\section{Wetting Agents}

Wetting agents are typically employed prior to wool dyeing to "wet out" a dyepack, that is to ensure that all of the wool fibres are wet. Wool fibres are naturally hydrophobic, and any wool fibres that remain dry will not come into contact to the dye molecules in an aqueous dyebath. This is particularly important in large samples. Wetting agents associate to the surface of wool fibres, and as such can also slow the migration of dye molecules to wool fibres. As gold nanoparticles also associate to the surface of wool fibres, wetting agents are believed to gradually exchange with gold nanoparticles during their uptake in the ex-situ synthesis of gold 
nanoparticle-wool fibre composites.

The wetting agent Albaflow FFA was recommended by Professor James Johnston's contacts at Wool Yarns Ltd to improve the colour uniformity of dyed yarns. The uptake of the TA-mauve colloid to Crossbred, Ashford and Cariaggi top form wool and TU yarn was studied with UV-vis spectroscopy. The standard TA-mauve conditions were used with Albaflow FFA $\left(1 \mathrm{~g} \mathrm{~L}^{-1}\right)$ added to both the glycine buffer and the colloid. The uptake curves are presented in Figure 4.32. An identical experiment without the wetting agent was previously presented in Figure 4.23 in Section 4.3.1. The wetting agent increases the absorption of the colloidal solution across the entire visible region, and as such the curves are normalised so that the original colloid at zero time $=0.22$ absorbance.

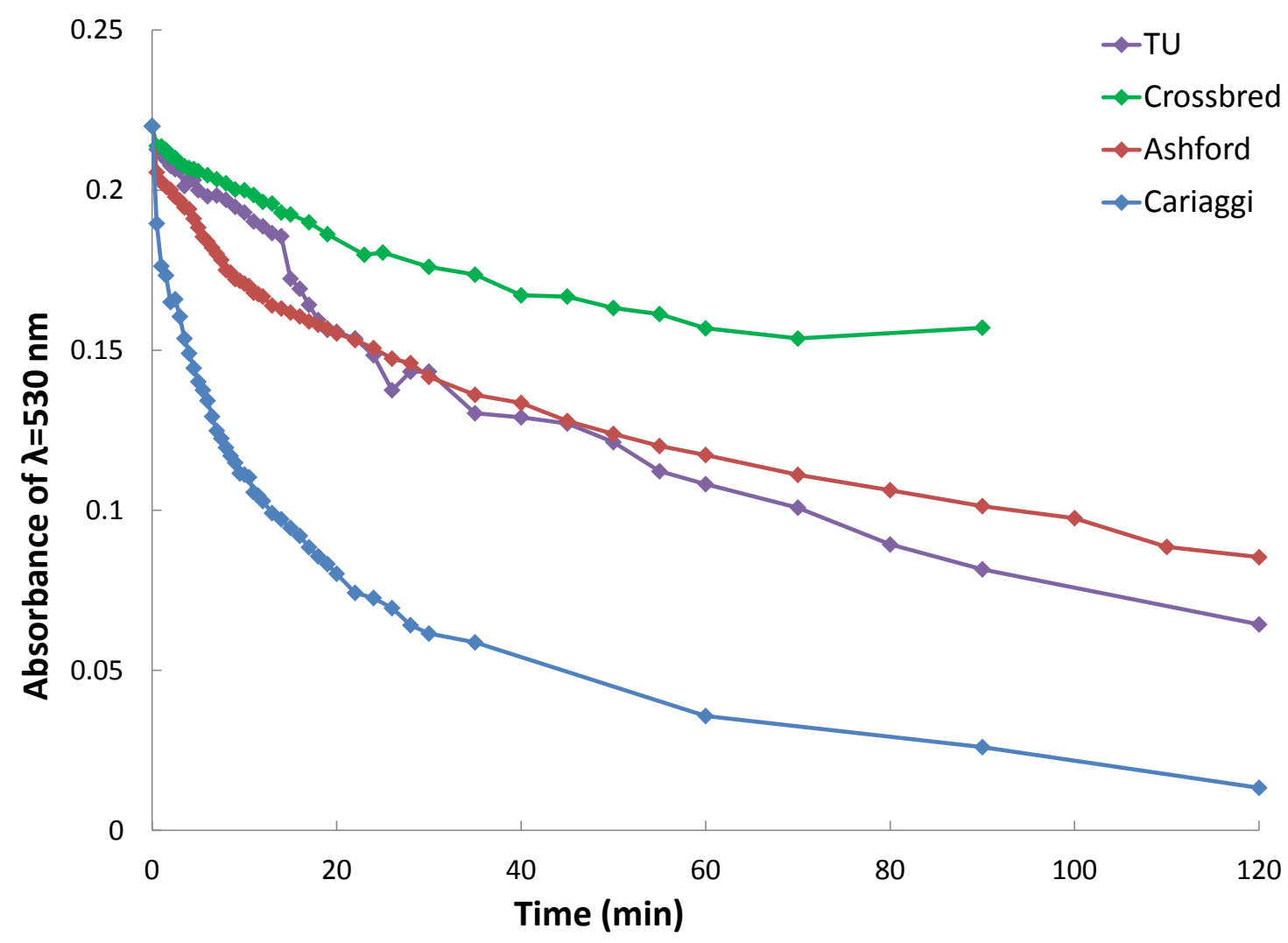

Figure 4.32 Uptake of TA-mauve to different wool types with $1 \mathrm{~g} \mathrm{~L}^{-1}$ of Albaflow FFA in the $\mathrm{pH} 2.2$ buffer and $\mathrm{pH} 2.2$ colloid (normalised so that the original SPR peak $\left(\lambda_{\max }\right.$ of $\left.\left.530 \mathrm{~nm}\right)=0.22 \mathrm{abs}\right)$.

The presence of Albaflow FFA has substantially slowed the uptake of TA-Au NPs to each wool type. Again, Cariaggi exhibits the fastest uptake, and the rate of uptake decreases when thicker wool fibres are used. The TU uptake curve is relatively jagged, indicating re-equilibration of either the TA-Au NPs in solution or the wetting agent from the surface of the wool fibres and the dye solution. The uptake of the TAmauve colloid is incomplete after $120 \mathrm{~min}$ for each wool type, which is significantly slower than when no wetting agent is used. The uptake was left overnight and is eventually complete for each wool type. 
The composites that result at the completion of the uptake reactions in Figure 4.32 are shown in Figure 4.33. The uniformity of each is improved with the presence of the wetting agent (particularly so with the TU composite), and the hue and intensity of colouration is not significantly altered by the presence of the wetting agent.

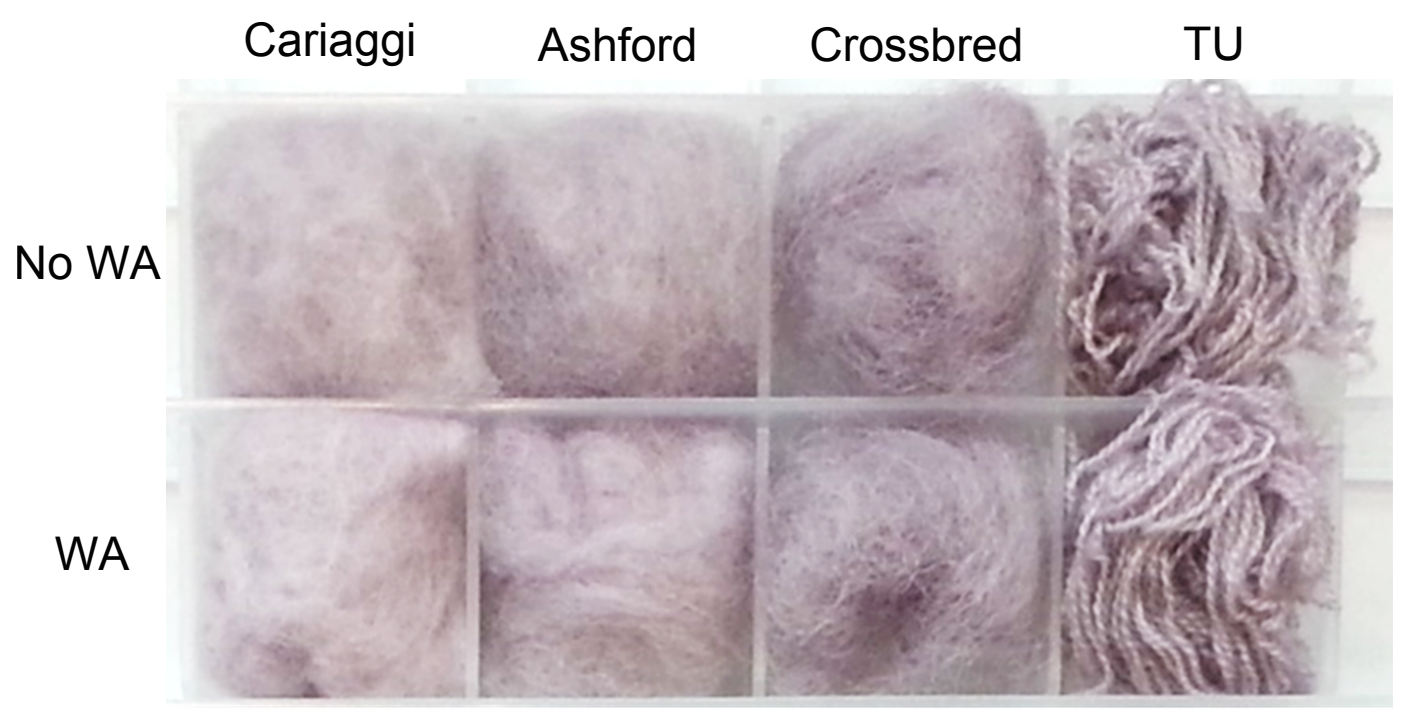

Figure 4.33 TA-mauve composites of different wool types with and without $1 \mathrm{~g} \mathrm{~L}^{-1}$ Albaflow in the glycine buffer and colloid.

CIE colour coordinates of the above TA-mauve composites are shown in Table 4.9. The presence of Albaflow FFA in the colloid has resulted in a general increase in the $\mathrm{L}^{*}, \mathrm{a}^{*}$ and $\mathrm{b}^{*}$ values of the composites of each wool type. This illustrates a lighter and more pink colouration of the composites when the wetting agent is employed in their synthesis. This is understandable as a more even distribution of gold nanoparticles across the composite fibres will result in a higher proportion of individual nanoparticles compared to agglomerates, and relatively pink coloured composites.

Table 4.9 CIE colour coordinates of TA-mauve composites of different wool types different amounts of Albaflow FFA (WA) in the buffer and colloid.

\begin{tabular}{ccccccccc}
\hline Wool type & \multicolumn{2}{c}{ Cariaggi } & \multicolumn{2}{c}{ Ashford } & \multicolumn{2}{c}{ Crossbred } & \multicolumn{2}{c}{ TU } \\
\hline WA $\left(\mathrm{g} \mathrm{L}^{-1}\right)$ & 0 & 1 & 0 & 1 & 0 & 1 & 0 & 1 \\
\hline $\mathrm{L}^{*}$ & 65.79 & 67.43 & 62.12 & 64.09 & 57.14 & 58.99 & 56.14 & 57.62 \\
$\mathrm{a}^{*}$ & 6.39 & 6.95 & 6.24 & 6.16 & 5.88 & 6.28 & 5.28 & 6.62 \\
$\mathrm{~b}^{*}$ & -0.88 & -1.10 & -1.56 & -0.40 & -1.60 & -1.49 & -2.30 & -1.24 \\
\hline \hline
\end{tabular}

The significant effect of Albaflow FFA on slowing the rate of uptake of TA-Au NPs to wool fibres and enhancing the uniformity of the synthesised composites was a very positive result. This led to optimising the amount of the wetting agent used in the TA-mauve synthesis of TU yarn composites. Figure 4.34 shows the uptake of the TA-mauve colloid to TU yarn with $0-4 \mathrm{~g} \mathrm{~L}^{-1}$ Albaflow FFA. 


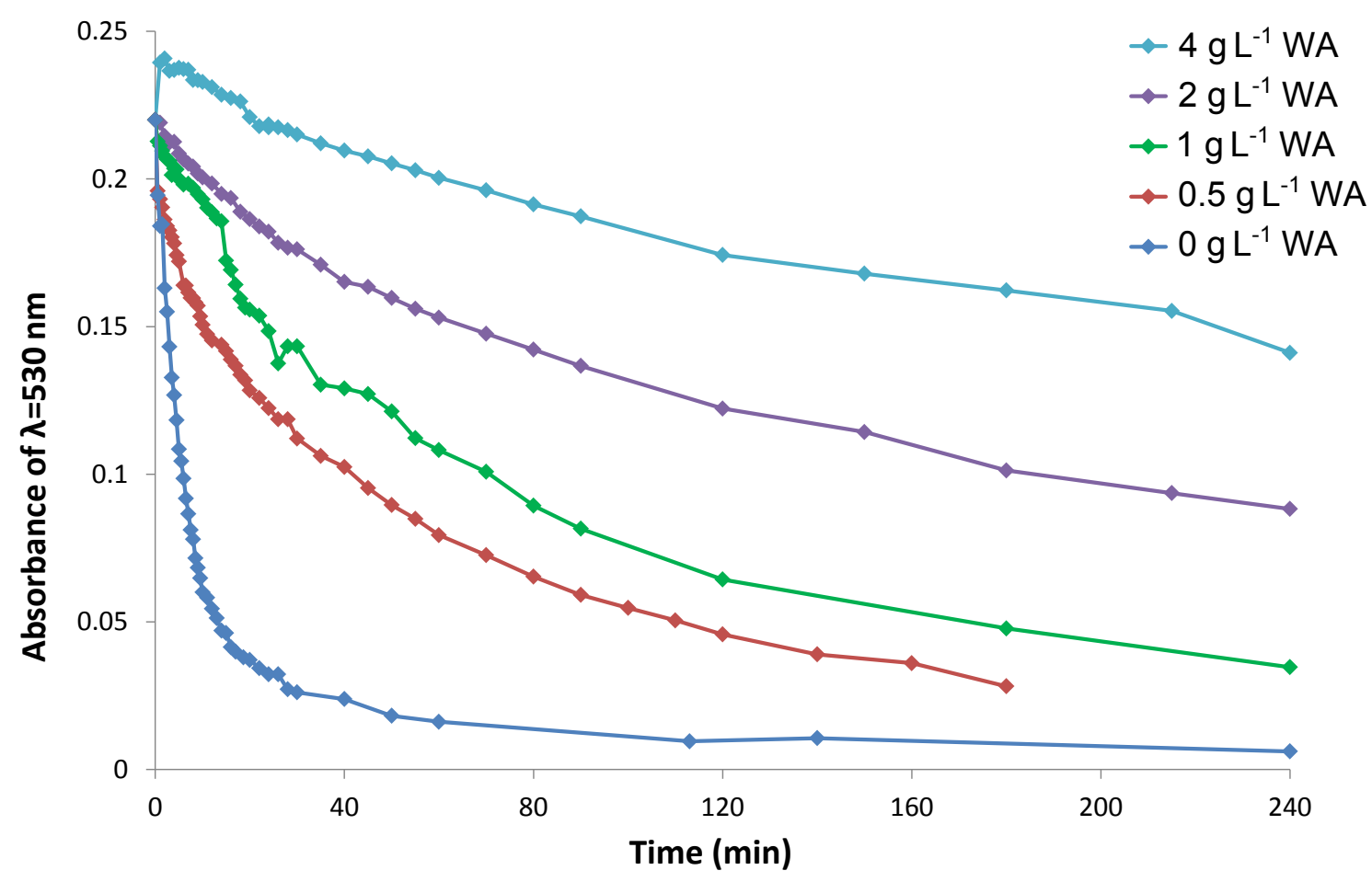

Figure 4.34 Uptake of the TA-mauve colloid to TU yarn different amounts of Albaflow FFA in the $\mathrm{pH} 2.2$ buffer and pH 2.2 colloid.

The rate of uptake is greatly influenced by Albaflow FFA; increasing the amount of the wetting agent in the synthesis has both slowed the uptake and caused the uptake curves to become flatter in shape. A flatter uptake profile is desirable for scale-up reactions, in order to maximise the colour uniformity of the gold nanoparticle-wool fibre composites. Interestingly, when $4 \mathrm{~g} \mathrm{~L}^{-1}$ Albaflow FFA is used, there appears to be an initial increase in the absorbance of the SPR peak of TA-Au NPs at the beginning of the uptake. This is likely due to wetting agent that was associated to the wool after soaking in the buffer leaching into the dyeing solution, thereby increasing the intensity of the entire visible region, including the SPR peak.

The uptake was monitored for a period of $240 \mathrm{~min}$, after which only the uptake to the sample without Albaflow FFA was approximately complete. The samples were left in the uptake solutions for 7 days, after which they absorbed all of the TA-Au NPs in the uptake solution. This time scale is too slow for scale-up procedures, but as will be shown in Section 5.4.1, dye circulation rapidly increases the rate of uptake. The TA-mauve TU yarn composites synthesised with different amounts of Albaflow FFA are shown in figure Figure 4.35.

CIE colour coordinates of the TU yarn TA-mauve composites are shown in Table 4.10. The $0.5 \mathrm{~g} \mathrm{~L}^{-1} \mathrm{WA}$ composite is lighter and more pink coloured compared to the composite synthesised without the wetting agent. Further increases in the amount of Albaflow FFA employed in the synthesis result in incrementally more 


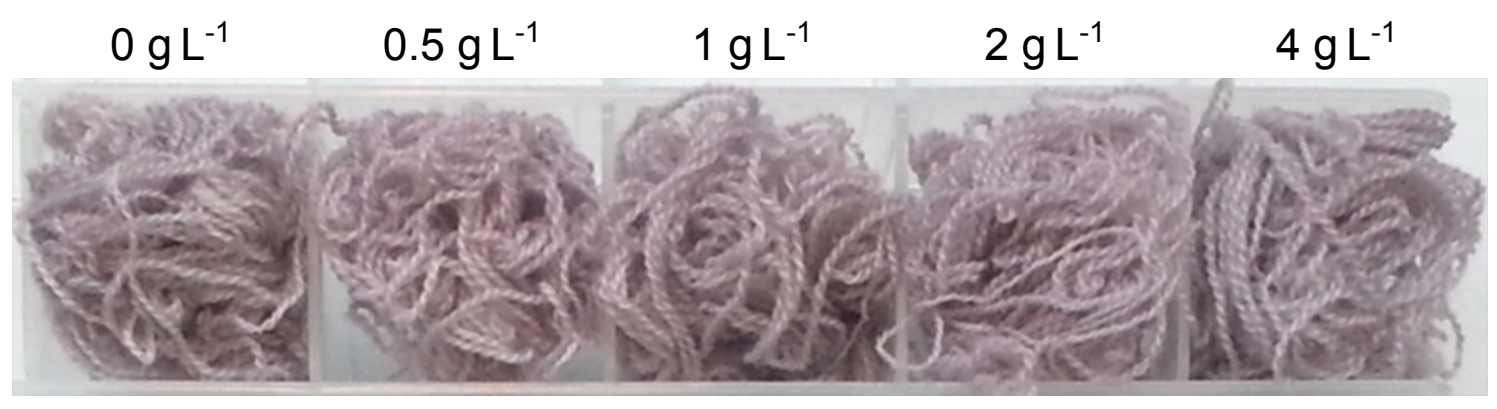

Figure 4.35 TA-mauve composites of TU yarn synthesised with different amounts of Albaflow FFA in the pH 2.2 buffer and colloid.

purple composites of the same approximate darkness (with decreasing $\mathrm{a}^{*}$ and $\mathrm{b}^{*}$ values). This implies that an increase in the concentration of Albaflow FFA beyond a certain threshold in the synthesis may somewhat destabilise the colloid.

Table 4.10 CIE colour coordinates of TU yarn TA-mauve composites synthesised with different amounts of Albaflow FFA (WA) employed in the buffer and the colloid.

\begin{tabular}{cccccc}
\hline $\mathrm{WA}\left(\mathrm{g} \mathrm{L}^{-1}\right)$ & 0 & 0.5 & 1 & 2 & 4 \\
\hline $\mathrm{L}^{*}$ & 56.14 & 60.06 & 59.75 & 60.26 & 58.92 \\
$\mathrm{a}^{*}$ & 5.28 & 7.24 & 6.97 & 6.39 & 5.28 \\
$\mathrm{~b}^{*}$ & -2.30 & -0.34 & -1.42 & -1.92 & -1.99 \\
\hline \hline
\end{tabular}

Due to the success of using Albaflow FFA to control the uptake of the TA-mauve colloid to a range of wool types, it was then used in the TSC-grey method to attempt to enhance the control of the agglomeration of TSC-Au NPs.

\section{Controlling the TSC-grey Method}

The TSC-grey method involves the controlled agglomeration of TSC-Au NPs, which results in a grey colour of the composites. The agglomeration typically occurs in the dye solution, after which agglomerates of gold nanoparticles are absorbed by the wool fibres. Alternatively, individual gold nanoparticles are absorbed by the wool and then aggregate together on the surface of the wool during the dyeing and/or drying of the composites post-dyeing. Albaflow FFA was used in the TSC-grey method to attempt to direct the agglomeration of TSC-Au NPs on the surface of the wool rather than in the colloid. This was hoped to enhance the reproducibility of the TSC-grey method.

The uptake of TSC-Au NPs to TU yarn with different amounts of Albaflow FFA present in the colloid $\left(0-4 \mathrm{~g} \mathrm{~L}^{-1}\right)$ was studied with UV-vis spectroscopy. A glycine buffer was not used and the pH of the TSC-grey colloid was adjusted to 2.0 after the addition of Albaflow FFA. Figure 4.36 shows the UV-vis spectra of the TSC- 
grey colloids after 2 min of uptake. The typical agglomeration of TSC-Au NPs that typically occurs in the uptake solution is observed in the $0 \mathrm{gL}^{-1}$ spectrum, where the long wavelength absorption is more intense than the original SPR peak. Interestingly, the Albaflow FFA has largely prevented the destabilisation of the TSC-Au NPs in the dyeing solution (which typically occurs due to the $\mathrm{pH}$ adjustment and interaction with the wool fibres). The spectra of the colloids containing Albaflow FFA are relatively broad, exhibiting a slight shoulder from $625-700 \mathrm{~nm}$. This indicates some destabilisation of the colloid, however, Albaflow FFA has prevented most of the agglomeration of TSC-Au NPs in the uptake solution.

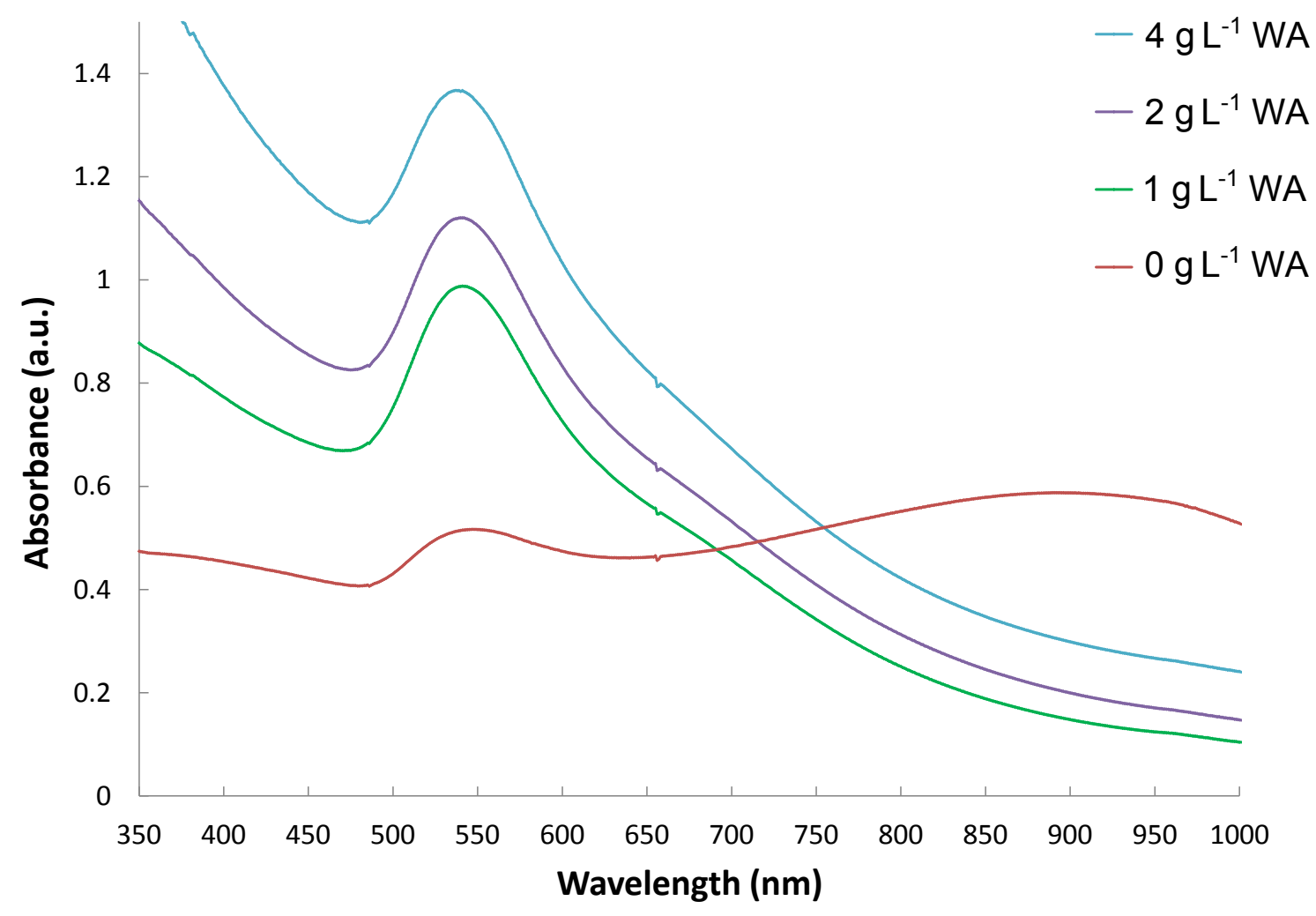

Figure 4.36 UV-vis spectra of TSC-grey colloids after 2 min of uptake to TU wool with different amounts of Albaflow FFA in the uptake solutions.

The stabilisation of the TSC-grey colloid provided by the wetting agent is illustrated in Figure 4.37, where the colloids that encompass Albaflow FFA remain mostly purple, while the colloid without the wetting agent is grey-coloured. The agglomeration evidenced in the UV-spectrum of the colloid without Albaflow FFA is clearly seen, and the different amounts of the wetting agent result in different shades of purplegrey colloid, with the $4 \mathrm{~g} \mathrm{~L}^{-1}$ colloid appearing the most purple.

The rate of uptake of each of the Albaflow FFA containing colloids is substantially slowed. Remarkably, after 4 days of the uptake reaction, a low intensity SPR absorbance remains in each dye solution which incorporated Albaflow FFA, as seen 


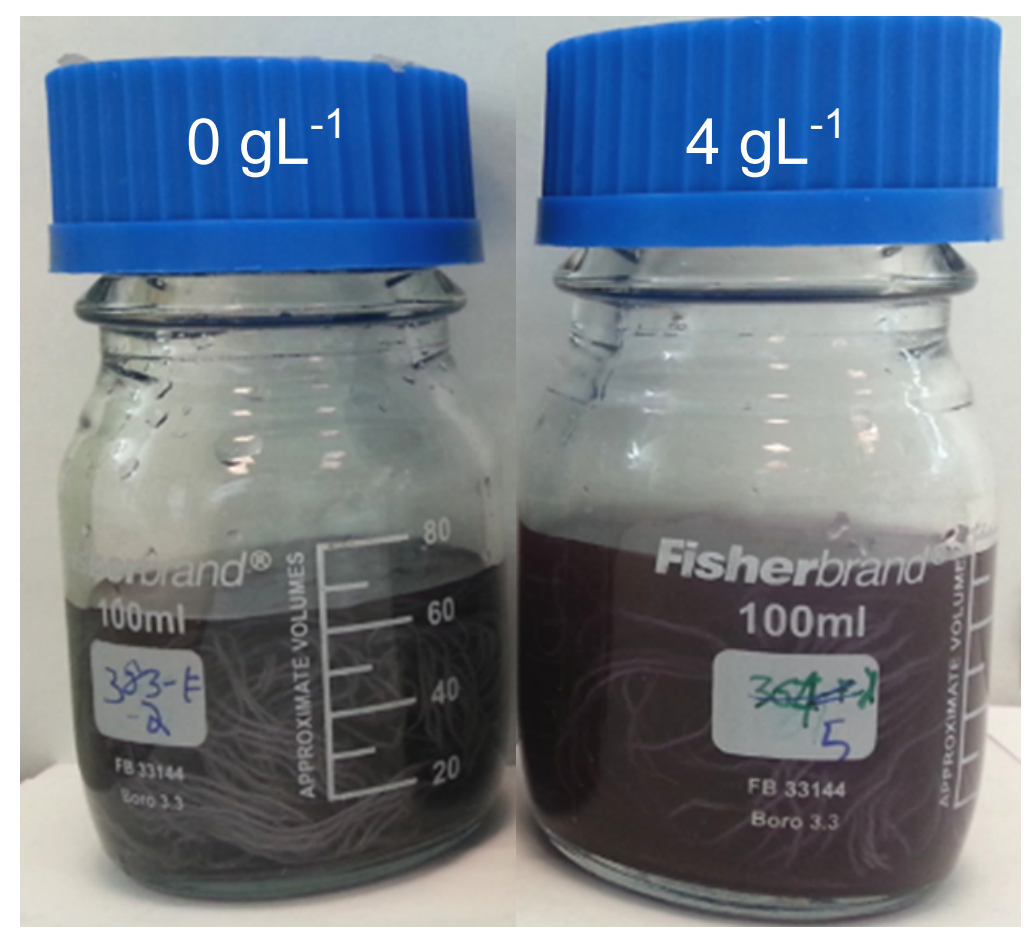

Figure 4.37 The reaction solutions after $10 \mathrm{~min}$ of uptake of the TSC-grey colloid to TU yarn with different amounts of Albaflow FFA in the colloids.

in Figure 4.38. This shows that the stability of the TSC-grey colloid is greatly enhanced by the presence of the wetting agent.

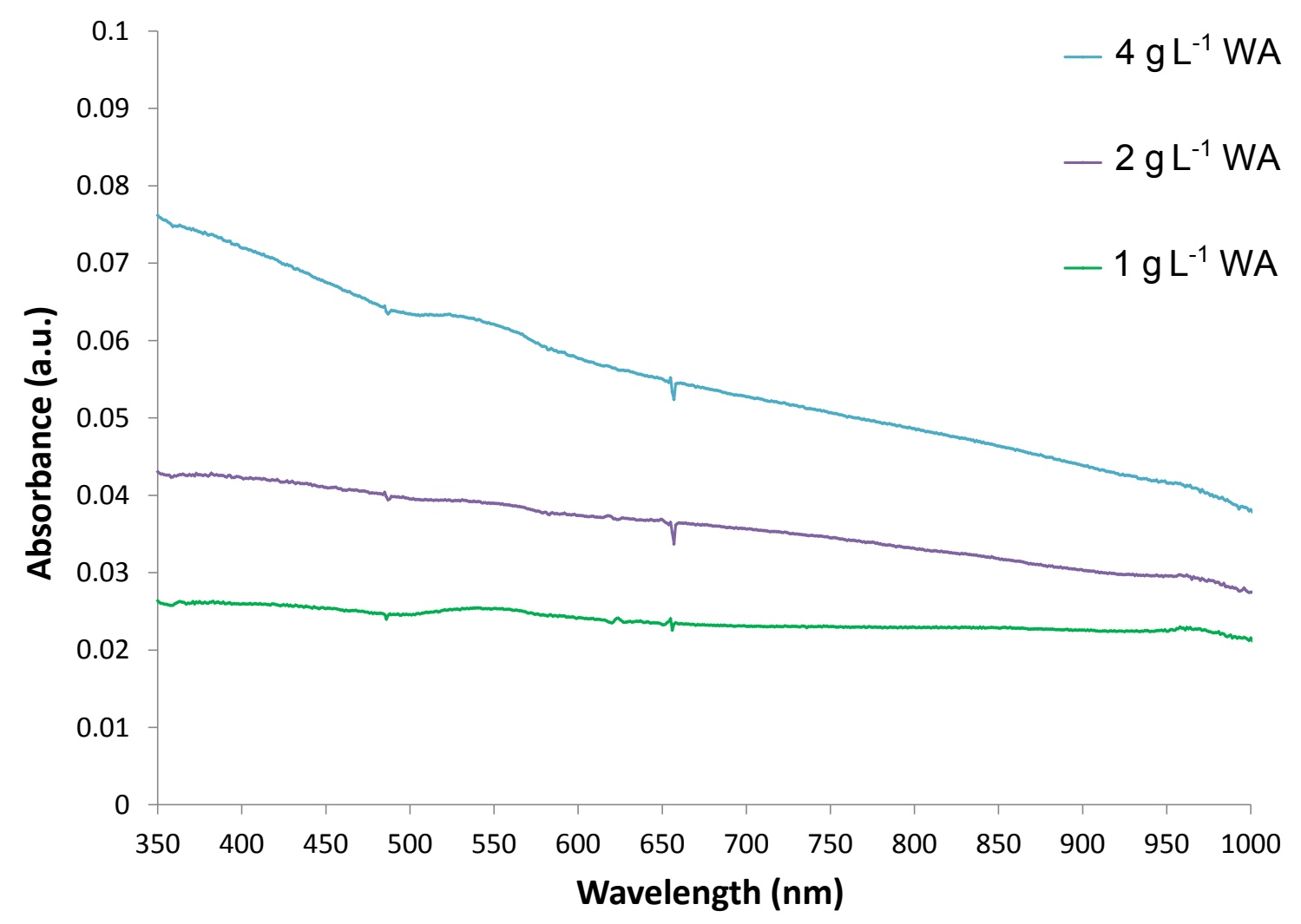

Figure 4.38 UV-vis spectra of TSC-grey colloids after 4 days of uptake to TU wool with different amounts of Albaflow FFA in the uptake solutions.

The TSC-grey TU yarn composites that were synthesised with different amounts of 
Albaflow FFA in the colloid are shown in Figure 4.39. The composites are different shades of grey, with some exhibiting a slight purple tinge (this is difficult to observe in the image).

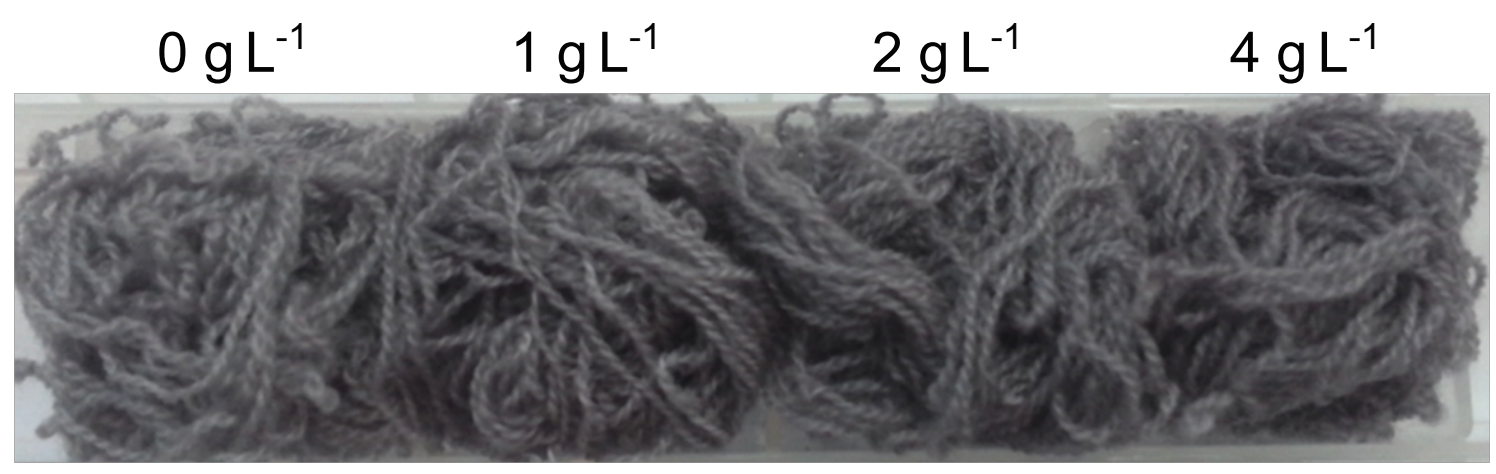

Figure 4.39 A TSC-grey TU yarn composites synthesised with different amounts of Albaflow FFA in the uptake solution.

The CIE colour coordinates of the composites are shown in Table 4.11. Increasing the amount of Albaflow FFA in the colloid results in darker and more purple-coloured composites, as evidenced by the decreasing $\mathrm{L}^{*}$, increasing $\mathrm{a}^{*}$ and more negative $\mathrm{b}^{*}$ values when more of the wetting agent is employed. The composites synthesised with higher amounts of Albaflow FFA also appear more uniformly coloured.

Table 4.11 CIE colour coordinates of TU yarn TSC-grey composites synthesised with different amounts of Albaflow FFA (WA) added prior to the $\mathrm{pH}$ adjustment of the colloid.

\begin{tabular}{ccccc}
\hline WA $\left(\mathrm{g} \mathrm{L}^{-1}\right)$ & No WA & 1 & 2 & 4 \\
\hline $\mathrm{L}^{*}$ & 54.15 & 48.80 & 46.72 & 46.03 \\
$\mathrm{a}^{*}$ & 1.10 & 2.01 & 2.34 & 2.44 \\
$\mathrm{~b}^{*}$ & -3.46 & -3.86 & -4.65 & -4.70 \\
\hline \hline
\end{tabular}

SEM was used to investigate the stabilising nature of Albaflow FFA on the TSC$\mathrm{Au}$ NPs. SEM micrographs of the surfaces of TU yarn TSC-grey composite fibres synthesised with 0, 1 and $4 \mathrm{~g} \mathrm{~L}^{-1}$ Albaflow FFA are shown in Figure 4.40. When no wetting agent is employed in the synthesis, the gold nanoparticles are typically present as agglomerates on the surface of the composite. Using $1 \mathrm{~g} \mathrm{~L}^{-1}$ Albaflow FFA in the synthesis appears to have resulted in a relatively higher proportion of discrete gold nanoparticles relative to agglomerates of gold nanoparticles on the surface of the composites. The high magnification image of the $1 \mathrm{gL}^{-1}$ Albaflow FFA composite indicates that the agglomerates contain gold nanoparticles that are located further apart than those on the surface of the composite synthesised without the wetting agent.

When $4 \mathrm{~g} \mathrm{~L}^{-1}$ of the wetting agent is used, the inter-nanoparticle spacing is greatly 


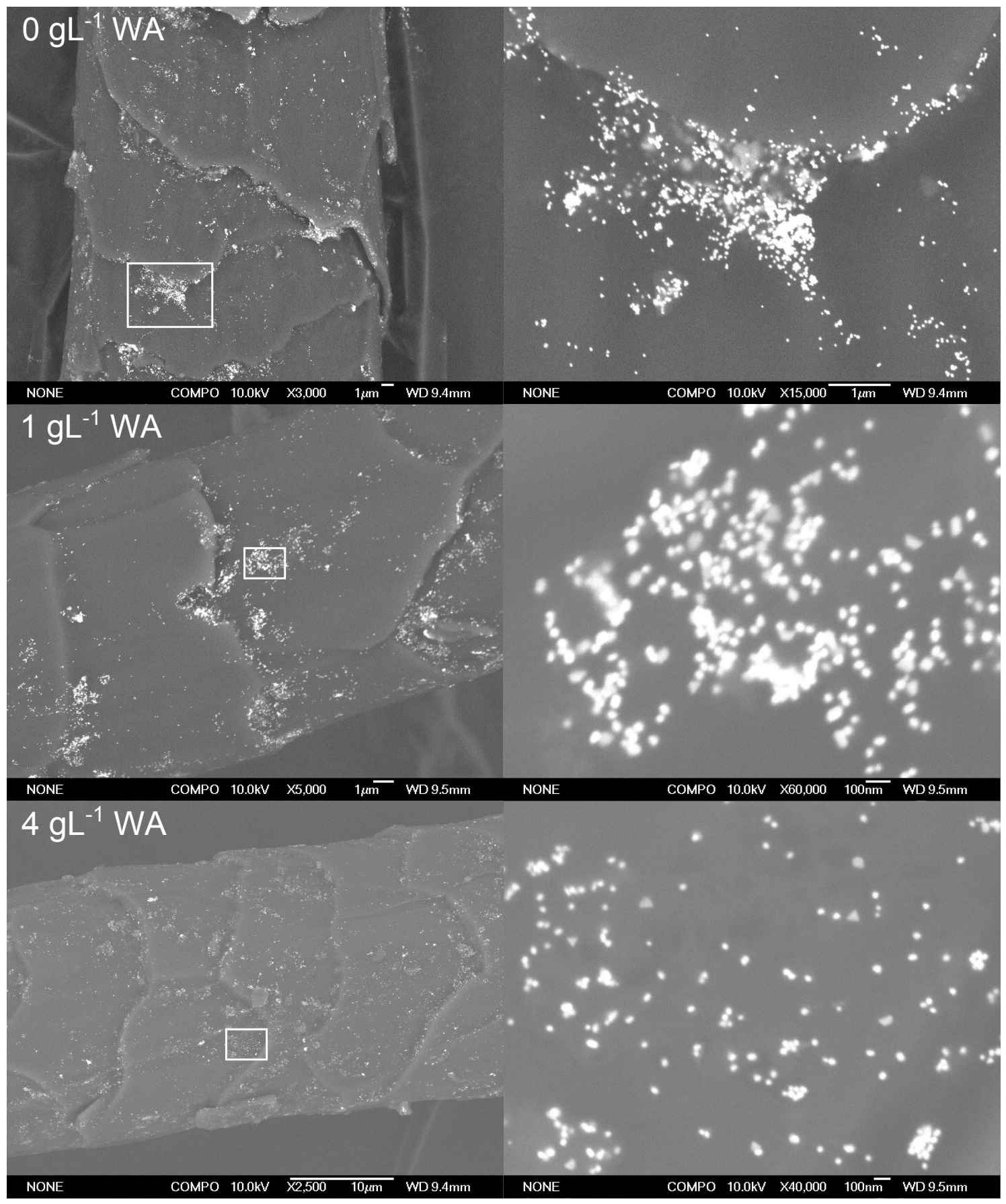

Figure 4.40 SEM micrographs in backscatter mode of TSC-grey composites synthesised with different amounts of Albaflow FFA in the TSC-grey colloid. 
enhanced. The relatively low magnification micrographs show much more uniformly distributed gold nanoparticles across the fibre surface, with little to no preferential attachment at the edges of the cuticle scales. The areas of relatively high density of gold nanoparticles exhibit agglomerates of only a few particles that are located distinctly away from other agglomerates. This is likely the cause of the slight purple tinge of the $4 \mathrm{~g} \mathrm{~L}^{-1}$ Albaflow FFA composite.

The use of Albaflow FFA in the TSC-grey method has slowed the rate of uptake of TSC-Au NPs to TU yarn, and allowed for more uniformly coloured composites to be synthesised. Next, the combination of Albaflow FFA and the glycine buffer was used to further investigate the chemistry of the TSC-grey method.

\section{Albaflow FFA and Glycine Buffers with the TSC-grey Method}

In an effort to further enhance the control and reproducibility of the TSC-grey method, glycine buffers were used in conjunction with the wetting agent. As with the TA-mauve synthesis, the $\mathrm{pH}$ of the dye solution is increased due to the absorption of $\mathrm{H}^{+}$by the wool. Thus, the glycine buffer is used to keep the reaction $\mathrm{pH}$ constant throughout the dyeing process, so that the electrostatic driving force for the uptake of TSC-Au NPs to wool is retained.

In the following study, TU yarn was soaked in a pH 2.0 glycine buffer $\left(0.1 \mathrm{~mol} \mathrm{~L}^{-1}\right.$, 1:60 wool to liquor ratio, $120 \mathrm{~min}$ ) before being immersed in a TSC-grey colloid also at $\mathrm{pH}$ 2.0. Albaflow FFA $\left(0-2 \mathrm{~g} \mathrm{~L}^{-1}\right)$ was added to both the buffer and colloid (which is equivalent to the $0-4 \mathrm{~g} \mathrm{~L}^{-1}$ Albaflow FFA added to the colloid only in the previous experiments). Figure 4.41 shows the uptake of the TSC-grey colloid to TU yarn over time, without the presence of the wetting agent. The typical destabilisation of TSC$\mathrm{Au}$ NPs occurs to a lesser extent than when the buffer is not employed (as was shown in Figure 4.36). This could be due to the wool leaching destabilising components (for example processing lubricants) into the buffer solution, or the buffer molecules stabilising the TSC-grey colloid. The longer wavelength absorbance of agglomerated gold nanoparticles increases in intensity relative to the SRP peak of stabilised TSC$\mathrm{Au}$ NPs during the first $10 \mathrm{~min}$ of the reaction. After this point, both peaks steadily decrease as nanoparticles and agglomerates are absorbed by the wool.

The uptake of the TSC-grey colloid to TU yarn with $0.5-2 \mathrm{~g} \mathrm{~L}^{-1}$ of Albaflow FFA in both the buffer and colloid are presented in Figure 4.42. The stabilising presence of the wetting agent prevents the agglomeration of the TSC-Au NPs, which allows the uptake to be monitored with UV-vis spectroscopy. 


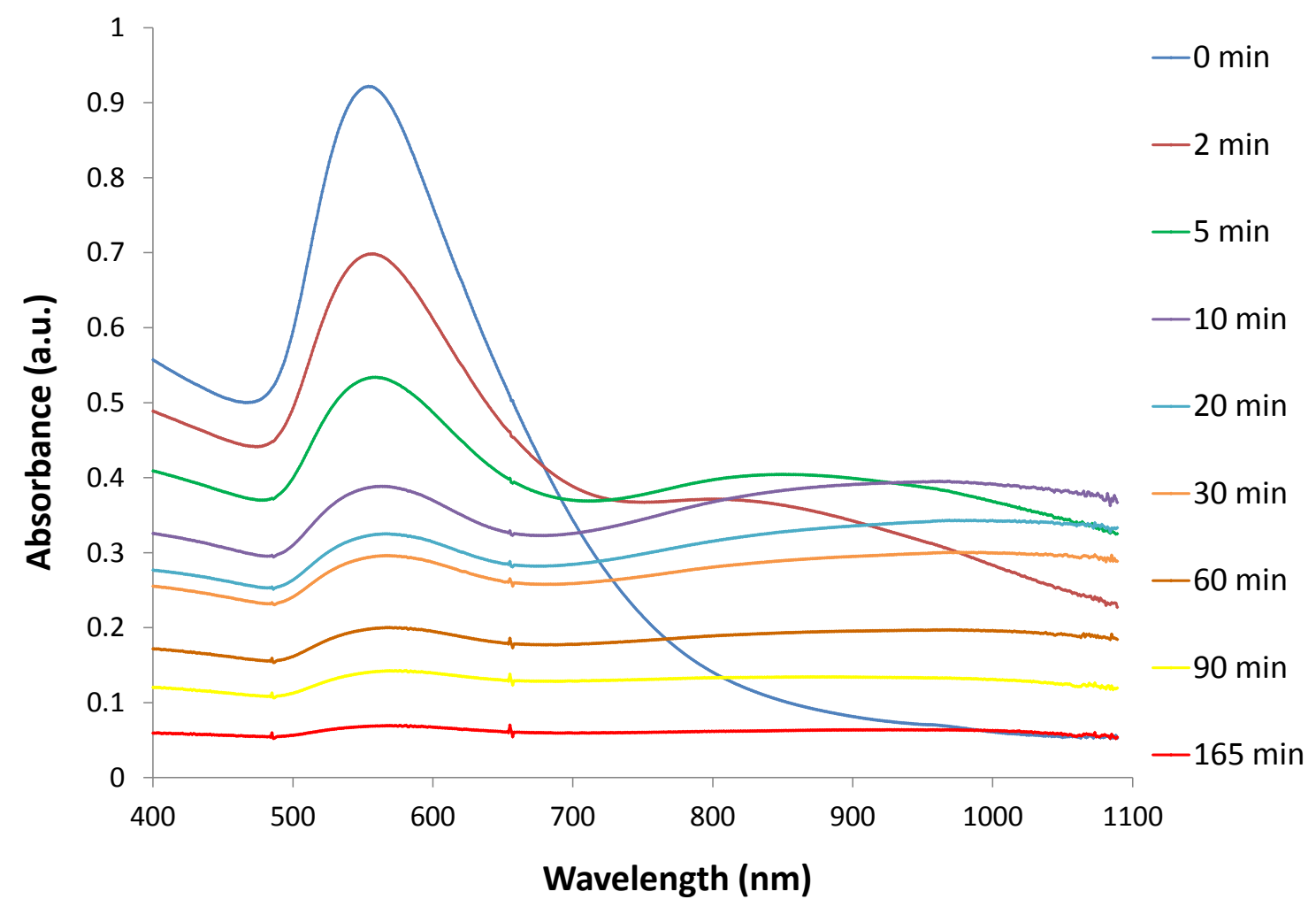

Figure 4.41 UV-vis spectra of the uptake of TSC-grey to TU yarn of TSC-grey colloid after pre-soaking in a pH 2.0 glycine buffer (without Albaflow FFA).

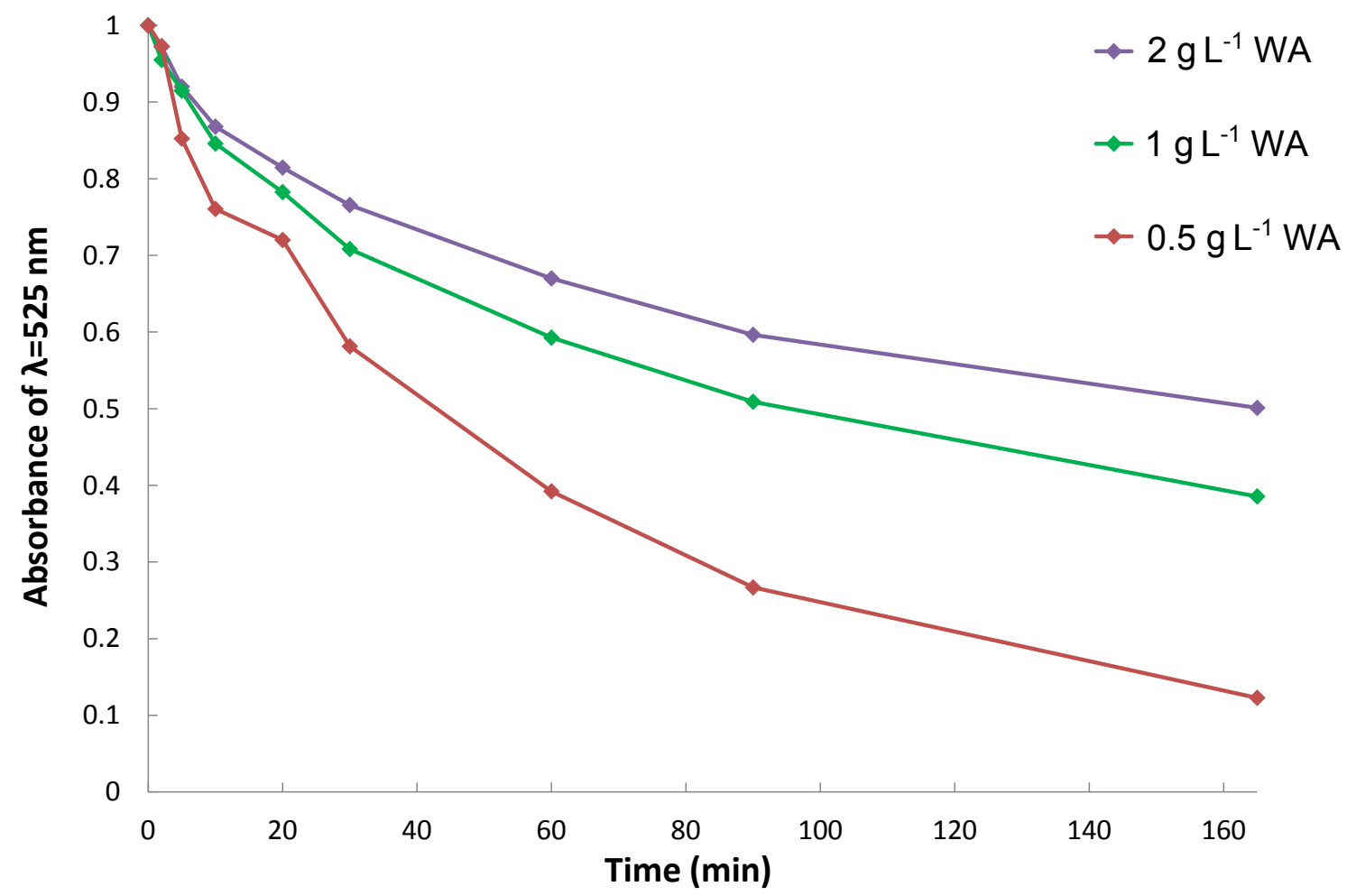

Figure 4.42 Uptake of the TSC-grey colloid to TU yarn after pre-soaking in a pH 2.0 glycine buffer with different amounts of Albaflow FFA in both the buffer and colloid. 
The composites that resulted at the end of the uptake of the TSC grey colloid after pre-soaking in a pH 2.0 glycine buffer are shown in Figure 4.43. The colour range displays different shades of grey, where the composite synthesised without Albaflow FFA is a much lighter grey. Again the composites synthesised with higher amounts of the wetting agent exhibit a purple tinge, which relatively more pronounced than the composites synthesised in without the glycine buffer. This implies more enhanced stabilisation of the TSC-Au NPs when the both Albaflow FFA and the buffer are used together.

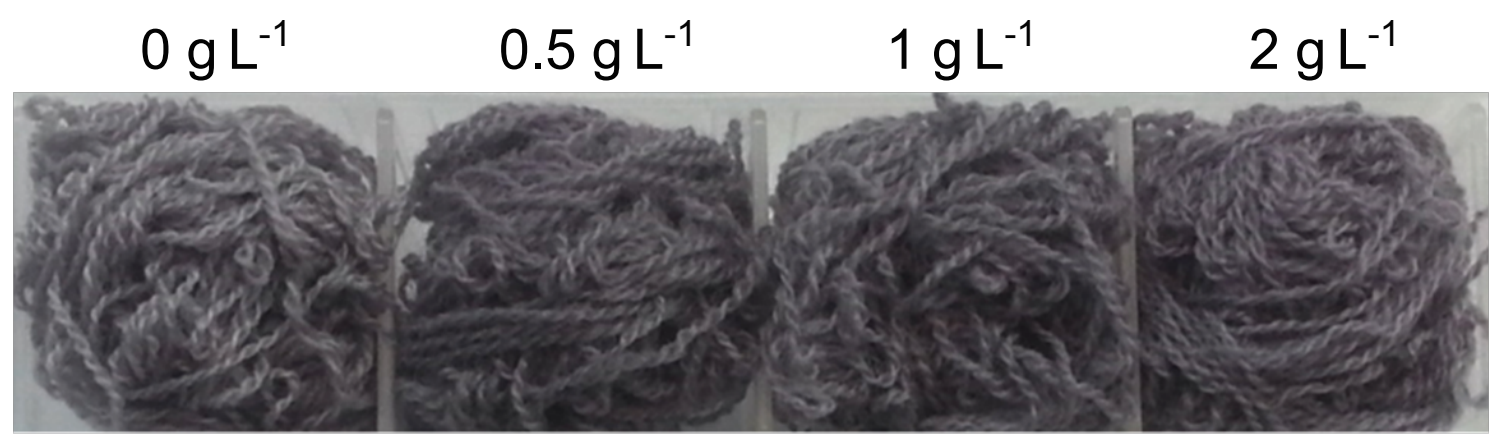

Figure 4.43 TSC-grey composites of TU yarn synthesised with different amounts of Albaflow FFA in the pH 2.0 buffer and colloid.

Table 4.12 presents the CIE colour coordinates of the TU yarn TSC-grey composites shown in Figure 4.43. Increasing the amount of Albaflow FFA in the synthesis has resulted in darker, more red and bluer coloured composites. The $2 \mathrm{~g} \mathrm{~L}^{-1}$ Albaflow FFA composite is slightly lighter and less blue than the $1 \mathrm{gL}^{-1}$ composite, which represents the purple tinge of this composite.

Table 4.12 CIE colour coordinates of TU yarn TSC-grey composites synthesised with different levels of Albaflow FFA (WA) in the buffer and colloid.

\begin{tabular}{ccccc}
\hline WA $\left(\mathrm{g} \mathrm{L}^{-1}\right)$ & 0 & 0.5 & 1 & 2 \\
\hline $\mathrm{L}^{*}$ & 50.93 & 45.33 & 42.66 & 43.86 \\
$\mathrm{a}^{*}$ & 2.64 & 3.60 & 3.68 & 3.89 \\
$\mathrm{~b}^{*}$ & -3.82 & -5.06 & -5.69 & -5.06 \\
\hline \hline
\end{tabular}

The TSC-grey composites synthesised utilising both the glycine buffer and Albaflow FFA exhibited a more intense purple tinge than those synthesised without the buffer. Considering that scale-up reactions employ a range of different concentrations and combinations of auxiliary agents to promote uniform colouring, the above study was repeated at an elevated uptake temperature to attempt to enhance the greyness of the composites. 


\section{Albaflow FFA and Glycine Buffers with the TSC-grey Method at $80^{\circ} \mathrm{C}$}

TSC-grey TU yarn composites synthesised with $\left(0-2 \mathrm{~g} \mathrm{~L}^{-1}\right)$ Albaflow FFA in the $\mathrm{pH}$ 2.0 buffer and colloid, and an uptake temperature of $80^{\circ} \mathrm{C}$ are shown in Figure 4.44. Interestingly, the composites are more grey coloured (less purple), indicating that the heat has increased the agglomeration of the TSC-Au NPs on the surface of the composites. However, the resulting composites are a significantly lighter grey colour and less uniform than those synthesised at RT. Additionally, the rate of uptake is much more rapid at $80^{\circ} \mathrm{C}$, which likely caused the relatively uneven colouration of the composites.

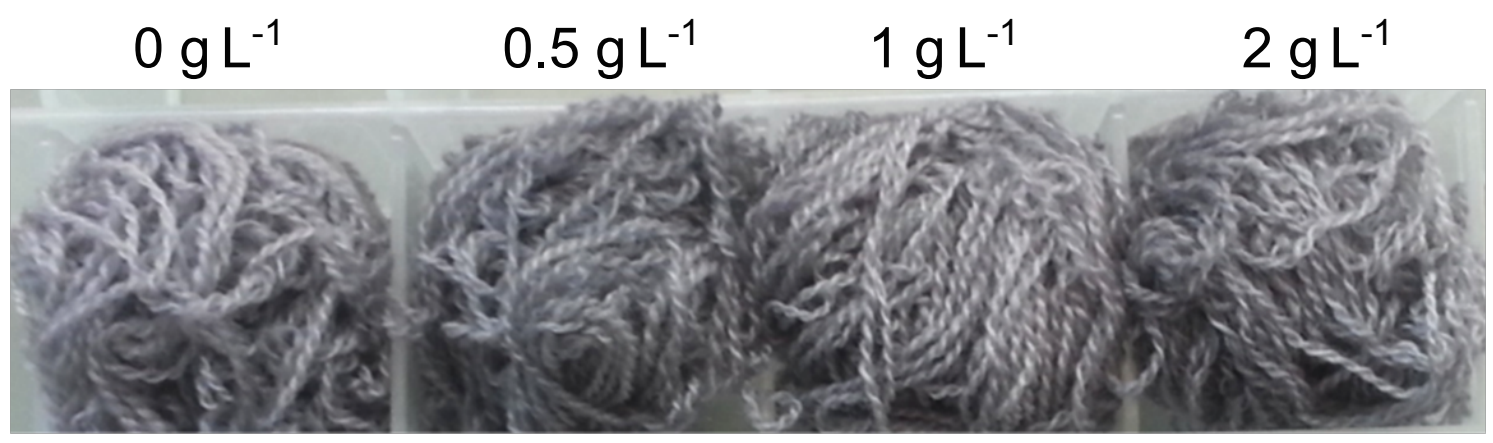

Figure 4.44 TSC-grey composites of TU yarn synthesised at an uptake temperature of $80^{\circ} \mathrm{C}$ with different amounts of Albaflow FFA in the $\mathrm{pH} 2.0$ buffer and colloid.

The CIE colour coordinates of these composites are shown in Table 4.13. Increasing the amount Albaflow FFA in the reaction results in the synthesis of darker and bluer composites. The redness values are significantly lower than those of the composites synthesised at RT, as is synonymous with greyer composites. The $80^{\circ} \mathrm{C}$ composites also exhibit much lower $\mathrm{L}^{*}$ values than the RT composites due to their lighter grey colouration.

Table 4.13 CIE colour coordinates of TU yarn TSC-grey composites synthesised with different amounts of Albaflow FFA (WA) added after pH adjustment.

\begin{tabular}{ccccc}
\hline WA $\left(\mathrm{g} \mathrm{L}^{-1}\right)$ & 0 & 0.5 & 1 & 2 \\
\hline $\mathrm{L}^{*}$ & 52.77 & 52.07 & 48.59 & 46.00 \\
$\mathrm{a}^{*}$ & 2.70 & 2.15 & 1.89 & 2.02 \\
$\mathrm{~b}^{*}$ & -3.64 & -3.85 & -3.92 & -4.62 \\
\hline \hline
\end{tabular}

Elevating the uptake temperature has increased the relative greyness of the composites, however, the composites are also relatively light and unevenly coloured. Given the positive effects upon slowing the rate of uptake and increasing the colour uniformity of the ex-situ composites synthesised with the use of Albaflow FFA, a range of levelling agents were next investigated. 


\section{Levelling Agents}

As mentioned in Section 1.1.4, the structure of levelling and wetting agents are generally similar, however, levelling agents are typically more effective at controlling the uptake of dye molecules to wool. As such, a range of levelling agents were used to attempt to slow the uptake of gold nanoparticles to wool. The levelling agents that were recommended by Professor James Johnston's industry contacts at Tararua Yarns in Levin, New Zealand are listed in Table 4.14.

Table 4.14 Levelling agents that were employed in the ex-situ procedure.

\begin{tabular}{ccc}
\hline Levelling agent & Recommended level & Amount employed \\
\hline Albegal B & $2 \mathrm{~g} \mathrm{~L}^{-1}$ & $0.5 \mathrm{~g} \mathrm{~L}^{-1}$ \\
Lyogen MF & $2 \mathrm{~g} \mathrm{~L}^{-1}$ & $0.5 \mathrm{~g} \mathrm{~L}^{-1}$ \\
Croscolor DH2 & $0.33 \mathrm{~g} \mathrm{~L}^{-1}$ & $0.0825 \mathrm{~g} \mathrm{~L}^{-1}$ \\
Tecarapid CBF & $2 \mathrm{~g} \mathrm{~L}^{-1}$ & $0.5 \mathrm{~g} \mathrm{~L}^{-1}$ \\
\hline \hline
\end{tabular}

The uptake TA-Au NPs to TU yarn with different levelling agents was studied with UV-vis spectroscopy. The synthesis was originally carried out with the recommended amounts of levelling agents added to the glycine buffer prior to soaking the wool in the colloid. However, the recommended amounts of each of the levelling agents completely prevented the uptake of TA-Au NPs to TU yarn. As such, the uptake of TA-Au NPs was repeated with one quarter of the recommended amounts of the different levelling agents in the buffers, the uptake curves of which are shown in Figure 4.45.

Each of the levelling agents have significantly slowed the uptake of TA-Au NPs to wool to different extents. The levelling agents also cause an increase in the intensity of the SPR absorbance at the beginning of the uptake. Similar to Albaflow FFA, the levelling agents associate to the surface of the wool fibres during the buffer stage, and some leach into the colloid at the beginning of the uptake. The levelling agents, especially Albegal B, cause a baseline contribution across the entire UVvisible spectrum, hence amplifying the intensity of the $530 \mathrm{~nm}$ SPR peak.

The uptake reactions were carried out at RT, a temperature at which only Croscolor DH2 and Tecarapid CBF exhibited significant absorption of the TA-Au NPs over time. As both Albegal B and Lyogen MF had almost completely prevented the uptake of the TA-Au NPs, these uptake solutions were then heated to $80^{\circ} \mathrm{C}$ for $24 \mathrm{~h}$. The resulting composites from these uptake reactions are shown in Figure 4.46 (the Croscolor DH2 and Tecarapid CBF composites were not heated). Despite the elevated uptake temperature, the Albegal B composite remains completely uncoloured. 


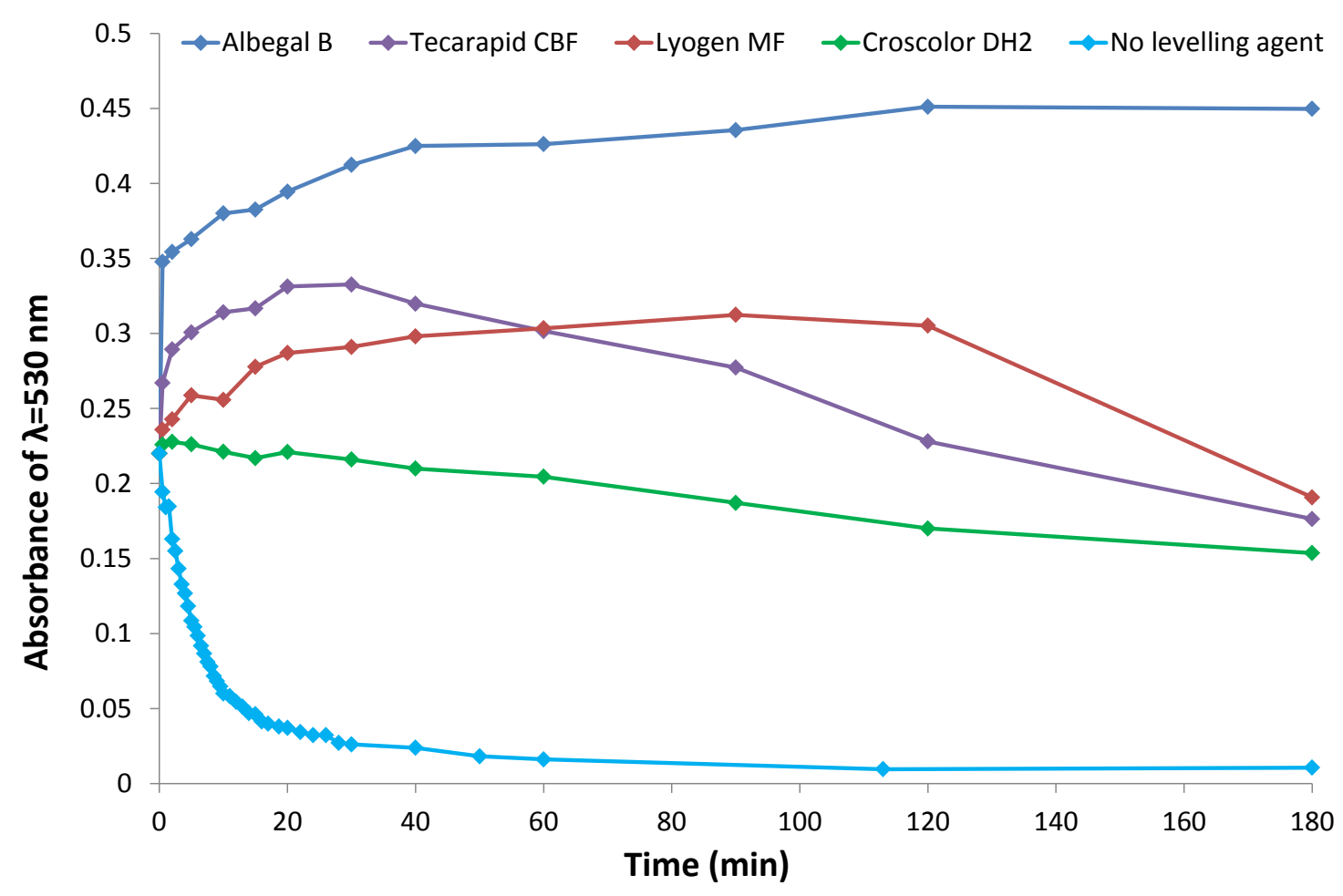

Figure 4.45 The uptake of the TA-mauve colloid to TU yarn, with one quarter of the recommended amount of the levelling agents present in the buffer only. The uptake with no levelling agent is provided for reference.

The heated Lyogen MF composite is light grey-mauve, and the Croscolor DH2 composite is a darker grey-mauve. Only the Tecarapid CBF composite exhibits the typical purple-mauve hue of the TA-mauve synthesis.

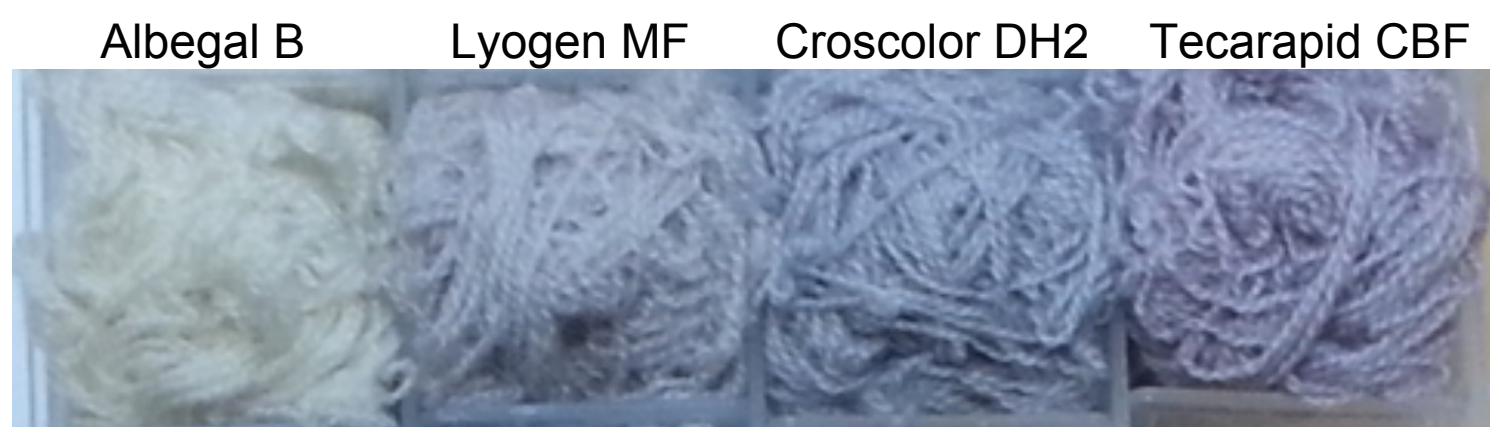

Figure 4.46 TA-mauve TU yarn composites synthesised with the addition of levelling agents to the uptake solutions, where the uptake of the Albegal B and Lyogen MF solutions was elevated to $80^{\circ} \mathrm{C}$.

CIE colour coordinates of the composites are presented in Table 4.15. The light yellow colour of the Albegal B composite is represented with its very high $\mathrm{L}^{*}$ and $\mathrm{b}^{*}$ values, indicating a lack of purple coloured gold nanoparticles. The relatively high $\mathrm{L}^{*}$ and $\mathrm{a}^{*}$ values of the Lyogen MF composite are due to its relatively light greymauve colour. The Croscolor DH2 composite exhibits a very low a* value, which is representative of its grey colouration. The purple-mauve hue of the Tecarapid 
composite exhibits the CIE colour coordinates similar to those that are typically observed in the TA-mauve method. Additionally, the composites are all very level in colour.

Table 4.15 CIE colour coordinates of TU yarn TA-mauve composites synthesised with different levelling agents in the buffer prior to the uptake of TA-Au NPs.

\begin{tabular}{ccccc}
\hline & Albegal B & Lyogen MF & Croscolor DH2 & Tecarapid CBF \\
\hline $\mathrm{L}^{*}$ & 79.91 & 68.09 & 62.35 & 57.99 \\
$\mathrm{a}^{*}$ & 0.65 & 2.06 & 0.91 & 4.88 \\
$\mathrm{~b}^{*}$ & 13.07 & 3.95 & -0.28 & -1.65 \\
\hline \hline
\end{tabular}

The investigated levelling agents either completely halted or substantially slowed the uptake of the TA-Au NPs to TU yarn, and resulted in evenly coloured composites. Croscolor DH2 and Tecarapid CBF showed promise for the large-scale synthesis of ex-situ gold nanoparticle-wool fibre composites. These levelling agents are investigated further in package dyeing reactions in Section 5.4.1.

\subsubsection{Concentration and Volume of Dye Solution}

Industrial wool dyeing reactions employ a diverse range of wool to liquor ratios, depending on the type of wool, dyestuffs and equipment used in the dyeing process. ${ }^{8}$ As such, it is important to determine the effects of both the concentration and volume of the colloid that is used in the ex-situ procedure. Lucas and Johnston had already found that varying the concentration of gold nanoparticles that were used in the ex-situ procedure determines the colour of the resulting composites (the main difference between the TSC-pink and grey methods is the concentration of TSC-Au NPs employed). ${ }^{5}$

The following study presents TA-mauve TU yarncomposites synthesised with different concentrations of TA-Au NPs (from 0.5-6 times the regular concentration). The concentrations of $\mathrm{Au}^{3+}$ that were used in the synthesis of TA-Au NPs are noted in Table 4.16, where $1 \mathrm{x}$ represents the typical concentration of TA-Au NPs used in the standard TA-mauve method.

The dyeing reactions used the conditions of the standard TA-mauve method with $1 \mathrm{~g} \mathrm{~L}^{-1}$ Albaflow FFA in the buffer and colloid, while equivalent ratios of $\mathrm{AuCl}_{4}^{-}$to TA were used in the colloidal syntheses. Figure 4.47 shows the resulting composites, where the colour range encompasses different shades of pink, mauve and purple. 
Table 4.16 The concentrations TA-Au NPs that were used to colour TU yarn.

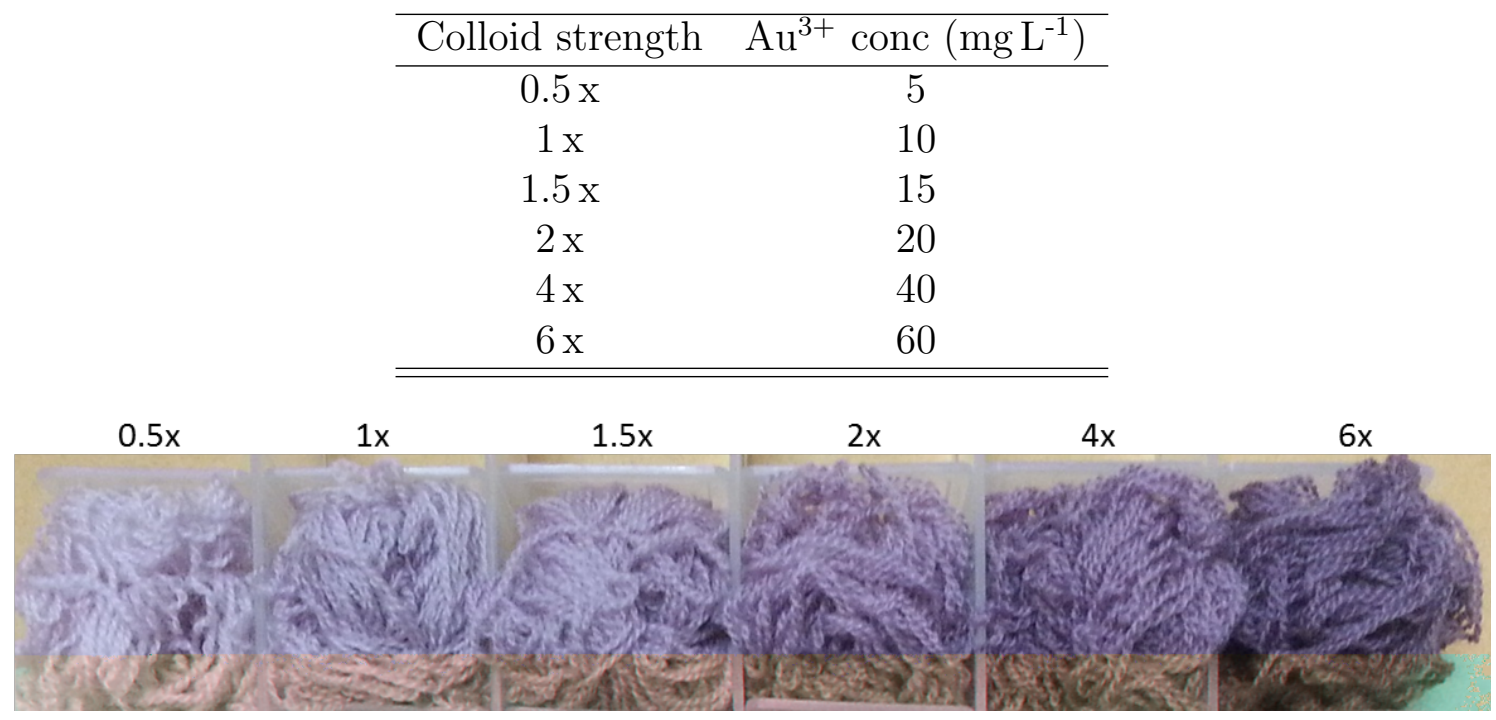

Figure 4.47 TA-mauve TU yarn composites synthesised with different concentrations of TA-Au NPs and $1 \mathrm{~g} \mathrm{~L}^{-1}$ Albaflow FFA.

Table 4.17 shows the CIE colour coordinates of the composites that are shown in Figure 4.47. Increasing the concentration of the TA-mauve colloid causes darker and bluer composites across the investigated concentration range. There is an increase in the $\mathrm{a}^{*}$ value of composites synthesised with the $0.5 \mathrm{x}$ to $2 \mathrm{x}$ concentrations of TA-Au NPs, as a darker mauve colour is produced. This is followed by a decreasing redness value when the concentration is increased from $2 \mathrm{x}$ to $6 \mathrm{x}$ as the darker purple composites are produced. This may be due to the higher concentration of the colloid increasing the agglomeration of the gold nanoparticles, which would result in slight grey colour contribution.

Table 4.17 CIE colour coordinates of TA-mauve TU yarn composites synthesised with different concentrations of the colloid.

\begin{tabular}{cccccccc}
\hline Colloid strength & $0 \mathrm{x}$ & $0.5 \mathrm{x}$ & $1 \mathrm{x}$ & $1.5 \mathrm{x}$ & $2 \mathrm{x}$ & $4 \mathrm{x}$ & $6 \mathrm{x}$ \\
\hline $\mathrm{AuCl}_{4}^{-}$conc $\left(\mathrm{mg} \mathrm{L}^{-1}\right)$ & 0 & 5 & 10 & 15 & 20 & 40 & 60 \\
\hline $\mathrm{L}^{*}$ & 84.83 & 69.21 & 59.75 & 55.25 & 49.24 & 43.29 & 37.49 \\
$\mathrm{a}^{*}$ & 0.41 & 6.06 & 6.97 & 9.76 & 10.99 & 10.44 & 9.81 \\
$\mathrm{~b}^{*}$ & 12.52 & 2.40 & -1.42 & -1.98 & -2.48 & -3.26 & -4.19 \\
\hline \hline
\end{tabular}

SEM and EDS analyses of yarn composites synthesised with $1 \mathrm{x}$ and $6 \mathrm{x}$ the concentration of the standard TA-mauve colloid are shown in Figure 4.48. The $1 \mathrm{x}$ concentration composite exhibits the expected distribution of (primarily individual) TA-Au NPs and agglomerates of a few nanoparticles on its surface.

SEM micrographs of the $6 \mathrm{x}$ composite show a much higher level of gold nanoparticles, with the gold nanoparticles preferentially congregating at the cuticle edges. The higher magnification image appears to show a relatively higher proportion of 


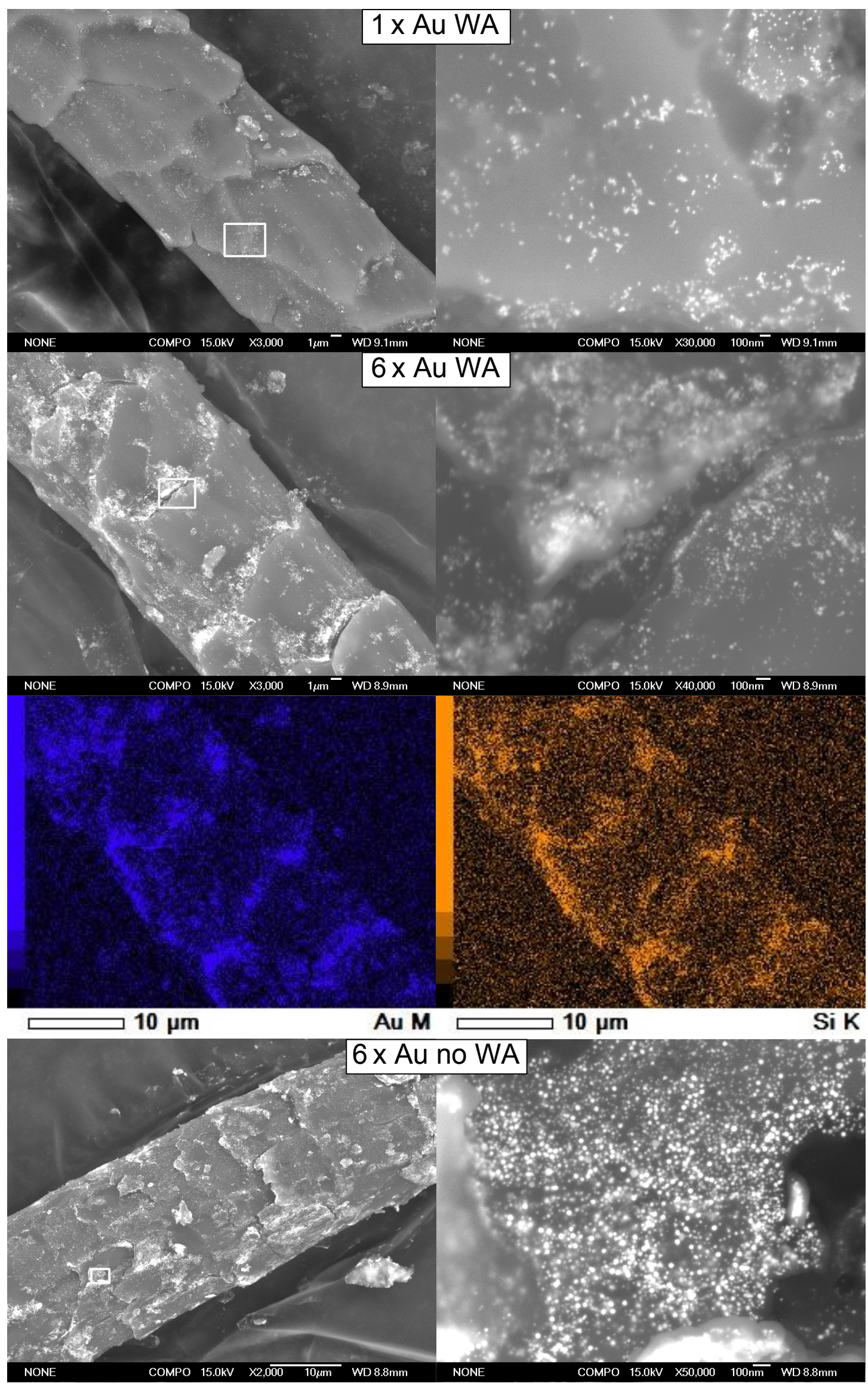

Figure 4.48 SEM and EDS analysis of TA-mauve TU yarn composites synthesised with 1 and $6 \mathrm{x}$ the concentration of TA-Au NPs in the standard TA-mauve method, and a composite with $6 \mathrm{x}$ the concentration synthesised without Albaflow FFA. 
agglomerates of gold nanoparticles compared to individual particles than the $1 \mathrm{x}$ composite. The cloudy areas in the SEM micrographs are composed of large agglomerates of gold nanoparticles (these are observable upon further magnification of these areas).

The Au EDS elemental map of the surface of the $6 \mathrm{x}$ concentration composite highlights the congregation of gold nanoparticles near the cuticle edges. EDS maps of $\mathrm{Si}$ and $\mathrm{O}$ also show a significant relative density of these elements near the cuticle edges, which shows the preferential association of Albaflow FFA at the cuticle edges.

The synthesis of the $6 \mathrm{x}$ TA-mauve TU composite was repeated without the use of Albaflow FFA. The SEM micrographs of the resulting composite show significantly less material located near the cuticle edges, with a relatively higher gold density across the cuticle scales. This indicates a propensity for Albaflow FFA to direct gold nanoparticle attachment to the cuticle edges. This may occur by blocking access to the cuticle edges, which results in more uniformly coloured composites.

\section{Volume of Colloid}

Industrial package dyeing reactors, which are used for colouring fine merino yarns, typically employ wool to liquor ratios that are between 1:7 and 1:20. This is a much lower volume of dye solution than the typical 1:60 wool to liquor ratio employed in the laboratory-scale synthesis of ex-situ composites. TA-Au NPs were used to colour S14, S19 and TU merino yarns with wool to liquor ratios of 1:7. 1:15 and 1:20 and $1 \mathrm{x}$ the concentration of the TA-mauve colloid $\left(10 \mathrm{mg} \mathrm{L}^{-1} \mathrm{Au}^{3+}\right)$. This was undertaken to investigate whether lower volumes of the same concentration of the TA-mauve colloid could produce suitably coloured composites.

Samples of S14, S19 and TU yarns (5 g) were added to 35, 75 and $100 \mathrm{~mL}$ of the TAmauve colloid, and transferred on a shaker table to promote uniform dye absorption. The conditions of the standard TA-mauve method were used, with $1 \mathrm{~g} \mathrm{~L}^{-1}$ Albaflow FFA added to the buffer and the colloid. The resulting composites are represented in Figure 4.49. The colours of the composites are relatively uneven for $5 \mathrm{~g}$ samples, due to the gold colloid barely covering the wool yarn in the dye solution. The colours of the composites range from light pink to mauve. 


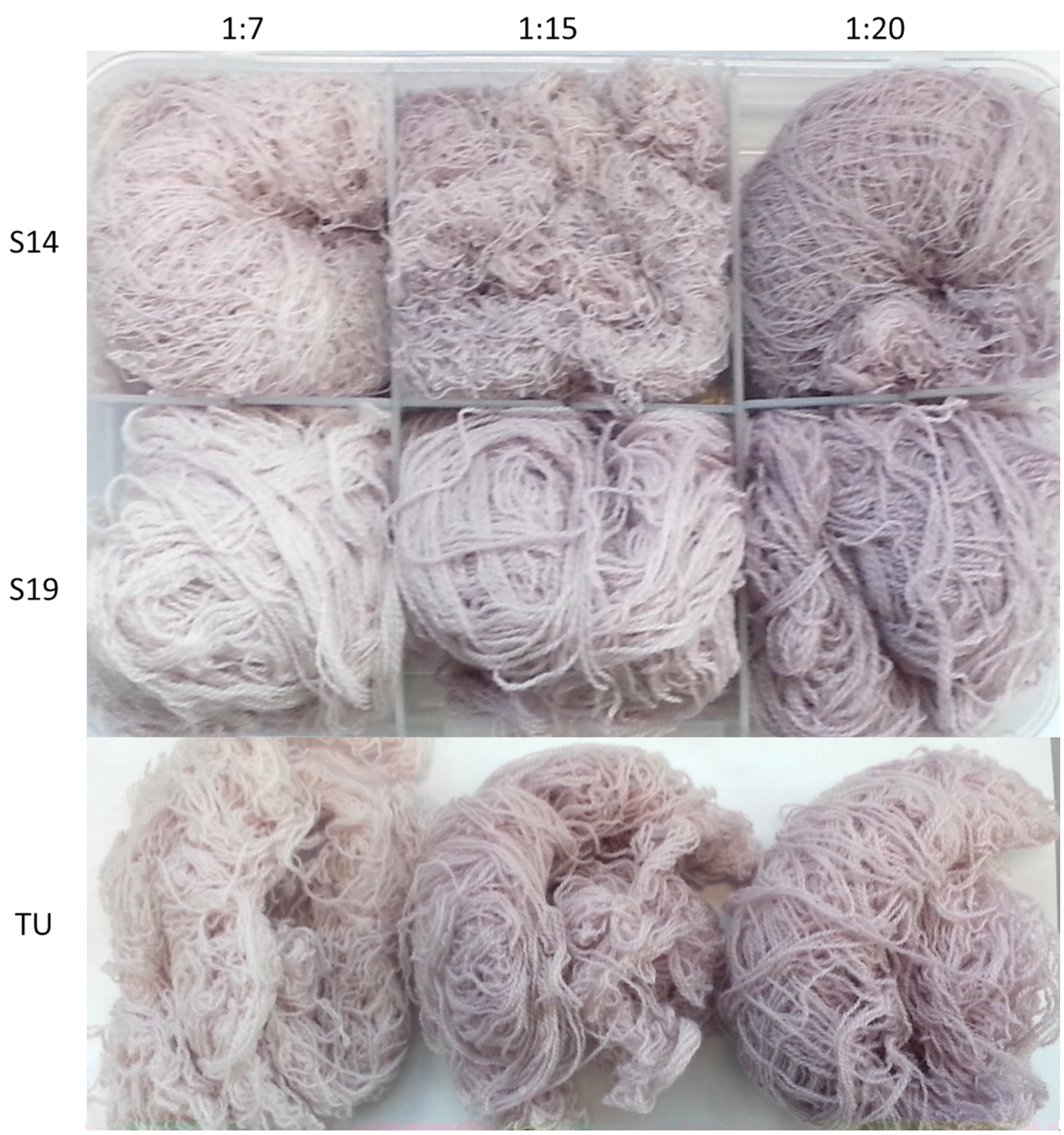

Figure 4.49 TA-mauve composites of different finished merino yarns synthesised with different volumes of the TA-mauve colloid.

CIE colour coordinates of the composites are shown in Table 4.18. Increasing the volume of TA-mauve colloid in the dyeing of each yarn type (or changing the wool to liquor ratio from 1:7, 1;15 and 1:20) results in sequentially darker, more red and blue coloured composites. This is associated with a higher loading of pink-purple coloured gold nanoparticles on the surfaces of the composite fibres.

Table 4.18 CIE colour coordinates of TU yarn TA-mauve composites synthesised with different colloid concentrations.

\begin{tabular}{cccccccccc}
\hline Yarn type & & S14 & \multicolumn{3}{c}{ S19 } & \multicolumn{3}{c}{ TU } \\
\hline Wool:liquor & $1: 7$ & $1: 15$ & $1: 20$ & $1: 7$ & $1: 15$ & $1: 20$ & $1: 7$ & $1: 15$ & $1: 20$ \\
\hline $\mathrm{L}^{*}$ & 74.14 & 66.42 & 58.08 & 74.67 & 66.45 & 59.58 & 76.09 & 69.16 & 62.14 \\
$\mathrm{a}^{*}$ & 6.08 & 5.07 & 5.79 & 6.24 & 7.32 & 7.96 & 6.47 & 7.75 & 8.37 \\
$\mathrm{~b}^{*}$ & 3.22 & -0.15 & -2.38 & 4.10 & 2.55 & 0.71 & 3.36 & 1.16 & -0.44 \\
\hline \hline
\end{tabular}


Interestingly, the S14 fibres exhibit the darkest, least red and bluest colour coordinates at each wool to liquor ratio, which is unexpected considering the higher effective surface area of the $14.5 \mu \mathrm{m}$ fibres. The thicker S19 and TU yarn composites exhibit sequentially lower $\mathrm{L}^{*}$, higher $\mathrm{a}^{*}$ and lower $\mathrm{b}^{*}$ values, indicating a lesser purple colouration, and fewer gold nanoparticles on the surface of the composites. This trend is in contrast to prior experiments, where yarns of thicker wool fibres are typically darker purple in colour. The effect may be due to the difference in the structures of the yarns or the presence of processing additives during yarn making, and may be compounded by the uneven colouring of the composites (as the CIE colour coordinates are less representative). The uneven colouration of the composites is not particularly concerning, as package dyeing reactions circulate dye liquors to promote uniform absorption.

The experiments in this section have shown that dyeing merino yarns with a range of different concentrations and volumes of TA-Au NPs generally results in different intensities of pink-mauve colouration, and typically does not influence the hue of the composites. This is in contrast to dyeing wool with TSC-stabilised colloids, where different concentrations and volumes of TSC-Au NPs results in pink, purple or grey composites. Varying the concentration and volume of gold colloids will allow for a range of composites to be synthesised in large-scale wool dyeing procedures.

\subsubsection{Drying of Composites}

A repeated observation of the ex-situ procedure was that the colour of the composites often darkens upon drying. TSC-pink composites typically appear pink when wet, and darken to a light purple upon drying. Additionally, dried TSC-grey composites appear a darker grey colour than when they are wet. It was considered that this could be due to aggregation of nanoparticles on the surface of the composites as they dry (rather than an optical effect of the water). Cryo-SEM was used to investigate this, where the composite fibres were frozen in liquid nitrogen at the completion of the uptake of TSC-Au NPs to wool (while still wet). Following this the frozen composite fibres were carbon coated and imaged at relatively low magnifications.

Figure 4.50 shows Cryo-SEM micrographs of two different TSC-pink Cariaggi composite fibres frozen at the completion of the uptake of TSC-Au NPs. The backscatter images were taken at 4000x magnification (at higher magnifications the images begin to distort). Both fibres appear to exhibit a slightly higher proportion of gold on the surface of the cuticles as compared to those located near the edges of the cuticle scales (circled in Figure 4.50), relative to the dried composites that were shown ear- 
lier in Figure 4.7. This indicates that gold nanoparticles may migrate towards the cuticle edges during the drying of the composites. However, the inability to image higher magnifications of the composite make it difficult to ascertain whether or not there are fewer agglomerates of TSC-Au NPs before the composites are dried.

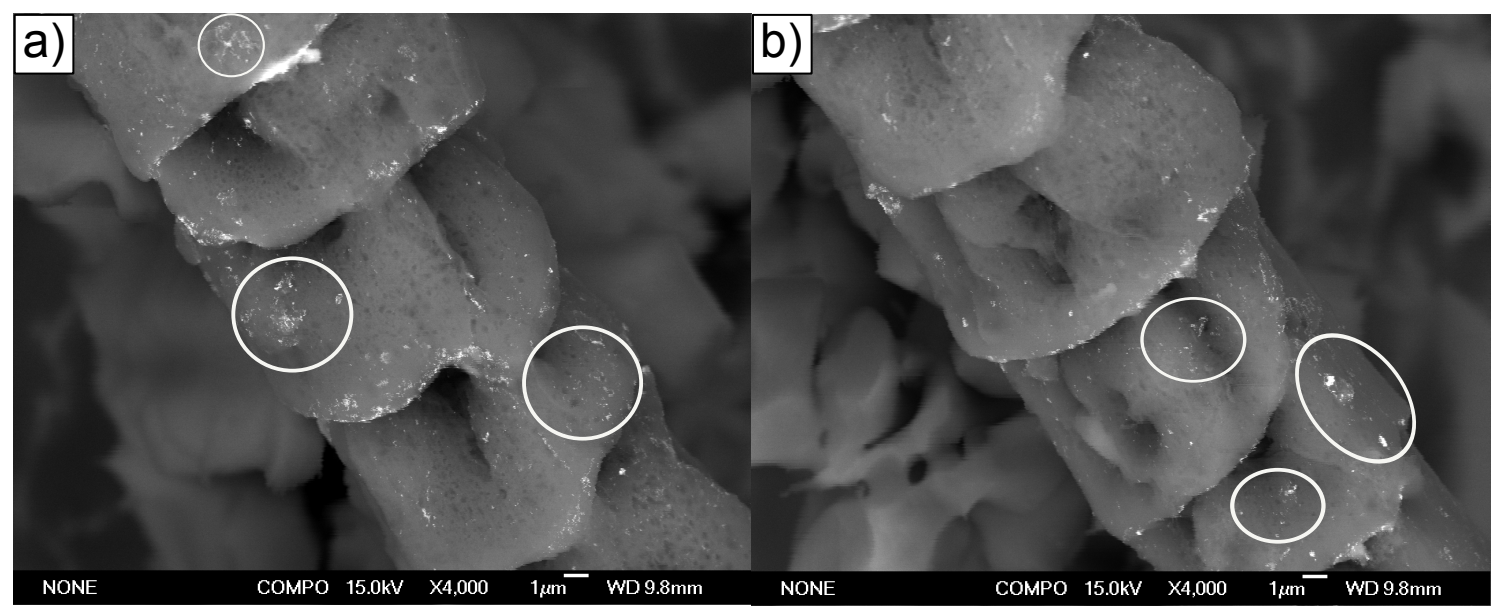

Figure 4.50 Cryo-SEM micrographs in backscatter mode of TSC-pink Cariaggi wool fibres frozen at the completion of the uptake (before drying) in (a) and (b).

Cryo-SEM analysis of a TSC-grey Ascend felted yarn composite was also undertaken. The backscatter micrograph in Figure 4.51 (a) shows that the bright areas which represent gold nanoparticles are distributed relatively evenly across the cuticle scales. There does not seem to be any preferential attachment of the gold nanoparticle at or near the cuticle edges. This is uncommon for TSC-grey composites, and shows more compelling evidence of a movement of gold nanoparticles towards the cuticle edges during the drying of the composites.

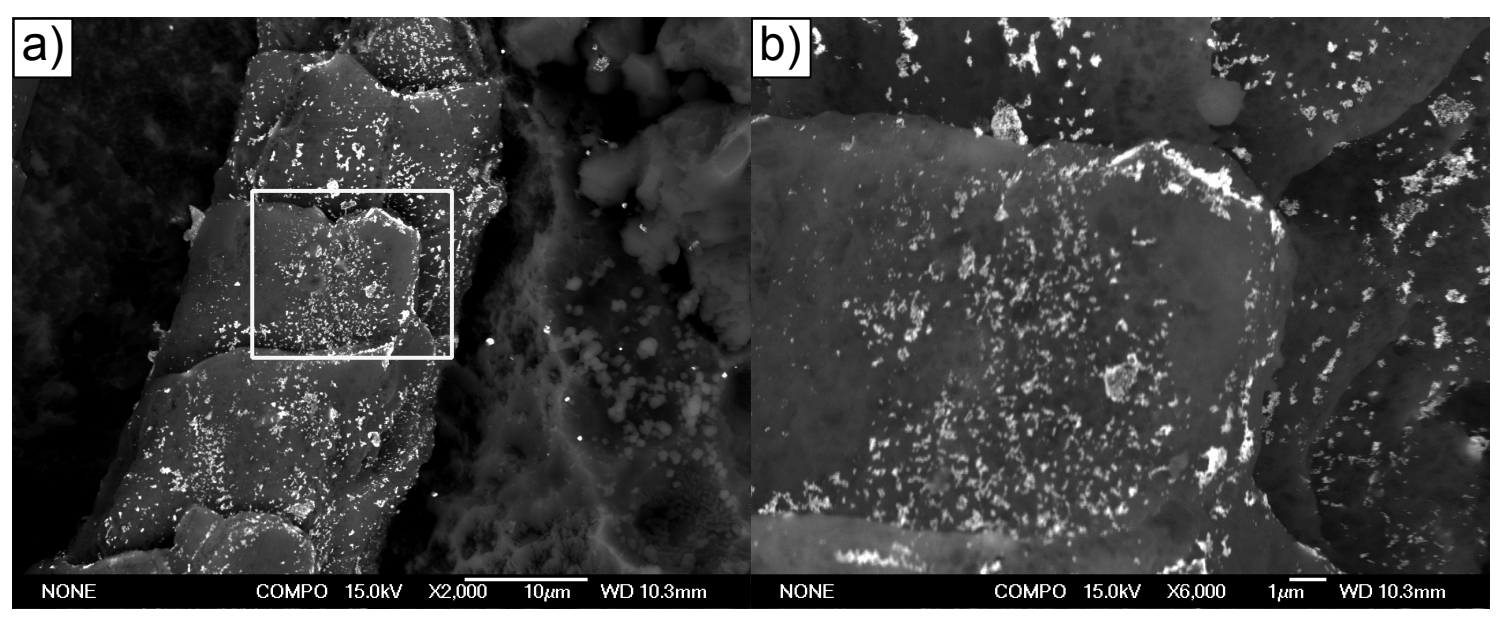

Figure 4.51 Cryo-SEM images of a TSC-grey Ascend felted yarn composite fibre frozen at the completion of the uptake at different magnifications in (a) and (b).

The higher magnification image (Figure 4.51 (b)) does show the presence of mostly agglomerates of TSC-Au NPs, with some individual gold nanoparticles also being present. This is often observed on the surface of TSC-grey composites however, and 
as such, it cannot be determined whether individual gold nanoparticles agglomerate further upon drying of the composites.

The Cryo-SEM analyses of TSC-pink and TSC-grey composites indicate that there may be a movement of gold nanoparticles and agglomerates from the cuticle scales towards the edges of the cuticle scales during the drying of the composites, however, they failed to provide conclusive evidence of this. The movement towards the cuticle edges is understandable considering that these are the kinetically favourable sites for attachment, and that the typical pathway for the absorption of chemicals into the interior of the wool occurs through the junctions between the cuticle scales.

\subsection{Conclusions}

Section 4.1 introduced the chemistry of the ex-situ procedure by detailing the synthesis and characterisation of ex-situ composites of Cariaggi wool. The syntheses of TA- and TSC gold nanoparticle colloids were then characterised in Section 4.2. The rate of formation and $\mathrm{pH}$ stability of different concentrations of gold colloids was studied with UV-vis spectroscopy. The ratios of $\mathrm{AuCl}_{4}^{-}$to stabilising agents were manipulated, and it was found that increasing the amount of TSC in the gold nanoparticle synthesis resulted in more stable gold colloids.

Characterisation of the effects of wool type, $\mathrm{pH}$, auxiliary agents, gold nanoparticle colloid concentration and volume, on the ex-situ procedure were presented in Section 4.3. The uniform colouring of merino yarns with Albaflow FFA was a very positive result, as yarn is optimal form of wool to dye from a commercial perspective. Each of the investigated reaction conditions influenced the rate uptake of gold nanoparticles to wool fibres and/or the colouration of the ex-situ composites. Thus, these reaction parameters are important to consider in scale-up dyeing procedures.

The reaction conditions that were optimal for ex-situ composites of Cariaggi wool and TU yarn for the TA-mauve and TSC-grey methods are listed in Table 4.19. It must be noted that new sources of wool that are used in the ex-situ procedure may require further optimisation of these parameters.

The chemistry that was presented in this Chapter 4 directly relates to the scale-up of ex-situ gold nanoparticle-wool fibre composites, and was an integral step towards the commercialisation of the novel composites. The ex-situ procedure is a relatively inexpensive and robust alternative to the in-situ procedure. As such, the scale-up 
Table 4.19 The conditions of the standard ex-situ methods.

\begin{tabular}{lcc}
\hline Approach & TA-mauve & TSC-grey \\
\hline $\mathrm{Au}^{3+}$ conc in colloid $\left(\mathrm{mg} \mathrm{L}^{-1}\right)$ & 10 & 40 \\
Molar ratio of $\mathrm{AuCl}_{4}^{-}$to stabiliser & $1: 0.1$ & $1: 12.5$ \\
Wool to liquor ratio & $1: 60$ & $1: 60$ \\
Uptake pH & 2.2 & 2.0 \\
Glycine buffer conc $\left(\mathrm{mol} \mathrm{L}^{-1}\right)$ & 0.1 & 0.1 \\
Wetting agent $\left(\mathrm{g} \mathrm{L}^{-1}\right)$ & 1 & 1 \\
$\mathrm{Au}$ content $(\mathrm{wt} \mathrm{\% )}$ & 0.06 & 0.24 \\
\hline \hline
\end{tabular}

experiments presented in Chapter 5 only involve the ex-situ approach. Following this, research into improving the washfastness properties of ex-situ composites is detailed in Chapter 6. 


\section{Chapter 5}

\section{Scale-up of Ex-situ Gold Nanoparticle-wool Fibre Composites}

This chapter details the pilot-scale preparation of ex-situ gold nanoparticle-wool fibre composites, which is an integral step towards the commercialisation of these novel nanocomposites. The scale-up research was carried out co-currently with the optimisation of the in-situ and ex-situ procedures that was presented in Chapters 3 and 4 respectively. Any advancements in these areas were then integrated into the scale-up procedures as appropriate. The development of demonstration products of significant size was required for market appraisal at different stages of the $\mathrm{PhD}$ research. As such, a range of larger-scale nanocomposite syntheses were carried out that utilise a range of gold colloids and wool types.

Although ex-situ composites are less colourfast than in-situ composites, the ex-situ procedure is the only economically viable method at this stage of the research. If the in-situ procedure could be made sufficiently cost-effective, then these composites would also be produced on a larger-scale. Likewise, when improvements in the washfastness of ex-situ composites were made, these would be integrated into the existing scale-up procedures. This research helped to gain a greater understanding of the chemistry involved in scale-up procedures, and produced composites of $0.5-$ $1.5 \mathrm{~kg}$, a scale that was sufficient to develop prototype apparel, rugs and carpets for market testing.

Section 5.1 details scale-up solution-phase syntheses of gold colloids using specialist equipment. Following this, the larger-scale dyeing of top form wool and felted yarn 
in the laboratory and with an industrial hank dyeing reactor is presented in Section 5.2. The colouring of fine merino yarns with conventional package dyeing equipment is then detailed in Section 5.3. Finally, Section 5.4 presents the development of novel flow reactors for the synthesise of ex-situ composites in the laboratory at Victoria University of Wellington.

\subsection{Scale-up of Gold Nanoparticle Synthesis}

The optimisation of the gold colloids used in the synthesis of ex-situ composites were detailed in Section 4.2. Once this had been achieved, it was important to develop reactors for the larger-scale synthesis of gold nanoparticles. This section details the use of reaction vessels composed of different materials to synthesise gold colloids, to determine their ability for use in scale-up reactors. Following this, a barrel reactor was used for the synthesis of 20-100 L volumes of TA- and TSC-stabilised gold nanoparticles for subsequent dyeing reactions.

\subsubsection{Materials for Gold Nanoparticle Syntheses}

The material that a reaction vessel is composed of is an important consideration for use in the synthesis of gold colloids and dyeing of wool with these colloids. Industrial wool dyeing reactors are typically composed of stainless steel, and the use of such reactors could allow the ex-situ synthesis to be integrated into existing dyeing procedures. However, stainless steel may detrimentally interact with $\mathrm{AuCl}_{4}^{-}$, and as such may not be suitable for the synthesis of gold nanoparticle-wool fibre composites.

Other materials that were considered for the ex-situ procedure were glass and plastics. Glass is an inert material that does not react with $\mathrm{AuCl}_{4}^{-}$or gold nanoparticles, however it is relatively expensive and complex to operate. Plastics that do not contain electron-rich chemical functionality, for example high density polyethylene (HDPE) or polyvinylchloride (PVC) were also investigated. Plastics that contain electron-rich functional groups may reduce $\mathrm{AuCl}_{4}^{-}$and form gold nanoparticles in the polymeric matrix, for example nylon 6,6 and polyurethane exhibit nitrogencontaining functionality that is able to reduce $\mathrm{Au}^{3+}{ }^{57} \mathrm{~A}$ summary of the advantages and disadvantages of the various materials is presented in Table 5.1. 
Table 5.1 Properties of different materials for the composition of gold nanoparticle synthesis reactors.

\begin{tabular}{cccc}
\hline Material & Stainless steel & Glass & Plastic \\
\hline Relative cost & mid-range & expensive & inexpensive \\
Durability & durable & fragile & durable \\
Ability to be heated & easy & easy & difficult \\
Interaction with gold & reactive & inert & inert \\
\hline \hline
\end{tabular}

The interaction between $\mathrm{AuCl}_{4}^{-}$and stainless steel reactors was investigated with atomic absorption spectroscopy (AAS). For this, an $\mathrm{AuCl}_{4}^{-}$solution of approximately $10 \mathrm{mg} \mathrm{L}^{-1}$ was stored in vessels composed of stainless steel and glass. Table 5.2 shows the change in concentration of $\mathrm{AuCl}_{4}^{-}$over time in these vessels. Interestingly, after 60 min of storing $\mathrm{AuCl}_{4}^{-}$in glass, there is a slight reduction of the concentration of $\mathrm{AuCl}_{4}^{-}$, and after 5 days the concentration drops from $9.2-7.9 \mathrm{mg} \mathrm{L}^{-1}$. The loss of concentration of $\mathrm{AuCl}_{4}^{-}$over time when stored in the glass vessel indicates a lower stability of the $\mathrm{AuCl}_{4}^{-}$complex in the aqueous solution (which is $\mathrm{pH}$ 4), in comparison to the $\mathrm{AuCl}_{4}^{-}$in the highly acidic $4 \mathrm{wt} \% \mathrm{Au}^{3+}$ stock solution.

Table 5.2 The concentration of $\mathrm{Au}^{3+}$ remaining in solution after storage in glass or stainless steel reaction vessels measured with AAS.

\begin{tabular}{cccc}
\hline Storage time & 0 min & 60 min & 5 days \\
\hline $\mathrm{Au}^{3+}$ in glass $\left(\mathrm{mg} \mathrm{L}^{-1}\right)$ & 9.2 & 9.0 & 7.9 \\
$\mathrm{Au}^{3+}$ in stainless steel $\left(\mathrm{mg} \mathrm{L}^{-1}\right)$ & 9.3 & 7.6 & 0.7 \\
\hline \hline
\end{tabular}

The storage of $\mathrm{AuCl}_{4}^{-}$in the stainless steel vessel over time results in the deposition of gold on the stainless steel surface. The attraction between the $\mathrm{AuCl}_{4}^{-}$and the stainless steel is illustrated in Figure 5.1 (a), where the gold colour is seen on the stainless steel surface. The propensity for $\mathrm{AuCl}_{4}^{-}$to coat stainless steel was considered to be due to comparable redox electrode potentials of the reduction of $\mathrm{Au}^{3+}$ and further oxidation of the stainless steel surface (which already exhibits an oxide surface). However, the $\mathrm{AuCl}_{4}^{-}$that coats the stainless steel vessel (Figure 5.1 (a)) does not change colour, indicating that it is not reduced to $\mathrm{Au}^{0}$ as it plates the stainless steel surface.

As the loss of concentration of $\mathrm{AuCl}_{4}^{-}$in the stainless steel reaction vessel is relatively low from 0-60 min, and the formation of TA-Au NPs is typically complete in the first 30-60 min of the standard TA-mauve synthesis, it may be possible to synthesise the TA-mauve colloid in stainless steel reactors. However, as the main advantage of using stainless steel in gold nanoparticle synthesis is its ability to be easily heated, plastic reactors are more suitable for the room temperature (RT) synthesis. The synthesis of TSC-Au NPs would ideally be carried out in stainless steel reactors, due to the 


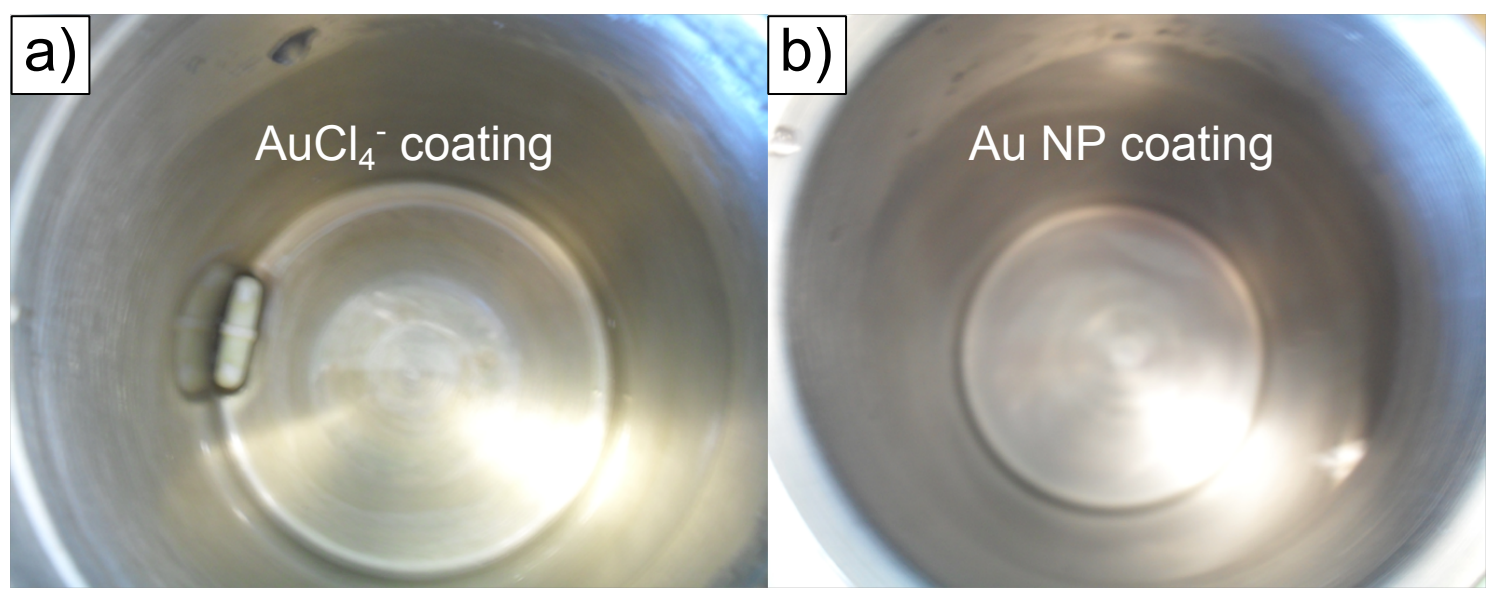

Figure 5.1 The interior of stainless steel containers that stored an aqueous solution of $10 \mathrm{mg} \mathrm{L}^{-1} \mathrm{AuCl}_{4}^{-}$(a) and the standard TA-mauve colloid for 5 days (b).

ease of heating this material. However, the interaction of $\mathrm{AuCl}_{4}^{-}$with the stainless steel reactors will likely be promoted at the higher temperatures required for this synthesis. As such, it was concluded that stainless steel reactors were undesirable for the synthesis of TSC-Au NPs, and alternative reactors were investigated.

The ability to store gold colloids in stainless steel containers was next investigated. It was considered that if metal ions could leach into the colloidal solution, it may have a lower stability. Alternatively, the gold nanoparticles may be electrostatically attracted to the stainless steel surface. Determining the stability of gold nanoparticles in stainless steel reaction vessels is integral to deducing whether or not existing commercial dyeing equipment can be used in the ex-situ procedure.

Figure 5.1 (b) shows the interior of a stainless steel reaction vessel that has had $200 \mathrm{~mL}$ of the TA-mauve colloid stored in it for 5 days. The reactor has a slight pink tinge, indicating the attachment of some of the TA-Au NPs in the solution with the stainless steel over time.

The UV-vis spectra of $200 \mathrm{~mL}$ volumes of the standard TA-mauve colloid that were stored in glass beakers and stainless steel containers over time are shown in Figure 5.2. For this, a $400 \mathrm{~mL}$ TA-mauve colloid was synthesised in a glass beaker, and half of it was then transferred to a stainless steel container. Interestingly, the shape and intensity of the TA-mauve colloids are not significantly altered when stored in the stainless steel vessel. The SPR peak of the colloid made in the glass beaker is slightly more intense after 5 days of storage. This implies either that there is some evaporation of the colloid (concentrating the TA-Au NPs in the solution) or the continued formation of TA-Au NPs after $60 \mathrm{~min}$ of the synthesis. The fact that the SPR peak of the stainless steel stored colloid is approximately as intense after 5 days implies that any evaporation or continued nanoparticle formation is balanced 
by the slight coating of the stainless steel vessel that occurs (as shown in Figure 5.1 (b)). The fact that the decrease in intensity of the SPR peak of TA-Au NPs after 5 days of storage in stainless steel is approximately negligible dictates that reactors composed of this material are likely suitable for the TA-mauve method.

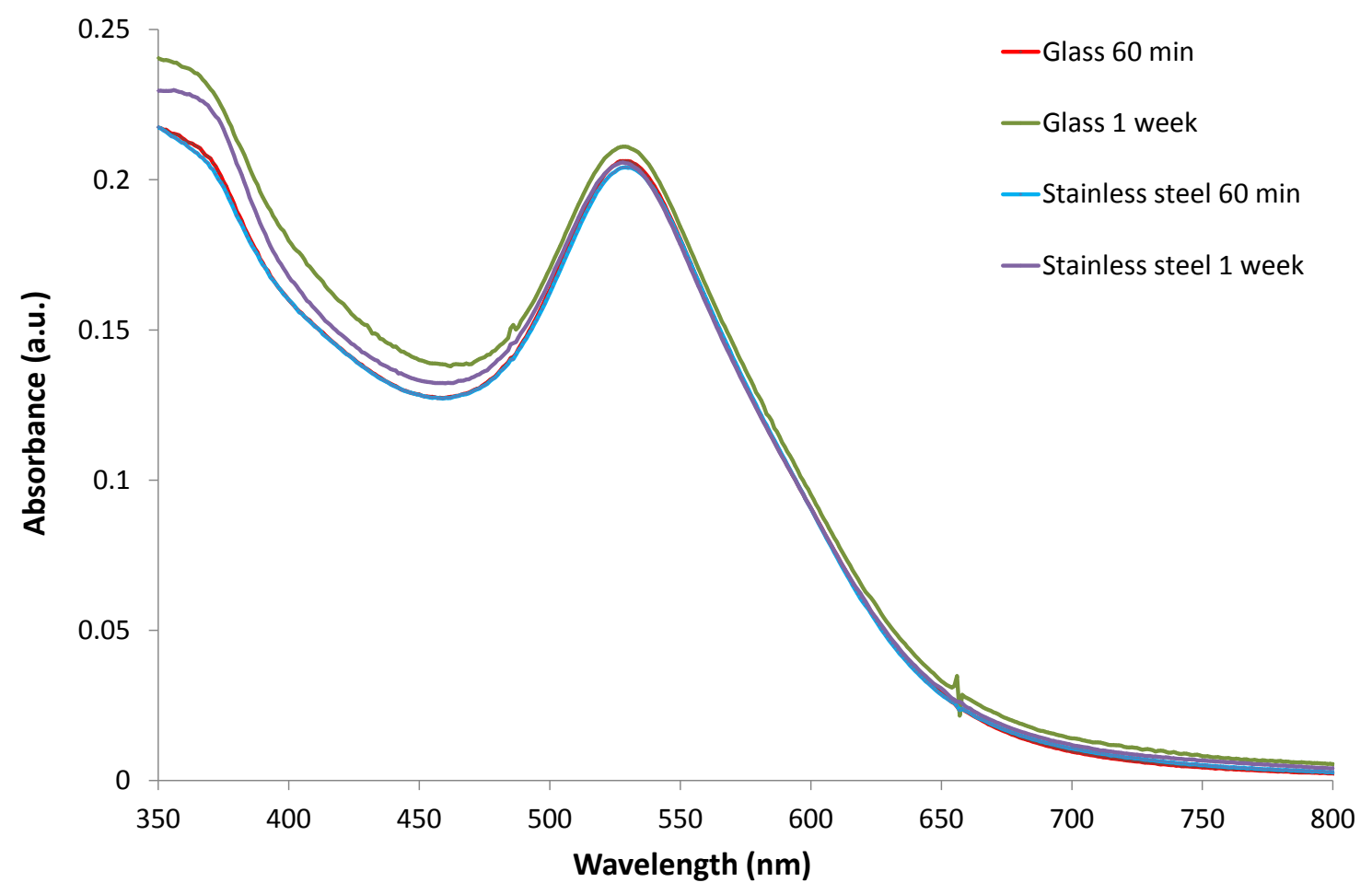

Figure 5.2 UV-vis spectra of a TA-mauve stored in glass or stainless steel vessels after $60 \mathrm{~min}$ and 5 days.

This experiment has shown that gold colloids may be stored in stainless steel equipment with only a slight eventual coating of the colloid on the stainless steel surface. As such, the TA-mauve colloid can be successfully used to colour wool in stainless steel reactors. Next, gold nanoparticle colloids of 5-90 L volumes are synthesised and characterised.

\subsubsection{Scale-up Gold Nanoparticle Syntheses}

The synthesis of TA-stabilised gold nanoparticles occurs at RT, and as such, the syntheses can be carried out in plastic buckets (HDPE). This involves no chemical interaction between the $\mathrm{AuCl}_{4}^{-}$or gold nanoparticles and the plastic reactor. As such, attaining a uniform mixing of reactants is the paramount concern associated with scaling-up the synthesis of TA-Au NPs.

The synthesis of TSC-Au NPs requires temperatures of up to $95^{\circ} \mathrm{C}$, which introduces 
a number of difficulties to larger-scale syntheses of the nanoparticles. TSC-stabilised colloids of up to $5 \mathrm{~L}$ volumes can be synthesised in glass beakers on hot plates with magnetic stirring capabilities. However, scale-up procedures in this project involve the synthesis of up to $90 \mathrm{~L}$ volumes of gold colloids. Given that a glass reactor of this size would be costly and complex to use, a plastic barrel reactor was first investigated in scale-up syntheses of TSC-Au NPs.

The barrel reactor was designed by Professor Johnston, Dr Aaron Small and Alan Rennie from the Victoria University Workshop for the syntheses of gold nanoparticle colloids of up to $100 \mathrm{~L}$. The barrel reactor is shown in Figure 5.3, the interior of the barrel reactor is equipped with three baffles to promote turbid mixing. An overhead high-velocity mixer with three wide blades is attached to stir the reaction mixture.

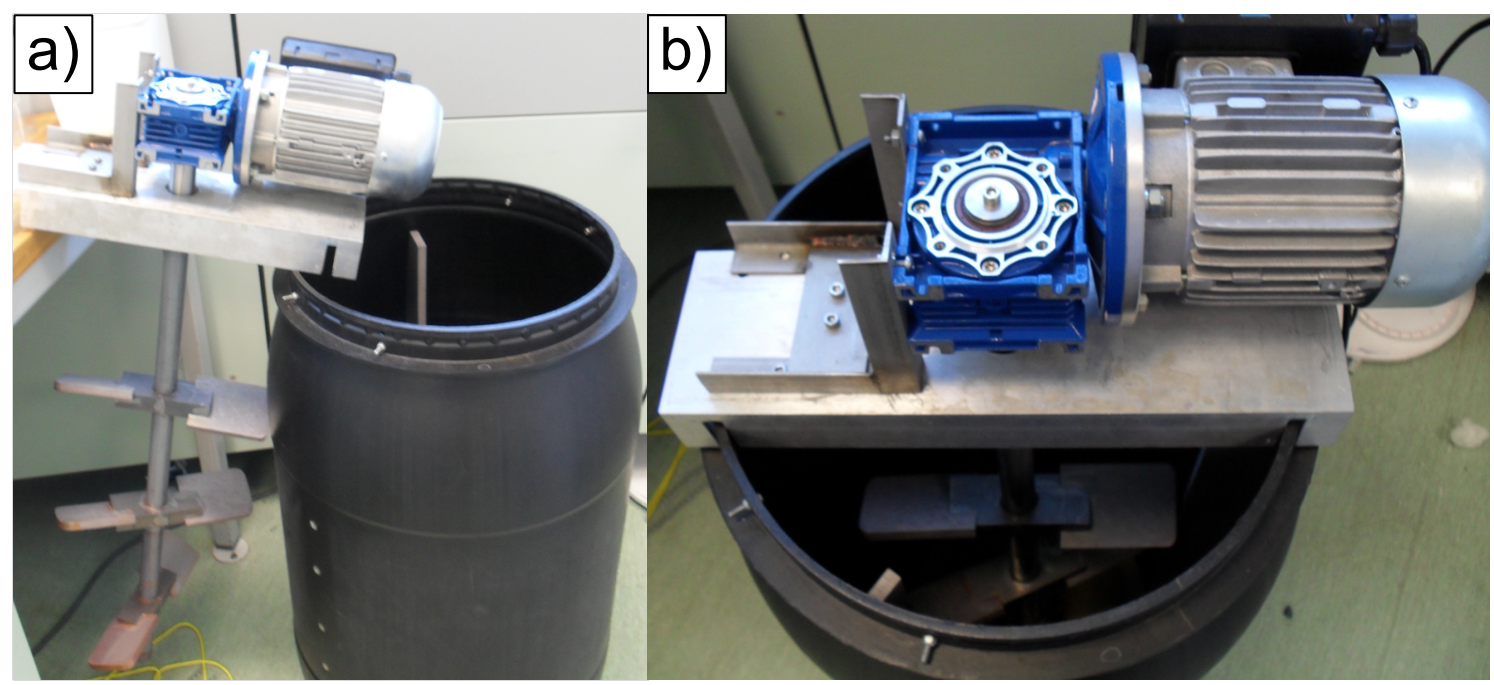

Figure 5.3 Images of the interior of the barrel reactor with the overhead stirrer beside it (a) and fitted (b).

\section{Barrel Reactor Synthesis of TA-Au NPs}

The production of volumes of up to $10 \mathrm{~L}$ of TA-Au NPs were carried out in either glass beakers or HDPE plastic buckets. The mixing of the reactants is provided by placing the buckets on magnetic stirrers. Syntheses of 20-90 L of TA-stabilised colloids were undertaken in the barrel reactor. Images taken during the synthesis of a $60 \mathrm{~L}$ volume of the standard TA-mauve colloid are shown in Figure 5.4. The synthesis involved the addition of $15 \mathrm{~mL}$ of $4 \mathrm{wt} \% \mathrm{Au}^{3+}$ to $60 \mathrm{~L}$ of double distilled water in the barrel reactor. The solution was stirred at $30 \mathrm{rpm}$, and $200 \mathrm{~mL}$ of $1 \mathrm{wt} \%$ TA was then added. The reaction solution was stirred for at least $60 \mathrm{~min}$ before it is used to colour wool fibres. This gold colloid was used to colour a $1 \mathrm{~kg}$ hank of Ascend felted yarn (the chemistry of which is detailed in Section 5.2.2). 


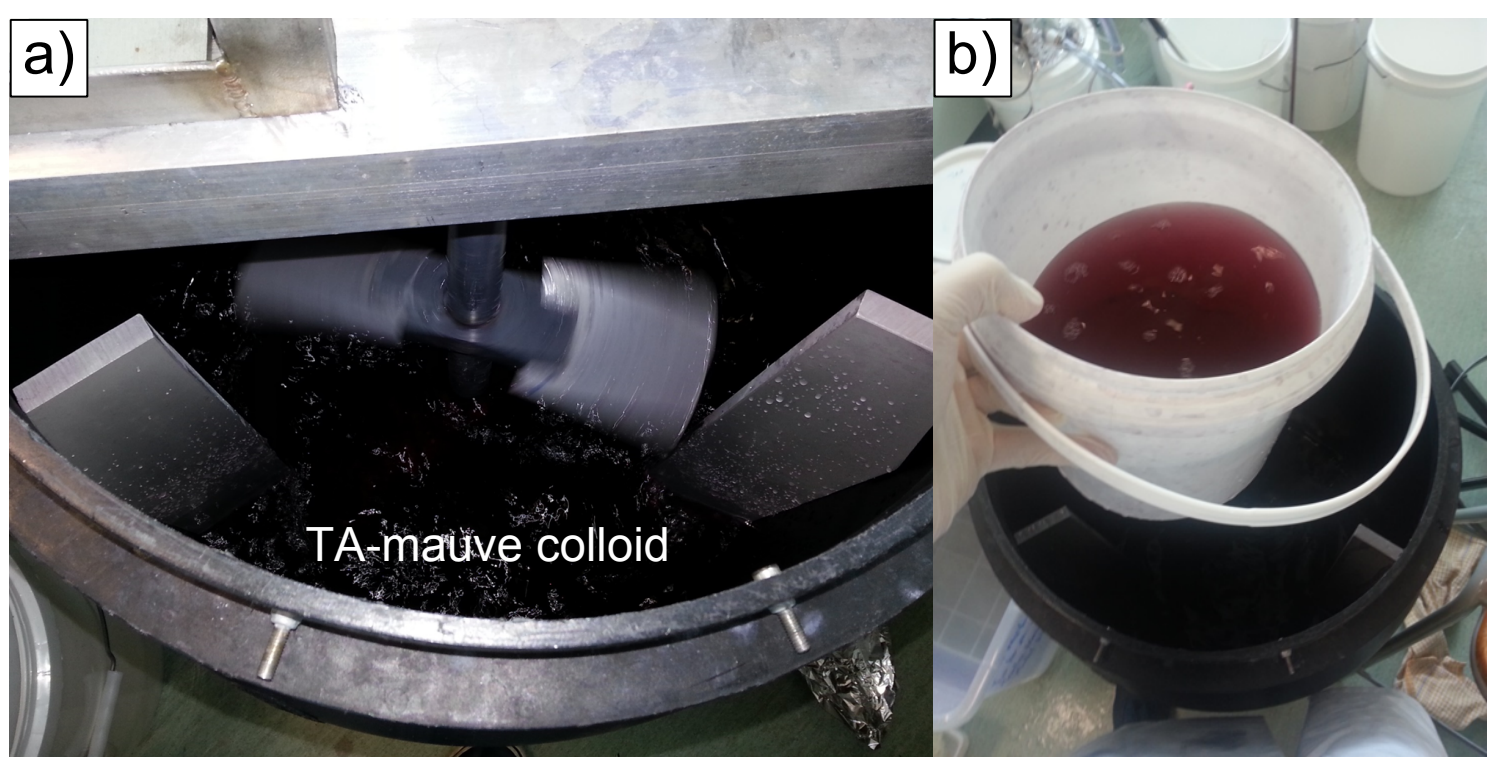

Figure 5.4 Images of the synthesis of a $60 \mathrm{~L}$ TA-mauve colloid in the barrel reactor during the preparation (a), and the TA-mauve colloid in a white bucket where the purple colour of the colloid is seen more easily (b).

The UV-vis spectrum of the TA-mauve colloid synthesised in Figure 5.4 is shown in Figure 5.5. The UV-vis spectrum of a $600 \mathrm{~mL}$ TA-mauve colloid is also presented to provide a comparison. Interestingly, the $60 \mathrm{~L}$ colloid exhibits a narrower SPR peak. This implies that a more homogeneous distribution of the sizes and shapes of gold nanoparticles have formed in the larger colloid. As such, the mixing achieved in the barrel reactor is likely as effective if not more so than that achieved in a $600 \mathrm{~mL}$ volume in a beaker on a magnetic stirrer.

Larger-scale syntheses TA-Au NPs are easily reproducible in the barrel reactor. The RT synthesis does not require the use of metallic surfaces, which is in contrast to the relatively difficult synthesis of TSC-Au NPs. 


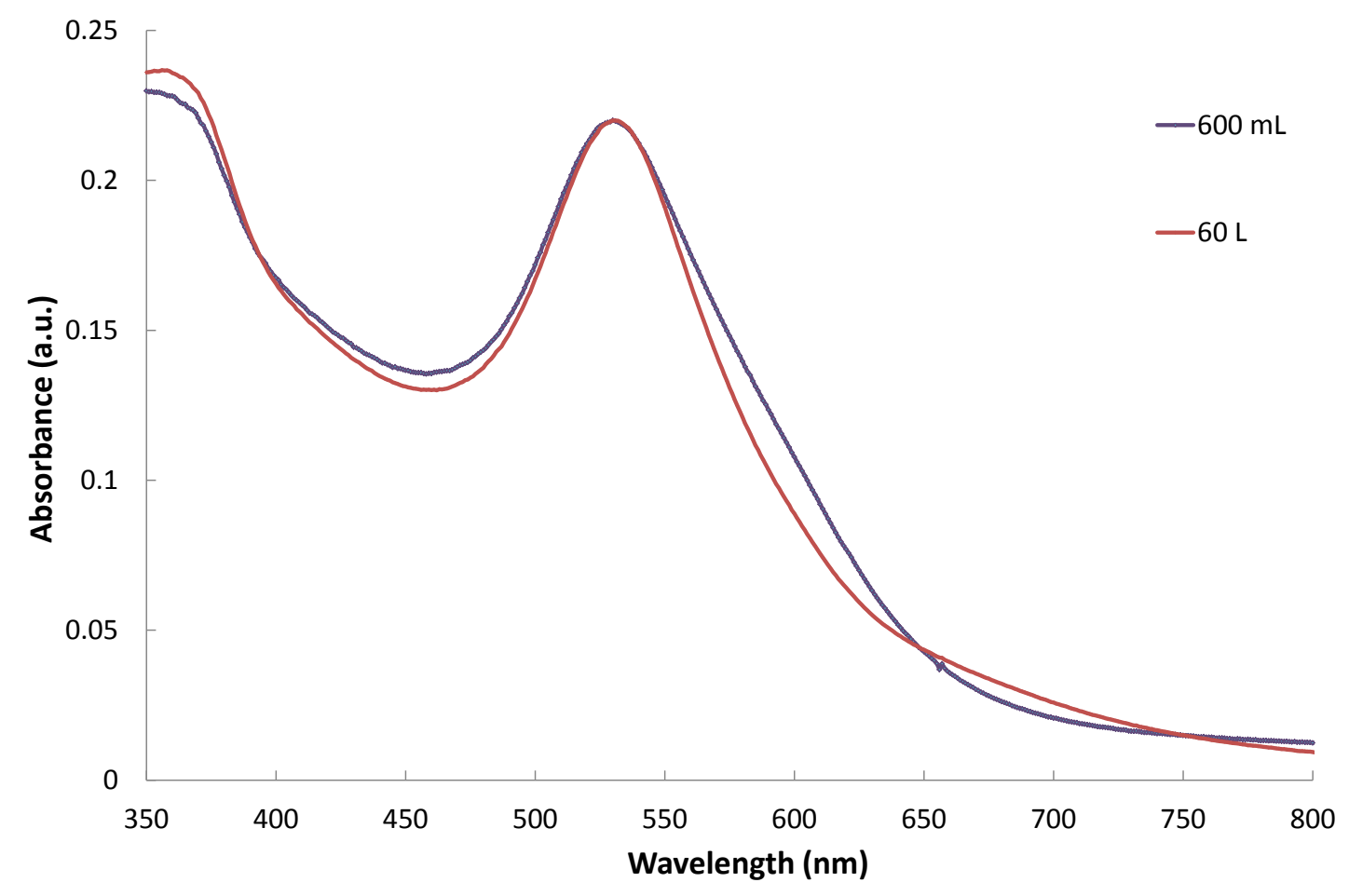

Figure 5.5 UV-vis spectra of a $600 \mathrm{~mL}$ TA-mauve colloid synthesised in a glass beaker and a $60 \mathrm{~L}$ TA-mauve colloid synthesised in the barrel reactor.

\section{Barrel Reactor Synthesis of TSC-Au NPs}

The TSC-grey method involves the synthesis of a TSC-stabilised gold colloid, which is used to colour wool. The dyeing reaction involves the "controlled" agglomeration of TSC-Au NPs, which impart a grey colour to the composites. The best results of the TSC-grey method are achieved when a stable gold colloid is used to colour the wool.

The synthesis of volumes of up to $5 \mathrm{~L}$ of TSC-stabilised colloids were carried out in glass beakers on magnetic stirring hotplates. In this, the contact between the reaction solution and metallic contacts is limited to the condensation of the colloid vapour on the aluminium foil which covers the beaker (the temperature probe of the hot plate is composed of Teflon). However, throughout this research project the TSC-grey method has proved to be less reproducible than the TA-mauve method.

An example of the reproducibility issues of synthesising the TSC-grey colloid is presented in Figure 5.6, where TSC-grey colloids of 2 and a $5 \mathrm{~L}$ volumes were synthesised in glass beakers. The $2 \mathrm{~L}$ colloid exhibits the typical deep-red colour and UV-vis spectrum of the TSC-grey colloid, while the $5 \mathrm{~L}$ colloid displays a red-shifted and broadened SPR peak and is brown-purple. This indicates larger and more polydisperse gold nanoparticles have formed in the $5 \mathrm{~L}$ colloid. The variability in the 
TSC-grey colloidal synthesis is discussed in more depth later in this section.

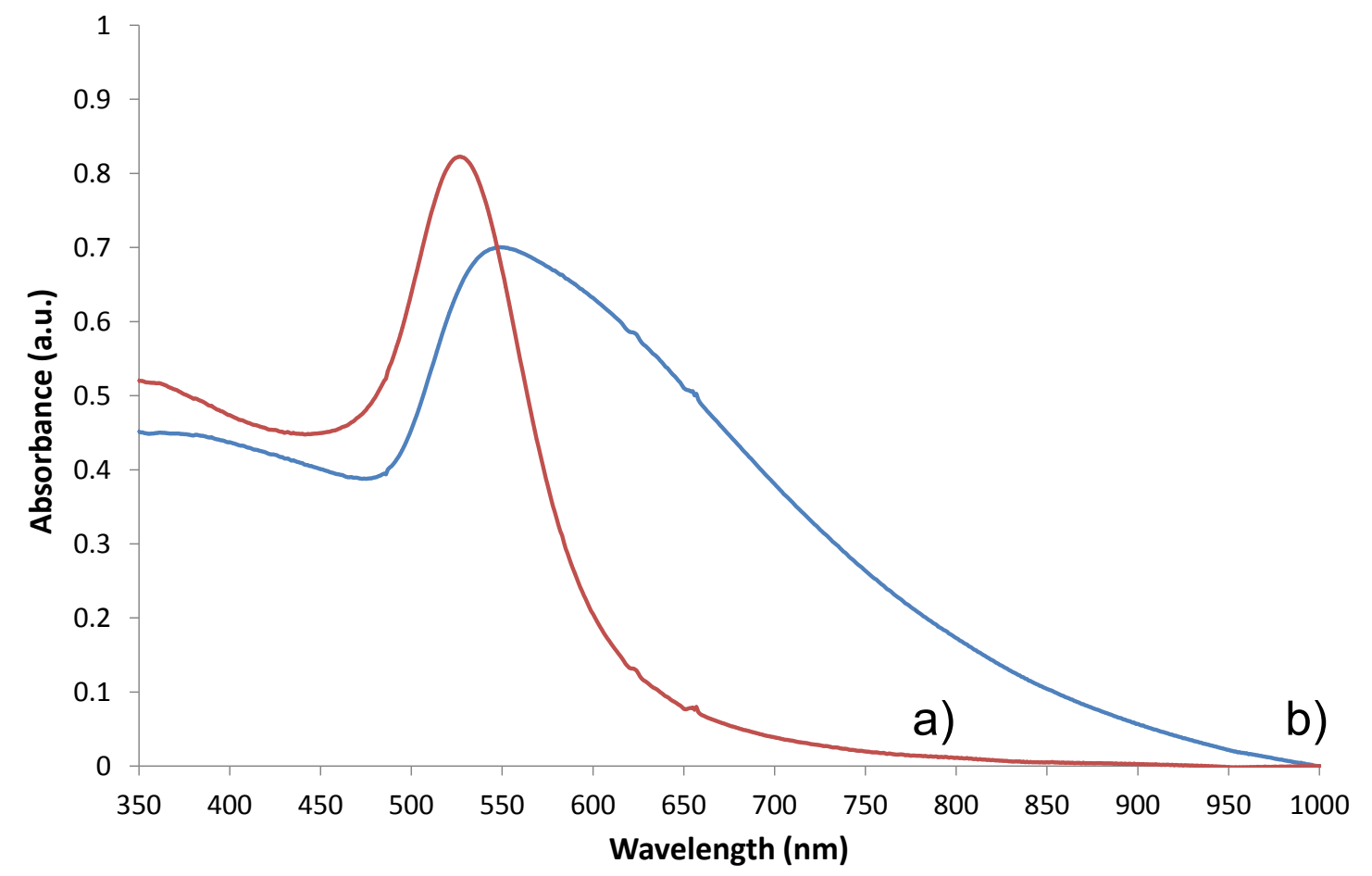

Figure 5.6 TSC-grey colloids synthesised in glass beakers: a 2 L stable colloid (a) and a $5 \mathrm{~L}$ destabilised colloid (b).

For the synthesis of $10 \mathrm{~L}$ of the TSC-grey colloid, two $5 \mathrm{~L}$ batches of TSC-Au NPs are typically synthesised in glass beakers. The synthesis of a $10 \mathrm{~L}$ TSC-grey colloid was attempted in a HDPE bucket with the use of immersion heaters, however, a stable colloid could not be formed. This was due to the leaching of a plasticiser into the reaction solution at the high temperature, which was believed to have destabilised the colloid.

The barrel reactor is a $125 \mathrm{~L} \mathrm{PE}$ drum that was employed for the synthesis of 20-90 L volumes of TSC-Au NPs. Plastic reactors require the immersion of metallic elements to heat the water. These elements were removed immediately prior to the addition of $\mathrm{AuCl}_{4}^{-}$and TSC, this was to obviate the potential gold plating of the metallic surfaces. As such, there is some loss of heat during the formation of TSC-Au NPs as the colloid is not heated during the synthesis.

The synthesis of a $60 \mathrm{~L}$ TSC-grey colloid in the barrel reactor is presented. Images of the synthesis are shown in Figure 5.7, where distilled water $(60 \mathrm{~L})$ was heated to $99^{\circ} \mathrm{C}$ in the barrel reactor with the immersion heater and element that are shown in Figure 5.3 (a) and (b). The temperature of the water was raised as high as possible prior to the reaction, as the elements were removed during the formation of TSC-Au 
NPs. When the maximum temperature had been reached, the immersion heater and element were quickly removed and the overhead stirrer was attached to the top of the barrel. The heated water was stirred at $30 \mathrm{rpm}, 60 \mathrm{~mL}$ of $4 \mathrm{wt} \% \mathrm{Au}^{3+}$ and then $150 \mathrm{~mL}$ of $5 \mathrm{wt} \%$ TSC were both added to the solution. The reaction was stirred for $60 \mathrm{~min}$ before it was left to cool. Aluminium foil was placed over the top of the barrel during the heating and stirring stages to slow the loss of heat of the solution. The temperature of the colloid cannot be measured during the formation due to the stirrer, but the colloid remained above $80^{\circ} \mathrm{C}$ after $60 \mathrm{~min}$ of synthesis.

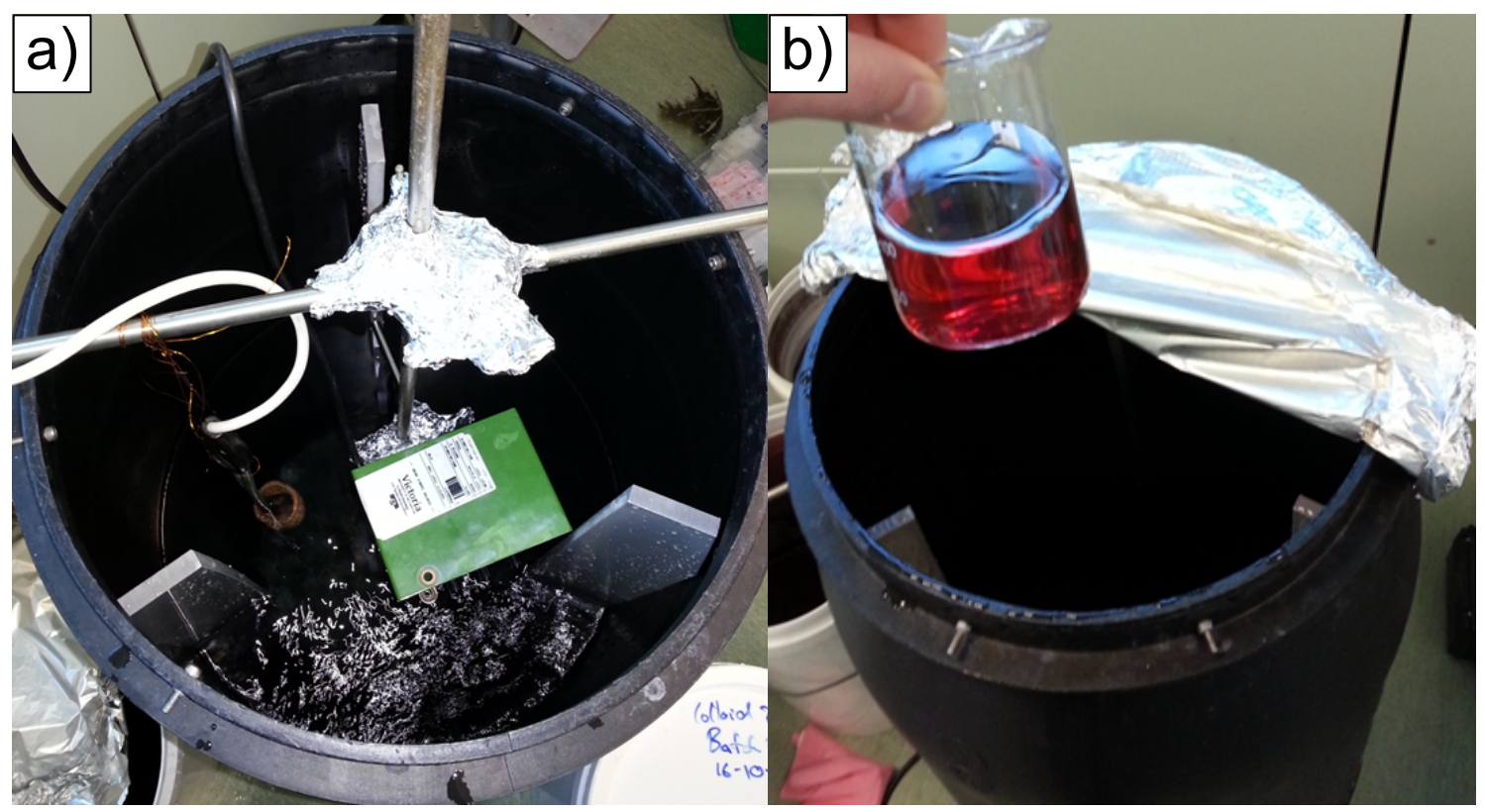

Figure 5.7 The synthesis of the TSC-grey colloid in the barrel reactor during the synthesis (a) and the resulting stable colloid (b).

Figure 5.7 presents images of the synthesis of a TSC-grey colloid which was used to successfully colour Ascend felted yarn. However, similar to the TSC-grey syntheses previously presented in Figure 5.6, this was not always the case; a destabilised colloid would sometimes result. In dyeing reactions, destabilised colloids coagulate significantly, settle out of the dye solutions and generally result in unevenly coloured composites. The cause of the destabilisation was considered to be due to one or more of the following factors:

- heat loss of the solution during the synthesis

- leaching of metal ions from the elements used to heat the reaction solution

- non-uniform heating and mixing of the reaction solution

The difficulty in reproducibly synthesising the TSC-grey colloid is considered to be due to a combination of the potential heat loss, non-uniform mixing and heating and 
contact with metallic surfaces that occurs in the barrel reactor syntheses. As such, there may be a narrow range of success for the colloidal synthesis. To overcome this problem, laboratory research that was detailed in Section 4.2.3 increased the amount of TSC used in the synthesis. This allowed more effective capping of the TSC-Au NPs to prevent agglomeration, and greatly enhanced the reproducibility of the TSC-grey synthesis. This was a very positive result, and TSC-grey colloids were reproducibly synthesised when a 1:12.54 molar ratio of $\mathrm{AuCl}_{4}^{-}$to TSC was used.

The research detailed in this section has shown that large volumes of TA- and TSC-stabilised gold colloids can be reproducibly synthesised in the barrel reactor. The stability of the gold colloids while being stored in stainless steel vessels implies that they may be used in industrial dyeing reactors to colour wool. The following section presents the use of large volumes of TA- and TSC-stabilised colloids to colour kilogram quantities of top form wool and felted yarn in static tanks and hank dyeing reactors.

\subsection{Scale-up Hank Dyeing Procedures}

In the synthesis of $0.1-1 \mathrm{~g}$ samples of ex-situ gold nanoparticle-wool fibre composites, wool samples are agitated to promote the uniform absorption of gold nanoparticles. This is achieved by placing the dyeing reaction vessels (with typical volumes of 6-60 mL) on an orbital shaker table. The homogeneous absorption of gold nanoparticles by the wool is typically achieved with this method. This results in the uniform colouring of the composites. Additionally, the matting of wool fibres of $0.1-1 \mathrm{~g}$ is uncommon. However, when larger quantities of wool (>100 g) are coloured, the propensity for wool fibres to felt or mat together upon external agitation is considerably elevated. As such, a number of alternative measures are used in the wool dyeing industry to promote the uniform absorption of dyestuffs by wool fibres, while preventing damage to the wool fibres.

The following section presents the first of these methods, which involved the soaking of top form wool in static reactors with the gentle agitation of wool by hand. Following this, conventional hank dyeing reactors were used in an attempt to colour Ascend felted yarn at Wool Yarns Ltd, in Levin, New Zealand. Finally, hank dyeing procedures were reproduced in the laboratory, utilising gentle agitation of the hank and gradual circulation of the dye liquor to colour Ascend felted yarn. 


\subsubsection{Hank Dyeing of Top Form Wool}

The preparation of ex-situ Cariaggi gold nanoparticle-wool fibre composites were the first scale-up procedures that were undertaken in this research project. The experiments were carried out before buffers or auxiliary agents were integrated into the ex-situ procedure. These composites were produced for the fabrication of demonstration products that were displayed at investment opportunities and trade expositions. The demonstration products were luxury jerseys, scarves and the pin stripes in woollen suits. The synthesis of ex-situ Cariaggi composites involved the synthesis of large volumes of gold colloids in the barrel reactor, using procedures that were detailed in Section 5.1.2. The colloids were then used to dye Cariaggi wool in static reactors (with no circulation of the dye liquor).

\section{TA-mauve Hank Dyeing of Top Form Wool}

The following experiments involved the synthesis of $100 \mathrm{~g}$ lots of TA-mauve Cariaggi composites. This involved the synthesis of $6 \mathrm{~L}$ of the standard TA-mauve colloid in HDPE buckets on a magnetic stirrer. The $\mathrm{AuCl}_{4}^{-}$concentration of $10 \mathrm{mg} \mathrm{L}^{-1}$ was achieved with the addition of $1.5 \mathrm{~mL} 4 \mathrm{wt} \% \mathrm{Au}^{3+}$ and $20 \mathrm{~mL} 1 \mathrm{wt} \%$ TA. The $\mathrm{pH}$ of the colloid was lowered to 2.7 with the addition of $\mathrm{HCl}$. Cariaggi wool (100 g) was soaked in hot tap water (to remove water soluble surface lubricants and lipids), drip dried and then simply immersed in the colloid. The top form wool was gently lifted up and down inside the dyebath to promote the movement of the dye liquor throughout the wool sample.

Figure 5.8 (a) shows a 100 g Cariaggi TA-mauve composite while drying soon after the dyeing reaction. The composite appears uniformly mauve coloured. However, when the composites are dry, some colour variation was observed between different areas of the composite. The dry composite is shown in Figure 5.8. The synthesis was repeated nine times so that a total of $1 \mathrm{~kg}$ of the composites was produced. The composites all exhibited a similar, reasonably uniform mauve colouration. These experiments showed that TA-mauve Cariaggi composites of $100 \mathrm{~g}$ size can be reproducibly synthesised with reasonable colour uniformity in static reactors. However, this method is time consuming and as such, larger ex-situ syntheses were next carried out. 


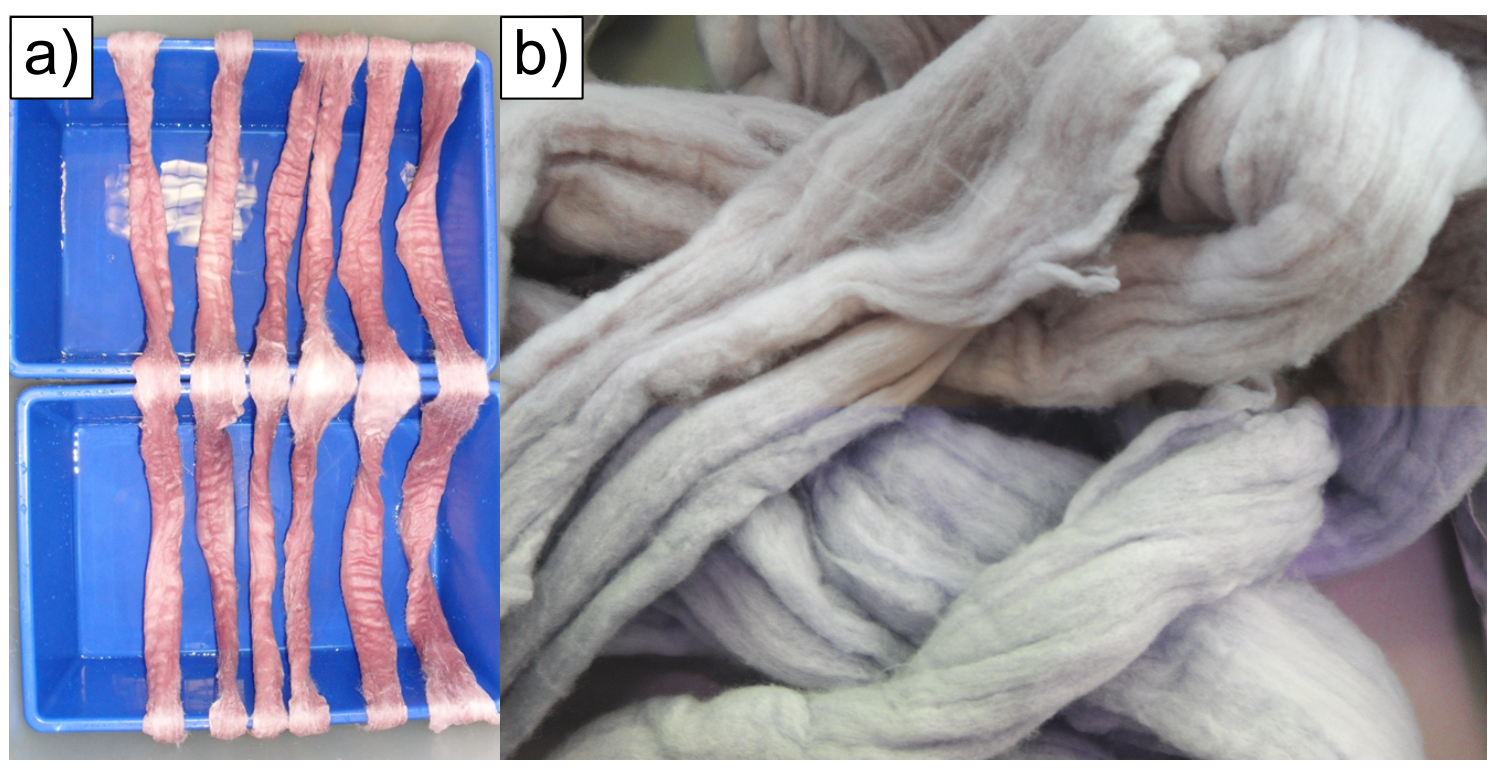

Figure 5.8 A 100 g TA-mauve Cariaggi composite before (a) and after (b) drying after the dyeing reaction.

\section{TSC-pink Cariaggi Composites}

The synthesis of a $500 \mathrm{~g}$ TSC-pink Cariaggi composite was carried out in a static reactor. The TSC-pink colloid was synthesised in the barrel reactor $(7.5 \mathrm{~mL} 4 \mathrm{wt} \%$ $\mathrm{Au}^{3+}$ and $18.75 \mathrm{~mL} 5 \mathrm{wt} \%$ TSC), and again adjusted to $\mathrm{pH} 2.7$ with $\mathrm{HCl}$ addition. $500 \mathrm{~g}$ of Cariaggi wool was soaked in hot tap water, drip dried and immersed in the TSC-pink colloid. Again, the top form wool was gently agitated during the dyeing reaction. The resulting composite is presented in Figure 5.9. Interestingly, the composite is an uneven purple-grey colour and exhibits some completely white areas that have not absorbed TSC-Au NPs. The grey areas indicate the agglomeration of TSC-Au NPs on the surface of the composite.

This experiment highlights the difficulty of attaining uniformly coloured composites of $500 \mathrm{~g}$ size with static dyeing reactions. As such, it is believed that the uniform absorption of gold nanoparticles requires more than the gentle agitation of top form wool. The following experiments present the synthesis of TSC-grey Cariaggi composites in static reactors.

\section{TSC-grey Cariaggi Composites}

The following experiment involved the synthesis of a $1.5 \mathrm{~kg}$ TSC-grey Cariaggi composite. A $90 \mathrm{~L}$ volume TSC-grey colloid was synthesised in the barrel reactor (with $90 \mathrm{~mL} 4 \mathrm{wt} \% \mathrm{Au}^{3+}$ and $225 \mathrm{~mL} 5 \mathrm{wt} \%$ TSC), and was adjusted to $\mathrm{pH} 2.7$ with the 


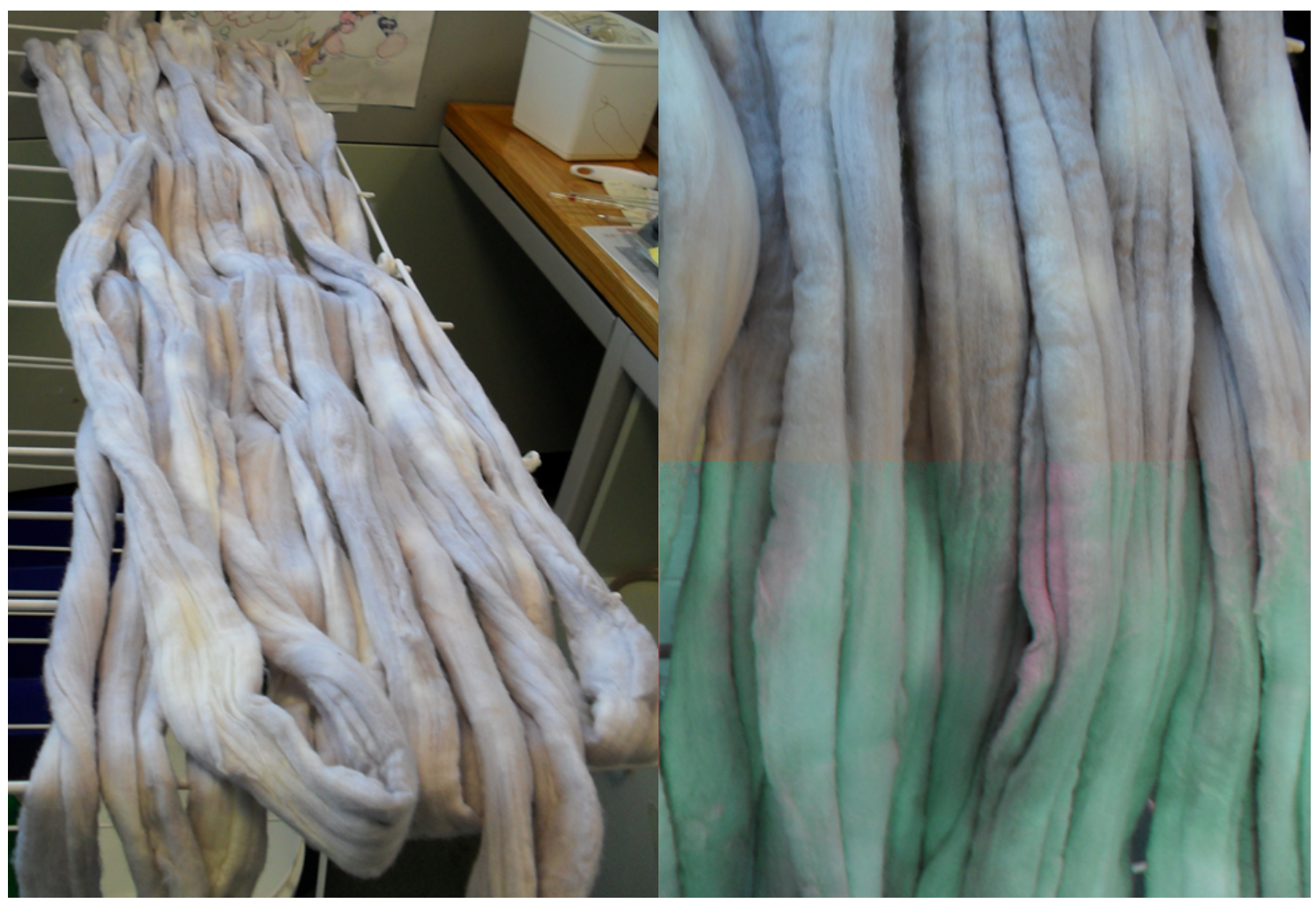

Figure 5.9 A $500 \mathrm{~g}$ 'TSC-pink' Cariaggi composite synthesised in a hank dyeing reaction.

addition of $\mathrm{HCl}$. Images of the dyeing reaction and the resulting composite are shown in Figure 5.10. At the beginning of the dyeing, the colloid is purplish-grey in colour. As the reaction continues the TSC-Au NPs agglomerate both in the dye solution and on the surface of the wool fibres. The resulting composite is patchy grey in colour and exhibits some completely white areas. Interestingly, the $1.5 \mathrm{~kg}$ TSC-grey composite appears to be more uniformly coloured than the $500 \mathrm{~g}$ TSCpink composite. This is likely due to the higher concentration of the TSC-grey colloid allowing gold nanoparticles to move throughout the dyepack to a greater extent before they are absorbed by the Cariaggi wool.

The synthesis of a $1 \mathrm{~kg}$ TSC-grey Cariaggi composite was then carried out with vigorous agitation of the wool during the dyeing reaction. This was undertaken to attempt to mix the gold colloid and the wool fibres, to result in a more uniformly coloured composite. The $60 \mathrm{~L}$ TSC-grey colloid was again synthesised in the barrel reactor (with $60 \mathrm{~mL} 4 \mathrm{wt} \% \mathrm{Au}^{3+}$ and $150 \mathrm{~mL} 5 \mathrm{wt} \%$ TSC) and adjusted to $\mathrm{pH}$ 2.7. The Cariaggi wool was agitated (in an up and down motion) while in the TSC-grey colloid with significantly more force. The resulting composite is shown in Figure 5.11. The grey colouration of the composite appears to be more uniform in colour than the $1.5 \mathrm{~kg}$ composite in Figure 5.10. However, unfortunately the agitation has also caused exorbitant composite fibre damage. Additionally, pulling 


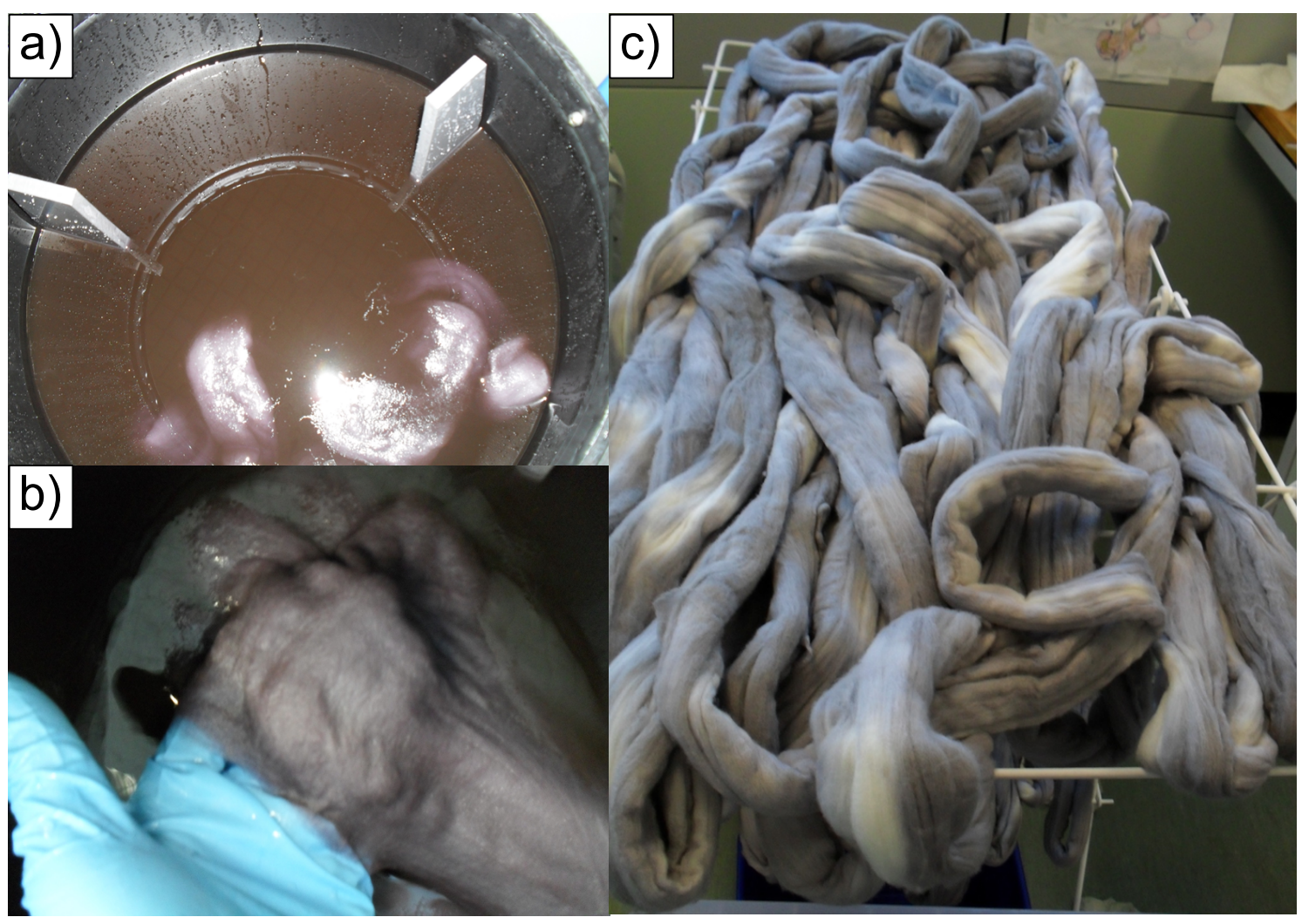

Figure 5.10 Images of the synthesis of a $1.5 \mathrm{~kg}$ TSC-grey Cariaggi composite: the wool soaking in the barrel reactor early in the reaction (a), after the agglomeration of TSC-Au NPs (b) and the resulting composite (c).

apart of some of the matted regions of the composite (as seen in Figure 5.11 (b)) exposes undyed areas in the interior of the top form wool. The resulting composite was too matted to be used in the fabrication of demonstration products. As such, this method is unsuitable for the ex-situ synthesis of composites.

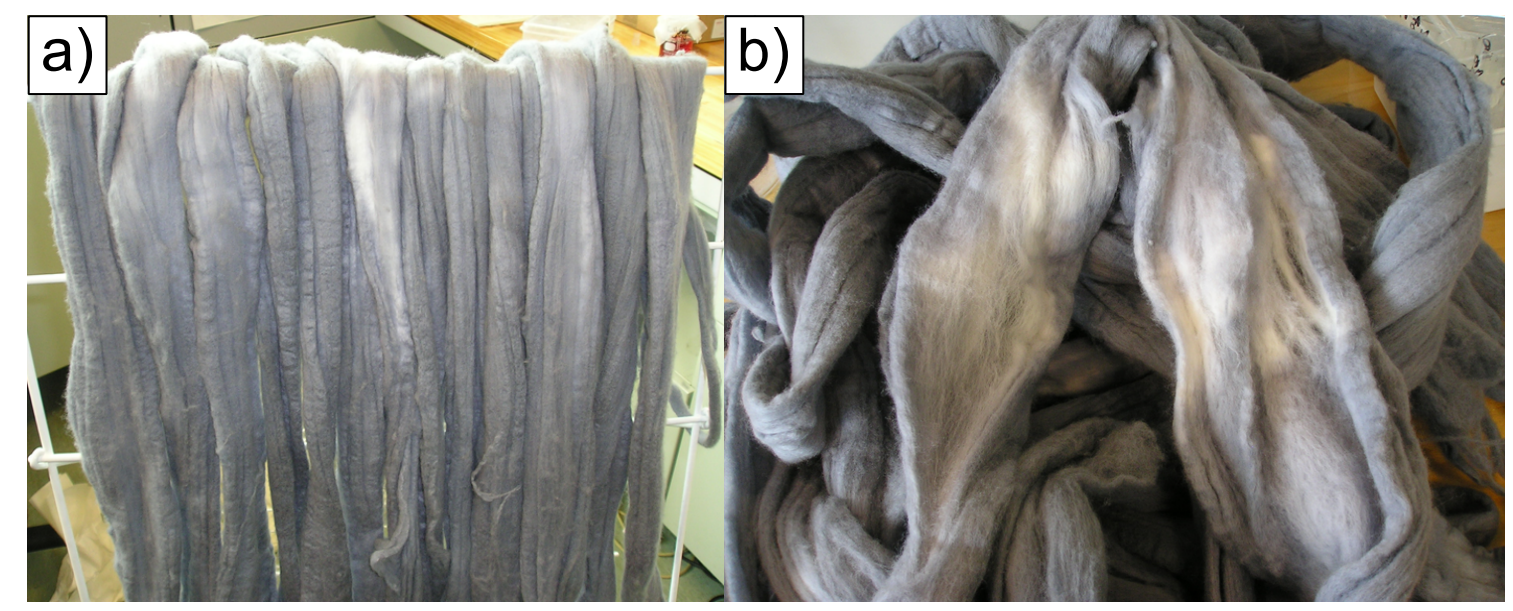

Figure 5.11 A $1 \mathrm{~kg}$ TSC-grey Cariaggi composite synthesised with vigorous agitation in the fish tank reactor (a) and exposing the interior of some of the more matted areas (b). 
The experiments presented in this section have shown that the colouring of top form wool in static reactors results in unevenly coloured composites of sample sizes of $>100 \mathrm{~g}$. More vigorous agitation of the top form wool increased the colour uniformity somewhat, but resulted unacceptable composite fibre damage. The composites required blending and combing processes to atone for the uneven colouration of the composites. This was carried out by Design Spun Ltd, a wool processing entity in Hawkes Bay, New Zealand. The processed composites were spun into yarn and knitted into demonstration products, examples of which are presented in Figure 5.12.

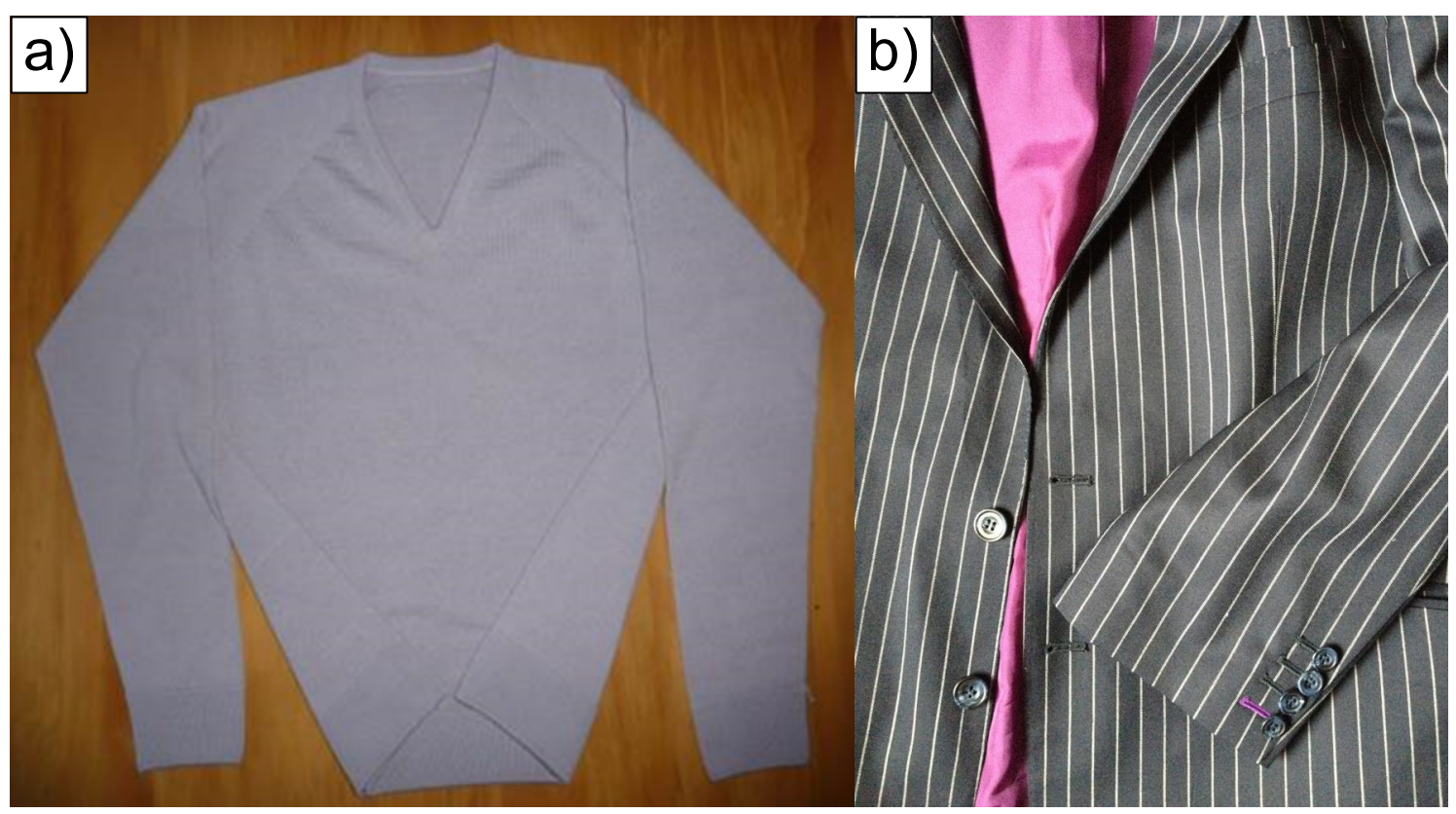

Figure 5.12 Aulana demonstration products fabricated from Cariaggi ex-situ composites, a TA-mauve merino jersey (a) and a bespoke suit with TA-mauve pinstripes.

The blending and gilling of the ex-situ Cariaggi composites was an expensive process and resulted in the loss of a significant amount of the composite fibres. As such, the dyeing of top form wool in static reactors is not suitable for the scaling-up the synthesis of ex-situ gold nanoparticle-wool fibre composites. These results were important for product exposure and provided an excellent starting point to improve upon. This led to extensive laboratory research on slowing the rate of uptake of gold nanoparticles to wool fibres and the investigation of alternative wool dyeing methods, such as the conventional hank dyeing of wool. 


\subsubsection{Hank Dyeing of Felted Merino Yarn}

Felted yarns were provided by Ascend Ltd, a luxury rug manufacturer located in Christchurch, New Zealand. The felted yarn was to be coloured with gold nanoparticles and fabricated into luxury rugs to be presented at international trade expositions. This required the synthesis of $8 \mathrm{~kg}$ of ex-situ felted yarn composites of different colours. The felted yarn dyeing reactions were carried out 12 months after the Cariaggi dyeing reactions, after which time a significant amount of laboratory optimisation research had been undertaken. The experiments were carried out after the glycine buffer had been integrated into the synthesis, and led to the discovery of the need to use wool dyeing auxiliaries.

The hank dyeing of Ascend felted yarns was first carried out at Wool Yarns Ltd in Lower Hutt, New Zealand in collaboration with Professor James Johnston's industry contacts and Dr Kerstin Lucas. Following this, hank dyeing procedures were simulated in the laboratory at Victoria University of Wellington. The wetting agent (WA) Albaflow FFA was used in many of the dyeing of reactions.

\section{Conventional Hank Dyeing of Felted Yarn}

The hank dyeing reactor at Wool Yarns Ltd that was used to colour felted yarn is shown in Figure 5.13. The reactor consists of a tank that holds up to $150 \mathrm{~L}$ of dye solution, into which hanks of wool were lowered. The dye liquor was gradually pumped from the bottom of the tank to overflow at the back end at the top of the tank. A hank of polypropylene yarn was hung at the back of the reactor nearest the overflow to prevent the possible entanglement of the wool hanks in the circulating dye liquor.

The first experiment in the hank dyeing reactor involved colouring two $1 \mathrm{~kg}$ hanks of Ascend felted yarn with the TA-mauve colloid. The felted yarn hanks were soaked in a glycine buffer (40 L, $0.1 \mathrm{~mol} \mathrm{~L}^{-1}$, pH 2.2, no Albaflow FFA (WA)) overnight prior to the dyeing reaction. $120 \mathrm{~L}$ of the standard TA-mauve colloid was synthesised in the barrel reactor in two $60 \mathrm{~L}$ lots (each used $15 \mathrm{~mL} 4 \mathrm{wt} \% \mathrm{Au}^{3+}$ and $200 \mathrm{~mL} 1 \mathrm{wt} \%$ TA). The colloid was diluted to $150 \mathrm{~L}$ with tap water, and circulated around the reactor. Albaflow FFA $\left(0.5 \mathrm{~g} \mathrm{~L}^{-1}\right)$ was added to the dye solution for the first time.

The resulting hanks are shown in Figure 5.13 (b) soon after they were removed from the dye solution. The composites are very light pink in colour. Interestingly and unfortunately, the polypropylene hank absorbed a significant amount of the TA-Au 
NPs in the dye solution, thus allowing fewer nanoparticles to be absorbed by the wool hanks and the light pink colour of the composites. The porous nature of the polypropylene hank may be the reason for the absorption of gold nanoparticles, as polypropylene does not exhibit chemical functionality that can be protonated to electrostatically attract the negatively charged TA-Au NPs.

The dry composites are shown in Figure 5.14. There is a significant colour difference between the inside and outside of the hanks. As the dye solution must migrate through the outside of the hank to reach the inside, the local concentration of TA$\mathrm{Au}$ NPs in the dye liquor will be lower when it comes into contact with the inside of the hank. Additionally, the wool hank that was located closer to the overflow of the dye solution is a relatively darker pink-mauve colour than the hank located furthest from the overflow. Again, the colour difference is likely due to the local concentration of TA-Au NPs in the dye solution being lower after it has flowed through both the polypropylene hank and the wool hank that was closest to the overflow.

The synthesis of a $1 \mathrm{~kg}$ TSC-pink felted yarn composite was also attempted in the hank dyeing reactor. In this synthesis the hanks were raised out of the reactor and pulled around at different time intervals during the dyeing in an attempt to promote the uniform absorption of TSC-Au NPs throughout the hank. However the resulting composite was again very uneven in colour. Thus, it became clear that the colouring of felted yarn hanks in the hank dyeing reactor was not conducive to producing evenly coloured composites. This showed that conventional hank dyeing reactors are unsuitable for use in the ex-situ synthesis of gold nanoparticle-wool fibre composites.

Conventional hank dyeing reactions employ an excess of organic dyestuffs. These dye molecules do not immediately fix to the wool, and are able to migrate throughout the hank during the dyeing. The temperature of the dyeing reaction is gradually raised to fix the dye molecules. In contrast, the uptake of gold nanoparticles to wool involves the immediate fixing or 'striking' of gold nanoparticles when they electrostatically interact with the wool protein. Thus, they are considered to be unable to migrate through the dyepack after the initial electrostatic attachment. Additionally, there is an excess of the possible attachment sites on the wool hank than there are gold nanoparticles in the dye solutions. As such, achieving a homogeneous distribution of gold nanoparticles throughout the wool matrix is extremely challenging. The conventional hank dyeing experiments were an important step towards scalingup the synthesis of the ex-situ composites; the research introduced the concepts of auxiliary agents and dye circulation, which were then utilised in the laboratory to 

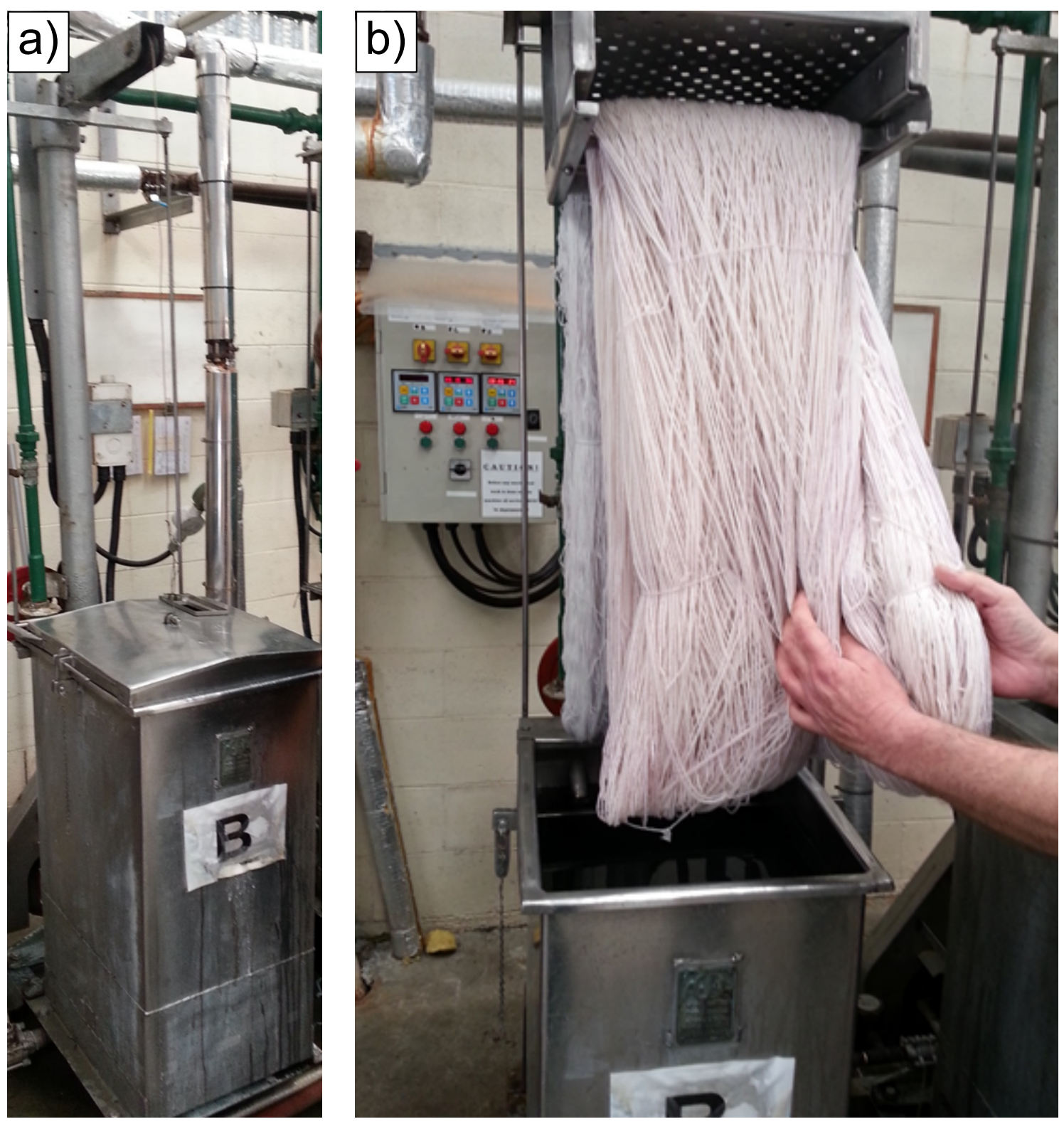

Figure 5.13 The hank dyeing reactor at Wool Yarns Ltd (a), where hanks of wool are held in place (b) before being lowered in to a gently circulating dye solution.

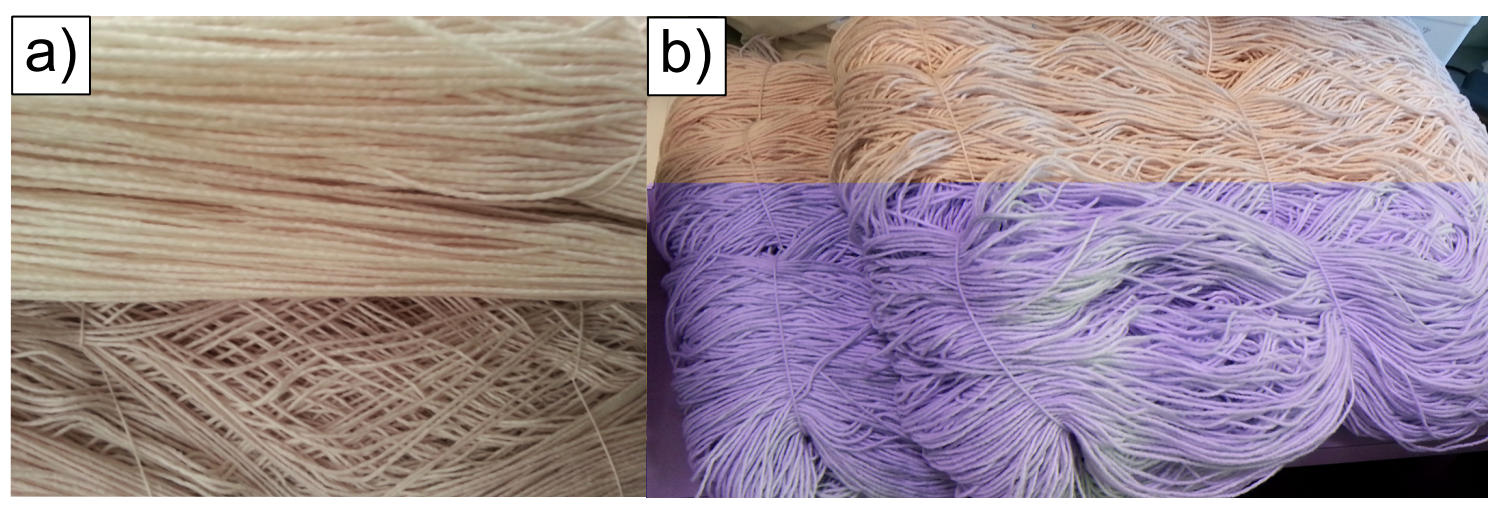

Figure 5.14 $1 \mathrm{~kg}$ TA-mauve felted yarn composites synthesised in the hank dyeing reactor: illustrating the colour differences between the inside and outside of the relatively dark hank (a) and between the two different hanks (b). 
dye hanks of felted yarn with remarkable colour uniformity.

\section{Hank Dyeing of Felted Yarn in the Laboratory}

The ex-situ syntheses of Ascend felted yarn composites were carried out with simulated hank dyeing procedures in the laboratory at Victoria University of Wellington. The syntheses employed a combination of auxiliary agents, gentle dye circulation and physical agitation of the hanks.

The following dyeing reactions employed large volumes of gold colloids that were synthesised in the barrel reactor, using procedures detailed in Section 5.1. The gold colloids were used to colour felted yarn in a large plastic container, which is named the "fish tank" reactor. Immersion heaters with pumping capabilities were used to heat and gently circulate the dye solutions. The felted yarn hanks were agitated by physically dunking the hanks, and turning the hanks inside out at different stages during the dyeing reactions. This was anticipated to promote the uniform absorption of gold nanoparticles throughout the wool yarn matrix.

The desired colour range encompassed mauve and three different shades of grey (light, middle and dark) composites for the fabrication of luxury rugs. The different shades of grey were achieved by altering the concentration of TSC-Au NPs in the dye solution. The reaction conditions of the respective syntheses are detailed in Table 5.3 and are significantly different from those of the Cariaggi dyeing reactions in Section 5.2.1.

Table 5.3 The reaction conditions of the dyeing of Ascend felted yarn hanks in the laboratory (*the gold cost is based on the world gold price as at 23-9-14 ${ }^{58}$ ).

\begin{tabular}{lcccc}
\hline Synthesis & Mauve & Light grey & Middle grey & Dark grey \\
\hline $\mathrm{Au}^{3+}$ conc $\left(\mathrm{mg} \mathrm{L}^{-1}\right)$ & 10 & 10 & 20 & 40 \\
Volume of colloid $(\mathrm{L})$ & 60 & 40 & 60 & 60 \\
Gold content $(\mathrm{g})$ & 0.6 & 0.4 & 1.2 & 2.4 \\
$\quad$ per kg of composite) & & & & \\
Approximate Au cost $(\$)^{*}$ & 29 & 19 & 58 & 116 \\
$\quad$ per kg of composite) & 30 & 50 & 50 & 50 \\
Uptake temperature $\left({ }^{\circ} \mathrm{C}\right)$ & 2.2 & 2.2 & 2.0 & 2.0 \\
Uptake pH & 2 & 2 & 3 & 1 \\
Total mass produced $(\mathrm{kg})$ & & &
\end{tabular}

The notable differences of the ex-situ syntheses of Ascend yarn composites compared to the syntheses of Cariaggi composites include the relatively low reaction $\mathrm{pH}$, the heat and auxiliary agents employed. The reaction conditions were optimised to 
promote the migration of gold nanoparticles throughout the thick felted yarn, where many wool fibres are located in the interior of the yarn.

\section{TA-mauve felted yarn composites}

The synthesis of a $1 \mathrm{~kg}$ TA-mauve felted yarn composite was undertaken in the fish tank reactor. The felted yarn hank was soaked in a glycine buffer $\left(20 \mathrm{~L}, 0.1 \mathrm{~mol} \mathrm{~L}^{-1}\right.$, $\mathrm{pH} 2.2$ ) for 120 min prior to immersion in the standard TA-mauve colloid (60 L, pH 2.2). $0.5 \mathrm{~g} \mathrm{~L}^{-1}$ Albaflow FFA was added to both the buffer and the colloid. The uptake of the TA-mauve colloid to Ascend felted yarn is presented in Figure 5.15. The uptake was relatively slow, requiring $110 \mathrm{~min}$ to be approximately complete. The solution was gradually heated during the uptake to promote the diffusion of the dye solution throughout the felted yarn hank. The uptake was slow for the first $60 \mathrm{~min}$ of the reaction, then rate of uptake was enhanced after the dye solution was heated to $30-35^{\circ} \mathrm{C}$.

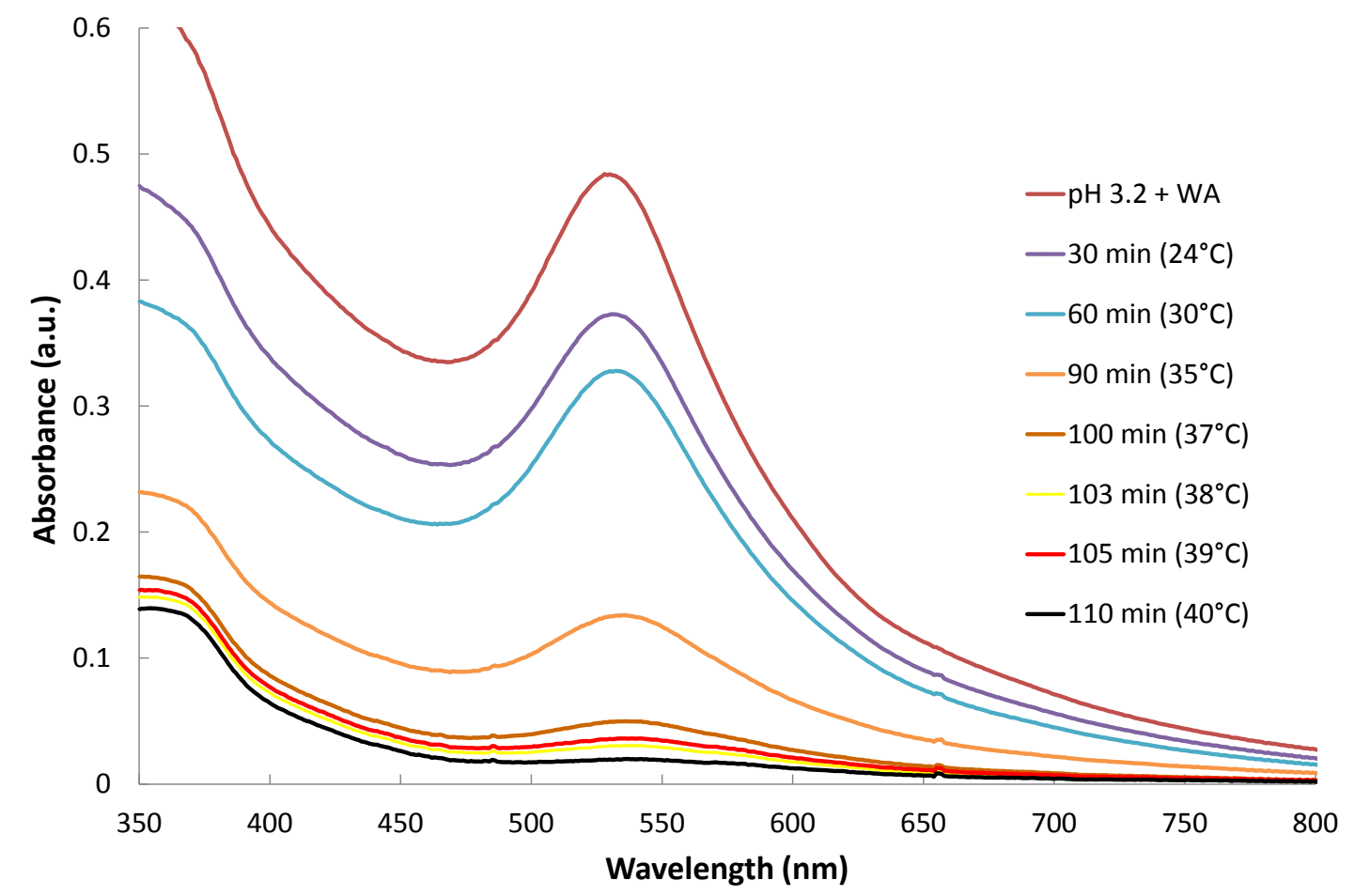

Figure 5.15 UV-vis spectra of the uptake of the TA-mauve colloid to felted yarn in the fish tank reactor.

The structure of the Ascend yarn consists of merino wool fibres that are felted together to form thick yarn of approximately $2 \mathrm{~mm}$ diameter. As such, the uptake profile is considered to involve the rapid saturation of the exterior of the yarn with TA-Au NPs. This may be followed by the penetration of TA-Au NPs into the wool fibres located in the interior of the yarn after the attachment sites on the 
exterior fibres exhausted. Additionally, the wetting agent would likely preferentially associate to the exterior of the yarn, and hence may slow the uptake of TA-Au NPs to these areas sufficiently to allow the migration into the centre of the felted yarn.

Images of the dyeing of Ascend felted yarn with the TA-mauve colloid and the resulting composite are presented in Figure 5.16 (a), and shows the felted yarn hank near the beginning of the reaction where it is light pink in colour. Further into the dyeing reaction (Figure 5.16 (b)), the now mauve coloured hank begins to loosen, and care is required to prevent fibre entanglement. The resulting composite is purple-mauve and is significantly more uniform in colour compared to the light pink composites synthesised in the hank dyeing reactor (Figure 5.14). Again the inside of the hank is relatively light compared to the outside of the hank.

The above reaction was repeated to produce an additional $1 \mathrm{~kg}$ composite. The two Ascend felted yarn TA-mauve composites can be seen in Figure 5.19, which encompasses the entire range of synthesised in these experiments. The composites are remarkably similar in colour, with both exhibiting a relatively light shade in the interior of the hank. This is an excellent result, showing promising reproducibility of dyeing felted yarn in the fish tank. Next, TSC-grey composites were synthesised with this method.

\section{TSC-grey felted yarn composites}

The success of synthesising TA-mauve Ascend felted yarn composites in the fish tank reactor lead to the synthesis of TSC-grey composites with this method. The synthesis of a TSC-middle grey composite is first presented. This method utilises a lower concentration of the TSC-grey colloid than was used in the Cariaggi TSCgrey synthesis, to achieve a similar hue. This is due to the relatively lower effective surface area of the felted yarn compared to the relatively open structure of top form wool.

The TSC-middle grey colloid $(60 \mathrm{~L})$ was synthesised in the barrel reactor and used to colour felted yarn in the fish tank reactor. The wool hank was soaked in warm tap water and drip dried before the dyeing reaction. The TSC-middle grey colloid was adjusted to pH 2.0 after the addition of $0.5 \mathrm{~g} \mathrm{~L}^{-1}$ Albaflow FFA into the colloid. UV-vis spectra of the TSC-middle grey colloid during the uptake to felted yarn is presented in Figure 5.17. The typical agglomeration of the colloid begins to occur after the $\mathrm{pH}$ of the colloid is adjusted to 2.0. The agglomeration continues during the dyeing reaction. The reduction in intensity of the original SPR peak $(\lambda=525 \mathrm{~nm})$

and the longer wavelength absorbance of the agglomerates indicates that both are 


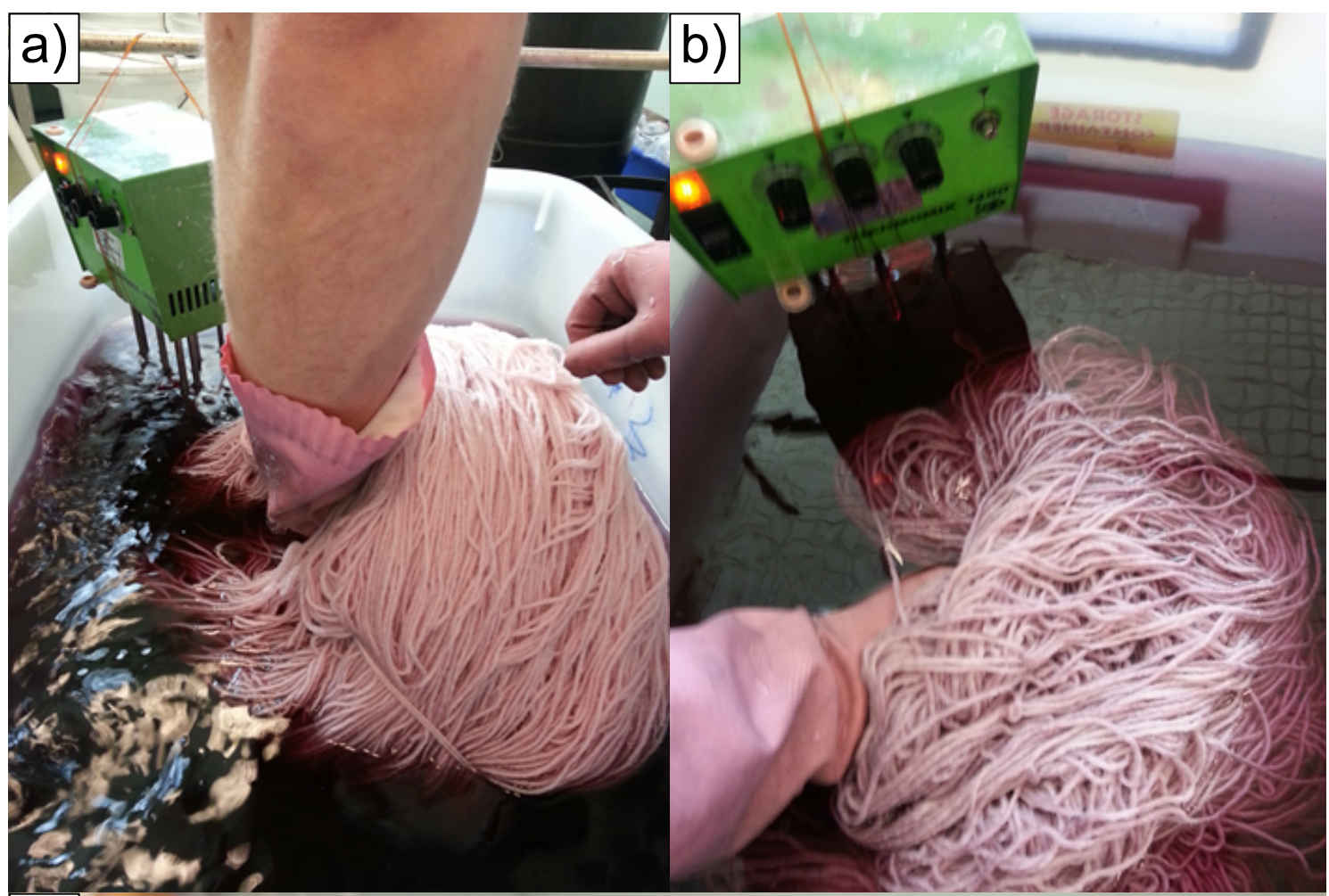

c)

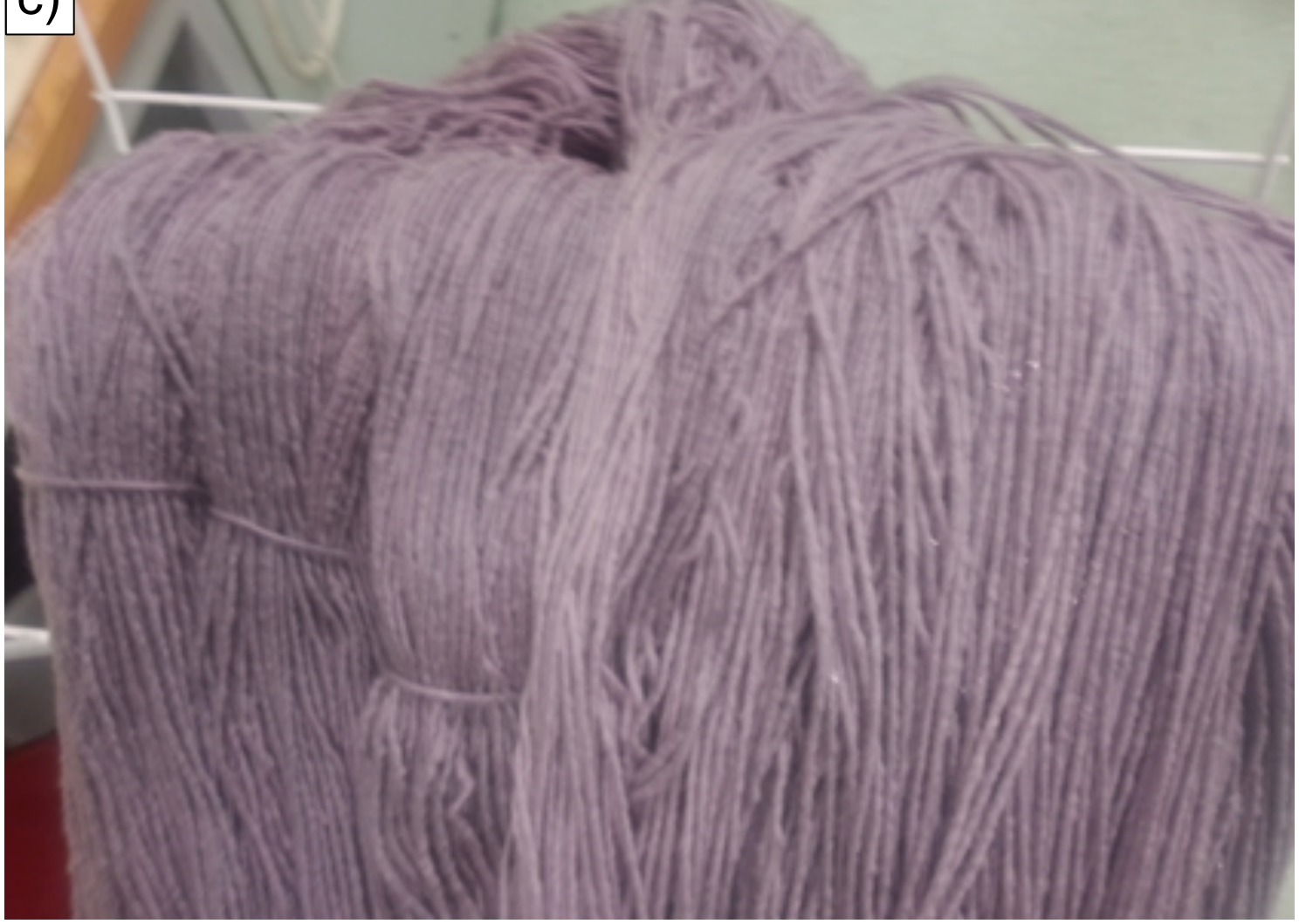

Figure 5.16 The synthesis of a $1 \mathrm{~kg}$ TA-mauve felted yarn composite: the dyeing reaction near the beginning of the uptake (a), after approximately $60 \mathrm{~min}$ of the uptake (b) and the resulting composite (c). 
absorbed by the wool during the dyeing reaction.

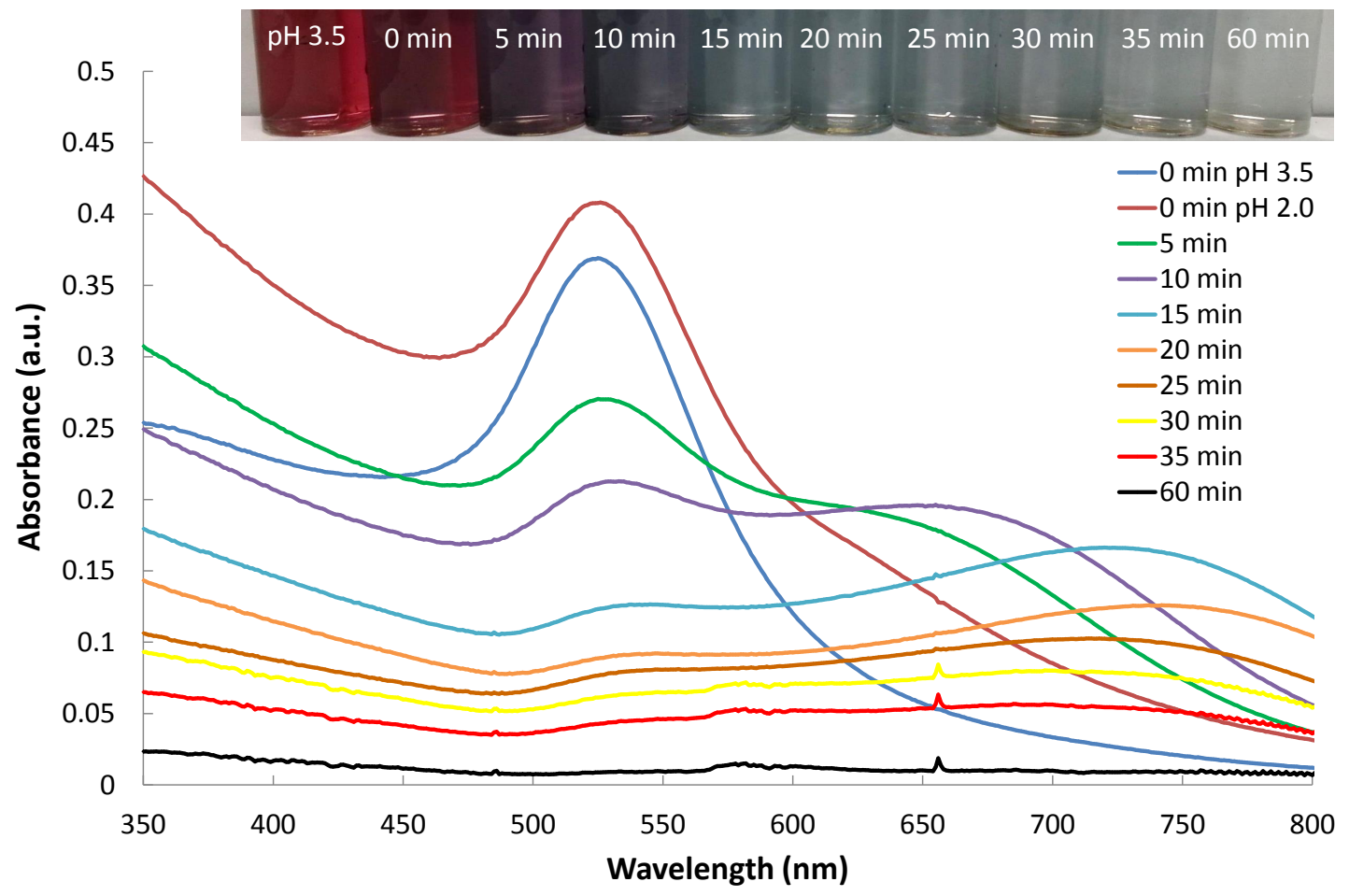

Figure 5.17 The uptake of the TSC-middle grey colloid to Ascend felted yarn $(1 \mathrm{~kg})$ in the fish tank reactor.

The resulting TSC-middle grey composite was remarkably uniform. The above process was repeated twice more to produce a total of $3 \mathrm{~kg}$ of middle grey composites, all of which are shown in Figure 5.19. Similar to the TA-mauve composites, there is again some variation in the colour of the composites between the inside and outside of the hanks. Additionally, the three different TSC-middle grey hanks are slightly different shades of grey, which implies that this method is not as reproducible as the TA-mauve method.

The TSC-dark grey synthesis employs the equivalent concentration of TSC-Au NPs that was used in the TSC-grey Cariaggi method $\left(60 \mathrm{~L}, 60 \mathrm{~mL} 4 \mathrm{wt} \% \mathrm{Au}^{3+}, 75 \mathrm{~mL}\right.$ 5 wt $\%$ TSC). Again, $0.5 \mathrm{~g} \mathrm{~L}^{-1}$ of Albaflow FFA was added to the colloid before the $\mathrm{pH}$ was lowered to 2.0. Images of the TSC-dark grey dyeing reaction are shown in Figure 5.18, where the immersion heater slowly heated the colloid to $50{ }^{\circ} \mathrm{C}$ during the uptake. An additional pump was employed to increase the circulation of the dye liquor around the system.

The immersion of the felted yarn hank into dye solution resulted in the rapid agglomeration of TSC-Au NPs, to result in a dark grey-black coloured solution. The agglomerates of TSC-Au NPs then began to coat the fish tank reactor and the tubing of the pump in addition to being absorbed by the wool hank. The coating is observed in the circled area in Figure 5.18 (c) and this represents a significant 
amount of wasted gold. The extent of the agglomeration of TSC-Au NPs may be enhanced by the heat and dye circulation of this method. The structure of the felted yarn slows the access of the agglomerates of TSC-Au NPs to the wool located in the centre of the felted yarn. As such, lower concentrations of TSC-Au NPs were used in subsequent dyeing reactions. Despite the gold wastage, the TSC-dark grey method did produce a reasonably uniformly coloured composite, which can be seen in Figure 5.19.

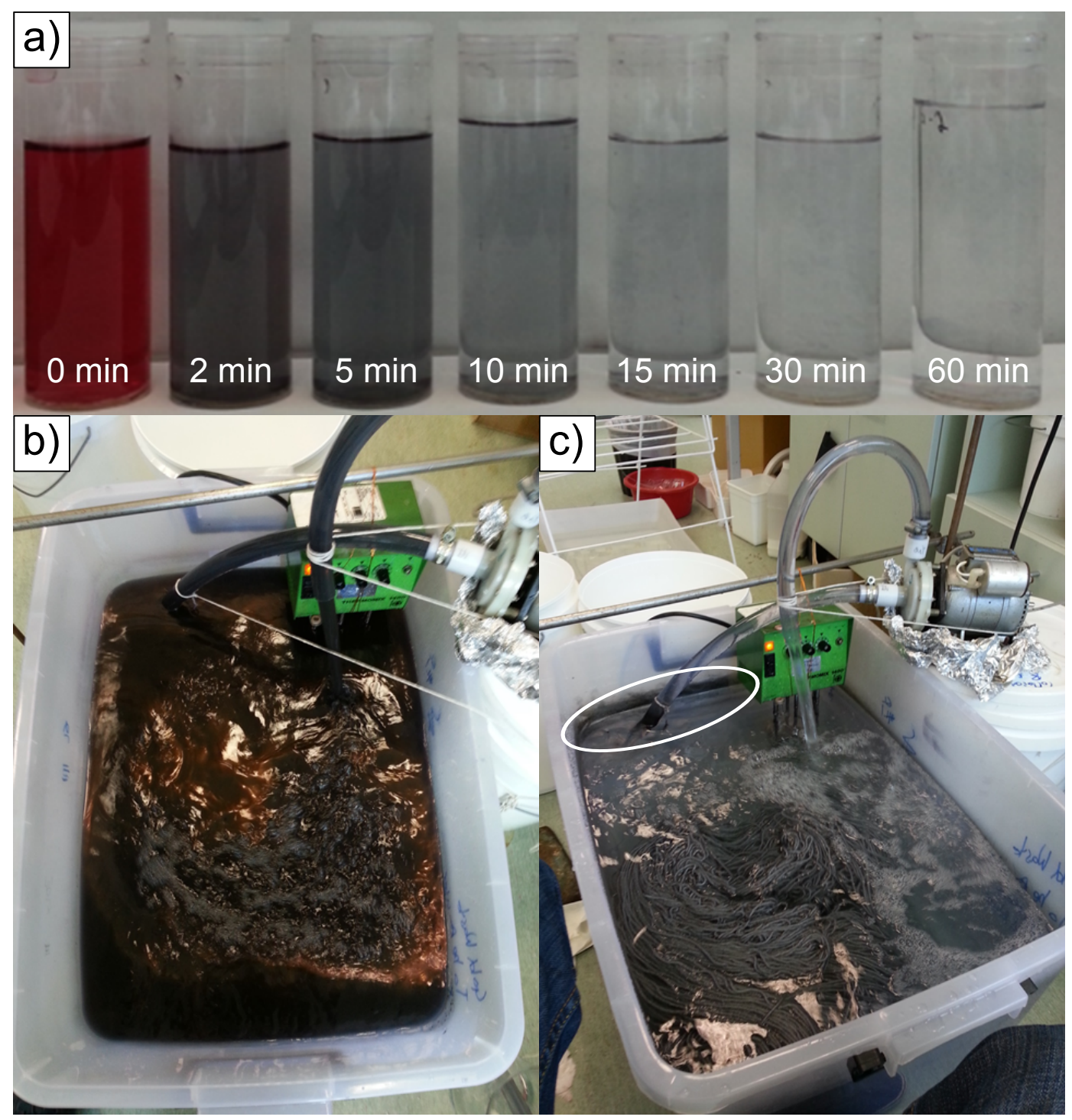

Figure 5.18 The TSC-dark grey composite synthesised in fish tank: the uptake solutions (a), the dyeing reaction soon after the addition of the felted yarn hank (b) and the dyeing reaction near the completion of the uptake (c).

TSC-light grey felted yarn composites were next synthesised. The TSC-light grey method uses two thirds of the volume of the standard TSC-pink colloid (40 L, $10 \mathrm{~mL}$ $4 \mathrm{wt} \% \mathrm{Au}^{3+}$ and $25 \mathrm{~mL} 5 \mathrm{wt} \%$ TSC). The gradual heating of the colloid (up to $50{ }^{\circ} \mathrm{C}$ ) during the dyeing process was employed both to aid the penetration of TSC$\mathrm{Au}$ NPs into the centre of the felted yarn and to facilitate the agglomeration of the 
nanoparticles (and hence impart a light grey colour to the composites). Two TSClight grey composites of $1 \mathrm{~kg}$ size were synthesised and are shown in Figure 5.19. Both are light-grey coloured, however one of the hanks appears to exhibit a pink tinge, indicating the presence of individual gold nanoparticles on the surface of the composite. This is another example of the difficulty in controlling the agglomeration of TSC-Au NPs in these methods.

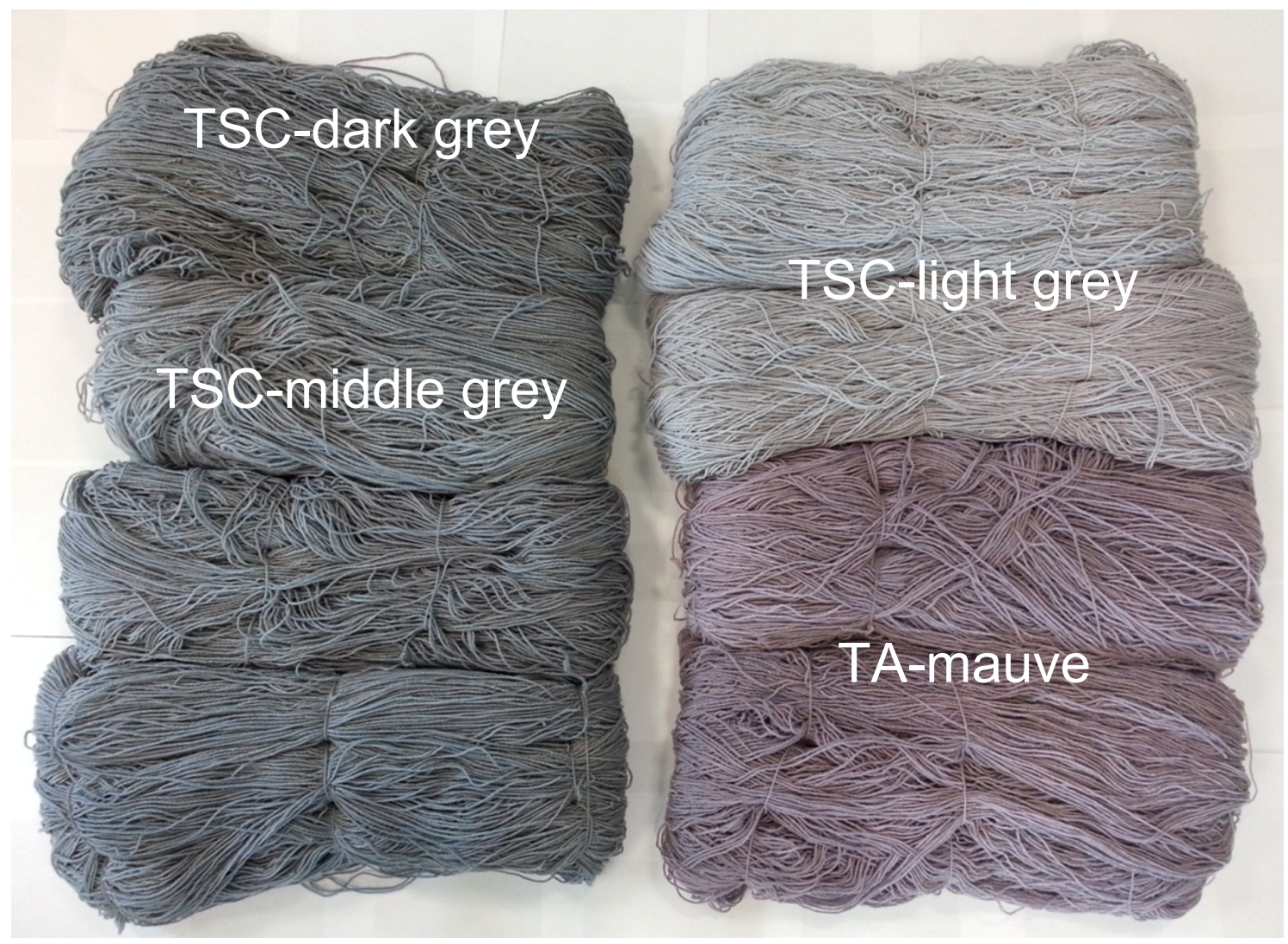

Figure 5.19 TA-mauve and different shades of TSC-grey Ascend felted yarn composites $(1 \mathrm{~kg})$ synthesised in the fish tank reactor with modified hank dyeing procedures.

The Ascend felted yarn ex-situ composites synthesised with modified hank dyeing procedures are presented in Figure 5.19. CIE colour coordinates of the ex-situ felted yarn composites are shown in Table 5.4. As the CIE colour coordinates are measured on a very small area of a $1 \mathrm{~kg}$ hank of felted yarn, their values represent only an approximation of the colour of the entire composite (the colour was measured on felted yarn that was tied to the outside of the hank, as yarn from the hanks could not be taken from different areas of the hanks). The TA-mauve composites exhibit relatively similar $\mathrm{L}^{*}, \mathrm{a}^{*}$ and $\mathrm{b}^{*}$ values compared to the repeated TSC-grey composites. The light grey composites are expectedly brighter than the middle grey composites, however the dark grey composite is of a similar darkness to the middle grey composites. This is understandable given the significant deposition of TSC-Au NPs on the fish tank during the synthesis of the dark grey composite. Addition- 
ally, the differences in $\mathrm{L}^{*}$ value between the repetitions of the middle grey syntheses highlights the relative diversity in colour of this method.

Table 5.4 CIE colour coordinates of the Ascend felted yarn composites.

\begin{tabular}{ccccccccc}
\hline & \multicolumn{2}{c}{ TA-mauve } & \multicolumn{2}{c}{ Light grey } & \multicolumn{3}{c}{ Middle grey } & Dark grey \\
\hline Run & 1 & 2 & 1 & 2 & 1 & 2 & 3 & 1 \\
\hline $\mathrm{L}^{*}$ & 52.55 & 52.23 & 69.02 & 67.80 & 52.41 & 54.47 & 56.46 & 53.11 \\
$\mathrm{a}^{*}$ & 4.31 & 3.91 & 0.73 & -0.02 & -0.16 & -0.64 & 0.03 & -0.29 \\
$\mathrm{~b}^{*}$ & -3.08 & -3.80 & -0.77 & -1.54 & -3.10 & -2.33 & -2.78 & -1.39 \\
\hline \hline
\end{tabular}

Mixtures of the different coloured felted yarn composites presented in Figure 5.19 were used to fabricate the luxury rugs by the designers at Ascend Rugs Ltd, and are shown in Figure 5.20. The creation of the surface of the rug involves aligning the yarn and cutting through it, thus exposing the interior of the yarn. The colouration of the rugs shows the successful penetration of gold nanoparticles throughout the interior of the yarn. The rugs were exposed to international audiences at showcases in Los Angeles and Frankfurt, that were organised by Ascend Rugs Ltd and Wools of New Zealand Ltd.

The successful colouring of Ascend felted yarns with gold nanoparticles was an important step towards scaling-up the synthesis of ex-situ gold nanoparticle-wool fibre composites. Utilising the hank dyeing reaction conditions of gentle dye circulation and auxiliary agents, together with physical agitation in the fish tank reactor, resulted in composites with impressive colour uniformity. The fabrication of gold nanoparticle-coloured rugs provided an excellent opportunity to showcase the novel Aulana composites to an international audience. This was an important step towards the commercialisation of these novel nanocomposites.

While the modified hank dyeing procedures resulted in composites that were suitable for direct use in the manufacture of rugs, this method was deemed to be unsuitable for the colouring of other wool types. The dye circulation and agitation of the hanks during the synthesis would result in damaged top form wool and entangled hanks of fine merino yarns. Additionally, it was a hands-on approach that can produce variable results (particularly with the TSC-grey methods). The development of a more reproducible method that is applicable for the colouring of top form wool and fine yarns is desired. To achieve this, alternative types of dyeing were explored in this research, including the conventional package dyeing of merino yarns. 

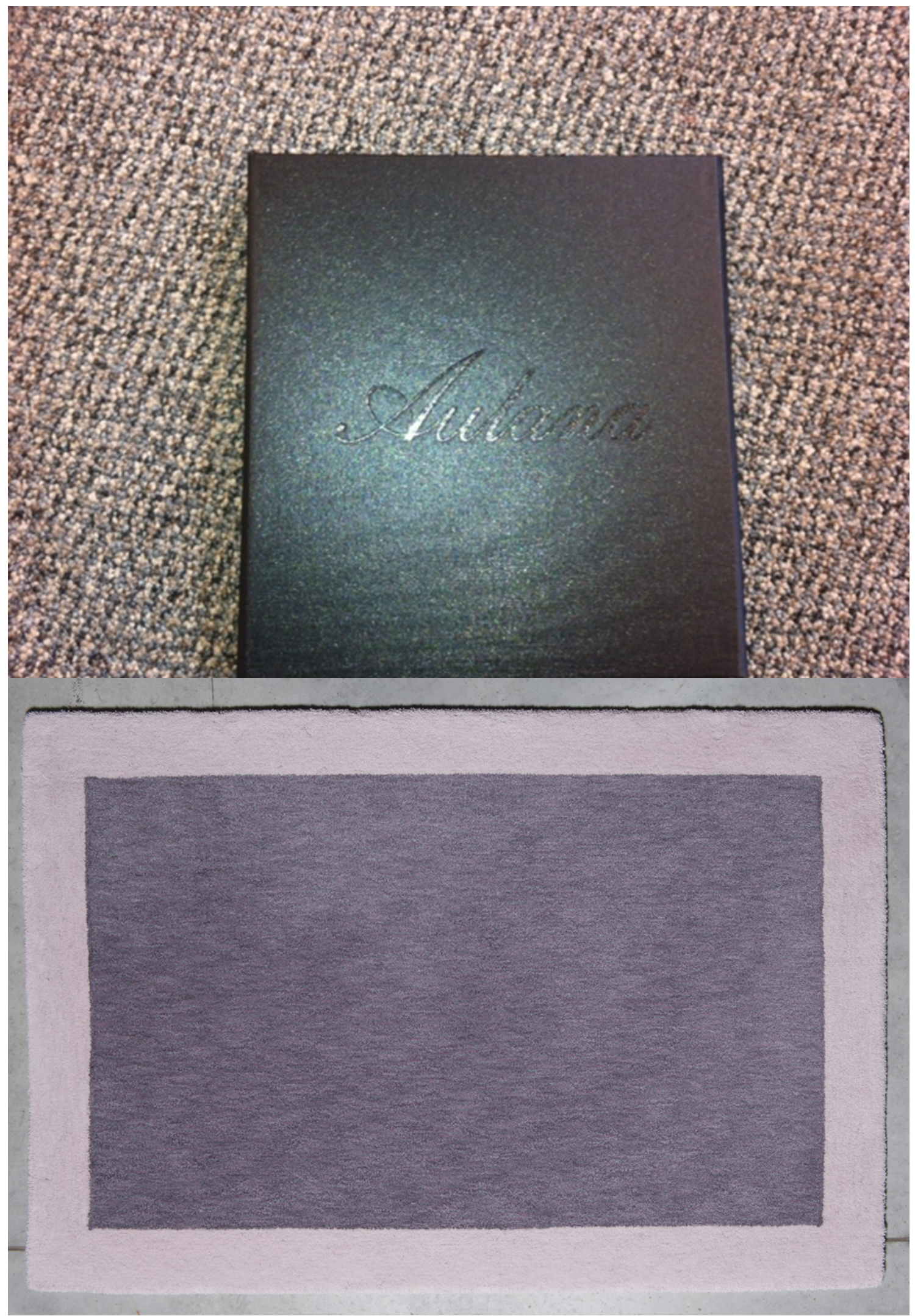

Figure 5.20 Aulana rugs fabricated from ex-situ felted yarn composites that were synthesised in modified hank dyeing procedures. 


\subsection{Package Dyeing of Merino Yarn}

The wool dyeing industry utilises package dyeing equipment to colour fine yarns of merino wool. Package dyeing involves the tight winding of fine yarns around perforated cones, through which dye liquor is forced during the dyeing reaction. Package dyeing reactors are pressurised, to promote the movement of the dye liquor throughout the dyepack. The yarn is tightly held in place to prevent movement during the dye circulation (Figure 5.21 (b), which prevents the potential matting and entanglement of the wool yarns.

The hank dyeing of fine merino yarns results in entangled and unevenly coloured yarns, and as such is confined to hanks of relatively thick yarn. The fine yarns are knitted into finished products directly after they are dyed. Hence, achieving colour uniformity is integral to this process, as colour differences cannot be atoned for with further processing of the yarns.

This section presents the integration of the ex-situ procedure into conventional industrial package dyeing processes. The research was carried out at Tararua Yarns Ltd in Levin, New Zealand, in collaboration with Professor James Johnston's industrial contacts, who shared technical knowledge and expertise on the dyeing of merino yarns. ${ }^{54}$

The industrial pilot-scale package dyeing reactor used in the synthesis of ex-situ gold nanoparticle-wool fibre composites is presented in Figure 5.21. The reactor can be used to dye up to $2 \mathrm{~kg}$ of wool yarn at a time and holds $8-9 \mathrm{~L}$ of dye liquor. The package dyeing reactions involve placing a cone of wool in the dyeing tank, the top of which is seen in Figure 5.21 (c) and (d). Dye liquor is then added to the overflow tank, where it is pumped into the dyeing tank, through the wool cone and out the outlet tap back into the overflow tank.

The interior of the package dyeing reactor is seen in Figure 5.21 (c) and (d). A perforated cone of $250 \mathrm{~g}$ of Tararua Untreated (TU) yarn (Figure 5.21 (b)) is placed inside the dyeing tank. The cone is clamped down so that the dye liquor is forced through the wool yarn as it is pumped around the system. The flow may be directed either from the inside to the outside of the cone (from the central pole) or vice versa. The flow directions are termed in-out or out-in flow respectively, and may be alternated during the dyeing reactions. The package dyeing reactor was first used to synthesis TA-mauve ex-situ gold nanoparticle-wool fibre composites of TU yarn. 


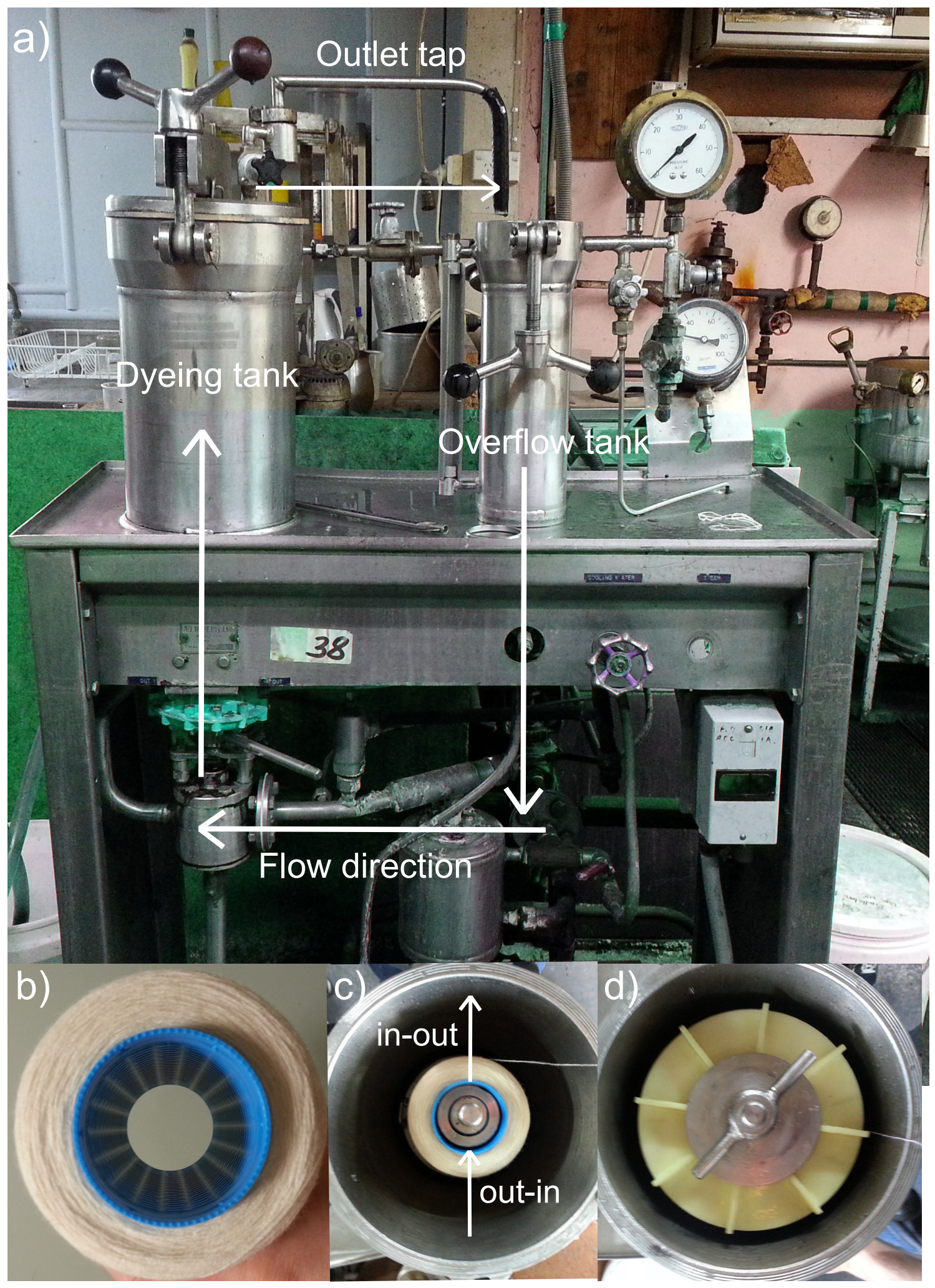

Figure 5.21 The package dyeing reactor from Tararua Yarns Ltd, where a cone of fine yarn is compressed in the tank and a dye solution is circulating through the cone and out into the overflow tank (a), a $250 \mathrm{~g}$ cone of Tararua Untreated (TU) yarn (b), the possible flow directions in the dyeing tank (c) and the clamped top of the dyeing tank $(d)$. 


\subsubsection{Package Dyed TA-mauve TU Yarn Composites}

The first package dyeing reaction involved the synthesis of a $750 \mathrm{~g}$ TA-mauve TU yarn composite. Given the maximum $9 \mathrm{~L}$ capacity of the reactor, this equates to a wool to liquor ratio of 1:12. The equivalent conditions of the standard TA-mauve method were replicated in the package dyeing reactor $\left(0.1 \mathrm{~mol} \mathrm{~L}^{-1}\right.$ glycine, $\mathrm{pH} 2.2$, $0.5 \mathrm{~g} \mathrm{~L}^{-1}$ Albaflow FFA). This involved increasing the concentration of the buffer and TA-mauve colloid to account for the lower volume of the synthesis.

Hot tap water was cycled around the system to ensure that all of the yarn in the dyepack was wet and to remove processing lubricants and lipids from the wool prior to the dyeing reaction. These lubricants were believed to detrimentally influence the chemistry of the ex-situ procedure. A significant amount of lubricants and wool grease leached from the TU yarn during the washing step, as is seen in Figure 5.22 (a). Following this, the water was drained and the glycine buffer was circulated around the system for $120 \mathrm{~min}$ and then drained prior to the circulation of the TAmauve colloid. An image of the overflow tank near the beginning of the dyeing reaction is shown in Figure 5.22 (b). The TA-mauve colloid is relatively cloudy and foam appears at the top of the overflow tank. This is likely due to the pressure and flow causing the Albaflow FFA in the solution to foam.

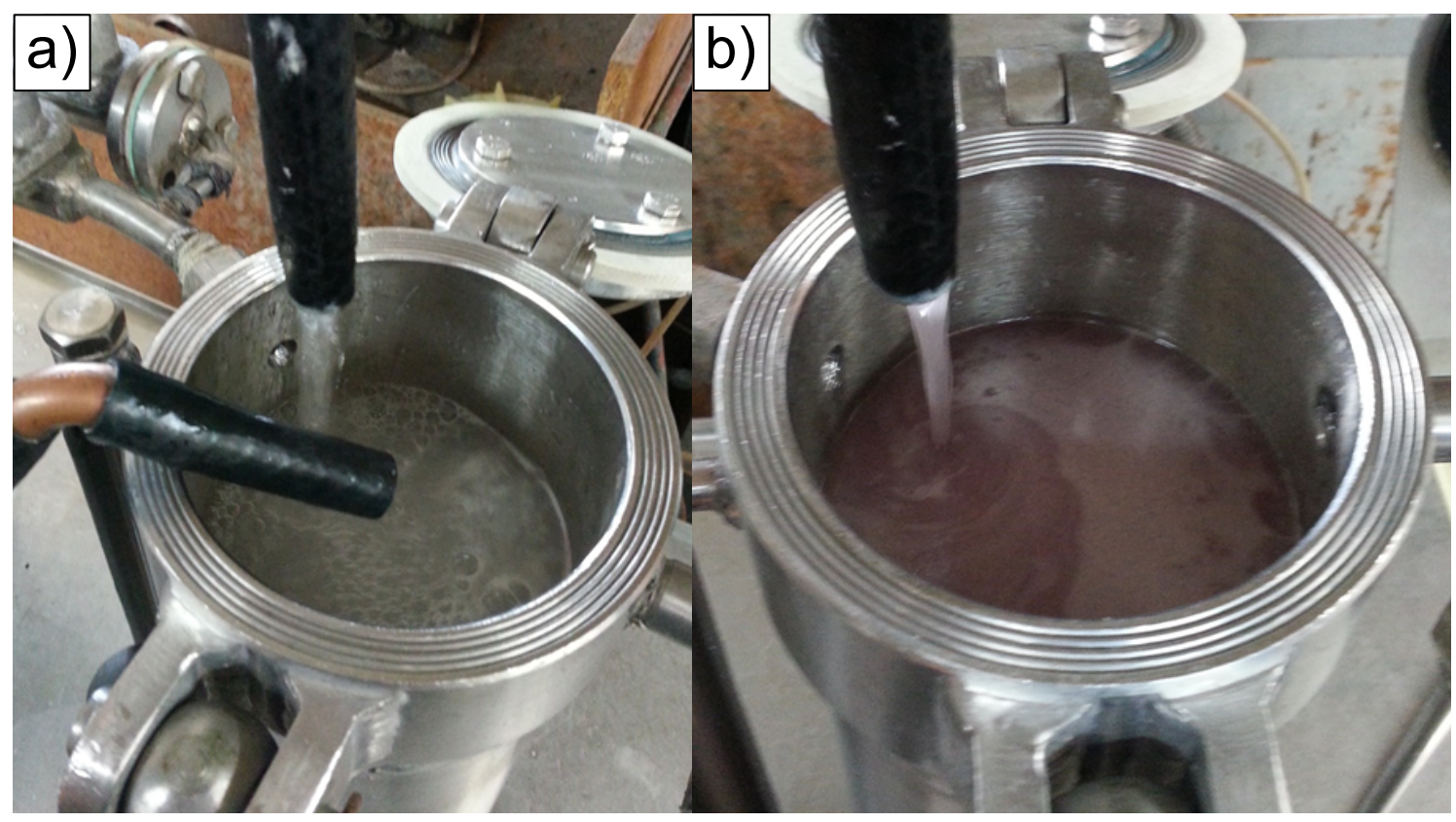

Figure 5.22 Images of the overflow tank of the package dyeing reactor during the hot water washing step (a) and near the beginning of the uptake of the TA-mauve colloid (b). 
The uptake was extremely rapid, with the reaction being complete in less than 5 min. The flow and pressure increased the interaction between the wool and the colloid, and had a substantial influence on the rate of uptake. The uptake behaviour was very different from the uptake chemistry observed in static dyeing reactions. The composite that resulted from this dyeing reaction is shown in Figure 5.23, together with a levelness test. Levelness tests involve the knitting of a piece of fabric from yarn that was removed from the inside, middle and outside of a dyepack. This was carried out to observe potential colour differences throughout the composite (which are difficult to observe when looking at the dyepack as a whole).

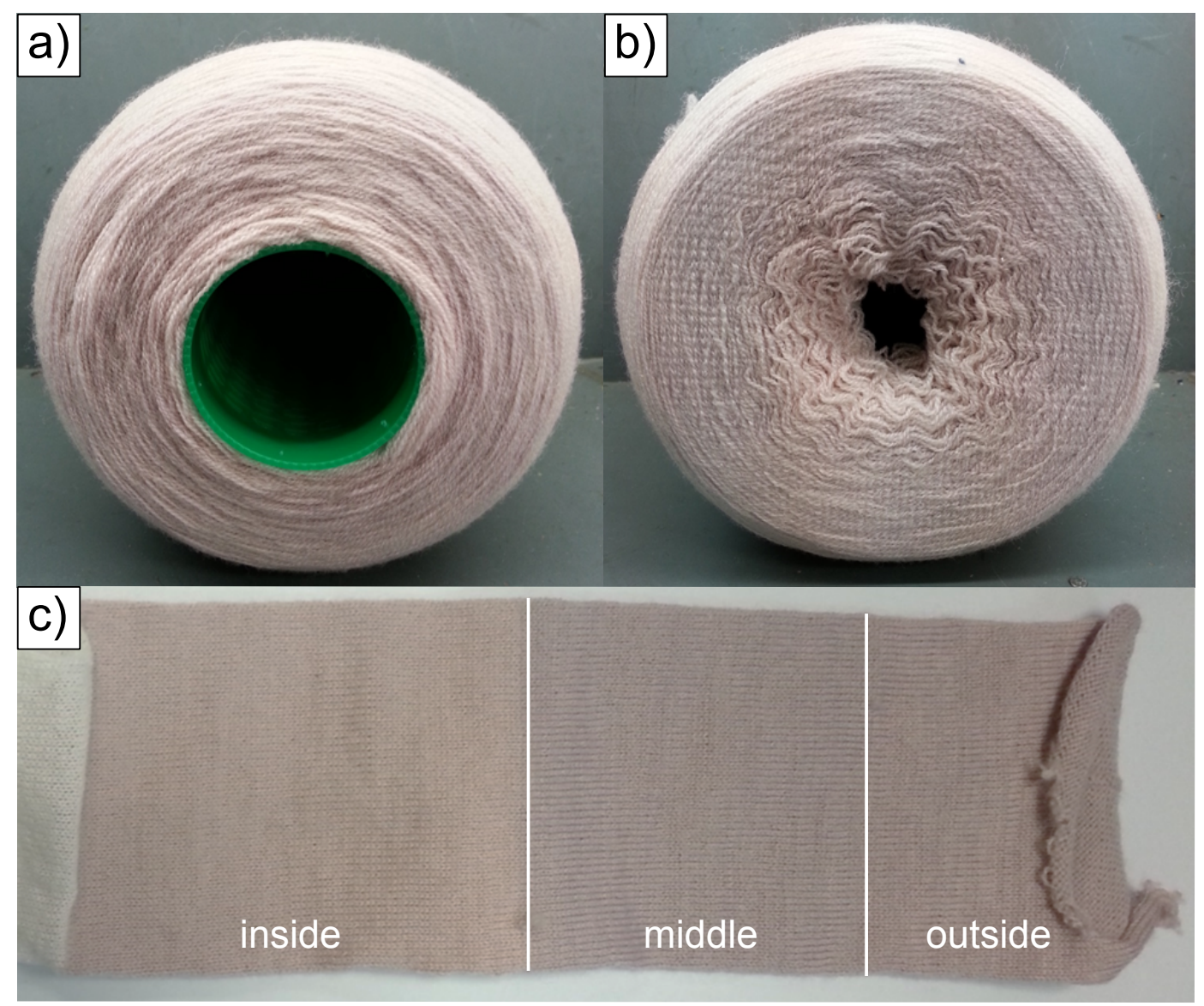

Figure 5.23 Images of a $750 \mathrm{~g}$ TA-mauve TU yarn composite that was synthesised in the package dyeing reactor and a levelness test showing areas of yarn from the inside, middle and outside of the dyepack.

The levelness test of the TA-mauve TU yarn composite shows significant colour variation throughout the dyepack, with the inside of the dyepack being relatively light. These colour differences were quantified by measuring the CIE colour coordinates of the different areas of the dyepack, which are presented in Table 5.5. There are significant differences in colour throughout the dyepack: the inside is much lighter than the middle and outside, indicating that fewer TA-Au NPs were absorbed in this area. Also, the middle is relatively dark and blue. This is interesting given that the flow was set to in-out. A possible explanation of the lighter inside of the dyepack may be the preferential association of Albaflow FFA to the inside of the dyepack 
under the applied flow. This would slow the absorption of gold nanoparticles to the inside of the dyepack, but depends on the how fast the Albaflow FFA is taken up by the wool. This would be interesting to investigate in future studies.

Table 5.5 CIE colour coordinates of inside, middle and outside of the $750 \mathrm{~g}$ TAmauve TU yarn composite dyepack synthesised in the package dyeing reactor (the areas of the dyepack are separated by white lines).

\begin{tabular}{cccc}
\hline Area of dyepack & Inside & Middle & Outside \\
\hline $\mathrm{L}^{*}$ & 71.62 & 67.98 & 69.65 \\
$\mathrm{a}^{*}$ & 3.72 & 3.25 & 4.02 \\
$\mathrm{~b}^{*}$ & 4.60 & 2.48 & 2.84 \\
\hline \hline
\end{tabular}

The first package dyeing reaction resulted in a TA-mauve TU yarn composite with significant colour variation throughout the dyepack. The rate of uptake was much more rapid than that seen in static reactions. This result led to the chemistry presented in Section 5.4.1 to better characterise the influence of dye circulation. The amount of Albaflow FFA used in the synthesis was then manipulated to attempt to slow the uptake and increase the colour uniformity of the resulting composites.

\section{Albaflow FFA Amount}

There are a number of ways to increase the colour uniformity of composites that are synthesised in package dyeing reactors, by either slowing the rate of uptake or by minimising the residence time of dye circulation. Both of these methods increase the number of circulations of dye liquor that occur during the uptake. The rate of uptake can be changed by manipulating the reaction temperature, the concentration of dyestuffs and by employing wool dyeing auxiliaries. The residence time is the time it takes for the dye liquor to cycle around the reactor, and may be reduced by employing lower volumes or faster flow rates of dye circulation. One of the aims of this research was to maximise the number of circulations of the dye solutions that occurred during the package dyeing reactions.

To attempt to slow the uptake of TA-Au NPs and enhance the colour uniformity of the composites, the TA-mauve TU yarn composites were synthesised with 1, 2 and $4 \mathrm{gL}^{-1}$ Albaflow FFA in the glycine buffer and TA-mauve colloid. Composites of $250 \mathrm{~g}$ size were synthesised in these experiments, to reduce the required amount of expensive gold reagents and TU yarn (of which there was a limited supply). The $250 \mathrm{~g}$ TU wool equated to a wool to liquor ratio of 1:36 with $9 \mathrm{~L}$ of dye solution. As such, the concentrations of the standard TA-mauve buffer and colloid were manipulated to account for this. These reactions employed a 3:2 min ratio of in-out to 
out-in flow direction (beginning at in-out flow).

The uptake of the TA-mauve colloid to TU yarn in the package dyeing reactor with 1-4 $\mathrm{g} \mathrm{L}^{-1}$ Albaflow FFA in the buffer and colloid is presented in Figure 5.24, where the intensity of the SPR peak of TA-Au NPs $(\lambda=530 \mathrm{~nm})$ decreases over time. Again, the rate of uptake is much more rapid than that which is typically observed in static reactors in the laboratory. Increasing the amount of Albaflow FFA in the synthesis does increase the uptake time. The uptake curves are relatively non-linear compared to those observed in static reactors, where increasing the amount of Albaflow FFA in the synthesis caused the uptake curves to become increasingly linear. The foaming of the wetting agent in the package dyeing reactions may be contributing to its relative ineffectiveness of slowing the rate of uptake of TA-Au NPs.

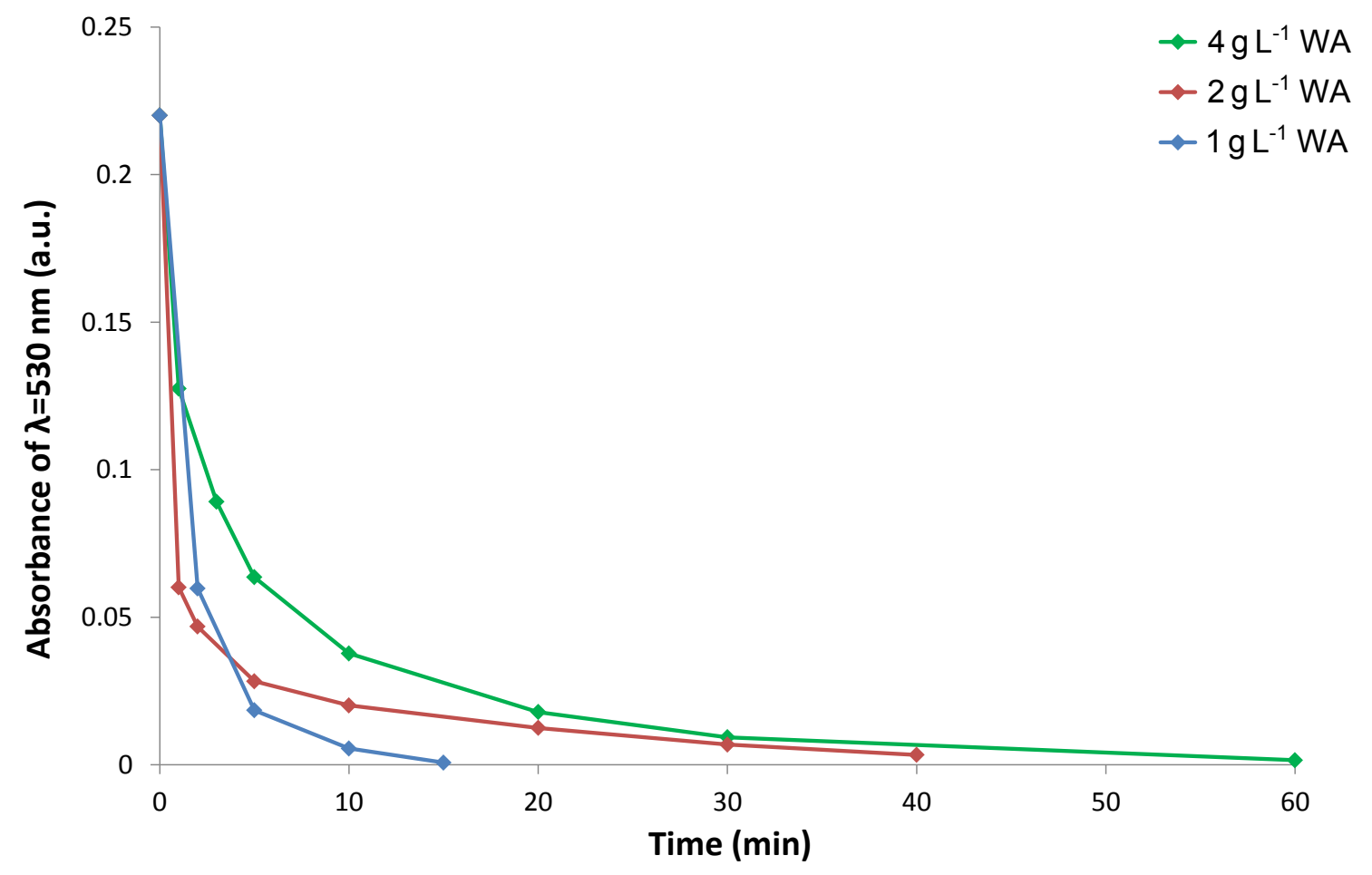

Figure 5.24 The uptake of the TA-mauve colloid to TU yarn in package dyeing reactions with different amounts of Albaflow FFA in the $\mathrm{pH} 2.2$ glycine buffer and colloid.

The levelness tests of the resulting composites are presented in Figure 5.25. Surprisingly, the $1 \mathrm{~g} \mathrm{~L}^{-1} \mathrm{WA}$ composite exhibits the lowest colour variation throughout the dyepack. Increasing the amount of Albaflow FFA to 2 and then $4 \mathrm{~g} \mathrm{~L}^{-1}$ has resulted in increased discolouration throughout the dyepack. 

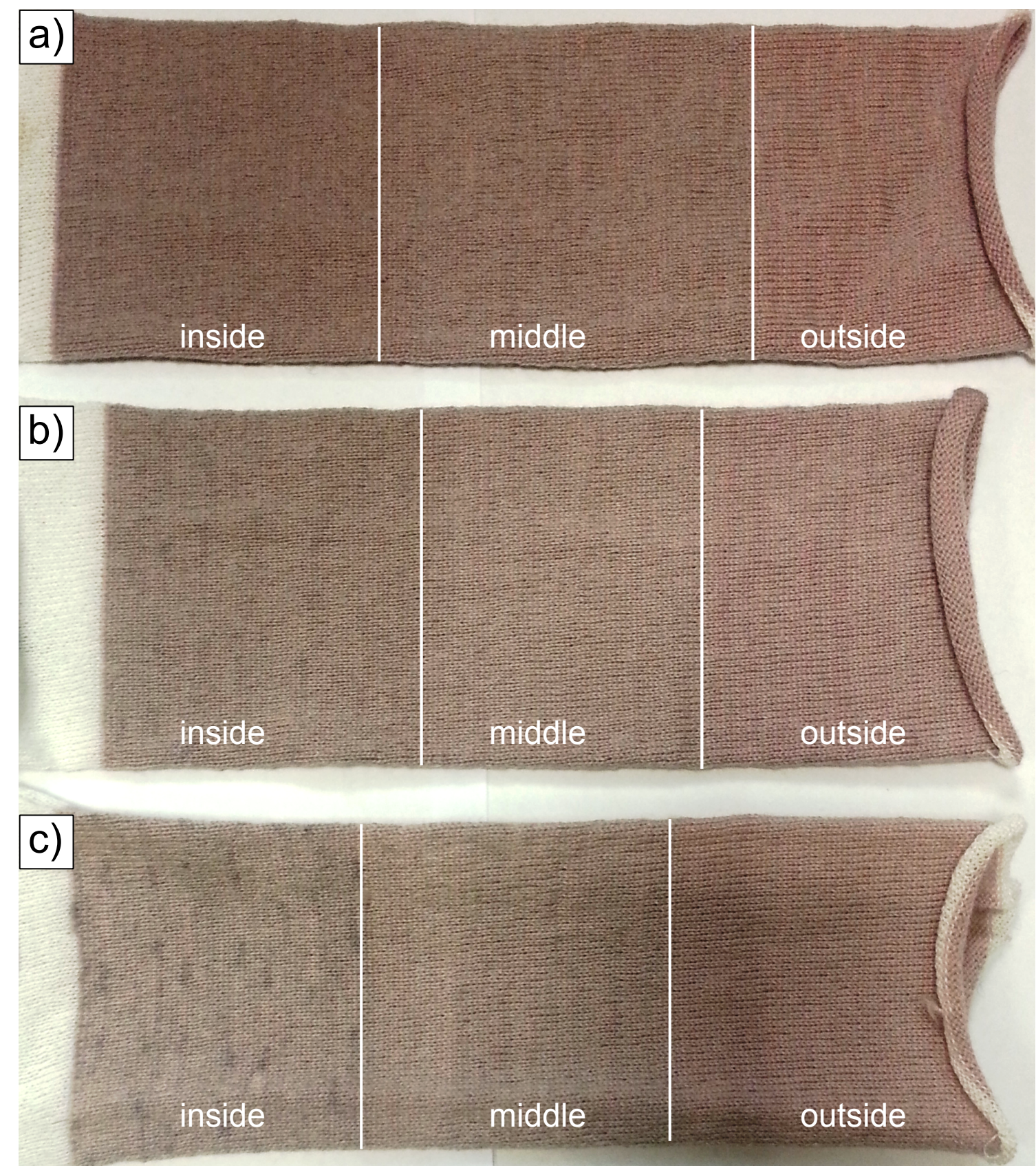

Figure 5.25 Levelness tests of TA-mauve TU yarn composites synthesised in the package dyeing reactor with $1 \mathrm{gL}^{-1}$ (a), $2 \mathrm{~g} \mathrm{~L}^{-1}$ (b) and $4 \mathrm{~g} \mathrm{~L}^{-1}$ (c) Albaflow FFA in the buffer and colloid.

CIE colour coordinates of the inside, middle and outside of the dyepack of composites synthesised with different amounts of Albaflow FFA are presented in Table 5.6. Each composite exhibits different $\mathrm{L}^{*}, \mathrm{a}^{*}$ and $\mathrm{b}^{*}$ values throughout the dyepack. The relative variation throughout the dyepack is more pronounced when the amount of Albaflow FFA is increased, which agrees with the colour differences observed in Figure 5.25.

Interestingly, each composite generally exhibits a higher a* value and lower $\mathrm{L}^{*}$ value as we move from the inside to the outside of the dyepack. The relatively dark 
Table 5.6 CIE colour coordinates measured from different areas of the $250 \mathrm{~g}$ TAmauve TU yarn composites synthesised with different amounts of Albaflow FFA.

\begin{tabular}{cccccccccc}
\hline WA & \multicolumn{3}{c}{$1 \mathrm{~g} \mathrm{~L}^{-1}$} & \multicolumn{3}{c}{$2 \mathrm{~g} \mathrm{~L}^{-1}$} & \multicolumn{3}{c}{$4 \mathrm{~g} \mathrm{~L}^{-1}$} \\
\hline Area & In & Mid & Out & In & Mid & Out & In & Mid & Out \\
\hline $\mathrm{L}^{*}$ & 60.72 & 62.81 & 63.12 & 61.18 & 63.18 & 62.76 & 60.96 & 65.30 & 66.21 \\
$\mathrm{a}^{*}$ & 4.49 & 4.57 & 5.67 & 3.13 & 3.67 & 4.82 & 3.12 & 3.52 & 5.07 \\
$\mathrm{~b}^{*}$ & 0.82 & 0.85 & 1.05 & 1.48 & 1.00 & 0.48 & 0.88 & 1.18 & 1.89 \\
\hline \hline
\end{tabular}

coloured inside of each dyepack indicates the presence of more gold nanoparticles. This is understandable given that the uptake began on the in-out flow direction for the first $3 \mathrm{~min}$, after which time more than half of the nanoparticles in the dye solutions had been absorbed by the TU yarn. When the flow direction is from inout, the local concentration of TA-Au NPs in the dye solution will be lower as the dye liquor migrates from the inside to the middle and then outside of the dyepack.

The above experiments showed that increasing the amount of Albaflow FFA in the synthesis from 1 to $4 \mathrm{~g} \mathrm{~L}^{-1}$ increases the colour variation throughout the dyepack. As such, $0.5-1 \mathrm{~g} \mathrm{~L}^{-1}$ of Albaflow FFA was employed in future package dyeing reactions. Next, the flow rate and direction were manipulated to attempt to increase the colour uniformity of the TA-mauve composites.

\section{Flow Rate and Direction}

The rate of dye circulation in the package dyeing reactor is controlled by the outlet tap. The pump runs at one speed, and as such the flow rate is dictated by the outlet tap. If the tap is fully open, the flowrate is maximised and the pressure inside the dyeing tank is minimised. Alternately, when the outlet tap is almost fully closed, the pressure inside the dyeing tank builds as the dye liquor is forced throughout the dyepack to a greater extent. As such, there is a trade-off between flow rate and pressure.

In the prior experiments, the flow rate was set at a "medium" level which was recommended by the dyeing. Additionally, the flow direction was set to a 3:2 min ratio of in-out to out-in, and after the initial $3 \mathrm{~min}$ of in-out flow the uptake was at least half complete. In the following experiments, the rate and direction of the flow is manipulated in synthesis of TA-mauve TU yarn composites. The reaction conditions that were employed in the package dyeing experiments are summarised in Table 5.7. 
Table 5.7 Summary of the reaction conditions that were manipulated to attempt to increase the colour uniformity of TA-mauve TU yarn composites in the package dyeing reactor.

\begin{tabular}{ccccc}
\hline Experiment & 1 & 2 & 3 & 4 \\
\hline Mass of TU yarn (g) & 250 & 250 & 250 & 500 \\
Albaflow FFA $\left(\mathrm{g} \mathrm{L}^{-1}\right)$ & 1 & 1 & 0.5 & 1 \\
Flow rate & fast & medium & fast & medium \\
Flow direction & in-out & out-in & out-in & out-in \\
\hline \hline
\end{tabular}

The uptake of each of the above TA-mauve syntheses was very rapid, with each of the dyeing reactions complete in approximately $10 \mathrm{~min}$. The resulting levelness tests for the composites are presented in Figure 5.26. Experiment 1 resulted in a much lighter outside of the dyepack, as far more TA-Au NPs were absorbed by the yarn located in the inside and middle of the dyepack. Experiment 3 exhibited relatively lower colour variation throughout the dyepack, which showed that the out-in flow increased the colour uniformity of the composites. The conditions of Experiment 2 resulted in the most uniform coloured composites, indicating that out-in flow and a medium flowrate result in the most uniformly coloured composites.

The success of Experiment 2 led to the synthesis of a $500 \mathrm{~g}$ TA-mauve TU yarn composite utilising the same reaction conditions (Experiment 4). The resulting composite was approximately as uniform in colour as the $250 \mathrm{~g}$ composite, indicating that the reaction is scalable. This was a very positive result, and future dyeing reactions were to involve the synthesis of $1 \mathrm{~kg}$ and then $2 \mathrm{~kg}$ TA-mauve TU yarn composites using these reactions conditions.

The colouring of Ascend felted yarn with the TA-mauve colloid was also attempted with the package dyeing reactor. However, the resulting felted yarn composite was very unevenly coloured and exhibited flattened and damaged areas of the yarn. It was considered that the relatively thick felted yarn was unable to be wound tightly enough to prevent its movement during the dyeing reaction. The dye solution may have channeled through the areas between the yarn which would have less resistance than moving through the felted in the dyepack. Thus, felted yarn was deemed to be unsuitable for use in package dyeing reactions. Next, TSC-grey syntheses were attempted in the package dyeing reactor. 

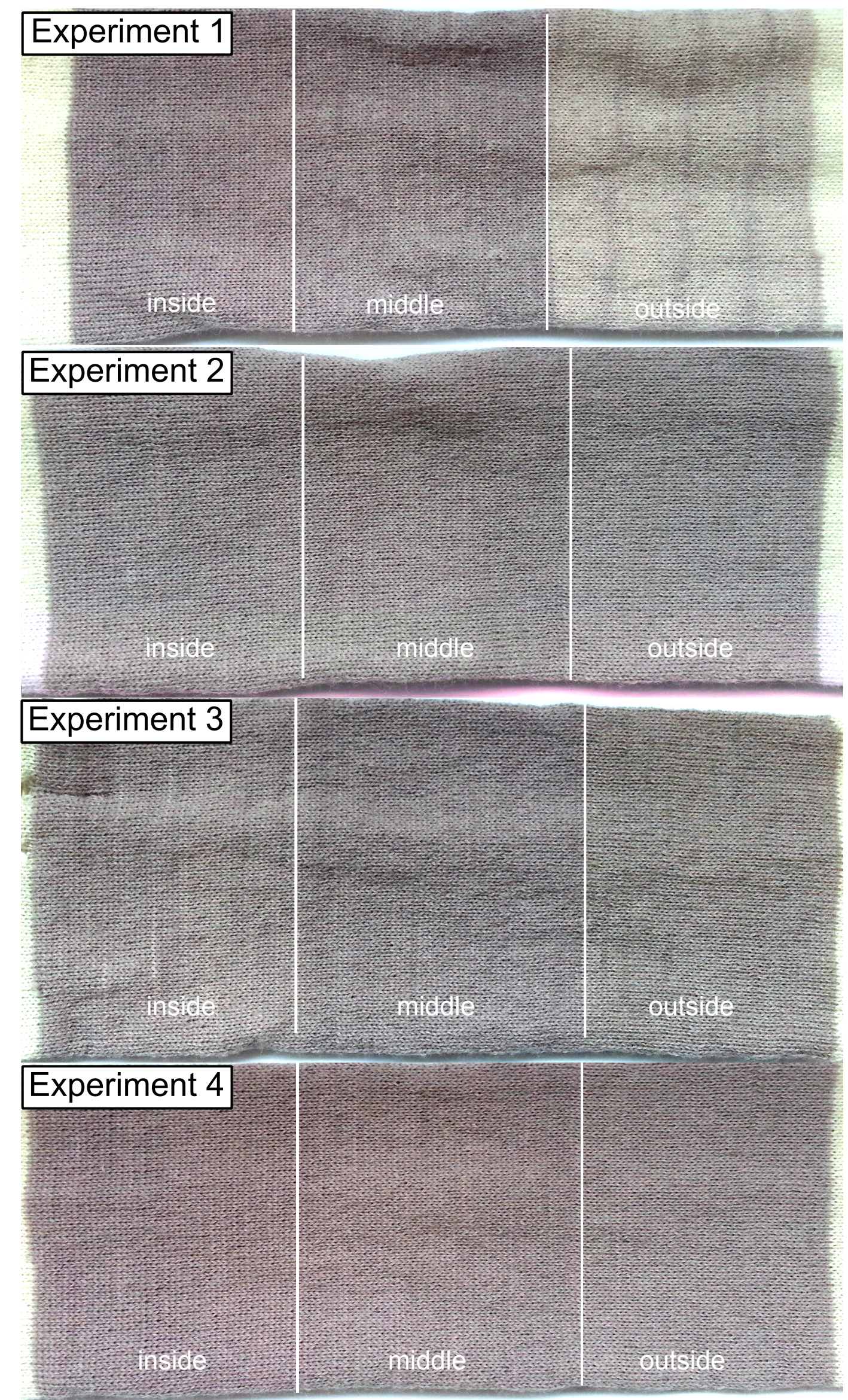

Figure 5.26 Levelness tests of TA-mauve TU yarn composites resulting from Experiments $1,2,3$ and 4 in the package dyeing reactor. 


\subsubsection{TSC-grey TU Yarn Composites}

The first TSC-grey synthesis involved the dyeing of a $250 \mathrm{~g}$ TU yarn in the package dyeing reactor. The equivalent concentration of the typical TSC-grey colloid was used. The glycine buffer was not employed, and as such $2 \mathrm{~g} \mathrm{~L}^{-1}$ Albaflow FFA was added to the colloid (the equivalent of $1 \mathrm{~g} \mathrm{~L}^{-1}$ in the buffer and colloid in the TAmauve synthesis). The TU yarn was washed with hot tap water, drained and then the TSC-grey colloid (at pH 2.0) was circulated around the system. The dyeing reaction involved the in-out flow direction only. An image of the TSC-grey TU yarn composite just removed from the package dyeing reactor is shown in Figure 5.27.

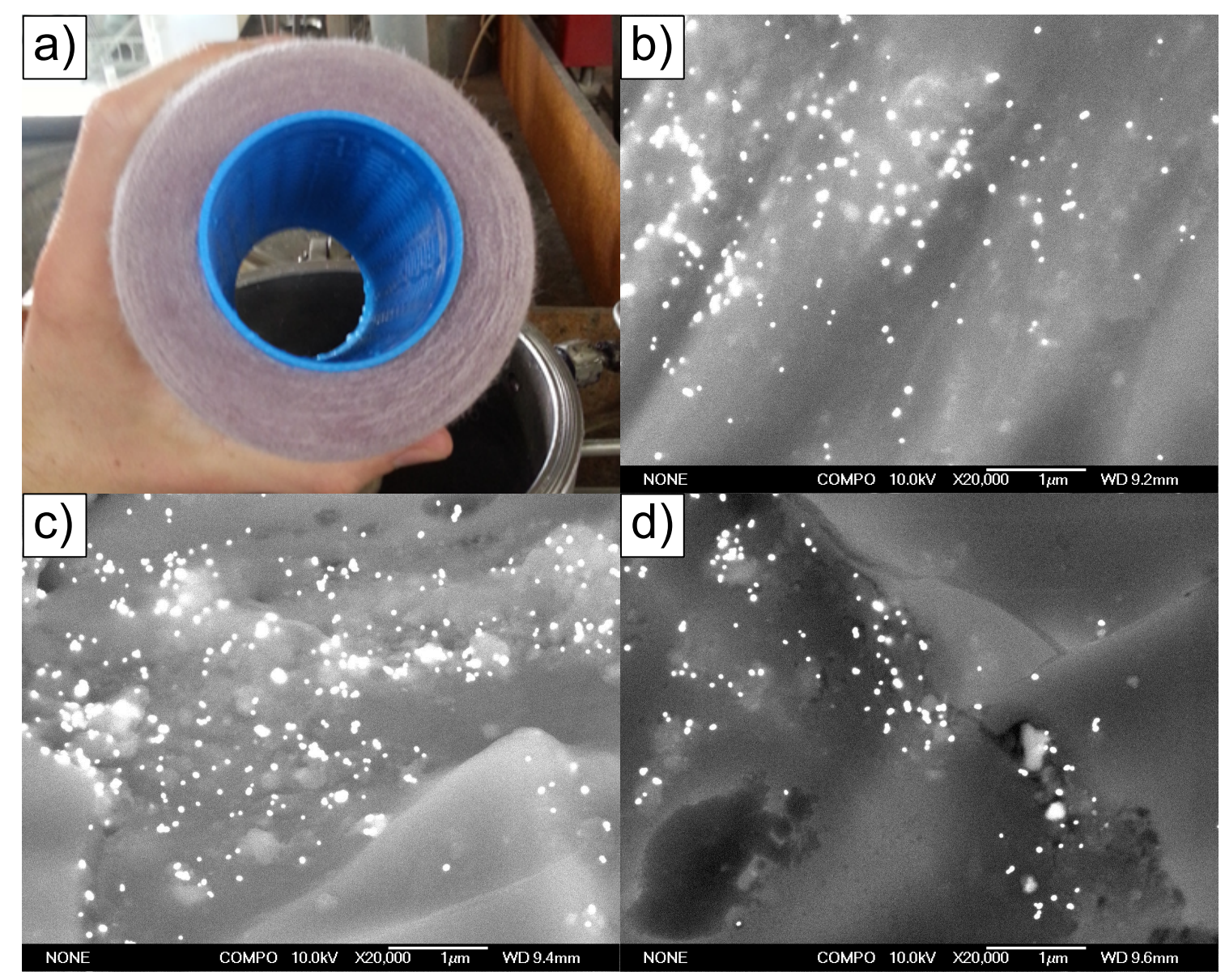

Figure 5.27 A TU yarn TSC-grey composite synthesised in the package dyeing reactor (a) and SEM micrographs in fibres from the inside (b), middle (c) and outside (d) of the dyepack.

Interestingly, the resulting composite was purple coloured, indicating little agglomeration of the gold nanoparticles on the TU yarn. The pressure and flow of the package dyeing reactor was originally believed to promote the agglomeration of TSC-Au NPs. However, the flow and pressure have seemed to have distributed the TSC-Au NPs in the dye solution more homogeneously throughout the dyepack than which is typical in static dyeing reactions. Thus, the gold nanoparticles are likely 
located too far from one another for there SPR bands to couple and give the desired grey colouration of the composites.

Figure 5.27 also presents SEM micrographs of the surfaces of composite fibres that were located in different areas of the dyepack. The micrographs reveal a relatively higher proportion of individual gold nanoparticles compared to agglomerates of TSC$\mathrm{Au}$ NPs on the surface of the composite fibres than is typical for this method. This indicates that the package dyeing conditions result in more homogeneous distribution of TSC-Au NPs throughout the dyepack, giving the relatively purple colour of the composite.

The TSC-grey synthesis was repeated in the package dyeing reactor without the use of Albaflow FFA, to attempt to promote the agglomeration of the TSC-Au NPs in the colloid. This was expected to result in grey-coloured composites. The resulting TSC-grey composite and a levelness test of different areas of the dyepack are shown in Figure 5.28. The composite is grey coloured, however the colour is also very uneven. The uptake was complete in less than $2 \mathrm{~min}$ and resulted in the most intense colour gradient throughout the dyepack of all of the experiments so far.

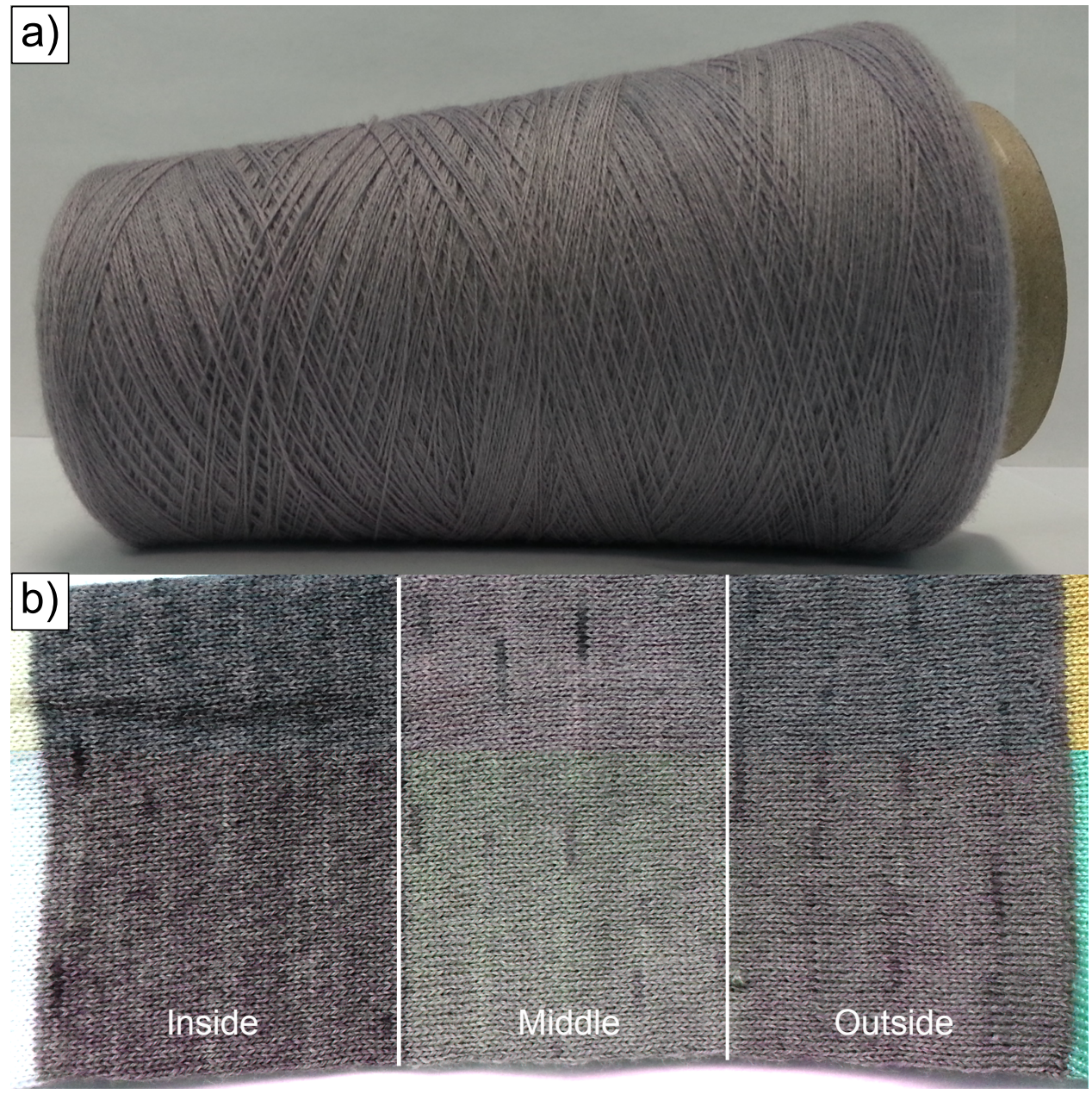

Figure 5.28 A TU yarn TSC-grey composite synthesised in the package dyeing reactor (a) and a levelness sock of the dyepack (b). 
This experiment has indicated that using Albaflow FFA in the TSC-grey colloid is integral to attaining a uniform colour of the composites in the package dyeing reactor. If possible, future experiments would have incorporated less Albaflow FFA $\left(0.5-1 \mathrm{~g} \mathrm{~L}^{-1}\right)$ to attempt to form a uniform grey composite.

In summary, this section investigated and discussed the potential of using conventional package dyeing reactors to synthesise ex-situ gold nanoparticle-wool fibre composites. The colour uniformity of TA-mauve TU yarn composites was improved by manipulating the amount of Albaflow FFA and the flow rate and direction of the dye liquor. The TSC-grey method was relatively difficult to integrate into the package dyeing process, and resulted in non-uniform purple-grey coloured composites. Unfortunately, Tararua Yarns Ltd closed down for business at this point of the research project, and as such the process could not be optimised further. Future experiments would have included increasing the scale of the syntheses of TA-mauve composites (up to $2 \mathrm{~kg}$ ) and attempting to attain uniformly coloured TSC-grey composites.

This work yielded positive results for successfully integrating the ex-situ synthesis (particularly the TA-mauve method) into conventional packages dyeing procedures. The results led to the development of a suite of novel flow reactors that were based upon similar dyeing principles. Additionally, the significant differences between the uptake behaviour of the package dyeing reactions (compared to those of static dyeing reactions) observed in this research facilitated further studies of the uptake chemistry of the ex-situ procedure. Next, a range of novel flow reactors were designed and used in the ex-situ synthesis of gold nanoparticle-wool fibre composites.

\subsection{Novel Hybrid Flow Reactors for Wool Dyeing in the Laboratory}

This section details the development of a suite of novel flow reactors that were used in the synthesis of ex-situ composites of different wool types in the laboratory. The reactors utilise the package dyeing principle of circulating dye liquor around a tightly packed quantity of wool, and employ vastly different flowrates, volumes and masses of wool. A summary of the relevant parameters of the different flow reactors is detailed in Table 5.8.

The Benchtop flow reactor was developed to study the uptake of gold colloids to TU yarn with different auxiliary agents, to elucidate uptake differences between static and flow dyeing reactions. The first flow reactor involved the dyeing reactions 
Table 5.8 The reaction conditions and function of the suite of novel flow reactors for the ex-situ synthesis of gold nanoparticle-wool fibre composites.

\begin{tabular}{|c|c|c|c|}
\hline Reactor(s) & Benchtop flow & First flow & Pressurised flow \\
\hline Function & Uptake behaviour & $\begin{array}{c}\text { Prototype } \\
\text { development }\end{array}$ & $\begin{array}{l}\text { Scale-up } \\
\text { syntheses }\end{array}$ \\
\hline Mass of wool (g) & 5 & 50 & $300-660$ \\
\hline Dye volumes (L) & 0.3 & 12 & $6-8$ \\
\hline Flowrate $\left(\mathrm{L} \mathrm{min}^{-1}\right)$ & 0.34 & 8 & 30 \\
\hline Wool type & Fine yarn & $\begin{array}{l}\text { Top form wool } \\
\& \text { fine yarn }\end{array}$ & $\begin{array}{c}\text { Top form, felted } \\
\text { \& fine yarns }\end{array}$ \\
\hline
\end{tabular}

with relatively gentle flowrates and pressure employed an open system. Finally, pressurised flow reactors in a closed system were designed to allow for rapid flowrates and higher pressure to synthesise composites with enhanced colour uniformity.

\subsubsection{Benchtop Flow Reactor}

The Benchtop flow reactor was designed to better simulate the reaction conditions of the package dyeing of merino yarns in the laboratory. The reactor was developed to better understand the uptake chemistry occurring in these procedures, which was vastly different from that observed in static reactors (the laboratory-scale chemistry of which was detailed in Section 4.3.3). The research was carried out con-currently with the package dyeing reactions at Tararua Yarns Ltd.

The Benchtop flow reactor is shown in Figure 5.29. The set-up involves $5 \mathrm{~g}$ quantities of TU yarn that were held inside a piece of tubing that protrudes from the pump outlet. The dye solution is circulated through the tubing into the beaker, which acts as an overflow tank. The flowrate is approximately $342 \mathrm{~mL} \mathrm{~min}^{-1}$ with this set-up, which equates to a residence time of $53 \mathrm{~s}$ for the circulation of a $300 \mathrm{~mL}$ gold colloid (which is used to colour $5 \mathrm{~g}$ of yarn).

The flowrates employed are relatively slow compared to those of the package dyeing reactor, and the pressure cannot be increased (as it is an open system). As such, the rate of uptake will likely be slower than those observed in package dyeing reactions or scale-up reactions in the pressurised flow reactors. However, the Benchtop flow reactor did provide a more accurate representation uptake chemistry of these reactors compared to the static experiments (that are detailed in Section 4.3.3).

A study of the uptake of TA-Au NPs to TU yarn with different amounts of Albaflow FFA in the buffer and colloid is presented in Figure 5.30. The uptake curves show 


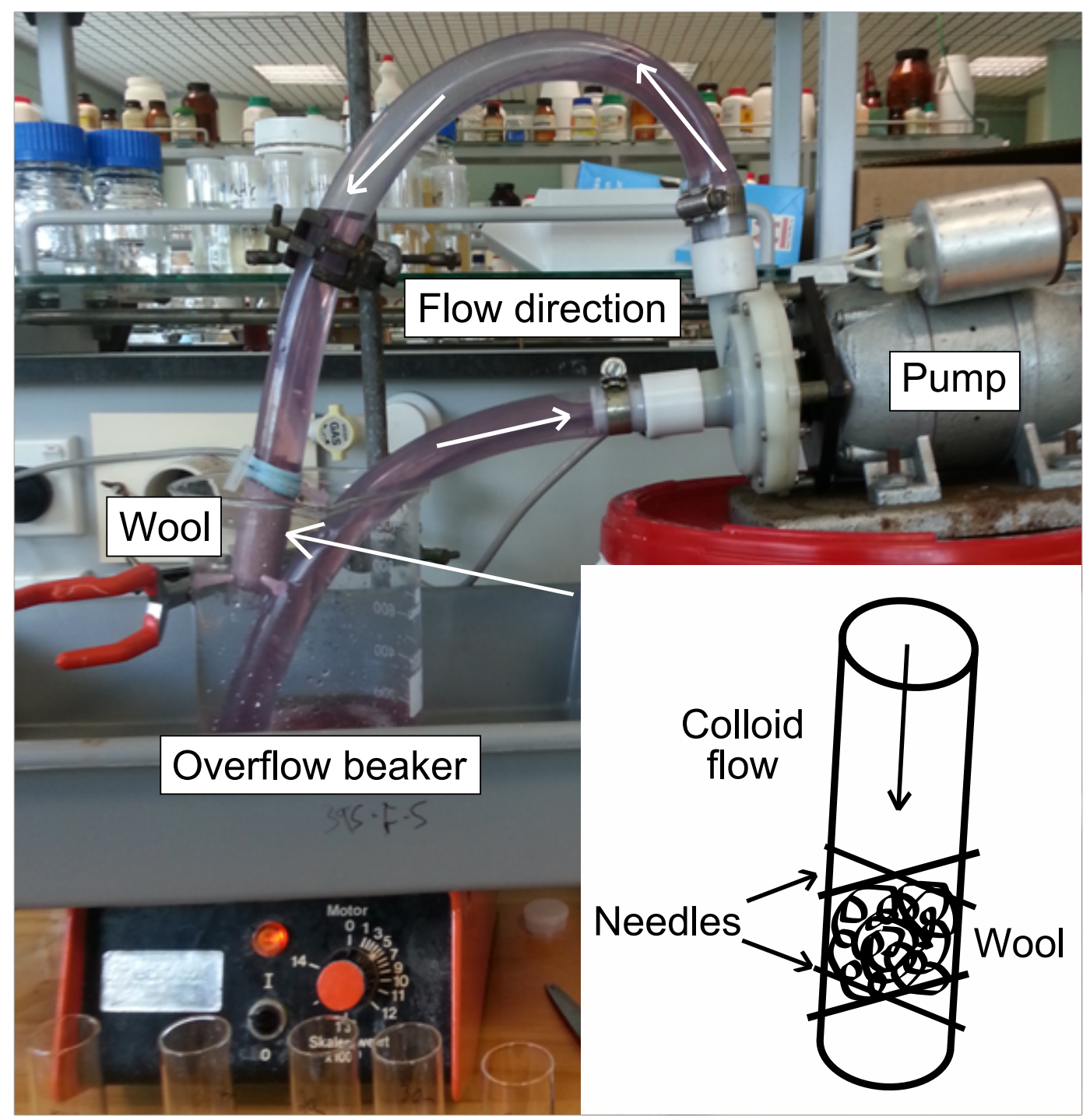

Figure 5.29 The Benchtop flow reactor that was used to study the uptake of gold nanoparticles to TU yarn, where the colloid flows through wool that is compressed between needles in PVC tubing, into the overflow beaker and back up through the pump. The inset is a diagram of the wool in the tubing.

the decrease in the intensity of the SPR peak of TA-Au NPs $(\lambda=525 \mathrm{~nm})$ in the dye solution over time. The standard TA-mauve reaction conditions were employed in the uptake reactions (1:60 wool to liquor ratio, $0.1 \mathrm{~mol} \mathrm{~L}^{-1}$ glycine buffer, $\mathrm{pH} 2.2$ buffer and colloid).

Similar to that which was seen in Section 4.3.3, the presence of Albaflow FFA significantly slowed the uptake of TA-Au NPs to wool. The uptake in the Benchtop flow reactor was substantially faster than the uptake of TA-Au NPs in static reactors (Figure 4.33). When no Albaflow FFA was used in the Benchtop flow reactor, the uptake of the TA-mauve colloid was complete in $5 \mathrm{~min}$, and more than half of the uptake occurred in the first $30 \mathrm{~s}$ of the reaction. When the amount of Albaflow FFA was increased from $0-2 \mathrm{~g} \mathrm{~L}^{-1}$, there was a significant decrease in the rate of the uptake. However, when $4 \mathrm{~g} \mathrm{~L}^{-1}$ of Albaflow FFA was employed, the uptake was more 


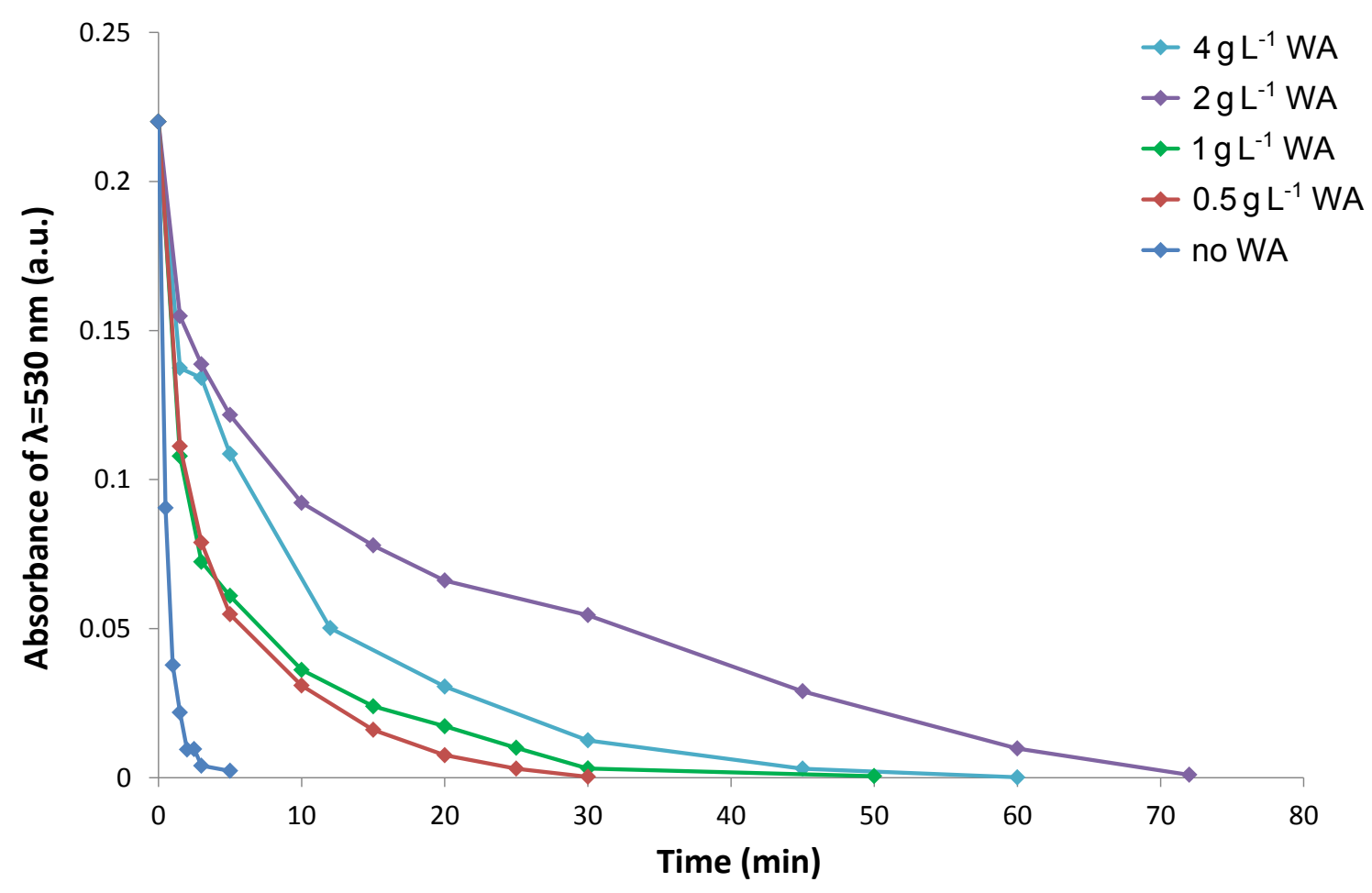

Figure 5.30 Uptake of TA-mauve colloid to TU in the Benchtop flow reactor with different amounts of Albaflow FFA (WA) in the buffer and colloid.

rapid than when $2 \mathrm{gL}^{-1}$ is used. This was due to the wetting agent coagulating and foaming under the applied flow, and destabilising the TA-mauve colloid, from which point it coated the tubing and the overflow beaker. This behaviour was also observed in package dyeing reactions in Section 5.3.1.

An image of the composites that resulted at the end of the uptake reactions are presented in Figure 5.31. The composites are aligned as they were in the tubing of the Benchtop flow reactor. There is a significant colour gradient through the composite synthesised without Albaflow FFA, showing preferential uptake of TA$\mathrm{Au}$ NPs at the top of the composite. The top is first in the flow direction of the colloid, and the concentration of the TA-mauve colloid should decrease as it passes through the TU yarn sample.

The colour gradient through the TA-mauve composites appears to decrease as the amount of Albaflow FFA in the synthesis is increased from $0-2 \mathrm{~g} \mathrm{~L}^{-1}$. The CIE colour coordinates of measured at the top, middle and bottom of the 0,1 and $2 \mathrm{gL}^{-1}$ Albaflow FFA TA-mauve TU yarn composites are presented in Table 5.9. The variance between the $\mathrm{L}^{*}, \mathrm{a}^{*}$ and $\mathrm{b}^{*}$ throughout the different areas of the composites decreases as the Albaflow FFA amount is increased from $0-2 \mathrm{~g} \mathrm{~L}^{-1}$. As such, slowing the rate of uptake of TA-Au NPs to TU yarn has increased the colour uniformity of the composites. A further increase in the amount of Albaflow FFA to $4 \mathrm{~g} \mathrm{~L}^{-1}$ resulted in the destabilisation of the TA-mauve colloid and an unevenly coloured 


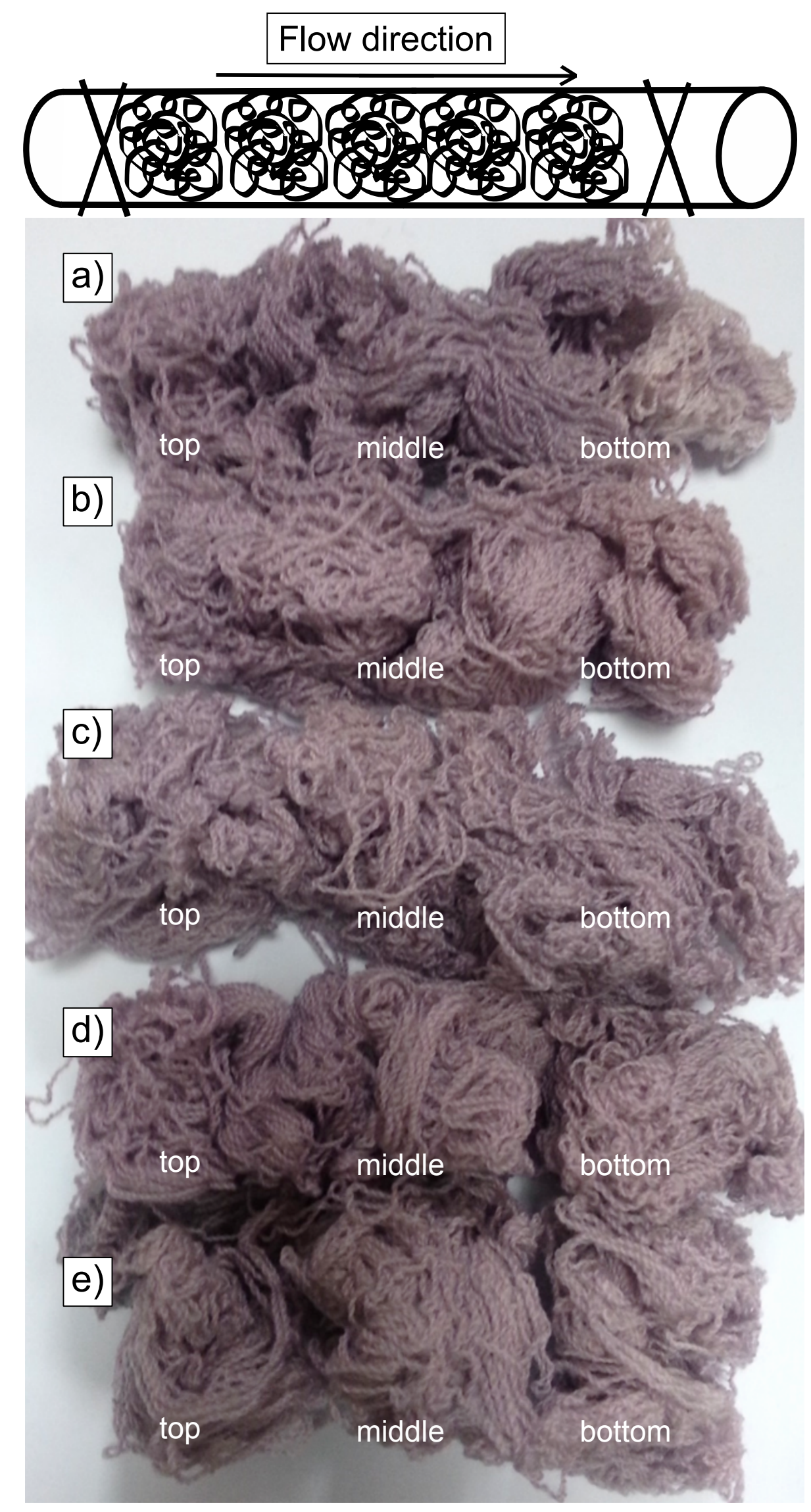

Figure 5.31 TA-mauve TU yarn composites synthesised in the Benchtop flow reactor without Albaflow FFA (a), and with 0.5, 1, 2, and $4 \mathrm{~g} \mathrm{~L}^{-1}$ (b)-(e) Albaflow FFA in the buffer and colloid, and the composites are aligned as they were in the Benchtop flow reactor. 
composite. The $\mathrm{L}^{*}$ values of the 1 and $2 \mathrm{~g} \mathrm{~L}^{-1}$ composites are lower at the top of the composite, which indicates that the wool has absorbed a relatively higher amount of TA-Au NPs at these areas.

Table 5.9 CIE colour coordinates of different areas of the $5 \mathrm{~g}$ TA-mauve TU yarn composites synthesised in the Benchtop flow reactor.

\begin{tabular}{cccccccccc}
\hline WA & \multicolumn{3}{c}{ no WA } & \multicolumn{3}{c}{$1 \mathrm{~g} \mathrm{~L}^{-1}$} & \multicolumn{3}{c}{$2 \mathrm{~g} \mathrm{~L}^{-1}$} \\
\hline Area & Top & Mid & Bottom & Top & Mid & Bottom & Top & Mid & Bottom \\
\hline $\mathrm{L}^{*}$ & 60.82 & 58.33 & 65.09 & 54.48 & 57.29 & 59.64 & 56.55 & 58.75 & 58.16 \\
$\mathrm{a}^{*}$ & 4.85 & 7.71 & 5.78 & 6.33 & 7.22 & 7.45 & 7.95 & 7.81 & 7.40 \\
$\mathrm{~b}^{*}$ & -1.63 & -2.00 & -1.28 & -2.74 & -1.91 & -0.97 & -1.57 & -1.67 & -1.03 \\
\hline \hline
\end{tabular}

This experiment has shown both the remarkable effect that dye circulation has on the rate of uptake of gold nanoparticles to wool. This led to the use of levelling agents in the dyeing of TU yarn in the Benchtop flow reactor. TA-mauve synthesis. Croscolor DH2 and Tecarapid CBF were both utilised in the following study, as these two levelling agents showed the most promise in the static reactors (the chemistry of which was detailed in Section 4.3.3. The standard TA-mauve method was used without the presence of Albaflow FFA unless it is noted otherwise.

The uptake of TA-Au NPs to TU yarn with different amounts of Croscolor DH2 is shown in Figure 5.32 below. The presence of Croscolor DH2 in the synthesis has slowed the of TA-Au NPs to the TU yarn. When $0.33 \mathrm{~g} \mathrm{~L}^{-1}$ Croscolor DH2 was added to the buffer, the uptake is slightly slower than when it is not used and is complete in approximately $15 \mathrm{~min}$. This implies that that the TU yarn retains some of the Croscolor DH2 that was present in the buffer solution. Incorporating $0.083 \mathrm{~g} \mathrm{~L}^{-1}$ Croscolor DH2 into the colloid has more significantly influenced the uptake, with the uptake incomplete after $120 \mathrm{~min}$. The addition of $0.33 \mathrm{gL}^{-1}$ Croscolor DH2 to the colloid has caused a significant change in the shape of the uptake curve, compared to the $0.083 \mathrm{~g} \mathrm{~L}^{-1}$ uptake. There appears to be a rapid saturation of the TU yarn with the Croscolor DH2 in the TA-mauve colloid at the beginning of the uptake. This is followed by a period of relative inactivity from 5-45 min, after which the uptake is approximately linear from 45-110 min.

The TA-mauve TU yarn composites that were synthesised in the Benchtop flow reactor with different amounts of Croscolor DH2 are shown in Figure 5.33. The composite synthesised with $0.033 \mathrm{~g} \mathrm{~L}^{-1}$ in the buffer is unevenly mauve coloured, as this amount of Croscolor DH2 has not significantly enhanced the colour uniformity of the composite. Unfortunately, incorporating Croscolor DH2 into the TA-mauve colloid has resulted in increasingly grey coloured composites. The $0.33 \mathrm{~g} \mathrm{~L}^{-1}$ colloid composite is grey-brown coloured, indicating that the Croscolor DH2 has caused 


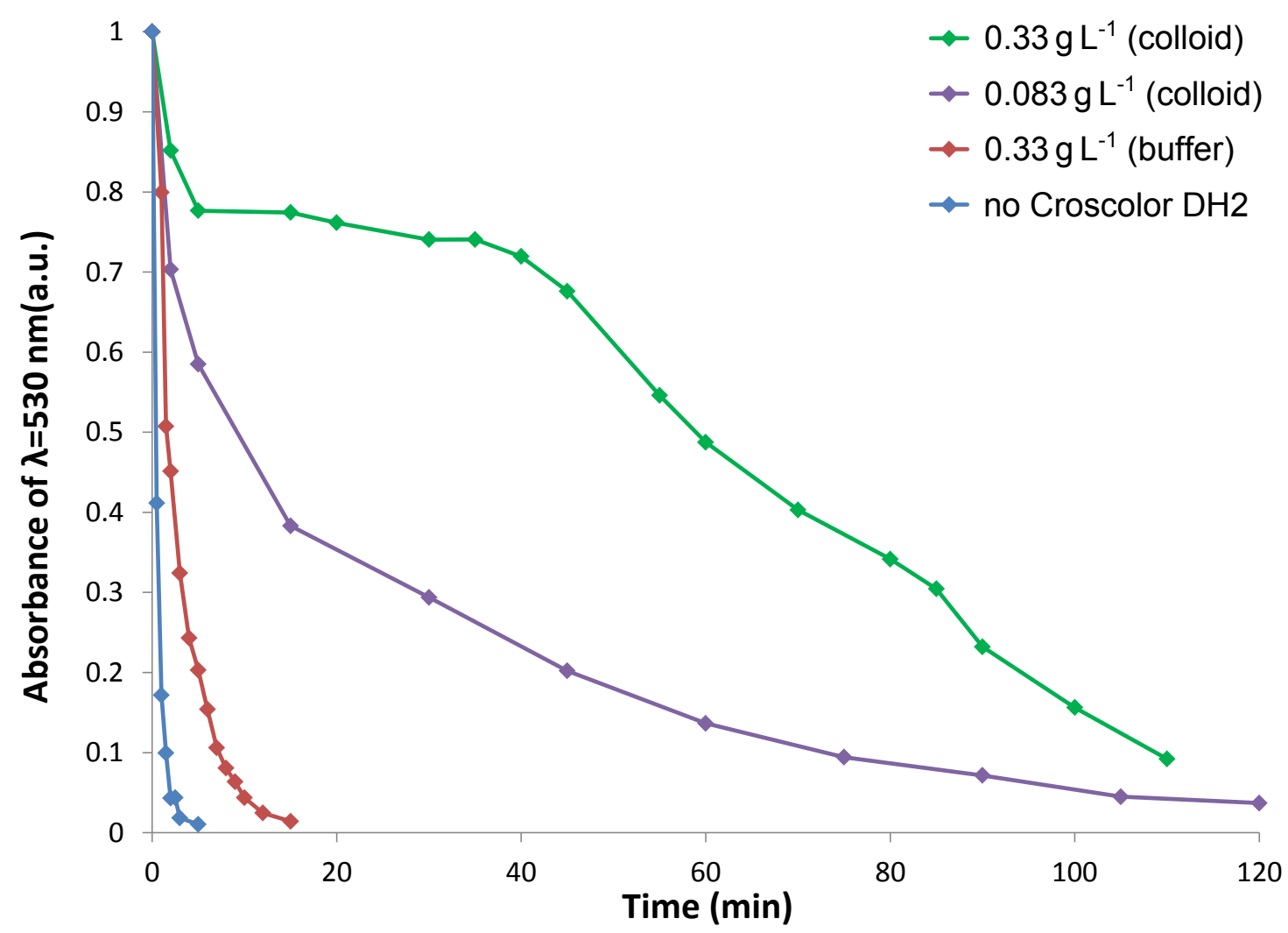

Figure 5.32 Uptake of TA-mauve colloid to TU with different amounts of Croscolor DH2 in the Benchtop flow reactor.

the agglomeration of TA-Au NPs and damage to the wool fibres in the TU yarn. The destabilisation of the TA-mauve colloid occurred approximately $45 \mathrm{~min}$ into the uptake, which coincided with the beginning of the linear area of the uptake curve seen in Figure 5.32.

The CIE colour coordinates of the different areas of the Croscolor DH2 composites are shown in Table 5.9. Increasing the amount of Croscolor DH2 in the dyeing reactions has generally caused more level colours of the composites. However, increasing amounts of Croscolor DH2 also results in lower $a^{*}$ values and higher $b^{*}$ values of the composites. The grey-brown colour of the $0.33 \mathrm{~g} \mathrm{~L}^{-1}$ Croscolor DH2 composite is evidenced by its high $b^{*}$ value.

Table 5.10 CIE colour coordinates of TA-mauve TU yarn composites that were synthesised in the Benchtop flow reactor with different amounts of Croscolor DH2.

\begin{tabular}{cccccccccc}
\hline Cros & \multicolumn{3}{c}{$0.33 \mathrm{~g} \mathrm{~L}^{-1}$ in buffer } & \multicolumn{3}{c}{$0.083 \mathrm{~g} \mathrm{~L}^{-1}$ in colloid } & \multicolumn{3}{c}{$0.33 \mathrm{~g} \mathrm{~L}^{-1}$ in colloid } \\
\hline Area & Top & Mid & Bottom & Top & Mid & Bottom & Top & Mid & Bottom \\
\hline $\mathrm{L}^{*}$ & 55.26 & 57.48 & 71.56 & 56.72 & 57.02 & 58.91 & 65.74 & 65.10 & 67.54 \\
$\mathrm{a}^{*}$ & 5.13 & 3.63 & 1.73 & 2.47 & 2.41 & 2.12 & 0.79 & 0.54 & 0.78 \\
$\mathrm{~b}^{*}$ & -2.20 & -0.95 & 2.94 & -0.41 & -1.02 & -0.83 & 3.51 & 3.67 & 4.07 \\
\hline \hline
\end{tabular}




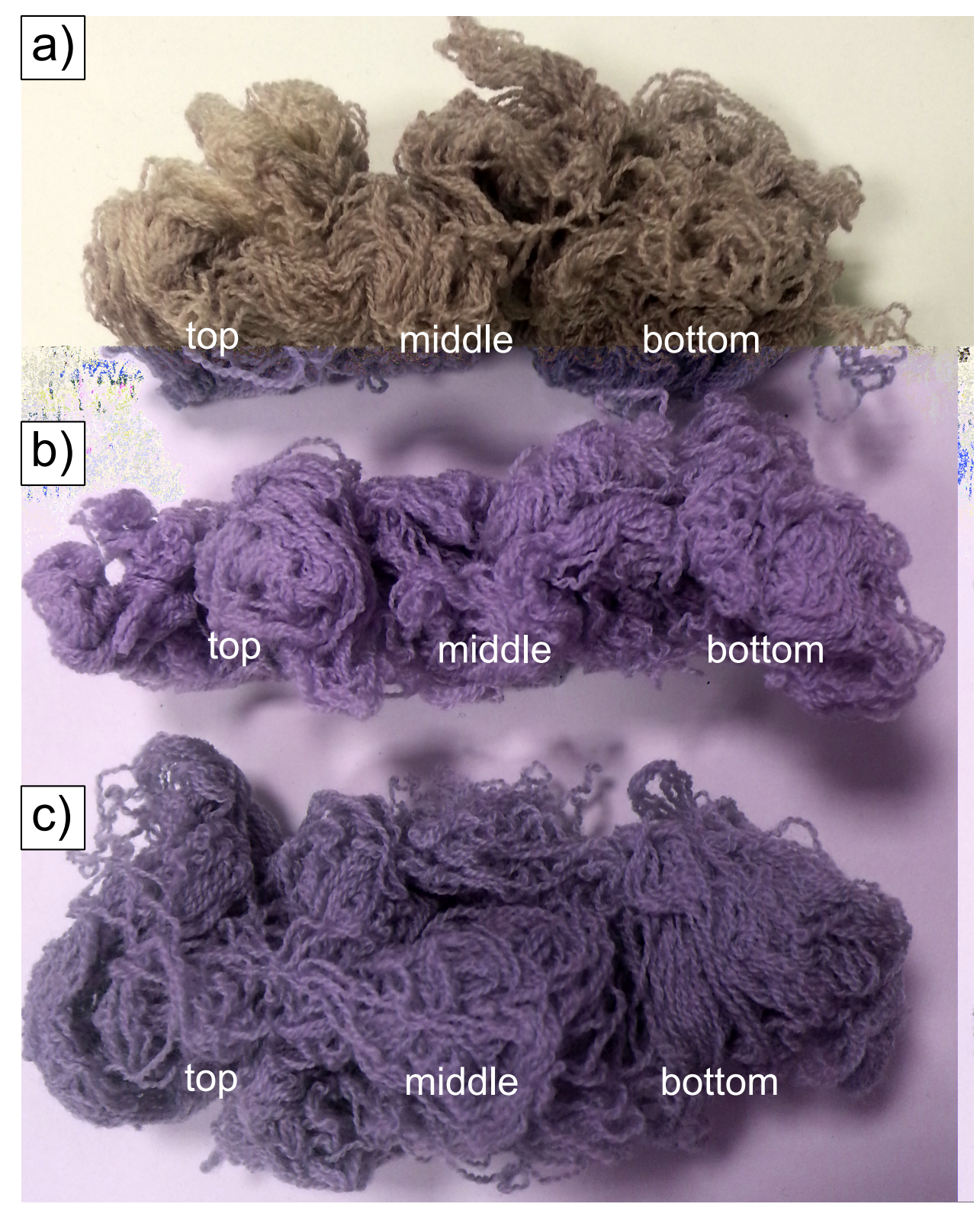

Figure 5.33 TA-mauve TU yarn composites synthesised in the Benchtop flow reactor with different amounts of Croscolor DH2 in the synthesis: $0.33 \mathrm{~g} \mathrm{~L}^{-1}$ in the buffer (a), $0.33 \mathrm{~g} \mathrm{~L}^{-1}$ in the colloid (b) and $0.083 \mathrm{~g} \mathrm{~L}^{-1}$ in the colloid (c).

These results show that Croscolor DH2 is not suitable for controlling the uptake in the TA-mauve synthesis. The amount of Croscolor DH2 required to significantly slow the uptake of TA-Au NPs resulted in the discolouration of the resulting composites. Next, Tecarapid CBF was used in the synthesis of TA-mauve TU yarn composites in the Benchtop flow reactor.

The uptake of the TA-mauve colloid to TU yarn in the Benchtop flow reactor with different amounts of Tecarapid CBF is presented in Figure 5.34. Incorporating increasing amounts of Tecarapid CBF in the TA-mauve colloid (from 0-2 $\mathrm{g} \mathrm{L}^{-1}$ ) has both slowed the uptake time and resulted in a more linear uptake curve. The 0.5 and $1 \mathrm{gL}^{-1}$ Tecarapid CBF uptake curves exhibit areas where the rate of uptake 
increases as the uptake time increases. This is an interesting occurrence, which is more pronounced in the $1 \mathrm{gL}^{-1}$ Tecarapid CBF uptake curve. It may demonstrate the degradation of the Tecarapid in the colloid after a certain point, after which the uptake of TA-Au NPs to the TU yarn becomes increasingly unimpeded.

The $2 \mathrm{~g} \mathrm{~L}^{-1}$ Tecarapid CBF uptake exhibits a period of relatively rapid absorption for the first $30 \mathrm{~min}$ of the uptake. This is followed by the gradual and approximately linear uptake, where the uptake is less than half complete after $240 \mathrm{~min}$. The initial 30 min of the uptake may represent the initial saturation of the TU yarn with the Tecarapid CBF and TA-Au NPs in the dye solution, where approximately 15-30\% of the colloid is absorbed. Following this there is the uptake may involve the gradual absorption of TA-Au NPs, which is slowed by the Tecarapid that has adsorbed onto the surface of the TU yarn.

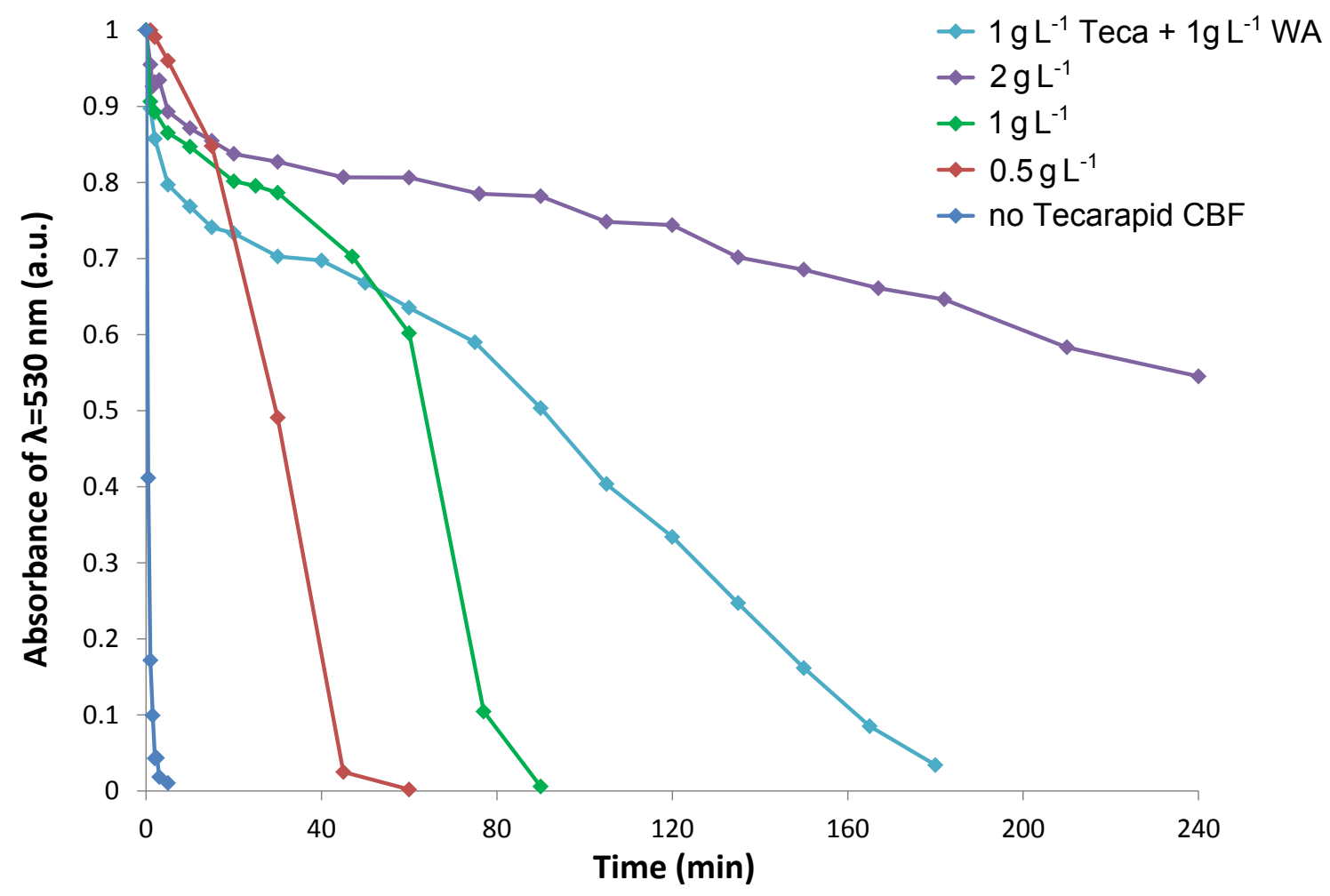

Figure 5.34 Uptake of TA-mauve colloid to TU with different amounts of Tecarapid CBF in the colloid.

A combination of Tecarapid CBF and Albaflow FFA was also employed in the Benchtop flow reactor, the uptake curve of which is also presented in Figure 5.34. The uptake of the two auxiliary agents involves an initial saturation period, followed by the gradual uptake from 30-90 min and finally a more rapid and approximately linear uptake region from 90-240 min. This may represent the exhaustion (or destruction) of the Tecarapid after $90 \mathrm{~min}$ of the uptake, from which point the uptake is considered to be controlled by the Albaflow FFA remaining in the dye solution. 
The TA-mauve composites synthesised in the Benchtop flow reactor with different amounts of Tecarapid CBF in the colloid are presented in Figure 5.35. Increasing the amount of Tecarapid CBF in the uptake solution appears to have resulted in increasingly lighter mauve coloured composites. The composite synthesised with $1 \mathrm{gL}^{-1}$ Tecarapid CBF and Albaflow FFA, but is a similar mauve colour to that which typically results from the TA-mauve method.

The CIE colour coordinates of the composites that were synthesised with $0.5-2 \mathrm{~g} \mathrm{~L}^{-1}$ Tecarapid CBF in the uptake solutions are shown in Table 5.11. The use of 0.5 or $1 \mathrm{gL}^{-1}$ Tecarapid in the TA-mauve colloid has resulted in composites with only slight differences in $\mathrm{L}^{*}, \mathrm{a}^{*}$ and $\mathrm{b}^{*}$ values at the different areas of the dyepack. This indicates that the composites are very uniform in colour. The $2 \mathrm{gL}^{-1}$ Tecarapid CBF composite is also evenly coloured, but exhibits significantly higher $\mathrm{L}^{*}$ and $\mathrm{b}^{*}$ values. This indicates that $2 \mathrm{gL}^{-1}$ of Tecarapid CBF is too high an amount for the TA-mauve method. The combination of up to $1 \mathrm{gL}^{-1}$ Tecarapid CBF and Albaflow FFA represents the most promising combination of auxiliary agents for use in scale-up dyeing procedures that utilise dye circulation.

Table 5.11 CIE colour coordinates of $5 \mathrm{~g}$ TA-mauve TU yarn composites that were synthesised in the Benchtop flow reactor with different amounts of Tecarapid $\mathrm{CBF}$.

\begin{tabular}{cccccccccc}
\hline Teca & \multicolumn{3}{c}{$0.5 \mathrm{~g} \mathrm{~L}^{-1}$} & \multicolumn{3}{c}{$1 \mathrm{~g} \mathrm{~L}^{-1}$} & \multicolumn{3}{c}{$2 \mathrm{~g} \mathrm{~L}^{-1}$} \\
\hline Area & Top & Mid & Bottom & Top & Mid & Bottom & Top & Mid & Bottom \\
\hline $\mathrm{L}^{*}$ & 56.98 & 56.50 & 55.41 & 56.57 & 56.30 & 57.54 & 64.66 & 63.10 & 65.25 \\
$\mathrm{a}^{*}$ & 8.78 & 8.52 & 7.83 & 5.83 & 6.84 & 6.55 & 6.76 & 6.65 & 6.83 \\
$\mathrm{~b}^{*}$ & -1.49 & -1.67 & -2.12 & -2.65 & -1.67 & -1.63 & 1.30 & 1.94 & 1.54 \\
\hline \hline
\end{tabular}

Due to the propensity for Tecarapid CBF to slow the uptake of TA-Au NPs to TU yarn, it was also employed in the synthesis of TSC-grey TU yarn composites in the Benchtop flow reactor. Again, the agglomeration of TSC-Au NPs that occurs during the uptake reaction makes it difficult to characterise the uptake chemistry. The resulting TSC-grey composites synthesised in the Benchtop flow reactor with Tecarapid CBF (and in some cases Albaflow FFA) are shown in Figure 5.36.

The significant colour variation across the TSC-grey composite is evident when no auxiliary agents are employed, where the bottom of the $5 \mathrm{~g}$ dyepack is only slightly dyed. The use of Tecarapid CBF has resulted in more uniformly coloured composites, but the colours are also relatively purple. CIE colour coordinates of TSC-grey TU yarn composites synthesised with and without Tecarapid CBF are shown in Table 5.12. The use Tecarapid CBF has reduced the variation in the $\mathrm{L}^{*}$, 




Figure 5.35 TA-mauve TU yarn composites synthesised in the Benchtop flow reactor with $0.5 \mathrm{gL}^{-1}$ (a), $1 \mathrm{gL}^{-1}$ (b) and $2 \mathrm{gL}^{-1}$ (c) Tecarapid $\mathrm{CBF}$ and $1 \mathrm{gL}^{-1}$ Tecarapid CBF and Albaflow FFA in the buffer and colloid (d). 


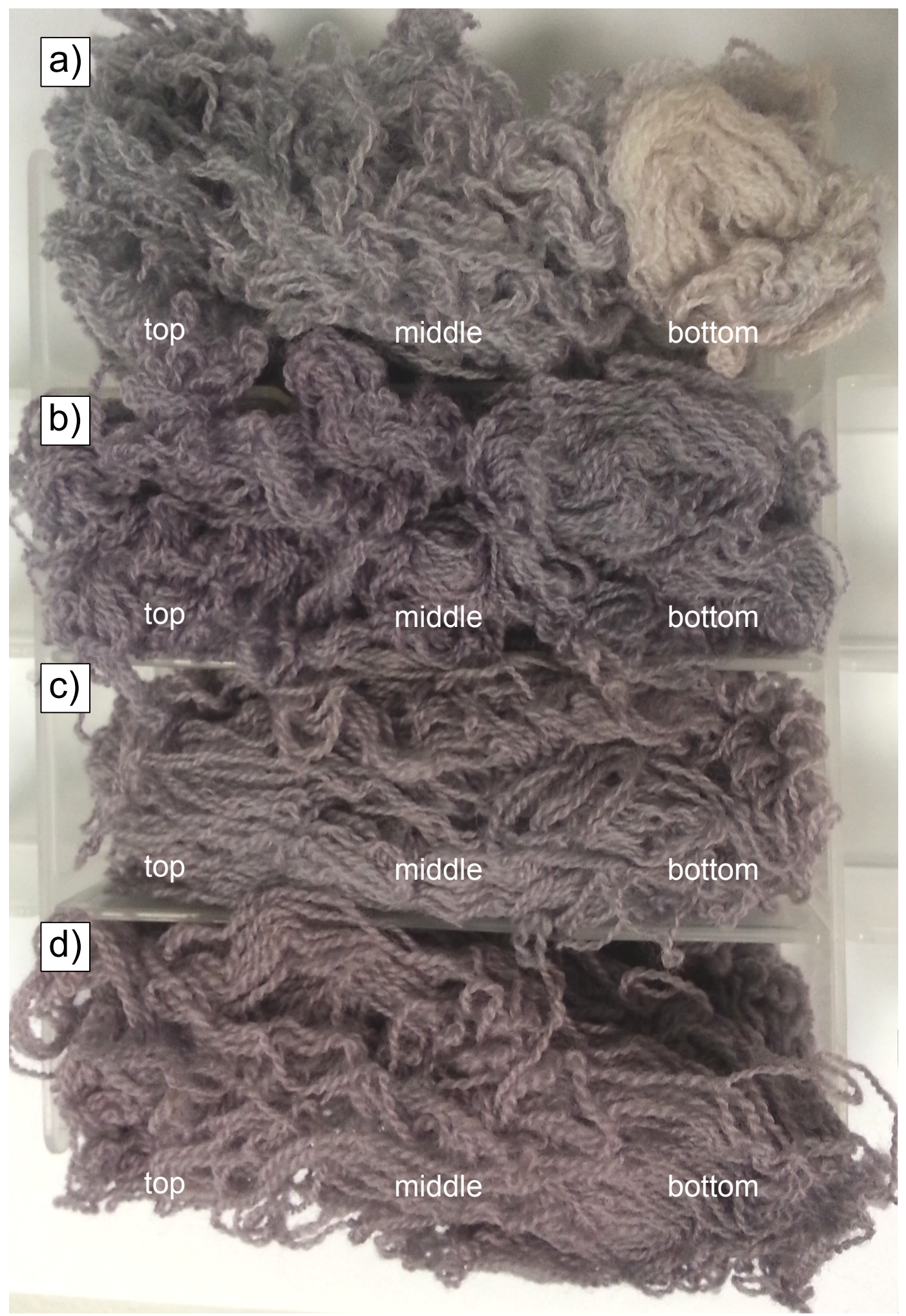

Figure 5.36 TSC-grey TU yarn composites synthesised in the Benchtop flow reactor with no auxiliary agents (a), $1 \mathrm{~g} \mathrm{~L}^{-1}$ Tecarapid CBF (b), $0.5 \mathrm{~g} \mathrm{~L}^{-1}$ Tecarapid $\mathrm{CBF}$ and Albaflow FFA (c) and $1 \mathrm{gL}^{-1}$ Tecarapid CBF and Albaflow FFA (d). 
$a^{*}$ and $b^{*}$ values of the different areas of the dyepack of each sample. The use of Tecarapid CBF has also significantly increased the redness values of the composites, which accounts for the purple-grey colours of the composites. These results imply that there is a balance between stabilising the TSC-Au NPs enough to slow the rate of their uptake to wool, while retaining the ability for the nanoparticles to aggregate on the surface of the TU yarn (which is required for the grey colour). This was also observed when a combination of Albaflow FFA and Tecarapid CBF resulted in purple-grey coloured composites.

Table 5.12 CIE colour coordinates of $5 \mathrm{~g}$ TSC-grey TU yarn composites that were synthesised in the Benchtop flow reactor with different amounts of Tecarapid CBF.

\begin{tabular}{cccccccccc}
\hline Teca & \multicolumn{3}{c}{$0 \mathrm{~g} \mathrm{~L}^{-1}$} & \multicolumn{3}{c}{$1 \mathrm{~g} \mathrm{~L}^{-1}$} & \multicolumn{3}{c}{$1 \mathrm{~g} \mathrm{~L}^{-1}+1 \mathrm{~g} \mathrm{~L}^{-1} \mathrm{WA}$} \\
\hline Area & Top & Mid & Bottom & Top & Mid & Bottom & Top & Mid & Bottom \\
\hline $\mathrm{L}^{*}$ & 49.08 & 57.54 & 65.44 & 43.75 & 46.46 & 47.62 & 45.47 & 49.39 & 49.20 \\
$\mathrm{a}^{*}$ & -0.99 & 0.87 & 1.20 & 2.32 & 3.59 & 3.55 & 3.68 & 4.23 & 4.53 \\
$\mathrm{~b}^{*}$ & -5.56 & -3.14 & 0.88 & -5.88 & -4.93 & -5.69 & -3.36 & -2.28 & -2.64 \\
\hline \hline
\end{tabular}

In summary, the Benchtop flow reactor provided uptake information on the uptake of gold nanoparticles to TU merino yarn that is believed to more accurately reflect the uptake chemistry of large-scale flow reactors. A range of wool dyeing auxiliaries were investigated, and the combination of Tecarapid CBF and Albaflow FFA looks particularly promising for integration into scale-up reactions in the flow reactors.

\subsubsection{First Flow Reactor}

The first flow reactor was designed by Professor James Johnston and Dr Aaron Small for initial scale-up syntheses of gold nanoparticle-wool fibre composites. The reactor builds upon the principles of conventional package dyeing procedures, involving the circulation of dye liquor around a system that contains wool that is compressed between two perforated discs. The first flow reactor is shown in Figure 5.37. A dye solution flows from the overflow bucket into inlet tap, rises up through the cylindrical reactor and is cycles back into the overflow bucket through one or more of the outlet taps.

Images of the interior of the first flow reactor are also shown in Figure 5.37 (c)-(e). Wool is placed on the perforated disc inside the reactor and the movable stamp is pushed down to compress the wool. The compression prevents the movement of the wool during the dyeing reaction. The first flow reactor incorporates a movable seal, which located at the top of the reactor. The total volume of dye liquor in the system may be adjusted by moving the seal down the cylindrical reactor (which can force 
the dye solution to exit through a different outlet tap).

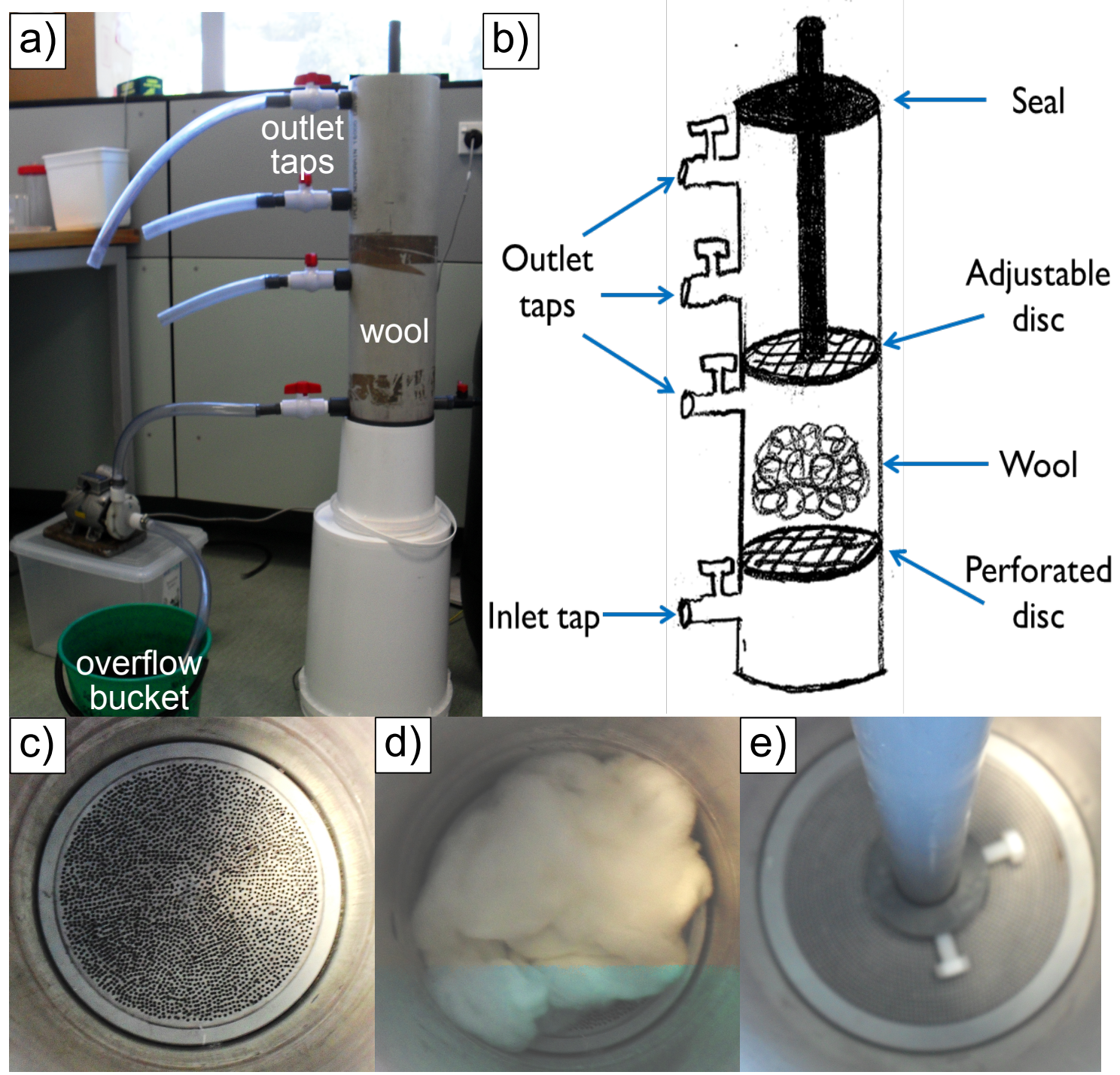

Figure 5.37 The first flow reactor (a), a cartoon of the interior of the reactor (b) and images of the interior perforated disc (c), which wool is placed on (d) before it is compressed with the stamp (e).

The first flow reactor was used to synthesise ex-situ composites of top form wool and fine merino yarn. Cariaggi and Ashford TA-mauve composites that were synthesised in the first flow reactor are presented in Figure 5.38. The composites were $40 \mathrm{~g}$ in size, which is the minimum mass required to cover the perforated disc in the reactor. The top form wool syntheses employed $2.4 \mathrm{~L}$ of the standard TA-mauve colloid, which was diluted to $12 \mathrm{~L}$ with distilled water and $\mathrm{pH}$ adjusted to 2.6. This equates to a wool to liquor ratio of 1:300, which is exceptionally high compared to those employed in industrial dyeing procedures. The flow rate of the reaction is approximately $4.3 \mathrm{~L} \mathrm{~min}^{-1}$, which equates to a residence time of dye circulation of $2.8 \mathrm{~min}$. The top form wool dyeing reactions were carried out prior to the incorporation of glycine buffers or auxiliary agents into the ex-situ procedure. 


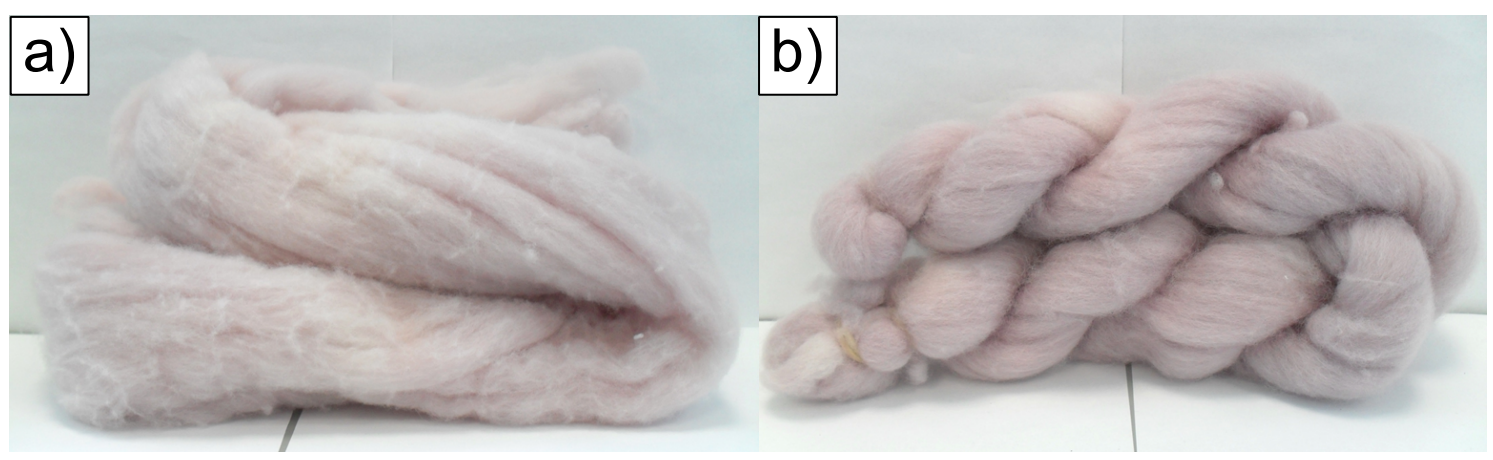

Figure 5.38 Cariaggi (a) and Ashford (b) TA-mauve ex-situ composites of $40 \mathrm{~g}$ size that were synthesised in the first flow reactor.

The resulting composites are relatively uniform in colour compared to composites of a similar size that were synthesised in static dyeing reactions. Additionally, the top form composites are not significantly damaged. This was a very positive result for the use of novel flow reactors for synthesis of ex-situ composites, and implies that the wool is held in place tightly during the dyeing reaction.

The first flow reactor was next used to synthesise TA-mauve TU yarn composites, to assess the feasibility of dyeing fine merino yarns this way. The following experiment involved the uptake of the equivalent concentration of the standard TA colloid (diluted from $3 \mathrm{~L}$ to $12 \mathrm{~L})$. The glycine buffer $\left(0.025 \mathrm{~mol} \mathrm{~L}^{-1}, \mathrm{pH} 2.6\right)$ was cycled around the reactor for $120 \mathrm{~min}$ prior to the colloid. Albaflow FFA $\left(1 \mathrm{~g} \mathrm{~L}^{-1}\right)$ was incorporated in the diluted buffer and colloid.

The uptake of TA-Au NPs to TU yarn in the first flow reactor is presented in Figure 5.39. The relatively low intensity of the SPR absorbance (due to the dilution of the colloid) results in more pronounced artefacts of the UV-vis spectrophotometer. The combination of the glycine buffer, Albaflow FFA and the diluted colloid (which equates to a wool to liquor ratio of 1:240) have resulted in the gradual uptake of TA$\mathrm{Au}$ NPs in the first flow reactor. The UV-vis spectra relate to an uptake curve that is relatively linear, and the uptake is almost complete after $60 \mathrm{~min}$ of the reaction. The low intensity absorbance of the 60 min UV-vis spectrum (relating to the SPR peak of TA-Au NPs) remains when the uptake is left for longer. This indicates an associated waste of gold nanoparticles with the method, which is likely enhanced due to the excessive wool to liquor ratio of the synthesis.

The resulting TA-mauve TU yarn composites are presented in Figure 5.40. Interestingly, the colour of the composite is very uneven; the bottom face of the composite exhibits dark purple coloured regions and the remainder of the composite is a light mauve colour. The CIE colour coordinates measured of relatively light and dark areas of the composite are shown in in Table 5.13. The CIE colour coordinates are 


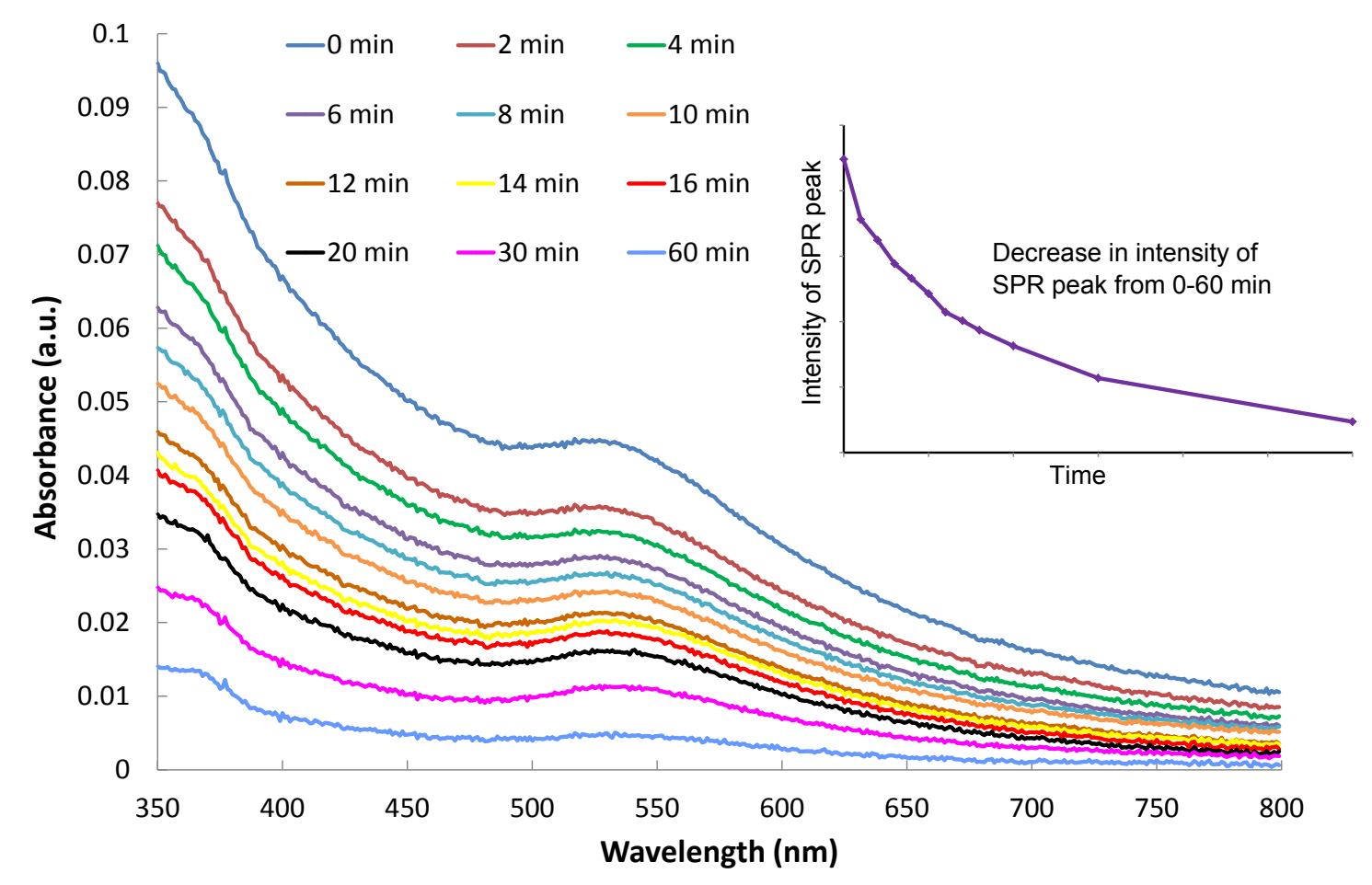

Figure 5.39 UV-vis spectra of the uptake of a diluted TA-mauve colloid to TU yarn in the first flow reactor. The inset represents the uptake curve from these spectra

very different, particularly the $\mathrm{L}^{*}$ value of the dark area is much lower. This highlights the differences in colour of the dyepack. The light regions of the composite are significantly brighter than that which is typically measured for the standard TA-mauve method, which is understandable given the amount of TA-Au NPs that remains in the uptake solution and is confined in the dark regions of the composite.

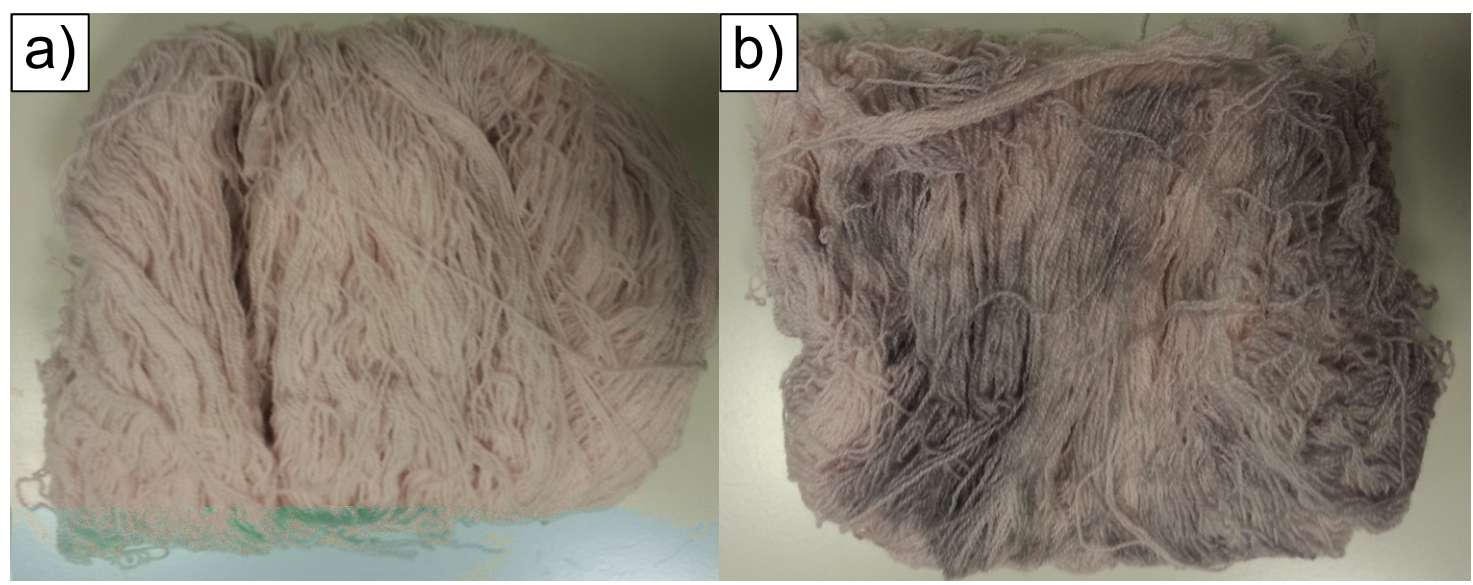

Figure 5.40 The top (a) and bottom (b) of a $50 \mathrm{~g}$ TA-mauve composite synthesised in the first flow reactor with the equivalent reaction conditions of the standard TA-mauve method. 
Table 5.13 CIE colour coordinates a TA-mauve TU yarn composite synthesised in the first flow reactor.

\begin{tabular}{ccc}
\hline Area of dyepack & Top & Bottom (dark area) \\
\hline $\mathrm{L}^{*}$ & 73.01 & 59.11 \\
$\mathrm{a}^{*}$ & 3.16 & 1.22 \\
$\mathrm{~b}^{*}$ & 2.81 & 0.96 \\
\hline \hline
\end{tabular}

SEM micrographs of the surfaces of composite fibres from the relatively dark and light areas of the TA-mauve TU yarn composite are shown in Figure 5.41. The relatively dark areas exhibit areas of very large agglomerates of TA-Au NPs on the surface. These areas are circled in Figure 5.41, and indicate the potential destabilisation of the TA-mauve colloid during the first flow dyeing reaction. It is considered that these agglomerates preferentially associate to the bottom of the dyepack because the dye liquor comes into contact with these areas first. In contrast, the SEM micrographs of the relatively light composite fibres (taken from the top of the dyepack) show the presence of significantly fewer individual and agglomerates of TA-Au NPs.

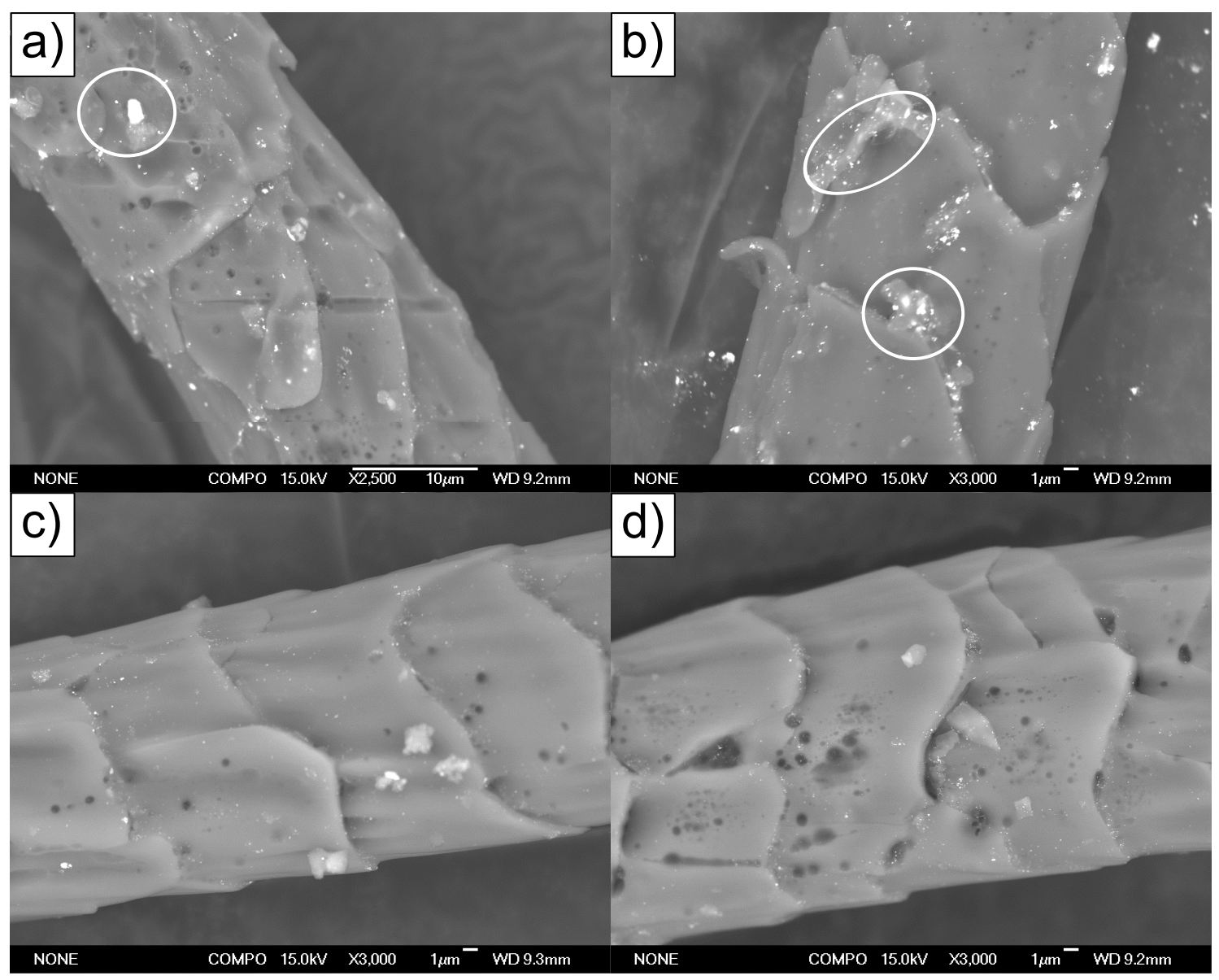

Figure 5.41 SEM micrographs in backscatter mode of the surface of TA-mauve composite fibres synthesised in the first flow reactor from relatively dark (a)-(b) and light (c)-(d) areas of the dyepack. 
The TA-mauve synthesis of TU yarn composites was repeated with the gradual lowering of the $\mathrm{pH}$ of the dye liquor during the uptake of TA-Au NPs. The glycine buffer was not employed in this experiment and $1 \mathrm{gL}^{-1}$ Albaflow FFA was added to the diluted equivalent of the standard TA-mauve colloid. The gradual $\mathrm{pH}$ adjustment was considered to slow the uptake of TA-Au NPs to the TU yarn and increase the colour uniformity of the resulting composites. The experiment involved the drop-wise addition of $1 \mathrm{~mol} \mathrm{~L}^{-1} \mathrm{HCl}$ into the overflow bucket, where the $\mathrm{pH}$ of the dye solution was slowly lowered from 3.5-2.0 over 180 min. Figure 5.42 presents the UV-vis spectra of the uptake reaction, where samples were removed from the system 20 min after the $\mathrm{pH}$ adjustment. The uptake was successfully slowed during this method, with evidence of the SPR peak of TA-Au NPs remaining in the dye solution at pH 2.0 and after approximately 180 min of total dye circulation.

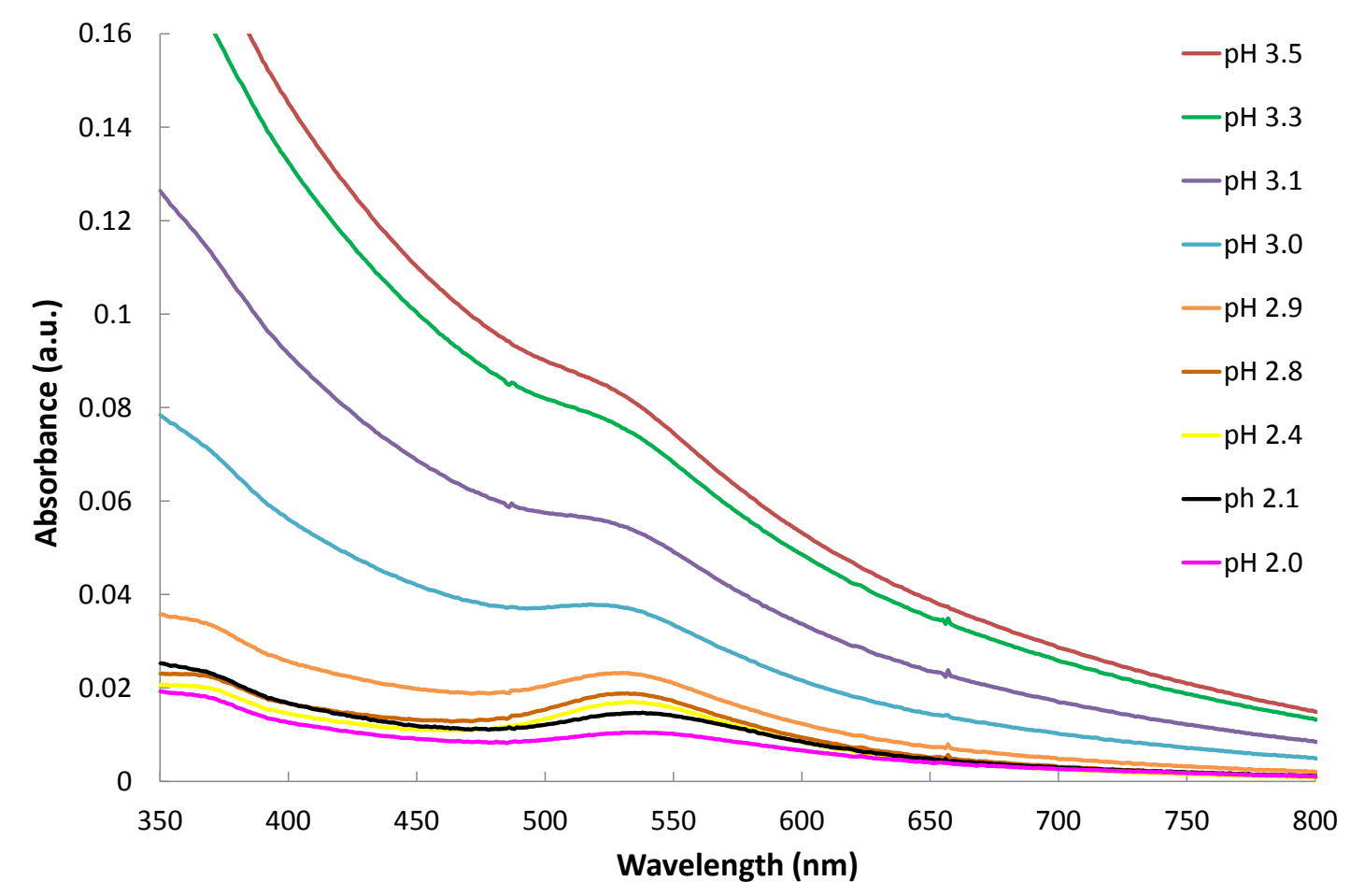

Figure 5.42 UV-vis spectra of the uptake of a diluted TA-mauve colloid to TU yarn in the first hybrid reactor with the gradual lowering of the $\mathrm{pH}$ of the uptake solution with $\mathrm{HCl}$ addition $\left(1 \mathrm{~g} \mathrm{~L}^{-1}\right.$ Albaflow FFA).

Images of the top and bottom faces of the resulting composite are shown in Figure 5.43. Interestingly, again there are some dark purple coloured regions on the bottom face of the dyepack, while the remaining yarn is a relatively light mauve colour. The CIE colour coordinates of the top face and a dark purple region of the bottom face are presented in Table 5.14 and highlight the colour differences between the areas. The dark purple areas exhibit expectedly lower $L^{*}$ and $b^{*}$ values compared to the lighter mauve areas. 


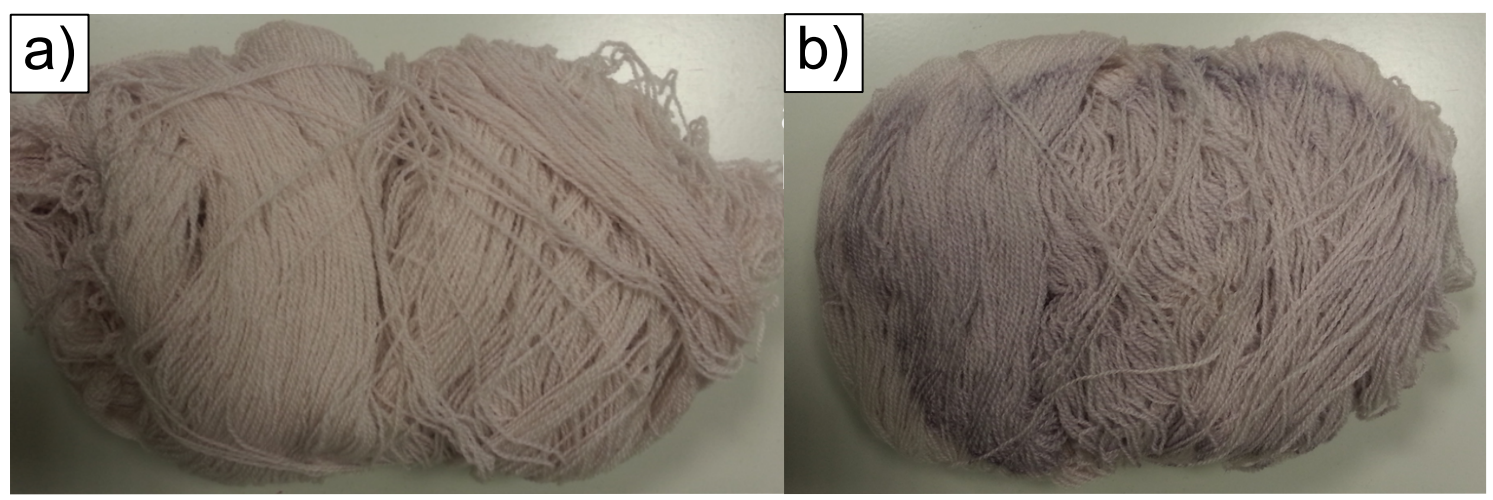

Figure 5.43 The top (a) and bottom (b) of a $50 \mathrm{~g}$ TA-mauve composite synthesised in the first flow reactor with the gradual $\mathrm{pH}$ lowering of the dye solution.

Table 5.14 CIE colour coordinates a TA-mauve TU yarn composite synthesised in the first flow reactor with gradual $\mathrm{pH}$ adjustment.

\begin{tabular}{ccc}
\hline Area of dyepack & Top & Bottom (dark area) \\
\hline $\mathrm{L}^{*}$ & 72.69 & 62.52 \\
$\mathrm{a}^{*}$ & 1.93 & 2.36 \\
$\mathrm{~b}^{*}$ & 3.93 & 0.49 \\
\hline \hline
\end{tabular}

The gradual lowering of the $\mathrm{pH}$ of the diluted TA-mauve colloid resulted in the gradual uptake of TA-Au NPs over 180 min, however the TA-Au NPs were unevenly distributed throughout the TU yarn composite. The relative preference for attachment at the bottom face is understandable considering that when the $\mathrm{pH}$ of colloid is lowered, the number of reactive sites for the electrostatic binding of gold nanoparticles increases. When the $\mathrm{pH}$ adjustment is gradual, the gold nanoparticles that may attach to the wool preferentially interact with the first wool fibres that they come into contact with, in this case being the TU yarn on the bottom face of the dyepack. As such, the gradual lowering of the $\mathrm{pH}$ of the dye solution is not a viable option for increasing the colour uniformity of ex-situ composites.

The previous reaction was repeated, this time with the rapid lowering of the $\mathrm{pH}$ of the dye solution. This involved the circulation of the standard concentration of the TA-mauve colloid ( $3 \mathrm{~L}$ diluted to $12 \mathrm{~L}$ ) with $1 \mathrm{gL}^{-1}$ Albaflow FFA at the unadjusted $\mathrm{pH}$ of the TA-mauve colloid. The $\mathrm{pH}$ was then rapidly dropped to 1.7 with the addition of $\mathrm{HCl}$ into the overflow bucket. This resulted in the rapid uptake of TA-Au NPs to the TU yarn over $10 \mathrm{~min}$. The resulting composite is presented in Figure 5.44. It is much more uniform than those synthesised with the $\mathrm{pH} 2.6$ glycine buffer or the gradual lowering of the $\mathrm{pH}$ of the dye solution. The colour differences between the top and bottom faces of the dyepack are minimal, and are highlighted by the CIE colour coordinates which are presented in Table 5.15. 


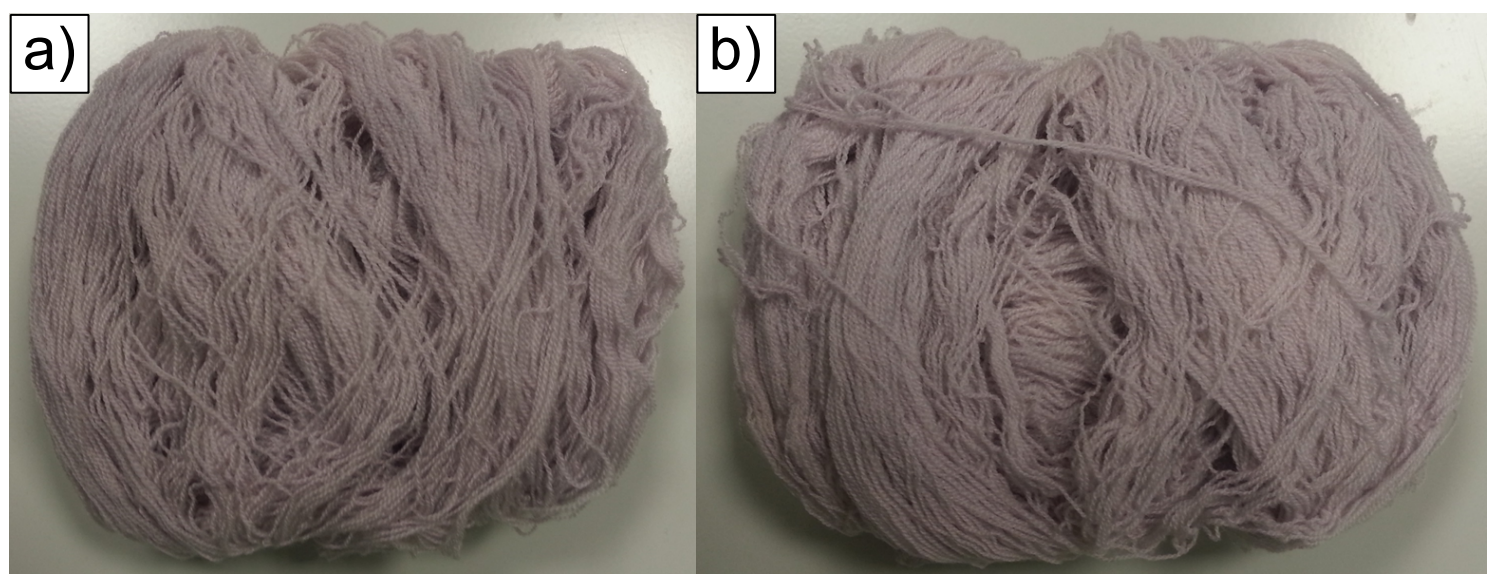

Figure 5.44 The top (a) and bottom (b) of a $50 \mathrm{~g}$ TA-mauve composite synthesised in the first flow reactor with a pH 2.2 buffer and colloid $\left(1 \mathrm{~g} \mathrm{~L}^{-1}\right.$ Albaflow FFA).

Table 5.15 CIE colour coordinates a TA-mauve TU yarn composite synthesised in the first flow reactor with a $\mathrm{pH} 2.2$ buffer and colloid $\left(1 \mathrm{~g} \mathrm{~L}^{-1}\right.$ Albaflow FFA).

\begin{tabular}{ccc}
\hline Area of dyepack & Top & Bottom \\
\hline $\mathrm{L}^{*}$ & 72.69 & 62.52 \\
$\mathrm{a}^{*}$ & 1.93 & 2.36 \\
$\mathrm{~b}^{*}$ & 3.93 & 0.49 \\
\hline \hline
\end{tabular}

In summary, the research presented in this section showed that the first flow reactor can be used to dye top form wool and fine merino yarns without damaging the wool fibres. Optimising the reaction conditions allowed for the uniform colouring of TU yarn with TA-Au NPs, but only of quantities of up to $50 \mathrm{~g}$.

The minimum volume of dye liquor that was used in the first flow reactor was $12 \mathrm{~L}$. It was originally thought that the volume of the reactor depend on whether the dye liquor was forced to exit out of the bottom, middle or top outlet tap. However, the movable seal had a propensity to leak if the pressure in the system reached a certain threshold, and because of this all of the outlet taps were required to be open during the dyeing reactions. Increasing the quantity of wool in the reactor or increasing the flowrate of dye circulation both elevate the internal pressure of the reactor. As such, the quantities of wool dyed and the flowrates employed in the dyeing reactions were limited. This limit led to the development of a suite of relatively robust novel flow reactors that could withstand higher pressure and flowrates for large-scale syntheses of ex-situ composites, as is discussed next. 


\subsubsection{Pressurised Flow Reactors}

The first flow reactor was used to dye quantities of up to $50 \mathrm{~g}$ of top form wool or fine merino yarn with reasonable colour uniformity. However, the propensity for the first flow reactor to leak through the movable seal meant that larger quantities of wool or faster flowrates could not be employed in the dyeing reactions. As such, more robust flow reactors that could withstand higher internal pressure were designed for larger-scale syntheses of ex-situ gold nanoparticle-wool fibre composites. The reactors employed faster flowrates, lower volumes of dye liquor and larger quantities of wool than the first flow reactor.

Three reactors with different volumes were developed to colour varying masses of wool. The reactors are sealed and do not employ overflow tanks, thus constituting closed systems. The pressurised flow reactors were described in greater depth in Section 2.2.4, and the relevant parameters of the reactors are summarised in Table 5.16.

Table 5.16 A summary of the reaction parameters of the pressurised flow reactors.

\begin{tabular}{cccc}
\hline Reactor & Baby Guinness & Half Pint & Full Pint \\
\hline Abbreviation & BG & HP & FP \\
Volume of reactor $(\mathrm{L})$ & 6 & 8 & 10 \\
Weight of wool $(\mathrm{g})$ & $300-360$ & 500 & $600-660$ \\
\hline \hline
\end{tabular}

The set-up of the Baby Guinness (BG) reactor is shown in Figure 5.45. Similar to the first flow reactor, the dye solution is pumped through the inlet at the bottom of the reactor, up through the cylinder and out the top of the reactor. A screw cap is used to close the system, which prevents the leakage of dye solutions and allows the system to be pressurised. There is an outlet tap located at the bottom of the reactor to allow the sampling of uptake solutions during the dyeing reactions. Additionally, a stronger pump that is capable of higher volume flow is used to circulate the dye liquor. The pump runs at one speed, and thus the rate of dye circulation is controlled by a valve which restricts the flow.

The interior of the BG reactor is shown in Figure 5.46. Similar to the first flow reactor, the BG reactor contains a perforated disc on to which the wool is placed. The wool is coiled around in a spiral configuration before being compressed inside the reactor. A stamp with a perforated disc on the bottom (Figure $5.46(\mathrm{~b})$ ) is again used to tightly pack the wool inside the reactor, to prevent the agitation of the wool fibres during the dyeing process. The pressurised flow reactors were used to synthesise ex-situ composites of top form wool, felted yarn and fine merino yarns with TA- and TSC-stabilised gold colloids. 


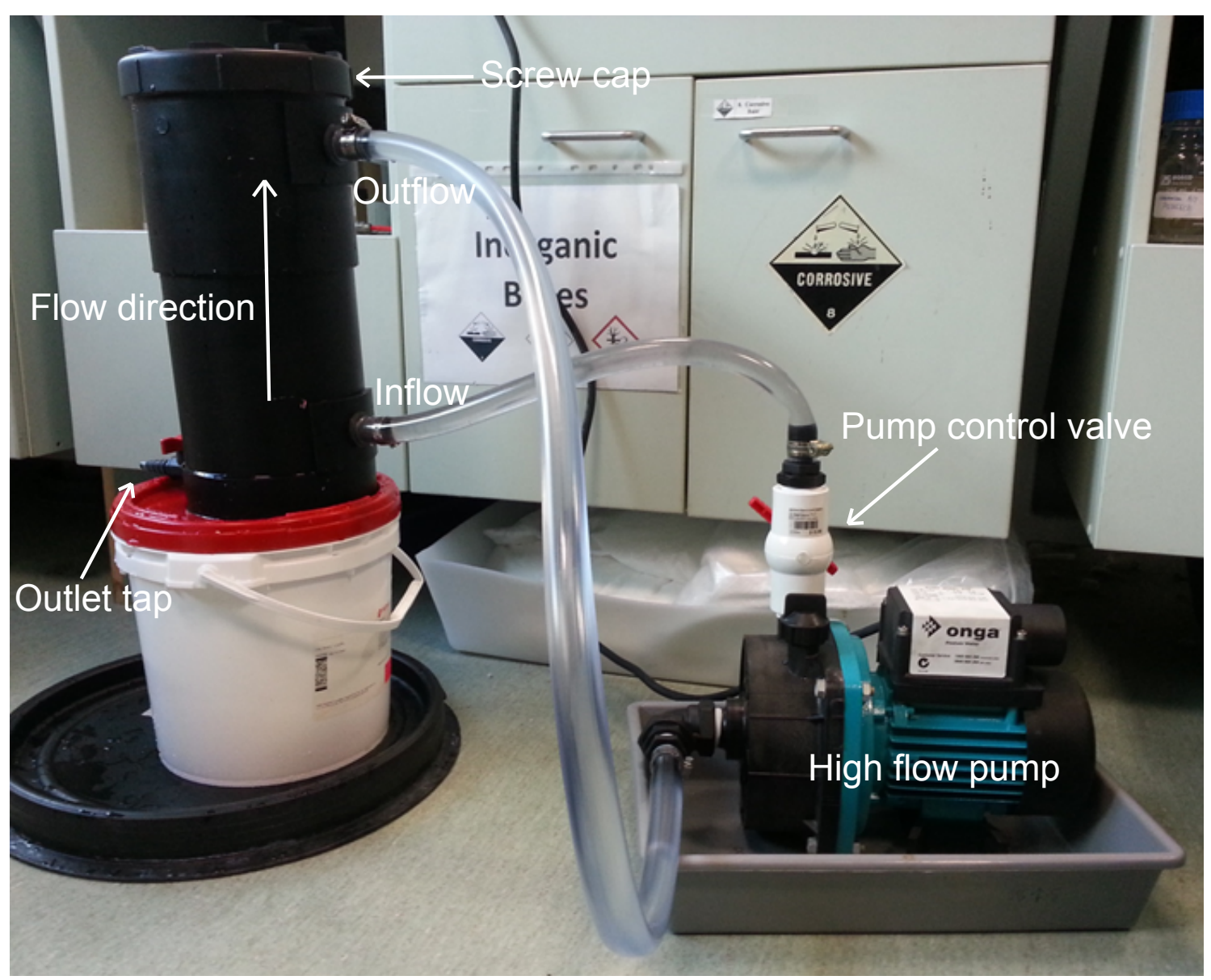

Figure 5.45 The Baby Guinness (BG) reactor set-up, where dye liquor is pumped through the inflow, up the cylindrical reactor and exits through the outflow. The outlet tap allows sampling of the dye solution during the dyeing reactions and the rate of dye circulation is dictated by the control valve. 


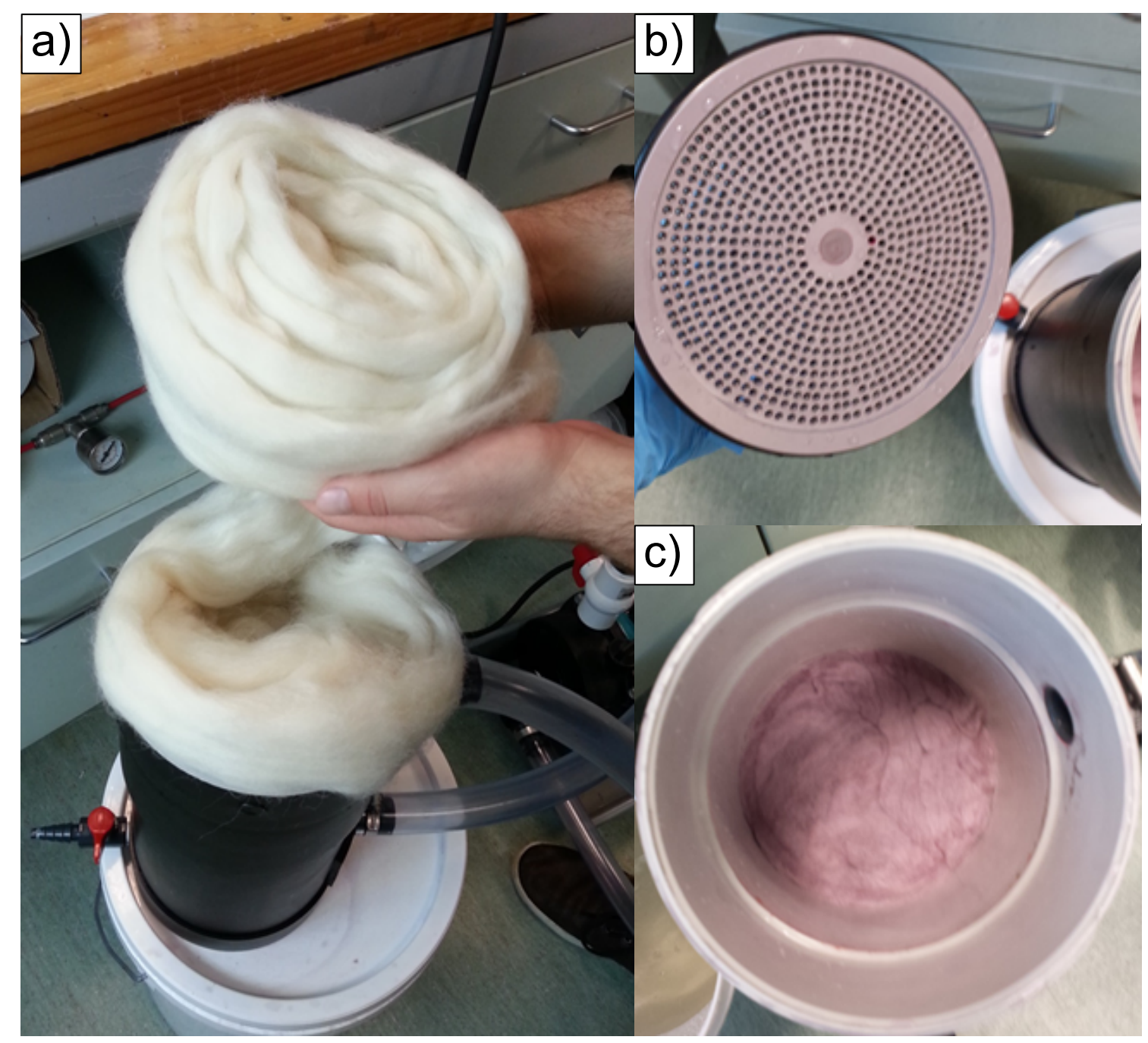

Figure 5.46 Images of the interior of the Baby Guinness reactor: the coiled configuration of the top form wool before dyeing (a), the stamp used to compress the wool (b) and the open reactor after a TA-mauve dyeing reaction (c). 


\section{TA-mauve Composites of Wool in Top Form}

The BG reactor was first used to colour $320 \mathrm{~g}$ of top form wool (sourced from an Italian wool dyeing company) with TA-Au NPs. The synthesis involved the cycling of hot tap water $(6 \mathrm{~L})$ around the system for $15 \mathrm{~min}$, followed by the glycine buffer (6 L, $0.1 \mathrm{~mol} \mathrm{~L}^{-1}$, pH 2.2, $0.5 \mathrm{~g} \mathrm{~L}^{-1}$ Albaflow FFA) for $120 \mathrm{~min}$. The $6 \mathrm{~L}$ capacity of the $\mathrm{BG}$ reactor equates to a wool to liquor ratio of $1: 18.75$, and as such the amount of glycine and Albaflow FFA employed is relatively low compared to that of static dyeing reactions (which employ a typical wool to liquor ratio of 1:60). The glycine buffer was drained from the system, and then $6 \mathrm{~L}$ of the equivalent concentration of the TA-mauve colloid ( $\mathrm{pH} 2.2,0.5 \mathrm{~g} \mathrm{~L}^{-1}$ Albaflow FFA) was pumped around the system. This involved concentrating the TA-mauve colloid more than 3 times that of typical static dyeing reactions.

The uptake of TA-Au NPs to loose wool is presented in Figure 5.47 and Figure 5.49. The uptake is very fast; it is complete after $5 \mathrm{~min}$ of the dyeing reaction, and approximately two thirds of the uptake occurs in the first minute. The rapid uptake illustrates the significant influence of the relatively higher pressure, flowrate and concentration of the reaction on the rate of the uptake of TA-Au NPs. The estimated flowrate of $30 \mathrm{Lh}^{-1}$ equates to a residence time of the circulation of $6 \mathrm{~L}$ of the dye liquor of approximately $12 \mathrm{~s}$. This equates to approximately 25 cycles of dye circulation during the first 5 min of the dyeing reaction.

Images of the resulting TA-mauve loose wool composite that was synthesised in the BG reactor both immediately after dyeing and when dried are shown in Figure 5.48. The composite immediately post-dyeing appears to be remarkably uniformly purplemauve coloured. However, the top form wool is twisted and matted in some areas. The dry composite is a darker, uniform mauve colour, but exhibits areas with significant fibre matting. The colour uniformity of this sample is an extremely positive result, however the damage to the loose wool is undesirable and may require additional combing and aligning of the fibres before it can be processed into yarn. As such, some of the reaction parameters were adjusted to attempt to minimise the potential wool fibre damage.

It was believed that the circulation of hot water around the pressurised flow reactor would facilitate the agitation of wool fibres and promote matting of the wool (when wet wool fibres are heated, they are able rub together more easily). As such, the rapid flowrates employed with the BG reactor were thought to require a relatively gentle approach. The TA-mauve synthesis of loose wool composites was repeated without the hot water washing step, and the glycine buffer was circulated for only 


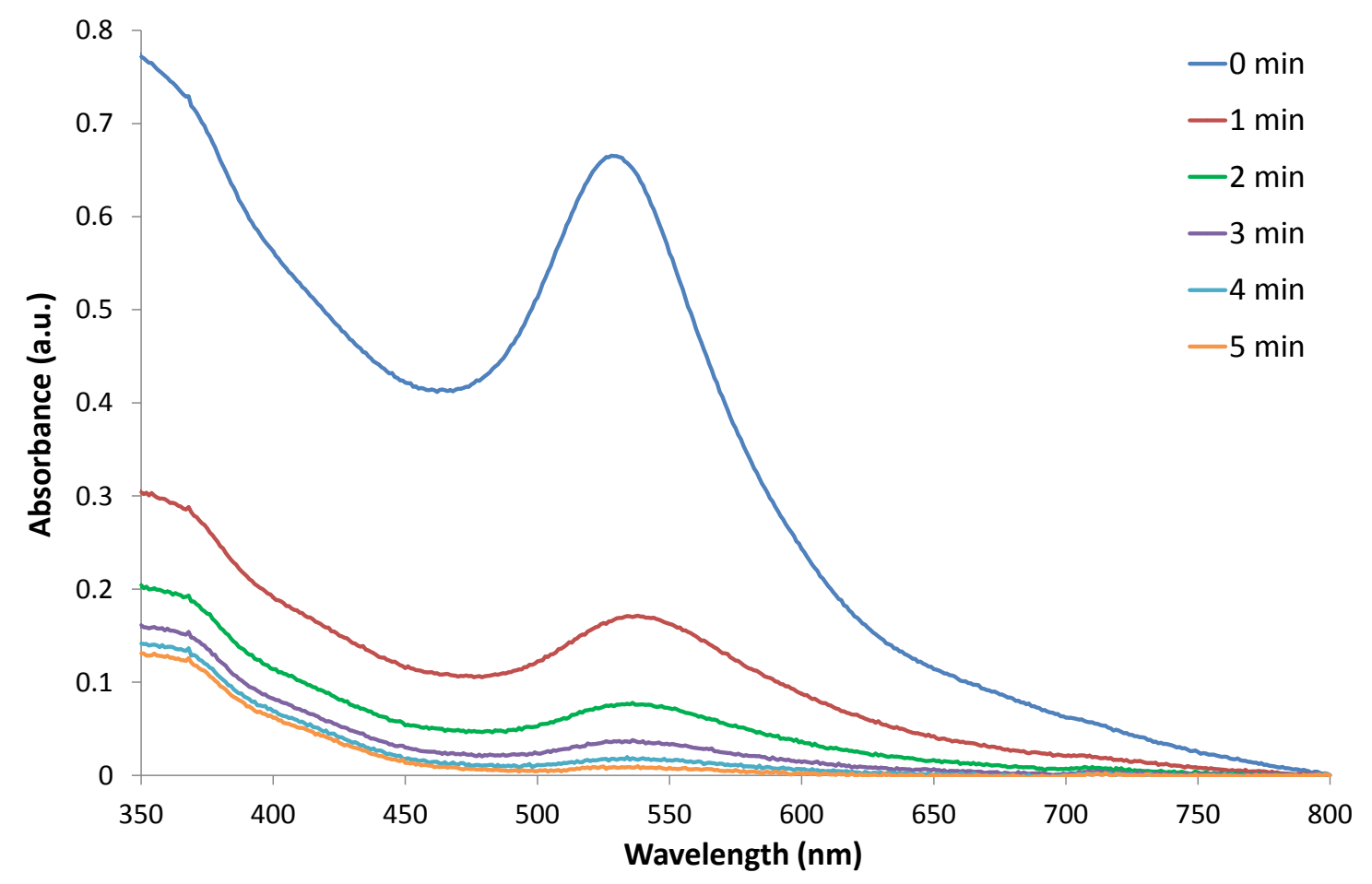

Figure 5.47 UV-vis spectra and images of the uptake of the concentrated TA-

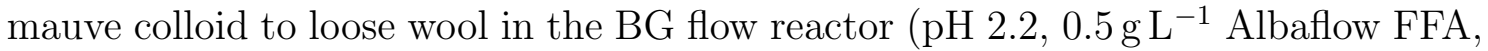
$0.1 \mathrm{~mol} \mathrm{~L}^{-1}$ glycine buffer $120 \mathrm{~min}$, hot water wash).

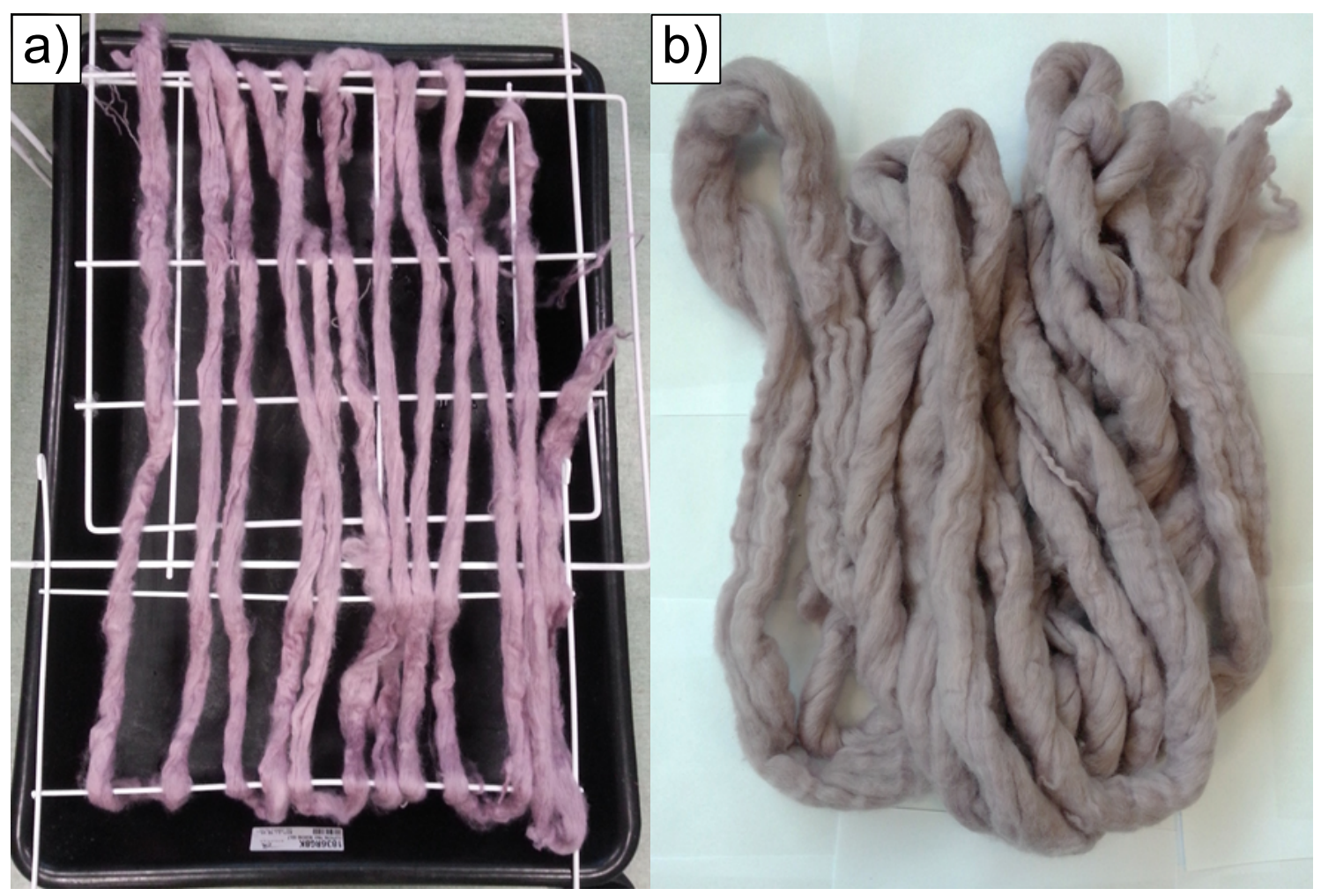

Figure 5.48 TA-mauve loose wool composites synthesised in the BG flow reactor with the conditions of the standard TA-mauve method ( $\mathrm{pH} 2.2,0.5 \mathrm{~g} \mathrm{~L}^{-1}$ Albaflow FFA, glycine buffer circulation for $120 \mathrm{~min}$, hot water wash) before (a) and after (b) drying. 
15 min prior to the gold colloid. All of the other reaction conditions were consistent with the previous experiment.

The uptake of TA-Au NPs to loose wool in the BG reactor under different reaction conditions is presented in Figure 5.49. The hot water pre wash uptake curve relates to the previous experiment (the UV-vis spectra of which were shown in Figure 5.47). The "no hot water" uptake curve pertains to this dyeing reaction, where the uptake is significantly slower, requiring approximately $22 \mathrm{~min}$ for the complete absorption of TA-Au NPs. This equates to approximately 110 cycles of the dye liquor. The relatively slow rate of uptake may be due to the presence of residual processing lubricants on the wool interfering with the absorption of TA-Au NPs. Additionally, the ion exchange reaction between the glycine buffer and the wool may not be complete in just $15 \mathrm{~min}$. The uptake profile is both slower and more linear than that of the previous experiment, which is desired to increase the homogeneity of absorption of TA-Au NPs throughout the dyepack.

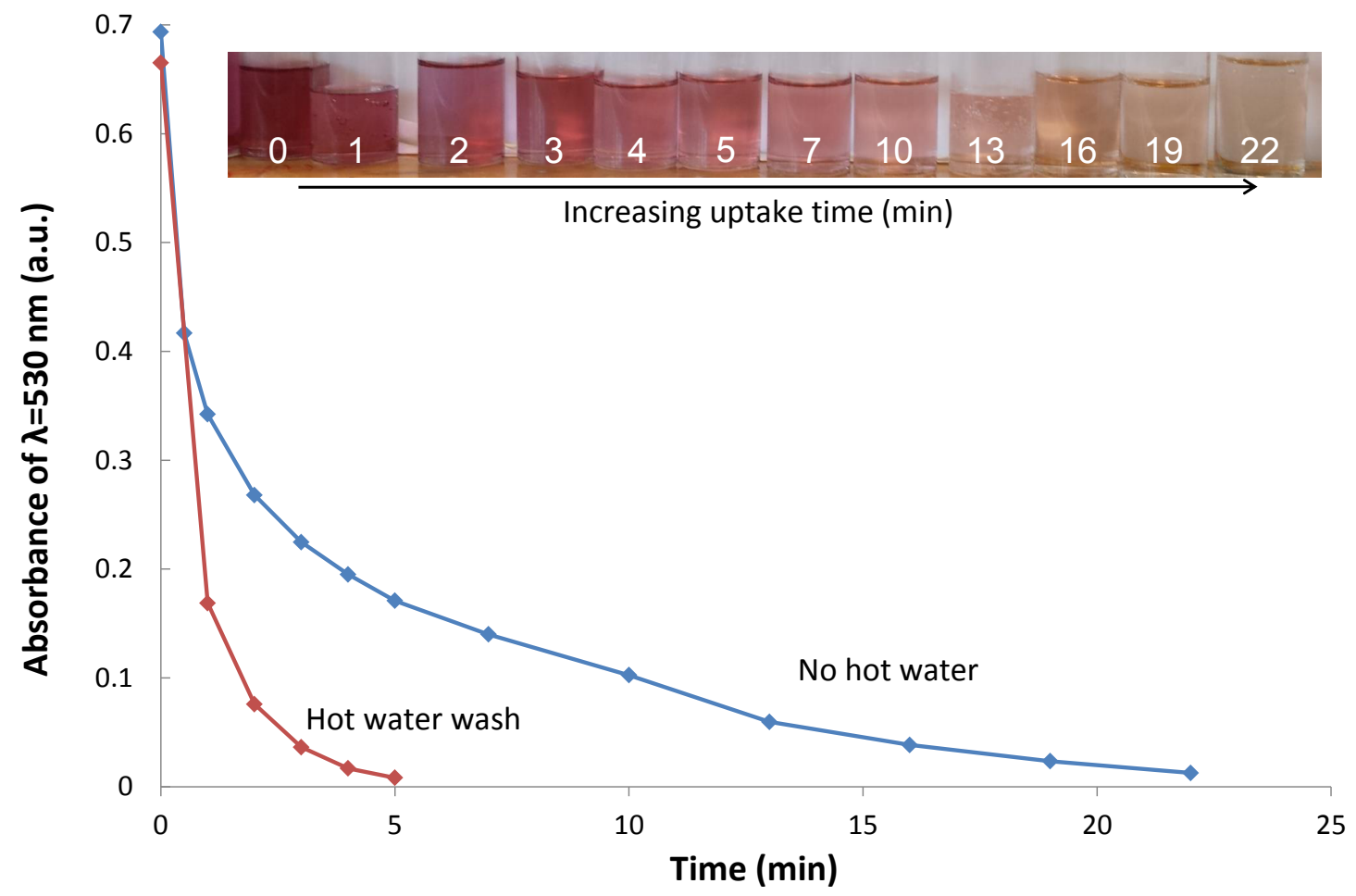

Figure 5.49 Uptake curves of the dyeing reactions of loose wool with the TAmauve colloid in the BG flow reactor with a hot water wash and $120 \mathrm{~min}$ buffer circulation or no hot water wash and 15 min buffer circulation.

Images of the resulting TA-mauve loose wool composite synthesised without hot water (and 15 min buffer circulation) is shown in Figure 5.50. The composite appears to be relatively less twisted and damaged than composite resulting from the previous experiment. When the composite is dried, it exhibits significantly less fibre damage, however there are still some areas of slight matting. This indicates that the wool 


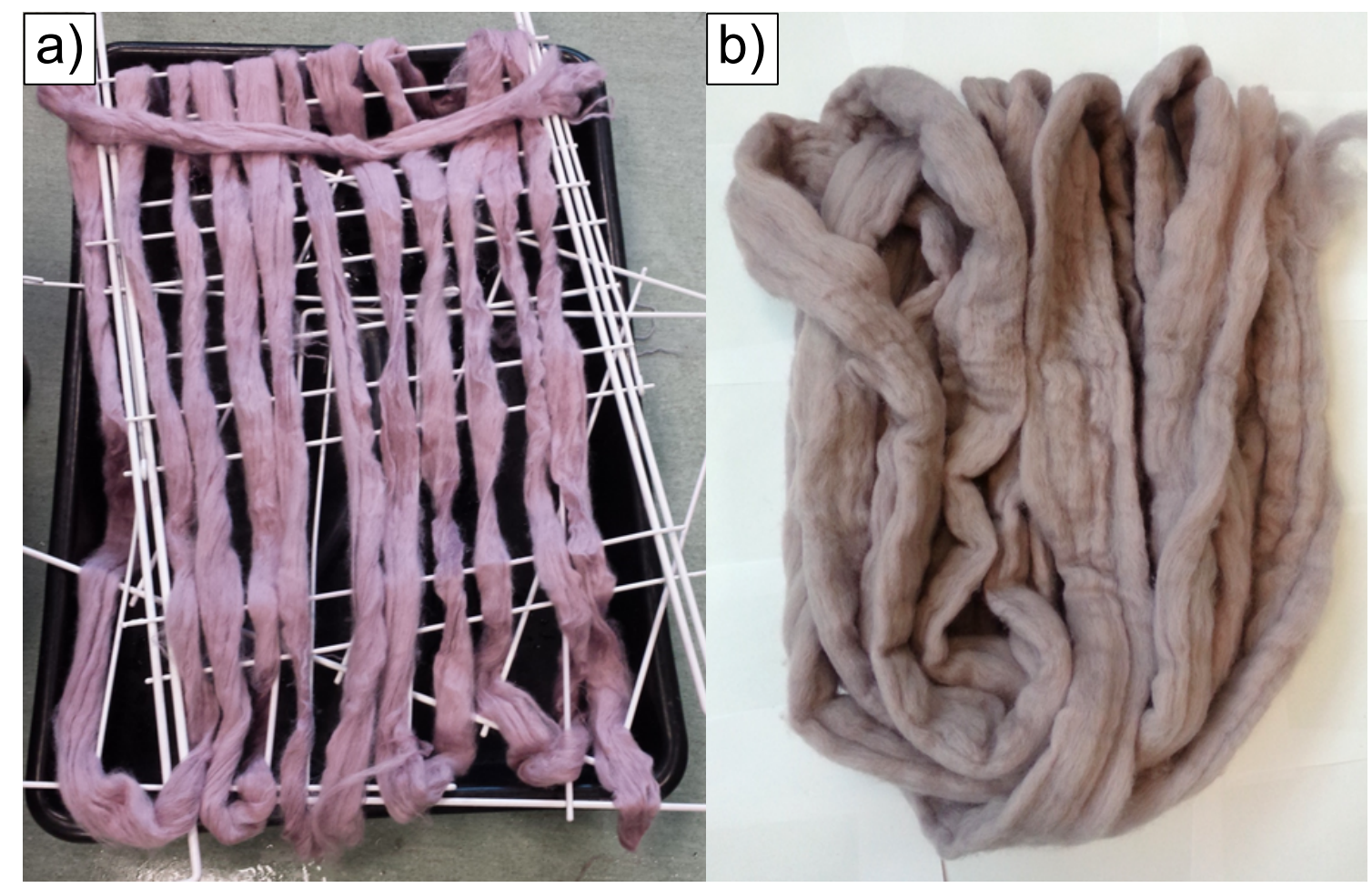

Figure 5.50 TA-mauve loose wool composites synthesised in the BG flow reactor immediately after dyeing (a) and when dried (b).

damage has been reduced by removing the hot water washing cycle and reducing the time of buffer circulation. As such, future dyeing reactions with the pressurised flow reactors did not involve a hot water washing cycle.

SEM analysis of the composite in Figure 5.50 was undertaken to determine whether the relatively fast flowrates of the pressurised flow dyeing reaction had influenced the distribution of gold nanoparticles on the surface of the composite fibres. The SEM micrographs of two different composite fibres are presented in Figure 5.51. The composite fibres appear to exhibit the typical amount and distribution of gold nanoparticles on their surfaces, that is expected with the TA-mauve method, including a relatively higher proportion of gold nanoparticles located near the cuticle edges. Additionally, the higher magnification images do not seem to show any deviation from the typical ratio of individual nanoparticles to agglomerates of TA-Au NPs on the composites. The BG reactor allowed for synthesis of TA-mauve loose wool composites of 320-360 g size with remarkable colour uniformity. This promising result lead to the use of pressurised flow reactors to colour Ascend felted yarn, the chemistry of which is next detailed. 


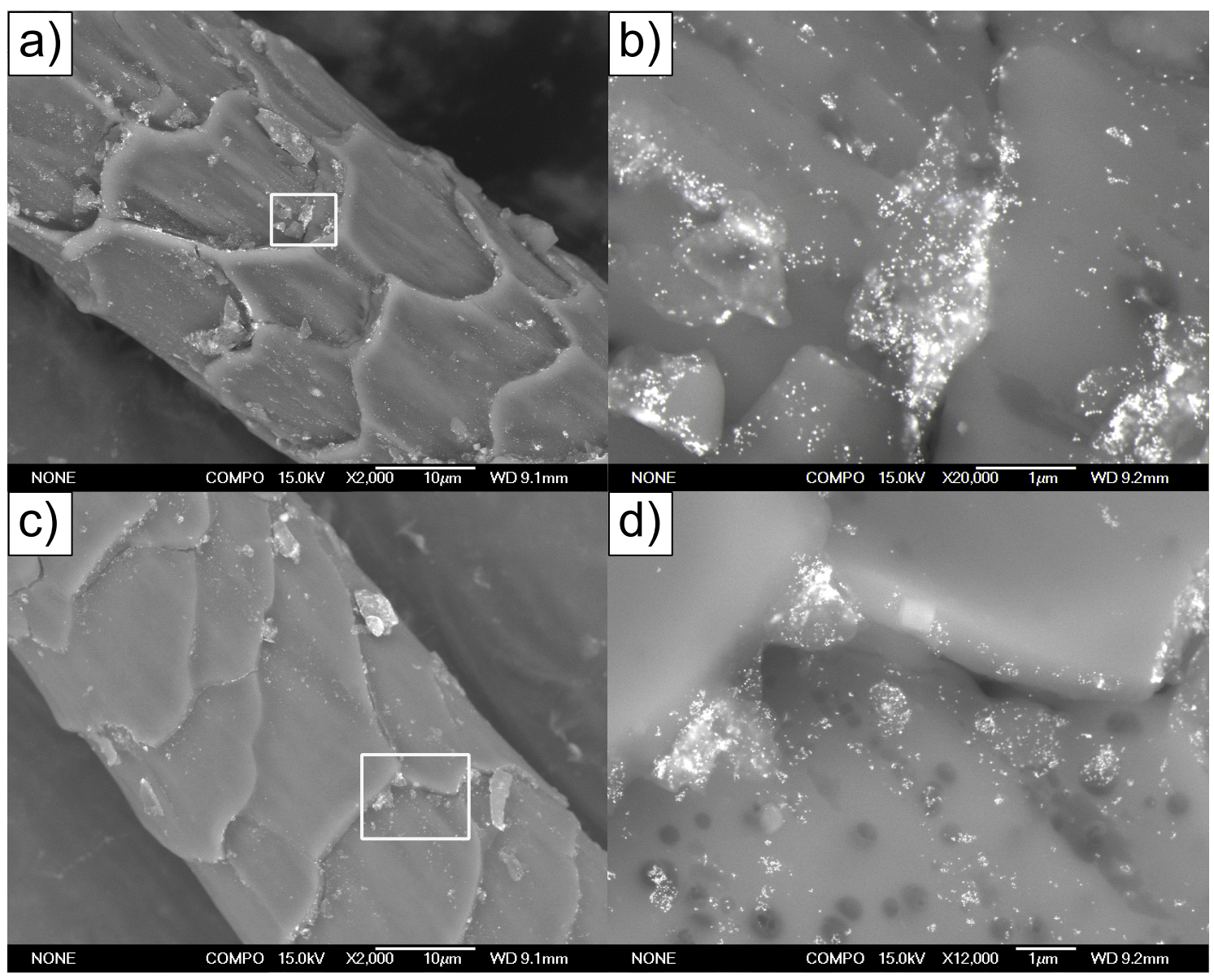

Figure 5.51 SEM micrographs in backscatter mode of a TA-mauve loose wool composite synthesised in the BG flow reactor (with no hot water wash and $15 \mathrm{~min}$ buffer circulation).

\section{TA-mauve Ascend Felted Yarn Composites}

The following experiments represent the synthesis of TA-mauve composites of Ascend felted yarn in the BG reactor under a range of reaction conditions. Each of the dyeing reaction involved the use of $5 \mathrm{~L}$ of the standard TA-mauve colloid $(2.5 \mu \mathrm{L}$ $\mathrm{AuCl}_{4}^{-}$per $10 \mathrm{~mL}$ colloid, $\mathrm{pH} 2.2$ glycine buffer, hot water wash, $120 \mathrm{~min}$ buffer circulation). The relatively low concentration of the colloid was anticipated to impart a light mauve hue to the felted yarn. The selection of reaction conditions were manipulated in the syntheses to attempt to optimise the colour uniformity of the resulting composites Table 5.17.

The composites synthesised in the BG reactor with the above reaction conditions are shown in Figure 5.52. Each of the composites seem to be more uniform in colour than those synthesised in hank dyeing procedures (which were detailed in Section 5.2.2). This is a very promising result (even considering the small size of the samples). Composites 1 and 2 involved different masses of felted wool yarn that were coloured with different ratios of TA-Au NPs to wool. As such, Composite 1 exhibited 
Table 5.17 Details of reaction parameter employed in different experiments carried out in the $\mathrm{BG}$ reactor.

\begin{tabular}{ccccc}
\hline Composite & 1 & 2 & 3 & 4 \\
\hline Mass of yarn (g) & 120 & 270 & 270 & 270 \\
Wool to liquor ratio & $1: 50$ & $1: 22$ & $1: 22$ & $1: 22$ \\
Albaflow FFA in buffer and colloid $\left(\mathrm{g} \mathrm{L}^{-1}\right)$ & 0.5 & 0.5 & 1 & 1 \\
Tecarapid CBF in colloid $\left(\mathrm{g} \mathrm{L}^{-1}\right)$ & 0 & 0 & 1 & 1 \\
Form of wool & loose & loose & loose & coiled \\
Uptake time (min) & 30 & 5 & 120 & 180 \\
\hline \hline
\end{tabular}

a slower uptake and is a significantly darker mauve colour than Composite 2. Both composites are uniform in colour, indicating that using different concentrations of the TA-mauve colloid can result in different shades of mauve coloured composites.

Composite 3 was synthesised with $1 \mathrm{gL}^{-1}$ of both Albaflow FFA and Tecarapid CBF in the TA-mauve colloid. This resulted in an uptake time of $120 \mathrm{~min}$, which is substantially slower than that of Composite 2 (which involved $0.5 \mathrm{~g} \mathrm{~L}^{-1}$ Albaflow FFA only). Composite 3 is a slightly lighter mauve colour than Composite 2 and interestingly, is also less uniformly coloured than Composite 3. This indicates that such a combination of auxiliary agents is detrimental to the colour uniformity of the composites.

The synthesis of Composite 4 involved the same reaction conditions as Composite 3, except for the felted yarn being wound into a coiled cylinder structure, as opposed to the typical loose configuration of felted yarn. This experiment was carried out to investigate the importance of the form of the wool. The coiled structure is tightly wound and hence was seen to restrict the access of gold nanoparticles into the centre of the structure. As such, the uptake is even slower, taking approximately 180 min to complete, and the exterior of the composite is much darker in colour than the interior of the composite (and Composite 3 which was both lighter and more uniformly coloured). Hence, the form of the wool is important to consider in relation to the dyeing reactions in pressurised flow reactors.

The most promising results of colouring Ascend felted yarn with gold colloids were achieved with Composites 1 and 2, these were the most uniformly coloured, despite the relatively rapid rates of uptake of these experiments. Manipulating the concentration of the colloid (or the wool to liquor ratio of a colloid of equal concentration) allows for different shades of mauve to be achieved with the BG reactor. 


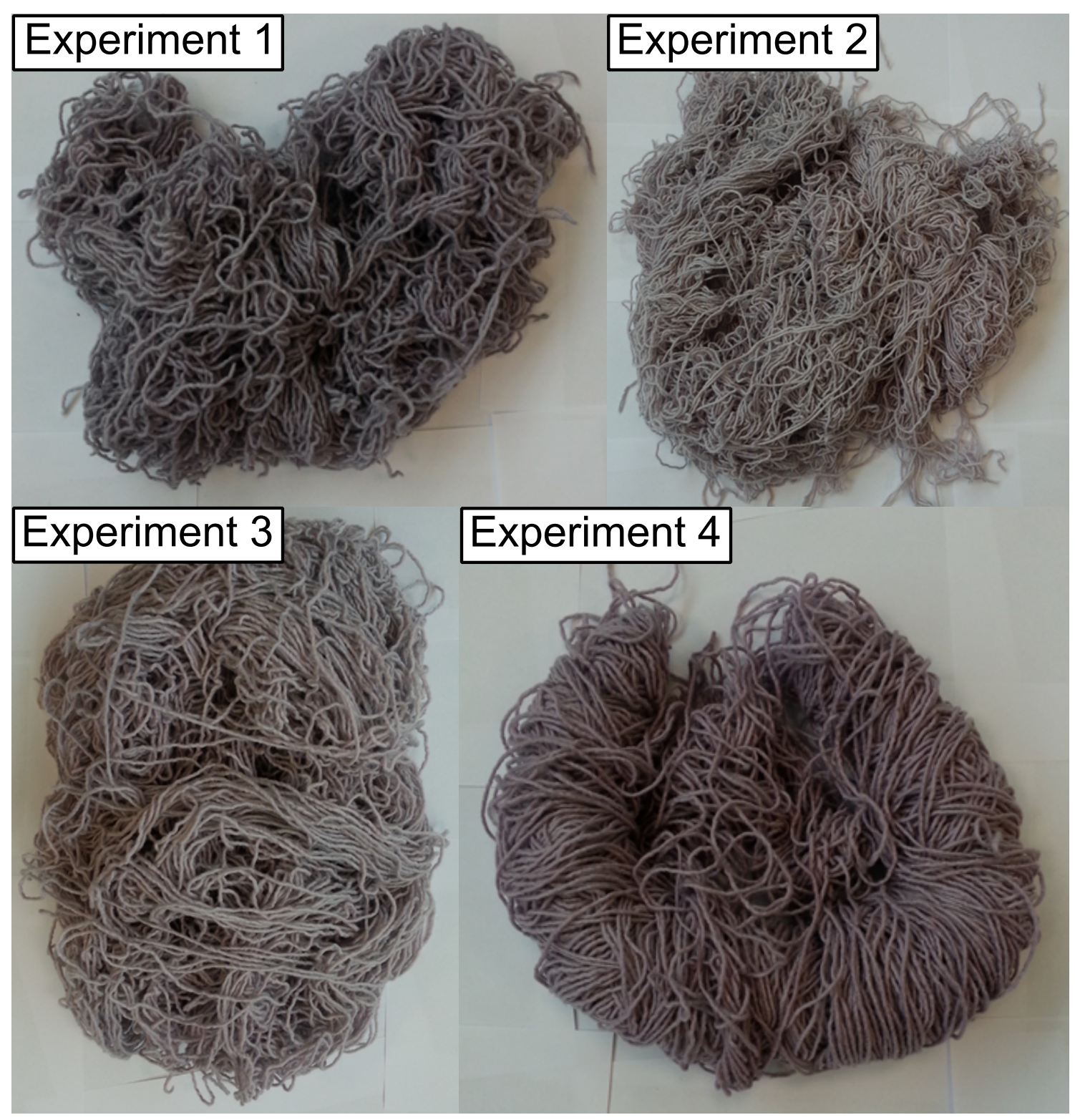

Figure 5.52 Ascend felted yarn TA-mauve composites synthesised in the BG flow reactor, where Experiment 1 is dark mauve, Experiments 2 and 3 are light mauve and Experiment 4 is purple-mauve in colour.

\section{TSC-grey Loose Wool Composites}

The success of synthesising uniformly coloured TA-mauve composites in the pressurised flow reactors led to integrating the TSC-grey method with the BG reactor. The first synthesis involved colouring $360 \mathrm{~g}$ of loose wool with half of the equivalent concentration of the TSC-grey colloid, which is referred to as the TSC-light grey method in this section. The lower concentration was employed both to reduce the expense of the dyeing reactions and due to difficulties that were encountered in increasing the concentration of the TSC-grey colloid. The wool to liquor ratio of the reaction was 1:16.7, and as such the typical TSC-grey colloid was almost twice as concentrated as it is in the standard TSC-grey method (which employs a 1:60 wool 
to liquor ratio, as was detailed in Section 4.3.4). Additionally, due to the success of using the glycine buffer in the TSC-grey synthesis in laboratory-scale research (that was detailed in Section 4.3.3), the buffer was also employed in initial dyeing runs in the $\mathrm{BG}$ reactor.

The first reaction involved the circulation of a $\mathrm{pH} 2.0$ glycine buffer $\left(0.1 \mathrm{~mol} \mathrm{~L}^{-1}\right.$, $15 \mathrm{~min}, 1 \mathrm{~g} \mathrm{~L}^{-1}$ Albaflow FFA) prior to the $\mathrm{pH} 2.0$ TSC-light grey colloid. A hot water wash cycle was not employed to minimise the agitation of the wool that occurs in the dyeing procedure. The uptake of TSC-Au NPs at pH 2.0 was extremely rapid; it was complete in $1 \mathrm{~min}$ (which equates to less than 5 circulations of the dye liquor around the reactor). The resulting composite is shown in Figure 5.53. Interestingly, the $\mathrm{pH} 2.0$ composite is a reasonably uniform light grey in colour, which is surprising considering the rate of the uptake.

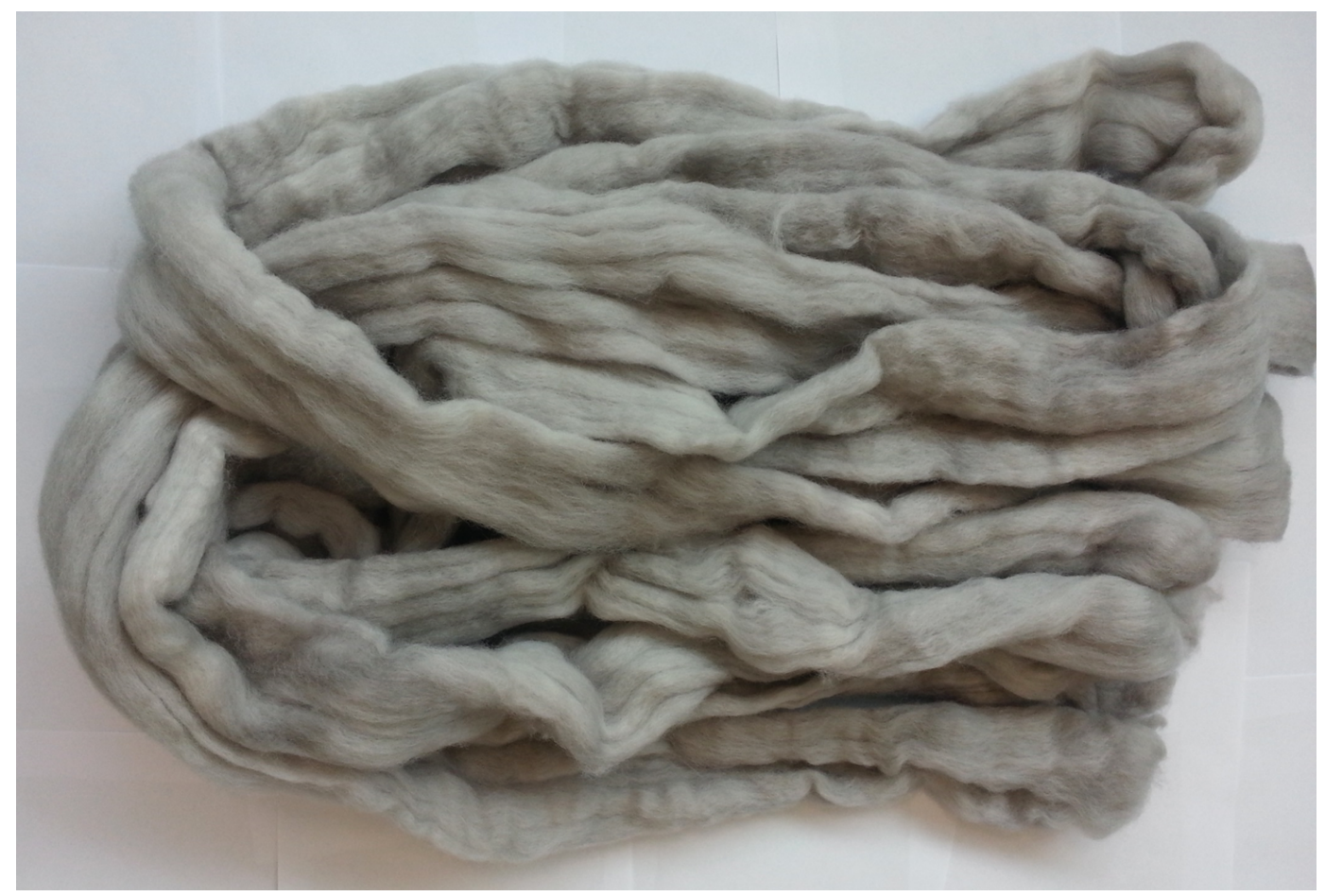

Figure 5.53 A TSC-light grey loose wool composite synthesised in the BG reactor with a $\mathrm{pH} 2.0$ glycine buffer and colloid ( $\mathrm{pH} 2.0,1 \mathrm{~g} \mathrm{~L}^{-1}$ Albaflow FFA).

It was considered that slowing the uptake of TSC-Au NPs to loose wool would result in more uniformly coloured TSC-light grey composites. As such, the above synthesis was repeated with a $\mathrm{pH} 2.8$ glycine buffer and colloid. The uptake was significantly slower, taking approximately $25 \mathrm{~min}$ for the full absorption of TSC-Au NPs. However, as is seen in Figure 5.54, the resulting composite is very unevenly coloured. There are some very dark grey coloured areas, while the remainder of the dyepack is either light grey or completely uncoloured. The colour distribution looks similar to the composites synthesised in the first flow reactor with the gradual $\mathrm{pH}$ 
lowering of the system. The dyeing reaction at this $\mathrm{pH}$ likely involves the continual agglomeration of the TSC-Au NPs in the dye solution during the uptake. It is considered that after the agglomerates become sufficiently large and unstable, they then attach to the wool. In contrast, at the lower reaction $\mathrm{pH}$ of 2.0 , the uptake occurs so rapidly that the agglomeration does not occur during the circulation of the dye solution to this extent. Additionally, the dark grey areas overlap around where the holes in the stamp are present, an observation which will be elaborated on later.

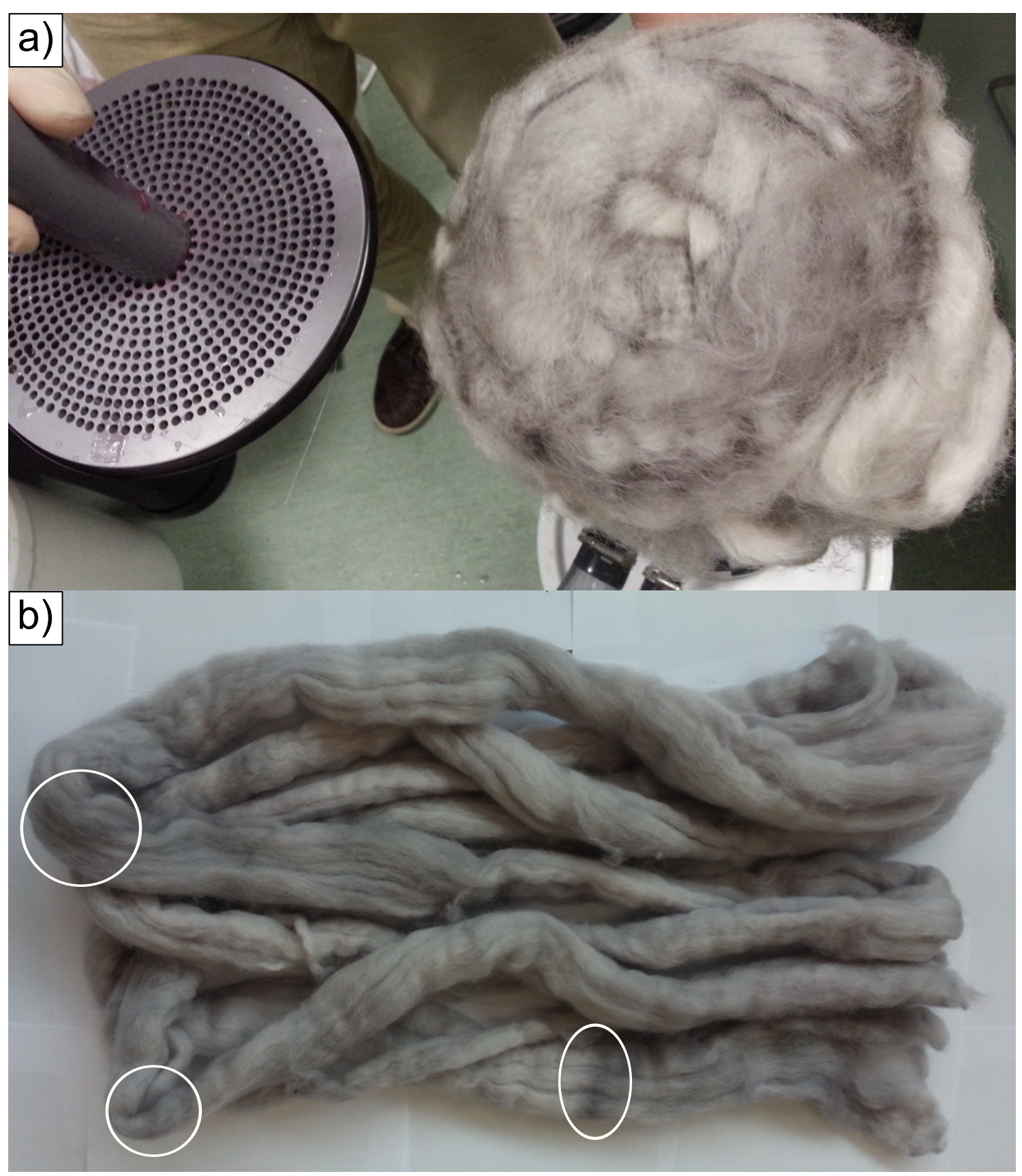

Figure 5.54 Images of a TSC-light grey loose wool composite synthesised in the $\mathrm{BG}$ reactor with a pH 2.8 glycine buffer and colloid directly removed from the reactor after the dyeing reaction (a) and the resulting composite (b), where the darker grey areas are circled. 
Due to the substantial differences in rate of uptake of TSC-Au NPs and the colour of the resulting TSC-light grey composites that occurs when the $\mathrm{pH}$ of the reaction is changed from 2.0 to 2.8, the TSC-light grey synthesis was repeated at $\mathrm{pH} 2.2$, 2.4 and 2.6 (for both the buffer and colloid). The uptake of composites with a reaction $\mathrm{pH}$ of 2.0-2.8 is shown in Figure 5.55. When the reaction $\mathrm{pH}$ is maintained at 2.0-2.2, the uptake is complete in $<1 \mathrm{~min}$. At $\mathrm{pH} 2.4$, the uptake is complete after $5 \mathrm{~min}$, and further increasing of the reaction $\mathrm{pH}$ sequentially slows the rate of uptake. The uptake curves relate to the intensity of the SPR peak of individual TSC-Au NPs $(\lambda=525 \mathrm{~nm})$ only, and hence provide only an indication of the rate of uptake, which however does occur on a similar timescale.

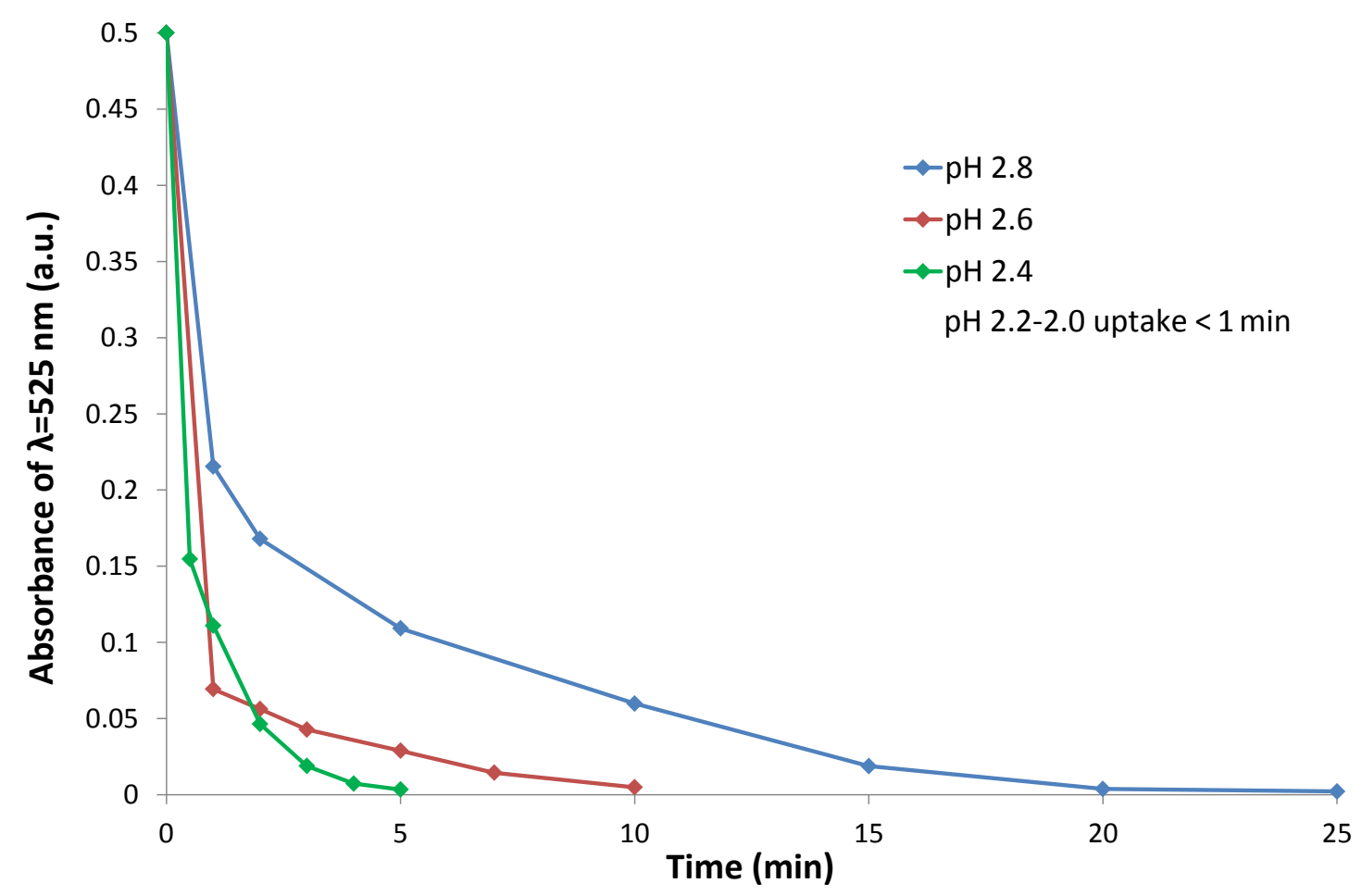

Figure 5.55 Uptake of the TSC grey colloid to loose wool with different reaction $\mathrm{pH}$ levels of the glycine buffer and colloid.

Interestingly, the composites synthesised at $\mathrm{pH}$ 2.4-2.8 are all relatively unevenly light grey in colour. All of these composites exhibit areas of dark grey-black colouration near the top of the dyepack. The pH 2.4 TSC-light grey composite is presented in Figure 5.56. The top face of the dyepack looks similar to that of the $\mathrm{pH} 2.8$ composite (shown in Figure 5.54), where the dark grey areas are present where the holes in the stamp are located. However, as is seen in Figure 5.56 (b), the remainder of the dyepack is relatively more evenly light-grey coloured than that of the $\mathrm{pH} 2.8$ composite. The darker areas relate either to the areas near the stamp, or other areas where the loose wool is more tightly compressed in the spiral conformation. Thus, the faster rate of uptake of the $\mathrm{pH} 2.4$ dyeing reaction resulted in more evenly 
coloured composites. This was an interesting and unexpected result.

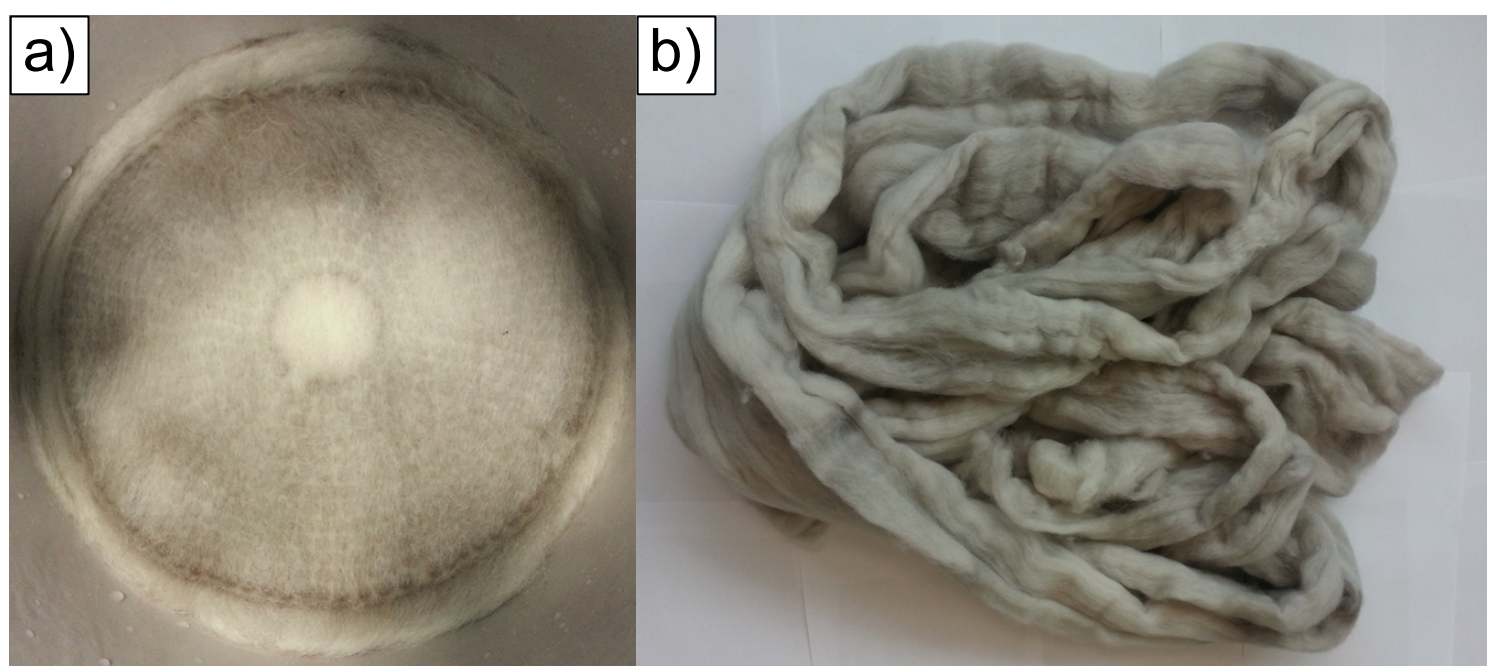

Figure 5.56 Images of the pH 2.4 TSC-light grey loose wool composite synthesised in the BG reactor: the top face of the dyepack immediately after the dyeing reaction (a) and the resulting composite (b).

The dark grey coloured areas on the composite correspond with regions where the wool fibres are relatively tightly compressed in the dyepack. These regions occur where the wool is pushed up into the holes in the stamp, where the flow of dye liquor is forced through a smaller area (through a relatively faster flowrate). These areas where the wool is more tightly packed are thought to act as filters, which halt the movement of large agglomerates of TSC-Au NPs that were circulating the in the dye solution. This phenomenon is not observed in the TA-mauve synthesis, likely because the TA-Au NPs do not aggregate in the dye solution, and as such are able to pass through these areas without being filtered out of the dye solution. Another possible effect is the increased turbulence of the flow of the colloid around the wool:solid support interface, which would increase the agglomeration of TSC-Au NPs in these areas.

The $\mathrm{pH}$ 2.4-2.8 composites all exhibited dark grey areas near the holes in the stamp. The pH 2.0 and 2.2 composites likely would also have exhibited a similar colour distribution if the uptake had lasted longer than $1 \mathrm{~min}$. The relatively weaklystabilised TSC-grey colloid is considered not to be able to withstand the relatively high flowrates and pressure of the BG dyeing reactions, thus aggregating to a size which can be filtered from the solution. Next, a stamp with wider holes was used to attempt to reduce the colour variation in TSC-grey composites. 


\section{New Stamp}

To investigate the cause of the dark grey areas on the top face of the dyepack in BG dyeing reactions, a new stamp was developed and implemented in the TSC-grey synthesis of top form wool composites (Figure 5.57 (a)). The equivalent concentration of the TSC-grey colloid was used in the following experiment, as the darker grey colour was anticipated to show colour differences in the dyepack more clearly. The reaction parameters were equivalent to the previous TSC-light grey syntheses, and a pH 2.4 glycine buffer and TSC-grey colloid was adjusted to 2.4.

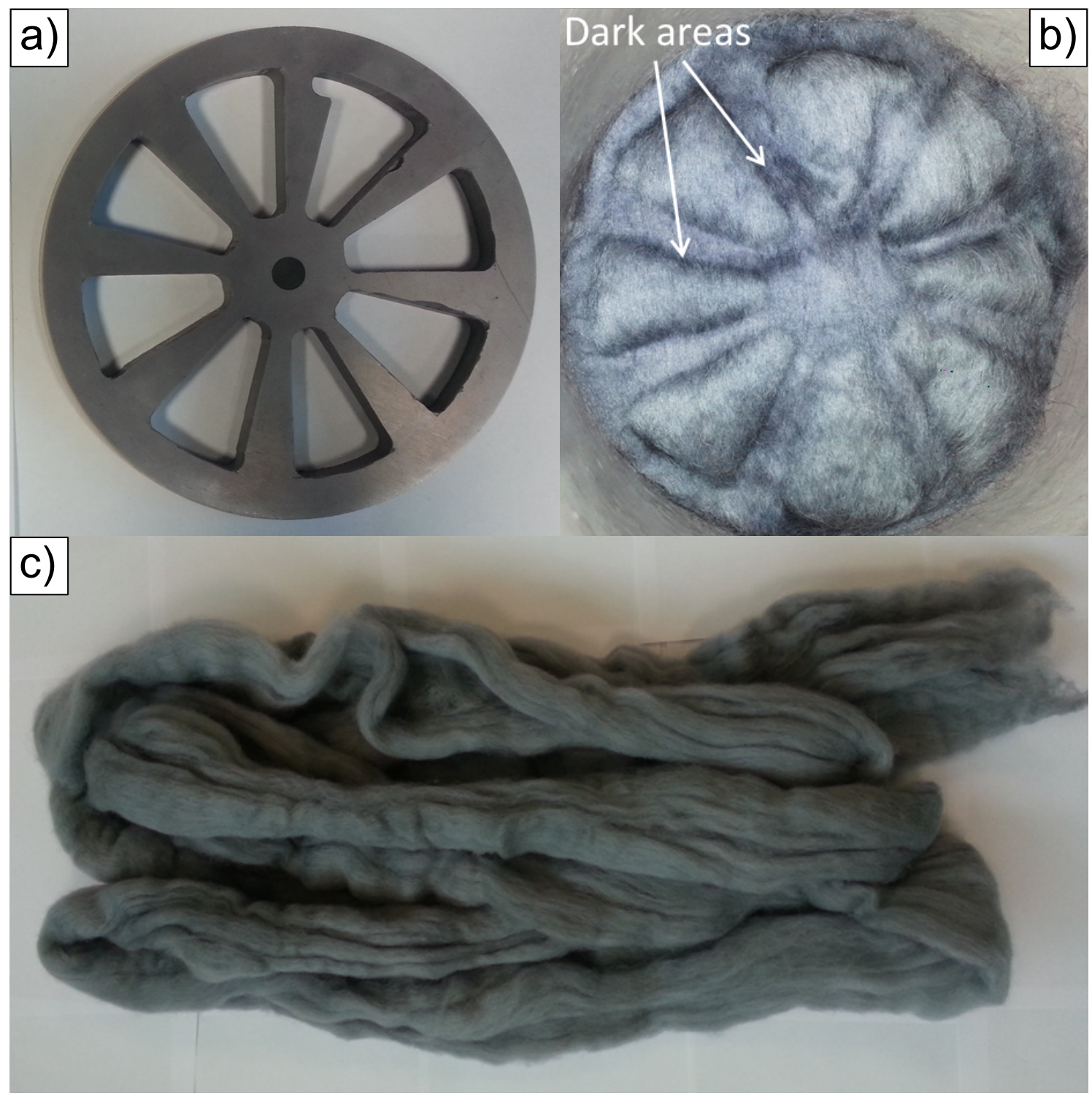

Figure 5.57 Images of a new stamp (a), the wet TSC-grey composites in the BG reactor post-dyeing (b) and the resulting composite (c).

The new stamp exhibits larger, triangular-shaped spaces through which the dye solution flows. Figure 5.57 (b) shows the top face of the dyepack after the TSCgrey dying reaction. Again, the image of the TSC-grey composite remaining in the 
$\mathrm{BG}$ reactor displays dark areas at the edges of the spaces in the stamp, while the other areas are a relatively light grey colour. The areas that outline the spaces in the stamp likely constitute tightly compressed wool fibres, which are again considered to act as a filter to remove large agglomerates of TSC-Au NPs from dye solution. The turbulence of the flow through these areas would again promote the agglomeration of TSC-Au NPs. The presence of the large agglomerates of TSC-Au NPs explains the dark grey colour of these areas.

The resulting TSC-grey loose wool composite synthesised with the new stamp is presented in Figure 5.57 (c). The composite is a very uniform grey colour, apart from the dark grey areas near the top of the dyepack. Thus, using the new stamp presents a significant improvement in the colour uniformity of the TSC-grey composites.

\section{Sacrificial Filter}

Many methods were considered to prevent the undesirable dark grey coloured areas that are observed on the top face of the dyepack. It was considered that if the agglomeration of the TSC-grey colloid in the solution could be prevented, then the relatively compressed areas would not filter out the larger agglomerates. However, the rapid flowrates of the pressurised flow reactors proved too harsh to prevent the agglomeration of TSC-Au NPs in the BG reactors. This approach provided no improvement. Different types and amounts of wool dyeing auxiliaries were also investigated in the dyeing reactions, but these did not prevent the agglomeration of TSC-Au NPs (too high a concentration of auxiliary agents causes their coagulation and the destabilisation of the colloid). This phenomenon was also observed in dyeing reactions involving the relatively gentle flowrates of the Benchtop flow reactor, where $4 \mathrm{~g} \mathrm{~L}^{-1}$ Albaflow FFA foamed and collapsed the relatively robust TA-mauve colloid (detailed in Section 5.4.1). The agglomeration of TSC-Au NPs during a dyeing reaction is shown Figure 5.58, where the TSC-grey colloid changed from deep-red to grey at the beginning of the uptake.

As the TSC-grey colloid could not be sufficiently stabilised to prevent its agglomeration during dyeing reactions in the pressurised flow reactors, a sacrificial filter was designed. The filter was composed of felted merino yarn, and was placed at the top of the dyepack to prevent the dark grey colouring of the top of the dyepack. Ideally, the agglomeration of TSC-Au NPs would be confined to the surface of the wool fibres and not occur in the dye solution. Although the use of the sacrificial filter has an associated loss of gold, it was considered such wastage of gold would be an acceptable outcome if it resulted in uniformly coloured composites. 


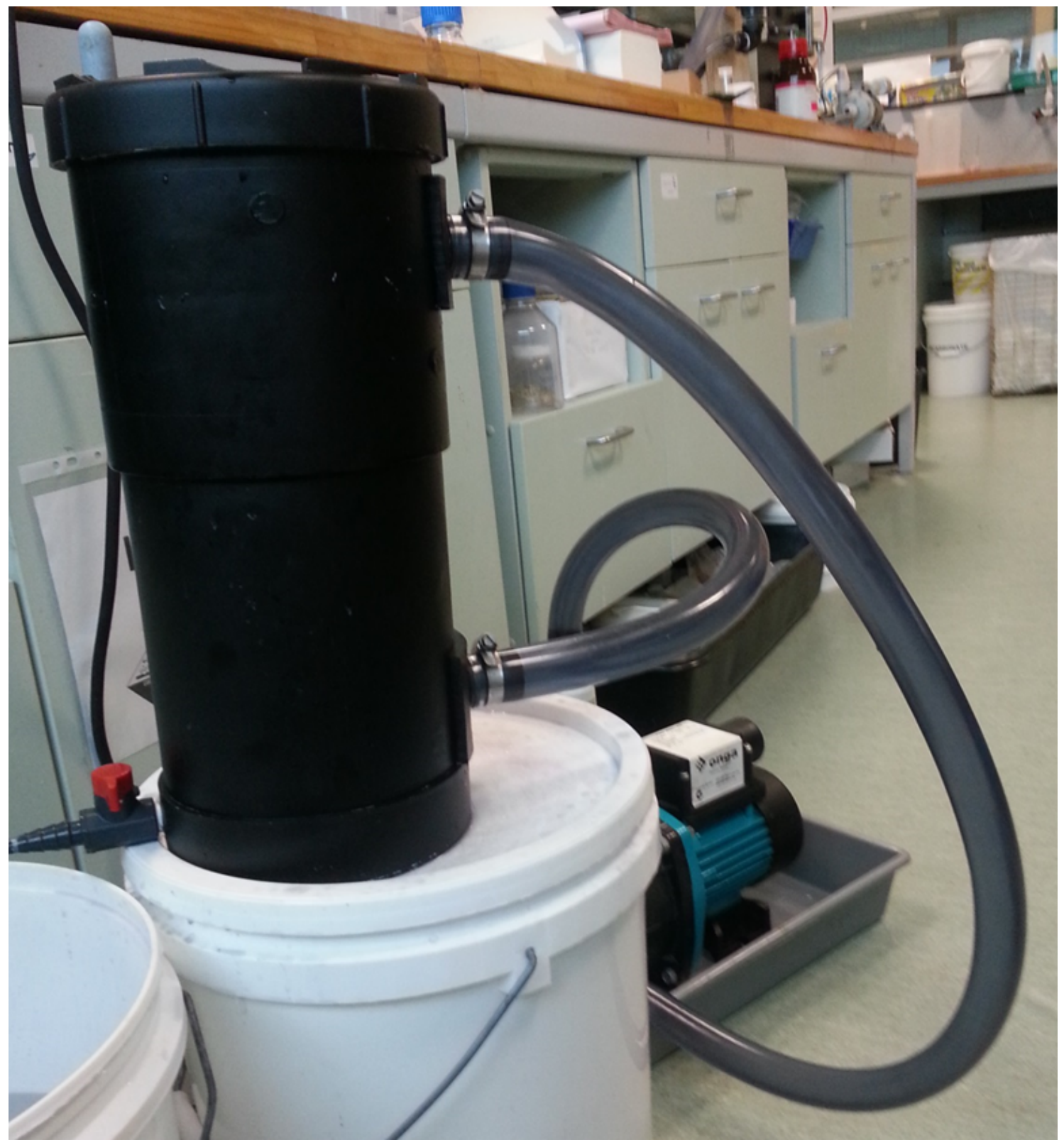

Figure 5.58 Image of the circulation of a TSC-grey colloid during the dyeing reaction in the $\mathrm{BG}$ reactor (approximately 1 min after the beginning of the reaction). 
The TSC-grey loose wool composites synthesised in the pressurised flow reactors with the sacrificial filter are shown in Figure 5.60. A pH 2.4 glycine buffer with $1 \mathrm{gL}^{-1}$ Albaflow FFA was cycled around the system for $15 \mathrm{~min}$ followed by the standard concentration of the TSC-grey colloid also at $\mathrm{pH} 2.4$. At $\mathrm{pH} 2.4$, the uptake of TSC-Au NPs was complete in 5 min, which equates to approximately 110 circulations of the dye solution during the reaction. The sacrificial filter in the BG reactor has produced the most evenly coloured TSC-grey composites of $360 \mathrm{~g}$ size to date. The filter has absorbed a significant quantity of TSC-Au NPs during the dye reaction, as is seen in Figure 5.59.

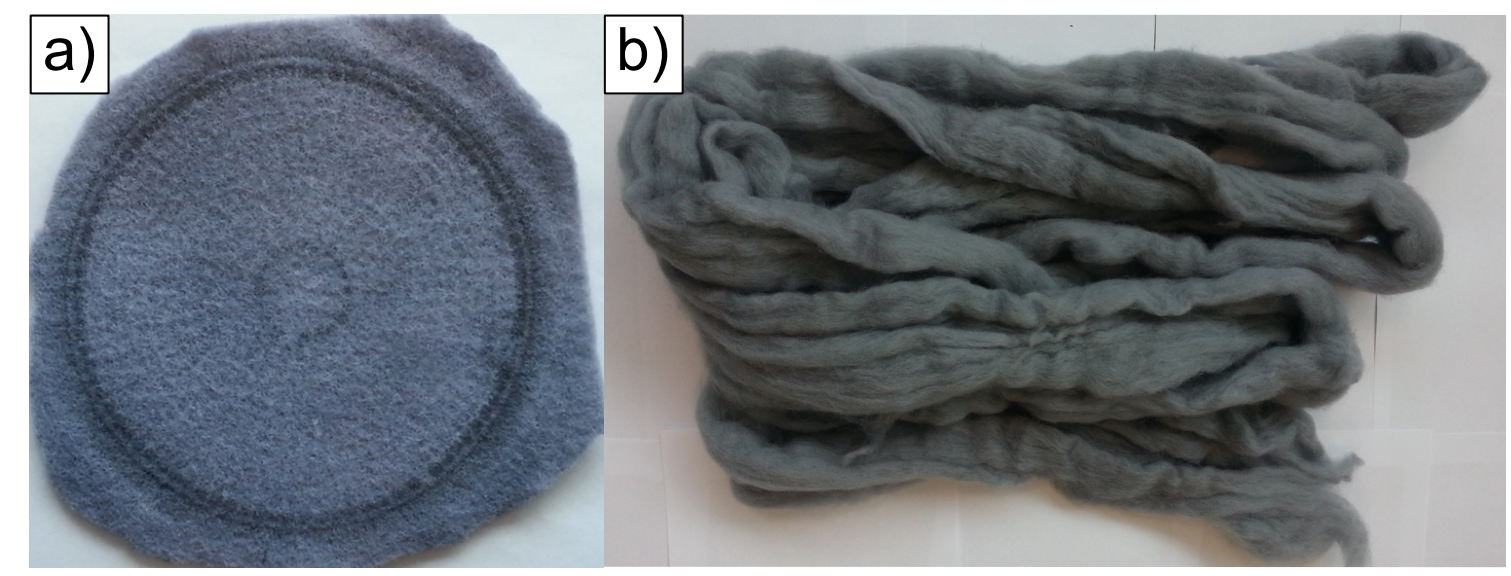

Figure 5.59 Images of the sacrificial filter used in the synthesis of the TSC-grey loose wool composite in the BG reactor after the dyeing reaction (a) and the resulting composite (b).

The successful use of the sacrificial filter in the TSC-grey synthesis of a $360 \mathrm{~g}$ TSCgrey loose wool composite lead to its use in the Full Pint (FP) reactor. This involved $660 \mathrm{~g}$ of loose wool being dyed with the equivalent concentration of the TSC-grey colloid (10 L, 1:16.7 wool to liquor ratio, $\mathrm{pH} 2.4,1 \mathrm{~g} \mathrm{~L}^{-1}$ Albaflow FFA). The resulting composite is shown in Figure 5.60 (b). The uptake time was approximately $10 \mathrm{~min}$. Again the sacrificial filter prevented the dark-grey colouration at the top of the dyepack. The $600 \mathrm{~g}$ composite synthesised in the FP reactor is not as uniformly coloured as the composite in the BG reactor, the top area of the dyepack appears to be a slightly lighter shade of grey than the middle and bottom of the dyepack. This is understandable given that many of the nanoparticles in the dye solution will be absorbed before they come into contact with the top area of the dyepack. The middle of the composite is the darkest grey colour, and may be due to the uneven packing of the wool in the FP reactor.

The ability to synthesise TSC-grey composites of 360-660 g size is a hugely promising and exciting result. This is where the research with the pressurised flow reactors concludes to date. As of this writing, strategies are currently being employed to 


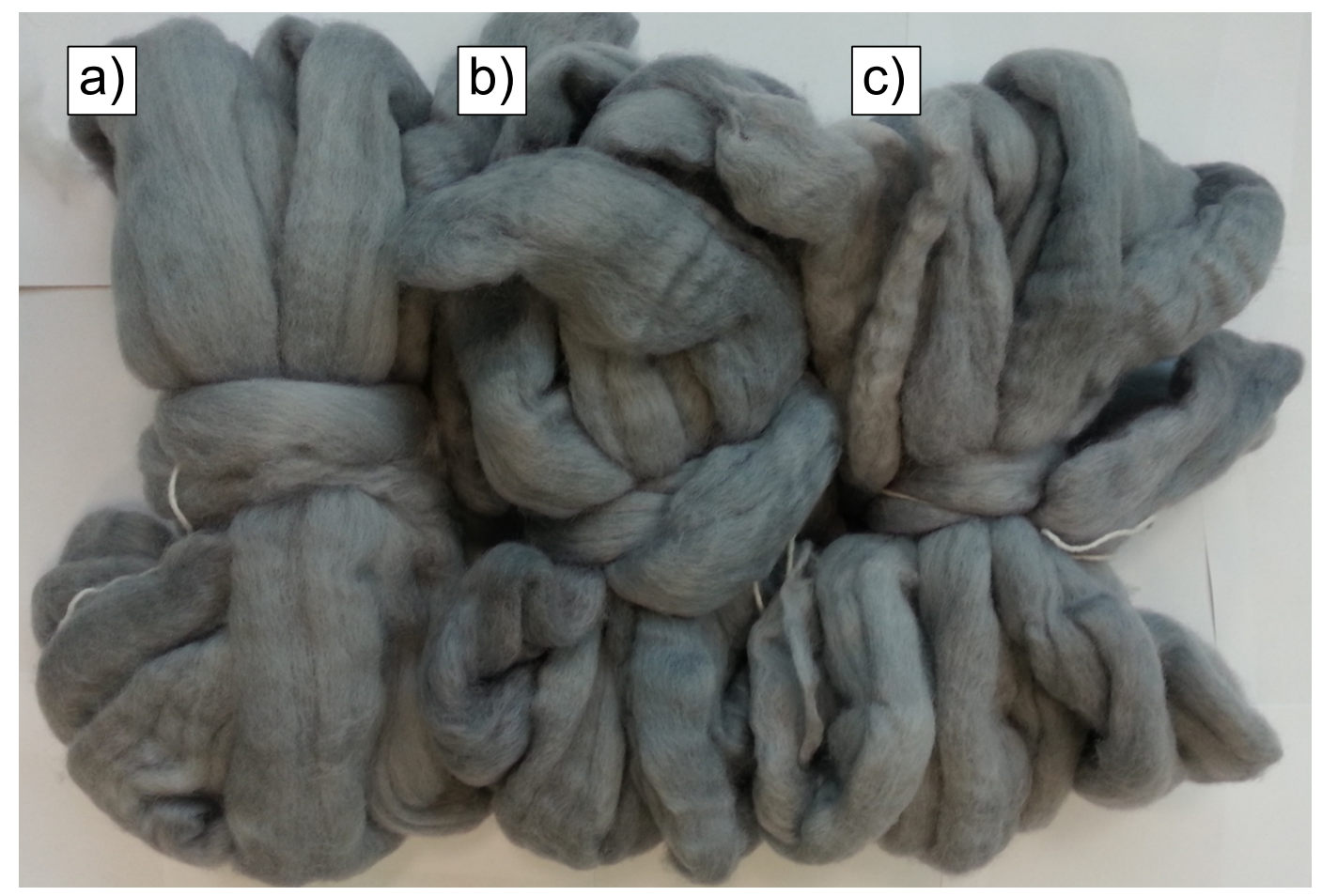

Figure 5.60 A 660 g TSC-grey loose wool composites synthesised with the sacrificial filter in the Full Pint (FP) reactor, where the wool relates to the bottom (a), middle (b) and top of the dyepack (c).

enhance the uniformity of the TSC-grey composites by increasing the rate of dye circulation, and looking to alternative measures of preventing the agglomeration of TSC-Au NPs in the dye solution. Additionally, the process has since been extended to colour $20 \mathrm{~kg}$ of carpet yarn by Ahmet Kitap and Dr Kerstin Lucas, for the manufacture of luxury rugs. The dyeing runs were undertaken in the FP reactor with $500 \mathrm{~g}$ hanks of the carpet yarn, and was used to synthesise light pink, mauve, light grey and middle grey composites with impressive colour uniformity.

\subsection{Conclusions}

This chapter has detailed extensive research into the large-scale synthesis of gold nanoparticle-wool fibre composites. First, large volumes of gold nanoparticle colloids were synthesised. A reproducible approach for the synthesis of 20-90 L of TA- and TSC-stabilised gold colloids was developed with the use of the barrel reactor. The difficulties of reproducing the synthesis of the TSC-grey colloid were alleviated by incorporating higher amounts of excess TSC into the synthesis and maximising the uniformity of heating and mixing of the reactants.

The gold nanoparticle colloids were used to colour top form wool, fine merino yarns 
and felted merino yarns with the following dyeing methods:

- Static dyeing

- Hank dyeing

- Conventional package dyeing

- Dyeing with novel flow reactors

Static dyeing reactions provided an excellent starting point for the development of the nanotechnology and contributed to the knowledge base for scale-up procedures. The uniform colouring of top form wool samples of $>100 \mathrm{~g}$ was not possible with static dyeing procedures. The top form wool was agitated during the dyeing process to attempt to increase the colour uniformity, however, this engendered significant felting of the wool fibres. The Cariaggi ex-situ composites were used to produce demonstration prototypes.

Next, hank dyeing procedures were replicated in the laboratory to colour kilogram hanks of Ascend felted yarn with remarkable colour uniformity. The modified hank dyeing of felted yarn involved a combination of dye circulation, physical agitation of the hanks and the incorporation of wool dyeing auxiliaries into the dyeing reactions. The felted yarn composites were used to fabricate luxury rugs for presentation at international expositions.

Fine merino yarns were dyed in an industrial pilot-scale package dyeing reactor at Tararua Yarns Ltd. The colour uniformity of TA-mauve TU yarn composites was improved by using different amounts of Albaflow FFA and manipulating the flow speed and direction. Unfortunately, the closing down of the wool dyeing plant occurred before the TSC-grey method could be optimised.

This led to the development of a series of novel flow reactors for use in the laboratory. These flow reactors reactors replicated the pumping of dye liquor around a mass of wool that was tightly packed together that occurs in the industrial package dyeing of wool. The Benchtop flow reactor investigated the influence of wool dyeing auxiliaries on the rate of uptake of gold nanoparticles to $5 \mathrm{~g}$ samples of TU yarn. The first flow reactor was used to colour $50 \mathrm{~g}$ quantities of TU yarn, however, this leaked when faster flowrates or larger masses of wool were dyed.

Finally, pressurised flow reactors, which employed relatively low volumes of dye liquor and relatively fast rates of dye circulation. These reactors could withstand 
high internal pressure, and resulted in uniformly coloured TA-mauve composites. The reproducibility of the TSC-grey method was enhanced by optimising the reaction $\mathrm{pH}$ and employing a sacrificial filter prevent discolouration near the top of the dyepack.

Currently we have a reproducible process methodology for the production of ex-situ composites of up to $300-660 \mathrm{~g}$ size in the pressurised flow reactors. The reactors are suitable for the colouring of top form wool, felted merino yarns and fine merino yarns. Future work is aimed at constructing larger pressurised flow reactors that can be used to uniformly colour kilogram quantities of wool. If additional research or investment funding is obtained, then the scale of this will further be increased.

Thus far, uniformly coloured TA-mauve composites can readily be produced, however the TSC-grey method requires further research to understand and control the agglomeration of TSC-Au NPs, and hence the grey colour. Additionally, the washfastness properties of the ex-situ composites is a concern in regard to their commercial potential. Research into improving the washfastness properties of the ex-situ procedure is next presented in Chapter 6 . 


\section{Chapter 6}

\section{Improving the Washfastness Properties of Ex-situ Composites}

The ex-situ procedure was developed as a relatively robust and comparatively costeffective alternative to the in-situ procedure for the large-scale synthesis of gold nanoparticle-wool fibre composites. The laboratory-scale optimisation and scale-up of the ex-situ procedure was previously detailed in Chapters 4 and 5 respectively. The synthesis of ex-situ composites uses only approximately $10 \%$ of the $\mathrm{AuCl}_{4}^{-}$ that is used in the in-situ procedure. However, ex-situ composites also have poorer washfastness properties compared to in-situ composites.

In-situ composites exhibit covalently bound gold nanoparticles both on the surface and throughout the interior of the wool fibres. In contrast, ex-situ composites encompass gold nanoparticles that are attached to the surface of the wool fibres only. When ex-situ composites are washed with detergents, some of the gold nanoparticles leach from the composites into the wash solutions. This implies that the gold nanoparticles are not covalently bound to the wool fibre matrix. The ex-situ procedure exploits a $\mathrm{pH}$ related driving force for the absorption of gold nanoparticles, and thus, the nanoparticles are considered to be electrostatically attached to the wool.

The leaching of gold nanoparticles during washing processes is a significant problem that relates to the commercialisation of the ex-situ composites. The optimal method of overcoming this issue was believed to be improving the washfastness properties of the composites (rather than, for example, developing a detergent that avoids the leaching of gold nanoparticles). As such, a range of experiments were carried out with the aim of improving the washfastness properties of the ex-situ composites. The experiments involved chemically treating wool fibres before or after the dye- 
ing, or utilising alternative reducing and stabilising agents in the gold nanoparticle synthesis. The experiments are summarised in Table 6.1 below.

Table 6.1 Types of washfastness experiments.

\begin{tabular}{ll}
\hline Treatment & Description \\
\hline Chemical Pre-treatments & Wool fibre modification prior to dyeing \\
Chemical Post-treatments & $\begin{array}{l}\text { Encapsulating gold nanoparticles on the wool } \\
\text { fibre surface after dyeing }\end{array}$ \\
Alternative reducing & $\begin{array}{l}\text { Stabilising Au NPs with reactive groups that } \\
\text { and stabilising agents }\end{array}$ \\
\hline \hline
\end{tabular}

If the washfastness properties of the ex-situ composites could not be sufficiently improved, then the nanocomposites would have to be sold with the caveat of washing with water only. As the washing of fine merino garments and rugs is often necessarily gentle, there is still significant market interest in luxury products that are not completely colourfast. However, improving the washfastness properties of the gold nanoparticle-wool fibre composites was deemed to be very important, and necessitated both the research presented in this chapter and the research that was carried out to attempt to reduce the gold content of the in-situ procedure (that was presented in Chapter 3).

Chapter 6 begins by characterising the washfastness properties of TA-mauve, TSCpink and TSC-grey composites of Cariaggi wool and TU yarn in Section 6.1. The use of chemical pre- and post-treatments to attempt to improve the washfastness properties of the ex-situ composites are described in Section 6.2. Finally, Section 6.3 details the use of alternative reducing and stabilising agents to attempt to synthesise gold nanoparticles that can covalently bond to wool.

\subsection{Washfastness of Ex-situ Composites}

The TA-mauve, TSC-pink and TSC-grey methods were commonly employed in the laboratory optimisation experiments and scale-up procedures that were detailed in Chapters 4 and 5, respectively. As such, the washfastness properties of Cariaggi wool and TU yarn composites coloured with TA- and TSC-stabilised gold nanoparticles are first presented.

The washing processes utilised a consumer wool fabric washing product called "Kindness wool wash". Kindness wool wash contains a mixture of anionic, cationic and non-ionic surfactants, and is used to wash wool in cold tap water without damaging 
the wool fibres. The concentration of Kindness wool wash that was used in washfastness tests was the commercially recommended; $3.125 \mathrm{~mL} \mathrm{~L}^{-1}$, or approximately $0.3 \mathrm{wt} \%$.

Two different washing processes were employed in the washfastness experiments. One method involved soaking a composite sample in the minimal amount of the recommended dilution of Kindness wool wash $(0.5 \mathrm{~g}$ in a $10 \mathrm{~mL}$ wash solution, wool to liquor ratio of 1:20), and agitating the sample for $24 \mathrm{~h}$ on the orbital shaker table. The UV-vis spectra of the resulting wash solutions were measured to determine the amount of leaching of gold nanoparticles from the composites.

The alternative washing process involved agitating composite samples (typically of 1-2 g mass) in excess Kindness wool wash, and comparing the CIE colour coordinates of the composites before and after the washing process. For this, either the Chiltern shaker or the dunking machine were used to simulate hand washing; involving shaking the composites in $500 \mathrm{~mL}$ wool wash for $24 \mathrm{~h}$ or dunking the composites in $20 \mathrm{~L}$ wool wash solution for $120 \mathrm{~min}$. The Chiltern shaker was used early on in the $\mathrm{PhD}$ project, before the dunking machine was developed, and the washing processes are described in greater detail in Section 2.2.5.

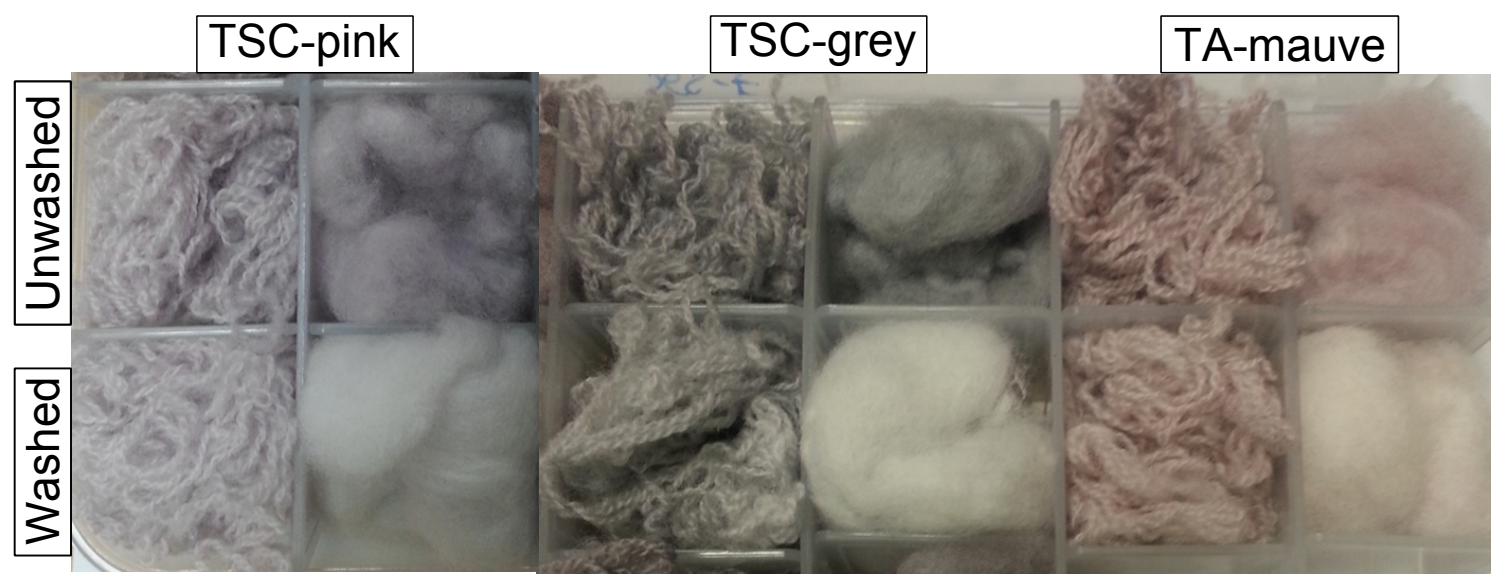

Figure 6.1 Cariaggi and TU yarn ex-situ composites of $1 \mathrm{~g}$ size before and after washing on the Chiltern shaker for $24 \mathrm{~h}$.

TA-mauve, TSC-pink and TSC-grey composites of Cariaggi wool and TU yarn before and after washing on the Chiltern shaker for $24 \mathrm{~h}$ are presented in Figure 6.1. The synthesis of the composites involved the standard methods (which were outlined in Section 2.2.2), without the use of wool dyeing auxiliary agents. Each of the Cariaggi composites display some loss of colour after the washing process, and the TU yarn composites show only a slight colour change. The Cariaggi composites are comparatively brighter than the TU yarn composites (the TSC-grey Cariaggi composite appears almost completely undyed), which indicates that a higher amount of gold nanoparticles have leached into the wash solutions. The Cariaggi composites 
are also significantly matted after the washing process, due to the agitation. The wet Cariaggi wool fibres are considered to frequently rub together during the washing, to cause the matting of the fibres. This rubbing together of the fibres likely causes more significant displacement of gold nanoparticles on the Cariaggi composites fibres compared to the TU yarn, which is not significantly matted. In practice with hand washing of sensitive garments this matting is largely avoided.

The CIE colour coordinates of the Cariaggi ex-situ composites before and after the washing process are shown in Table 6.2. The brightness and yellowness values of each composite are markedly higher for the Cariaggi ex-situ composites after the washing process. Additionally, the redness values of the TA-mauve and TSC-pink Cariaggi composites are far lower and the yellowness values of all of the composites are significantly higher after the washing. The changes in CIE colour coordinates all indicate a significant reduction in the number of gold nanoparticles on the Cariaggi composites as a result of the washing process.

Table 6.2 CIE colour coordinates of Cariaggi ex-situ composites before and after washing on the Chiltern shaker for $24 \mathrm{~h}$.

\begin{tabular}{ccccccc}
\hline Method & \multicolumn{2}{c}{ TA-mauve } & \multicolumn{2}{c}{ TSC-pink } & \multicolumn{2}{c}{ TSC-grey } \\
\hline Washing & before & after & before & after & before & after \\
\hline L* $^{*}$ (brightness) & 65.50 & 82.38 & 72.41 & 86.16 & 65.21 & 80.68 \\
$\mathrm{a}^{*}$ (redness) & 7.01 & 2.90 & 4.61 & 0.58 & -0.45 & -0.60 \\
$\mathrm{~b}^{*}$ (yellowness) & -0.87 & 5.14 & -1.26 & 3.28 & -2.51 & 1.87 \\
\hline \hline
\end{tabular}

The CIE colour coordinates of TU yarn ex-situ composites are shown in Table 6.3. While the $\mathrm{L}^{*}, \mathrm{a}^{*}$ and $\mathrm{b}^{*}$ values of each of the composites have all changed significantly as a result of the washing, the magnitude of the change is significantly less for the TU yarn composites, compared to that of the Cariaggi composites. The TAmauve TU yarn composite exhibits a far smaller increase in brightness and decrease in redness value than the TSC-pink and TSC-grey composites. This may be due to the stronger stabilising nature of TA preventing the leaching of a greater number of nanoparticles from the composites, compared to the relatively weakly stabilising TSC.

Table 6.3 CIE colour coordinates of TU yarn ex-situ composites before and after washing on the Chiltern shaker for $24 \mathrm{~h}$.

\begin{tabular}{ccccccc}
\hline Method & \multicolumn{2}{c}{ TA-mauve } & \multicolumn{2}{c}{ TSC-pink } & \multicolumn{2}{c}{ TSC-grey } \\
\hline Washing & before & after & before & after & before & after \\
\hline $\mathrm{L}^{*}$ & 62.33 & 66.66 & 67.86 & 74.50 & 56.27 & 65.86 \\
$\mathrm{a}^{*}$ & 6.03 & 5.81 & 4.11 & 4.47 & 0.14 & -0.24 \\
$\mathrm{~b}^{*}$ & 0.19 & 2.08 & 0.05 & 1.16 & -1.76 & -0.84 \\
\hline \hline
\end{tabular}


SEM analysis of the ex-situ TU yarn composites after washing was undertaken to investigate the effect of this washing process on the distribution of gold nanoparticles on the surface of the composites. SEM micrographs of TA-mauve, TSC-pink and TSC-grey after washing are presented in Figure 6.2.

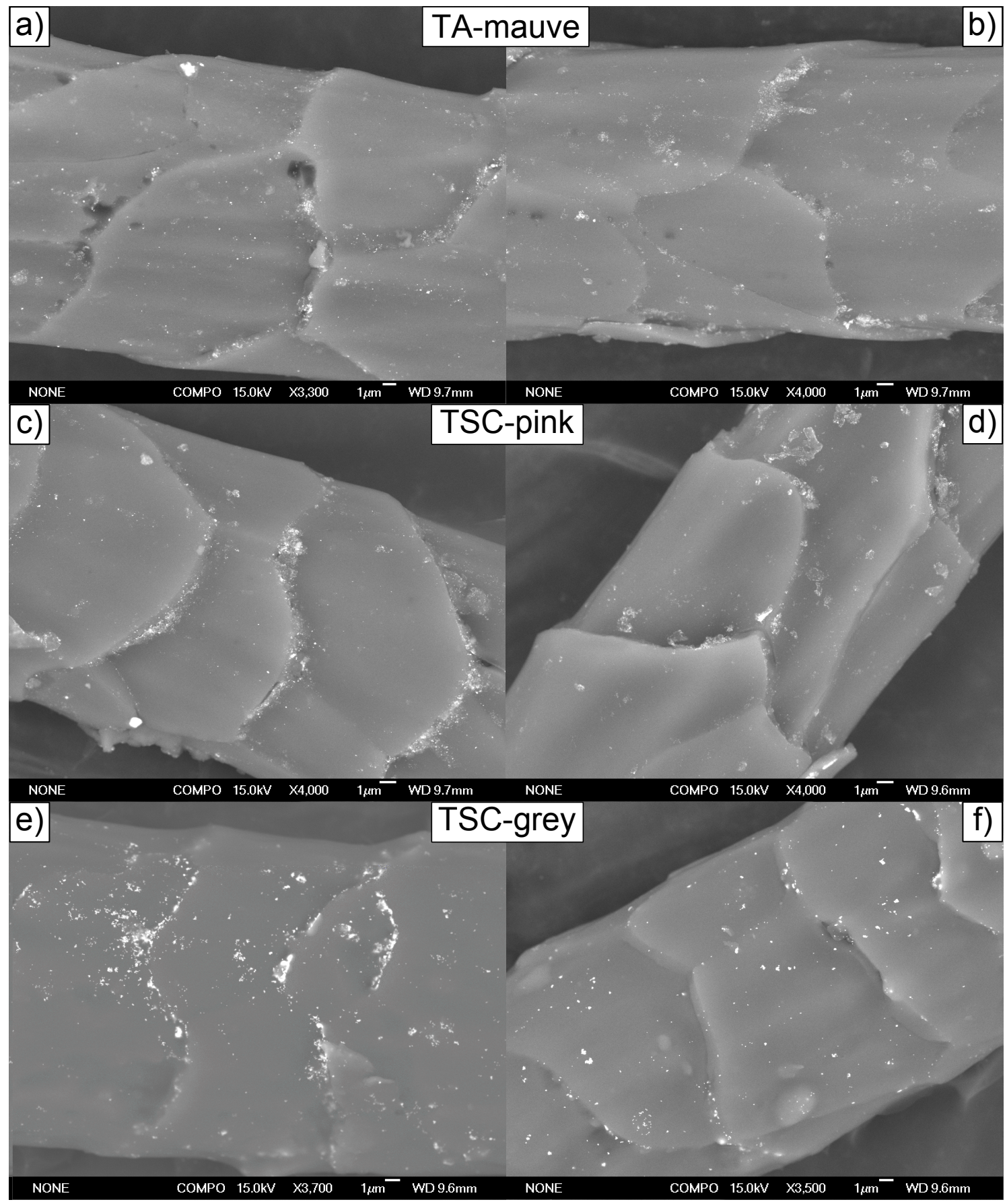

Figure 6.2 SEM micrographs in backscatter (BS) mode of TA-mauve (a)-(b), TSC-pink (c)-(d) and TSC-grey (e)-(f) TU yarn composites after washing on the Chiltern shaker for $24 \mathrm{~h}$.

The washed composites appear to exhibit a distribution of gold nanoparticles that is typical of ex-situ composites that have not been washed. There is considerable variation in the amount of gold nanoparticles present on different composite fibres, 
which is also seen in unwashed composites. This is particularly easily observable in the SEM images of the TSC-pink and TSC-grey composites, where the SEM micrographs on the left side (Figure 6.2 (c) and (e) exhibit comparatively more gold nanoparticles on the surfaces compared to those in Figure $6.2(\mathrm{~d})$ and (f). This variation in gold distribution may be due to some fibres being more readily accessible to the surfactants in the wash solution (for example those located on the outside of the TU yarn structure. Alternatively, it may be a function of the variability of gold nanoparticle distribution that is typically observed in the ex-situ procedure. It was also anticipated that the gold nanoparticles located near the junctions between the cuticle scales would be less likely to be displaced by surfactants than those present on the other areas of the cuticle scales (due to greater contact between the gold nanoparticles and wool fibre at the cuticle edges). However, no such preference was observed in Figure 6.2.

The following experiment investigates the leaching of gold nanoparticles from TAmauve and TSC-pink composites of Cariaggi, Ashford and Crossbred wool with the minimal amount of Kindness wool wash. This involved soaking the composites in Kindness wool wash (wool to liquor ratio of 1:20) overnight on the orbital shaker table. The process was repeated 3 times to determine the change in the leaching of gold nanoparticles over time. Figure 6.3 presents the composites of wool in top form before and after the 3 washing steps. The washed composites are lighter as a result of the washing; however, they are also significantly darker than Cariaggi composites in Figure 6.1 (that were washed for $24 \mathrm{~h}$ ). It is also worth noting that these composites are relatively less matted, as the this washing process is comparatively gentle.

UV-vis spectra of the wash solutions of the TA-mauve and TSC-pink Cariaggi composites are presented in Figure 6.4. The SPR absorbance of TSC-stabilised gold nanoparticles (between $500-600 \mathrm{~nm}$ ) is clearly observed in each of the wash solutions of the TSC-pink composites. The intensity of the SPR absorbance appears to decrease from the first to the third wash solution, which indicates that fewer TSC-Au NPs leach from the composites in sequential washing processes. In contrast, the UV-vis spectra of the TA-mauve wash solutions display significantly less evidence of the SPR absorbances of TA-Au NPs. This indicates that relatively fewer gold nanoparticles are leaching from the TA-mauve Cariaggi composites compared to the TSC-pink Cariaggi composites. This is understandable considering that the TA-mauve composites are also considerably darker after the washing (as is seen in Figure 6.3). 


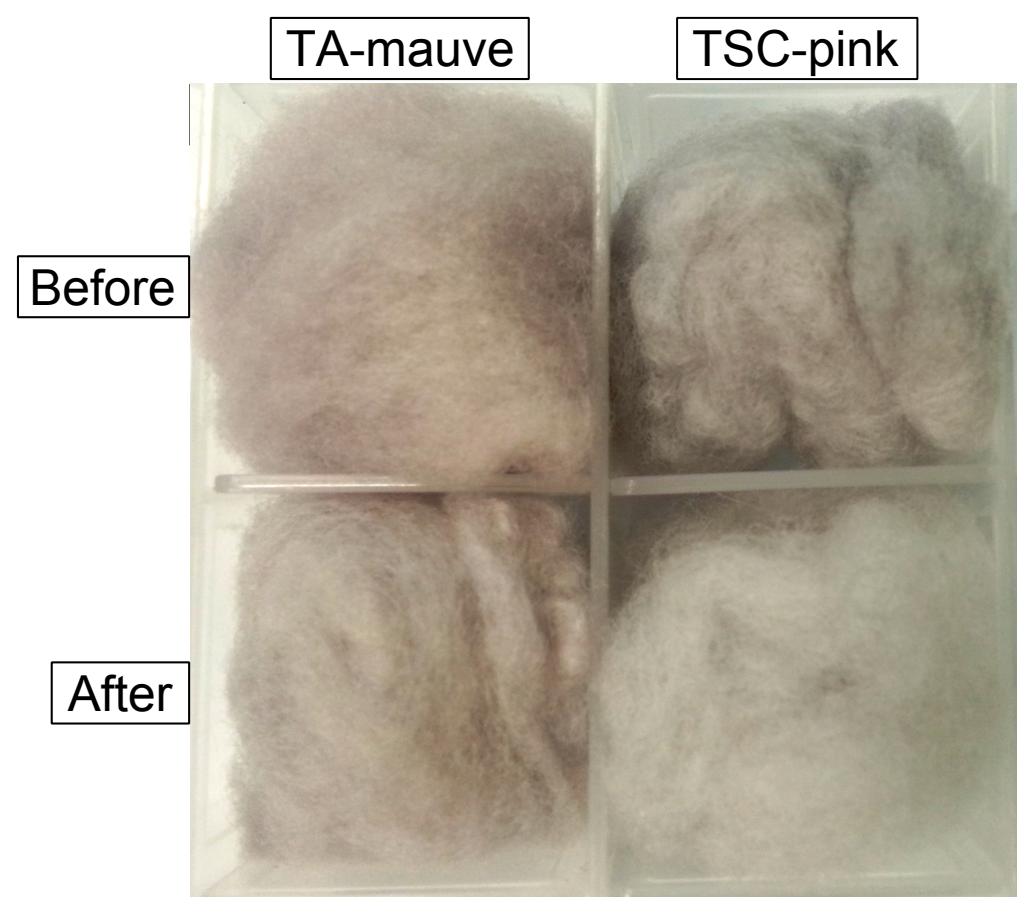

Figure 6.3 TA-mauve and TSC-pink composites of Cariaggi wool, before and after washing (three times with a 1:20 wool to liquor ratio of Kindness wool wash).

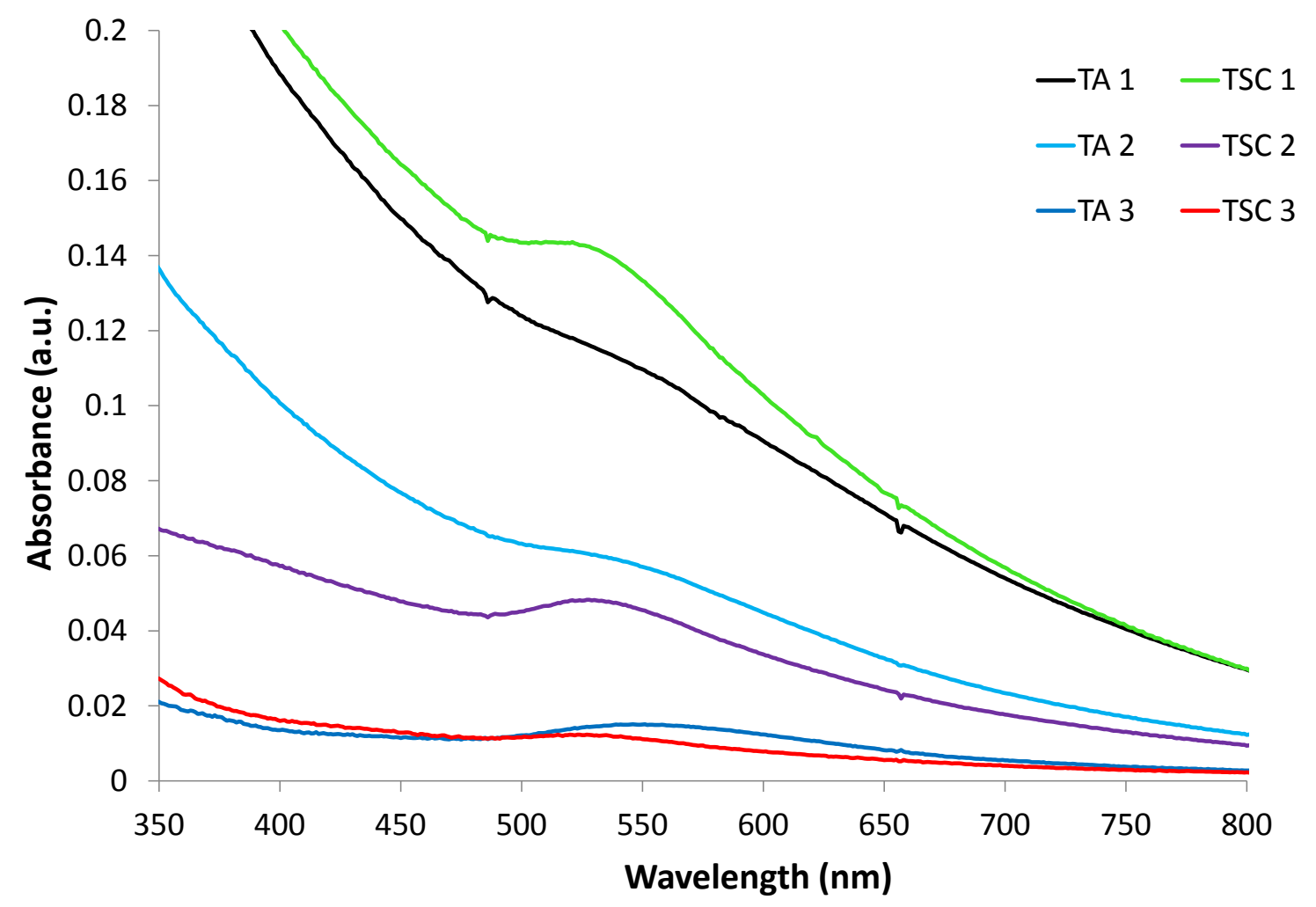

Figure 6.4 UV-vis spectra of solutions resulting from the washing of TA-mauve and TSC-pink composites of Cariaggi wool 3 times.

This section has shown that significant leaching of gold nanoparticles occurs when washing TA-mauve, TSC-pink and TSC-grey composites of wool in top form and merino yarn. The characterisation techniques that are used to determine the washfastness properties of ex-situ composites were also introduced. The experiments 
have shown that the washfastness properties of the composites are influenced by the method of gold nanoparticle synthesis and the type of wool fibres that are dyed.

\subsection{Chemical Treatments}

A range of chemical treatments were employed in attempts to improve the washfastness properties of ex-situ composites, by modifying the chemistry of the wool fibres either before or after the dyeing process. The optimal outcome of these experiments was gold nanoparticles that were covalently bonded to the wool fibres.

\subsubsection{Chemical Pre-treatments}

Chemical pre-treatments were used to chemically modify wool fibres prior to being dyed with gold nanoparticles. The range of chemicals that were employed in the pre-treatment experiments and the reaction conditions of their use are outlined in Table 6.4 below.

Table 6.4 Chemical reagents used for pre-treating wool fibres prior to dyeing with gold nanoparticles.

\begin{tabular}{lcccc}
\hline Reagent & Formula & Conc (wt \%) & Temp & $\mathrm{pH}$ \\
\hline Mercaptosuccinic acid (MSA) & $\mathrm{C}_{4} \mathrm{O}_{4} \mathrm{H}_{6} \mathrm{~S}$ & 1 & $\mathrm{RT}-50{ }^{\circ} \mathrm{C}$ & 3.2 \\
Thioglycollic acid (TGA) & $\mathrm{C}_{2} \mathrm{O}_{2} \mathrm{H}_{4} \mathrm{~S}$ & 1 & $\mathrm{RT}-50{ }^{\circ} \mathrm{C}$ & 3.2 \\
Sodium metabisulfite & $\mathrm{Na}_{2} \mathrm{~S}_{2} \mathrm{O}_{5}$ & 0.2 & $\mathrm{RT}-85^{\circ} \mathrm{C}$ & $2-8$ \\
$\begin{array}{l}\text { 1,3-propane dithiol } \\
\text { Polyethylenimine (PEI) }\end{array}$ & $\mathrm{C}_{3} \mathrm{H}_{8} \mathrm{~S}_{2}$ & 0.05 & $60{ }^{\circ} \mathrm{C}$ & $2-10$ \\
$\begin{array}{l}\text { Polydiallyldimethylammonium } \\
\text { chloride (PDADMAC) }\end{array}$ & $\left(\mathrm{C}_{2} \mathrm{H}_{5} \mathrm{~N}\right)_{n}$ & 1 & $\mathrm{RT}-50^{\circ} \mathrm{C}$ & $2-11$ \\
\hline \hline
\end{tabular}

As was mentioned in Section 1.1.2, sodium metabisulfite $\left(\mathrm{Na}_{2} \mathrm{~S}_{2} \mathrm{O}_{5}\right)$ is commonly used in the industrial processing of wool, sometimes after the oxidative shrinkproofing or bleaching of wool. ${ }^{7} \mathrm{Na}_{2} \mathrm{~S}_{2} \mathrm{O}_{5}$ causes the sulfitolysis of cystine residues, and results in thiol and S-sulfonate anions in place of the disulfide linkage (this is illustrated in Equation 6.1, where $\mathrm{W}$ is a polypeptide chain in wool). These functional groups were considered to be more reactive to gold nanoparticles than the disulfide bridge. The interaction with the gold nanoparticles could involve direct bonding to the stabiliser of the gold nanoparticle, or to the gold directly, after displacing the stabiliser.

$$
\mathrm{WSSW}+\mathrm{SO}_{3}^{2-} \rightleftharpoons \mathrm{WS}^{-}+\mathrm{WSSO}_{3}^{-}
$$


The use of MSA, TGA and 1,3-propane dithiol was proposed to achieve the reductive cleavage of the disulfide linkages in cystine residues of the wool fibre. This is illustrated in Equation 6.2, and results in two thiol groups in place of the disulfide linkage. ${ }^{7}$

$$
\begin{aligned}
& \mathrm{WSSW}+\mathrm{RS}^{-} \rightleftharpoons \mathrm{WSSR}+\mathrm{WS}^{-} \\
& \mathrm{WSSR}+\mathrm{RS}^{-} \rightleftharpoons \mathrm{RSSR}+\mathrm{WS}^{-}
\end{aligned}
$$

The reduction of cystine is more effective when the $\mathrm{pH}$ of the solution is higher than the pKa of the sulfur-containing reagents (which are typically between $\mathrm{pH}$ 9-10). Thus, the wool fibre modification with sulfur-containing reagents was carried out under both acidic and alkaline conditions. Increasing the number of thiol groups on the surface of the wool fibres was considered to provide additional reactive sites to bond to the gold nanoparticles.

PEI and PDADMAC were proposed to graft different types of reactive moieties onto the wool fibres. Branched PEI contains primary, secondary and tertiary amines, which were considered to be able to bond to the wool, thereby providing additional amine sites that could potentially encapsulate or bond to the gold nanoparticles. In contrast, PDADMAC, the chemical structure of which is shown in Figure 6.5, is a coagulant that is able to neutralise and coagulate negatively charged colloids. PDADMAC and was used to attempt to encapsulate gold nanoparticles on the surface of wool fibres.

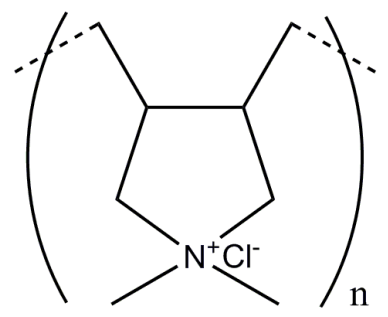

Figure 6.5 Polydiallyldimethylammonium chloride (PDADMAC).

Table 6.5 presents the effects of the different pre-treatment reagents on the synthesis of TA-mauve composites of Cariaggi wool. The experiments did not employ the glycine buffer or wool dyeing auxiliaries. The experiments involved soaking Cariaggi wool samples in the pre-treatment solutions for $120 \mathrm{~min}$, after which time the wool was removed, quickly rinsed with cold tap water, patted dry with a paper towel, and 
soaked in the TA-mauve colloid. The $\mathrm{pH}$ of the colloid that was used in the uptake was dependent on the pre-treatment. In some cases, the pre-treatments completely prevented the uptake of gold nanoparticles to the wool in the subsequent dyeing reactions. The 1,3-propane dithiol pre-treatment caused the gold nanoparticle colloid to agglomerate during the uptake, and resulted in purple-grey coloured composites.

Table 6.5 The effects of pre-treatment chemical reagents on the uptake of the TA-mauve colloid to Cariaggi wool (where $\mathrm{X}=$ prevented uptake, $\mathrm{Y}=$ complete uptake, $\mathrm{A}=$ agglomerated colloid and $-=$ not performed).

\begin{tabular}{ccccccc}
\hline Colloid $\mathrm{pH}$ & $\mathrm{Na}_{2} \mathrm{~S}_{2} \mathrm{O}_{5}$ & MSA & TGA & 1,3-propane dithiol & PDADMAC & PEI \\
\hline $\mathrm{pH} 2.7$ & $\mathrm{Y}$ & $\mathrm{X}$ & $\mathrm{X}$ & - & $\mathrm{X}$ & $\mathrm{X}$ \\
$\mathrm{pH} 8$ & - & - & - & $\mathrm{A}$ & $\mathrm{X}$ & $\mathrm{X}$ \\
$\mathrm{pH} 11$ & $\mathrm{X}$ & $\mathrm{Y}$ & $\mathrm{Y}$ & $\mathrm{A}$ & $\mathrm{X}$ & $\mathrm{X}$ \\
\hline \hline
\end{tabular}

The Cariaggi wool that was pre-soaked in PEI or PDADMAC did not absorb any gold nanoparticles under any of the dyeing conditions that were employed. This may be due the polymeric reagents leaching from the wool fibres during the uptake and interacting with the gold nanoparticles in the dye solutions. The leached polymers could encapsulate the Au NPs or neutralise their negative charge. Alternatively, if PEI and PDADMAC are chemically bound to the wool fibres, then it appears that they do not provide attractive sites for the attachment of gold nanoparticles. Either way, it was concluded that these polymeric pre-treatments were not suitable for the ex-situ procedure.

Figure 6.6 presents TA-mauve Cariaggi composites that were synthesised after pretreating the wool with $\mathrm{Na}_{2} \mathrm{~S}_{2} \mathrm{O}_{5}$, TGA and MSA. The complete uptake of the TAmauve colloid was achieved at the typical reaction $\mathrm{pH}$ of 2.7 for the $\mathrm{NaS}_{2} \mathrm{O}_{5}$ pretreatment (and the untreated wool). This is expected, and exploits the $\mathrm{pH}$ driving force for the absorption of negatively charged gold nanoparticles by the positively charged wool fibres. In contrast, the Cariaggi wool only absorbed the TA-mauve colloid at pH 11 after the MSA or TGA pre-treatments. The solutions of TGA and MSA (1 wt \%) were highly acidic, and when the Cariaggi wool was immersed in the $\mathrm{pH} 11$ TA-mauve colloid, the $\mathrm{pH}$ of the colloid dropped to 3.2, at which full uptake occurred. 


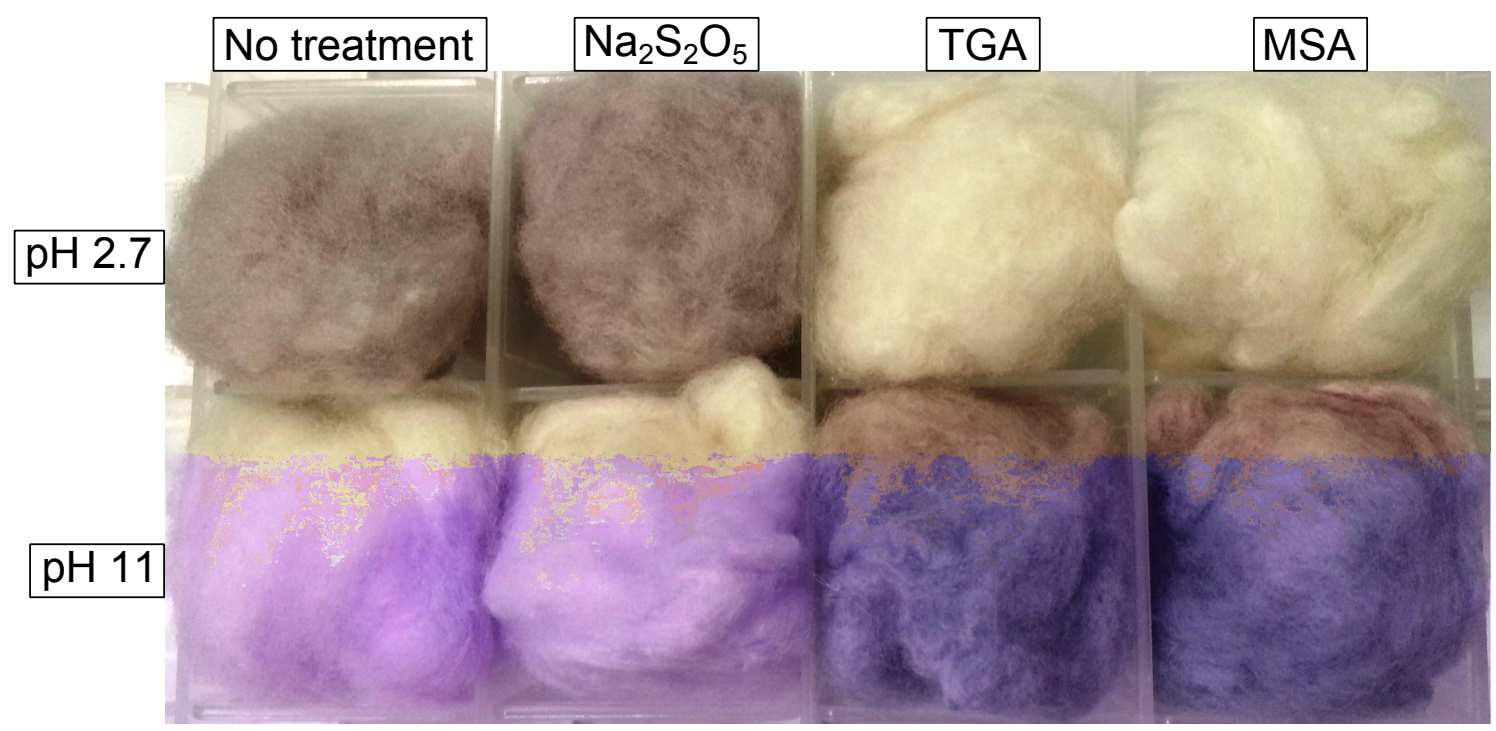

Figure 6.6 TA-mauve composites of Cariaggi wool that were synthesised after different pre-treatments and with a colloid $\mathrm{pH}$ of 2.7 or 11 .

Figure 6.7 presents the TA-mauve Cariaggi composites before and after two washing cycles, that employed the minimum amount of Kindness wool wash on the orbital shaker table. The TGA and MSA pre-treated composites appear more pink than the untreated and $\mathrm{NaS}_{2} \mathrm{O}_{5}$ treated composites, both before and after the washing processes. This implies that the additional thiol groups on the surface of the wool fibres, provided by the TGA or MSA treatment, have prevented the agglomeration of the TA-Au NPs on the surface of the composite fibres to some extent. Each of the washed composites appear comparatively lighter in colour than the unwashed composites; however, the TGA and MSA composites also seem to have leached relatively fewer gold nanoparticles from their surfaces.

The MSA and TGA pre-treatments in Figure 6.7 appeared to have improved the washfastness properties of the TA-mauve composites. As such, the MSA pre-treated composites were washed in a relative excess of Kindness wool wash on the Chiltern for $24 \mathrm{~h}$. CIE colour coordinates of TA-mauve Cariaggi composites synthesised with and without the MSA pre-treatment, before and after the washing process are shown in Table 6.6. The washing of the MSA-treated composite exhibited a change in CIE colour coordinates as a result of the washing process that was comparable to that of the untreated composite. Additionally, the TGA-treated composite exhibited similar washfastness properties to the MSA-treated composite. As such, this treatment was not investigated further.

The washfastness properties of the 1,3-propane dithiol pre-treated Cariaggi TAmauve composites are now discussed. The 1,3-propane dithiol pre-treatment resulted in the agglomeration of TA-Au NPs in the uptake solution, after which point the 


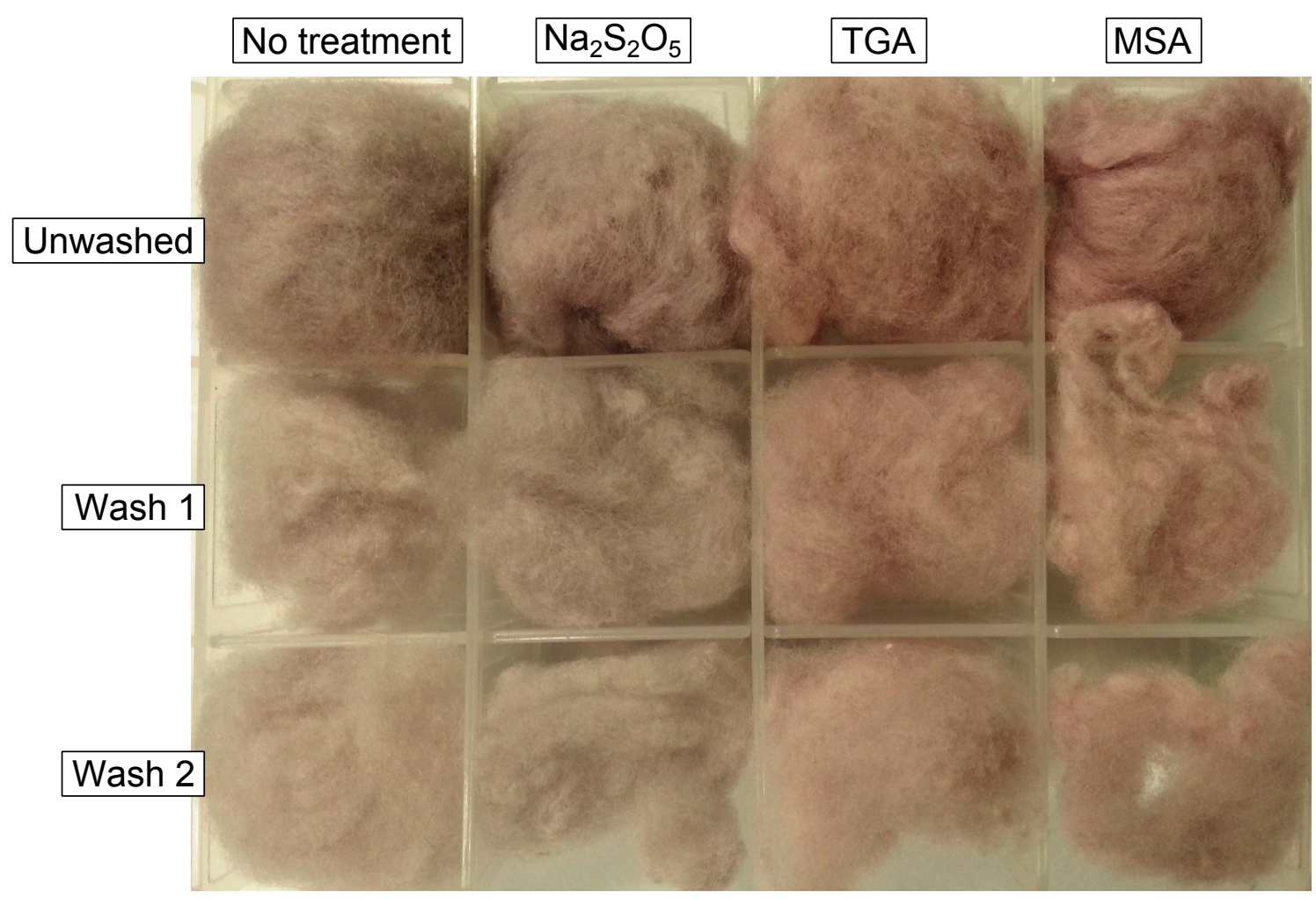

Figure 6.7 Cariaggi TA-mauve composites synthesised after pre-soaking the wool in $1 \mathrm{wt} \%$ solutions of different reagents prior to the dyeing process (not washed).

Table 6.6 CIE colour coordinates of TA-mauve Cariaggi composites with and without an MSA pre-treatment before and after washing in Kindness wool wash.

\begin{tabular}{ccccc}
\hline Method & \multicolumn{2}{c}{ Untreated } & \multicolumn{2}{c}{ MSA-treated } \\
\hline Washing & before & after & before & after \\
\hline $\mathrm{L}^{*}$ & 64.46 & 70.67 & 67.92 & 74.13 \\
$\mathrm{a}^{*}$ & 7.52 & 6.08 & 6.25 & 5.35 \\
$\mathrm{~b}^{*}$ & -0.10 & 0.62 & -1.10 & -0.15 \\
\hline \hline
\end{tabular}

agglomerated nanoparticles were absorbed by the wool fibres. This was evidenced by the purple-grey colour change, and implied that excess 1,3-propane dithiol leached from the wool fibres into the gold nanoparticle colloid, and bound the gold nanoparticles together. This agglomeration of gold nanoparticles by the 1,3-propane dithiol was undesirable for the ex-situ procedure. Even so, the washfastness properties of these composites were tested, to deduce whether the 1,3-propane dithiol treatment resulted in covalent bonds between the TA-Au NPs and the wool fibres.

Figure 6.8 presents the UV-vis spectra of the wash solutions of the TA-mauve Cariaggi composites that were pre-treated with 1,3-propane dithiol. The wash solutions of the composites synthesised at both $\mathrm{pH} 8$ and 10 exhibit only a slight absorbance between $500-600 \mathrm{~nm}$ that relates to the SPR of gold nanoparticles. In contrast, the SPR peak of TA-Au NPs is far more prominent in the wash solutions of the untreated TA-mauve composite. 


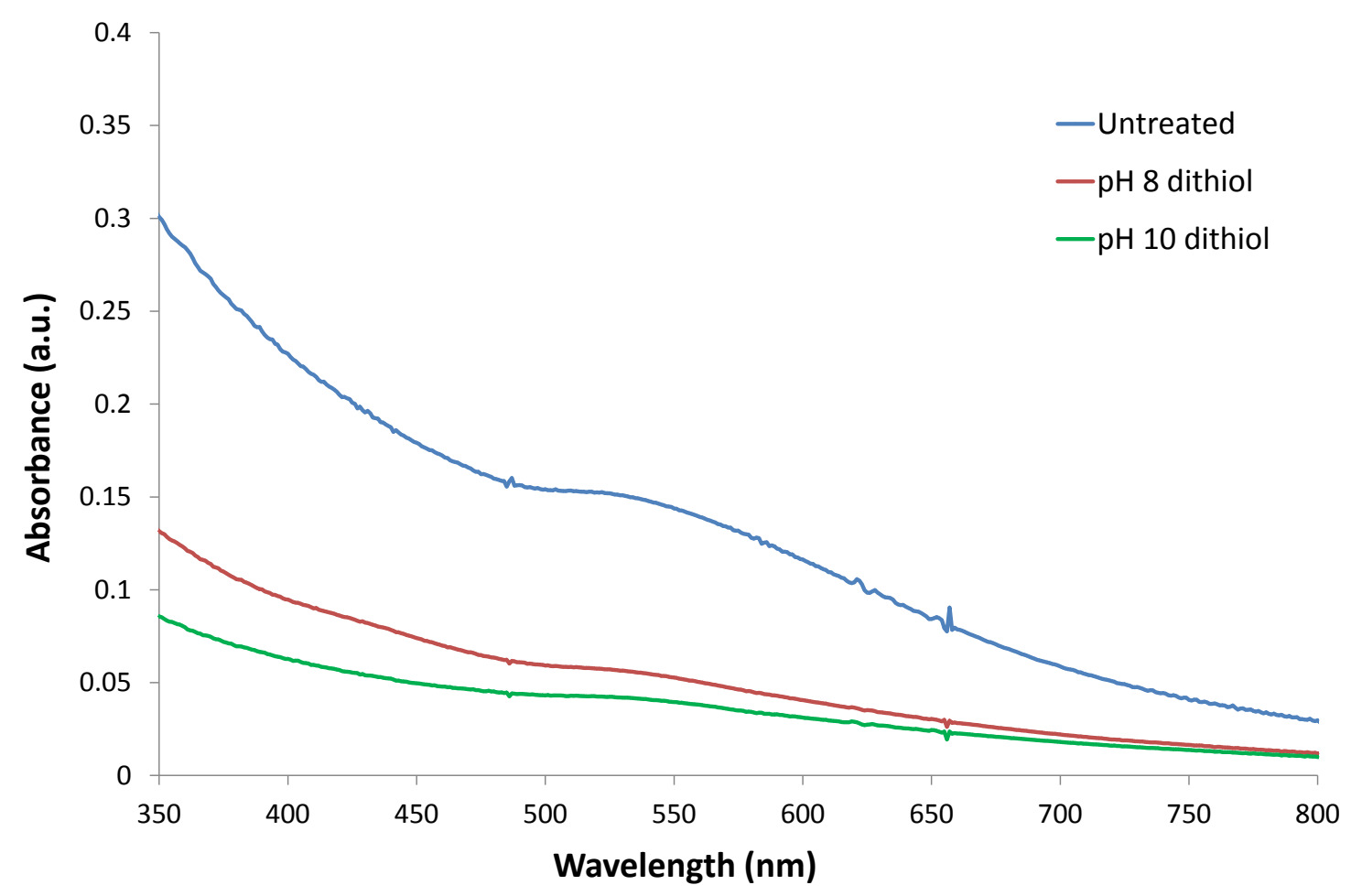

Figure 6.8 UV-vis spectra of the wash solutions of Cariaggi TA-mauve composites synthesised after pre-soaking the wool in 1,3-propane dithiol at pH 8 or 10 and an untreated composite.

The enhanced washfastness properties of the 1,3-propane dithiol compared to that of the other sulfur-containing reagents indicates that 1,3-propane dithiol was able to form more thiol groups on the surface of the wool fibres, which may have covalently attached gold nanoparticles. This is understandable when it is considered that any interaction of 1,3-propane dithiol with the wool protein provides additional thiol groups on the surface, whereas the cleavage of the disulfide linkage of cystine requires two equivalents of MSA or TGA.

Additionally, placing the wool into an acidic dyebath can result in the protonation of the Wool- $S^{-}$groups on the wool surface and reformation of the disulfide linkages. If this occurs before gold nanoparticles can bond to these areas, then the pre-treatment will not aid the attachment of gold nanoparticles. Thus, the observed increase in washfastness likely indicates that the thiol reactive groups are effective to some degree.

In summary, the pre-treatment experiments showed that the soaking of wool fibres in the investigated reagents was not able to completely prevent the leaching of gold nanoparticles into the wash solutions, but in some cases the washfastness properties were improved significantly. PEI and PDADMAC pre-treatments completely prevented the uptake of gold nanoparticles in subsequent dyeing reactions. $\mathrm{Na}_{2} \mathrm{~S}_{2} \mathrm{O}_{5}$ did not significantly increase the washfastness properties of the TA-mauve compos- 
ites. MSA and TGA initially appeared to increase the washfastness properties of the composites. However, the obseved colour differences upon extensive washing were similar to those of untreated composites. 1,3-propane dithiol saw a marked increase in the washfastness properties of the resulting composites at the cost of agglomerating the gold nanoparticles and resulting in more grey-coloured composites. None of the pre-treatments were able to prevent the leaching of gold nanoparticles in subsequent washing processes.

\subsubsection{Chemical Post-treatments}

A range of post-treatments were applied to ex-situ composites to attempt to improve their washfastness properties, by fixing the gold nanoparticles to wool fibres after the dyeing process. Again, the aim of this research was to facilitate the formation of covalent bonds between the gold nanoparticles and the wool fibres. Table 6.7 lists the investigated chemical reagents, and the reaction conditions of their use.

Table 6.7 Chemical reagents that were used in post-treatment experiments, and the reaction conditions of their use.

\begin{tabular}{lcccc}
\hline Reagent & Composition & Amount & Temp & $\mathrm{pH}$ \\
\hline MSA & $\mathrm{C}_{4} \mathrm{O}_{4} \mathrm{H}_{6} \mathrm{~S}$ & $1 \mathrm{wt} \%$ & $50-80{ }^{\circ} \mathrm{C}$ & 2 \\
TGA & $\mathrm{C}_{2} \mathrm{O}_{2} \mathrm{H}_{4} \mathrm{~S}$ & $1 \mathrm{wt} \%$ & $50-80{ }^{\circ} \mathrm{C}$ & 2 \\
PEI & $\left(\mathrm{C}_{2} \mathrm{H}_{5} \mathrm{~N}\right)_{n}$ & $1 \mathrm{wt} \%$ & $\mathrm{RT}-80^{\circ} \mathrm{C}$ & $2-11$ \\
PDADMAC & $\left(\mathrm{C}_{8} \mathrm{H}_{16} \mathrm{NCl}\right)_{n}$ & $1 \mathrm{wt} \%$ & $50-80^{\circ} \mathrm{C}$ & 4 \\
\hline \hline
\end{tabular}

The post-treatment experiments involved the chemical modification of TA-mauve Cariaggi composites, which were produced in scale-up hank dyeing procedures (the synthesis of which was previously detailed in Section 5.2.1). The composites were shortened fibres that were a waste product from the blending and combing of largescale composites, the synthesis of which was previously presented in Section 5.2.1.

MSA and TGA were employed to attempt to increase the number of thiol reactive sites on the wool fibres. These thiol sites were considered to be able to displace the stabiliser surrounding the gold nanoparticles, and bond to the gold nanoparticles directly. In this, the action was assumed to be the similar to that of the pretreatment experiments. In contrast, PDADMAC and PEI were employed to attempt to encapsulate the gold nanoparticles on the wool fibres. If the polymers were successfully bound to the composite fibres, they may then provide a protective barrier that could prevent the displacement of gold nanoparticles from the wool fibres when the composites were laundered. 
The post-treatments involved soaking the composites in solutions containing the different chemical reagents for $120 \mathrm{~min}$, at different temperatures and $\mathrm{pH}$ levels. However, it was observed that the post-treatments often caused the leaching of gold nanoparticles from the composites, or the greying of the TA-mauve Cariaggi composites, which indicated the agglomeration of gold nanoparticles on the wool fibre surfaces. Table 6.8 summarises the results of the post-treatment experiments on the Cariaggi TA-mauve composites.

Table 6.8 The effects of pre-treatment chemical reagents on the TA-mauve Cariaggi composites (where $\mathrm{L}=$ leaching of $\mathrm{Au}$ NPs and $\mathrm{A}=\operatorname{agglomerated} \mathrm{Au}$ NPs).

\begin{tabular}{ccccc}
\hline Conditions & MSA & TGA & PDADMAC & PEI \\
\hline $50^{\circ} \mathrm{C}$ & $\mathrm{A}$ & $\mathrm{A}$ & $\mathrm{L}$ & $\mathrm{L}$ \\
$80^{\circ} \mathrm{C}$ & $\mathrm{A}$ & $\mathrm{A}$ & $\mathrm{A}$ & $\mathrm{L}$ \\
\hline \hline
\end{tabular}

Soaking the TA-mauve Cariaggi composites in MSA and TGA caused the composites to change from mauve to purple-grey colour under each of the investigated reaction conditions, which indicated that the TA-Au NPs had agglomerated on the surface fibres. This agglomeration may be indicative of MSA and TGA having displaced the relatively large TA that surrounded the nanoparticles. If the TA was replaced by the comparatively small MSA or TGA molecules, then the gold nanoparticles would have the potential to move closer together, due to less steric repulsion between the particles. This would result in grey-coloured composites as observed.

The PDADMAC post-treatment caused extensive leaching of gold nanoparticles into the reaction solutions under each of the investigated conditions, and was not investigated further. It is proposed that PDADMAC has displaced the gold nanoparticles in a similar way to that of the surfactants in the Kindness wool wash. PEI also caused the leaching of gold nanoparticles. However, in some cases only minimal leaching of TA-Au NPs occurred, and the treatment also appeared to improve the washfastness properties of the resulting composites. Figure 6.9 presents TA-mauve composites that were subjected to a range of PEI post-treatments. After the PEI treatments, the composites all exhibited some extent of colour variation compared to the untreated composites. The PEI treatments may have also affected the distribution of the gold nanoparticles on the surface of the composites, as the treated composites were all relatively more pink in colour compared to the untreated composites. This was possibly due to preferential displacement of agglomerates of gold nanoparticles.

Figure 6.9 also shows the PEI post-treated composites after washing in excess Kindness wool wash on the Chiltern shaker for $24 \mathrm{~h}$. The washfastness properties of the PEI-treated composites were all significantly improved compared to those of the un- 


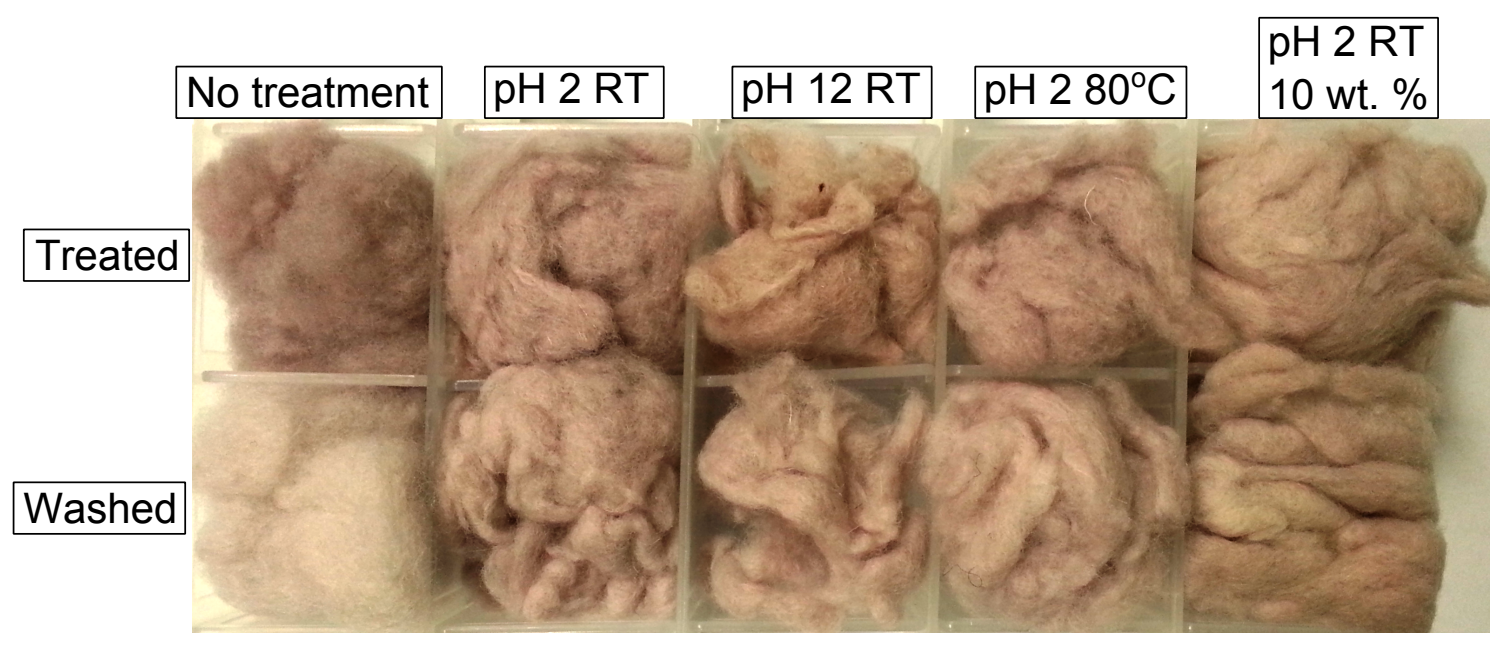

Figure 6.9 TA-mauve composites of Cariaggi wool that were synthesised after PEI pre-treatments with different reaction conditions, before and after washing on the Chiltern shaker for $24 \mathrm{~h}$.

treated composite. The leaching caused by the PEI post-treatment is not desirable. However, the improved washfastness properties of these composites warrants further investigation of this method.

All of the chemical post-treatments were found to cause either leaching of gold nanoparticles from the composites or greying of the composite fibres. Despite this, the use of PEI enhanced the washfastness properties of the composites, and was the best post-treatment thus far. The investigated post-treatments were unable to completely prevent the leaching of gold nanoparticles, and as such, alternative methods were investigated.

\subsection{Alternative Reducing and Stabilising Agents}

A number of dual reducing and stabilising agents were investigated for use in the synthesis of gold nanoparticles. It was considered that if reactive groups that were attracted to the wool protein could be present on the exterior of the gold nanoparticles, covalent bonds between the gold nanoparticles and the wool fibres could be formed, and would result in composites with excellent washfastness properties. The different chemical reagents that were employed in the synthesis of gold nanoparticles are listed in Table 6.9.

MSA and 1,3-propane dithiol were used in earlier treatments to chemically modify the wool fibres. In the following experiments, the sulfur-containing reagents were used to to grow and stabilise gold nanoparticles from solutions of TSC-capped "seed" 
Table 6.9 Chemical reagents used in the synthesis of gold nanoparticles for washfastness experiments.

\begin{tabular}{lcc}
\hline Reagent & Composition & Group attached to gold \\
\hline MSA & $\mathrm{C}_{4} \mathrm{O}_{4} \mathrm{H}_{5} \mathrm{~S}$ & thiol \\
1,3-propane dithiol & $\mathrm{C}_{3} \mathrm{H}_{8} \mathrm{~S}_{2}$ & thiol \\
PEI & $\left(\mathrm{C}_{2} \mathrm{H}_{5} \mathrm{~N}\right) \mathrm{n}$ & amine \\
Reactive dyes & variable & variable \\
\hline \hline
\end{tabular}

nanoparticles. PEI and reactive dyes were employed as dual reducing and stabilising agents in gold nanoparticle syntheses, as they can readily reduce $\mathrm{Au}^{3+}$ to $\mathrm{Au}^{0}$.

\subsubsection{Sulfur-containing Stabilisers}

MSA and 1,3-propane dithiol, were employed as stabilisers in the solution-phase synthesis of gold nanoparticles. For this, TSC-stabilised "seed" gold nanoparticles were first synthesised, and MSA or 1,3-propane dithiol were then introduced to the seed particle colloids. The use of MSA was based upon the method of Nui and coworkers, ${ }^{59}$ which involved the addition of TSC-stabilised seeds to a growth solution containing a 1:0.6 molar ratio of $\mathrm{AuCl}_{4}^{-}$:MSA at RT. MSA is a mild reducing agent, and is able to facilitate the growth of gold nanoparticles. The seed particles were synthesised via the addition of $70 \mu \mathrm{L}$ of TSC ( $5 \mathrm{wt} \%$ ) to a $10 \mathrm{~mL}$ solution of $40 \mathrm{mg} \mathrm{L}^{-1} \mathrm{AuCl}_{4}^{-}$at $100^{\circ} \mathrm{C}$. The colour of the seed particle colloid changed from the original deep-red colour to purple upon the addition of the TSC-Au NPs into the growth solution, which indicated the growth of the seed nanoparticles into larger, MSA-capped particles.

A schematic that illustrates the possible stabilisation of the MSA-capped gold nanoparticles is depicted in Figure 6.10. The attraction of the thiol group of MSA is believed to be greater than that of the carboxylate groups, because of the strong affinity of sulfur for gold. Additionally, the carboxylate groups of MSA may also aid the stabilisation of the nanoparticles. Thus, similar to TSC-Au NPs, the MSA-Au NPs are thought to exhibit carboxylate groups on the exterior of the particles. However, the $\mathrm{Au}-\mathrm{S}$ bond between the MSA and the gold nanoparticle will be stronger than the attraction between the TSC stabiliser and the TSC-Au NPs.

The addition of the TSC-capped seed particles into the $\mathrm{MSA}: \mathrm{AuCl}_{4}^{-}$growth solution resulted in a red-shift of the $\mathrm{SPR}$ peak to $\lambda_{\max }=560 \mathrm{~nm}$, indicating the formation of larger gold nanoparticles. However, when the MSA-capped gold nanoparticles were used to colour Cariaggi wool, the resulting composites exhibited washfastness 

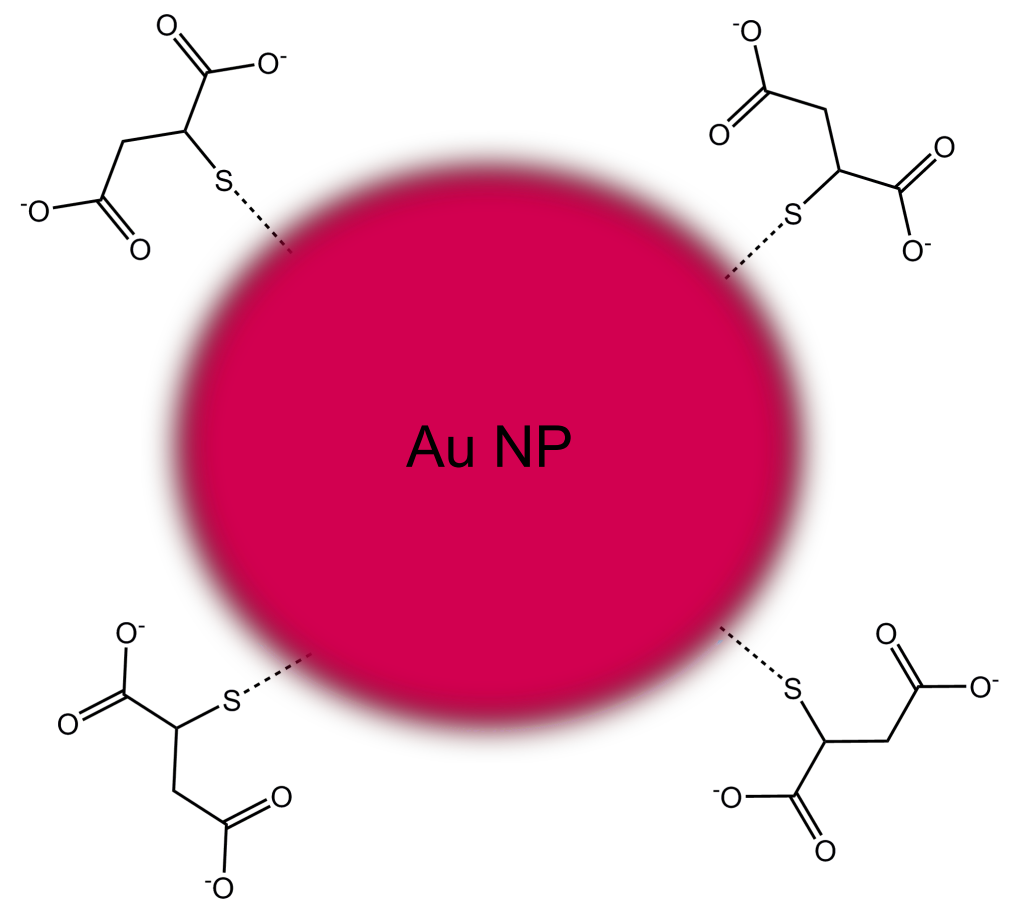

Figure 6.10 A schematic illustrating the possible interaction between MSA and gold nanoparticles in their synthesis.

properties that were similar to those of the TSC-pink composites. As such, this method failed in improving the washfastness properties of the ex-situ composites. Considering that the exterior of the TSC-Au NPs and MSA-Au NPs are the same, and that the composites of these colloids exhibit similar washfastness properties, the leaching of gold nanoparticles from composites likely involves the displacement of stabilised gold nanoparticles from the surface of the wool fibres. The alternative possibility is that the stabilising agents are displaced from the gold nanoparticles, leaving bare gold nanoparticles to leach from the composites.

The MSA synthesis method was repeated with 1,3-propane dithiol as the stabilising agent. This utilised the same TSC-Au NPs seeds as the MSA method, which were added to a growth solution containing a 1:0.6 molar ratio of $\mathrm{AuCl}_{4}^{-}$to 1,3-propane dithiol. Interestingly, the addition of 1,3-propane dithiol to the seed solution caused the colour of the colloid to change from red to colourless, and led to the growth of red specks in the solution. The red specks aggregated into larger clumps during this colour change, and eventually settled out on the bottom of the reaction vessel. This indicated that the 1,3-propane dithiol bound the seed nanoparticles together. However, the colour of the aggregates of 1,3-propane dithiol-capped gold nanoparticles remained red, and did not change to grey. This implies that the SPR bands of the nanoparticles are too far apart to interact together, as the absorption energy of the SPR did not appear to change. Thus, the spacing between the nanoparticles is larger than approximately twice the diameter of the particles, and a network structure may have formed. A schematic depicting such a configuration is presented in Figure 6.11, 
where 1,3-propane dithiol molecules form $\mathrm{Au}-\mathrm{S}$ bonds with the nanoparticles and S-S bonds with themselves to link the gold nanoparticles.

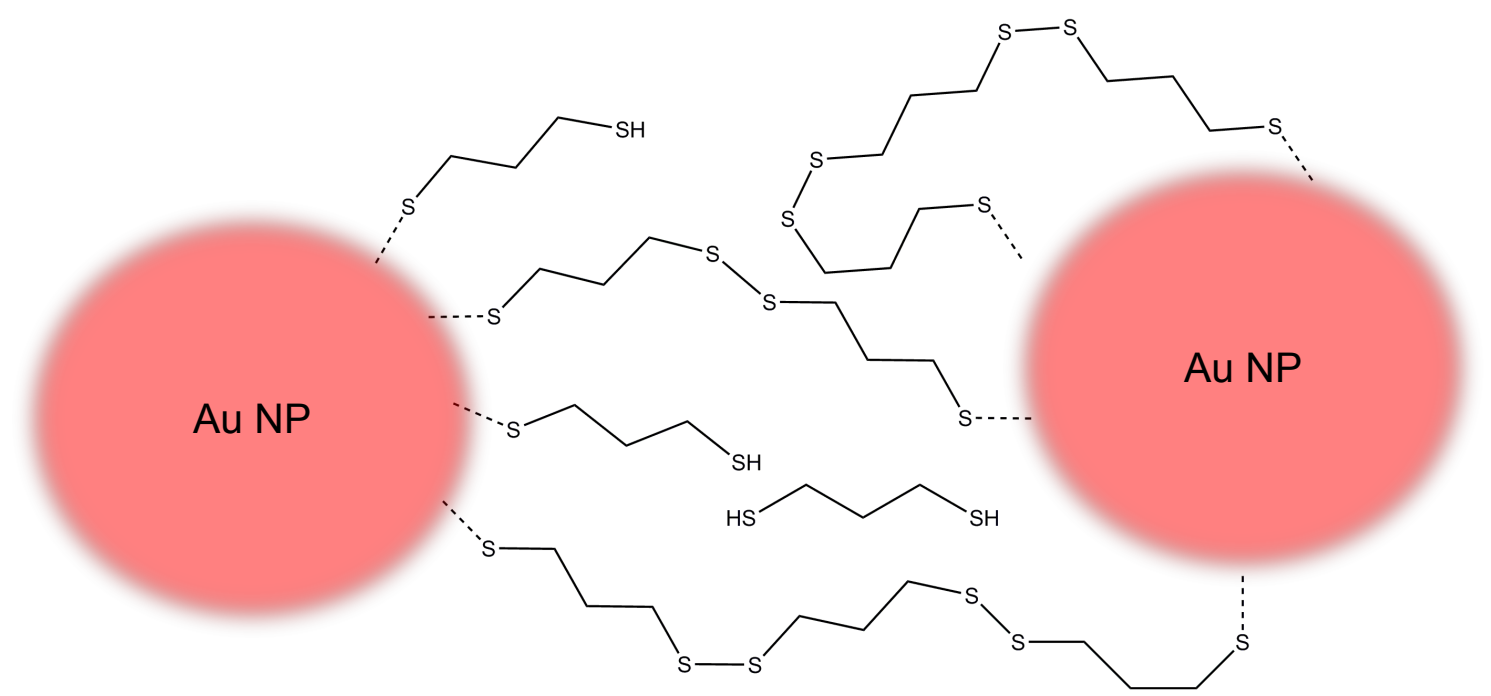

Figure 6.11 A schematic illustrating the possible interaction between 1,3-propane dithiol and gold nanoparticles in their synthesis.

The propensity for 1,3-propane dithiol to form red coloured aggregates of gold nanoparticles has shown that this reagent was not suitable for the synthesis of gold nanoparticles in the ex-situ procedure. The $\mathrm{pH}$ of the colloid was approximately 3.5, a $\mathrm{pH}$ at which the thiols are protonated. Carrying out the synthesis at a higher $\mathrm{pH}$ may prevent the disulfide bond formation, and the aggregation of the gold nanoparticles in the solution. However, the dyeing of wool with negatively charged gold nanoparticles requires a $\mathrm{pH}$ below the isoelectric region of wool, and as such, this aggregation would likely still occur during the dyeing process.

The use of stabilisers with thiol-functionality to covalently link gold nanoparticles to wool fibres was investigated. However, the propensity for thiols to react together under acidic conditions to form disulfide bonds resulted in the formation of aggregates of 1,3-propane dithiol-stabilised gold nanoparticles. Additionally, composites coloured with MSA-Au NPs did not exhibit improved washfastness properties. These experiments indicated that using stabilising agents that contain distinct chemical functional groups that are attracted to only either the gold nanoparticles or the wool fibres would be desirable for use in the ex-situ procedure. This would prevent the aggregation of gold nanoparticles during or prior to dyeing reactions. 


\subsubsection{Polyethylenimine}

Branched polyethyleneimine (PEI) was used as a dual reducing and stabilising agent in the synthesis of gold nanoparticles. The chemical structure of a possible fragment of branched PEI contains primary, secondary and tertiary amines, and is shown in Figure 6.12. While the chemical nature of PEI may be attractive to both the gold and the wool matrix, it is unlikely to crosslink and cause the aggregation of gold nanoparticles in the reaction solution. The large polymer was considered to be able to encapsulate the gold nanoparticles and potentially form an amide bond between the amine functionality of the PEI and the carboxylate groups of the wool fibres. The synthesis of PEI-stabilised gold nanoparticles has been detailed in an earlier study by Dr Michael Richardson and Professor James Johnston. ${ }^{60}$ In this, solutions of $\mathrm{AuCl}_{4}^{-}$and PEI are heated to $70^{\circ} \mathrm{C}$, and a stable gold colloid forms after $180 \mathrm{~min}$. A PEI to $\mathrm{AuCl}_{4}^{-}$molar ratio of 33:1 was found to be optimal for the nanoparticle synthesis. These gold colloids were also used to colour wool fibres in another study. ${ }^{50}$

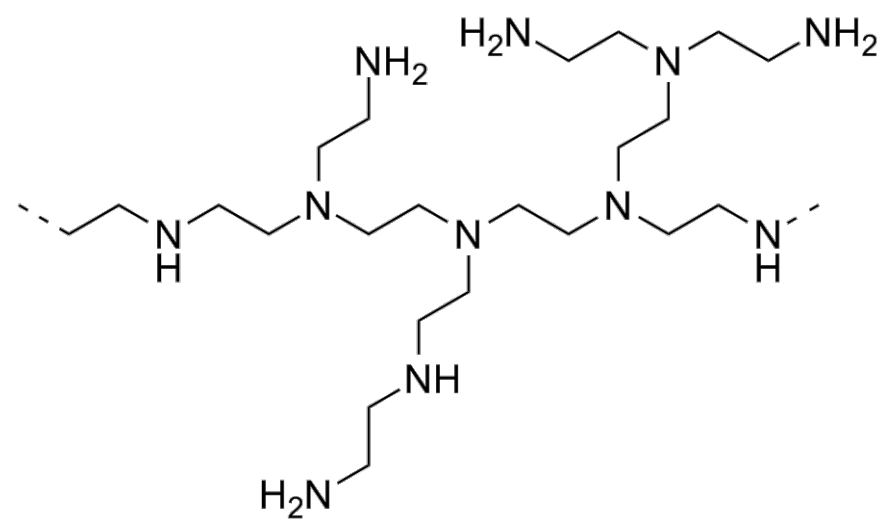

Figure 6.12 An illustration of the chemical structure of a possible fraction of branched PEI.

Figure 6.13 presents the UV-vis spectra of PEI-stabilised gold colloids at different reaction $\mathrm{pH}$ levels. The synthesis involved the addition of PEI to boiling solutions of $\mathrm{AuCl}_{4}^{-}$in distilled water. The reaction temperature of $100^{\circ} \mathrm{C}$ allowed for the formation of a stable gold nanoparticle colloid in approximately $30 \mathrm{~min}$ (as opposed to the $180 \mathrm{~min}$ formation time at $70^{\circ} \mathrm{C}$ ). The SPR peak of PEI-Au NPs centres at approximately $525 \mathrm{~nm}$ at each reaction $\mathrm{pH}$. The colloid that was synthesised at $\mathrm{pH}$ 2.2 , resulted in the narrowest SPR peak, and as such, this colloid was utilised in the dyeing of wool fibres.

The dyeing of wool with PEI-stabilised gold colloids is referred to as the PEI-grey method. The PEI-grey method involved the same concentration of $\mathrm{AuCl}_{4}^{-}$as the TSC-grey method; $10 \mu \mathrm{L}$ of $\mathrm{AuCl}_{4}^{-}$(4 wt \%) was added to $10 \mathrm{~mL}$ of boiling distilled water, which was quickly followed by the addition of $1.728 \mathrm{~mL}$ of branched PEI 


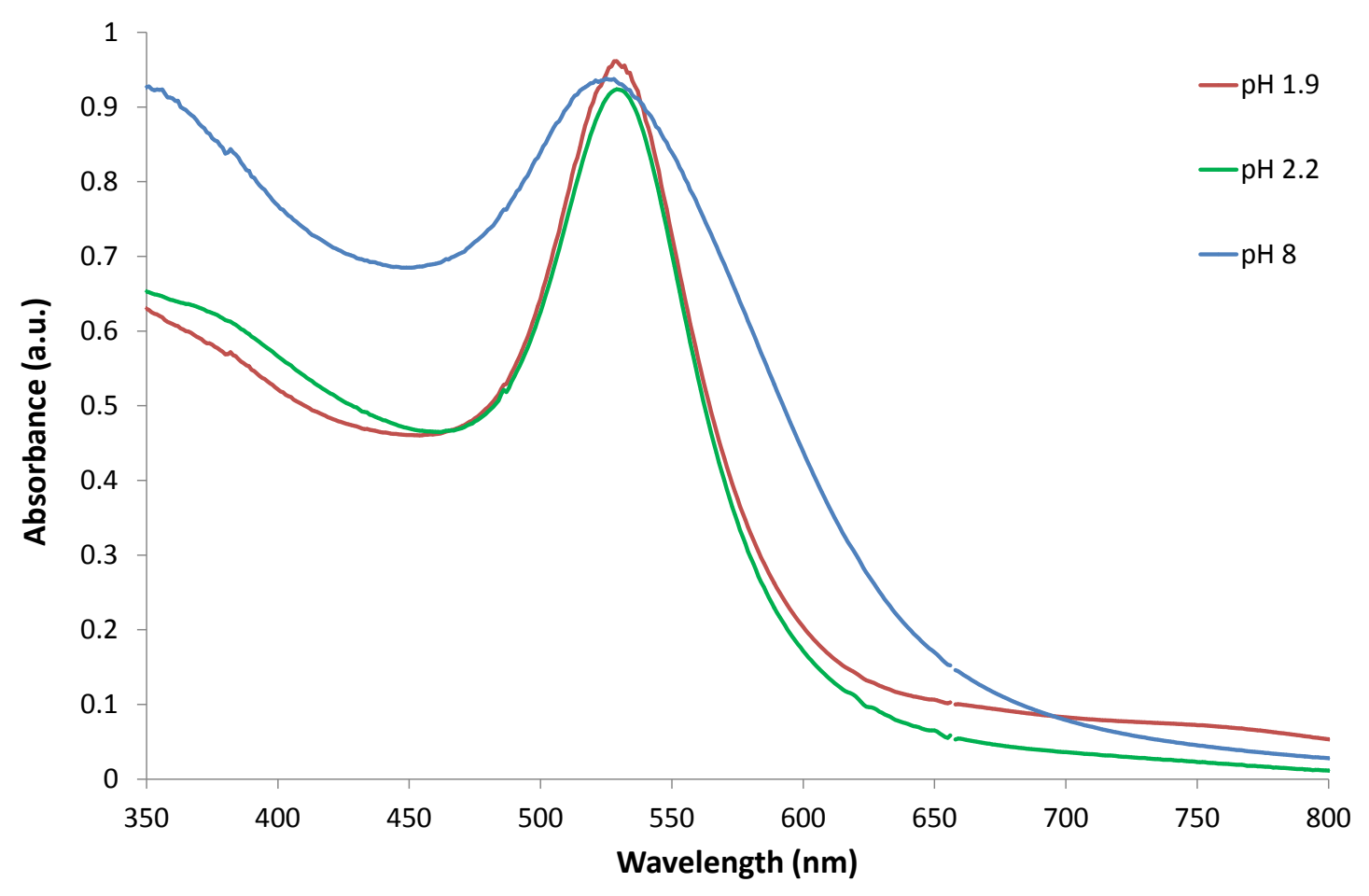

Figure 6.13 UV-vis spectra of PEI-stabilised gold colloids that were synthesised at different reaction $\mathrm{pH}$ levels.

(1 wt \%). The $\mathrm{pH}$ of the colloid was modified by altering the $\mathrm{pH}$ of the PEI stock solution; using a PEI stock solution at $\mathrm{pH} 1.9$ in the synthesis results in a $\mathrm{pH} 2.2$ colloid.

When wool fibres were soaked in the PEI-grey colloid at its formation $\mathrm{pH}$, the uptake was incomplete; approximately only half of the gold nanoparticles were absorbed by the wool fibres. The amount of uptake of PEI-Au NPs did not increase significantly when the temperature of the dye solution was elevated to 50 or $100{ }^{\circ} \mathrm{C}$. When the $\mathrm{pH}$ of the PEI colloid was increased to 7 , above the isoelectric region of wool, complete uptake occurred at RT. Thus, the PEI surrounding the gold nanoparticles are positively charged $\left(\mathrm{pK}_{a 1}=5\right)$, and are attracted to wool at a $\mathrm{pH}$ above the isoelectric region of the wool, when the wool is negatively charged.

PEI-grey composites of Cariaggi wool and TU yarn synthesised at $\mathrm{pH} 7$ with different uptake temperatures are presented in Figure 6.14. The uptake temperature was increased to promote amide bond formation between the PEI surrounding the gold nanoparticles and the chemical functionality of the wool fibres, such as the carboxylic acid groups of the 18-MEA fatty acid layer. Each of the Cariaggi composites were grey with a distinct purple tinge, while the composites of TU yarn were grey. Due to the structure of the TU yarn, the absorption of PEI-Au NPs was concentrated at the wool fibres located on the outside of the yarn, which are more readily accessible. Hence, the TU yarn composite was observed to be relatively more grey. 
Additionally, the uptake temperature does not seem to have influenced the colour of the composites.

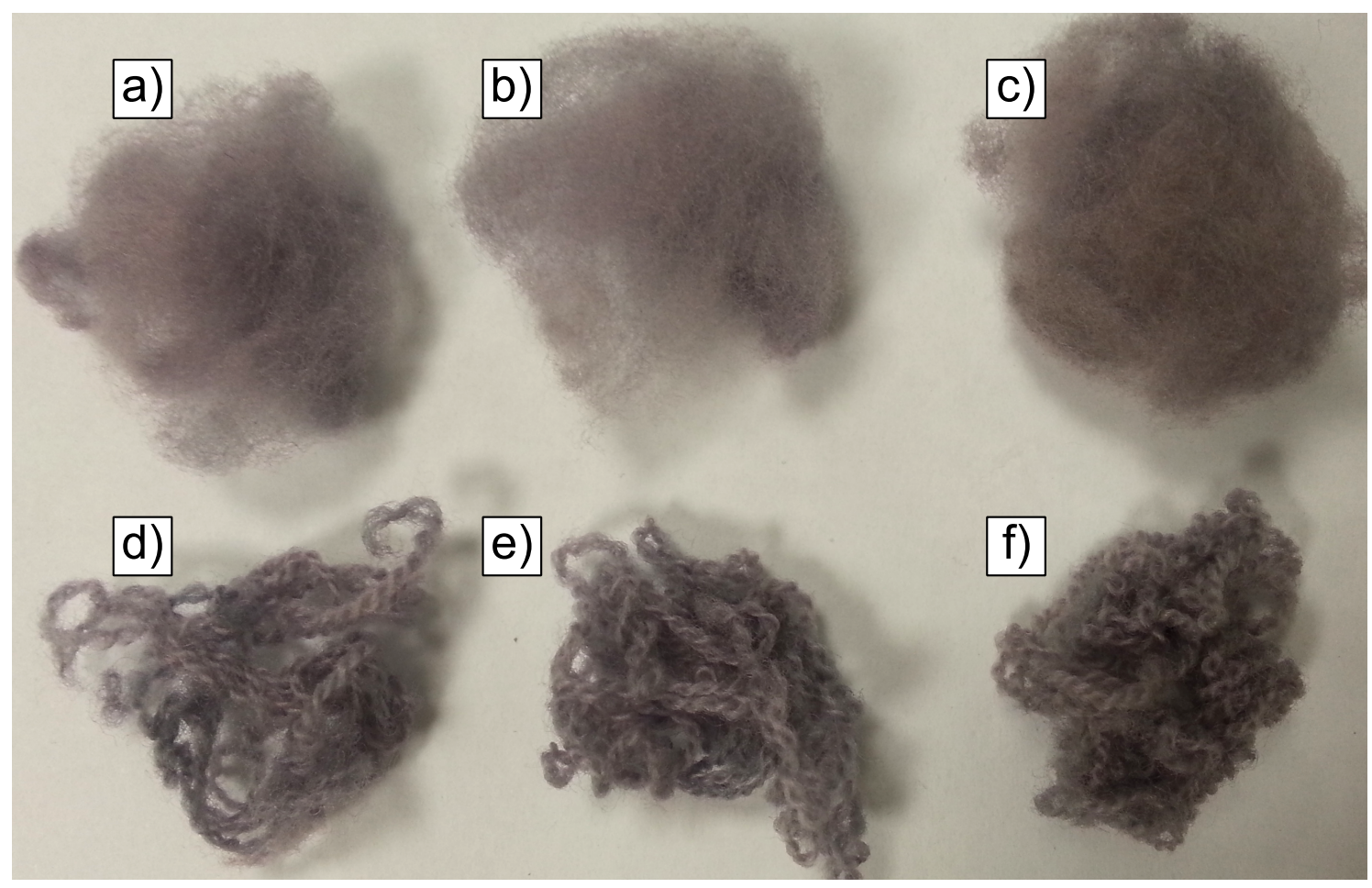

Figure 6.14 PEI-grey Cariaggi and TU yarn composites synthesised at RT (a) and $(\mathrm{d}), 50^{\circ} \mathrm{C}(\mathrm{b})$ and $(\mathrm{e})$ and $100{ }^{\circ} \mathrm{C}(\mathrm{c})$ and (f).

The washfastness properties of the composites shown in Figure 6.14 were first tested with a 1:20 wool to liquor ratio of Kindness wool wash. Figure 6.15 presents the UV-vis spectra of the wash solutions of the PEI-grey composites that resulted from these washing processes. The wash solutions of the TU yarn and Cariaggi wool PEIgrey composites synthesised at RT exhibited the most prominent SPR peaks, which indicated that the most significant leaching of PEI-Au NPs had occurred during this washing process. When the temperature of the reaction was increased to $50^{\circ} \mathrm{C}$ and $100^{\circ} \mathrm{C}$, the SPR peak of the wash solutions became less intense. Thus, increasing the temperature of the uptake improved the washfastness properties of the composites. This is considered to be due to the higher temperature facilitating amide bonding between the wool fibres and the PEI stabilising the gold nanoparticles. Additionally, the amount of leaching was similar between the Cariaggi and TU yarn composites.

The promising results of the previous experiment led to further testing of the washfastness properties of PEI-grey composites with excess Kindness wool wash. For this, Cariaggi and TU yarn PEI-grey composites of $2 \mathrm{~g}$ mass were synthesised (with an uptake $\mathrm{pH}$ of 7 and temperature of $100^{\circ} \mathrm{C}$ ). The resulting composites were immersed in Kindness wool wash $(500 \mathrm{~mL})$ and shaken on the Chiltern shaker for $24 \mathrm{~h}$. An image of the composites before and after washing is shown in Figure 6.16. The Cariaggi and TU yarn composites are significantly lighter grey in colour after the 


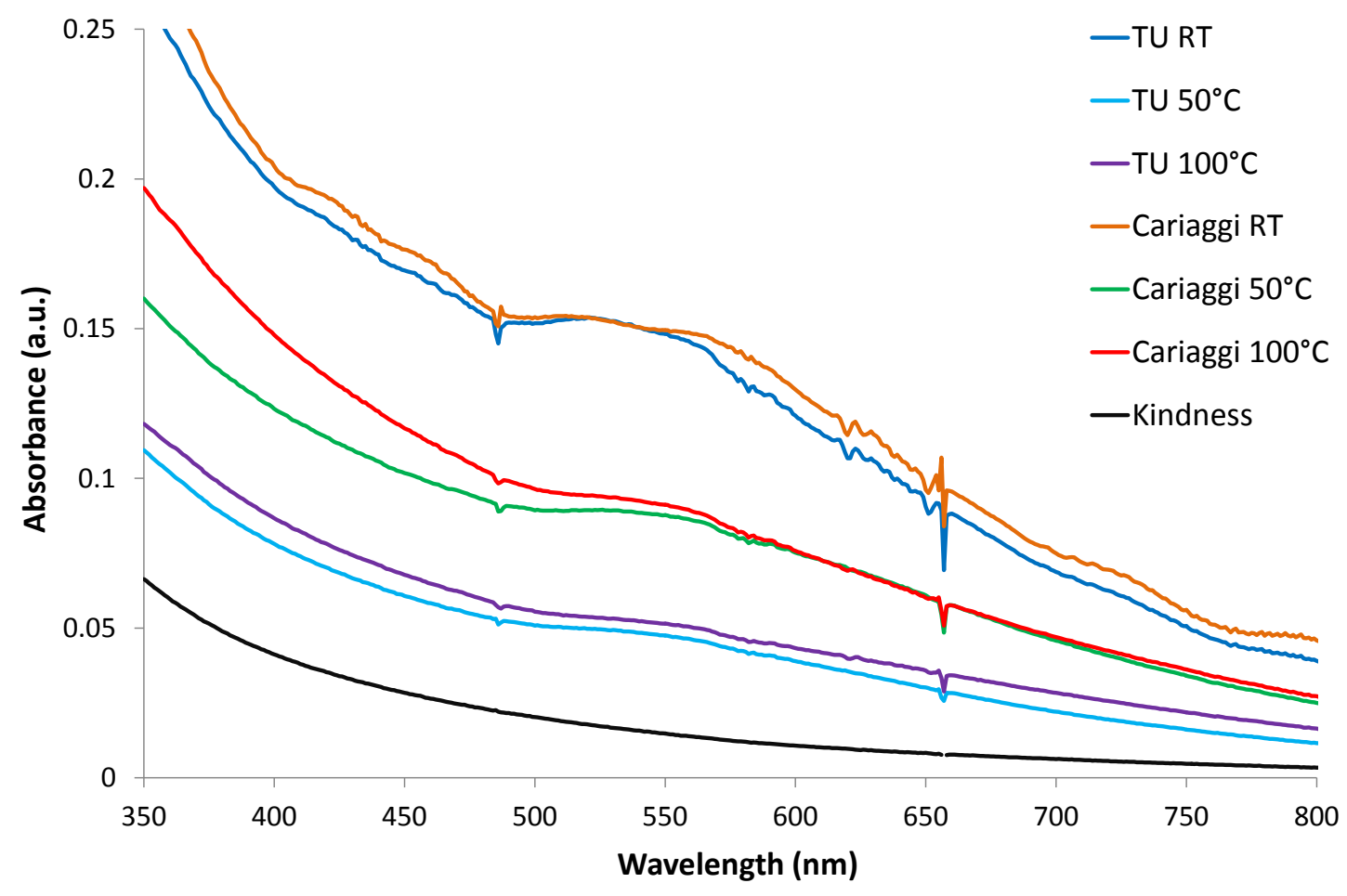

Figure 6.15 UV-vis spectra of the wash solutions of PEI-grey Cariaggi and TU yarn composites synthesised at different temperatures (1:20 ratio of wool to Kindness wool wash).

washing process. However, the washed Cariaggi composite appears to retain far fewer gold nanoparticles. Additionally, the vigorous agitation of the Chiltern shaker has matted the Cariaggi composite. This was also observed earlier in Figure 6.1, and again is thought to be due to the rubbing of wet wool fibres against one another displacing the gold nanoparticles from the composites. In contrast, the relatively rigid structure of the TU yarn does not allow for such matting.

CIE colour coordinates of the PEI-grey composites before and after washing are presented in Table 6.10, which quantifies the colour differences that were observed in Figure 6.16. The Cariaggi composite exhibits large increases in brightness and yellowness values, and a slight reduction in redness value upon washing. The differences in CIE colour coordinates of the TU yarn composites before and after washing are comparatively small, as fewer nanoparticles leach from the TU yarn composite.

Table 6.10 CIE colour coordinates of Cariaggi and TU yarn PEI-grey composites before and after washing in Kindness wool wash on the Chiltern shaker for $24 \mathrm{~h}$.

\begin{tabular}{ccccc}
\hline Composite & \multicolumn{2}{c}{ Cariaggi } & \multicolumn{2}{c}{ TU yarn } \\
\hline Washing & before & after & before & after \\
\hline $\mathrm{L}^{*}$ & 54.86 & 71.88 & 49.04 & 51.22 \\
$\mathrm{a}^{*}$ & 2.54 & 1.58 & 2.03 & 2.20 \\
$\mathrm{~b}^{*}$ & -0.87 & 2.86 & -0.95 & -0.54 \\
\hline \hline
\end{tabular}




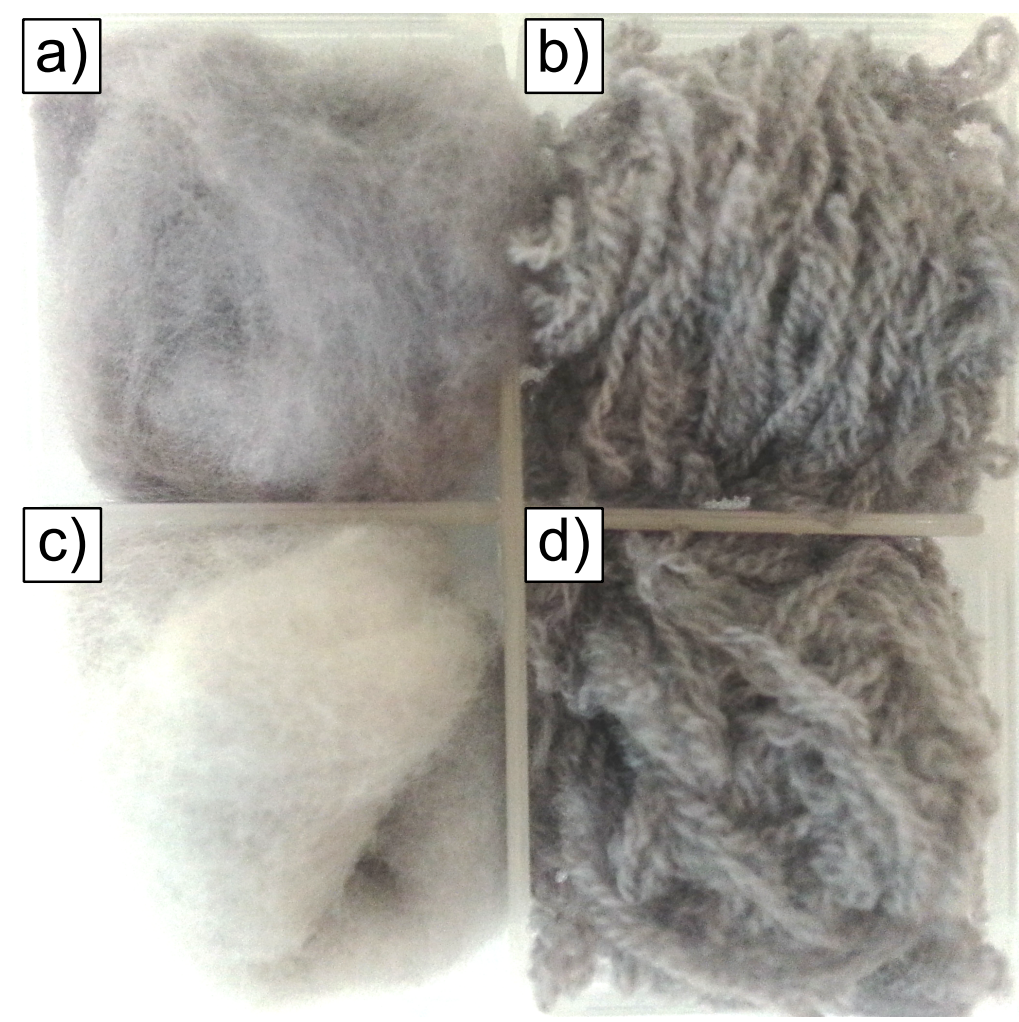

Figure 6.16 PEI-grey Cariaggi (a) and TU (b) composites and the composites after washing and TU yarn after washing on the Chiltern shaker for $24 \mathrm{~h} \mathrm{(c)}$ and (d).

SEM and EDS analysis was carried out on the Cariaggi PEI-grey composites before and after washing, and is presented in Figure 6.17. The surface of the unwashed composite at relatively low magnification (in Figure 6.17 (a)) appears to exhibit both individual gold nanoparticles and large agglomerates of particles. The amount of gold nanoparticles on the surface of the PEI-grey composite appears to be similar to that of a typical TSC-grey composite. The relatively high magnification image in Figure 6.17 (b) shows that the large agglomerates of gold consist of a number of very small nanoparticles that are located very close together. This conformation and distribution of gold nanoparticles would contribute to the grey colouration of the composites, as the SPR bands of the gold nanoparticles would be close enough to couple. The Au EDS elemental map in Figure 6.17 (d) confirms the gold content of the nanoparticles, as the areas of high concentration of $\mathrm{Au}$ correspond to the bright areas identified as gold nanoparticles in Figure 6.17 (c).

Figure 6.17 (e) and (f) are SEM micrographs of the surfaces of two different PEIgrey composite fibres after the washing process (in excess Kindness wool wash). The fibres exhibit vastly different concentrations of gold nanoparticles on their respective surfaces. The fibre in Figure 6.17 (e) has a similar gold distribution to the unwashed composite fibre in Figure 6.17 (a). It is supposed that this fibre may have been located at a relatively free area of the wool that has not matted during washing. If 


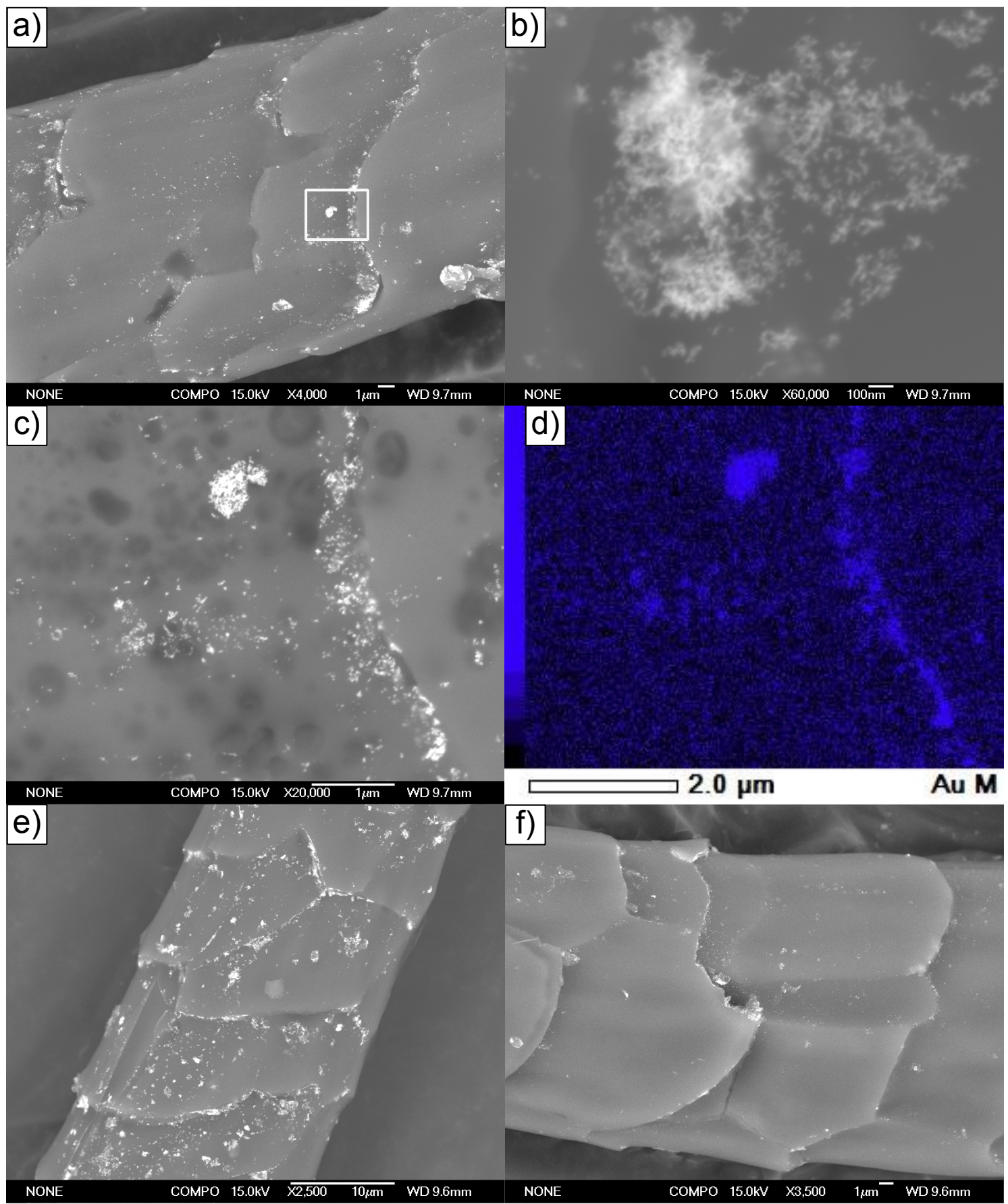

Figure 6.17 SEM micrographs in backscatter mode of an unwashed Cariaggi PEIgrey composite (a)-(c), an EDS elemental Au map (d), and SEM images of two composite fibres after washing on the Chiltern shaker for $24 \mathrm{~h}(\mathrm{e})$ and (f). 
this is so, this is an extremely positive result, and implies that the rubbing together of fibres has caused the bulk of the leaching of gold nanoparticles. Thus, the gentle hand washing of PEI-grey composites may cause only minimal leaching of gold nanoparticles from the PEI-grey composites, and would be a desirable composite for large-scale procedures.

\section{Amide Formation Catalyst}

The following study presents the use of a catalyst to attempt to promote the formation of amide bonds between wool fibres and the stabilising agents that surround the gold nanoparticles. The experiments were first carried out with the PEI-grey method, and later were extended to the TA-mauve and TSC-grey approaches, where an amide bond could potentially form between an amine in the wool protein and a carboxylate group in TA or TSC. The catalyst, 4(4,6-dimethoxy-1,3,5-triazin-2-yl)4-methylmorpholinium chloride hydrate (DMTMM) was utilised, and its structure is shown in Figure 6.18. DMTMM is an activating agent for amide bond formation, which has been used in the grafting of chemical functionality to wool and nylon $6,6 .{ }^{61}$<smiles>COc1nc(OC)nc([N+]2(C)CCOCC2)n1</smiles>

Figure 6.18 4(4,6-dimethoxy-1,3,5-triazin-2-yl)-4-methylmorpholinium chloride (DMTMM).

TU yarn was utilised in the following experiments, as its structure prevents excess agitation of wool fibres during laundering. Thus, the washing of TU yarn was considered to be more representative of the laundering of finished products, compared to the washing of wool in top form. The experiments involved either adding DMTMM (0.03 g per gram of wool) into the dye solution during the uptake, or immersing the wool in a boiling aqueous solution of DMTMM immediately after the dyeing process.

The PEI-grey composites that resulted from these experiments are shown in Figure 6.19 , where each composite appears to be a similar dark grey colour. The 
composites were washed on the dunking machine for $120 \mathrm{~min}$ in excess Kindness wool wash. The washed composites are also shown in Figure 6.19 (d)-(f), where each composite is a significantly lighter grey colour.

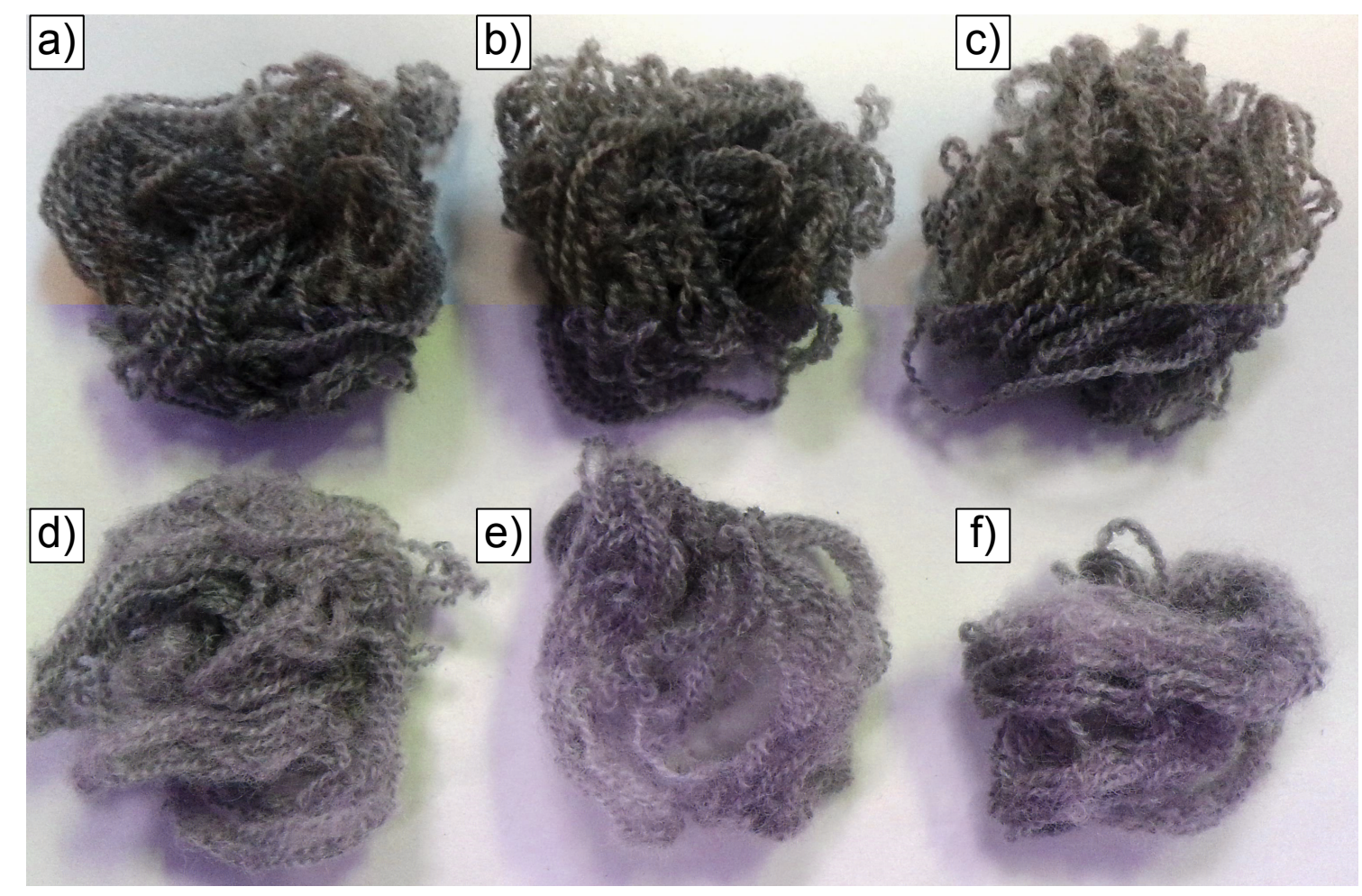

Figure 6.19 PEI-grey composites of TU yarn before (top) and after (bottom) washing, synthesised without DMTMM (a) and (d), with DMTMM in the uptake solution (b) and (e) and with a DMTMM post-treatment after dyeing (c) and (f).

The CIE coordinates of the PEI-grey TU yarn composites before and after washing on the dunking machine are presented in Table 6.11. The washing process resulted in significantly higher brightness and yellowness values and slightly lower redness values for each of the composites. Interestingly, the differences in CIE colour coordinates of the composites before and after washing are similar regardless of whether the catalyst was employed. Additionally, the composite synthesised without the catalyst exhibits the darkest grey colour. Thus, there is no evidence to suggest that the use of DMTMM has influenced the level of amide bond formation in the PEI-grey method.

Table 6.11 CIE colour coordinates of PEI-grey TU yarn composites synthesised with and without the amide formation catalyst DMTMM before and after simulated washing.

\begin{tabular}{ccccccc}
\hline Catalyst & \multicolumn{2}{c}{ No catalyst } & \multicolumn{2}{c}{ During } & dyeing & \multicolumn{2}{c}{ Post-dyeing } \\
\hline Washing & before & after & before & after & before & after \\
\hline L $^{*}$ & 47.48 & 59.00 & 49.72 & 61.27 & 49.00 & 63.67 \\
$\mathrm{a}^{*}$ & 0.29 & -0.22 & 0.47 & -0.35 & -0.46 & -0.81 \\
$\mathrm{~b}^{*}$ & -1.92 & -0.82 & -1.87 & -0.46 & -2.22 & -0.42 \\
\hline \hline
\end{tabular}


The washing process on the dunking machine is thought to be relatively harsh compared to gentle hand washing of the composites. Thus, this washing process may exaggerate the leaching of gold nanoparticles from the PEI-grey TU yarn composites. However it does show that while some of the PEI-Au NPs may be covalently bound to the wool fibres, a significant proportion of the gold nanoparticles are likely not covalently bound, and are able to be displaced from the surface of the fibres in washing processes.

The DMTMM experiments were repeated with the TSC-grey and TA-mauve methods, to investigate the effect of DMTMM on the washfastness properties of these composites. It was proposed that an amide bond would form between carboxylate groups on the TSC or TA stabilising the gold nanoparticles and the amine functionality of the wool protein, thereby improving the washfastness properties of the composites. These experiments again involved increasing the uptake temperature to $100{ }^{\circ} \mathrm{C}$ with or without DMTMM in the uptake solution, or soaking the composites in a boiling aqueous solution of DMTMM immediately after the uptake.

It was found that this resulted in the greying of the TA-mauve composites, and the leaching of TSC-Au NPs into the post-treatment solution. However, increasing the temperature of the uptake successfully improved the washfastness properties of the composites. Figure 6.20 presents the TSC-grey, TA-mauve and PEI-grey composites of TU yarn that were synthesised at an uptake temperature of $100{ }^{\circ} \mathrm{C}$ before and after washing. The TA-mauve composite in (Figure 6.20 (c)) exhibits a slight grey tinge, indicating that the elevated temperature of the uptake has caused a slight agglomeration of the TA-Au NPs on the surface of the composite fibres.

The CIE colour coordinates of the composites in Figure 6.20 are presented in Table 6.12. Each of the composites exhibit similar changes in CIE colour coordinates as a result of the washing process. The fact that significant colouration remains after the washing process indicates that some amide bonds have formed between the respective stabilising agents that surround the gold nanoparticles, and the wool fibres. The PEI-grey composite is the darkest composite after the washing process, as it leached the fewest gold nanoparticles into the wash solution, which could be due to a comparatively high number of amide bonds forming between the PEI and the wool fibres. This is understandable given the prevalence of carboxylic acid groups on the 18-MEA layer and the wool protein, and may illustrate a difficulty of the TSC and TA nanoparticles from coming into contact with the amine functionality that is present in the wool protein. Future washfastness experiments involving the heating of TA-mauve and TSC-grey composites of 18-MEA stripped wool would be interesting to undertake. 


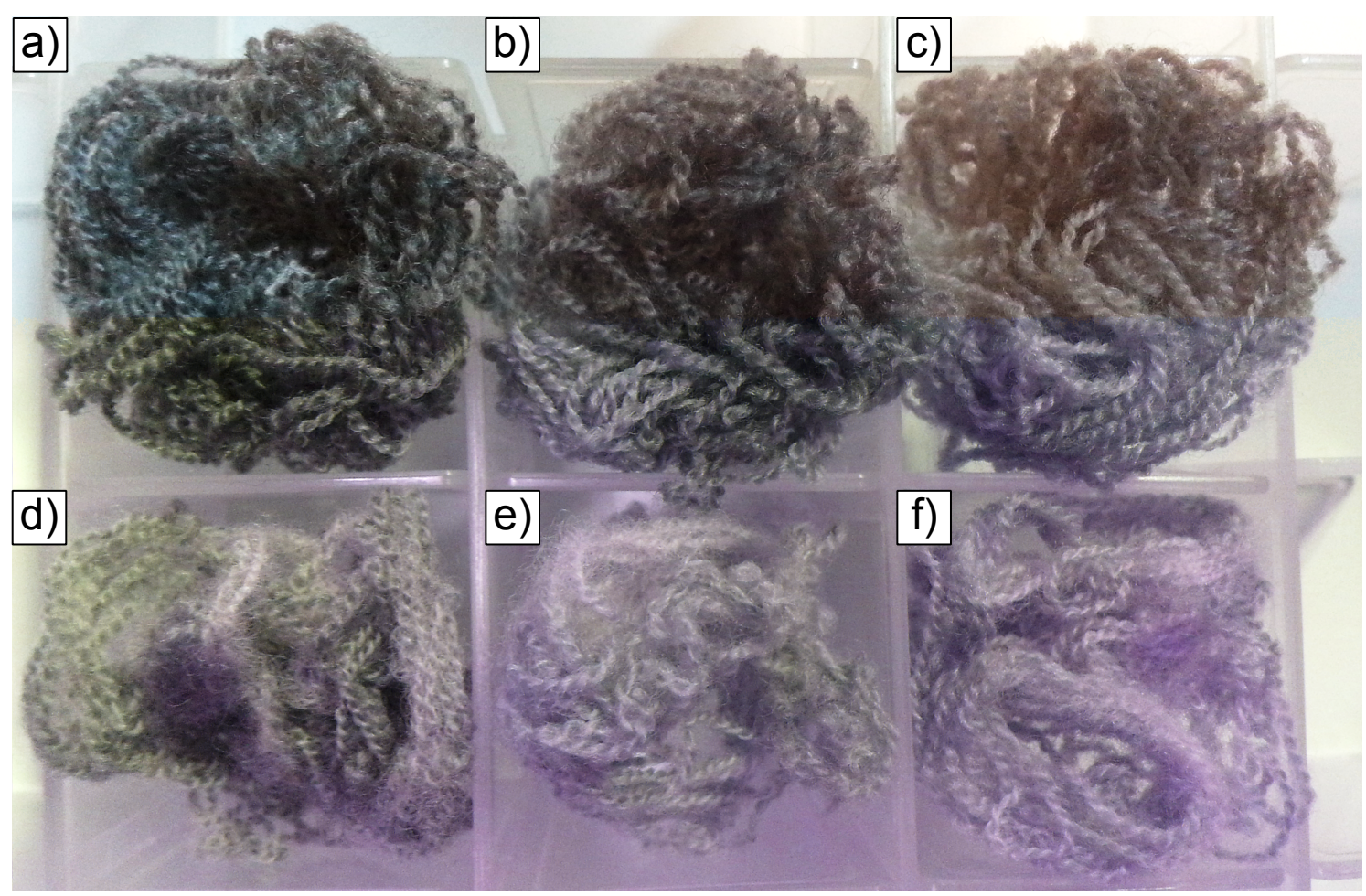

Figure 6.20 TU yarn composites synthesised PEI-grey (a) TSC-grey (b) and TAmauve (c) colloids with an uptake temperature of $100{ }^{\circ} \mathrm{C}$ (without DMTMM), and the composites after washing on the dunking machine for $120 \mathrm{~min}$ (d)-(f).

Table 6.12 CIE colour coordinates of TU yarn ex-situ composites synthesised with an uptake temperature of $100^{\circ} \mathrm{C}$ before and after washing.

\begin{tabular}{ccccccc}
\hline Method & \multicolumn{2}{c}{ PEI-grey } & \multicolumn{2}{c}{ TSC-grey } & \multicolumn{2}{c}{ TA-mauve } \\
\hline Washing & before & after & before & after & before & after \\
\hline $\mathrm{L}^{*}$ & 47.48 & 59.00 & 53.69 & 68.87 & 61.75 & 70.24 \\
$\mathrm{a}^{*}$ & 0.29 & -0.22 & 1.47 & 0.51 & 3.50 & 1.56 \\
$\mathrm{~b}^{*}$ & -1.92 & -0.82 & -3.27 & -1.06 & -3.27 & -1.06 \\
\hline \hline
\end{tabular}

In summary, PEI-capped gold nanoparticles were successfully used to colour merino wool fibres and resulted in composites with impressive washfastness properties. While the PEI-grey composites are not completely colourfast, they have been shown to have improved washfastness properties compared to TA-mauve and TSC-grey composites, which is a very promising result. It is highly recommended that scaleup dyeing procedures that allow for the boiling of the dye solutions during the uptake are investigated, so that larger-scale PEI-grey composites can be produced.

\subsubsection{Reactive Dyes}

Reactive dyes were investigated as potential dual reducing and stabilising agents in the solution phase synthesis of gold nanoparticles. As mentioned in Section 1.1.4, 
reactive dyes encompass a chromophoric component and a distinct reactive group. The reactive moiety is designed to covalently bond with the wool fibres, thereby preventing their leaching during laundering. It was considered that reactive dyes may be able to facilitate the reduction of $\mathrm{Au}^{3+}$ to $\mathrm{Au}^{0}$ and stabilise the formed nanoparticles. If gold nanoparticles could be synthesised using the chromophore of the reactive dye, without involving the reactive moiety, gold nanoparticles with a reactive group on the exterior would result. Ideally, the colour of the reactive dye would be destroyed in the process. These "reactive" gold nanoparticles could potentially covalently bond to the chemical functionality of the wool fibres. A covalent bond linking the wool fibre surface and the stabiliser of gold nanoparticles is highly desirable for the ex-situ procedure, and was anticipated to result in composites with excellent washfastness properties.

Table 6.13 details the relevant reactive dyes that were investigated for the solution phase synthesis of gold nanoparticles. The reactive dyes were chosen based upon the recommendation of Professor James Johnston's industry contacts at Tararua Yarns Ltd. ${ }^{54}$ The industrial colouring of wool with these reactive dyes typically operates under acidic conditions at elevated temperatures of up to $120^{\circ} \mathrm{C}$ (where temperatures above $100^{\circ} \mathrm{C}$ require pressurised reactors).

Table 6.13 Reactive dyes that were used in the gold nanoparticle syntheses.

\begin{tabular}{ccccc}
\hline Reactive dye & Remazol Blue & Lanasol Red & Lanasol Yellow & Acomet Yellow \\
\hline Abbreviation & RB & LR & LY & AY \\
Reactive group & vinyl sulfone & $\alpha$-bromoacrylamido & triazine \\
$\begin{array}{c}\text { Formed stable } \\
\text { Au NPs? }\end{array}$ & Yes & Yes & No & Yes \\
\hline \hline
\end{tabular}

The following experiments involved the addition of different amounts of the reactive dyes (from $1 \mathrm{wt} \%$ aqueous stock solutions) into boiling solutions of $40 \mathrm{mg} \mathrm{L}^{-1} \mathrm{AuCl}_{4}^{-}$, which is the equivalent concentration of $\mathrm{AuCl}_{4}^{-}$that is used in the synthesis of the TSC-grey and PEI-grey colloids. The chemical structures of the reactive dyes are presented together with the normalised UV-vis spectra of the reaction solutions. The ratios of $\mathrm{AuCl}_{4}^{-}$to reactive dyes that were employed are weight-based.

Figure 6.21 presents the UV-vis spectra of gold colloids that were synthesised with Remazol Blue (RB), and the chemical structure of RB. The chromophoric peak of $\mathrm{RB}$ centres at approximately $590 \mathrm{~nm}$, and also has a large shoulder at around $620 \mathrm{~nm}$. In the attempted synthesis of gold nanoparticles with a weight ratio of 1:1.25 of $\mathrm{AuCl}_{4}^{-}$to $\mathrm{RB}$, the chromophoric peak is significantly lower in intensity, and it appears as though an agglomerated gold colloid has formed. When a ratio of 
1:2.5 of $\mathrm{AuCl}_{4}^{-}$to $\mathrm{RB}$ was employed, a stable gold colloid resulted. The colloid is a deep-red colour, which demonstrates that the blue colour of RB is destroyed during the synthesis. Thus, it is possible that the reactive part of the dye is not involved in the formation of gold nanoparticles, and as such RB-stabilised gold nanoparticle colloids were used in subsequent wool dyeing reactions.

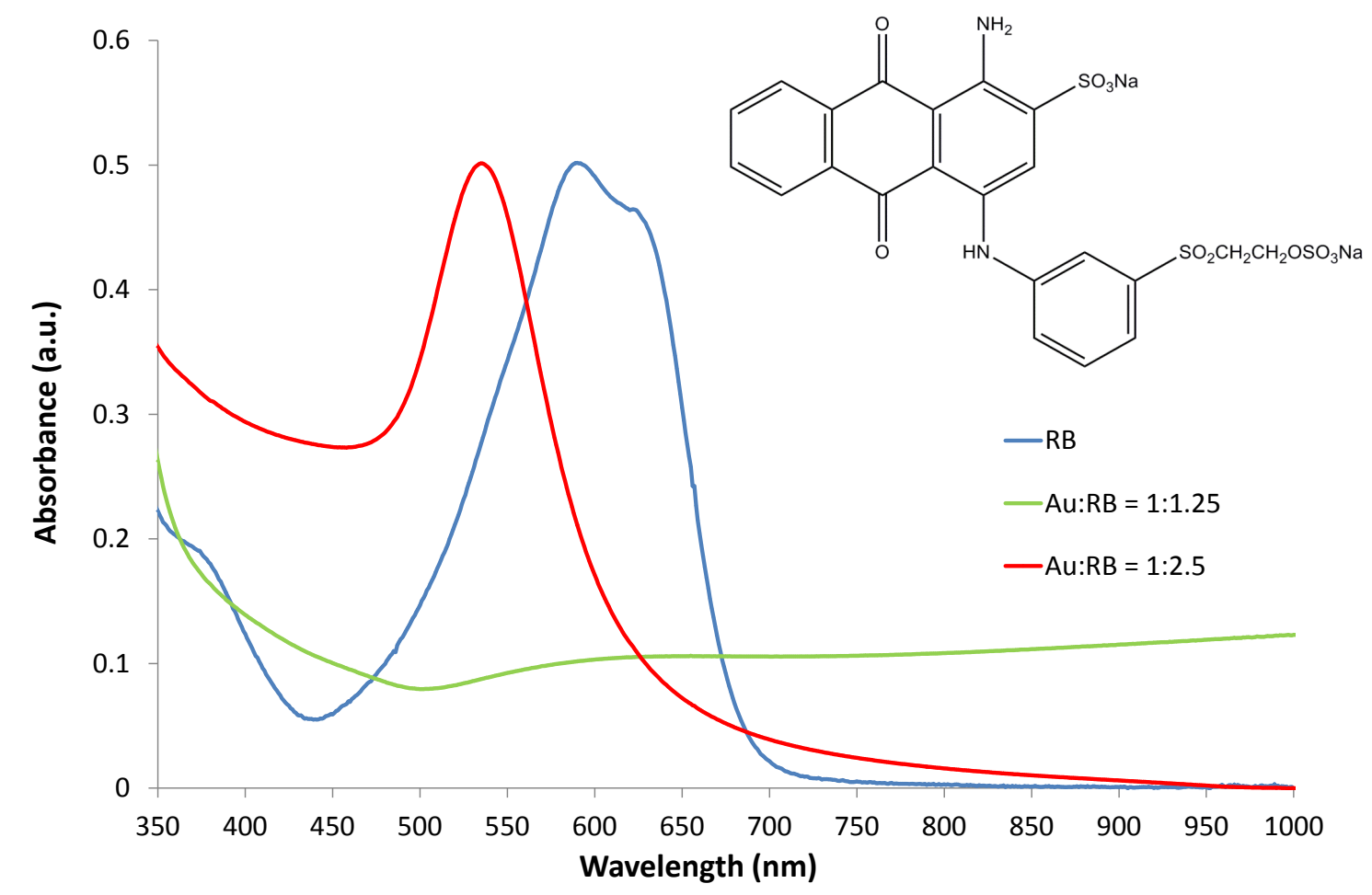

Figure 6.21 The UV-vis spectrum and chemical structure of Remazol Blue (RB), and the UV-vis spectra of gold colloids that were synthesised with different ratios of $\mathrm{AuCl}_{4}^{-}$to RB.

The UV-vis spectra of Lanasol red (LR) and LR gold colloids are shown in Figure 6.22. The LR dye exhibits its UV-vis peak at approximately $500 \mathrm{~nm}$. The UVvis spectrum of the 1:1.25 ratio of $\mathrm{AuCl}_{4}^{-}$:LR colloid resembles that of a destabilised gold colloid. When an $\mathrm{AuCl}_{4}^{-}$to $\mathrm{LR}$ ratio of 1:2.5 was used, the colloid exhibited a narrower SPR peak $(\lambda=550 \mathrm{~nm})$, which indicates the formation of a stable colloid. The use of LR in the synthesis of gold nanoparticles resulted in the destruction or change of the chromophoric absorbance, and hence, a change in the chemical environment of the chromophore has occurred. Such a change in energy indicates that the chromophore of the reactive dye is used in the reduction of $\mathrm{Au}^{3+}$. Hence, the reactive moiety may not be involved in the formation of gold nanoparticles, and as such, LR-stabilised gold colloids were used to dye wool. 


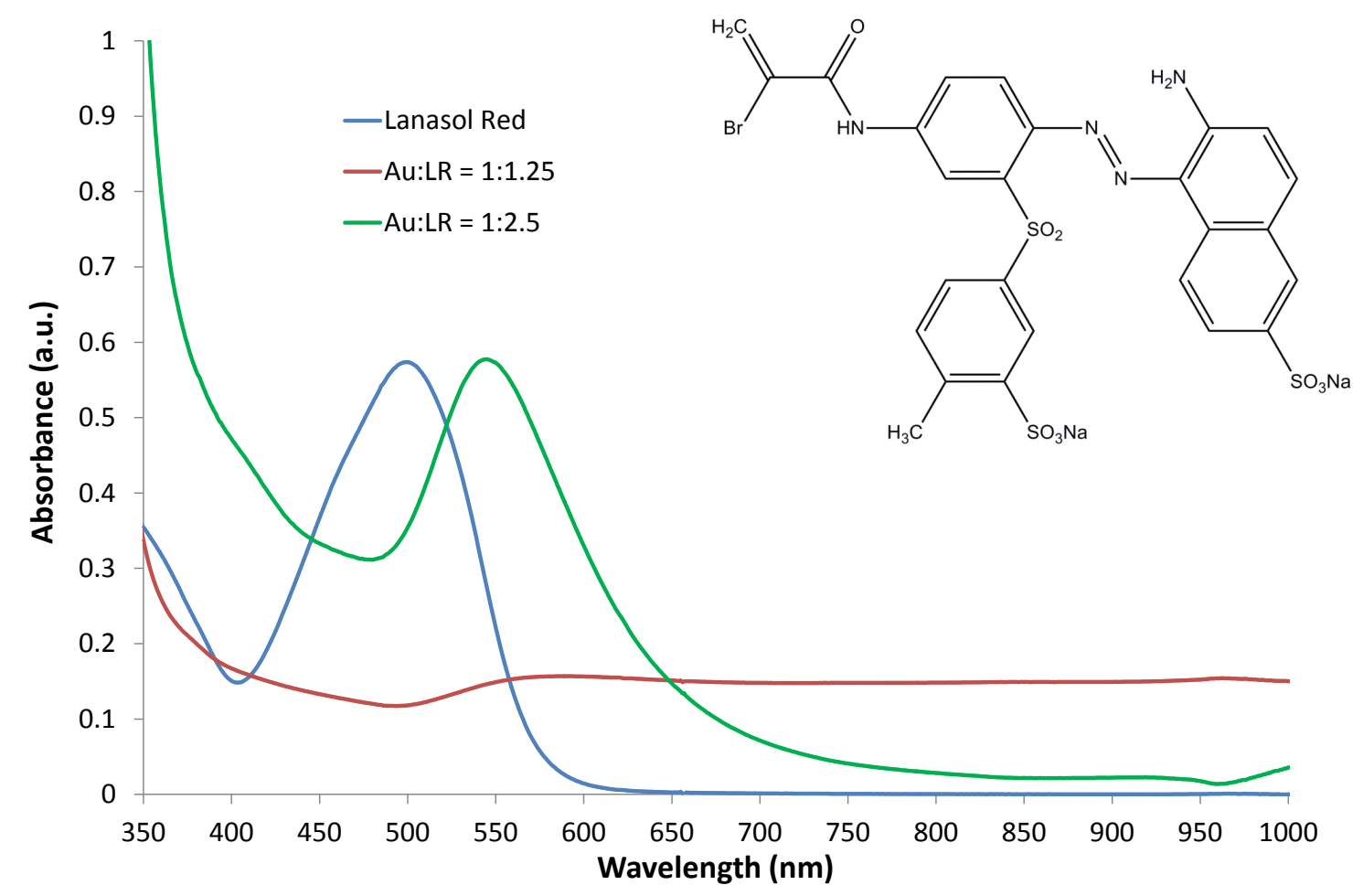

Figure 6.22 The UV-vis spectrum and chemical structure of Lanasol Red (LR), and the UV-vis spectra of gold colloids that were synthesised with different ratios of $\mathrm{AuCl}_{4}^{-}$to LR.

Lanasol Yellow (LY) exhibits the same $\alpha$-bromoacrylamido reactive component as LR, with a chromophore that exhibits a peak absorbance at approximately $400 \mathrm{~nm}$. The UV-vis spectra presented in Figure 6.23 show the development of a broad SPR peak from 500-650 nm. This shows that the use of LY has caused some reduction of $\mathrm{Au}^{3+}$ to $\mathrm{Au}^{0}$, however, a stable gold colloid was not successfully formed. The yellowcoloured absorption of the LY dye molecule almost disappears in the spectrum of the 1:1.25 ratio of $\mathrm{AuCl}_{4}^{-}$to $\mathrm{LY}$, but it remains in the 1:2.5 spectrum. This indicates that the chromophore may be involved in the reduction of $\mathrm{Au}^{3+}$ to $\mathrm{Au}^{0}$. It is also possible that the reactive moiety is involved in the formation of the gold nanoparticles. The inability of LY forming a stable gold colloid meant that it was not used in the ex-situ procedure of gold nanoparticle-wool fibre composites.

Finally, Acomet yellow (AY) was used in the attempted synthesis of gold nanoparticle colloids, the UV-vis spectra of which are shown in Figure 6.24. The colloid is able to form gold nanoparticles, but the absorption energy of the yellow chromophore appears unaffected by the process. The resulting gold colloids exhibit SPR peaks that centre at approximately $560 \mathrm{~nm}$, in addition to the original yellow coloured peak from $375-475 \mathrm{~nm}$. The absorption may be due to excess AY, otherwise the synthesis likely involves the reactive pyridine moiety of the dye molecule, while not affecting the yellow colour of the dye. As such, AY was not used in future gold nanoparticle syntheses. 


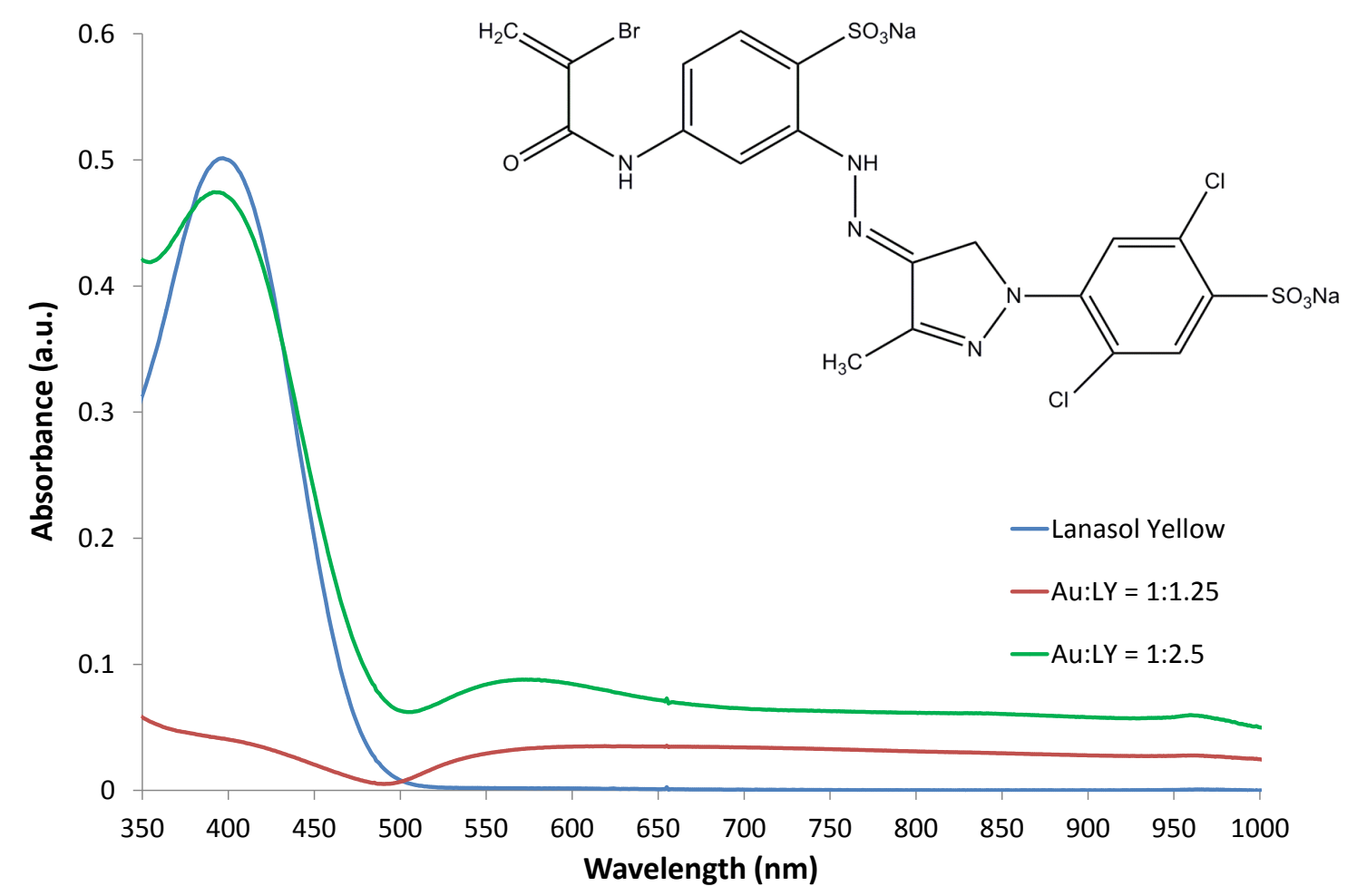

Figure 6.23 The UV-vis spectrum and chemical structure of Lanasol Yellow (LY), and the UV-vis spectra of gold colloids that were synthesised with different ratios of $\mathrm{AuCl}_{4}^{-}$to LY.

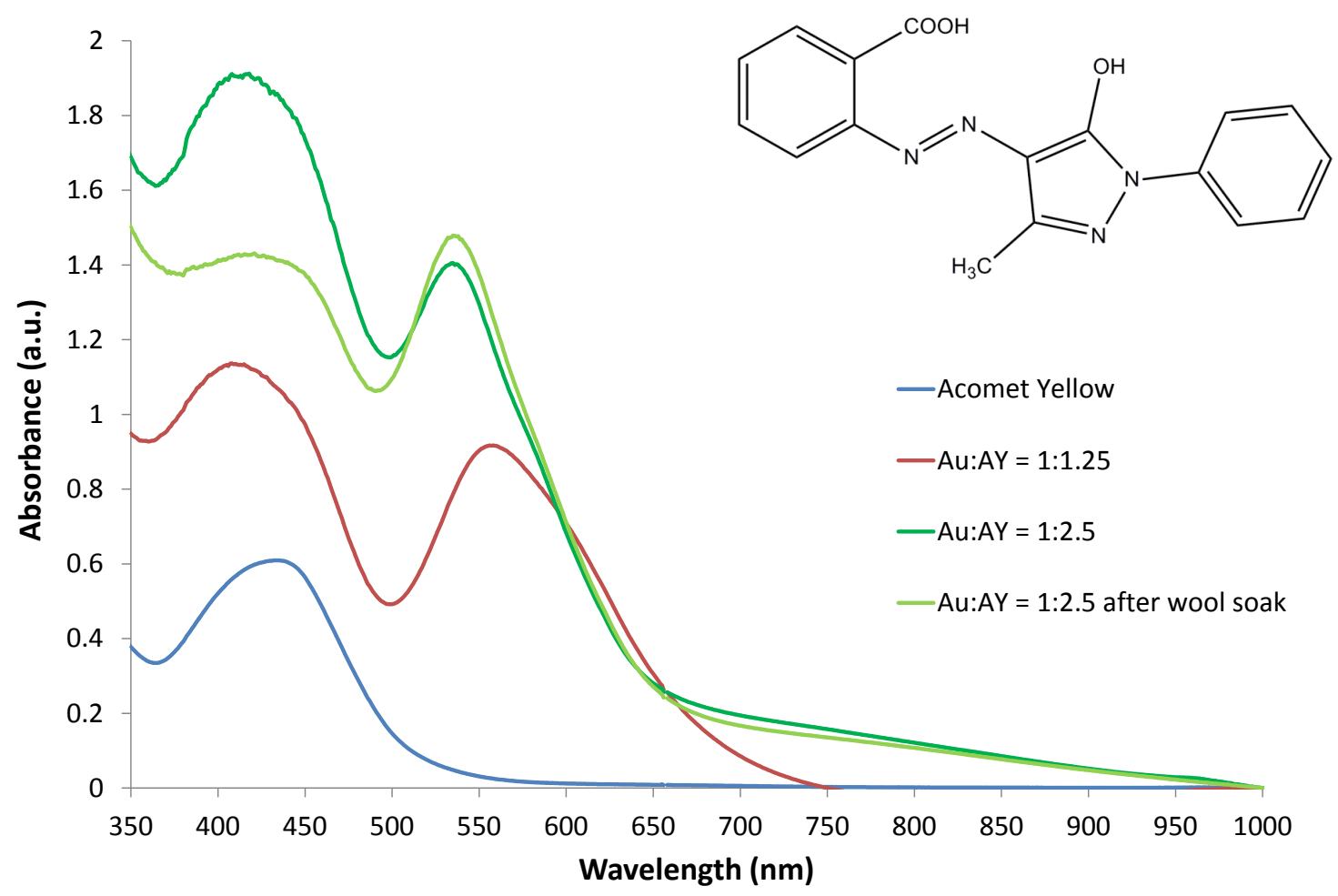

Figure 6.24 The UV-vis spectrum and chemical structure of Acomet Yellow (AY), and the UV-vis spectra of gold colloids that were synthesised with different ratios of $\mathrm{AuCl}_{4}^{-}$to $\mathrm{AY}$. 
Both RB and LR showed promise for use in the synthesis of gold nanoparticles that contain reactive groups on their exterior. The syntheses of these gold nanoparticle colloids were first optimised to minimise the amount of excess dye remaining in the solution. Following this, the colloids were used to colour wool fibres under a range of conditions and the washfastness properties of the composites were tested.

\section{Remazol Blue}

A weight ratio 1:2.5 of $\mathrm{AuCl}_{4}^{-}$to $\mathrm{RB}$ was found to be optimal for the synthesis of $\mathrm{RB}$ stabilised gold nanoparticles. Using lesser amounts of RB formed a destabilised gold colloid, and using more RB resulted in excess dye molecules in the colloidal solution. As such, the RB-colloid with an $\mathrm{AuCl}_{4}^{-}$: $2.5 \mathrm{RB}$ ratio was used to colour Cariaggi wool and TU yarn. When the dyeing reaction was carried out at $\mathrm{pH} 7$ and $100^{\circ} \mathrm{C}$, full uptake of the colloid occurred. The composites that resulted from these uptake conditions, before and after washing for $24 \mathrm{~h}$ on the Chiltern shaker are presented in Figure 6.25. The washing process was found to cause significant leaching of RB-Au NPs from both the Cariaggi and TU yarn composites. Additionally, although the composites comprise the equivalent amount of gold that is used in the TSC-grey method, the RB composites are purple-grey in colour. This was originally thought to be due to the presence of excess blue coloured RB molecules in the colloid.

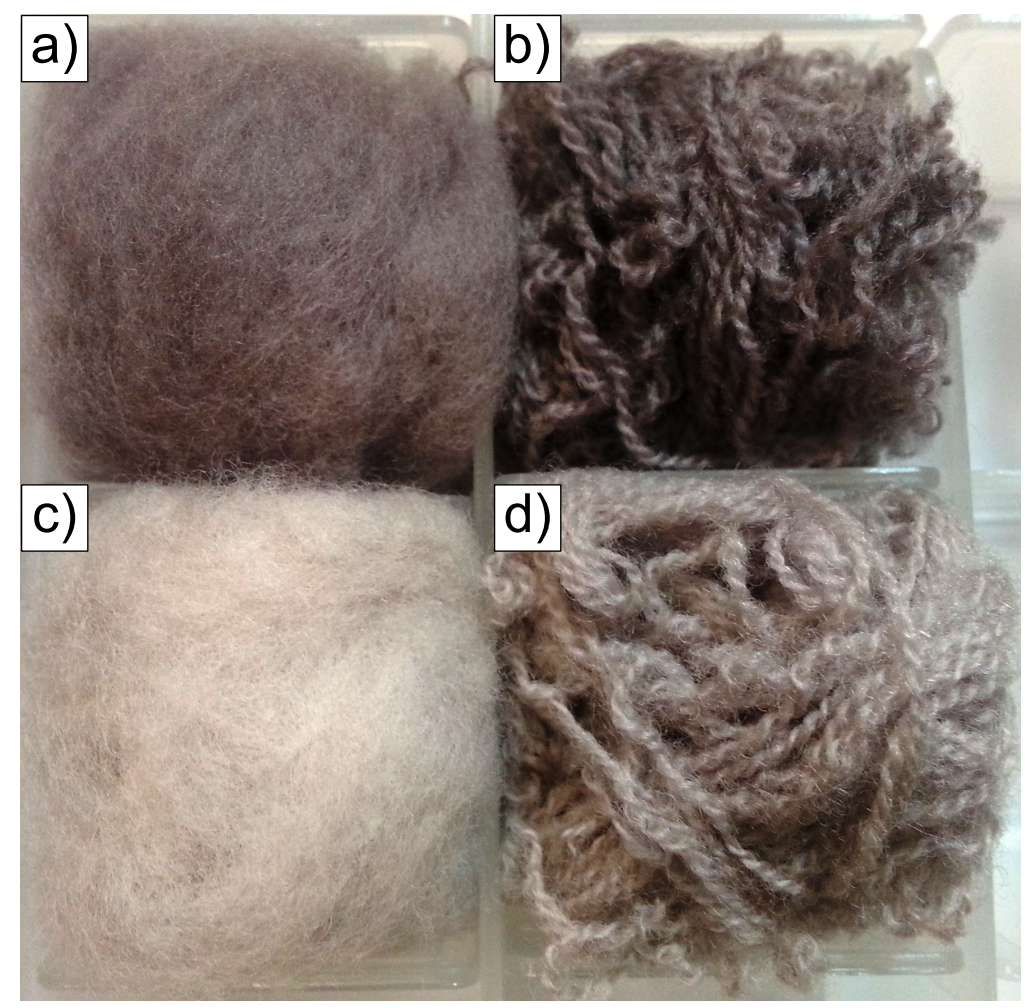

Figure 6.25 RB composites of Cariaggi wool (a) and TU yarn (b), and the composites after washing on the Chiltern shaker for $24 \mathrm{~h}(\mathrm{c})$ and (d). 
CIE colour coordinates of the RB composites before and after washing are presented in Table 6.14. However, the $\mathrm{b}^{*}$ values of the unwashed Cariaggi and TU yarn composites are only slightly negative, which indicates that excess RB molecules are not present in the dye solution. The CIE colour coordinates of the unwashed RB composites are similar to those of purple-mauve ex-situ composites; as such, the purple colour of the composites is thought to be due to the size, shape and distribution of the gold nanoparticles, rather than any residual RB dye molecules.

Table 6.14 CIE colour coordinates of Cariaggi and TU yarn RB composites before and after washing.

\begin{tabular}{ccccc}
\hline Wool type & \multicolumn{2}{c}{ Cariaggi } & \multicolumn{2}{c}{ TU yarn } \\
\hline Washing & before & after & before & after \\
\hline $\mathrm{L}^{*}$ & 54.01 & 71.67 & 47.66 & 62.14 \\
$\mathrm{a}^{*}$ & 5.61 & 4.19 & 4.69 & 5.38 \\
$\mathrm{~b}^{*}$ & -0.36 & 3.31 & -0.20 & 3.04 \\
\hline \hline
\end{tabular}

The washing process caused significant leaching of the gold nanoparticles from the RB composites. The washed composites appear to exhibit washfastness properties that are similar to those of other ex-situ composites. Thus, the attraction between the RB-stabilised gold nanoparticles and the wool fibres is likely only electrostatic in nature, not covalently bound RB-Au NPs. The assumed lack of the formation of covalent bonds between the RB-Au NPs and the wool fibres may be due to the vinyl sulfone reactive component of the $\mathrm{RB}$ molecule being attracted to the gold nanoparticles, and hence unable to bond to the wool. Alternatively, it may be difficult for the reactive dye to gain access to the electron-rich functionality of the wool protein (much of which is located in the fibre interior), as the RB-Au NPs can only attach to the surfaces of the wool fibres.

SEM analysis of the surfaces of the unwashed Cariaggi RB composite, and the TU $\mathrm{RB}$ composite before and after washing are presented in Figure 6.26. The RBstabilised gold nanoparticles are typically spherical in shape, however there are also the occasional nanorod and triangular platelet shaped gold nanoparticles present. Additionally, there is a significant size distribution of the gold nanoparticles, some of which are up to $50 \mathrm{~nm}$ in diameter. The larger nanoparticles and the agglomerates will contribute to the grey colour spectrum of the composites.

The RB TU yarn composite after washing still exhibits a significant coverage of gold nanoparticles on the surface of the fibres. Thus, it is possible that some of the RB-Au NPs are covalently bound to the surface of the wool fibres. However, as colouring wool with RB-stabilised gold colloids did not result in composites with improved washfastness properties (compared to other ex-situ composites), this method was deemed to be unsuitable for integration into scale-up procedures. 


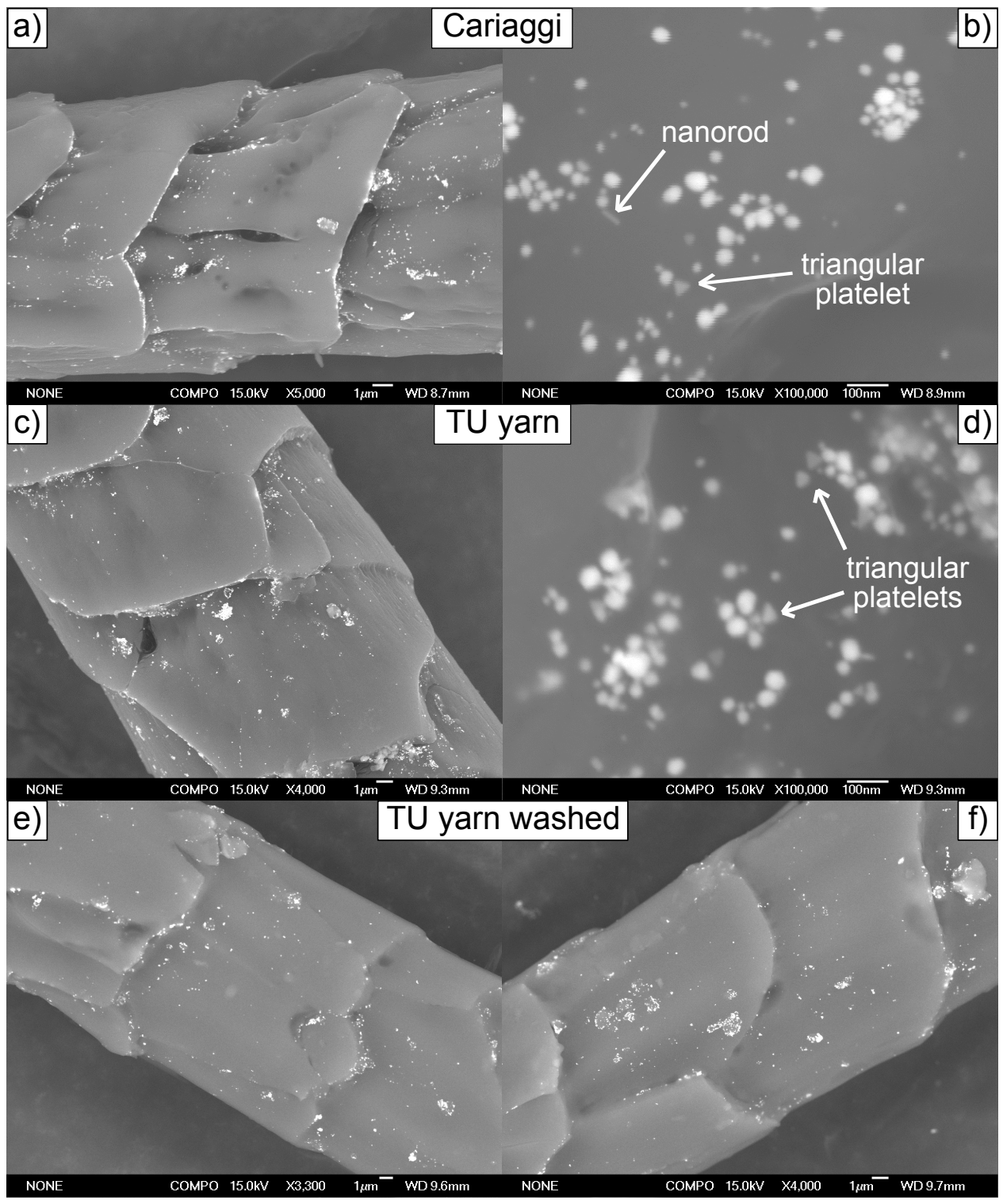

Figure 6.26 SEM micrographs in backscatter mode of the surfaces of RB-Au NPs composites of Cariaggi wool before washing (a)-(b) and TU yarn (c)-(d) before washing, and TU yarn composite fibres after washing in excess Kindness wool wash on the Chiltern shaker for $24 \mathrm{~h}(\mathrm{e})-(\mathrm{f})$.

\section{Lanasol Red}

Lanasol red (LR) also showed significant promise as a dual reducing and stabilising agent for use in the synthesis of gold nanoparticles. The red colour of LR made it difficult to determine whether or not the chromophore was involved in the synthesis of gold nanoparticles (due to the overlap of the deep-red SPR band of gold nanoparticles and red absorbance of the dye molecule). However, as Lanasol Yellow (LY) (which contains the same reactive $\alpha$-bromoacrylamido moiety) was unable to form a stable gold colloid, it is possible that the reactive component of the dye is not involved in the synthesis, and could potentially be located on the exterior of the gold nanoparticles. 
Figure 6.27 presents TU yarn composites that were coloured with LR-stabilised gold colloids, which incorporated different weight ratios of $\mathrm{AuCl}_{4}^{-}$: $\mathrm{LR}$ in their syntheses. The LR colloid that employed $\mathrm{AuCl}_{4}^{-}: 2.5 \mathrm{LR}$ resulted in purple-grey coloured composites. When higher amounts of LR were employed in the synthesis of gold nanoparticles, stable colloids were formed. However, the resulting composites were very red in colour, which indicates that excess LR dye molecules remained in the colloidal dye solutions, and imparted the red hue to the composites.

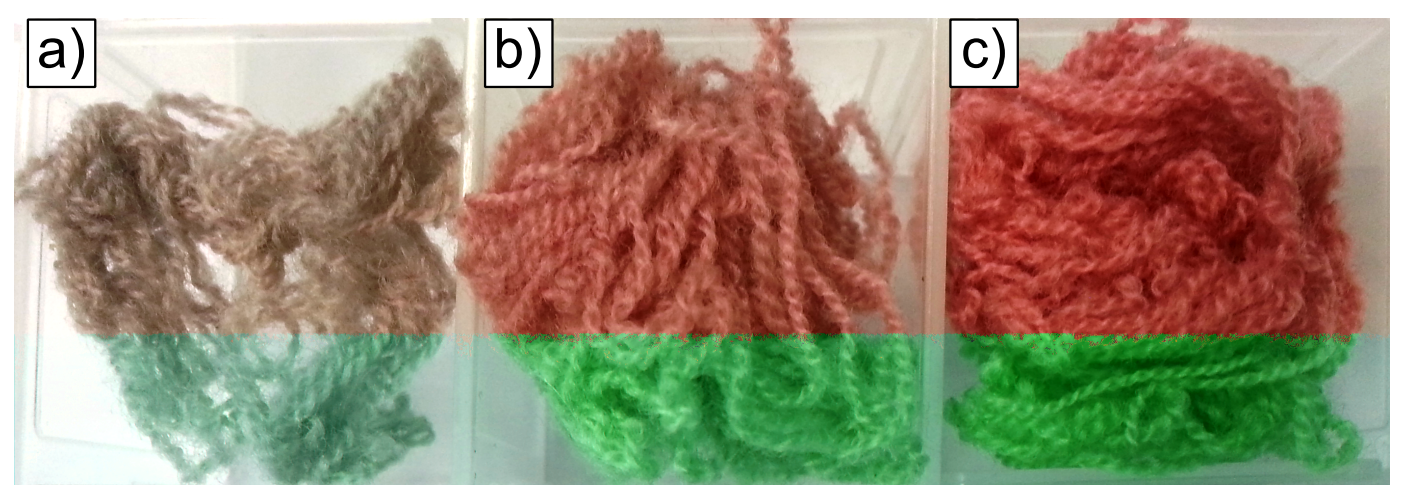

Figure 6.27 TU yarn composites dyed with LR-stabilised gold colloids that were synthesised with weight ratios of $\mathrm{AuCl}_{4}^{-}$to LR of 1:2.5 (a), 1:3.33 (b) and 1:5 (c).

CIE colour coordinates of the LR-composites are presented in Table 6.15. When the amount of LR used in the synthesis of the gold colloids was increased, the resulting composites saw a remarkable increase in redness values, in addition to being darker and more yellow. The high red values of the 1:3.33 and 1:5 $\mathrm{AuCl}_{4}^{-}$:LR composites indicate that they have absorbed LR molecules in the dye solutions.

Table 6.15 CIE colour coordinates of TU yarn dyed with LR colloids that were synthesised with different weight ratios of $\mathrm{AuCl}_{4}^{-}$:LR.

\begin{tabular}{cccc}
\hline $\mathrm{AuCl}_{4}^{-}$to LR ratio & $1: 2.5$ & $1: 3.33$ & $1: 5$ \\
\hline $\mathrm{L}^{*}$ & 62.65 & 56.71 & 53.35 \\
$\mathrm{a}^{*}$ & 4.02 & 16.35 & 25.63 \\
$\mathrm{~b}^{*}$ & 4.51 & 7.19 & 10.19 \\
\hline \hline
\end{tabular}

SEM analysis was carried out on the LR TU yarn composites in Figure 6.27 (a) and (b), to investigate the size, shape and distribution of the LR-stabilised gold nanoparticles, and is presented in Figure 6.28. At relatively low magnification, the grey-coloured LR composite appears to exhibit a distribution of gold nanoparticles that is common with the TSC-grey method. However, the higher magnification image in Figure 6.28 (b) shows that the gold is typically present as spherical and triangular platelet-type nanoparticles, in addition to some rod and string like entities which appear to link the nanoparticles together. The diverse range of sizes and shapes of the LR-Au NPs would absorb a range of different energies of visible light, and would account for the purple-grey colour of this composite. 


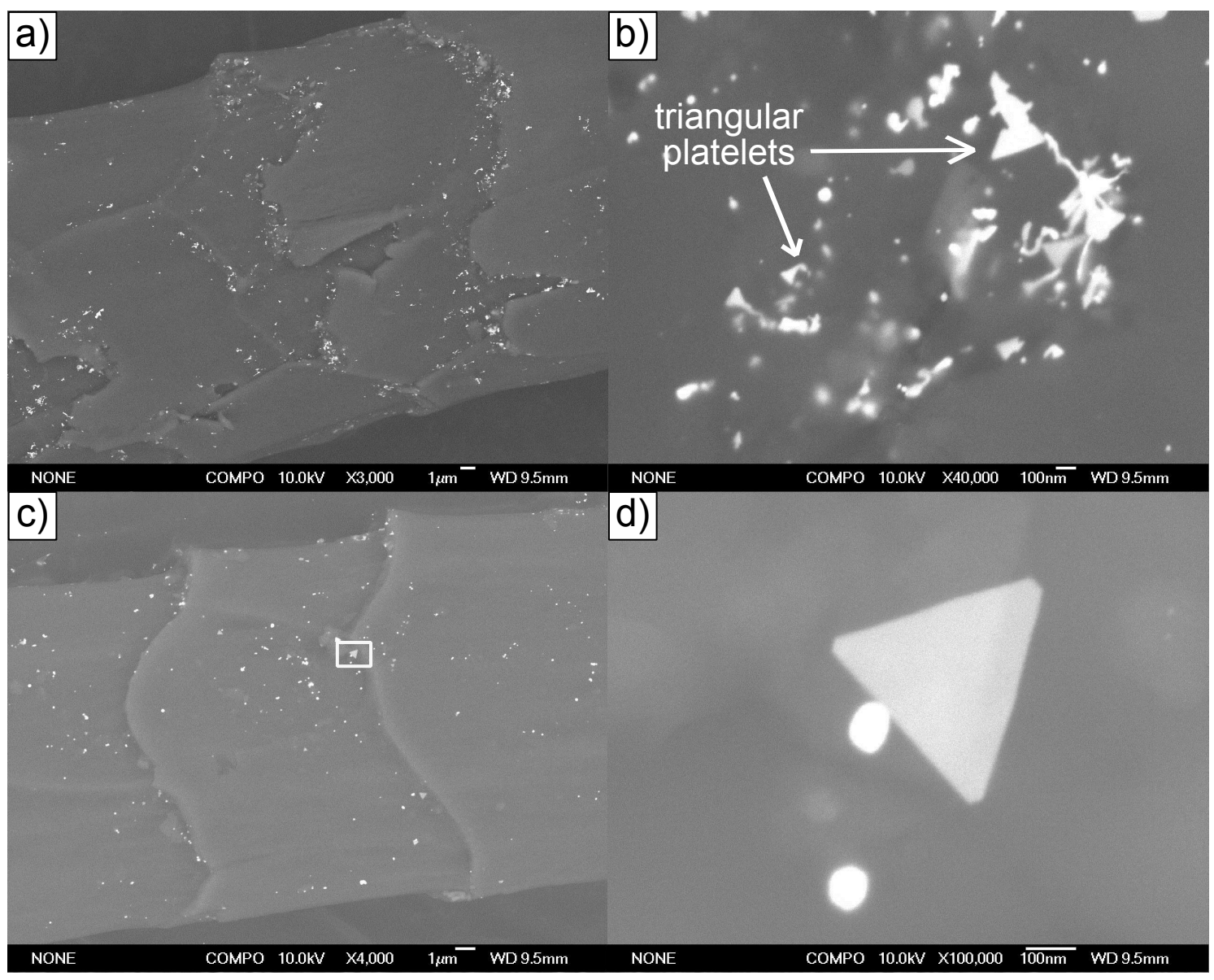

Figure 6.28 SEM micrographs in backscatter mode of the surfaces of LR TU yarn composites synthesised with an $\mathrm{AuCl}_{4}^{-}$:LR ratio of 1:2.5 (a)-(b) and 1:3.33 (c)-(d).

In contrast, the purple-red coloured composite that was synthesised with a ratio of $\mathrm{AuCl}_{4}^{-}$to LR of 1:3.3 does not exhibit these string-like particles. The micrographs in Figure 6.28 (c) and (d) show that the gold nanoparticles are typically spherical particles of approximately $50 \mathrm{~nm}$ in diameter, or much larger triangular platelets with edges of up to $500 \mathrm{~nm}$ length. The lack of the string-like gold entities is considered to be due to higher amount of LR molecules aiding the stabilisation of this gold colloid, and allowing complete nanoparticles to be formed.

It was determined that the synthesis of a stable LR-stabilised gold colloid required a $\mathrm{AuCl}_{4}^{-}$to LR ratio of 1:3.33. However, these gold colloids impart a red colour to wool fibres in dyeing reactions, indicating that they contain residual LR molecules. To attempt to reduce the excess LR dye molecules that remain in the colloidal solutions, gold nanoparticle syntheses were attempted with a combination of lesser amounts of LR and an additional stabilising agent. For this, different amounts of polymethacrylate (PMA) were employed in the synthesis of LR-stabilised gold nanoparticles. TU yarn composites that resulted from these colloids are presented in Figure 6.29. While the composites are different shades of purple-red in colour, the red colour of LR has remained in each of the composites. 


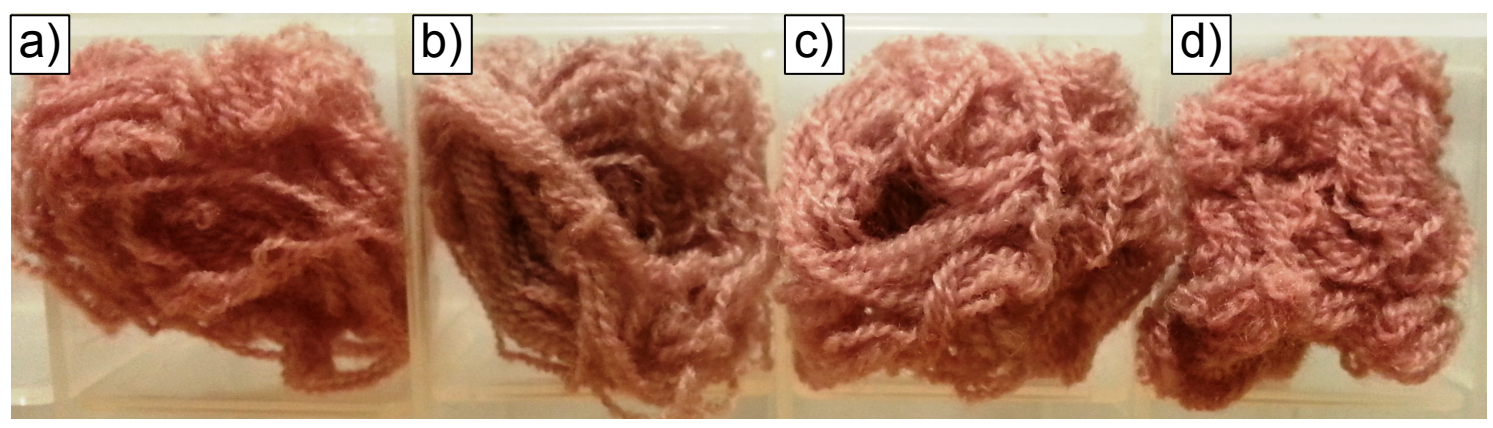

Figure 6.29 LR TU yarn composites dyed with colloids that incorporated different amounts of PMA in their syntheses (and a ratio of $\mathrm{AuCl}_{4}^{-}$to LR of 1:3.33): no PMA (a), $10 \mathrm{mg} \mathrm{L}^{-1}$ (b), $25 \mathrm{mg} \mathrm{L}^{-1}$ (c) and $50 \mathrm{mg} \mathrm{L}^{-1}$ (d).

CIE colour coordinates of the TU LR composites that were presented in Figure 6.29 are shown in Table 6.16. The LR TU yarn composites exhibit very high redness values, which is considered to be due to excess LR molecules in the colloid being absorbed by the wool fibres.

Table 6.16 CIE colour coordinates of LR TU yarn composites dyed with colloids synthesised with different amounts of PMA in the colloid synthesis.

\begin{tabular}{ccccc}
\hline Amount of PMA $\left(\mathrm{mg} \mathrm{L}^{-1}\right)$ & 0 & 10 & 25 & 50 \\
\hline $\mathrm{L}^{*}$ & 56.71 & 58.58 & 58.32 & 51.97 \\
$\mathrm{a}^{*}$ & 16.35 & 12.65 & 15.21 & 17.30 \\
$\mathrm{~b}^{*}$ & 7.19 & 3.73 & 3.92 & 5.74 \\
\hline \hline
\end{tabular}

LR-stabilised gold colloids were successfully used to colour wool fibres. However, the amount of LR that was required to form a stable gold colloid resulted in the red colouration of the composites due to excess LR molecules in the dye solutions. As such, this is currently not a viable method for producing ex-situ composites. Future experiments that utilise alternative stabilisers in addition to LR could potentially result in fewer excess LR molecules, and would be worthwhile to investigate. 


\subsection{Conclusions}

Chapter 6 has detailed experiments that attempted to improve the washfastness properties of ex-situ composites. First, the washfastness properties of TA-mauve, TSC-pink and TSC-grey composites were characterised. The ex-situ composites were seen to leach significant amounts of gold nanoparticles as a result of the washing processes.

A range of chemical pre-treatments were then investigated to attempt to provide additional reactive sites for the attachment of gold nanoparticles. Many of the pre-treatments prevented the uptake of gold nanoparticles in subsequent dyeing reactions. However, the thiol-containing reagents appeared to significantly enhance the washfastness properties of Cariaggi TA-mauve composites.

Next, a range of chemical post-treatments were enacted on TA-mauve Cariaggi composites. These treatments all caused either the agglomeration of gold nanoparticles on the surfaces of the composites, or the leaching of gold nanoparticles into the post-treatment solutions. However, although the PEI post-treatments caused the slight leaching of gold nanoparticles during the treatment, they also significantly improved the washfastness properties of the composites.

Finally, a range of alternative reducing and stabilising agents were employed in the synthesis of gold nanoparticles. Of these reagents, PEI showed the greatest promise for improving the washfastness properties of the composites. Further investigation of this method is recommended.

While many of the presented experiments appeared to significantly improve the washfastness properties of the composites, none of the experiments exhibited covalently bound gold nanoparticles exclusively, as they all leached gold nanoparticles to some extent. This may be too difficult to realise, given that there are experimental constraints that are related to the commercial viability of the production of the composites. However, given that these nanocomposites are to be fabricated into luxury garments and carpets that will require gentle laundering, and that some of these methods result in only the minimal leaching of gold nanoparticles from the composites, the ex-situ methods are still considered to be commercially viable, if not optimal, for scale-up production. 


\section{Chapter 7}

\section{Conclusions}

This $\mathrm{PhD}$ research programme involved the optimisation and scale-up of the synthesis of gold nanoparticle-wool fibre composites. The research was concerned with the commercialisation of the novel composites.

Chapter 3 detailed the research of the in-situ procedure, with the aim of reducing the gold content of in-situ composites. The chemistry of the in-situ procedure was introduced by characterising in-situ composites of Cariaggi wool. Following this, the effects of a range of reaction conditions on the uptake and ageing processes were determined. It was found that the colouration of the composites was highly dependent on the type of wool that was used in the synthesis.

External energy sources and reducing agents were then used to increase the rate of gold nanoparticle formation in the wool fibres and promote the formation of gold nanoparticles near the surface of the wool fibres. The use of external reducing agents resulted in composites of the novel colours of yellow, brown and black. This was an excellent finding, however, the gold content of in-situ composites remained higher than that of ex-situ composites. As such, scale-up syntheses of in-situ composites have not been carried out to date. These results led to the laboratory-scale optimisation of the ex-situ procedure, which is a cost-effective alternative to the in-situ procedure.

Chapter 4 was concerned with the laboratory-scale optimisation of the ex-situ procedure. The chemistry of the ex-situ procedure was introduced by characterising ex-situ composites of Cariaggi wool. The synthesis of gold nanoparticle colloids was then optimised. The formation and $\mathrm{pH}$ stability of TA- and TSC-stabilised gold colloids was studied with UV-vis spectroscopy. The synthesis of TSC-Au NPs was 
improved by increasing the amount of TSC that was employed.

The uptake of gold nanoparticle colloids to wool was characterised. The wool type, $\mathrm{pH}$, use of auxiliary agents, gold nanoparticle concentration and volume of the gold colloid used were found to influence to rate of uptake and the colouration of the resulting composites. Merino yarns were able to be uniformly coloured with the use of auxiliary agents. This was a very positive result, as wool yarns are the optimal form of wool to dye. The ex-situ procedure is a relatively inexpensive and robust alternative to the in-situ procedure. A summary of advantages and disadvantages of in-situ and ex-situ procedures are listed in Table 7.1. The chemistry that was presented in this Chapter 4 was an integral step towards commercialising these novel composites, and led to the pilot-scale production of ex-situ gold nanoparticle-wool fibre composites.

Table 7.1 The advantages and disadvantages of the in-situ and ex-situ procedures.

\begin{tabular}{lccc}
\hline Approach & In-situ purple & TA-mauve & TSC-grey \\
\hline Au content of a purple comp & $1.6 \mathrm{wt} \%$ & $0.06 \mathrm{wt} \%$ & $0.24 \mathrm{wt} \%$ \\
$\mathrm{Au}$ cost per kg of comp* & $\$ 852$ & $\$ 32$ & $\$ 128$ \\
Washfastness & Excellent & Leaching & Leaching \\
\hline \hline
\end{tabular}

*Based upon the gold price as at June 2015

Chapter 5 detailed the scale-up production of ex-situ gold nanoparticle-wool fibre composites. The stability of $\mathrm{AuCl}_{4}^{-}$and gold nanoparticles in reactors that were composed of different materials was determined. A plastic barrel reactor was used to achieve uniform mixing of reactants. The barrel reactor was used to reproducibly synthesise volumes of up to $90 \mathrm{~L}$ of TA- and TSC-stabilised gold colloids. Difficulties in reproducibly synthesising the TSC-grey colloid were overcome by increasing the amount of TSC in the synthesis.

The gold nanoparticle colloids were used to colour wool in top form, fine merino yarns and felted merino yarns with the following dyeing methods:

- Static dyeing of wool in top form

- Hank dyeing of felted merino yarn

- Conventional package dyeing of fine merino yarn

- Pressurised flow reactors for dyeing of wool in top form and felted merino yarn

Static dyeing reactions provided an excellent starting point for the development of 
the nanotechnology and contributed to the knowledge base of scale-up procedures. Hank dyeing procedures were replicated in the laboratory, where dye circulation, wool dyeing auxiliaries and physical agitation was used to colour kilogram hanks of Ascend felted yarn with remarkable colour uniformity. Fine merino yarns were dyed in an industrial pilot-scale package dyeing reactor at Tararua Yarns Ltd, but the wool dyeing plant closed for business before the process could be optimised.

A suite of novel flow reactors that were based upon industrial dyeing reactors were developed for colouring a range of wool types in the laboratory. A Benchtop flow reactor was employed to study the uptake of gold nanoparticles to wool with dye circulation. Pressurised flow reactors were used to colour top form wool, felted yarn and fine merino yarn with remarkable colour uniformity.

Chapter 6 detailed experiments that were carried out to improve the washfastness properties of ex-situ composites. The washfastness properties of TA-mauve, TSCpink and TSC-grey composites were first characterised. Following this, a range of chemical treatments were investigated to provide additional reactive sites for the attachment of gold nanoparticles. Many of these treatments caused the leaching of gold nanoparticles from the composites, or agglomeration of gold nanoparticles on the surfaces. However, the use of polyethylenimine significantly improved the washfastness properties of ex-situ composites.

A range of alternative reducing and stabilising agents were used in gold nanoparticle synthesis. Reactive dyes were used to synthesise stable gold nanoparticle colloids, but the chromophore of the dye molecules was not sufficiently degraded during this process. Polyethylenimine-stabilised gold colloids were used to produce composites with significantly improved washfastness properties. This is a very positive result and warrants further investigation.

The work in this thesis has shown that the methodology is specific to wool type and the method of processing. Therefore, it is not possible to unequivocally state the best conditions for the dyeing of each wool type, and the process must be optimised for each different source of wool. In addition, in scale-up syntheses, the colour uniformity of composites is dependent on the homogeneity of the processing technology of the wool source. This represents an ongoing challenge, due to the variability of the natural fibres. In general, TA-stabilised gold colloids are used to produce pink and mauve colours, and TSC-stabilised colloids result in grey coloured composites. The pressurised flow reactors gave the most uniformly coloured composites to date.

Following on from this work, $500 \mathrm{~g}$ hanks of fine merino yarn have been coloured with 
the pressurised flow reactors. Uniform pink and mauve colours were achieved with different concentrations of the TA-mauve colloid, and different shades of grey were synthesised with improved colour uniformity, however, the process is not completely uniform. The sacrificial filter is no longer used, and as such, recovery of gold from the dyeing procedures is not required. The development of larger pressurised flow reactors that can be used to dye multiple kilograms of wool at a time is currently under way.

Further work will involve developing a reproducible method for the large-scale synthesis of grey coloured ex-situ composites. This represents a major challenge in the commercialisation of gold nanoparticle-wool fibre composites. Research into alternative grey coloured methods, such as scale-up syntheses of PEI-grey composites is recommended. This will require the development of dyeing reactors that may be heated during the synthesis. Further research into improving the washfastness properties of ex-situ composites is also currently being carried out. 


\section{References}

1. Off the sheep's back: a look at historical wool export prices and volumes. http://www.stats.govt.nz/browse-for-stats/economic-indicators/pricesindexes/historical-wool-export-prices-volumes-2011.aspx. 2011.

2. Peden, R. http://www.teara.govt.nz/en/farming-in-the-economy/page-8. 2012.

3. Statistics New Zealand, Wool and Sheepmeat Export Values. 2011.

4. Johnston, J. H.; Lucas, K. A. Gold Bull. 2011, 44, 85-89.

5. Burridge, K. A. Gold and silver nanoparticles as high-value colourants and multifunctional entities for natural fibres and minerals. Ph.D. thesis, Victoria University of Wellington, 2010.

6. Johnston, J. H.; Lucas, K. A. http://www.aulana.co.uk/. 2014.

7. Simpson, W. S., Crawshaw, G. H., Eds. Wool: Science and Technology; Woodhead Publishing Limited, 2002.

8. Lewis, D. M., Ed. Wool Dyeing; Society of Dyers and Colourists, 1992.

9. Popescu, C.; Hocker, H. Chem. Soc. Rev. 2007, 36, 1282-1291.

10. Leeder, J.; Bradbury, J. Text. Res. J. 1971, 41, 563-568.

11. Negri, A.; Cornell, H.; Rivett, D. Text. Res. J. 1993, 63, 109-115.

12. MacLaren, J. A., Milligan, B., Eds. Wool Science The Chemical Reactivity of the Wool Fibre; Science Press, 1981.

13. Davidson, R. J. Photochem. Photobiol., B 1996, 33, 3-25.

14. Smith, G. J. Photochem. Photobiol., B 1992, 12, 173-178.

15. Meade, S. J.; Dyer, J. M.; Caldwell, J. P.; Bryson, W. G. Text. Res. J. 2008, 78, 943-957.

16. Eustis, S.; El-Sayed, M. Chem. Soc. Rev. 2006, 35, 209-217.

17. Novo, C.; Funston, A. M.; Mulvaney, P. Nat. Nanotechnol. 2008, 3, 598-602.

18. Huang, X.; El-Sayed, I. H.; Qian, W.; El-Sayed, M. A. J. Am. Chem. Soc. 2006, 128, 2115-2120.

19. Kim, Y.; Johnson, R. C.; Hupp, J. T. Nano Lett. 2001, 1, 165-167. 
20. Kabashin, A.; Meunier, M.; Kingston, C.; Luong, J. J. Phys. Chem. B 2003 , 107, 4527-4531.

21. Navaladian, S.; Viswanathan, B.; Viswanath, R. P.; Varadarajan, T. K. Nanoscale Res. Lett. 2007, 2, 44-48.

22. Yin, Y.; Alivisatos, A. Nature 2005, 437, 664-670.

23. Philip, D. Spectrochim. Acta, Part A 2008, 71, 80-85.

24. Daniel, M.; Astruc, D. Chem. Rev. 2004, 104, 293-346.

25. Turkevich, J.; Stevenson, P. C.; Hillier, J. Discuss. Faraday Soc. 1951, 11, 5575.

26. Brust, M.; Walker, M.; Bethell, D.; Schiffrin, D. J.; Whyman, R. J. Chem. Soc., Chem. Commun. 1994, 7, 801-802.

27. Murphy, C.; San, T.; Gole, A.; Orendorff, C.; Gao, J. et al. J. Phys. Chem. B 2005, 109, 13857-13870.

28. Shankar, S.; Rai, A.; Ankamwar, B.; Singh, A.; Ahmad, A. et al. Nat. Mater. 2004, 3, 482-488.

29. Senthil Kumar, P.; Pastoriza-Santos, I.; Rodriguez-Gonzalez, B.; Garcia de Abajo, F. J.; Liz-Marzan, L. M. Nanotechnol. 2008, 19, 1-7.

30. Freestone, I.; Meeks, N.; Sax, M.; Higgitt, C. Gold Bull. 2007, 40, 270-277.

31. https://ancientglass.wordpress.com/technical-question-about-historicalglass/what-is-the-difference-between-opal-dichroic-and-opalescentglass/lycurgus-cup-roman-4th-c/. 2014.

32. Faraday, M. Philos. Trans. R. Soc. London, A 1857, 147, 145-181.

33. http://www.hps.cam.ac.uk/whipple/explore/microscopes/faradaysslide/. 2014.

34. Mie, G. Annalen der Physik 1908, 25, 377-445.

35. Link, S.; El-Sayed, M. J. Phys. Chem. B 1999, 103, 4212-4217.

36. Aherne, D.; Ledwith, D. M.; Gara, M.; Kelly, J. M. Adv. Funct. Mater. 2008, $18,2005-2016$.

37. Lu, X.; Rycenga, M.; Skrabalak, S. E.; Wiley, B.; Xia, Y. Annu. Rev. Phys. Chem. 2009, 60, 167-192.

38. Link, S.; Ei-Sayed, M. Annu. Rev. Phys. Chem. 2003, 54, 331-366.

39. Myroshnychenko, V.; Rodriguez-Fernandez, J.; Pastoriza-Santos, I.; Funston, A. M.; Novo, C. et al. Chem. Soc. Rev. 2008, 37, 1792-1805.

40. Burda, C.; Chen, X.; Narayanan, R.; El-Sayed, M. Chem. Rev. 2005, 105, 10251102.

41. Mulvaney, P. Langmuir 1996, 12, 788-800. 
42. Liz-Marzan, L. M. Langmuir 2006, 22, 32-41.

43. Lok, C. N.; Ho, C. M.; Chen, R.; He, Q. Y.; Yu, W. Y. et al. J. Biol. Inorg. Chem. 2007, 12, 527-534.

44. Kelly, F. M.; Johnston, J. H.; Borrmann, T.; Richardson, M. J. J. Nanosci. Nanotechnol. 2008, 8, 1965-1972.

45. Kelly, F. M.; Johnston, J. H. ACS Appl. Mater. Interfaces 2011, 3, 1083-1092.

46. Burridge, K. A.; Johnston, J. H.; Borrmann, T. J. Nanosci. Nanotechnol. 2009, 9, 6813-6822.

47. Burridge, K.; Johnston, J.; Borrmann, T. J. Mater. Chem. 2011, 21, 734-742.

48. Kelly, F. M.; Johnston, J. H.; Borrmann, T.; Richardson, M. J. Eur. J. Inorg. Chem. 2007, 4, 5571-5577.

49. Johnston, J. H.; Nilsson, T. J. Mater. Sci. 2012, 47, 1103-1112.

50. Richardson, M. J.; Johnston, J. H. J. Colloid Interface Sci. 2007, 310, 425-430.

51. Johnston, J. H.; Richardson, M. J.; Burridge, K. A. Nanotechnology Conference and Trade Show (Nanotech 2008), Boston, MA, JUN 01-05, 2008.

52. Johnston, J. H.; Burridge, K. A.; Kelly, F. M.; Small, A. C. Provisional Patent. 2009.

53. Johnston, J. H.; Lucas, K. A. Personal Communication.

54. McLaren, B. Personal Communication.

55. https://www.gold.org/investment/interactive-gold-price-chart. 2014.

56. Luty-Blocho, M.; Fitzner, K.; Hessel, V. Chem. Eng. J. 2011, 171, 279-290.

57. Parry, M. Nanogold and nanosilver hybrid polymer materials. Ph.D. thesis, Victoria University of Wellington, 2013.

58. http://goldprice.org/. 2014.

59. Niu, J.; Zhu, T.; Liu, Z. Nanotechnol. 2007, 18, 325607.

60. Richardson, M. J.; Johnston, J. H.; Borrmann, T. Eur. J. Inorg. Chem. 2006, 2618-2623.

61. Montalbetti, C.; Falque, V. Tetrahedron 2005, 61, 10827-10852. 